\title{
PARTITIONS AND ATOMS OF CLAUSE STRUCTURE
}

This collection brings together some of Dominique Sportiche's best work, including essays that are published here for the first time. The articles build on the theory of Principles and Parameters and its Economy/Minimalist descendants. They progressively develop a view of syntactic structures in which syntactic properties are increasingly analyzed as atomized in progressively smaller elementary components and partitioned in the way these elementary components are represented. As a consequence of this view, it is suggested that languages do not differ at all in their syntactic organization.

The author develops his argument through the analysis of a variety of syntactic configurations. Successive articles examine what it means to be a subject, how Case-marking functions, how it relates to agreement, and how pronominal clitic constructions should be analyzed. In each case, it is concluded that the notions involved should be broken down into smaller elements. In turn, these elements form subgroups of syntactic structures. The composition of these subgroups obeys a partitioning principle that groups together elements having similar interpretative functions.

After specializing in Mathematics and Physics at the University of Paris 7, Dominique Sportiche studied Linguistics at the Massachusetts Institute of Technology in Cambridge, where, in 1984, he received a Ph.D. supervised by Professor Noam Chomsky. After working for a few years in Montreal, Canada, mainly at the University of Quebec in Montreal, he is now Professor of Linguistics at UCLA. 


\title{
ROUTLEDGE LEADING LINGUISTS Series editor: Carlos Otero
}

\author{
1 On Syntax and Semantics \\ James Higginbotham
}

2 Partitions and Atoms of Clause Structure Subjects, agreement, case and clitics Dominique Sportiche 


\section{PARTITIONS AND ATOMS OF CLAUSE STRUCTURE}

Subjects, agreement, case and clitics

Dominique Sportiche

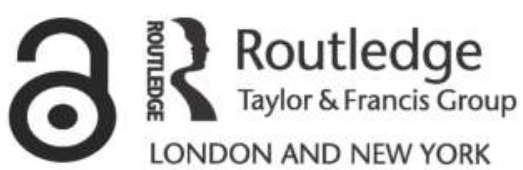




\section{First published 1998 \\ by Routledge \\ Published 2017 by Routledge \\ 2 Park Square, Milton Park, Abingdon, Oxon OX14 4RN \\ 711 Third Avenue, New York, NY 10017, USA \\ Routledge is an imprint of the Taylor \& Francis Group, an informa business}

Chapters 2 and $8 \odot 1998$ Dominique Sportiche and Hilda Koopman All other chapters (C) 1998 Dominique Sportiche

Typeset in Garamond by Keystroke, Jacaranda Lodge, Wolverhampton

The Open Access version of this book, available at www.tandfebooks.com, has been made available under a Creative Commons Attribution-Non Commercial-No Derivatives 4.0 license.

British Library Cataloguing in Publication Data A catalogue record for this book is available from the British Library

Library of Congress Cataloging in Publication Data Sportiche, Dominique.

Partitions and atoms of clause structure : subjects, agreement, case, and clitics / Dominique Sportiche.

A collection of the author's essays, most of which were previously published in various sources.

Includes bibliographical references and index.

1. Grammar, Comparative and general-Clauses. I. Title. P297.S68 1998

$415-\mathrm{dc} 21 \quad 97-30512$

ISBN 978-0-415-16926-4 (hbk)

Publisher's Note

The publisher has gone to great lengths to ensure the quality of this reprint but points out that some imperfections in the original may be apparent 


\section{CONTENTS}

Acknowledgements

vii

Introduction

1 A theory of floating quantifiers and its corollaries for constituent structure

2 The position of subjects

with Hilda Koopman

3 Movement, agreement and case

4 Clitic constructions

5 Subject clitics in French and Romance: Complex Inversion and clitic doubling

6 French predicate clitics and clause structure

7 Sketch of a reductionist approach to syntactic variation and dependencies

8 Appendix: Theta theory and extraction

with Hilda Koopman

Index 



\section{ACKNOWLEDGEMENTS}

Chapter 1 first appeared in the journal Linguistic Inquiry 1988, 19:3, 425-49 (C) 1988 the Massachusetts Institute of Technology and is reproduced here with the kind permission of MIT Press Journals, Massachusetts.

Chapter 2 first appeared in the journal Lingua 1991, 85: 1, 211-58 and is reproduced here with the kind permission of Elsevier Science, Amsterdam.

Chapter 4 first appeared in L. Zaring and J. Rooryck, Pbrase Structure and the Lexicon (1995), and is reproduced here with the kind permission of Kluwer Academic Publishers, Dordrecht.

Chapter 6 first appeared in A. Cardinaletti and M.T. Guasti (eds), Small Clauses (1995), and is reproduced here with the kind permission of Academic Press Inc., Florida.

Chapter 7 first appeared in H. Campos and P. Kempchinsky, Evolution and Revolution in Linguistic Theory: Essays in Honor of Carlos Otero (1995), and is reproduced here with the kind permission of Georgetown University Press, Washington.

Chapter 8 is taken from the GLOW abstract of a paper given at the 1985 GLOW colloquium in Brussels.

Chapters 3 and 5 are previously unpublished. 



\section{INTRODUCTION}

In this introduction, I will provide a survey of what I think the central ideas presented in each of these articles are and how they relate to each other, together with comments on how these ideas developed in my own research. I will also put some of these ideas in the perspective of more recent research.

These articles are written within a general framework largely inspired by the work of Noam Chomsky (cf. Chomsky 1995) that has been called successively the Extended Standard Theory (EST), the Government and Binding Theory (GB) and the Economy/Minimalist Program (E/M), reflecting various hypotheses that were at one time or other perceived as playing an important role in ongoing research. These theories make certain very general assumptions about the study of Universal Grammar. They assume, recognizing it as a substantial idealization, that it is fruitful and epistemologically sound to study linguistic structure in isolation from other manifestations of human cognitive capacities such as smell, vision, intentions, scientific reasoning, beliefs, feelings of pleasure or sadness, face recognition, or even mental language processing (on-line understanding), and so on. They take the main problem to be that of accounting for the existence of the rich mental system of linguistic structure manifested by adults, given the nature and structure of the (linguistic) environment humans grow up in.

In practice, grammarians adopting this perspective work on constructing models of these mental linguistic structures in children or adults, address the question of how these models are acquired, or used by (idealized) mental processing systems. My work belongs to the first category, i.e. the construction of explicit models of mental linguistic structures. This work takes its central problem to be that of explaining how physical expressions such as articulated sounds or signs relate systematically to the meanings which speakers attribute to them; this is the sound/meaning (or sign/meaning) problem. The way this extremely complex problem is addressed is by segmenting it into a series of more manageable subproblems in ways that seem warranted empirically and over which there seems to exist a very large consensus in our field. This is achieved by postulating that a syntactic 
component mediates relations between a mental dictionary of lexical units with their atomic properties and the interpretative properties (semantic or phonological) of strings of such units concatenated according to syntactic rules. Beyond these very general assumptions about linguistic theory, there is no constant body of assumptions which distinguishes theoretical approaches such as EST, GB or E/M from many other approaches that are entertained by others such as Head Driven Phrase Structure Grammar, Lexical Functional Grammar, etc. and it is very unclear to what extent all these theories really differ from each other, once terminological and notational differences are set aside.

The following chapters, written between 1985 and 1994, adopt as a point of departure the notations and explicit assumptions presented in Chomsky's Lectures on Government and Binding (1981) and subsequent work. I view this period as having been a theoretically extremely fertile period in syntactic theory during which many underlying assumptions about what drives syntactic representations have come under scrutiny. It is difficult to assess what has driven theoretical progress as opposed to what has made it possible. Work on head movement seems to me one of the central theoretical developments that has made many others possible. But what seems to me to have driven and to be driving innovations now is primarily semantic interpretative properties, both of lexical items and of constructions.

These chapters reflect their times. They address various questions dealing with phrase structure and its relation to lexico-semantic information, and the nature and properties of syntactic dependencies, i.e. of co-occurrence restrictions within a linguistic expression.

\section{About subjects and floating Qs}

The first two chapters ("The Position of Subjects" and "A Theory of Floating Quantifiers") are strongly related. The first one was circulated in different versions for several years, the latest version, Koopman and Sportiche (1988) entitled "Subject Positions," being the most complete. The second one was presented to the North Eastern Linguistic Society (NELS) in the Fall of 1986 and a short version was published as Sportiche (1987) in the NELS proceedings. The main idea explicitly discussed in Chapter 2 and in these articles is what has come to be known as the VP-Internal Subject Hypothesis or, more generally the Predicate Internal Subject Hypothesis. Put in its most general form, this hypothesis suggests that, contrary to what had been assumed up to then, the syntactic dependency between a passivized object and its predicate as in (1a) is of the same nature as the dependency between the subject of a clause and its predicate as in (1b):

(1)(a) This lion was tamed by a serious trainer.

(b) A serious trainer was taming this lion. 
Adopting a notation for this dependency in terms of traces, this analogy can be made explicit as below:

(2)(a) $\left[_{D P} \text { This lion }\right]_{i}$ was [ tamed $\left[_{D P} t_{i}\right.$ ] by a serious trainer].

(b) $\left[_{D P} A \text { serious trainer }\right]_{i}$ was $\left[\mathrm{L}_{\mathrm{DP}} \mathrm{t}_{\mathrm{i}}\right]$ taming this lion].

The position $t_{i}$ in both cases is now seen as the position in which these DPs are licensed as arguments of their respective predicates, and sometimes it seems that they do show up precisely in these positions as in impersonal passives for objects or small clause constructions for subjects:

(3)(a) Il a été dressé un lion.

It has been tamed a lion.

(b) I saw [a serious trainer tame a lion].

It was only a matter of time before a proposal such as the VP-Internal Subject Hypothesis came to be made as all the ingredients were already theoretically in place for its consequences to be drawn. Thus, passive and raising-to-subject constructions showed the necessity to distinguish the position in which an argument is licensed qua argument, that is, its thematic position, which is somewhere within the $\alpha$ constituent below, from the position in which it surfaces, which is described as being the position in which it receives Case:

(4)(a) $\left[_{\mathrm{DP}}\right.$ This lion] was $\mathrm{L}_{\alpha}$ tamed by a serious trainer].

(b) $\left[_{\mathrm{DP}}\right.$ This serious trainer] seems $\mathrm{L}_{\alpha}$ to have tamed this lion].

The very simple and straightforward logic of this state of affairs is that:

1 objects in general should also be associated with two positions: a thematic position (the VP internal object position) and a Case position outside VP. This second consequence is the Case-in-AGRobject proposal to which we return below.

2 subjects should be associated with two positions: a thematic position (the VP internal subject position) and a Case position. This is the VP-Internal Subject Hypothesis. It is worth noting in passing that this conclusion is independent from the precise location of this thematic position. Paradoxically, it is not essential to the VP-Internal Subject Hypothesis that this position should actually be in VP.

This is very much how Hilda Koopman and I have presented the VPInternal Subject Hypothesis in our paper Koopman and Sportiche (1991) included in this volume (Chapter 2). But these considerations are not those which led us to it initially (although once the idea was here, it was an easy jump). The talk we gave in the Spring of 1985 at the Generative Linguist of the Old World (GLOW) conference in Brussels, Belgium, some salient 
points of which the abstract (Koopman and Sportiche (1985)) reprinted in this volume (Chapter 8) is meant to summarize, was keeping closer to the origins of this idea. We were trying to account for the observed variation between English/French type languages, which show strong asymmetries between subjects and objects under $W h$-extraction and Chinese/Japanese type languages which apparently did not. We suggested that the difference had to do with whether or not $W h$-extraction was proceeding from a theta position. Given the VP-Internal Subject Hypothesis, in an English tensed clause for example, the subject is never in a theta position, we claimed, while the object is, hence the asymmetry. In Chinese, this subject raising from its thematic position to a VP external Case position would not take place hence the easiness to extract it. We further claimed that if a language had a similar routine raising of a ( $w h$-)object to a non-thematic position, extraction of objects should also be difficult: we argued this was the case in Dutch. The idea that (relevant) Dutch objects are found outside VP is now a widely accepted position, referred to as object scrambling. ${ }^{1}$

This same GLOW presentation contained as a supporting argument for this VP-Internal Subject Hypothesis an analysis of the distribution of floated quantifiers in French and English, which grew into the article Sportiche (1988) included in this volume (Chapter 1), and which took the distribution of these stranded quantifiers to reveal these VP internal positions as below:

(5)(a) [Serious trainers $]_{\mathrm{i}}$ have $\left[_{\beta}\right.$ all $\left.\mathrm{t}_{\mathrm{i}}\right]$ tamed lions.

(b) $[\text { Les dompteurs }]_{\mathrm{i}}$ sérieux ont $\left[{ }_{\beta}\right.$ tous $\left.t_{\mathrm{i}}\right]$ dompté des lions.

This analysis claimed that assigning the structures indicated, in which a stranded quantifier was adjacent to a trace of its restriction (then called an NP, now a DP) was advantageous in that the relative distribution of stranded quantifiers could be largely derived from movement theory. Again, it is worth noting that the specific structure assumed in the article (taking the DP subject and the stranded $Q$ to form an underlying constituent $\beta$ ) is not necessary for this analysis. In fact, I make some suggestions later (for example in Sportiche, 1993a) that the structure might alternatively be as in (6) without affecting the central conclusions:

$$
\ldots \text { have }\left[t_{i} \text { [all }\left[t_{i} \text { [tamed lions }\right]\right.
$$

As its title ("A Theory of Floating Quantifiers and its Corollaries for Constituent Structure") indicates, Chapter 1 also contains the logical suggestion that, given this analysis, the distribution of stranded quantifiers can function as a sharp probe for exploring constituent structures going well beyond the VP internal subject proposal. This is explored to a certain extent in the article but far from the full extent to which it could have been, which would lead to much more articulated syntactic structures than were 
postulated then and even, although to a lesser extent, than are postulated today (cf. Sportiche (1995b; 1995c) discussed below).

One reason for this is due to the fact that, although $I$ argued that the distribution of a stranded quantifier provides a necessary condition for the nearby presence of its restriction DP, it clearly does not provide a sufficient condition. Thus, if there is a floated Q, there should be an associated DP nearby. But the presence of a DP (of the right kind) does not necessarily allow a stranded $Q$ next to it. This remained unexplained in this paper, and suggests that some other condition enters into play as well to license a floated Q. This is where I think the alternative structure suggested in (6) might be crucially important since it supposes that stranded Qs are not generated as forming constituents with their restricting DP (crucially however, they do require an adjacent DP at some point of the derivation) and therefore points to the existence of a type of licensing condition $\mathrm{L}$ bearing on the underlying distribution of quantifiers in general. This suggestion is actually pursued in my ongoing work .

These ideas were explicitly discussed but they do not in my present view constitute the most general ideas contained in these papers. The most general ideas have to do with the very way in which the basic buildings blocks of constituent structures ought to be looked at.

One idea is developed in the course of arguing against the view that stranded Qs appear where they do (VP initially, for example) because they are adverbs and this is where adverbs occur. The argument I provide is that the notion of adverb (or more generally adjuncts) is an heterogeneous wastebasket and that these stranded Qs fit none of a variety of adverbial types that occur in roughly the same pre-VP position. In fact I show that no two categories of adverbs have exactly the same distribution and none has the distribution of stranded Qs. In trying to understand what governs this distribution, I postulated (adapting Zubizarreta (1982)) that:

(7) Adverbials and modifiers are generated to the periphery of the constituents over which they take scope, as determined by their meaning.

This suggests a strong correlation between "syntactic properties" and "semantic properties." In fact it suggests that, to a very large extent, there is no difference in kind between the two, but rather, just a difference in the nature of the evidence (semantic interpretative, phonological or formal - e.g. word order or Case) that is used to reach conclusions about the nature of the very same linguistic structures (it is likely that a lot of phonological evidence has the same status, e.g. prosodic domains and it should be taken as strong evidence about syntactic structure not about readjusted syntactic structures). It also strongly suggests that there is something fundamentally right in the standard model in which semantic interpretation is performed off deep structure: 
(8) Grammatical objects are generated exactly where they are interpreted.

The idea in (8) is conceptually related to the preceding idea. It is based on an answer to the question not whether the VP-Internal Subject Hypothesis is correct but rather if it is correct, why it is correct. What general principle of constituent structure construction would have the VP-Internal Subject Hypothesis as a consequence? What the VP-Internal Subject Hypothesis illustrates is that when a predicate is projected in syntax, its thematic properties are satisfied first, to the exclusion of the Case properties of its argument. This in turn suggests that the properties that must be satisfied when a syntactic structure is constructed are partitioned into subtypes (thematic, Case, etc.) and that all properties of certain types must be satisfied before properties of a second type can begin to be satisfied and so on and so forth.

(9) Partitioning of syntactic properties

A syntactic structure is a succession of layers of substructures, each one embedded in the next one and each projected to license one particular subtype of property to the exclusion of all others.

Such a proposal is made at the very end of Chapter 4, "Clitic Constructions" (Sportiche 1992). It is not carried out to its fullest consequences in any of the essays included here. I return to it below.

\section{About movement, agreement and case}

Chapter 3, "Movement, Agreement and Case" (Sportiche 1990) has remained unpublished although some subparts appear scattered among other published articles. It contains two distinct subparts.

The first part (Secrions 1,2 and 3) is the most theoretical part of the chapter dealing with general questions of syntactic architecture. It grew out of a certain dissatisfaction with the particular form that the Barrier Theory took in Chomsky (1986) which was itself an attempt to synthesize a variety of attempts to derive the constraints on movement and much else as is found for example in Stowell (1981) or Kayne (1984). I tried to implement the belief that a simpler and more elegant system could be constructed out of the same building blocks. This led to a series of progressively larger unpublished manuscripts, successively the "Unifying Movement Theory" (1987), the "Conditions on Silent Categories" (1988) (a version of which was published in French as Sportiche (1989)) and parts of the article under discussion.

The research agenda this first part explores takes as central the question of why these constraints exist on movement dependencies rather than others. It seeks to answer this question by constructing a theoretical edifice in which all 
these constraints reduce to one: each individual constraint can be thought of as a view of a same general prohibition from a different angle, metaphorically a projection of the same multidimensional geometrical object on a different plane.

In order to implement this program most elegantly, a number of simplifying assumptions were necessary many of which have since become widely accepted. For example, I accepted Koopman's 1986 idea (published as Koopman (1992)) that agreement is always a specifier/head relationship. I postulated total structural uniformity across categories. Naturally, the VPInternal Subject Hypothesis was adopted, and generalized to all categories, an updated echo of Stowell's (1983) "subject across categories" proposal. I also accepted Larson's (1988) VP-shell proposal, and extended it to NPs/DPs in Section $6 .^{2}$

From these assumptions, it becomes possible to restrict movement landing sites either to specifiers or to adjunct positions and to require movement always to be strictly local. This is the essence of the Condition on Chain Link (CCL) informally stated below:

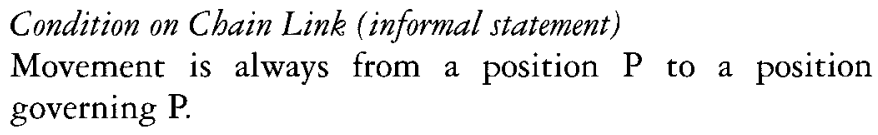

The discussion of participle agreement in Section 5 leads to a loosening of this requirement by allowing the local government domain of a head to be extended to that of the chain containing it. This means that if $\mathbf{P}$ is governed by a head $\mathrm{H}$ and $\mathrm{H}$ moves up the tree, direct movement from $\mathrm{P}$ can be to a higher position than if $\mathrm{H}$ had not moved. This idea is essentially identical to Baker's Government Transparency Corollary (1988) or to the notion of Dynamic Minimality explored in Deprez (1989).

The second part of this manuscript (Sections 4,5 and 6) was written in 1989 and 1990 and presented in seminars at UCLA. It comprises a detailed examination of a variety of constructions.

Sections 4 and 5 investigate clitic movement and participle agreement phenomena, primarily in French, within the rather constrained system of movement developed in the first part. Modulo basic assumptions originating to a large extent with the work on these topics of Richard Kayne (Kayne $(1972 ; 1975 ; 1989 b))$, such as the head nature of clitics and the existence of clitic movement, the idea of these sections is to let the theory of movement decide how clitic movement proceeds and, as a result, to predict the pattern of agreement found in participle constructions.

A number of conclusions regarding the specific syntax of clitics are reached in Section 4:

(a) Non-reflexive clitic movement is done in two steps: object XP movement 
possibly triggering agreement followed by head movement (incorporation of the clitic into its host) (thus arguing against a view of clitic movement as head movement throughout adopted by others).

(b) Participle agreement is obligatory when object movement must transit through [Spec, AGRo]. This happens in the case of A-movement of objects (passive) (due to the strict locality of movement) but not in the case of A-bar movement (object clitics, $W b$ Movement). This also happens in reflexive clitic constructions which are argued to involve object to subject movement (the reflexive clitic itself being base generated in the inflectional complex of its clause), an idea implicit in Marantz (1984) and explicit in a footnote of Bouchard (1984) and also pursued in Kayne (1988).

Section 5 examines the relationship between agreement and Case. There, the fundamental question asked is why participle agreement is restricted to accusative clitics (or otherwise accusative objects). The answer given and the central idea of this section is one which is by now familiar:

(11) Both accusative Case and participle agreement are a property of the same position, namely [Spec, AGRo].

In my own thinking, this idea finds its source in some data noted in Kayne (1989a). This article was devoted to participle agreement and introduces the existence of an intermediate AGR projection that has come to be known as AGRo. In this article, Kayne also discussed the fact that participle agreement with objects is impossible in constructions with expletive subjects:

(12)(a) Il a vu beaucoup de femmes/Combien de femmes a-t-il vuES. $\mathrm{He}$ has seen many women/How many women has he seen-FEM-PL.

(b) Il est arrivé beaucoup de femmes/Combien de femmes est-il arrivé(*ES).

It is arrived many women/How many women is it arrived (*FEM-PL).

Kayne accounted for this impossibility by the necessity of the object replacing the expletive. Upon reading his article, I thought that instead, this could be related to the assumption that the object in impersonal constructions does not take the accusative Case (a part of Burzio's Generalization, discussed in Belletti (1988)). This correlation could be instantiated by making accusative Case and participle agreement a property of the same position, namely [Spec, AGRo].

Given Koopman's proposal that agreement is always a specifier/head relation and the widespread assumption at the time that AGRs was respon- 
sible for nominative Case, it is not surprising that the same conclusion could be reached independently by regularizing the paradigm: if AGRs is responsible for the nominative, it is natural to take AGRo to be responsible for the accusative. This is the way in which the same conclusion is reached in Chomsky (1991), and in Mahajan (1990) (who, however, also provides extensive empirical grounding for this idea based on Hindi). ${ }^{3}$

There is an important difference between the view of AGRo presented here (and also in Koopman and Sportiche (1991)) and Chomsky's or Mahajan's work. They assume that AGRo is VP external, i.e. higher than the thematic positions of the subject and of the object. I propose (primarily on grounds of locality of movement) that it is VP internal, i.e. higher than the thematic position of the object but lower than the thematic position of the subject. The first approach would seem more consistent with the partitioning idea given in (9): first a thematic layer, then a Case/inflectional Layer. For this reason, I have changed my mind on these questions over the years and later articles such as Sportiche (1992) "Clitic Constructions" published in this volume as Chapter 4 assume this first approach. More recently however, I have come to realize that consistency with (9) could be achieved under the second approach if it were made more radical. The layering and partitioning of properties is interrupted by clause boundaries (and for sound partitioning reasons not discussed here). Thus in Sue thinks that Jobn saw Mary, the nominative position of Sue is separated from that of John by the thematic position of Jobn: an embedded clause starts a new cycle of layers. If the accusative and thematic positions of direct objects were part of a new clause, not containing the Case and thematic position of subjects, the second approach mentioned above would just be an instance of clausal embedding. The germ of this idea is already found in the lexical decomposition approach discussed in Sportiche (1993b) "Sketch of a Reductionist Approach to Syntactic Dependencies and Variations," and in my ongoing work on reconstruction.

The rest of Section 5 is devoted to exploring this idea about Case and agreement in conjunction with the Condition on Chain Link (CCL). This leads to discussions of:

(a) Agreement in what I call anti-movement constructions (which overlap with what are now called transitive expletive constructions).

(b) Burzio's Generalization (which is argued to be derivable).

(c) Movement and agreement in reflexive constructions, particularly the problem raised by indirect object reflexive constructions.

Finally Section 6 returns to the question of locality of movement, when it comes to extraction from NP. It articulates a view of the Abney (1987)/ Szabolcsi (1983) DP Hypothesis and integrates it with a Larsonian view of the internal structure of NPs to derive the properties of movement out of NPs/DPs. 


\section{About clitics}

The three chapters entitled "Clitic Constructions," "Subject Clitics in French and Romance: Complex Inversion and Clitic Doubling" and "French Predicate Clitics and Clause Structure" (Chapters 4, 5 and 6) deal with various aspects of the syntax of pronominal clitic constructions (primarily in French with obvious consequences elsewhere). The first one, Sportiche (1992), was written between late 1991 and early 1992 and circulated as a manuscript then. The last two assume the framework put in place in the first one which contains three central ideas.

\subsection{Clitic constructions}

The first idea arises essentially from taking $W b$ Movement as the paradigm case for movement: $W b$ Movement takes place to a designated projection, and in order to license a particular property of the moving item. This view of Wh Movement, which goes back to May (1985), is clearest in Rizzi's (1991) biconditional criterion formulation in terms of specifier/head relationship. Since Case/agreement is checked in a specifier/head relationship and my construal (in "Movement, Agreement and Case," published in Chapter 3 Section 5 of this volume) of Larson's VP shell idea also makes theta assignment a specifier/head relationship, it appeared natural to assume that:

(13) All licensing conditions hold in specifier/head relationships.

So that indeed, $W b$ Movement is a paradigm case. The idea expressed in (13) is, in my own work, explored furthest in Sportiche (1993b), "Sketch of a Reductionist Approach" (published in this volume as Chapter 7). "Clitic Constructions" (Chapter 4) explores this idea in the case of pronominal clitic constructions. It thus assumes the existence of a "clitic projection" involved in clitic movement constructions in the same way that a Q(uestion) projection is involved in $\mathrm{Wh}$ Movement constructions. It departs from the Wh Movement model in that it assumes there are as many clitic projections in a clause as there are pronominal clitics (one for nominative clitics, one for accusative clitics, one for dative clitics, and so on, hence the perhaps misleading names of NomP, AccP, etc. given to various clitic projections). Clitic movement can then be viewed as movement of an XP to a particular [Spec, CIP].

This view is independent of a subsidiary issue also dealt with in Chapter 4: is the clitic itself moving into CIP or not? In this chapter and in Sportiche (1993a) ("Subject Clitics in French and Romance: Complex Inversion and Clitic Doubling" published in this volume as Chapter 5), I suggest that it is not and instead is generated as the head of the CIP itself (whence it may incorporate to a higher head). This, I argue among other things would allow a simple treatment of clitic doubling constructions. Alternatively, it is 
possible that the clitic is the head of the XP moving into [Spec, CIP] (whence it would incorporate to a higher head).

The second idea is to relate the properties of (a subset of pronominal) clitic constructions to Scrambling in the Germanic languages (and in Hindi). The CIP approach allows the treatment of Scrambling as simply being movement of a full phrase into [Spec, CIP]. This reasoning assumes that Scrambling and clitic "placement" involve the satisfaction of the same property of the moving XP: in Chapter 4, I suggest with moderate confidence that it is specificity. ${ }^{4}$ What I considered important in this result is the reduction to the same underlying phenomenon of two classes of constructions, clitics and Scrambling, one in Romance, the other in Germanic, with no apparent counterpart in the other. Why such conclusions should be reached at all suggests the view, further articulated in Chapter 7 ("Sketch of a Reductionist Approach") that, despite appearances, syntax is strongly invariant, i.e. that reductions of this sort is an absolute norm.

The third idea, perhaps the most important one when it comes to syntactic analysis, and already alluded to above, is the idea of layering and partitioning given in (9). It is based on the specific conclusions reached on the syntax of pronominal clitics.

\subsection{Subject clitics and French predicate clitics}

The second and third papers exploit this framework of analysis. Sportiche (1993a) was written shortly after Sportiche (1992). It is an exploration of how the distinction between clitic projections and agreement projections, particularly NomP (for nominative clitics) and AGRsP can be profitably put to use to describe the complex array of data found in Subject Clitic Inversion and Complex Inversion in French.

The third paper, written in 1994 and published as Sportiche (1995b) attempts to extend this kind of approach to the predicate clitic le (Jean l'est, malade/"John is it, sick") to try to derive some of the rather surprising properties of this construction. It argues that a proper analysis of its distribution leads naturally to the proposal that small clauses in Stowell's terminology should be analyzed as full clauses. It also capitalizes on Kayne's 1993 analysis of the bavelbe alternation and on the analysis of reflexive clitics found in 'Movement, Agreement and Case' to explain intriguing distributional restrictions predicate le is subject to (it cannot occur without the verb be and is incompatible with reflexive clitics).

One idea this paper contains is merely outlined but ties in with the general point of view according to which there is a close parallelism between syntactic structures and semantic properties. The idea is put forward to explain why adjunct (secondary) predicates never cliticize as le. Thus the secondary predicate debout cannot cliticize as $l e$ in the following sentence: 
(14) Jean lit le journal debout $/ *$ Jean le lit le journal.

John reads the newspaper standing.

The idea is that the predicate of the main clause is the secondary predicate standing and not the verb read reflecting what this sentence means: John stands when he reads the newspaper. Cliticizing the secondary predicate onto the verb read in French would amount to lowering the main predicate into an adverbial clause, a process prohibited on general syntactic grounds. This kind of approach to adjuncts, viewing their syntax as strongly guided by the interpretive import of their presence, and doing away with the notion of adjunct altogether was developed in my 1994 "Adjuncts and Adjunction," not included in this volume. ${ }^{5}$

\section{Sketch of a reductionist approach to syntactic variation and dependencies}

The first part of this last paper, Sportiche (1993a) published as Sportiche $(1995 \mathrm{c})$ is a programmatic attempt to gather the general ideas implicit or explicit in all these previously discussed papers in a coherent theoretical perspective. It puts forth a variety of general ideas that I saw a posteriori as having guided my linguistic work, all roughly pushing for ever greater unification of properties and principles both in a given language and crosslinguistically and all assuming, when relevant, greater parallelism between syntax and interpretation. It is inspired by some of the Economy proposals of Chomsky (1991) and ends up sharing a number of conclusions with Chomsky's (1995) Minimalist Program. Thus it states that or argues for the proposals that:

(a) There is no syntactic variation: syntactic representations are built according to universal principles of phrase structure and to satisfy properties of lexical items, which I take to be crosslinguistically invariant.

(b) The parts of a lexical item relevant to syntax (i.e. influencing syntactic structures containing it) are compounds of elementary predicates or operators drawn out of a small (probably finite) universal pool of such elementary items individually visible to syntax. This leads to general lexical decomposition.

(c) All (binary) syntactic dependencies (e.g. government, selection, etc.) are movement dependencies.

(d) Movement dependencies are either XP movement to specifier or head movement.

(e) There are no adjuncts; there is no adjunction. ${ }^{6}$

The second part is a very preliminary case study of wh-questions in French and English from this perspective, trying in particular to deal with observed 


\section{INTRODUCTION}

variation. Of course this leads to some retreat from the maximalist position in (a) above. One variation that is potentially rich in consequences concerns the options for the size of moving constituents (pied-piping) exercised in French as opposed to English. I suggest that pied-piping can be rather more extensive than was previously assumed and is at work in French intonational yes/no questions of the sort:

(15) Jean est parti (with rising intonation)?

Has John left?

For which I argue that the entire clause (IP) has moved into [Spec, CP]. Once the existence of massive pied-piping is acknowledged, many familiar assumptions about even very basic syntactic structures have to be critically re-examined and, I think, substantially modified. ${ }^{7}$

\section{Notes}

1 These ideas were pursued elsewhere in Koopman and Sportiche (1986), and in the case of Dutch in the work of Koopman (1986). Sportiche (1992) "Clitic Constructions" also builds on them.

2 The original manuscript also contained some notes extending this approach to APs.

3 It is however unclear whether what is called participial agreement morphology here corresponds to what Mahajan calls agreement. Agreement in Hindi is triggered by Scrambling, which is itself limited to specific direct objects. This leads Mahajan to propose that the accusative Case is reserved for specific objects, a conclusion that does not appear tenable in a variety of languages marking the accusative Case overtly. This property of Hindi agreement morphology makes it closer to French (and Romance) clitic heads. This is what I argue extensively in Chapter 4 (which would mean that the proper parallelism would take Hindi agreement morphology to be incorporated clitics). If participial agreement morphology on accusative licensing verbs is not intimately linked to Case as I suggest, an alternative explanation will be needed as to why, with such verbs, agreement is limited to (preposed) accusative objects.

4 In a slightly different version of this paper, namely Sportiche (forthcoming), I suggest instead that the property in question is whatever the definite article encodes.

5 These ideas included doing away with adjunction under movement and base generated adjunction. The latter leads to some suggestions concerning the treatment of adverbs and adverbial expressions (PPs, etc.). See Barbiers (1995) or Cinque (1997) for further discussion along these or similar lines.

6 This idea, the basis of Sportiche (1994), is of course quite close to Kayne's (1995) proposal that there are no specifiers but instead that former specifiers are all left adjuncts. His prohibition against multiple adjunctions can be seen as equivalent to allowing no more than one specifier per category.

7 That much larger constituents can systematically move than previously thought is explored in Nkemji (1995), Kayne (1995), Koopman (1996). 


\section{PARTITIONS AND ATOMS}

\section{References}

Abney, S (1987) "The noun phrase in its sentential aspect," unpublished doctoral dissertation, MIT.

Baker, M. (1988) Incorporation, Chicago, Illinois: University of Chicago Press.

Barbiers, S. (1995) The Syntax of Interpretation, Holland Institute of General Linguistics.

Belletti, A. (1988) "The case of unaccusatives," Linguistic Inquiry 19: 1-34.

Bouchard, D. (1984) On the Content of Empty Categories, Dordrecht: Foris.

Chomsky, N. (1981) Lectures on Government and Binding, Dordrecht: Foris. (1986) Barriers, Cambridge, Mass.: MIT Press.

- (1991) "Some notes on economy of derivation and representation," in R. Freidin (ed.) Principles and Parameters in Comparative Grammar, Cambridge, Mass.: MIT Press.

(1995) The Minimalist Program, Cambridge, Mass.: MIT Press.

Cinque, G. (1997) "Adverbs and the Universal Hierarchy of Functional Projections," unpublished book manuscript, Universita di Venezia.

Deprez, V. (1989) "On the typology of syntactic positions and the nature of chains," doctoral dissertation, MIT.

Kayne, R. (1972) "Subject inversion in French interrogatives," in J. Casagrande and B. Saciuk (eds) Generative Studies in Romance Languages, Rowley, Mass.: Newbury House.

(1975) French Syntax: The Transformational Cycle, Cambridge, Mass.: MIT Press. - (1984) Connectedness and Binary Branching, Dordrecht: Foris Publications.

- (1988) "Romance se/si," paper given at the GLOW conference, Budapest, GLOW Newsletter 20.

(1989a) "Facets of romance past participle agreement," in Dialect Variation and the Theory of Grammar, (ed.) P. Beninca, Dordrecht: Foris Publications.

(1989b) "Null subjects and clitic climbing," in O. Jaeggli and K. Safir (eds) The Null Subject Parameter, Dordrecht: Kluwer.

- (1993) "Toward a modular theory of auxiliary selection," Studia Linguistica 47 (1): 3-31.

- (1995) The Antisymmetry of Syntax, Cambridge, Mass.: MIT Press.

Koopman, H. (1986) "On the distribution of Dutch objects," lectures given at the Universities of Ottawa, Washington at Seattle, MIT, Geneva, and the University of California, San Diego.

- (1992) "On the absence of case chains in Bambara," Natural Language and Linguistic Theory, Vol. 10.4: 555-95.

- (1996) "The spec/head configuration," in E. Garrett and F. Lee (eds) Syntax at Sunset, vol. 1, UCLA.

Koopman, H. and Sportiche D. (1985) "Theta theory and extractions," in GLOW Newsletter 14: 57-58, reprinted in this volume as Chapter 8.

- (1986) "A note on long extraction in vata and the ECP," Natural Language and Linguistic Theory 4.3: 357-74.

- (1988) "Subject positions," published in part as Koopman and Sportiche (1991).

(1991) "The position of subjects," Lingua 85 (2/3): 211-58, reprinted in this volume as Chapter 2. 


\section{N T R ODUCTION}

Larson, R. (1988) "On the double object construction," Linguistic Inquiry 19, 335-92. Mahajan, A. (1990) "The A/A-bar distinction and movement theory," unpublished doctoral dissertation, MIT.

Marantz, A. (1984) On the Nature of Grammatical Relations, Cambridge, Mass.: MIT Press.

May, R. (1985) Logical Form, Cambridge, Mass.: MIT Press.

Nkemji, M. (1995) "Heavy pied piping in nweh," unpublished doctoral dissertation, UCLA.

Rizzi, L. (1991) "Residual verb second and the wh-criterion," in Technical Report in Formal and Computational Linguistics 2, Faculty of Letters, University of Geneva.

Sportiche D. (1987) "A theory of floating quantifiers," in Proceedings of the 1986 NELS Conference, 581-94, reprinted as Chapter 1 in this volume.

(1988) "A theory of floating quantifiers and its corollaries for constituent structure," Linguistic Inquiry 19(3): 425-49, reprinted in this volume as Chapter 1. (1989) "Le mouvement syntaxique: contraintes et paramètres," Langages 95: $35-80$.

(1990) "Movement, agreement and case," unpublished manuscript reprinted as Chapter 3 in this volume.

- (1992) "Clitic constructions," ms., UCLA, reprinted as Sportiche (1995a) and as Chapter 4 in this volume.

(1993a) "Subject clitics in French and Romance, complex inversion and Clitic Doubling," ms., UCLA, reprinted as Chapter 5 in this volume.

- (1993b) "Sketch of a reductionist approach to syntactic variations and dependencies," published as Sportiche (1995b) and as Chapter 7 in this volume.

- (1994) "Adjuncts and adjunction," keynote address delivered at the 1994 Linguistic Symposium on Romance Languages, UCLA (and also at Cornell University).

— (1995a) "Clitic constructions," in L. Zaring and J. Rooryck (eds) Pbrase Structure and the Lexicon, Dordrecht: Kluwer, reprinted in this volume as Chapter 4.

- (1995b) "Sketch of a reductionist approach to syntactic variation," in $\mathrm{H}$. Campos and P. Kempchinsky (eds) Evolution and Revolution in Linguistic Theory: Essays in Honor of Carlos Otero, Washington, D.C.: Georgetown University Press, reprinted as Chapter 7 in this volume.

- (1995c) "French predicate clitics and clause structure," in A. Cardinaletti and M.T. Guasti (eds) Syntax and Semantics, vol. 28, New York: Academic Press, reprinted as Chapter 6 in this volume.

- (forthcoming) "Pronominal clitic dependencies," to appear in Henk van Riemsdijk (ed.) Language Typology: Clitics in the European Languages, Berlin: Mouton de Gruyter.

Stowell, T. (1981) "The Origins of Phrase Structure," doctoral dissertation, MIT. (1983) "Subjects across categories," The Linguistic Review 2: 285-312.

Szabolcsi, A. (1983) "The possessor that ran away from home," The Linguistic Review 3: 89-102.

Zubizarreta, M.-L. (1982) "On the relationship of the lexicon to syntax," doctoral dissertation, MIT. 


\section{A THEORY OF FLOATING QUANTIFIERS AND ITS COROLLARIES FOR CONSTITUENT STRUCTURE*}

Koopman and Sportiche $(1985 ; 1987)$ propose that the structure of $S=I P$ is always as in (1), linear order aside, ${ }^{1}$

(1)

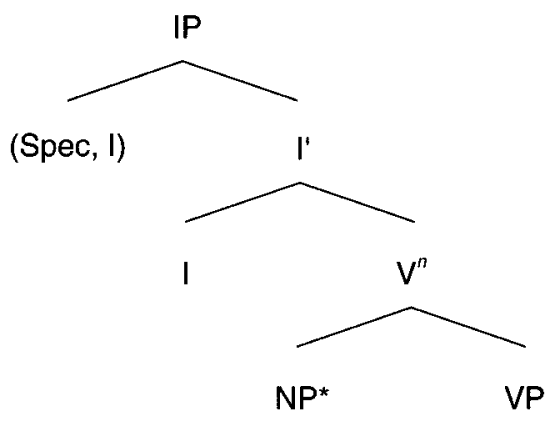

where $\mathrm{NP}^{*}$ is the canonical subject position (of VP), and $\mathrm{V}^{\mathrm{n}}$ some projection of $\mathrm{V}$ in the $\mathrm{X}$-bar system such that $\mathrm{V}^{\mathrm{n}}$ is a small clause with VP as predicate and $\mathrm{NP}^{*}$ as subject. ${ }^{2}$

Koopman and Sportiche argue further that in languages like French, English and Dutch (but not in languages like Japanese, Chinese, Irish and Italian) an overt NP subject, although base-generated in the position NP*, must appear in (Spec, I) position at S-Structure. In other words, it must be "moved" from NP* to (Spec, I). In effect, then, they propose treating Infl as a raising category in languages of the first type. The parametric variation between the two sets of languages is due to whether or not this movement is obligatory - that is, to whether or not Infl is obligatorily a raising category.

Here, I outline a treatment of the floating quantifiers phenomenon. I claim that certain of its properties receive an explanatory account if the structure in (1) is assumed. Though I discuss floating quantifiers mostly in French, I hope that this treatment will be found to have much wider relevance. In particular, I outline in Section 6 how it could be extended to English. 


\section{A THEORY OF FLOATING QUANTIFIERS}

\section{The problem}

Floating quantifiers in French are illustrated in (2):

(2)(a) Tous les enfants ont vu ce film.

All the children have seen this movie.

(b) Les enfants ont tous vu ce film.

The children have all seen this movie.

In (2b) a quantifier - $\mathrm{Q}$ or the floating $\mathrm{Q}$ - appears non-adjacent and to the right of some NP (which we will note $\mathrm{NP}^{\wedge}$ - here les enfants). Floating quantifiers are so called because they appear in structures like (2a) and (2b). This juxtaposition is not accidental: any syntactic analysis of this construction holds some implicit or explicit view about the semantic properties of tous in each sentence of (2). For some syntactic analyses, including those juxtaposing (2a) and (2b), the two sentences are closely related or identical at some level of syntactic representation precisely because $Q$ universally quantifies over the set denoted by $\mathrm{NP}^{\wedge}$ in both of these sentences; the $\mathrm{Q}$ is of the same logical type in both. This is the most common view held by generative grammarians, and it is in fact the view I adopt.

However, another course is conceivable. One could maintain that even though the quantificational properties of $(2 \mathrm{a})$ and $(2 \mathrm{~b})$ are identical, there is no syntactic correlate of this identity. The very same conclusion can be reached from a different point of view: if it is held that no semantic identity of the relevant sort relates (2a) and ( $2 b$ ), it would then seem natural to assume that no syntactic identity of the relevant sort holds either. The truth conditions of the members of pairs related as in (2) are in most instances identical (more on this in Section 5), but it could be claimed that their semantic representations are nevertheless different. This is the view put forth by Dowty and Brodie (1984), for example, who propose that determiner Qs are NP quantifiers, whereas floating Qs are VP quantifiers, a different logical type.

Summing up, then, I assume there is a syntactic dependency between $\mathrm{Q}$ and $\mathrm{NP}^{\wedge}$ and will address the problem of how best to characterize it. In Section 7 I briefly discuss the view according to which there is no syntactic relation between (2a) and (2b), that is, according to which there is no syntactic dependency between $\mathrm{Q}$ and $\mathrm{NP}^{\wedge}$ in sentences like (2b).

I have used the term determiner $Q s$. It ambiguously refers to Qs like each in both Each man left and Each of the men left. In the latter use we might call these partitive $Q s$. French suggests that floating structures correspond to partitive structures: the first each translates as chaque, which does not float, whereas the second corresponds to chacun, which does float. This correlates with the fact that the latter may appear as a partitive $Q$, whereas the former may not. I will therefore assume throughout that the related pairs are of the type Each of the 
men left/The men each left and not Each man left/The men each left. Consequently, I treat examples like (2a) as partitive structures, without de Insertion, an assumption supported by the fact that tous is followed by a full NP.

\section{The linear distribution of Qs}

Let us begin with the relevant facts about floating Qs. Typically, Qs appear (in French) in initial position (perhaps specifier position) of NPs presumably within NP. As noted above, floating Qs are followed by a full NP either with or without de Insertion, as in tous les enfants "all the children," chacun de mes amis "each of my friends." 3 As we will see, the exact structure of these phrases is not important for our discussion. For concreteness, we might assume that the $\mathrm{Q}$ in each case is adjoined to the left of the NP it modifies - in other words, that in each case the structure is $\left[_{\mathrm{NP}} \mathrm{Q} N \mathrm{NP}^{*}\right]$. Minimally, then, we need the statement in (3):

(3) (Floating) Qs may appear in NP-initial position.

This statement might itself be partly derivable from other considerations regarding the lexicosemantic properties of Qs. I put this question aside here, however. Regardless of what is thought of the semantic properties of (2a) and (2b), it is quite clear that an optimal analysis of floating $Q$ structures is one in which nothing essential needs to be said beyond (3). This is precisely what I will argue the adoption of (1) makes possible. In effect, then, I will argue that there is no process of Q-Float. Qs simply appear NP-initially.

In (2b) Q appears between Infl material and VP material. This is illustrated by the following forms:

(4)(a) Les enfants (*tous) ont (tous) vu (*tous) ce film (tous). The children all have seen this movie.

(b) Les enfants (*tous) verront (tous) ce film (tous). The children all see (future) this movie.

Emonds (1978) shows on independent grounds that French tensed verbs appear in Infl at S-Structure. In both examples of (4) the tensed verb is in Infl: $\mathrm{Q}$ may appear between Infl and VP. It may also appear sentence-finally, although this is less natural for a bare $Q$ than for a modified $Q$ (for example, presque tous "almost all"). The awkwardness of the postparticipial position in (4a) shows that the postverbal Q in (4b) is also between Infl and VP. ${ }^{4}$ (I will return to the sentence-final position in section 4.) 


\section{A THEORY OF FLOATING QUANTIFIERS}

\subsection{Floating $Q s$ and subjects in $V^{n}$}

If nothing else than (3) needs to be stipulated, we are led to postulate the presence of an empty NP to the right of Q in all of these cases. Therefore, we are led to postulate that $(2 \mathrm{~b})$ contains the following substructure:

(5)

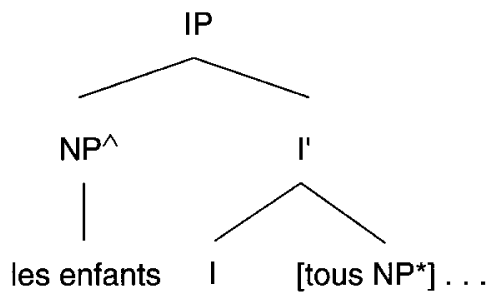

namely, a substructure in which an empty NP* appears between I and VP following the Q. It is clear that Koopman and Sportiche's hypothesis mentioned in (1) provides a rationale for such a structure, namely, (6):

(6)

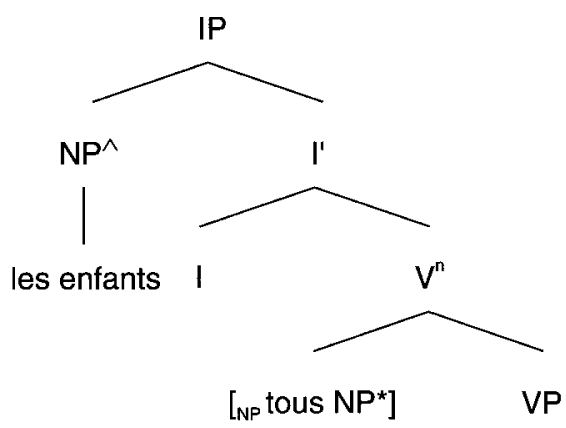

In other words, given (1), we expect, quite independently of the distribution of Qs, to find an NP immediately following Infl. Consequently, nothing needs to be added to (3) concerning the position of Q in (2b). Assuming that there is such an NP position, this specific conclusion rests on two premises. First, the assumption that NP* and VP are sisters as in (1) must be independently motivated. I believe that it is, and that the independent motivations are compelling (see Koopman and Sportiche (1985; 1987), Sportiche (1987)). Second, the assumption that in French NP* may be a left sister of VP must also be independently motivated. This is simply the question of what the relation is in (1) between $\mathrm{NP}^{*}$ and $\mathrm{V}^{\mathrm{n}}$ and between NP* and VP. Again, it is argued in the references cited that NP* is the subject of a small clause with VP head, and the subject of a small clause may precede its predicate. ${ }^{5}$ This approach is consistent with the remark that floating Qs correspond to partitive Qs, since only partitive Qs are followed by full NPs. In the case of determiner non-partitive $Q s$ the $Q$ is followed by some non-maximal projection of $\mathrm{N}$ that cannot be left empty, as witnessed by *Chaque est venu 
PARTITIONS AND ATOMS

"Each has come," *Il a vu chaque "He saw each." (Recall that cbaque cannot appear in partitive structures.)

\subsection{The adverbial analysis of floating $Q s$}

Let us now examine the alternatives. In order to explain the appearance of $\mathrm{Q}$ between I and VP in French, it is usually suggested that a $\mathrm{Q}$ has adverbial properties (see, for example, Kayne (1975), Belletti (1982), Jaeggli (1982), Klein (1976)) ${ }^{6}$ and that Q appears between I and VP because adverbs appear there. The merit of such a proposal cannot be evaluated before further questions are answered: (a) What kind of adverb is a $Q$ ? (b) What is the distribution of each kind of adverb? (c) What is the distribution of the class of adverbs that Qs belong to? and finally and most importantly, (d) What governs the distribution of each type of adverb (that is, why do adverbs appear where they do)?

There are several types of adverbs. Borrowing terminology and observations from Jackendoff (1972), let us restrict our attention to the following interpretive classes:

Sentential adverbs: probably, certainly, possibly

Manner adverbs: slowly, painstakingly

Subject-oriented adverbs: cleverly, intelligently

If we consider the distribution of these adverbs in French, we discover that they may all appear between I and VP. Consequently, assimilating Q to any such adverb does seem to provide at least a descriptively adequate account, no matter what kind of adverb Qs are assimilated to. However, if we ask why adverbs appear where they do, further elaboration is required, which casts doubt on the adverbial status of $Q$. Consider for example a sentential adverb like probably. From the equivalence of Jobn will probably leave and It is probable that John will leave, it appears that probably should be considered as modifying some constituent equal to or larger than IP, since the propositional content and the modals and Tense are in its scope. From this, we can predict its distribution by adopting the principle in (7), a principle implicitly or explicitly assumed by most syntacticians (see Travis (1984), Zubizarreta (1982) for explicit formulations). Using modify as a general term for relations such as qualification of and quantification over, we can state this principle as follows:

(7) Adjunct Projection Principle

If some semantic type $X$ "modifies" some semantic type $Y$, and $X$ and $Y$ are syntactically realized as $a$ and $b, a$ is projected as adjacent either to $b$ or to the head of $b$. 


\section{A THEORY OF FLOATING QUANTIFIERS}

This principle establishes a direct connection between the lexical meaning of modifiers and the syntactic configuration in which they appear. It is the analogue of the Projection Principle for predicate argument structure: an argument of some predicate is projected as a sister of this predicate (because theta-marking requires sisterhood). The Adjunct Projection Principle states a similar generalization for non-arguments. Some principle like (7) for nonarguments is necessary if, following Chomsky (1981) and Stowell (1981), we assume that there are no phrase structure rules, since there must be a systematic way to insert non-arguments into constituent structure. ${ }^{7}$ It can be seen as part of the language learner's apparatus for the projection of syntactic structures from word properties. (7) thus appears both natural on syntactic grounds and conceptually plausible.

Three points about (7) deserve mention. First, (7) states a necessary but not sufficient condition. It inventories possible positions for modifiers, but not actual positions. Certain positions could be predicted by principle (7) to be able to host a modifier but not allow it for other reasons (such as string adjacency for Case assignment, as discussed by Stowell (1981)). Second, (7) does not say anything about adverbs in general, but only about those whose lexical meaning makes it clear what they can modify (see Jackendoff's (1972) discussion of such adverbs as utterly). Third, (7) does not specify how adjacency is realized in terms of constituent structure. In general, it will be sisterhood: just as a direct object is sister to its verb, forming a $\mathrm{V}^{\prime}$, a nonargument will be sister to the constituent it modifies, forming an adjoined structure. For example, tous will be projected as sister to its NP (in fact adjoined, given the assumption of the text). ${ }^{8}$

Let us return to sentential adverbs. What do they modify? The fact that John will probably leave means "It is probable that John will leave" and not "John's leaving will be probable" suggests that they take scope over I. Consequently, they modify IP, and, by (7), they should appear as sister to I or IP. (8) illustrates the correctness of this prediction:?

(8)(a) Probably, John left.

(b) John probably will leave.

(c) John will probably leave.

(d)* John will buy probably shoes. (sister to IP)

(sister to I)

(sister to I)

(inside VP)

Next consider manner adverbials. Manner adverbials seem to be predicate modifiers: they can be subcategorized by verbs (such as to word), are often incorporated into verb meanings (for example, the meanings of electrocute, drown', and can also modify adjectives or nouns used predicatively. In the verbal projection system they will modify the predicate (VP). Consequently, when an adverb is interpreted as a manner adverb, it is predicted by (7) to be able in principle to appear adjoined to VP or V in a structure like (1), but not to $\mathrm{V}^{\mathrm{n}}$ (which is not predicative but propositional). The reader may 
check the validity of this prediction by consulting, for instance, the examples in Jackendoff (1972, Chapter 3) for English or those in Milner (1978) for French.

Returning now to our main concern, consider floating Qs. They have neither the distribution of sentential adverbs nor that of manner adverbs. In particular, they do not have the distribution of the subset of manner adverbs that impose restrictions on the nature of the subject, as do collectivement/"collectively," deux par deux/"two by two", which (like tous/"all") require plural subjects. Nor do they have the distribution of any other kind of adverb (temporal, speaker-oriented and so on). In terms of distribution, Qs in fact match more closely (but in fact not exactly) the class of subject-oriented adverbs. It would seem, then, that $\mathrm{Qs}$ could be assimilated to subject-oriented adverbs (although I think it is semantically implausible for the reasons mentioned below). However, it is immediately apparent that both subjectoriented adverbs and floating Qs constitute exceptions to (7), if the standard clausal structure is adopted, rather than (1). Qs modify the subject and, according to Jackendoff (1972), subject-oriented adverbs as well; yet they do not always appear adjacent to the subject. In other words, assimilating Qs to subject-oriented adverbs may perhaps yield a descriptively adequate account, but it fails to explain the nature of their distribution. ${ }^{10}$ If (1) is adopted, on the other hand, the distribution of Qs follows from (7). Qs appear adjacent to the NP they modify, namely, NP*. However, if (1) is adopted, there is no need to attribute any adverbial properties to Qs. (3) and (7) together suffice to predict their distribution. It also turns out that the a priori plausibility of considering floating Qs as adverbs in order to describe their distribution is an artifact of concentrating on French (or English). In other languages - for example, Moore, a Gur language from Burkina Fasso - a floating Q may appear between I and VP, but no adverb may, as Tellier (1986) discusses. Similarly, in Kilega, a Bantu language from Zaire, only subject modifiers (but no adverb) may appear between I and VP, as Kinyalolo (1986) observes. What about subject-oriented adverbs? If we follow Jackendoff (1972), subject-oriented adverbs "modify" both NP* and the propositional content of their clause; they should, according to (7), appear adjacent both to the subject (NP* or $\mathrm{NP}^{\wedge}$ in (1)) and to some syntactic constituent equal to or larger than $\mathrm{V}^{\mathrm{n}}$ (deciding exactly which depends on whether adverbs interpreted with subject orientation may take modals, Tense, and so on, in their scope). This is what they do. If the proposal of Jackendoff (1972) is correct, it makes the assimilation of Qs to subject-oriented adverbs quite suspicious, since subject-oriented adverbs and Qs would be of different semantic types. Note that if Jackendoff's description is correct, then adjacency of these adverbs to their modifiees cannot be straightforwardly realized as adjunction, since they would have to be adjoined both to the subject NP and to some propositional constituent $\left(\mathrm{V}^{\mathrm{n}}\right.$ or IP) at the same time. One possibility would be to assume parallel structures of the sort proposed by Williams 


\section{A THEORY OF FLOATING QUANTIFIERS}

(1980), Zubizarreta (1982) and Goodall (1984): the adverb would be adjoined to the subject in one structure and to some proposition in the other, provided that the two structures have identical strings, both in content and in order. For example, in a structure like (1) the adverb could be adjoined to NP* in one representation and to $\mathrm{V}^{\mathrm{n}}$ in the parallel representation. ${ }^{11}$

This account makes predictions about the relative ordering of sentential adverbs, subject-oriented adverbs, Qs and manner adverbs. Given the structure (1), repeated here in linear form,

$$
\left[\mathrm{I}^{\prime} \mathrm{I}\left[\mathrm{V}_{\mathrm{V}^{\mathrm{n}}} \mathrm{NP} * \mathrm{VP}\right]\right]
$$

if all the above-mentioned adverbs appear between I and VP, they must appear precisely in the order sentential adverbs/subject-oriented adverbs/ Qs/manner adverbs. Indeed, (7) requires that a sentential adverb appear adjacent (adjoined) to I and a floating Q adjacent (adjoined) to NP*, thus following the sentential adverb. A manner adverb will be adjoined to the predicate of the small clause; that is, it will be VP-initial and thus follow Q. As for subject-oriented adverbs, they must be adjacent (and perhaps adjoined) to both NP* and a propositional constituent. The only one possible is $\mathrm{V}^{\mathrm{n}}$. Therefore, they must precede $\mathrm{Q}$, since $\mathrm{Q}$ is adjacent to $\mathrm{NP}^{*}$ but inside $\mathrm{V}^{\mathrm{n}}$, and they must follow the sentential adverb, since the sentential adverb is inside $\mathrm{V}^{\mathrm{n}}$. Because manner adverbs and subject-oriented adverbs basically differ only in the position they occupy, some care is needed. Consider a case where the verb requires the presence of a manner adverb, as with formuler soigneusement "word carefully." Sentences like (9) result:

(9) Les enfants ont probablement intelligemment tous soigneusement The children have probably intelligently all carefully formulé leur demande. worded their request.

Of the possible $4 !=24$ orders of the italicized elements, (9) is the only possible one, if they all appear between I and VP and intelligemment has the subjectoriented reading.

\section{The nature of the relation $\mathrm{NP}^{\wedge} / \mathrm{Q}$}

The relation between $\mathrm{NP}^{\wedge}$ and $\mathrm{Q}$ seems to obey two conditions that antecedent-anaphor relations obey. First, $\mathrm{Q}$ must be c-commanded by $\mathrm{NP}^{\wedge}$. Corresponding to (10a), we do not find (10b):

(10)(a) L'auteur de tous ces livres a vu ce film.

The author of all these books has seen this movie.

(b)* Lauteur de ces livres a tous vu ce film. 
Second, the relation between $\mathrm{NP}^{\wedge}$ and $\mathrm{Q}$ must be local, as shown in (11), where $\mathrm{NP}^{\wedge}$ is not contained in the first clause containing $\mathrm{Q}$ :

(11)* Les enfants l'ont persuadé [de tous acheter ce livre]. The children him-have persuaded Comp all buy this book.

\subsection{Anapboric properties and subjects in $V^{n}$}

These two properties immediately follow from the analysis I am proposing. Adopting (1) in French means that there is an NP-Movement relation between $\mathrm{NP}^{\wedge}$ and NP*: NP* is the trace of $\mathrm{NP}^{\wedge}$ (naturally, if the Q-NP structure is $\left[\mathrm{NP}^{*} \mathrm{Q} \mathrm{NP}^{*}\right]$, I am referring to the internal $\mathrm{NP}^{*}$ ). This is where the antecedent-anaphor relation comes from. Because $\mathrm{Q}$ is adjacent to NP*, the illusion is created that antecedent-anaphor properties hold of the pair $\mathrm{NP}^{\wedge} / \mathrm{Q}$. Notice that this is independent from the specific structure of a phrase [Q NP]. What matters is that in a phrase like tous $\left[_{\mathrm{X}}\right.$ les enfants], when the constituent $\mathrm{X}$ is empty, it may count as an empty nominal category in the usual sense, here an "NP-trace."12

\subsection{Alternatives and Leftward Q-Movement in French}

Let us consider proposed alternatives.

Earlier analyses (such as that of Kayne (1975)) implicitly adopted principle (7). Q was assumed to be base-generated adjacent to its NP and moved rightward. Besides being incompatible with the current assumptions about possible movement rules, this approach has nothing to say about the anaphoric properties just mentioned.

More recent analyses usually consist in claiming in one form or another that $Q$ is an anaphor (see Jaeggli (1982), Kayne (1983, Chapter 4)). A priori, this is not desirable. Typically, anaphors are elements with a referential function that are referentially dependent upon another category (as, for example, $X$-self, each other). Q does not seem to fit this description. Belletti (1982) suggests that $\mathrm{Q}$ must move leftward and land next to $\mathrm{NP}^{\wedge}$, for independent reasons (to avoid vacuous quantification). She then suggests that the trace of $\mathrm{Q}$ is an anaphor.

The following argument suggests that holding that $\mathrm{Q}$ (or its trace) is an anaphor faces serious difficulties (as noted by Kayne (1983)). In French a Q like tous can not only "float" rightward but also appear to the left of the NP it quantifies over. This construction involving Leftward Q-Movement is discussed in Kayne (1975; 1983, Chapter 4), Quicoli (1976), and Pollock (1978). In the following example tous may appear in any position marked ${ }^{\wedge}$ :

12(a) Jean aurait aimé oser rencontrer tous les enfants. John would-have liked (to) dare meet all the children.

(b) Jean aurait ${ }^{\wedge}$ aimé $^{\wedge}$ oser $^{\wedge}$ les rencontrer ${ }^{\wedge}$. 


\section{A THEORY OF FLOATING QUANTIFIERS}

Several observations are relevant here. This process is not limited to tous. It may also affect NPs, provided that they exhaustively dominate a noun quantifier such as tout "everything" and rien "nothing," as in Elle a tout vul "She saw everything" or Il n'a rien compris "He did not understand anything," but not chacun/"every." 13 Tous (or tout or rien) can appear in its base position. If tous appears to the left of its "base" position, it appears at a left VPboundary. It seems rather clear that there is no way in which tous could be treated as an anaphor in these cases since it is not c-commanded by its antecedent. Note that no argument to the effect that the sequence of verbs in (12) has been reanalyzed as a single unit would be sufficient. First, the fact that the pronominal clitic les in (12b) cannot appear in the ${ }^{\wedge}$ positions argues against this kind of reanalysis. Second, other structures show that tous does not have to appear in the same clause as its antecedent. (13a-c) demonstrate this point:

13(a) Il faut tous $\left[_{\mathrm{VP}} \mathrm{e}_{\mathrm{i}}\left[_{\mathrm{CP}}\right.\right.$ qu'ils partent $\left.]\right\} .{ }^{14}$

It is-necessary all that-they leave.

(b) Il a tous $\left[_{\mathrm{VP}}\right.$ fallu $\left[_{\mathrm{CP}}\right.$ qu'ils partent $\left.]\right]$. It has all been-necessary that-they leave.

(c) Il a tous $\left[_{\mathrm{VP}}\right.$ fallu $\left[_{\mathrm{CP}}\right.$ qu'on les lise $]$. It has all been-necessary that-we them read.

(d)* Ils regrettent qu'il ait tous fallu que tu partes. They regret that-it was all necessary that you leave.

All the embedded clauses of (13a-d) are subjunctive. In (13a-c) $Q$ is outside the clause containing its antecedent. This suggests that it cannot be an anaphor. (13) also shows the difficulty of maintaining Belletti's proposal that the trace of $Q$ is an anaphor. In (13a-c) the $Q$ has been moved outside of the subjunctive clause. If the trace of $Q$ is an anaphor, the subjunctive clause must count as a transparent domain for anaphor binding. In this case (13d) is predicted to be grammatical: according to Belletti, $Q$ will move out of the embedded subjunctive clause and land next to the main subject. Because the subjunctive clause is transparent for anaphoric binding, (13d) should be good, contrary to fact. Note that our analysis provides no way to generate (13d).

Of course, two questions arise: How should we analyze this construction of Leftward Q-Movement? and Does it form a single phenomenon with Rightward Q-Float? Rightward Q-Float and Leftward Q-Movement clearly have different syntactic properties. For example, they affect different elements: chacun floats rightward but not leftward. Nouns such as tout "everything" float leftward but not rightward, as in $I l$ a tout mangéf" $\mathrm{He}$ ate everything." Another difference is that Leftward Q-Movement of tous can take place only if its NP is empty or a pronominal clitic. One suggestive observation is that although Rightward Q-Float is found in most Romance languages and many Indo-European languages, Leftward Q-Movement is 
more restricted. French seems to be the only Romance language that allows it (Norwegian, Swedish and Japanese might be cases from other language areas). A very natural analysis makes it a syntactic instance of May's (1977; 1985) rule of Quantifier Raising (QR). This would be an overt counterpart of a covert process, just like Wh Movement in English and Chinese (see Huang (1982)). It would also relate the fact that Leftward Q-Movement is "movement" to a left VP-boundary to the general theory of adjunction put forth in Chomsky (1986b) (see also Baltin's (1982) Like-Attracts-Like Constraint). This theory limits the adjunction sites of phrasal categories (QP, NP and so on) to phrasal categories of the same bar level that are not argument types (for example, VP but not $\mathrm{V}^{\mathrm{n}}$, which is an argument of I; see Sportiche (1987)). Since QR is an adjunction rule, it may adjoin the constituent containing the $\mathrm{Q}$ to VP, for example, but not $\mathrm{V}^{\mathrm{n}}$. Let me stress one important aspect of this analysis: there is no need to postulate different tous. Rightward Q-Float, I propose, is not a process affecting quantifiers. Leftward Q-Movement, on the other hand, is. Note, however, that we would want (3) to hold as well in Leftward Q-Movement cases: the null assumption is that a leftward-moved $Q$ is adjacent to its NP. The simplest instantiation is to claim that Leftward Q-Movement is movement of the NP containing the Q. This is consistent with the fact that leftward-moved Qs appear at VP-boundaries (they are phrasal categories and thus adjoined to VP) and the fact that the head noun of an object NP (tout) can also be subject to leftward movement, since it is in fact movement of an NP. ${ }^{15}$

This analysis makes a prediction the facts bear out: namely, that in a simple clause, if a rightward-floated $Q$ and a leftward-moved $Q$ occur between Infl and VP, the rightward-floated $Q$ will always precede the leftward-VPadjoined $\mathrm{Q}$ since a rightward-floated $\mathrm{Q}$ is adjacent to $\mathrm{V}^{\mathrm{n}}($ see $(1))$ :

(14)(a) Les enfants les ont tous tous lu. The children them have all all read.

(b) Ces livres, qu'ils ont tous tous lu... These books that-they have all all read ...

(c) Les enfants ont tous tout lu/*tout tous lu. The children have all everything read.

In these examples the first tous must be interpreted as being related to the subject, the second as being related to the (clitic or wh-) object. Note that this order is not due to some general crossing constraint. It is possible to find a $\mathrm{Q}$ associated with an object preceding a $\mathrm{Q}$ associated with an indirect object, as shown by this example due to R. Kayne: ?Je les leur $_{\mathrm{j}}$ ai tous $_{\mathrm{i}}$ toutes $_{\mathrm{j}}$ montrées $e_{i . j} /$.I showed all of them to all of them." A full account of Leftward QMovement as syntactic QR is beyond the scope of this chapter. I simply note here that the leftward movement of $Q$ is not free. The relation between a leftward-moved $\mathrm{Q}$ and its trace strongly resembles the relation between $n e$ 


\section{A THEORY OF FLOATING QUANTIFIERS}

and personne: subjunctive or infinitive clause boundaries become transparent in certain cases. This similarity strengthens the QR analysis of Leftward QMovement, given the properties of the ne-personne relation explored in Kayne (1983, Chapter 2).

\section{NPs Qs can float from}

What characterizes the set of NPs that Qs can float rightward from? The proposed analysis offers a straightforward answer: any time a $Q$ appears adjacent to an empty NP, the illusion of floating will be created. ${ }^{16}$ Indeed, this empty NP will usually require another $\mathrm{NP}^{\prime}$ as antecedent, and it will appear that floating has taken place from $\mathrm{NP}^{\prime}$. Reciprocally, if a $\mathrm{Q}$ cannot appear in a certain position, it suggests that no NP position is available there. (Recall that the latter implication is not necessary, since (7) is a necessary but not sufficient condition.)

In French a $Q$ a priori seems to be able to modify every available kind of empty category (except intermediate traces of $W b$ Movement or, more generally, empty categories in A-bar positions):

(15)(a) Les enfants ont tous mangé.

(NP-trace) The children have all eaten.

(b) Les enfants sont censés tous refuser. The children are supposed to all refuse.

(c) Les livres que j'ai lus?(presque) tous sont de ... (wh-trace) The books that I read almost all are by ...

(d) Il aurait fallu tous partir. (arbitrary PRO) It would have been necessary to all leave.

(e) Ils ont décidé de tous partir. (controlled PRO) They decided to all leave.

(f) Tous ont décidé de venir. All have decided to come.

(g) Je les ai vus presque tous. I them saw almost all. (see Sportiche (1983, Chapter 3))

Consider first (15f). Bare tous is not an NP, as the ungrammaticality of the following shows: *J'ai vu tous/"I saw all." I analyze the subject as being [tous pro]. Although a bare empty category is excluded in subject position in French (*e est parti "Has left"), it is possible if modified appropriately, as in (15f). What is the $e$ in expressions such as [tous e] or [trois $e$ ] that may occur in subject position? NP-trace is excluded (there is no A-binder), PRO would be governed and neither controlled nor arbitrary, and $w h$-trace is implausible since all diagnostics of $W h$ Movement fail (see also the judgments mentioned below concerning PRO and $w h$-traces in subject position). This leaves the 
possibility that $e$ is pro. This suggests that tous, trois play the necessary identificational role otherwise absent and thus permit pro to appear there. ${ }^{17}$

Consider next examples $(15 \mathrm{a}, \mathrm{b})$ (and $(15 \mathrm{~d}, \mathrm{e})$ ). The first two are raising cases, the last two control cases. They might raise the question, Why is a constituent of the form [tous e] permitted in a caseless position? The answer is straightforward: if $e$ is an NP-trace (or PRO), no Case is needed, since $e$ is the NP head of this constituent. Looking at other cases involving NP Movement, we predict (16a) to be grammatical, and (16c) not to be:

(16)(a) Les enfants ont été vus ?tous/presque tous. The children have been seen (almost) all.

(b) Les enfants sont venus ?tous/presque tous. The children came (almost) all.

(c) Les enfants ont dormi ?tous/presque tous. The children have slept (almost) all.

(16a) is a case of passive, and (16b) contains an unaccusative or ergative verb (Perlmutter (1978), Burzio (1985)); they both involve NP Movement. (16c) is an intransitive. Although I think that there is a subtle contrast in the indicated direction, all seem acceptable. ${ }^{18}$ In $(16 \mathrm{a}, \mathrm{b})$ it is plausible to posit a postverbal empty category in direct object position. The problem is (16c). The logic of our approach is to postulate an empty category post-verbally even in (16c). What could this empty category be?

Consider again the structure in (1). Why does NP* precede VP? If there are no phrase structure rules, the distribution of NPs follows from Theta-Theory and Case Theory (and, perhaps, X-bar theory). By Theta-Theory, NP* must be a sister of VP. But why must it precede the VP? We know that it may when NP* needs Case and NP* is the in-situ subject of a small clause with VP head, as Koopman and Sportiche $(1985 ; 1987)$ discuss. However, when NP* needs no Case, there is no reason to assume that it must either precede or follow VP. ${ }^{19}$ The null assumption seems to be that NP* is freely ordered with respect to VP. ${ }^{20}$ For the moment, let us assume just that. It follows that an example like (16c) may contain a postverbal trace (namely, NP*), to which presque tous is adjacent.

This now raises a new question concerning $(16 \mathrm{a}, \mathrm{b})$ : in each case the verb could be followed by two traces, namely, the object trace and the empty NP* (see Section 5 for more discussion of the NP Movement construction). I return to this question in Section 6.3.

Consider next the control examples (15d,e). Suppose first that French infinitival clauses behave like French finite clauses: they have the structure in (1) and movement from the NP* position is obligatory. Tous could be adjacent either to the PRO in (Spec, I) of the infinitival clause or to the NP* of this clause. If we could find material uncontroversially part of the tenseless Infl, we could determine which options are available. The discontinuous negative 


\section{A THEORY OF FLOATING QUANTIFIERS}

morphology ne-pas is one possibility. In tensed clauses the first morpheme immediately precedes Infl, and the second follows it. Adding negation in these two examples reveals the contrast: ${ }^{*}$ Ils ont décidé de tous ne pas partir à la même beure/Ils ont décidé de ne pas tous partir à la même beure/"They decided not to all leave at the same time/They decided to not all leave at the same time," ?*Il aurait fallu tous ne pas partir à la même beurelIl aurait fallu ne pas tous partir à la même beurel"It would have been necessary all not to leave at the same time/It would have been necessary not to all leave at the same time." Tous appears marginal or worse (but still much better than a Case Filter violation) in (Spec, I) position.

Again, the initial line we could follow is that there is no pre-Inf NP position in control structures or that movement of PRO to pre-Infl position is impossible or marginal. The course to follow depends on the status of the corresponding raising cases such as ??Les enfants ont semblé tous ne pas vouloir venir/Les enfants ont semblé ne pas tous vouloir venir/"The children seemed all not to want to come/The children seemed not to all want to come." The pre-Infl position does not yield perfect results, although these sentences seem comparatively better than the control cases. If the difference is not significant, we might want to argue that the pre-Infl position simply does not exist. If, on the other hand, the difference is significant, we can argue that the pre-Infl position is not available to PRO, although it is to NP-trace. This would be the case if, for example, the pre-Infl position is governed, thus excluding $\mathrm{PRO}$. In other words, movement from NP* to $\mathrm{NP}^{\wedge}$ in infinitivals is impossible with $\mathrm{NP}^{*}=\mathrm{PRO}$. That this movement is not necessary follows from the independently necessary assumption that a tenseless Infl is not a governor; hence, PRO does not have to move to avoid government. (See Koopman and Sportiche (1987) for further discussion.) If there is no PRO in the position $\mathrm{NP}^{\wedge}, \mathrm{Q}$ cannot appear there; that is, it cannot appear preceding Infl. However, Q can be attached to a post-Infl PRO.

Let us now turn to $w h$-traces. First, [Q wh-trace] seems easier with nonrestrictive relatives, but it is also possible with $w b$-questions or restrictive relatives. Consider the following situation in which children are divided in subgroups. There is only one subgroup such that all the children in it have gone to the movies. In order to identify this group, I can ask, "Which children have all gone to the movies?" The case of subject wh-trace as in *Quels enfants tous sont venus? /"Which children all have come?" is impossible. The object case (15d) or cases where the $Q$ does not immediately precede the subject trace (Quels enfants sont tous venus?!?Quels enfants sont venus tous?" Which children have all come?") are grammatical, as expected.

A suggestion I make and discuss in Sportiche (1987) would derive the fact that a bare $Q$ cannot be left behind in subject position. I propose that the that-t effect is due to the obligatory character of movement of Infl to Comp. Infl must move to Comp in order to head-govern (or, more precisely, to remove the barrier status of IP that prevents head government of) a subject 
trace, which would otherwise be illicit. If the complementizer already fills the $\mathrm{C}$ position, it prevents this movement of Infl to Comp. Otherwise, Infl is actually in Comp. ${ }^{21}$ The same is true for French. Infl must move to Comp position to permit head government of the subject trace. It follows that $\mathrm{Q}$ cannot immediately precede the tensed verb, since this verb is in Comp, not in $\operatorname{Infl}$.

Pursuing this matter and abstracting away from the effects of leftward QR, let us examine other cases where the presence of $Q$ reveals that of an empty category. If some independent support can be found for the existence of an empty category, it strengthens our contention that Qs only appear next to NPs.

Consider the following forms:

(17)(a) Jean a mis toutes les lettres dans la boîte. John put all the letters in the box.

(b) Jean a mis les lettres toutes dans la boîte.

This is a case of Rightward Q-Float from object position. Note first that the string toutes dans la bôtte forms a constituent (it can be clefted, coordinated and so on). We are led to postulate the following structure for (17b):

$$
\left.\left.\left.\left.\left[_{\mathrm{V}} \text { mis }\right] \mathrm{L}_{\mathrm{NP}} \text { les lettres }\right] \mathrm{L}_{\mathrm{X}} \text { toutes } \mathrm{L}_{\mathrm{NP} *} e\right] \mathrm{~L}_{\mathrm{Y}} \text { dans la boîte }\right]\right]
$$

What is this NP* position? Assume it is the analogue for PPs of what NP* is to VP in (1). Accordingly, $\mathrm{Y}=\mathrm{PP}$ and $\mathrm{X}=\mathrm{P}^{\mathrm{n}}$ (that is, some projection of $\mathrm{P}$ ); in other words, NP* is the subject of PP. ${ }^{22}$ Direct evidence for the existence of this NP position can be constructed on the basis of the following facts:

(19)(a) Les filles ont mis tous les ballons les uns près des The girls put all the balloons the ones next to the autres. others (next to each other).

(b) Les filles ont mis tous les ballons les unes près des autres.

(c) Les filles ont mis les ballons tous les uns près des autres.

(d)* Les filles ont mis les ballons tous les unes près des autres.

If the PP contains an anaphor (here les uns-les autres), it may be bound either by the masculine object - (19a) - or the feminine subject - (19b). However, in the presence of a $\mathrm{Q}$ like tous this anaphor must be bound by the NP that the $Q$ modifies (here the object). This is readily explained under our assumptions: the presence of tous forces the presence of NP* in the subject position of the PP. This NP* is in turn controlled by the direct object since tous is understood to modify this direct object in both (19c) and (19d). But then NP* acts as a subject for binding purposes; in other words, the anaphor les uns-les autres 


\section{A THEORY OF FLOATING QUANTIFIERS}

cannot be bound by anything but NP*. This predicts the ungrammaticality of (19d): the gender of the anaphor conflicts with its binding requirement. Recall that tous is not an NP and cannot be taken to be blocking the subject in (19d) from being the antecedent of the anaphor. Furthermore, the grammaticality of Les enfants $s_{\mathrm{i}}$ les. ont $\left[\right.$ tous ${ }_{\mathrm{i}}\left[\text { mis }[\text { les uns sur les autres }]_{\mathrm{i}}\right]_{\text { "The children }}$ have all put them on top of each other" also shows that tous by itself does not block anaphoric relations. ${ }^{23}$

In fact, a more general claim follows from this discussion: if a floating $Q$ forms a constituent with some XP, the XP must be understood as predicated of or bound by the NP over which the $\mathrm{Q}$ is understood to quantify. Indeed, the presence of the $\mathrm{Q}$, which quantifies over some $\mathrm{NP}^{\wedge}$, reveals the presence of an empty NP* to which $\mathrm{Q}$ is adjacent. In other words, the structure in question will have to be analyzed as in (20):

$$
\mathrm{NP}^{\wedge} \ldots\left[\left[_{\mathrm{X}^{\mathrm{n}}} \mathrm{Q}\left[_{\mathrm{NP}^{*}} e\right] \mathrm{XP}\right]\right.
$$

in which $\mathrm{NP}^{\wedge}$, the overt antecedent of $\mathrm{Q}$, is in fact the controller of NP*, itself the subject of XP. ${ }^{24}$ This explains a generalization made about English by Maling (1976: 716), who writes, "It appears that Q-Floating can apply only if the following phrase can reasonably be associated (semantically) with the NP that the quantifier binds." This generalization holds for French, too. Reanalyzing all these cases of floating as cases of "control," as we have done, predicts this generalization.

\section{Some problems and refinements}

In this section I discuss a few questions and problems that might arise in connection with this treatment of Q-Float. Once again consider (1). So far, I have used the terminology of movement to talk about the relation between $\mathrm{NP}^{\wedge}$ in (Spec, I) position and NP*. However, nothing in this treatment requires movement under its usual meaning that the moved phrase occupies different positions at different levels of representation. It could just as well be assumed (and this would be a position more consistent with Sportiche (1983)) that postmovement structures are "base-generated" and that NP-Movement relations are theta-role transmission relations. In other words, we will say that NP-Movement holds of two positions, $X$ and $Y$, if the theta-role assigned (or transmitted) to the position $Y$ is transmitted to $X$. We call $Y$ an $N P$-trace. It is a property of this kind of movement that NP-trace is an anaphor.

One objection often raised against a transformational analysis of Q-Float constructions concerns the fact that sentences containing floating Qs do not always correspond to sentences with partitive Qs. Taking examples from English, we find pairs such as Jobn, Bill and Mary all left versus *All of Jobn, Bill and Mary left. (Similar examples hold in French.) I see no real force to this argument. The alternative to a transformational analysis is to generate 
floating Qs and partitive Qs independently. Two observations must be explained: first, floating Qs can appear as partitive Qs; and second, floating Qs cannot always appear as partitive Qs. None of the alternative treatments of Q-Float accounts for both without additional machinery. In a transformational treatment (whether it involves physical movement or "base-generated" movement) some further condition is needed to exclude certain combinations of partitive $\mathrm{Q}+\mathrm{NP}$. Under a non-movement base-generated treatment, some reason must be provided to explain why partitive Qs and floating Qs partially overlap.

A second objection that might be raised against movement concerns differences in interpretation between floating Qs and determiner Qs. ${ }^{25}$ Consider for example the pairs in (21):

(21)(a) Tous les enfants ne sont pas partis.

All the children did not leave.

(b) Les enfants ne sont pas tous partis.

(c) All the children can do it.

(d) The children can all do it.

In $(21 \mathrm{a}, \mathrm{b})$ the universal quantifier can be in the scope of the negation: (not(all(...))). Only in (21a) can the negation be in the scope of the quantifier, although not very naturally so: ?(all(not (...))). The same observation holds of $(21 \mathrm{c}, \mathrm{d})$ with respect to the relative scope of the $\mathrm{Q}$ and the modal. It is clear that, a priori, no real argument can be constructed on the basis of this observation against the present proposal, or for that matter against any proposal embedded in a theory taking S-Structure representations as input for interpretive rules: a movement analysis yields richer structures than a base-generation analysis, because of the additional presence of traces. If the base analysis is sufficient to account for this kind of scope facts, it is always possible to claim that, under the movement analysis, these scope properties ignore NP-traces - that is, that they function as if the structures were basegenerated.

I have suggested that $\mathrm{Q}$ appears between $\mathrm{Infl}$ and a projection of $\mathrm{V}$. Further problems arise in more complex sentences. I will point out the general direction of my solution (regarding French and English, see Section 6); a full justification of my proposal would require a detailed discussion of the properties of the auxiliary system. Consider the following example:

(22) Les enfants ont ${ }^{\wedge}$ été ${ }^{\wedge}$ aperçus par les voisins. The children have been seen by the neighbors.

Q may appear in either ${ }^{\wedge}$ position. This arises when several auxiliary verbs are present, which only occurs in the passive in French. ${ }^{26}$ It is therefore difficult to decide whether we are dealing with a case of Leftward Q-Movement from 


\section{A THEORY OF FLOATING QUANTIFIERS}

the trace in object position or with a case of "Rightward" Q-Float. If in fact each ${ }^{\wedge}$ position precedes an empty NP position, it may be either. As mentioned earlier, I argue in Sportiche (1987) that every major $\mathrm{X}^{0}$ may contain a specifier position allowed to host NPs (although NPs with phonologically non-null heads usually cannot surface there because of lack of Case). Therefore, it is possible that both the verb apercevoir and the auxiliary verb être have a specifier position. In such a case the structure of (22) would be as follows, ${ }^{27}$

$$
\left[{ }_{\mathrm{I}} \text { ont }\right]\left[_ { \mathrm { XP } } \left[\left[_{\mathrm{NP} * *} e\right]\left[_{\mathrm{X}^{\prime}}\left[_{\mathrm{X}} \text { été }\right]\left[_{\mathrm{V}^{\mathrm{n}}} \mathrm{NP} * \mathrm{VP}\right] 1\right]\right.\right.
$$

and the "movement" of les enfants may proceed from object position to NP* to NP** to its surface position. ${ }^{28}$ As a matter of fact, Sportiche (1987) argues that movement must proceed through these intermediate positions for all types of NP Movement (passive, raising).

In conclusion, not only is Infl a raising category, but aspectual auxiliaries would be as well. Note that (23) with $\mathrm{X}=\mathrm{V}$ is basically the structure proposed by Emonds (1976), or by Chomsky (1986b) for English (augmented by specifier positions).

\section{Extension to English}

Does this analysis of Q-Float extend to English? ${ }^{29}$ Because a crosslinguistically uniform treatment is certainly preferable, the optimal assumption is that it does. In this section I briefly outline how this proposal might be extended to English.

\subsection{Q-float and auxiliaries}

One complication proceeds from the richer auxiliary system of English. Consider the English sentence (24):

(24) The carpets\#will ${ }^{\wedge}$ have ${ }^{\wedge}$ been $^{\wedge}$ being ${ }^{\wedge}$ dusted for two hours.

Transposing the proposal we just outlined for French to English would lead us to postulate the existence of (at least) one empty NP position in front of each verb (that is, in each ${ }^{\wedge}$-marked position). This would generalize the raising property of Infl to aspectual and passive auxiliaries. If this is the right approach, we expect floating Qs to be able to appear in any such ${ }^{\wedge}$-marked positions. ${ }^{30} \mathrm{By}$ and large, this seems to be true, although there is considerable variation among speakers concerning the degree of acceptability of a floating $\mathrm{Q}$ in each position. It is unclear what the facts are. The only position consistently and totally acceptable is the first ${ }^{\wedge}$ position. For the other positions, acceptability judgments depend on the individual speaker, on which auxiliary 
is present (progressive but not passive, perfective and passive but not progressive, etc.), and on the choice of the Q (all, each, both, etc.). However, it seems that no ${ }^{\wedge}$ position always prohibits $Q$. This suggests that we should accept the generalized raising hypothesis according to which every auxiliary verb is a raising verb.

In any case, whatever the facts, the options are clear: argue either that there is an NP position preceding each verb, or that there is none.

\subsection{The pre-Infl position}

The position marked \# raises another question. Such a position does not tolerate Q in French, but it does in English in tensed clauses. (In infinitives it seems marginal; see Section 6.3.) No obvious uniform treatment is available here. Let us speculate and examine where our assumptions would take us. In a sentence containing a floating $Q$ in this position,

$$
\left[\mathrm{NP}^{\wedge} \text { The children] all }\left[_{\mathrm{I}^{\prime}} \mathrm{L}_{\mathrm{I}} \text { will }\right] \text { leave }\right] \text {. }
$$

our proposal would minimally lead us to postulate that all immediately precedes an empty NP, call it NP*. What can NP* be? The simplest (but not the only possible) assumption is that NP* is (Spec, I). Since a bare Q can appear in subject position (as in All bave come, Both left), this is a priori plausible. This would also mean that $\mathrm{NP}^{\wedge}$ is not in the clause subject position. One possibility is that $\mathrm{NP}^{\wedge}$ is topicalized. ${ }^{31}$ This would have the advantage of reducing the difference between French and English concerning the availability of the position \# for $\mathrm{Q}$ to an independently existing difference, namely, the existence of topicalization in English, but its absence in French.

Alternatively, one could stipulate the difference, for example by postulating a rule (perhaps a limited version of Leftward Q-Movement, or a local rule in the sense of Emonds (1976)) adjoining Q to I. We want to treat the position \# differently from the other positions. The \# position seems to tolerate well only bare Qs (compare The men almost all will vote for him/The men will almost all vote for bim). This is consistent with an adjunction analysis (only an $\mathrm{X}^{0}$ can be adjoined to an $\mathrm{X}^{0}$ ) and gives credence to treating \# separately.

\subsection{Possible NP*s in English}

Is English like French in permitting $Q$ to appear freely next to an empty category?

First, a $\mathrm{Q}$ adjacent to a $w$-trace is in general not permitted at all in English, a fact for which I have no explanation.

Second, a $\mathrm{Q}$ immediately preceding a PRO in pre-Infl position seems marginal, thus mirroring the French situation. This is exemplified by pairs 


\section{A THEORY OF FLOATING QUANTIFIERS}

such as They promised all to leave/They promised to all leave, assuming to is in Inf. ${ }^{32}$ We can analyze this situation just like we did its French counterpart.

The most problematic case is the passive case: a bare $\mathrm{Q}$ in postverbal position in passive or ergative constructions is totally excluded (*The children were seen all, *The children bave arrived all). The logic of our approach leads us to deny the existence of a postverbal NP-trace. Williams (1982) and Sportiche (1983) argue that passive nominals like Rome's destruction do not involve NP Movement from object position of the head noun to its specifier position. Passive, they argue, consists in assigning the object theta-role directly to the specifier position of the NP. One problem with this approach is the loss of parallelism between NP and clauses: passive would still involve NP Movement from postverbal position in clauses but not in nominals.

Recall, however, the proposal in Sportiche (1987) that every XP has a specifier position allowed to contain an NP. In particular, VP has a VP-initial specifier position. ${ }^{33}$ We can therefore transpose this treatment of passive nominals to VPs and reestablish the lost parallelism: in passives, a theta-role otherwise assigned to an object is assigned to the (Spec, VP) position. Similarly, we can claim that the object theta-role of an ergative verb is assigned directly to the (Spec, VP) position: clause-internal NP Movement does not involve a postverbal trace. ${ }^{34}$

Since there are no postverbal traces in a regular passive, floating Qs cannot appear postverbally. Note that in Exceptional Case Marking passives a postverbal $\mathrm{Q}$ is possible: They were believed all to bave left at the same time. In this case the position of the postverbal NP is unaffected by the passive morphology since it is not theta-marked by the main verb.

We must now reconsider the French examples (16a) and (16b). We attributed the availability of a postverbal (modified) Q to the presence of NP-traces. There are several possibilities.

The preferred option assumes that French passives work like English passives. In this case the occurrence of postverbal Qs in passive structures cannot be due to post-verbal NP-traces. They must have the same source as in (16c), namely, the lack of fixed ordering between NP* and VP. ${ }^{35}$ But if NP* and VP are unordered, permitting postverbal Qs in French, why are they excluded in English? Let us assume that a covert NP* and VP are not always unordered. Rather, they are unordered only in languages that exhibit both orders overtly. In French, as mentioned, an overt NP* usually precedes VP.

An overt NP* can also follow VP: it has been argued that the rule of Inversion found in languages such as French and Italian is adjunction to VP (see Rizzi (1982: Chapter 4) and the references therein). There is a natural way to reinterpret this as simply being NP* following VP (see Koopman and Sportiche (1987) for discussion, especially of Italian). English does not have this sort of inversion.

In French a covert NP* and VP commute because of the existence of 
inversion; floating Qs can thus be postverbal. In English they do not; consequently, floating Qs are barred from postverbal position.

Some facts mentioned by Kayne (1975), Baltin (1980) and Jackendoff (1981) seem to support our basic thesis. They point out that units larger than a Q can float, as in (26a) and (26b), or even a full NP, as in (26c): ${ }^{36}$

(26)(a) Ces hommes avaient [tous les trois] connu Garbo.

(b) The men had [all three] known Garbo.

(c) The men had [all three of them] known Garbo.

Although (26b) has an "untransformed version" (All three of the men ...), this is not immediately clear for (26a) (*Tous les trois les bommes..., *Tous les trois des bommes...) or for (26c). Most important, it must be explained why the bracketed phrase - especially the bracketed NP in (26c) - may appear in these positions. Consider (26c). Why should an NP be allowed at all in this position? This follows directly from our proposal: the NP position in question exists independently of the facts of (26).

Consider more closely the bracketed NP [tous les trois]. Presumably, its structure is $\mathrm{L}_{\mathrm{NP}}$ tous les trois $\left.\mathrm{L}_{\mathrm{N}^{\mathrm{k}}} e\right]$. Under the current formalization of trace theory, this NP [tous les trois] cannot count as a trace of actual movement left behind by the movement of [ces hommes]. Under the base-generated movement account, it suffices to form a chain (ces bommes, [tous les trois e]), where a category can count as a trace if its head is of the appropriate kind (that is, empty (or spelled out)). A further question arises concerning this case: Why can this NP be lexical, when no lexical NP is usually allowed in this position? This is an issue for Case Theory. In (26a,b) the head of the NP is empty, so presumably it does not need Case. (26c) is different. The head of the NP in this example looks like a pronoun but is nevertheless necessarily bound by the subject in its governing category. Not only is it bound in its governing category, unlike a pronoun - it is in fact necessarily bound there, like an anaphor. This suggests that it might best be analyzed as an anaphor, that is, as a lexicalized (spelled-out) NP-trace. ${ }^{37}$ Furthermore, the ability of phrases like all three to trigger of Insertion might be the answer concerning Case: of is or assigns the Case this "pronoun" needs. The fact that neither tous nor tous les trois allows de Insertion then explains why there are no French sentences corresponding to (26c) with tous, though they do exist in some dialects with chacun, which does allow de Insertion:

(27) Les enfants ont chacun d'eux acheté une voiture. The children have each of them bought a car. 


\section{A THEORY OF FLOATING QUANTIFIERS}

\section{The semantic analysis}

Let us finally consider a possibility mentioned in Section 1, namely, that there is simply no syntactic dependency between $\mathrm{NP}^{\wedge}$ and $\mathrm{Q}$ in (2b). In the case of Rightward Q-Float the observations concerning the similarity between antecedent-anaphor relations and the $\mathrm{NP}^{\wedge}-\mathrm{Q}$ relation discussed in Section 3 remain. The alternative here is to build the locality and c-command properties into the semantic rule for floating $Q$ interpretation. Basically, a floating $Q$ will have to be treated as some kind of VP modifier. It is clear how the locality properties will arise. Roughly speaking, VP and Q combine in a larger VP predicated of the subject (see Dowty and Brodie (1984) for an example). Requiring predication under sisterhood (essentially) and requiring this VP modifier to be essentially sister or (immediate) daughter to VP will derive the desired locality effect. As Leftward Q-Movement shows, this approach will fail as a general treatment of stranded $Q$, since a $Q$ appears adjoined to a VP without "quantifying over" the subject of this VP. It is difficult to do justice to such alternatives when they do not try to address the questions under discussion. Specifically, I have tried all along not only to describe accurately the properties of floating Qs but also to provide reasons why they display their particular properties. These reasons derive partially from the simple fact that floating Qs are Qs and might therefore follow from the semantic rule needed for their interpretation. They also derive from the fact that Qs are of the same logical type in both floating and non-floating structures and consequently trigger the appearance of an adjacent NP. This explains why floating Qs display syntactic properties of determiner Qs. For example, it explains the fact that floating Qs, unlike any other VP modifiers, agree in number and gender with the subject NP, or the various distributional properties noted in Section 2. It also explains why the semantics of floating $Q$ constructions and partitive $Q$ constructions are so similar. Finally, it explains the binding effects found in cases where the floating $Q$ forms a constituent with PPs. These last cases would presumably require the assumption that floating Qs can also be of another type, namely, PP-modifiers. It seems a priori undesirable to assign the "same" $Q$ to two different logical types NP quantifiers and VP quantifiers - if it can be avoided, and even more so to assign it to yet other logical categories. These remarks suggest that the burden of proof rests on the proposals postulating no syntactic dependency between a floating $\mathrm{Q}$ and the NP it quantifies over.

\section{Conclusion}

I have examined constructions involving a quantifier $Q$ separated from and to the right of the NP $\left(\mathrm{NP}^{\wedge}\right)$ over which it seems to quantify. The literature contains three proposals, which differ with respect to the syntactic relation $\mathbf{R}$ that they posit between $\mathrm{NP}^{\wedge}$ and $\mathrm{Q}$ : 
(a) $\mathrm{R}$ does not exist.

(b) $\mathrm{R}$ is established by coindexing (construal).

(c) $\mathrm{R}$ is established by rightward movement of $\mathrm{Q}$.

I have argued in favor of a fourth alternative:

(d) $\mathrm{Q}$ is adjacent to $\mathrm{NP*}$ and $\mathrm{R}$ follows from the anaphoric relation between $\mathrm{NP}^{\wedge}$ and $\mathrm{NP}$.

I have shown that this proposal derives fundamental properties of the Q-Float construction: it reduces the properties of the distribution of floating Qs to the nature of constituent structure; it derives the anaphor-like behavior of the $\mathrm{NP}^{\wedge}-\mathrm{Q}$ relation; and it reduces the characterization of the set of NPs from which Qs may float to the theory of control and the theory of the movement of NPs.

\section{Notes}

* Comments and questions from H. Koopman, E. Keenan, R. Kayne and the audiences at the 1985 GLOW colloquium and the MIT and Amherst Linguistics Colloquium Series have helped improve and clarify many aspects of this article.

1 See Kuroda (1986) for an almost identical proposal based on many similar considerations, and Contreras (1987), Kitagawa (1986), Speas and Fukui (1986), Speas (1986), and Zagona (1982) for similar proposals.

2 This is actually a simplification that does not affect the argumentation of this article, in particular concerning the labeling of the $\mathrm{V}$ projections. For further details see Koopman and Sportiche (1985; 1987).

3 As in Vergnaud (1974) and Chomsky (1986a), I take de, of to be phonological spelling out of Case.

4 It has been reported to me that some speakers disagree with this judgment. The logic of my approach would suggest that these speakers can move the whole verbal complex to Infl. To my ear, this position can be forced. and only with an intonational break after the past participle.

5 See also the other references of Note 1 with respect to which these two points remain even though the node labeling of the equivalent of (1) and (6) is different.

6 Some equivalent is probably also true of the proposal of Dowty and Brodie (1984).

7 Notice incidentally that a principle like (7) basically requires the adoption of (1) independently of our present considerations since Infl material such as modals "modifies" (or, more precisely in this case, takes as complement) a proposition. This is realized as I taking $\mathrm{V}^{\mathrm{n}}$ as complement.

8 It is conceivable that certain non-arguments are not actually part of a tree structure but are instead only linearly related to its string. In such cases adjacency would be string adjacency. Such might be the case with parentheticals or perhaps subject-oriented adverbs (but see below in the text). This would explain why adverbs with the subject-oriented reading are not accessible to $W b$ Movement at all, as Chomsky (1986b) notes. 


\section{A THEORY OF FLOATING QUANTIFIERS}

9 The fact that, when they occur between I and VP, they must occur after the first tensed verb - both in French and in English - suggests that sisterhood to I and sisterhood to IP are the only two options. See Sections 5 and 6.1 for further remarks.

10 Strictly speaking, this shows only that these adverbs could not be generated where they appear. A movement analysis, however, is rather implausible.

11 Although I will continue to assume it, I am in fact not entirely convinced by Jackendoff's proposal. So as far as I can determine, with the exception of adverbs forming idiomatic units with a verb (such as surveiller étroitement "keep close tabs on") subject orientation and manner interpretations are available for exactly the same adverbs (even though it is not always easy to tell the two interpretations apart). It seems to me rather plausible to claim that subject orientation has nothing to do with subjects. It might be a case of an adverb modifying $\mathrm{V}^{\mathrm{n}}$ (or perhaps IP), where the appearance of subject modification is derivative: if John's answering the question was clever, the agent of the answering is clever. This would explain why (in French, at least) a manner adverbial in a subject-oriented adverb slot can qualify the actor or the agent and not the subject. A passive sentence like Jean a intelligemment été surveille can be paraphrased as "It was intelligent (of whoever) to keep close tabs on John" but not "It was intelligent of John to be kept close tabs on." The same observation holds of middle constructions.

12 In fact, French offers direct evidence that this is the case: for example, Je leur ai parlé $[$ à tous $e] /[$ à chacun $e] /$ "I them-spoke to all/to each," in which $e$ is the nominal category bound by the clitic pronoun.

13 Naturally, in these cases the structures corresponding to (12b) do not contain a pronoun.

14 Recall that tensed verbs appear in Infl at S-Structure in French.

15 If this is correct, it is necessary to explain why non-quantificational NPs are not subject to leftward movement of this sort.

16 Kuroda (1983) uses a similar idea to account for the asymmetric distribution of numeral quantifiers in Japanese.

17 Accepting the basic thesis of this article, we can now use the distribution of $Q$ to help determine what type of empty category appears in a particular construction.

18 In all these cases the preferred option is to adjoin a bare $\mathrm{Q}$ to $\mathrm{VP}$, as discussed above. As noted earlier, to my ear a bare $Q$ sentence-finally is slightly awkward. This does not bear on the conclusion based on examples such as *J'ai vu tous mentioned in connection with $(15 \mathrm{f})$, which are totally unacceptable.

19 Even theories such as Koopman's (1984), Travis's (1984) or Li's (1985) that invoke directionality of theta-role assignment do not extend it to subject or external theta-role assignment.

20 This idea is due to D. Pesetsky, who suggested it for the distribution of PRO. I apply it to NP-traces.

21 This explains why contraction of $Y$ on $X$, which requires government of $Y$ by $X$, is possible in Who do you think's leaving? with $X=$ think and $Y=i$. See Schachter (1984), Sportiche (1987).

22 Sportiche (1987) argues that every category (CP, IP, NP, VP, PP, AP) may contain a "specifier" position allowed to contain an NP (a version of Stowell's (1982) subject across categories proposal). Alternatively, NP* could be assumed 
to be in (Spec, $\mathrm{P}$ ) position, with $\mathrm{X}=\mathrm{PP}$ and $\mathrm{Y}=\mathrm{P}^{\prime}$. This difference does not affect the argument that follows.

23 I leave open here the position of les uns in (19), but see Belletti (1982). It would be tempting to say that it occupies $\mathrm{NP}^{*}$, whether NP* is under $\mathrm{Pn}^{n}$ or under PP, as in Note 22. Alternatively, given Note 22, it could be in (Spec, P) position, with $\mathrm{NP}^{*}$ under $\mathrm{P}^{\mathrm{n}}$. I also leave open what type of empty category $\mathrm{NP}^{*}$ is in (18). PRO and pro are the two plausible candidates.

24 In particular, this can be applied to the construction illustrated by Les professeurs

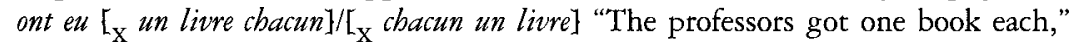
discussed in Burzio (1985, Chapter 3), explaining the same kind of binding effects.

25 See Williams (1982). Williams is concerned with the absence of Q-Float in NPs. Of course, since we assume raising of NP rather than Q-Float, this observation will follow if there is no raising in NPs. On this. see Section 6.3.

26 There is an implicit assumption here concerning the existence of "main verb" be, which I do not necessarily accept, but it will simplify the presentation.

27 It is perhaps even more complex: if the perfective auxiliary is generated outside Infl and has raised into Infl, as Emonds (1976) suggests, there is one more XP the head of which is the trace of the perfective auxiliary. Furthermore, it could be that each auxiliary verb is in fact head of a $\mathrm{V}^{\mathrm{n}}$ as in (1), and not just of a VP, adding one more possible empty NP position before an auxiliary-headed VP (see Koopman and Sportiche (1987) for further discussion).

28 Stowell (1978) and Burzio (1985) argue on independent grounds for the existence of the first step of this derivation - that is, that passivization of the object goes through an intermediate position after be.

29 See Maling (1976) for the distinction between Q-Float and Q-Postposing.

30 This would basically derive the facts handled by Baltin's (1982) landing site proposal in which floating Qs are stipulated to adjoin to the left of phrasal V projections. One exception is the \# position discussed in Section 6.3, which seems to be problematic for our proposal in English and for Baltin's proposal in French. Baltin also gives arguments supporting the assumption that $Q$ forms a constituent with the phrase following it, as must be the case under this account.

31 The intonation typical of topicalized structures is not observed in structures like (25). However it is not observed either in cases like (25) without a $Q$, which should exist, as a case of topicalized subject. This suggests that this intonation is not due to the topicalization alone.

32 English also seems to parallel French in raising structures. Thus, a pre-Infl Q seems better in raising structures than in comparable control structures: for example, consider They seemed all to have left at the same time/They seemed to all have left at the same time as compared with the control examples in the text.

33 So that the full structure of the $\mathrm{V}$ projection is $\left.\left[_{\mathrm{V}} \mathrm{n} N \mathrm{NP}_{\mathrm{VP}} \mathrm{NP} \ldots\left[_{\mathrm{V}}, \mathrm{V} \ldots\right]\right]\right]$ with two NP positions available. Sentences like I consider [John [Bill's friend]] actually show overtly the two (subject of a small clause and specifier) in the nominal system.

34 Something must be said about the fact that passive or ergative verbs do not assign Case. It is tempting to relate this phenomenon to the present proposal. If we assume that (Spec, VP) exists, it must be a Caseless position (which might follow from the directionality of Case assignment; see Koopman (1984), Travis 


\section{A THEORY OF FLOATING QUANTIFIERS}

(1984)). Indeed, in the absence of VP-external Case assigner, no "lexical" NP is permitted in (Spec, VP).

35 Note that, following Sportiche (1987), movement from (Spec, VP) out of VP must proceed through NP* for binding-theoretic reasons.

36 Note that they cannot float to the \# position discussed above, as we would expect under the analysis adjoining a $\mathrm{Q}$ to $\mathrm{I}$ in this position.

37 See Kayne (1983) for a similar proposal in French Complex Inversion constructions.

\section{References}

Baltin, M. (1980) "On the notion 'quantifier phrase'," Linguistic Inquiry 11: 247-49. (1982) "A landing site theory of movement rules," Linguistic Inquiry 13: 1-38.

Belletti, A. (1982) "On the anaphoric status of the reciprocal construction in Italian," The Linguistic Review 2: 101-38.

Burzio, L. (1985) Intransitive Verbs and Italian Auxiliaries, Dordrecht: Reidel.

Chomsky, N. (1981) Lectures on Government and Binding, Dordrecht: Foris. (1986a) Knowledge of Language: Its Nature, Origin, and Use, New York: Praeger. (1986b) Barriers, Cambridge, Mass.: MIT Press.

Contreras, H. (1987) "Small clauses in Spanish and English," Natural Language and Linguistic Theory 5: 225-44.

Dowty, D. and Brodie, B. (1984) "A semantic analysis of floated quantifiers in transformationless grammar," in Proceedings of the West Coast Conference on Formal Linguistics 3, Stanford, Calif.: Stanford Linguistics Association, Stanford University.

Emonds, J. (1976) A Transformational Approach to English Syntax, New York: Academic Press.

(1978) "The Verbal Complex V'-V in French," Linguistic Inquiry 9: 151-75.

Goodall, G. (1984) "Parallel structures in syntax," doctoral dissertation, UCSD, La Jolla, California.

Huang, C.-T. J. (1982) "Logical Relations in Chinese and the Theory of Grammar," doctoral dissertation, MIT, Cambridge, Mass.

Jackendoff, R. (1972) Semantic Interpretation in Generative Grammar, Cambridge, Mass.: MIT Press.

- (1981) "On the Constituent Structure of 'All Three of the Men'," Linguistic Inquiry 12: 150-51.

Jaeggli, O. (1982) Topics in Romance Syntax, Dordrecht: Foris.

Kayne, R. S. (1975) French Syntax, Cambridge, Mass.: MIT Press. (1983) Connectedness and Binary Branching, Dordrecht: Foris.

Kinyalolo, K. (1986) "Verb agreement in Kilega and its implication for sentence structure," unpublished manuscript UCLA, Los Angeles, California.

Kitagawa, Y. (1986) "Subject in Japanese and English," unpublished doctoral dissertation, University of Massachusetts, Amherst.

Klein, S. (1976) "A base analysis of the floating quantifier in French," in Proceedings of the Seventh Annual Meeting of NELS, Department of Linguistics and Philosophy, MIT.

Koopman, H. (1984) The Syntax of Verbs, Dordrecht: Foris. 


\section{PARTITIONS AND ATOMS}

Koopman, H. and Sportiche, D. (1985) "Theta theory and extraction," in GLOW Newsletter 14: 57-58, reprinted in this volume as Chapter 8. (1987) "Subjects," unpublished manuscript, UCLA, Los Angeles, California.

Kuroda, Y. (1983) "What can Japanese say about government and binding," in Proceedings of the West Coast Conference on Formal Linguistics 2, Stanford Linguistics Association, Stanford University, Stanford, Calif.

(1986) "Whether we agree or not," unpublished manuscript, UCSD, La Jolla, California.

Li, Y. A. (1985) "Abstract case in Chinese," unpublished doctoral dissertation, University of Southern California, Los Angeles.

Maling, J. (1976) "Notes on quantifier postposing," Linguistic Inquiry7: 708-18.

May, R. (1977) "The grammar of quantification," unpublished doctoral dissertation, MIT.

(1985) Logical Form, Cambridge, Mass.: MIT Press.

Milner, J. C. (1978) De la syntaxe à l'interprétation, Paris: Le Seuil.

Perlmutter, D. (1978) "Impersonal passives and the unaccusative hypothesis," in Proceedings of the Fourth Annual Meeting of the Berkeley Linguistic Society, UCLA, Los Angeles, California.

Pollock, J. Y. (1978) "Trace theory and French syntax," in S. J. Keyser, (ed.) Recent Transformational Studies in European Languages, Cambridge, Mass.: MIT Press.

Quicoli, A. C. (1976) "Conditions on quantifier movement in French," Linguistic Inquiry 10: 689-706.

Rizzi, L. (1982) Issues in Italian Syntax, Dordrecht: Foris.

Schachter, P. (1984) "Auxiliary reduction: an argument for GPSG," Linguistic Inquiry 15: 514-23.

Speas, M. (1986) "Adjunctions and projections in syntax," unpublished doctoral dissertation, MIT.

Speas, M. and Fukui, N. (1986) "Specifiers and projections," in MIT Working Papers 8, Department of Linguistics and Philosophy, Cambridge, Mass.: MIT.

Sportiche, D. (1983) "Structural invariance and symmetry in syntax," unpublished doctoral dissertation, MIT.

_ (1987) "Unifying movement theory," unpublished manuscript, University of Southern California, Los Angeles.

Stowell, T. (1978) "What was there before there was there?" in D. Farkas, W. M. Jacobsen, and K. W. Todrys, (eds) Papers from the 14th Regional Meeting of the Chicago Linguistic Society, Chicago, Ill: University of Chicago.

(1981) "Origins of phrase structure," unpublished doctoral dissertation, MIT. (1982) "Subjects across Categories," The Linguistic Review 2: 285-312.

Tellier, C. (1986) "Des quantificateurs flottants en français standard, en québécois et en moore," unpublished manuscript, McGill University and Université du Québec à Montréal.

Travis, L. (1984) "Parameters and effects of world order variations," doctoral dissertation, MIT.

Vergnaud, J.-R. (1974) "French relative clauses," unpublished doctoral dissertation, MIT.

Williams, E. (1980) "French causatives," unpublished manuscript, University of Massachusetts, Amherst.

(1982) "The NP cycle," Linguistic Inquiry 13: 277-95. 


\section{A THEORY OF FLOATING QUANTIFIERS}

Zagona, K. (1982) "Government and proper government of verbal projections," unpublished doctoral dissertation, University of Washington, Seattle.

Zubizarreta, M. L. (1982) "On the relationship of the lexicon to syntax," unpublished doctoral dissertation, MIT. 


\title{
THE POSITION OF SUBJECTS*
}

\author{
with Hilda Koopman
}

Grammatical theories all use in one form or another the concept of canonical position of a phrase. If this notion is used in the syntax, when comparing the two sentences:

(1)(a) John will see Bill.

(b) Bill John will see.

we say that Bill occupies its canonical position in (1a) but not in (1b). Adopting the terminology of the Extended Standard Theory, we can think of the canonical position of a phrase as its D-structure position.

Since the concept of canonical position is available, it becomes legitimate to ask of each syntactic unit in a given sentence what its canonical position is, relative to the other units of the sentence.

The central question we address in this article is: what is the canonical position of subjects. ${ }^{1}$ Starting with English, we propose that the structure of an English clause is as in (2):

(2)

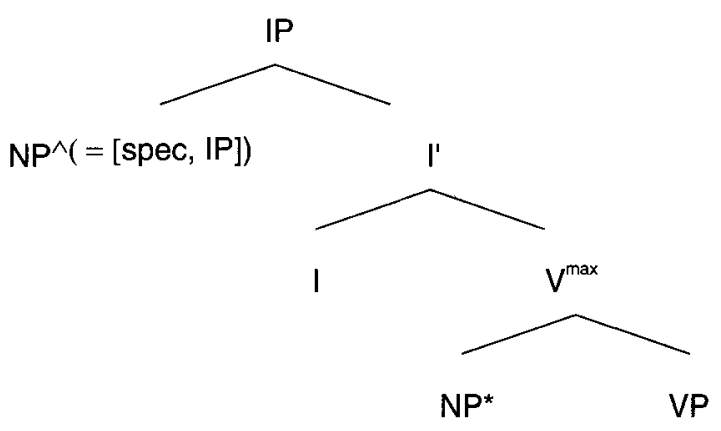

where NP* is the canonical or D-structure position of the subject, $\mathrm{NP}^{\wedge}=($ spec, $\mathrm{I})$ is its S-Structure position in simple declarative clauses, and $\mathrm{V}^{\max }$ is a small clause whose predicate is $\mathrm{VP}^{2}$

More generally, we propose that the constituent structure in (2) (linear 
order aside) can be generalized to hold for many (and perhaps all) languages, and that these languages fall in two classes. In Class 1 languages, such as English, French, Vata, a subject generated in position NP* must move to position $\mathrm{NP}^{\wedge}$. In a Class 2 language, such as possibly Italian, Welsh, Japanese, a subject generated in the NP* position does not have to raise to the position $\mathrm{NP}^{\wedge}$. We will suggest that raising may also be obligatory in such a language, although not necessarily to $\mathrm{NP}^{\wedge}$.

\section{Infl as a raising category}

Consider our proposal for English. It is equivalent to saying that Infl is a raising category. In this section, we argue that departure from this analysis of Infl needs arguments, arguments that are presently lacking.

\subsection{Raising verbs}

Start with a prototypical raising verb like seem. Why is it taken to be a raising verb?

\subsubsection{Syntactic arguments}

Here is a list of the classical arguments for analyzing the verb seem as a raising verb:

1 seem imposes no selectional restrictions on its subject;

2 seem can take expletive it as subject (it seems that Jobn sleeps all the time) or non-expletive subjects (Jobn seems to sleep all the time);

3 seem allows as subject an NP licensed by the predicate of the clause embedded under it:

weather it (it seems to rain)

idiom chunks (the cat seems to be out of the bag)

existential there (there seems to be a griffin on the 22nd level).

In contemporary terms, we see that all these properties have as necessary (but not sufficient) precondition the fact that seem does not assign an external theta role.

Why are all these observations considered arguments for treating seem as a raising verb? The answer is that the expression of certain lexical or grammatical relations requires a configurational or syntactic closeness: lexical relations such as selection, subcategorization or theta assignment can hold only of items that are structurally close. To put it differently, lexical relations are projected from the lexicon into the syntax subject to strict locality requirements.

Let us look at each case in turn: 
In John seems to sleep all the time, John is licensed by sleep, by virtue of the theta relation between the two. Theta relations are considered to be local in such a way as to exclude skipping over the verb seem.

In the weather verb case, the idiom chunk case or the existential sentence case the reasoning is the same, even though it does not have to do with theta relations. In each case, the subject of the main clause is licensed by the bottom predicate by syntactically projecting a property of the bottom verb according to rules that disallow skipping over the main verb.

\subsubsection{Semantic representation of raising verbs}

There is another, less straightforward reason why seem is treated like a raising verb. Consider a pair of sentences such as:

(3)(a) It seems that John sleeps all day.

(b) John seems to sleep all day.

Informally speaking, we want to say that their common semantic representation is SEEM(SLEEP(John)). It is quite straightforward to get this representation from the first sentence: Jobn appears as a syntactic argument of the verb sleep as well as an argument of the corresponding semantic predicate. This parallelism breaks down in the second case. How then is the structure of its semantic representation computed?

If seem is a raising verb, the relevant syntactic representation of (3), when looked at appropriately, is in fact seems Jobn to sleep all day (due to the presence of the trace of $J o b n$ ), i.e. essentially identical to the first sentence. The identity of semantic representations follows.

If seem is not a raising verb, two options arise for treating the second sentence. The first option consists in lowering the subject when computing the semantic representation of the sentence. This option is not really an alternative to the raising analysis. It is its mirror image. It is a notational variant that can be implemented consistently (e.g. locality conditions on projection of lexical properties will apply after lowering rather than before raising, etc.). The second option consists in denying identity of semantic representation for the two sentences and in arguing that the representation for the second sentence is (SEEM(SLEEP))(John). This comes down to treating seem as a function mapping a predicate $\mathrm{P}$ into a predicate $\mathrm{P}^{\prime}$ such that $\mathrm{P}^{\prime}$ inherits all the properties of $\mathrm{P}$ relating to its external argument if any. This option, although not impossible, requires strong empirical motivation, for it claims that there are two verbs seem. Ignoring the experiencer, the verb seem appearing in the second sentence would be a function mapping a predicate onto a predicate and taking one or no individual as argument, depending on whether or not the embedded verb has an external argument. The verb seem that appears in the first sentence would be a one-place predicate taking a 
proposition as argument. This empirical motivation is, to our knowledge, lacking.

\subsubsection{Further remarks on semantic properties of raising verbs}

Not all the predicates usually analyzed as raising allow an expletive subject and a tensed clause complement (e.g. tend, be liable). Nevertheless, they display all the other relevant properties of the verb seem, and we therefore want to treat them as raising predicates. Of course, appeal to locality of the syntactic expression of theta and other relevant relations will have the desired effect. Does the "semantic" argument apply here too?

A very strong claim which we could base on the discussion above is the following: there is no category $\mathrm{C}$ taking predicates as complements that is analyzed as a function mapping predicates onto predicates (except for the trivial case where the predicate in question has no external argument). This property would follow from the more general:

(4) No category takes as complement a syntactic category corresponding to a non-saturated predicate.

If this is correct, the argument above will extend to the raising predicates not taking the expletive subject/tensed clause complement combination.

In Case for Case, Fillmore (1968) proposed informally that clauses are made up of two constituents corresponding to a modality and a proposition. The intuition (also found in much generative semantics work) behind this proposal is rather clear. Consider modals, which, by assumption, appear in Infl. Semantically, it appears natural to suppose that they take saturated predicates or propositions as arguments. So a sentence like Mary might sleep all day can be paraphrased by it is possible that Mary sleeps all day, in which the semantic import of the modal in the first sentence is carried by the main clause in the second one. More specifically, it is carried by the adjective possible, which takes as argument a clause corresponding to the non-Infl material of the first sentence and in particular, containing the saturated predicate of the first sentence. To an extent that is theory particular but always substantial, syntactic theories all assume that there is a correspondence between semantics and syntax. The core of the intuition behind proposals such as Fillmore's seems to be that the null assumption is that there is as transparent a correspondence as possible between "semantic constituent structure" and syntactic structure: the strongest (and very possibly too strong) position would postulate the existence of a well-defined function from (the right class of) semantic types onto syntactic constituents and reciprocally. The principle in (4) is a particular case of this general transparency requirement. Applied to this case, if Infl takes a saturated predicate as argument, it is a priori reasonable to postulate that this semantic fact is reflected syntactically. 
Hence the naturalness of Fillmore's proposal. Proposing that the structure in (2) underlies clausal structure can now be seen as a contemporary way of embodying this intuition in syntactic theory: to Fillmore's modality constituent corresponds Infl. To Fillmore's proposition corresponds the constituent [NP VP].

Finally, if the semantic property of auxiliaries as taking a propositional complement is mirrored syntactically, it provides reasons for assuming that in (2) above NP and VP do form a constituent.

\subsection{Raising $\ln f$}

\subsubsection{The raising properties of Infl}

We now turn to the case of Infl. We want to show that the minimal analysis of tensed Infl is that it is a raising category. ${ }^{3}$ In order to make the point, let us consider some prototypical material in Infl, the modal will. Everything that follows applies equally well to anything else that might appear in tensed Infl. We have the following observations:

1 will does not assign an external theta role;

2 will allows as subject an NP licensed by the predicate embedded under it: external argument of a predicate (Jobn will sleep)

weather it (it will rain)

idiom chunks (the cat will be out of the bag)

existential there (there will be a griffin on the 22nd level).

These are diagnostic properties of raising items. We therefore conclude that will is a raising verb. By the same arguments all the modals are raising categories, do is a raising verb, and more generally tensed Infl is a raising category. By the same argument, if negation heads a NegP complement of Infl as recently suggested, negation is a raising category. By the same argument, if Infl is split between Tense heading a TP and AGR heading an AGRP (Pollock 1989), both $T$ and AGR are raising categories. By the same argument, aspectual verbs (perfective have and avoir, passive be and être, progressive be), which are analyzed as heading their own VP and taking VP complements are raising verbs.

This is a strong consequence. Suppose, as we claim, that aspect is determined outside the maximal projection of the verb, and that the maximal projection of the verb contains all its arguments. This would suggest that the thematic properties of a predicate are independent of the aspectual properties of the clause it appears in, although aspect could be dependent on some thematic properties. There are superficial examples of such dependencies: if a verb takes an agent, it can be put in the progressive. ${ }^{4}$ We expect no dependencies going the other way (although Campbell (1989) argues otherwise). 


\subsubsection{Locality, theta theory and selectional restrictions}

As we mentioned earlier, locality of theta assignment and selectional restrictions is the crucial assumption underlying our conclusion that Infl and other categories are raising categories. We discuss this now.

The standard analysis does not assume that Infl is a raising category. How is the distinction between raising verbs and Infl made? Consider the relation between thematic structure and constituent structure. Chomsky (1986) and many orhers have argued that a theta assignment relation between $X$ and $Y$ requires sisterhood between $\mathrm{X}$ and $\mathrm{Y}$. This proposal takes the realization of the theta relation between a verb and its direct object (sisterhood) to be the canonical way to realize theta relations. However, Chomsky (1986) notices that a complication arises in the case of assignment of theta role to subjects by VPs (on this see Chomsky (1981), Marantz (1984)). Indeed, given the standard clause analysis in which Infl is not a raising category:

$$
\left.\left.\left.\mathrm{E}_{\mathrm{IP}}[\mathrm{spec}, \mathrm{I}] \mathrm{L}_{\mathrm{I}^{\prime}} \mathrm{I}\left[_{\mathrm{VP}} \mathrm{L}_{\mathrm{V}}, \mathrm{V} \text { NP }\right]\right]\right]\right]
$$

sisterhood must be so defined as to ignore intermediate projections of Infl (we ignore here the technical problems that this proposal raises). Sisterhood has to be amended accordingly. Surely, this amendment is no simplification. No such complication arises under (2), i.e. if $\mathrm{I}$ is a raising category. We can take the syntactic requirement as being simple sisterhood throughout.

This argument is a simplicity argument: we show that a formally simpler notion of sisterhood is all we need. Such arguments however tend to be weak. We need the right notion of locality, whatever it is. But, given that Infl displays the diagnostic properties of raising, it takes an argument to go to the extra complication. We know of none. A couple of remarks might further strengthen this argument.

First, remember that we concluded that aspectual verbs are raising verbs too. They head their own verbal projections, taking VP complements. Since theta assignment is able to skip over them, we must conclude that sisterhood cannot be simply amended so that non-lexical projections are ignored. This is yet an extra complication, although not insurmountable. Second, it is quite clear that the same reasoning extends to selectional restrictions. If selectional restrictions also require sisterhood, the argument for simplicity is the same. However if selectional restrictions do not require sisterhood but a more permissive locality condition such as government, then again we will need to amend the usual notion of government just to take care of selectional restrictions imposed on external arguments if tensed Infl is not a raising category. 


\subsubsection{The existence argument}

So far, we have shown that there is some motivation for taking tensed Infl (and aspectual verbs) to be a raising verb, and furthermore, that not assuming this leads to some complications. We now show that the grammar of English (or French) as it stands generates raising structures with Infl already.

This argument is an elaboration of remarks found in Stowell (1983). Stowell shows that small clauses of the type $Y=\left\{N P X^{n}\right]$ exist for $X=A, P, N$ and $\mathrm{V}$. The interesting point is that he establishes directly (i.e. by exhibiting) and indirectly (i.e. by generalizing the structures of the type $\mathrm{Y}$ with all the lexical Xs) the existence of small clauses with verbal heads. In other words, the kind of constituent we postulate as complement of Infl has been argued to exist independently of our proposal.

In such small clause structures, the external argument of the predicate of the small clause is syntactically projected as sister of a projection of this predicate. This establishes that a subject (or, more precisely an external argument) can be projected as sister to its predicate.

Let us apply this to VPs. If a VP with an external argument can realize this argument as its sister in small clauses, nothing prevents this projection rule from applying in clauses as well. How can we avoid projecting the subject of a VP as sister to this VP in a clausal structure? In other words, the very existence of small clauses triggers the generation of structures like (2). The real question becomes whether the standard clause structure in (5) is ever justified. In the absence of arguments in favour of it, it has become superfluous. Note finally that the discussions in the previous subsections all point toward the same conclusion: the hypothesis that theta roles are assigned under sisterhood indicates that NP* and VP in (2) are indeed sister nodes and that I and NP* are not; the fact that small clauses exist independently indicates that the sequence NP VP in (2) forms a constituent excluding I, assuming the standard approach to small clauses according to which the predicate and its subject form a constituent excluding the rest (an assumption presumably having to do with the general relationship between semantic types and syntactic constituents). ${ }^{5}$ This is what we will continue to assume.

\subsection{Additional arguments}

\subsubsection{The X-bar theoretic argument: VSO languages}

First, we suppose that the null assumption concerning language variation is that it does not exist. In the absence of (learnable) evidence to the contrary, language structure does not vary. This puts an upper bound on how complex or remote from the primary linguistic data parameters can be. To be sure, detailed claims about the nature of this upper bound are intimately tied to the theory of how language acquisition actually proceeds. However, it appears 
plausible to suppose that covert structural differences such as that between the standard clause structure and the one we propose (or more generally differences of hierarchical organization of constituents) will be beyond this upper bound. Consequently, if some language can be shown to have a clausal structure of the type illustrated in (2), English will be assumed to have it too and reciprocally.

Consider a VSO (Verb-Subject-Object) language like Welsh or Irish. Assume the correctness of the standard clausal structure repeated below:

$$
\text { (5) } \quad\left[_{I P}[\text { Spec, IP }]\left[_{I^{\prime}} I\left[_{V P}\left[v_{v^{\prime}} \text { V NP }\right]\right]\right]\right]
$$

Then, $\mathrm{S}(\mathrm{ubject})$ is generated as the specifier of $\mathrm{I} ; \mathrm{V}$ and $\mathrm{O}$ are generated as part of a constituent in the VP excluding S, and the VP is the complement of I. This means that a VSO language cannot be base generated as VSO, since V and $\mathrm{O}$ must form a constituent excluding S. In other words, a VSO structure involves movement. This conclusion, which extrapolates constituent organization from the way it is in English or French to languages that look superficially different is supported empirically in various ways. As noted by many (Emonds (1981), Jones and Thomas (1977), Harlow (1981)), one supporting fact is that Welsh and Irish are also AuxSVO languages if there is an auxiliary. ${ }^{6}$ This suggests that the VSO structure involves movement of the $\mathrm{V}$ to the position that an overt Aux otherwise occupies. What is this position? Verb movement is by now fairly well documented. This documentation suggests that two landing positions for this verb movement are a priori plausible: it could be Infl, or it could be Comp. ${ }^{7}$ In the welldocumented cases (the Germanic languages, Den Besten and others, (1983)), movement of $\mathrm{V}$ to Comp takes place only in the absence of an overt complementizer. If a C-position is filled, it cannot be a landing site for $\mathrm{V}$ movement. If the $\mathrm{C}$-position is not filled, it is available as a landing site: this is why, in general, V to Comp (i.e. V-second) is only observed in root contexts. In Irish or Welsh, the VSO order is observed both in root clauses, and in non-root clauses. This in itself is not significant. However, it occurs in clauses containing overt complementizers, e.g. embedded clauses. This indicates that the simplest assumption is that the VSO order is derived by V-movement to Infl. This kind of V-movement, clearly found in French (cf. Emonds (1978)) or in Vata (cf. Koopman (1984)) is unaffected by the presence of complementizers. From this, we may conclude that the AuxSVO order is simply the base generated order, with $\mathrm{V}$ and $\mathrm{O}$ being part of a VP excluding $\mathrm{S}$. So the structure of Irish/Welsh is: Infl SVO. The problem with this is apparent. If structural variation is preferably avoided, $S$ should be specifier of Infl as in (5) above, and the VP containing VO the complement of I. If the Aux SVO is base generated as such, S, the specifier of I, intervenes between I and its complement VP. But this contradicts X-bar theory which claims that a head and its complement form a constituent excluding the specifier. It also 
contradicts the facts of Irish/Welsh, which do not allow a specifier to so intervene. Keeping to minimal assumptions, there are several possibilities:

$1 \mathrm{~S}$ is specifier of I, but the Inf S VO structure itself involves movement;

2 VP is not a complement of Infl;

$3 \mathrm{~S}$ is not specifier of $\mathrm{I}$.

The problem with (1) is that it is unclear what kind of movement it could be. For example, there is no plausible landing site between Infl and VP that would c-command the launching site given the right branching character of the language. ${ }^{8}(2)$ also raises questions. Infl is not a lexical category. A priori, then, we do not expect radical language variation in its complement structure. If Infl takes a VP complement in English or French, it should do so here too. Koopman (1987) provides independent evidence based on Bambara that Infl subcategorizes for an XP complement, and VP in particular.

Suppose then that $S$ is not specifier of I. We are led to a structure of the sort:

\section{(6) Infl NP VP}

It turns out that there is substantial evidence internal to Irish and Welsh supporting this conclusion as Chung and McCloskey (1987) have shown for Irish, and Harlow (1981) for Welsh (cf. also Sproat (1985); Koopman (1984)). Assume that more precisely, the structure is: Infl [NP VP], with NP and VP forming a constituent, as Chung and McCloskey propose. Given this conclusion we now face the reverse problem: if $S$ is not specifier of Infl in these languages, it should not be so in English either. If we cannot make Irish like English, we should, at the appropriate level of linguistic representation, try to make English like Irish: the simplest assumption seems to be that $S$ is not generated as specifier of Infl in English either. Rather, it is generated as a sister to VP. By the same reasoning as above, if we observe the surface word order S Infl VO, it must be because S moved from its base-generated position to pre-Infl position. Basically this leads to the adoption of the structure given in (2). S is base generated in the position NP*, and is moved to specifier of Infl position $\mathrm{NP}^{\wedge}$.

\subsubsection{Agreement in Arabic}

Standard Arabic is a language in which the orders VSO and SVO are both observed in simple clauses. The interesting feature here is the agreement pattern. In the order VSO, the verb only exhibits a default number agreement (third person singular). ${ }^{9}$ With the SVO order, the verb fully agrees with $\mathrm{S}$. How does agreement in clauses function? Let us assume the following standard version. Agreement is the morphological reflex of a relation between Infl and its specifier, or more generally, between a head and its specifier. This 
property of Infl is realized on the verb because the verb moves into Infl. As Mohammad (1989) observes, the Standard Arabic agreement pattern can be analyzed quite simply under the above treatment of VSO order coupled with assumption (2). VSO in Arabic, Mohammad argues, results from the obligatory movement of the verb to Infl, i.e. from an underlying Infl SVO. If nothing further takes place, Infl has a silent expletive specifier as Mohammad argues (or no specifier position at all) and agreement gets the default value, namely third person singular.

In the case of the SVO order, however, the derivation from an underlying Infl SVO comprises one more step. First, the $\mathrm{V}$ obligatorily moves to Infl position. In order to reestablish the SVO surface order, it must be assumed, just like in English, that $\mathrm{S}$ moves to specifier of I. Consequently, $\mathrm{V}$ in Inf has a specifier and fully agrees with it: we get subject/verb agreement (see Mohammad (1989) for a detailed analysis).

\subsubsection{Q-float}

The argument for structure (2) based on the distribution of Qs rightward "floated" from their NP is extensively developed in Sportiche (1988a). We limit ourselves here to a short outline of the logic of the argument.

In the first instance, such Qs appear between Infl and VP. It behaves with respect to the NP it is related to just like an anaphor does with respect to its antecedent. Yet, it can be shown that it is not an anaphor. These central observations can be explained by the following scenario.

1 The clausal structure (2) is correct.

2 Movement takes place from $\mathrm{NP}^{*}$ to $\mathrm{NP}^{\wedge}$, leaving a trace. ${ }^{10}$

3 This movement optionally pied pipes $\mathrm{Q}$. If not, we have a floated $\mathrm{Q}$ next to the trace NP*.

This explains why Q appears between Infl and VP: simply because NP* does (subjects of small clauses may precede their predicate). This also explains the anaphoric relation. This relation really holds between $\mathrm{NP}^{\wedge}$ and its trace NP*. Q, being adjacent to this trace, gives the illusion of being anaphorically dependent on $\mathrm{NP}^{\wedge}$. More generally, any time a structure contains an empty category $e$ dependent upon another $\mathrm{NP}^{* *}$, if $e$ is modified by $\mathrm{Q}$, the illusion that the $Q$ has floated off $\mathrm{NP}^{* *}$ will be created. Reciprocally, if a $\mathrm{Q}$ is stranded, we may suspect the existence of a covert NP adjacent to it.

\subsubsection{Q-lowering}

Consider the following sentence:

(7) A griffin seems to be lurking on the 25th level. 
In such an example, the indefinite subject can be understood either outside the scope of the verb seem (i.e. There is a griffin such that it seems to be lurking on the 25th level) or inside it (i.e. It seems that a griffin is lurking on the 25th level). As May $(1977 ; 1985)$ has discussed, this is due to the fact that the main verb is a raising verb, so that the second interpretation can be reconstructed by "lowering" the main subject into its base position. Aoun and Li (1988) propose a different treatment of this ambiguity. However, their approach shares with May's approach the idea that the crucial factor is the presence of a trace of the main subject in the embedded clause.

The same "lowering" effect is observed with the following structures:

(8) A griffin might be lurking on the 25th level.

The two interpretations here are one with the main subject outside the scope of the modal: there is a griffin and it might be lurking on the 25th level, and one with the main subject inside the scope of the modal: It might be that a griffin is lurking on the 25th level.

According to (2), might in Infl is a raising category, i.e. followed by a trace of the subject: the facts follow.

\subsubsection{Possible idioms}

We can elaborate on the argument for raising based on the distribution of idiom chunks. Idiomatic expressions are extremely common in English. What possible shape can they take? The semantics of idioms must be stated in some component of the grammar. The natural place is the lexicon, the repository of idiosyncratic information. It would appear rather natural at first that idioms are simply fully specified constituents with an idiomatic reading. It is quite easy to exhibit counterexamples to this generalization. Many idioms contain open positions: e.g. lose one's cool.

Surely however, there are constraints as to the open or variable positions that an idiom can contain. Otherwise, nothing would prevent an idiom only containing the italicized elements as part of it:

(9) A pale man slowly put flowers next to John.

Since such idioms do not exist, it is not obvious what this one could mean. Assume the following: only if pale modifies a subject and slowly co-occurs in the same proposition does the following idiomatic interpretation arise: pale means unknown to the speaker and the action was done in a roundabout way. In other words, when uttering (9), the speaker means that the man unknown to me put flowers next to John in a roundabout way. More generally, pale $X$ slowly verbed stands for $X$ unknown to speaker verbed in a roundabout way. 
In order to exclude impossible idioms, we may propose that idioms must at least meet a condition stronger than (10) below: ${ }^{11}$

(10) If $\mathrm{X}$ is the minimal constituent containing all the idiomatic material, the head of $\mathrm{X}$ is part of the idiom.

Applied to (9), this would mean that the head of $\mathrm{S}$ should be part of the idiom, which it is not. So this idiom is excluded. Similarly, the specifier of a noun and the complement of a noun cannot form an idiom together, with the head noun an open position. A verb taking an NP and a PP as complements cannot be an open position of the idiom made up of the NP and the PP.

Consider such idioms as the shit will hit the fan. For such idioms, (10) predicts that the minimal constituent containing the idiomatic material $\mathrm{S}=\mathrm{IP}$ should have its head fixed. But there is no constraint whatever on the content of Infl in such sentences. If the structure of S is Subject $\mathrm{I}_{\mathrm{I}^{\prime}} \mathrm{I}$ VP], these idioms constitute a systematic class of counterexamples to (10). Note that it is not the case that non-lexical categories cannot be part of idioms. For many French speakers examples such as les carottes sont cuites/"the carrots are cooked" "all is lost" in which Infl is fixed, or Que le diable l'emportel"let the devil take him away" "let him be damned," in which the complementizer is obligatory, are idioms. Of course, because of movement processes, a condition like (10) must be understood to hold at D-structure. Now, it is clear that adopting our proposal on clause structure in (2) removes this class of idioms as exceptions. At D-Structure, the minimal constituent containing all the idiomatic material is $\mathrm{V}^{\max }$, excluding $\mathrm{I}$.

\subsection{Conclusion: what is a subject?}

Consider the superficial properties of the subject of a clause in English (or French). A (non-derived) subject:

(1) occupies the position specifier of I, i.e. [NP, S] in usual terms;

(2) is the external argument;

(3) triggers agreement with the verb.

These three properties are usually all analyzed as a property of the specifier of Infl. Adopting (2), we see that the three properties do not correlate. Property (2) is a property of [NP, $\left.V^{\max }\right]$. Properties (1) and (3) do correlate due to the fact that we get specifier/head agreement, and we get a merger of $\mathrm{V}$ and I (by V to I or by Affix-hopping).

The distinction between (1) and (2) sheds a different light on the question of what the head of $S=I P$ is. Should the subject be contained in the maximal projection of $\mathrm{V}$, or is the standard clause structure (as in (4)) correct? (See Marantz (1979) who suggests that V is the head of S, and Hornstein 
(1977) who suggests that Aux is the head of S.) The answer according to (2) is positive, if we take subject as meaning external argument, ${ }^{12}$ negative, if we take subject as meaning the NP triggering agreement. In a sense, then, both positions are correct: the maximal projection of $\mathrm{V}$ contains the subject understood as external argument but does not contain the subject understood as the NP triggering agreement.

\section{Some implications}

\subsection{Summary and questions}

Summing up our conclusions: in a language like English or French, the internal structure of clauses is as in (2) repeated below.

(2)

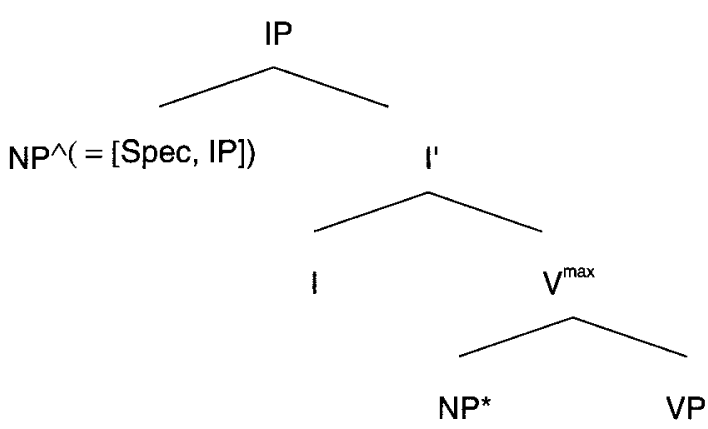

The canonical position of the external argument of $\mathrm{V}$, if any, is $\mathrm{NP}^{*}$, in which position it is initially generated. At S-structure in the simple cases we have discussed, i.e. basically tensed clauses, an overt external argument is realized in $\mathrm{NP}^{\wedge}$ : movement from $\mathrm{NP}^{*}$ to $\mathrm{NP}^{\wedge}$ must take place. English and French tensed Infl are raising categories.

Note that we have not shown that $\mathrm{I}$ is a raising category in general. Rather, we have shown that since (Spec, $\mathrm{I})=\mathrm{NP}^{\wedge}$ is always a theta-bar position, no selected or theta-marked NP can be base generated there. If a selected $\mathrm{NP}$ appears in NP position, it means it has moved there. In particular, we have established that when the external argument appears as specifier of Infl, raising must have taken place. Since this is always the case for French or English tensed clauses, tensed $\mathrm{I}$ is a raising category. This leaves open the possibility that movement from $\mathrm{NP}^{*}$ to $\mathrm{NP}^{\wedge}$ does not take place in certain cases.

A number of questions now arise:

1 What exactly is the nature of the difference between constructions in which raising from $\mathrm{NP}^{*}$ to $\mathrm{NP}^{\wedge}$ is obligatory and constructions in which it is not? We will suggest that the parameter has to do with Case Theory, 
and more specifically with the way in which nominative Case is assigned by Infl. We discuss this in Section 2.2.

2 Generally, we can ask whether a particular choice of Infl material in a particular language allows, requires or prohibits raising. In particular, we have argued that raising of $\mathrm{NP}^{*}$ to $\mathrm{NP}^{\wedge}$ in Irish, Welsh or Arabic tensed clauses is not obligatory (and perhaps impossible). Does this actually mean that external arguments in these languages surface in their D-structure position or does it mean that they do not raise to $\mathrm{NP}^{\wedge}$ ?

We will argue that the proper consequence of the parametric variation in the properties of Infl is whether or not raising to $\mathrm{NP}^{\wedge}$ is obligatory. We will suggest that there may be intermediate projections between the highest Infl projection (i.e. IP=S) and the highest VP projection. The relevant difference between constructions or languages will be whether or not raising to the specifier position of the highest Infl is required. We discuss this in Section 2.2.3.

3 More specifically, we can ask how English or French non-finite clauses behave with respect to raising. Is there raising in gerundival clauses, infinitival clauses with covert subjects or Exceptional Case Marking structures? We discuss this in Section 2.3.

4 Next, we consider the position NP*. In a number of articles that have adopted the so-called VP-Internal Subject Hypothesis (ISH), two views are found: the position of Koopman and Sportiche $(1985 ; 1988)$, and repeated at the beginning of this article is that $\mathrm{NP}^{*}$ is in the subject position of a small clause with VP predicate. Others (Kuroda (1988) Speas (1986)) have suggested that NP* is in the specifier position of the VP headed by the main verb. In Section 2.4 we will discuss these two alternatives and argue in favor of our position and show that it is compatible with the idea that NP* is the specifier of a VP.

5 Given that [Spec, IP] never receives a theta-role, Chomsky's (1981) definitions would characterize it as an A-bar-position. However, it is usually considered an A-position. Is it an A-position or an A-bar-position? What kind of movement is movement to $\mathrm{NP}^{\wedge}$, is it an instance of NP Movement or akin to $W h$ Movement? These questions are addressed in Section 2.5.

6 Then, there are questions relating to subjects in general. The extended Projection Principle requires the existence of the subject position of clauses. How is this to be interpreted within this new context? Looking at (2), does this mean that $\mathrm{NP}^{\wedge}$ is an obligatory position, or does it mean that NP* is an obligatory position? These questions which also relate to the distribution of expletives are not discussed in this article.

7 Finally, we can ask how the ISH relates to other properties of subjects. If some difference in parameter value is involved, how does it relate to other parametric variations involving subjects such as the possibility of having null subjects and subject/object asymmetries? We do not discuss these 
questions here. Some discussion can be found in Koopman and Sportiche (1988).

\subsection{The nature of the parameter}

\subsubsection{Case assignment and Case assignment to subjects}

Sometimes Infl forces raising (depending on the language and, possibly, in a given language, depending on the content of $\operatorname{Infl}$ ), sometimes it does not. Suppose that in a given construction, Infl forces raising. This means that in the structure (2), movement of $\mathrm{NP}^{*}$ to $\mathrm{NP}^{\wedge}$ is obligatory. This is reminiscent of the obligatory character of NP Movement in passive constructions in English or in raising constructions, suggesting a characterization of this effect in terms of Case Theory. Overt (and some covert) NPs need Case. If an NP needs Case, but is not in a Case-marked position, it can acquire Case if it moves to a Case-marked position. Adopting this idea means that when NP* is a Caseless position, an NP in it which needs Case must move. If on the other hand NP* is a Case position, movement is not necessary.

Let us again look at (2):

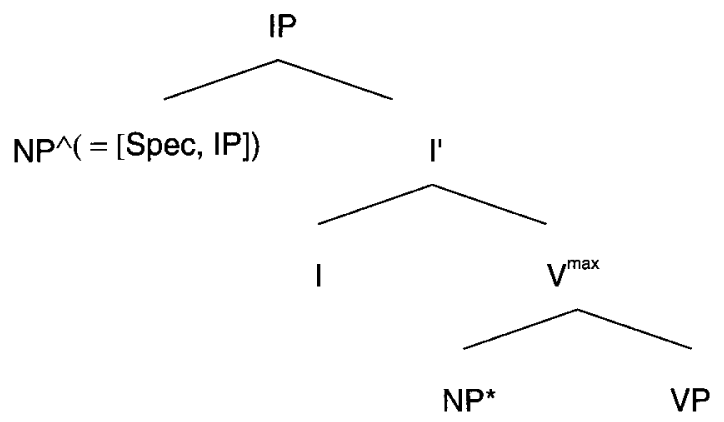

In English, we want to say that if Infl is tensed, NP* is not a Case-marked position, but $\mathrm{NP}^{\wedge}$ is. How can we implement this idea? Clearly, $\mathrm{NP}^{\wedge}$ receives Case by virtue of some property of Infl (presumably agreement). So let us say that tensed Infl is a Case assigner and assigns nominative Case. Case is usually assumed to be assigned under government. However, if Case is assigned under government, we need to say that I governs $\mathrm{NP}^{\wedge}$ but not $\mathrm{NP}^{*}$. This is in fact inconsistent with the notion of government that we would like to adopt. There is a good deal of evidence (see Chomsky (1986); Sportiche (1988b; 1990)) suggesting that if some $X^{0}$ governs $Y P$, it governs the specifier of $Y P$. Since clearly I governs $V^{\max }$ (sisterhood surely entails government), it should, by this definition govern its specifier, namely NP*. ${ }^{13}$ Furthermore, consider the case of Irish/Welsh or Arabic. There, we do want to say that NP* receives nominative Case from I. Presumably, then, I governs NP*. Under current 
assumptions about Case assignment, we face a problem: if Infl can assign nominative Case to its specifier $\mathrm{NP}^{\wedge}$, it should always be able to assign nominative Case to NP*. We would therefore expect no language to be like English in requiring raising to $\mathrm{NP}^{\wedge}$.

We adopt the following alternative suggested in Sportiche (1988b). The basic idea is to tie certain kinds of Case assignment (like nominative) with agreement or rather with agreement configurations.

Current theories distinguish between two kinds of Case assignment: structural Case and inherent Case. Structural Case is a configurational property: a tensed I assigns Case to its specifier in English regardless of the semantic or thematic relation of this specifier to the rest of the sentence. Nominative Case is typically a structural Case. Inherent Case on the other hand is lexically dependent or thematically dependent. Its assignment depends both on the lexical choice of the Case assigner and on the thematic relation between the Case assigner and the category receiving Case.

There is another difference, however. The structural configuration in which nominative Case assignment occurs is different from the configuration in which an object is inherently Case-marked. Nominative case assignment is a relation between a head, namely Infl, and its specifier. ${ }^{14}$ Inherent Case assignment is a relation between a head and a complement. This distinction would solve the problem just mentioned: if Infl can Case-mark NP ${ }^{\wedge}$, why can it not Case-mark NP* as well? Distinguishing between Case assignment to a complement or the specifier of a complement and Case assignment to a specifier would draw the required line.

We propose to implement this idea by distinguishing between Case assignment to an NP under government by a Case assigner and Case assignment under agreement of the NP with a Case assigning head.

Putting all this together, we distinguish between structural Case assignment and inherent Case assignment. Furthermore, we distinguish between Case by agreement and governed Case. Nominative is both structural Case and Case by agreement, while inherent Case is always governed Case. Where does objective Case fit in this picture? Objective Case is a structural Case as shown by the fact that it occurs in Exceptional Case Marking structures or on subjects of small clauses, when it bears no thematic relation with its Case assigner. Is objective Case a governed Case or is it a Case by agreement? We would expect objective Case to behave just like nominative Case. Since Infl can assign Case by agreement or governed Case, depending on the language, we expect the verb to either assign objective Case by agreement or governed Case as well, depending on the particular language. In English objective Case is apparently a governed Case. This is what we will assume here. (See Sportiche (1990) for a discussion of this issue.) We refer the reader to Section 2.5.2. for more discussion on languages that assign Case by agreement.

Case assignment by agreement is defined as follows. Let $\mathrm{H}$ be a Case assigning head. Then, if it is a Case assigner by agreement, it may assign Case 
to an NP in its specifier position, as a reflex of the general process of specifier head agreement.

Governed Case is assigned under government where government is provisionally defined in terms of X-command and in terms of barrier in the spirit of Chomsky (1986). The precise definition of barrierhood that we adopt is the one developed in Sportiche (1988b; 1990) and will not be of central concern here. It suffices to say that complement boundaries do not act as barriers:

(11)(a) Government

A governs $\mathrm{B}$ if $\mathrm{A} \mathrm{X}$-commands $\mathrm{B}$ and no barrier for $\mathrm{B}$ intervenes between $A$ and $B$.

(b) X-command

A X-commands $\mathrm{B}$ if the minimal constituent meeting property $\mathrm{X}$ containing A contains $\mathrm{B}$.

How should we define X-command? Look again at the structure in (2). If we take X-command to be max-command in the sense of Aoun and Sportiche (1983), this means that Infl governs its specifier. Infl, then, could in principle assign a governed Case to an NP in its specifier position. In particular, if Infl can assign governed Case, then it should be able to assign it either to the position NP* or to the position $\mathrm{NP}^{\wedge}$. Is this desirable? The Arabic data discussed earlier bear on this question. In Arabic, the subject can surface either in the postverbal position $\mathrm{NP}^{*}$ or in the position $\mathrm{NP}^{\wedge}$ and this correlates with the presence of number agreement. In terms of Case, we would say that NP* gets governed Case. In particular, given that there is no agreement of the verb with a postverbal subject, it shows, as expected, that governed Case does not correlate with agreement. If governed Case could be assigned to $\mathrm{NP}^{\wedge}$, we would expect agreement with $\mathrm{NP}^{\wedge}$ to be optional. It is not. This suggests that governed Case cannot be assigned to $\mathrm{NP}^{\wedge}$ in Arabic; only Case by agreement can. ${ }^{15}$ Consequently, we want to define government in such a way that a head does not govern its specifier. Again, we will adopt Sportiche's $(1988 \mathrm{~b} ; 1990)$ proposal according to which the correct notion of $\mathrm{X}$-command is $\mathrm{i}$-command defined as follows:

\section{i-Command}

A $\mathrm{i}$-commands (immediate command) $\mathrm{B}$ if the first constituent (distinct from A) containing A contains B.

A constituent will thus have a chance to be governed by a head only if it is included in a constituent sister to this head. In particular, a head does not govern its specifier.

Which of these two Case assigning options is realized depends on the particular category HP, the lexical content of the head $\mathrm{H}$ it contains, and the language $\mathrm{L}$. A priori, we want to minimize possible inter and intra language variations. Given that there is crosslinguistic variation, the strongest position 
would be one asserting that languages choose once and for all one and/or the other option, regardless of the head and its content. This seems too strong, however. Koopman (forthcoming) shows that in Dutch, P(repositions) are governed Case assigners, while $\mathrm{P}$ (ostpositions) are agreement Case assigners. In English Infl only assigns Case by agreement, while it seems that V only assigns governed Case.

\subsubsection{Formulating the parameter}

In English or French, the subject cannot surface as NP*. We construe this as meaning that NP* receives no Case. Rather, the subject surfaces in the specifier position of Infl exhibiting agreement with it (in a fairly impoverished way in English). We now construe this as meaning that when Infl is a Case assigner, it is a Case assigner by agreement only. ${ }^{16}$

In Arabic, the external argument can surface in either position. Furthermore, only subjects may move to [Spec, IP] suggesting that movement to $\mathrm{NP}^{\wedge}$ is A-movement (cf. Section 2.5 for discussion). Given the agreement facts, we now analyze this as arising from Infl being able to assign either governed Case or agreement Case.

In Irish, only the order VSO is allowed and the subject never agrees with the verb or the auxiliary in Infl. The only apparent case of agreement is found with (covert) pronominal subjects but McCloskey and Hale (1984) have argued that these cases are best analyzed as involving incorporation of a postverbal pronoun into Infl. We are then led to analyze a Case marking Infl in Irish as being only a governed Case assigner and never an agreement Case assigner.

In Welsh, only the order VSO is allowed, and non-pronominal NPs never agree with the verb or the auxiliary either. Pronominal NPs, however, must fully agree with the verb. Again, we analyze a Case-marking Infl in Welsh as being only a governed Case assigner, never an agreement Case assigner. This will account for the impossibility of non-pronominal NPs raising to $\mathrm{NP}^{\wedge} .{ }^{17}$

If it is a Case assigner, Infl is a structural Case assigner. The Case Infl (and, in fact, of other categories) varies as follows:

(13) (1) Infl is specified as a governed Case assigner or not.

(2) Infl is specified as an agreement Case assigner or not.

The various possibilities are illustrated in the following table:

$\begin{array}{lll} & + \text { Governed Case } & - \text { Governed Case } \\ + \text { Agr Case } & \text { Arabic finite clause } & \text { French finite clause } \\ & & \text { English finite clause } \\ & & \text { Portuguese inflected } \\ & \text { infinitive } \\ & & \text { English infinitive } \\ - \text { Agr Case } & \text { Irish finite clause } & \text { French infinitive }\end{array}$




\subsubsection{The case of Welsh}

Consider again a tensed clause. Given the way the parametric variation is formulated, we see that the effect of the parametric variation is not necessarily that in certain languages raising to $\mathrm{NP}^{\wedge}$ is obligatory, and in other languages, the subject NP may surface in the position NP*. Rather, the subject NP will have to surface either in the specifier position of the head assigning nominative Case or in a position that is governed by the head assigning nominative Case, so that governed Case can be assigned. If some intermediate projection intervenes between the Case assigning head and $\mathrm{V}^{\max }$, the subject NP will have to raise from NP* to the specifier of this intermediate projection in order to get nominative Case. To illustrate this point, consider the underlying structure of the English sentence Jobn will bave visited Paris, where ASPP is the projection of the aspectual auxiliary have:

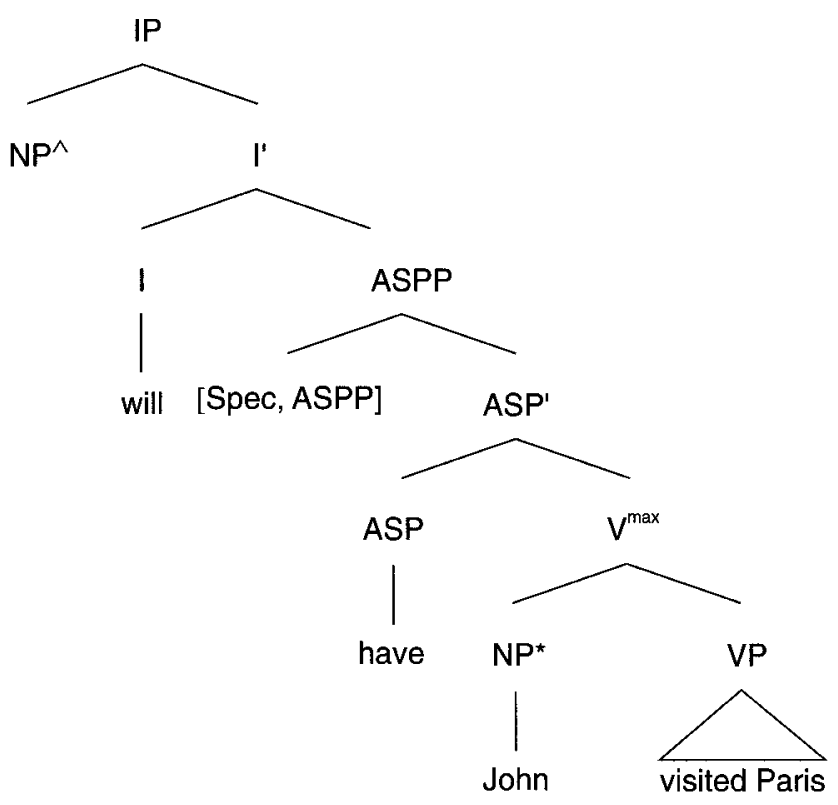

English requires John to raise to $\mathrm{NP}^{\wedge}$ (from now on, $\mathrm{NP}^{\wedge}$ will denote the highest specifier position of a clause; $\mathrm{NP}^{*}$ will continue to denote the NP position daughter of $\mathrm{V}^{\max }$ ). Suppose counterfactually that English Infl is a governed Case assigner instead of an agreement Case assigner. Then, raising of Jobn would be required to the specifier position of ASPP. This seems to happen in certain languages in which Infl assigns governed Case. We can illustrate this with a real example, from Welsh. As discussed above, nonpronominal subjects do not raise to $\mathrm{NP}^{\wedge}$, since they do not trigger agreement with the verb. Thus, according to our proposal, Infl is a governed Case 
assigner, not an agreement Case assigner. Let us consider evidence as to the position of the thematic subject at S-structure based on structures like (14), in which Infl is separated from NP* by intervening projections. We will discuss two cases: first, Infl is separated from NP* by aspectuals and, second, Infl is separated from NP* by negation.

Consider the following example which contains a modal and some periphrastic aspects:

(15) Dylai y plant fod wedi bod yn edrych ar y teledu. Should-3S the children be perf be at watch at the television. The children should have been watching television.

In this example, the modal in Infl does not agree with the plural subject which immediately follows it. If we assume that the periphrastic aspects project to ASPP, ${ }^{18}$ and that the modal is base generated in Infl then the example in (15) has the following D-structure:

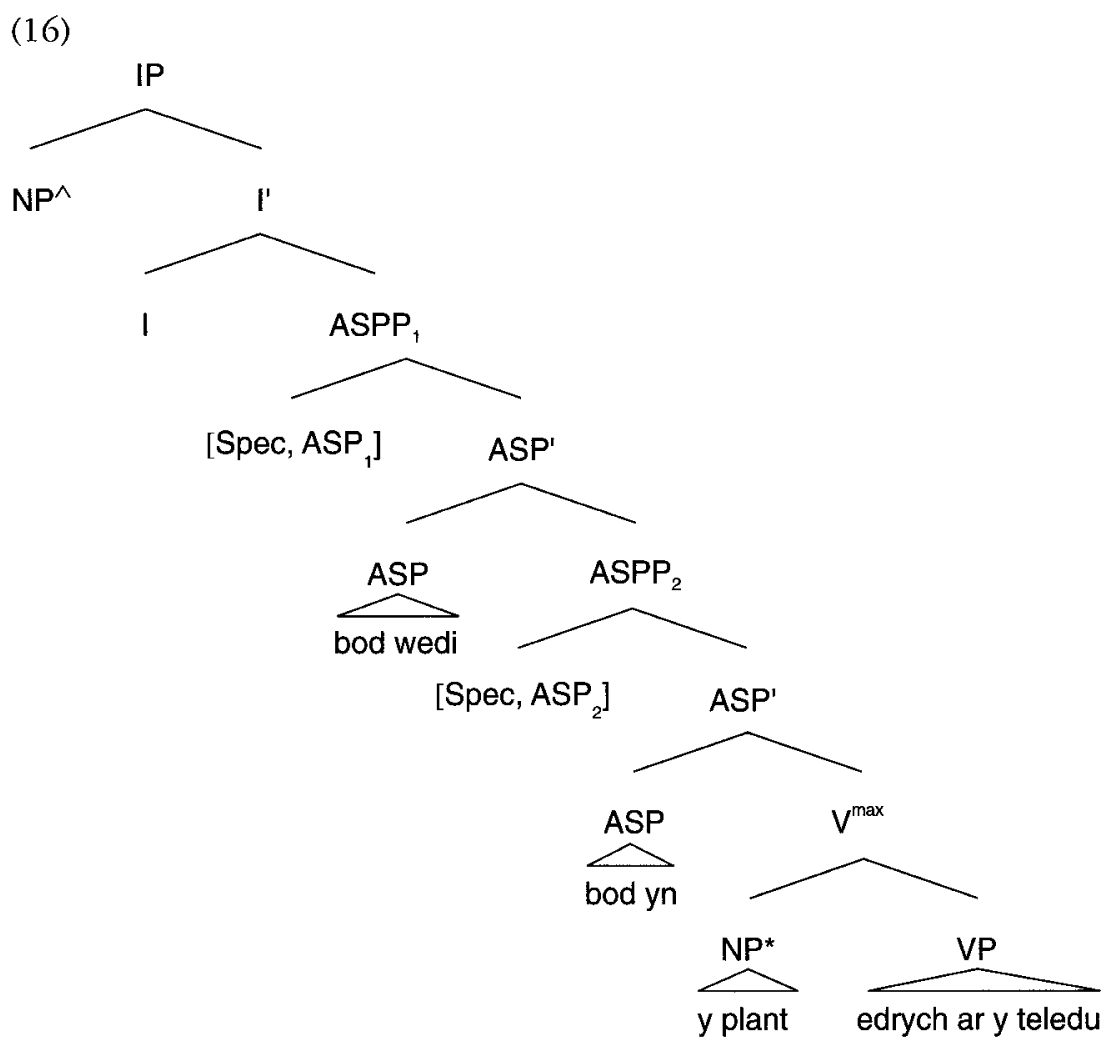


The thematic subject surfaces in a position governed by Infl, preceding all aspects, not in NP*, sister to VP, following all aspects. It must therefore have undergone raising from NP* via the intermediate $\left[S \mathrm{Spec}, \mathrm{ASPP}_{2}\right]$ into $[\mathrm{Spec}$, $\mathrm{ASPP}_{1}$ ] which is governed by Infl. The second type of evidence that shows that the thematic subject undergoes raising to be governed by Infl can be found in sentences with sentence medial negation:

$$
\begin{aligned}
& \text { Agorodd y dynion ddim y drws. } \\
& \text { Opened-3S the men not the door. } \\
& \text { The men didn't open the door. }
\end{aligned}
$$

Assuming that sentence medial negation is a modifier of negation - it does not block head movement of the main verb - and assuming negation is lower than Infl, but higher than ASP, will yield the following structure for such examples:

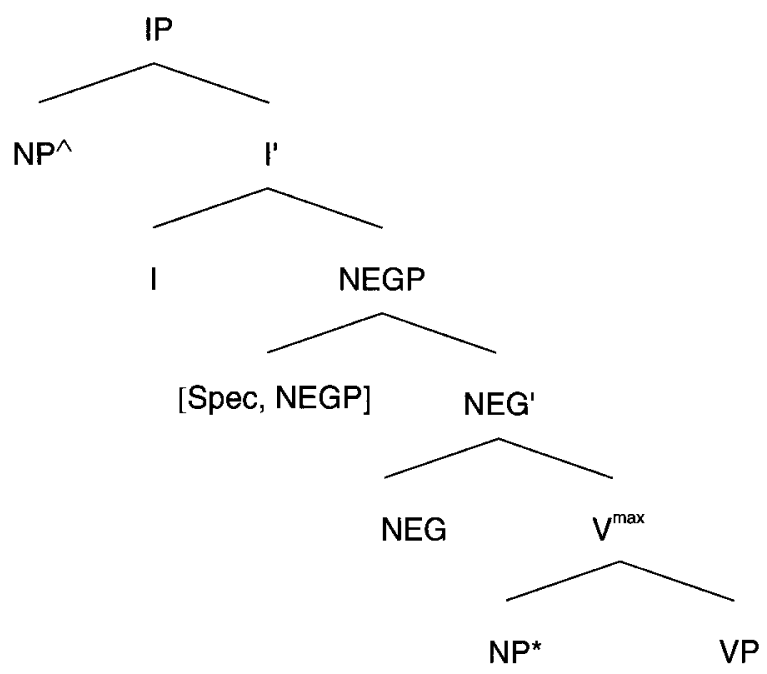

The verb has undergone head-to-head movement, and since the subject precedes negation, it must have raised to [Spec, NegP] where it can be assigned governed Case by Infl. In sum, then, NP* cannot remain in its underlying position, but must move in order to be governed by Infl, that is, Infl is a governed Case assigner.

\subsection{Infinitives and the distribution of PRO}

\subsubsection{Infinitives}

We now briefly examine the properties of subjects in non-finite clauses. Infinitival Infl is not a Case assigner. Since there is no Case available clause internally, no overt subject or covert subject requiring Case can surface there. 
If no Case is available from outside the non-finite clause, then the non-finite clause can have no overt subject or covert subject requiring Case ( $w$ b-trace or pro).

The case of ECM clauses is straightforward.

$$
\text { John believes }\left[N P \text { to }\left[t_{1} \text { have }\left[t_{2} \text { left }\right]\right]\right] \text {. }
$$

If NP is overt, it needs Case. It must therefore raise out of $t_{2}$ (to $t_{1}$ ) to a position where it gets Case from believe. In this respect, ECM clauses behave like tensed clauses. If NP in (19) is PRO, there should be no well formed output (*John believes to have left). If it has raised to a position governed by believe, it will be in violation of the requirement that PRO be ungoverned. If it has not raised at all, PRO will be governed by bave and will therefore be excluded. If it has partially raised to $t_{1}$, yielding (20), exclusion is not straightforward:

(20) John believes [NP to [PRO have [t left]]].

Given that infinitival Infl is usually considered not to be a governor and given that a head does not govern its specifier, PRO is ungoverned. The fact that the string of John believes it expletive to bave VP is well formed, but not with the reading John believes $i t_{\text {expletive }}$ to $P R O$ bave VP suggests that there is nothing wrong when NP in (20) is an expletive. If the position NP necessarily exists (possibly as a consequence of the Extended Projection Principle), we could invoke expletive replacement: at LF, PRO has to move to NP, a governed position, and is therefore excluded.

We will pursue a different suggestion. Specifying infinitival Infl as a non-governor is a stipulation. Why should tensed Infl be a governor and not tenseless Infl? If we assume that tenseless Infl is a governor, then (20) is excluded because PRO is governed. Quite generally, if the subject of an infinitive clause is PRO, it will not be able to occur as NP*.

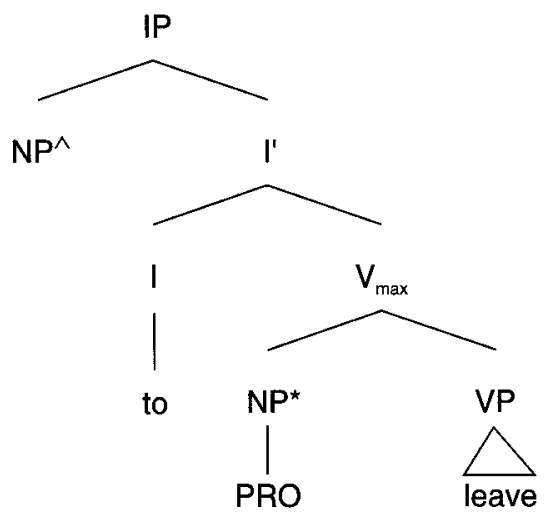


Given that tenseless Infl is now assumed to be a governor, PRO will be governed. There might be further reasons that support the governor status of tenseless Infl. Consider a raising structure:

(21)(a) John seems to sleep all day.

(b) John seems [t to [t sleep all day]].

There will be a trace following the tenseless Infl to in NP* of the embedded clause. If the conjunctive ECP requires that traces be both antecedentgoverned and head-governed, NP-traces must be governed by a head. Tenseless Infl will therefore have to count as a governor to avoid an ECP violation.

We will assume now that tenseless Infl is a governor. It is not an agreement category however. Then PRO must raise out of NP* to $\mathrm{NP}^{\wedge}$. One of the effects of defining government in terms of I-command, as we did earlier, is that $\mathrm{NP}^{\wedge}$ is no longer governed. ${ }^{19}$

This conclusion generalizes to structures in which one or several intermediate projections intervene between I and $V^{\text {max }}$ as in (14) earlier. PRO will always have to raise to the highest specifier of its clause whether this clause is finite or not. If it remains in some intermediate specifier position, it will be governed by the next head up. In particular, in control structures such as Jobn tried to sleep, the only allowable structure will be Jobn tried (PRO to (t sleep)).

\subsubsection{The distribution of PRO}

This formulation of government in terms of I-command allows a simple account of the possibility of having $\mathrm{PRO}$ as $\mathrm{NP}^{\wedge}$ of a tenseless clause despite the governing character of Infl. It shifts the problem to tensed clauses. Given that $\mathrm{NP}^{\wedge}$ is not a governed position, we now expect PRO to be able to appear in the $\mathrm{NP}^{\wedge}$ position of any clause.

As we just saw, this raises no problem in infinitivals. It shifts the problem. to tensed clauses, however, since we now expect sentences like $P R O$ is asleep to be grammatical. Following a suggestion of Sportiche (1990), where this is discussed in more detail, we would like to propose that this kind of sentence in English is ruled out in the following way.

Current theories distinguish two silent pronominal NPs: pro and PRO. PRO must be ungoverned while pro must be identified. PRO and pro are distinguished in terms of the features $+/$ - pronominal, $+/$-anaphoric.

Suppose instead that we distinguish between featured and non-featured silent pronouns. An NP is featured if it is in an agreement relation with a head. Otherwise it is non-featured. Let us now call pro the silent featured NP and PRO the silent non-featured NP. Because it has features, pro will have to be interpreted as an overt pronoun. Because it is featureless, PRO will be able to be controlled or receive an arbitrary interpretation (i.e. be assigned default features or features under control). 
The distribution of pro and PRO is then handled by the following conditions:

(22)(a) pro must be identified

(b) PRO must be ungoverned

In fact, this proposal differs minimally from the standard view. The major difference is that PRO cannot appear in an agreement position whether or not this position is governed. Summarizing, the standard view excludes PRO from $\mathrm{NP}^{\wedge}$ in tensed clauses because it is governed by tensed Infl, and permits $\mathrm{PRO}$ as $\mathrm{NP}^{\wedge}$ of infinitives because tenseless Infl does not count as a governor. According to our view, $\mathrm{NP}^{\wedge}$ is never governed from within its clause. PRO is permitted in $\mathrm{NP}^{\wedge}$ of an infinitive despite the fact that tenseless Infl is a governor. It is excluded from $\mathrm{NP}^{\wedge}$ of a tensed clause because this is an agreement position. Two consequences follow.

First, in languages in which there is no agreement between $\mathrm{NP}^{\wedge}$ and tensed Infl, $\mathrm{NP}^{\wedge}$ should be able to be PRO from the point of view of the theory of government. However, PRO might be excluded for other reasons. Indeed, take a language in which Inf is a governed Case assigner and [Spec, IP] is an A-position (see the discussion of this in Section 2.5). Then PRO in [Spec, IP] would be heading an A-chain with the Case position lower down, which is excluded (cf. Sportiche (1983)). In other words, not only do we need a language in which tensed Infl is not in an agreement relation with its Spec; we also need tensed Infl to not be a governed Case assigner. Otherwise we might end up with an ill-formed chain. Stenson (1989) proposes that the subject of Irish sentences with impersonal inflection actually is PRO. This is possible under our theory, if we assume that impersonal Infl in some sense absorbs the Case feature of Infl. Inf in Irish is not an agreement Case assigner, as discussed earlier. PRO, then, can seek refuge in $\mathrm{NP}^{\wedge}$. In main clauses, $\mathrm{PRO}$ can only be interpreted as arbitrary PRO, since there is no possible controller around. ${ }^{20}$

A second consequence of our proposal is that lexical NPs and PRO are not necessarily in complementary distribution. If a certain position is not governed, does not get Case under government and is not an agreement position, both lexical NPs and PRO should be allowed. Gerunds in English might illustrate this possibility, as witnessed by the grammaticality of both Jobn's reading this book and of $P R O$ reading this book.

\subsection{Properties of $N P^{*}$}

Since $[S$ pec, IP] is a position to which no theta-role is ever assigned, let us now discuss where the thematic position of the external argument is. We will contrast two proposals: NP* is generated in [Spec, VP] as sister to $\mathrm{V}^{\prime}$ (as suggested by Kitagawa (1986), Kuroda (1988), Speas (1986), and adopted by Guilfoyle et al. (1990)), or it is generated external to VP, as proposed by 
Koopman and Sportiche $(1985 ; 1988)$ or Manzini (1983). We will argue that NP* is not in [Spec, VP], but is external to VP. We will do so by showing that for some languages [Spec, VP] is a position in which objects occur. If it can be shown that $[S$ pec, VP] is a position reserved for objects in some languages - the thematic subject must therefore be generated elsewhere - it is reasonable to conclude that it cannot be the position for the external argument in any language.

\subsubsection{Three cases of object in Spec VP}

We will discuss three different cases, Bambara and Dutch, relying on work by Koopman (1987, and forthcoming), and French, as analyzed by Sportiche (1990).

B AMBARA

Bambara is a Mande language with an extremely rigid word order. The basic word order in tensed sentences is presented in (23):

$$
\text { (Adv/CP) NP }{ }_{1} \text { INFL (NP) V (PP) (ADV) CP }
$$

$\mathrm{NP}_{1}$ is the canonical subject position. It must be lexical. Consequently, Inf will be assumed to assign Case to its Spec.

Direct objects, or more precisely, NPs that depend on the verb for structural accusative Case, occur in an interesting position: they must precede the verb, and only they may do so; all other complements must follow. Moreover, only one NP may precede the verb. The assumption that the verb assigns its theta-role to the right, i.e. the VP is basically head initial (cf. Koopman (1984)), yields an account for the position of all non-NP complements. If we assume that verbs in Bambara assign Case in the same way that Infl does, i.e. the verb assigns structural Case to its Spec, we get an immediate account for the position in which they occur: given the X-bar schema, we would expect [Spec, VP] to be to the left of V, just as [Spec, IP] is to the left of I. An NP that needs structural Case moves from its thematic position to [Spec, VP] because it has to satisfy the Case filter; only one NP may move there, because X-bar theory only makes one Spec position available. Thus, structural Case assignment of subjects and "objects" is parallel in Bambara: both nominative and accusative are assigned to Spec positions. In sum, then, [Spec, VP] is the structural accusative position, the position in which objects occur, and cannot be the position of the underlying subject. 


\section{DUTCH}

We have so far seen that objects in Bambara occur in [Spec, VP]; it is also a standard assumption that objects in English are assigned governed Case (and occur under $\mathrm{V}^{\prime}$ ). Since UG allows for two possible Case-marked positions at $\mathrm{S}$-structure, the question arises for any language where objects are Casemarked, [Spec, VP] or $\left[\mathrm{NP}, \mathrm{V}^{\prime}\right]$. Let us now discuss Dutch objects. The situation in Dutch is much less transparent than that in Bambara, for various reasons: first, the Dutch VP is verb final, and thus both [Spec, VP] and [NP, $\left.\mathrm{V}^{\prime}\right]$ precede the verb. Second, there is extensive leftward scrambling in Dutch, which further obscures underlying order. And third, objects have a different distribution depending on whether they are pronominal or not, and whether they are specific or not. Pronominals are clitics, and occur on the highest functional projection in IP (Koopman, forthcoming). Specific NPs must scramble out of the VP, and in fact must occur in an (A-bar) position governed by the highest functional category in IP. Thus, neither pronominal objects nor specific objects tell us anything about the situation internal to VP. Non-specific objects, however, must remain rather close to the verb, and in fact must be assumed to occur in VP, since they must pied-pipe under VP preposing:

(24)(a) [Boekjes lezen] doet hij nooit.

Books read does he never.

He never reads books.

(b)* Lezen doet hij nooit boekjes.

Read does he never books.

The question thus arises where non-specific objects occur, i.e. where the structural accusative position is. We will now present an argument that the Case position is [Spec, VP]. We will do so by showing that the object is separated from the verb by all theta-marked elements, including optional arguments like instrumentals, i.e. it occurs exactly in the position where one expects to find [Spec, VP].

Given the possibility of Scrambling, we need a test to determine where the theta-positions in the VP are. Extraction out of PP, i.e. P-stranding, provides such a test. P-stranding in Dutch is possible under two conditions (cf. Van Riemsdijk (1978)): (1) R-movement to the [Spec, PP] is possible and, importantly, (2) the PP occurs in a theta-marked position. In particular, the $\mathrm{P}$ of a Scrambled PP can never be stranded. A stranded P therefore shows where the theta-position of the PP is.

Let us take an example that contains a verb c-selecting for an NP and PP, as well as an optional argument of the verb, an instrumental PP. Instrumental PPs behave as if being theta-marked by the verb, as shown by the possibility of stranding the instrumental P. What D- and S-structure orders would we expect? Assuming that all theta-marked elements occur under VP, that direct 
objects are projected as sister and adjacent to their verbs, and that optional arguments are projected higher than obligatory internal arguments, we would either expect (25a) (if the NP PP forms a small clause as proposed in Kayne (1984)), or (25b). In both cases the theta position of the NP follows the instrumental;
(25)(a) [Spec $\left[\mathrm{V}^{\prime},\left[\mathrm{PP}\right.\right.$ instr $\left.\left.\left.\left[\mathrm{NP} P \mathrm{P}_{\text {loc }}\right] \mathrm{V}\right]\right]\right]$
(b) $\left[\mathrm{Spec}_{\mathrm{V}}, \mathrm{PP}_{\text {instr }} \mathrm{PP}\right.$ loc $\mathrm{NP}$ V]] $]$
(c) $\left[N P_{i}\left[\begin{array}{lll}L_{v} & \ldots & t_{i} \ldots v\end{array}\right]\right.$

If Case is assigned to the [NP, $\left.V^{\prime}\right]$, we would furthermore expect (25a) or (25b) as surface order. If Case is assigned to [Spec, VP], we would expect the object to precede the instrumental PP.

As mentioned before, we will have to rule out the possibility that the instrumental PP has scrambled to the left of the VP. We therefore have to look at an example where the instrumental $P$ has been stranded:

(26) Dit is een ladder waar je (*mee) boekjes mee in de kast This is a ladder where you books with in the library kunt zetten. can put.

This is a ladder you can put books in the library with.

As this example illustrates, the order must be [NP P t PP V], i.e. the nonspecific object must precede the stranded P. It thus occurs exactly in the position where we would expect $[\mathrm{Spec}, \mathrm{VP}]$ to appear. We will take this as evidence for:

$$
\text { Dutch objects occur in }[\mathrm{Spec}, \mathrm{VP}] \text { at S-structure. }{ }^{21}
$$

This is related to (28)

$$
\mathrm{V} \text { assigns accusative Case to [Spec, VP]. }
$$

In sum, then, objects in Dutch are in [Spec, VP], which cannot be the position of subjects.

\section{FRENCH}

The third case we discuss is French participle agreement. We will adopt Kayne's (1985) central insight, as extensively discussed and modified in Sportiche (1990). The basic point here is that when participles in French agree, they always agree with a direct object, never with a subject. If agreement is a reflex of a Spec/head relation (in this case, the $\mathrm{V}$ in participial 
form and the direct object), the [Spec, VP] position cannot be the position of subjects.

Agreement is illustrated by the following sentences:

(29)(a) Cette écharpe, Jean l'a offertE $t_{i}$ à Pierre.

This scarf(FEM) John it has offered(FEM) to Peter.

As for this scarf, John offered it to Peter.

(b) L' écharpe que tu as offertE $t$ à Pierre.

The scarf Comp you have offered(FEM) to Peter.

The scarf that you offered to Peter.

Its most relevant property is that a participle agrees with a direct object only if the direct object precedes the participle. Objects in French normally follow the verb. In the two examples in (29) above, the direct object has been preposed by Wh Movement or clitic placement and has triggered agreement. Why should precedence play any role? Sportiche $(1988 \mathrm{~b} ; 1990)$ argues that the following derivation takes place:

(30)

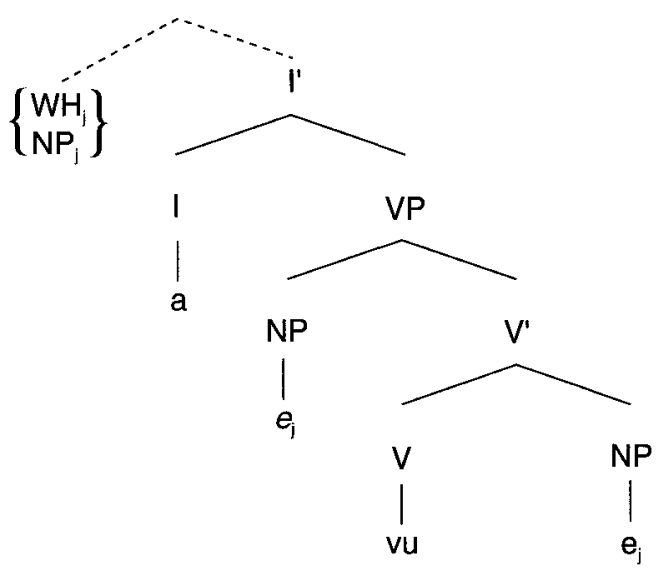

In the process of being preposed, the direct object moves through [Spec, VP] position. Its trace, now in a Spec/head relation with an agreeing head, triggers agreement.

\subsubsection{A note on $A G R_{0}$}

Kayne's (1985) analysis of participle agreement differs from that presented above in that he postulates an AGR projection to mediate agreement between the preposed object and the participle. Roughly there is an AGRP projection. The participle and the agreement affix merge and the object determines the shape of the agreement affix by transiting through [Spec, AGRP] or by adjoining to AGRP. Similarly, Chomsky (1989) proposes, partially based 
on the Bambara facts, that objects actually move to the Spec of a functional projection, $\mathrm{AGR}_{\mathrm{o}}$, triggering object agreement. According to this view, we would be dealing with a structure like that in (31).

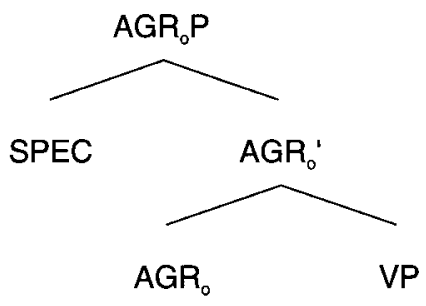

It might thus be argued that what we have called [Spec, VP], is really [Spec, AGR $\mathrm{P}$ ], and the question arises again where the subject is generated.

Note first that object movement is not coextensive with object agreement. Bambara and Dutch objects, we argue, appear in [Spec, VP] without triggering agreement. It is unclear what it means for an object to move to [Spec, AGRP] without triggering agreement. Why then would there be an agreement projection at all?

Suppose nevertheless that for the French case (and possibly others), we follow Kayne and Chomsky. There could be two possible structures: (1) NP* could be lower than AGR as in (32a), in [Spec, VP] or (2) it could be external to $A G R_{o}$ as in $(32 b)$ :

(32)(a) $\left[A G R_{0}\left[_{v P} N P *\left[_{V^{\prime}}, V \ldots\right]\right.\right.$

(b) $[\mathrm{NP} * \ldots[\mathrm{AGR}, \mathrm{VP}$

For (32a), it must be ensured that the object moves to [Spec, $A_{G R} P$ ], over the thematic subject, and the subject to [Spec, $A G R_{S} P$ ] (i.e. [Spec, IP]). If these movements count as $A$-movement, we would basically have to assume a Theory of NP Movement where an intervening subject does not block NP Movement since the object would move to [Spec, AGR $P$ ] over NP*: this is inconsistent with what is known of NP Movement. If these movements count as A-bar movements, it is unclear how to force the required derivation at all.

Even if we assume object movement (of whatever kind) over NP* to be possible, we still must prevent movement of the object to [Spec, $A_{G} R_{s}$ ] and of the subject to [Spec, $A G R_{\mathrm{O}} \mathrm{P}$ ]. Note that linking the position [Spec, $A R_{0} P$ ] with accusative Case and [Spec, $A_{G R}{ }_{S}$ ] with nominative Case (possibly a desirable move) is not sufficient. Both nominative and accusative Case are structural Cases, blind to thematic properties. How would we prevent a sentence like John believes Bill to bave left with two subjects to be interpreted with John the external argument of the embedded clause having raised to [Spec, $\left.A G R_{S} P\right]$ and Bill the external argument of the main clause having moved to $\left[\right.$ Spec, $A G R_{\mathrm{o}} \mathrm{P}$ ? $^{22}$ 
These difficulties argue against (32a) as a possible structure. (32b) will yield the desired derivations: simply assuming movement to be NP Movement, the external argument raises to $\left[\mathrm{Spec}, \mathrm{AGR}_{\mathrm{S}} \mathrm{P}\right.$, and the object to [Spec, AGR $\mathrm{P}$ ]. Both movements are legitimate cases of NP Movement. This conclusion is consistent with our proposal that NP* is not [Spec, VP].

Naturally, if there is an AGR projection as in (32b) above, it seems that we must modify the view that NP* is sister to VP for theta-theoretic reasons. Without further discussion here (but see Sportiche (1990)) we will suppose that there is no AGR projection at all in languages like Dutch and Bambara. In French, we identify $A_{G R}$ with participial (i.e. adjective-like) morphology: $A_{G R}=I_{o} f_{\text {participial }}$. Since participial morphology (unlike say Tense) does affect the syntactic expression of an external thematic role (e.g. passive participial morphology), it is consistent for us to assume that NP* is external to $\operatorname{Inf}_{\text {participial }} \mathrm{P}$.

In order to simplify further discussion, we will ignore this kind of projection in the remainder of this article. We will simply note NP* as external to VP rather than using the more precise "external to certain morphological projections taking VP as complement."

\subsubsection{Further remarks on $N P^{*}$}

We have established that NP* is external to VP. Exactly in what relationship does it stand with respect to VP?

We would like to continue to hold that the structural relationship between the external argument of a predicate and this predicate is identical across categories. Thus all the following phrases should basically have the same underlying internal structure:

(33)(a) (consider) $L_{\mathbb{W}}$ John $L_{z}$ very sick $\left.]\right\}$

(b) (saw) $\mathrm{L}_{\mathrm{W}}$ John $\mathrm{L}_{\mathrm{Z}}$ quickly leave $\left.]\right]$

(c) John will) $\left[_{W} \mathrm{NP} *\right.$ [ $_{\mathrm{Z}}$ quickly destroy his toys] $]$

(d) (witness) $\hbar_{W}$ John's $\left[_{Z}\right.$ quick destruction of his toys $\left.]\right]^{23}$

Extrapolating on the adjectival case (33a), it is clear the the constituent $\mathrm{Z}$ must be an XP, since it is subject to movement as exemplified by How sick do you consider Jobn? (this incidentally is a further argument that NP* is external to VP). What then is W?

There are three alternatives:

(34)(1) W $\mathrm{W}$ XP as suggested by Manzini (1983). In this case NP* is not configurationally distinguishable from an adjunct to XP, except for the fact that it gets an external theta role from $\mathrm{X}$. Presumably, it does not qualify as an adjunct.

(2) $\mathrm{W}=\mathrm{X}^{\max }$ as suggested in Koopman and Sportiche (1985; 
1988). In this case, every $X$ projects to $X P$ and possibly to one further level.

(3) $\mathrm{W}=\mathrm{XP}$ as suggested by Larson (1988) for VP and adopted and extended to other categories in Sportiche (1990).

In the last case, the projection rules from the lexicon to syntax are radically altered so that the underlying structure of $\mathrm{W}$ in $(33 \mathrm{c})$ for example would be:

(35)
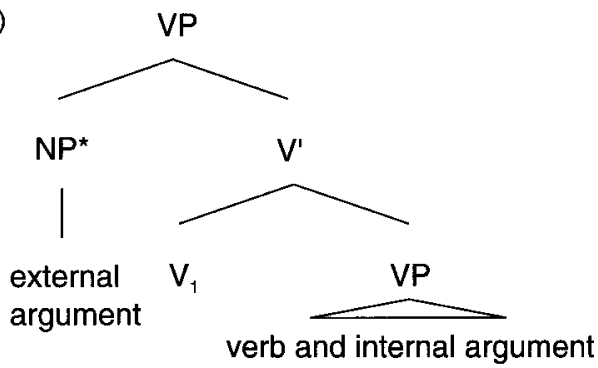

In derived structure, the verb presumably raises to $V_{1}$. In this structure, NP* is in [Spec, VP], but not of the minimal VP containing the verb in underlying structure. Deciding between these alternatives is not straightforward. One interesting argument against alternative (2) due to James Huang is not really conclusive. Because it provides independent support for the ISH, we will briefly present it and discuss it. This argument is based on a difference in behavior between the W constituent in (33a) and (33c).

An AP small clause as in (33a) cannot be preposed by movement:

(36)(a) You consider [John very sick].

(b) How sick do you consider John?

(c)* [John how sick] do you consider.

If it can be shown that the W constituent in (33c) can be preposed by movement, it would suggest that it is not a small clause.

The argument is based on the contrast between $W b$ Movement and VP-preposing with respect to reconstruction illustrated below:

(37)(a) Which paintings of each other do the girls say the boys like?

(b) Listen to each other, the girls say the boys do.

In (37a), the antecedent of the reciprocal can either be the main subject or the embedded subject. In other words, the binding theory can be satisfied either by the S-structure or by the reconstructed structure (i.e. as if the preposed phrase was still in its base position). In (37b), only one reading is possible, namely with the reciprocal taking the embedded subject as antecedent. Why 
is there a contrast? If VP preposing carries NP* along, we can derive this observation. Then the preposed VP is really [NP* listen to each other]. With the NP* counting as a subject for the binding theory, the reciprocal can only take it as antecedent to explain the lack of ambiguity of (36b). The subject of an AP small clause cannot be preposed but the subject of a VP must be: the conclusion then is that $\mathrm{NP}^{*}$ and VP do not form a small clause in the same way the subject of an AP small clause and the small clause do.

The first thing to notice is that the contrast in (37) and its analysis provide an argument for the ISH. The conclusion that NP* is internal to VP rather than a daughter to $\mathrm{V}^{\text {max }}$ holds only if the difference can only be accounted for by a difference in constituent structure. However, extending the range of data suggests that this argument is not conclusive. Consider reciprocal binding under $\mathrm{Wb}$ Movement of APs:

(38)(a) John considers [them proud of each orher].

(b) How proud of each other does John consider them?

(c)* They consider [John proud of each other].

(d)* How proud of each other do they consider John?

(e)* They say I am considered proud of each other.

(f)* How proud of each other do they say I am considered?

In order to account for the ungrammaticality of ( $38 \mathrm{~d}, \mathrm{f})$, we are led to assume that the AP preposed by $W b$ Movement also contains the subject of the AP small clause. By analogy with the VP case, we are led to the assumption that Jobn, the subject of the AP small clause, has raised out of the small clause (possibly for Case reasons):

(39)(a)* They consider John ${ }_{i} E_{W} t_{i}$ [proud of each other]].

(b)* $\left[_{\mathbb{W}} t_{i}\right.$ [How proud of each other $]$ do they consider John ${ }_{i}$.

This option is discussed in Sportiche (1990). Two consequences would follow. First, it would show that small clauses, that is the constituent W, can be preposed by movement. Second, it undermines the argument presented above against the $\mathrm{V}^{\max }$ hypothesis. Since the two authors of this article do not agree with each other on the underlying position of NP*, we will not try to argue one way or the other here. All three options of (34) are consistent with our discussions and basic thesis and we will continue using the notation $\mathrm{V}^{\max }$ as before.

\subsection{Properties of $N P^{\wedge}$ and of movement to $N P^{\wedge}$}

We now consider the status of the position $\mathrm{NP}^{\wedge}$.

Chomsky (1981) defines an A-position as a position to which a thetarole can be assigned. Given that $[\mathrm{Spec}$, IP $]$ never receives a theta-role, this 
definition would characterize it as an A-bar position. However, it is usually considered an A-position. Is it an A-position or an $\mathrm{A}$-bar position?

The distinction between $\mathrm{A}$ - and A-bar positions is mostly relevant to movement theory. Movement to an A-position has the properties of NP-movement, movement to an A-bar position of $W b$ Movement. Some of the relevant differences are listed below as $(40(1)-(6))$. Furthermore, the distinction is also relevant to the binding of lexical anaphors as indicated in $(40(7))$ :

(40)(1) Movement to an A-position obeys the SSC (cannot skip over a subject), movement to an $A$-bar position does not.

(2) Movement to an A-position must be from an A-position, movement from an A-bar position does not have to be.

(3) Movement to an A-position must be from a Caseless position. Movement to an A-bar position can be from a Case position (and must be if it is from an A-position).

(4) An A-position can be a Case position. An A-bar position cannot be. ${ }^{24}$

(5) Movement to an A-position does not license parasitic gaps, movement to an A-bar position does.

(6) Movement to an A-position does not create Weak Crossover Effects (viz. Everyone seemed to bis fatber t to be sick). Movement to an A-bar position may.

(7) A-positions may qualify as binders for lexical anaphors, A-bar positions may not (viz. *These boys, friends of each other $\left(i k e^{25}\right.$ ).

Since the ISH first appeared, various authors have addressed the question of the status of $\mathrm{NP}^{\wedge}$. For example, Diesing (1990) argues that $\mathrm{NP}^{\wedge}$ in Yiddish can be either an A-bar position or an A-position. Pesetsky (1990) has argued that $\mathrm{NP}^{\wedge}$ in English can be an A-bar position. Similarly, Guilfoyle et al. (1990) argue that objects can move to $\mathrm{NP}^{\wedge}$ over a subject, which entails that $\mathrm{NP}^{\wedge}$ may be an A-bar position. Koopman and Sportiche $(1985 ; 1988)$ took the position that $\mathrm{NP}^{\wedge}$ in English and French is always an A-position.

\subsubsection{NP^ can be an A-position}

In English, French or Arabic (with SVO order), we want to argue that NP ${ }^{\wedge}$ can be an A-position. Let us discuss whether or not it can be an A-bar position as well. Suppose $\mathrm{NP}^{\wedge}$ is an A-bar position. Then, by (40(4)) it cannot be a Case position. This means, contrary to what we have been arguing, that there is a way for $\mathrm{NP}^{*}$ to get Case in another way than by moving to $\mathrm{NP}^{\wedge}$. This is similar to Pesetsky's (1990) position. He argues that $\mathrm{NP}^{\wedge}$ in main clauses can be an A-bar position, but that $\mathrm{NP}^{\wedge}$ is an A-position in embedded clauses. According to him, sentence (41a) has the $w h$-phrase in $\mathrm{NP}^{\wedge}$ and the modal in Infl: 
(41)(a) Who will John see?

(b) John will see Bill.

(c)* Bill will John see.

He argues that Jobn in (41a) gets Case directly from Infl - what we call governed nominative case - and so does not have to move to $\mathrm{NP}^{\wedge}$. (41b) illustrates that $\mathrm{NP}^{\wedge}$ does not have to be a $w b$-phrase. Where does Jobn in (41b) get Case from? If it got governed Case from Infl, this would imply that $\mathrm{NP}^{\wedge}$ is an A-bar position here too, and that movement of the subject to $\mathrm{NP}^{\wedge}$ is $\mathrm{A}$-bar movement. The question then arises what would exclude (41c)? In (41c), the subject has remained in its Case position, and the object has moved to $\mathrm{NP}^{\wedge}$. Since this would be an instance of A-bar movement, the intervening subject is irrelevant. (41b) also shows that $\mathbf{N P}^{\wedge}$ (unlike $[\mathrm{Spec}$, $\mathrm{CP}$ ) tolerates non-wb NPs, albeit only subjects.

This situation is in fact reminiscent of the Arabic situation described earlier in Section 1.3.2. In Arabic, both orders Infl SOV and S Infl OV are allowed. In the derived VSO order (with V in Infl), there is no number agreement between $\mathrm{V}$ and $\mathrm{S}$. In the SVO order (with $\mathrm{V}$ in Infl and $\mathrm{S}$ in $\mathrm{NP}^{\wedge}$ ), $\mathrm{S}$ and $\mathrm{V}$ agree in number. Furthermore, nothing other than the subject can move to immediate preverbal position and trigger verbal agreement. If the subject can get Case postverbally, why is movement to preverbal position not A-bar movement, and why cannot anything other than the subject move to preverbal position?

The ungrammaticality of (41c) (and of parallel Arabic examples) suggests that $\mathrm{NP}^{\wedge}$ is an A-position in (41c) and (41b). (41c) would then be excluded as an SSC violation.

Let us suppose for a moment that Pesetsky's (1990) analysis for (41a) is correct. This would mean that $\mathrm{NP}^{\wedge}$ is an A-bar position in (41a), but an Aposition in $(41 \mathrm{~b}, \mathrm{c})$. How can we reconcile the two?

We could postulate that matrix Infl in English and Infl in Arabic assigns Case either by agreement or under government. If it assigns Case under government to a following $\mathrm{NP}, \mathrm{NP}^{\wedge}$ is an A-position. If it assigns Case by agreement to $\mathrm{NP}^{\wedge}, \mathrm{NP}^{\wedge}$ is an $\mathrm{A}$-position. In (41a), Case has been assigned under government. This is not quire sufficient. In (41c), we need to prevent Case from being assigned to Jobn under government. Otherwise, we face the same problem: $\mathrm{NP}^{\wedge}$ would be an A-bar position and the object could move there. We need an Auxiliary Hypothesis (AH) stipulating that when $\mathrm{NP}^{\wedge}$ is an A-bar position, only wh-phrases can occur there (much like [Spec, CP]). (41c) would violate either this last property if $\mathrm{NP}^{\wedge}$ is an A-bar position, or the $\mathrm{SSC}$, if $\mathrm{NP}^{\wedge}$ is an A-position. Movement to $\mathrm{NP}^{\wedge}$ in (41b) would then have to be interpreted as an instance of NP-movement.

Pesetsky's main argument is based on the distribution of auxiliary do. Unlike, say, the auxiliary have, do does not invert in counterfactuals: 
(42)(a) If John had eaten $\rightarrow$ Had John eaten

(b) If John did eat $\rightarrow *$ Did John eat

Pesetsky suggests that the ungrammaticality of $(42 \mathrm{~b})$ is due to the unexpected inability of $d_{0}$ to raise to $\mathrm{C} .{ }^{26} \mathrm{He}$ is then led to assume that do in who did John see too has failed to raise to $C$, remaining in Infl. This in turn leads to the analysis discussed above for (41a), according to which Infl in main clauses does not raise to $\mathrm{C}$ (in fact main clauses are never CPs, but always IPs). This hypothesis does not apply to embedded clauses. If it did, we would expect Subject-Aux Inversion to apply in embedded clauses as well: $\mathrm{NP}^{\wedge}$ would be an A-bar position, the subject would get governed Case from Infl and should therefore be able to appear in post-Infl position, contrary to fact: *I wonder who will John see.

Pesetsky's analysis leaves some questions unanswered however. One minor question concerns the status of $\mathrm{AH}$ : $\mathrm{AH}$ seems to reduplicate a stipulation that has to be made for [Spec, CP]. A second question concerns the difference between Arabic and English. Arabic shows that assigning governed Case is not a characteristic property of main clause Infl. In Arabic, all tensed Infl do. It seems then that we need to stipulate the difference between main clause Infl and embedded clause Infl in English. A third and more serious question concerns agreement. As Arabic shows, the failure of the subject to raise to $\mathrm{NP}^{\wedge}$ correlates with a lack of number agreement on Infl. However, this is not true of English:

(43)(a) Which children does John like?

(b)* Which children do John like?

Arabic, or Irish or Welsh show that assignment of (governed) nominative Case does not correlate with agreement. How then is the subject verb agreement in (43a) established? The well-supported view that agreement results from a spec/head relation is consistent with the standard analysis of (43a), Jobn is in [Spec, IP] receiving nominative Case from it and agreeing with it. Infl moves to $\mathrm{C}$ and the $w$ b-phrase is in [Spec, CP]. ${ }^{27}$ In Pesetsky's analysis, there never is a Spec/head relation between Infl and the subject. Summarizing: there is no question English $\mathrm{NP}^{\wedge}$ can function as an A-position. Whether it can also be an A-bar position is less clear, and depends on how one accounts for the distribution of $d o$, and the landing site of $W b$ Movement in main clauses.

Both alternatives are compatible with our basic views. We have argued that under each alternative, movement to $\mathrm{NP}^{\wedge}$ must be NP-movement-like in certain cases, for example when a non $w b$-phrase moves to $\mathrm{NP}^{\wedge}$ (as is required by e.g. our analysis of Q-float in Section 1.3.3).

We can distinguish two questions. The first question, which we addressed above, asks specifically whether $\mathrm{NP}^{\wedge}$ is sometimes an A-bar position in English. The second, more general question asks what can count as an A-bar position. Can $\mathrm{NP}^{\wedge}$ ever be an A-bar position? 
Given the definitions of A and A-bar positions in Chomsky (1981), A-bar positions are NP-positions which are either adjoined positions or [Spec, $\mathrm{CP}$ ]. We present in the next section some reasons to believe that this inventory is too restricted and that [Spec, ASPP] can count as an A-bar position in Kilega, and as an A-position in English. This conclusion suggests that the set of A-bar positions is larger than suggested by Chomsky's (1981) definitions, and that certain positions can in principle have either status. This lends further plausibility to Pesetsky's proposal for English, Diesing's for Yiddish or Guilfoyle, Hung and Travis's for Malayo-Polynesian languages.

\subsection{2 (Spec, ASPP\} as an A-bar position or an A-position}

Carstens and Kinyalolo's (1989), Kinyalolo's (1991) and Kinyalolo and Carstens's (1988) work on Kilega, a Bantu language spoken in Zaire, provides very strong support for the idea that [Spec, ASPP] can be an A-bar position. Here, we will outline the logic of the argument, referring to the original work for details.

Kilega displays a very extensive agreement system. If agreement reflects Spec/head relations, it can be used as a very reliable diagnostic of the path taken by moved phrases. Kilega is a head initial language. In main clauses, the verb is basically in second position: in a simple tense, the word order is XP V ... with V carrying Tense (and other) morphological affixes. Nouns are partitioned into classes. $\mathrm{V}$ agrees with XP. The agreement affix depends both on the the Class membership of XP and, for Class 1, on the kind of XP (e.g. wh-phrase or not). XP can be the subject, but it can also be an object from the same clause (a possibility dependent on the choice of the verb that we will ignore here), or a $w$ b-phrase either from the same clause or from some embedded clause (we omit tone markings here): ${ }^{28}$
(44)(a) Kasanganjo a-ku-bak-il-a
Kabisuba nyumba Kasanganjo 1AGR-PROG-build-APPL-FV Kabisuba house Kasanganjo is building a house for Kabisuba
(b) biki bi-a-kas-il-e bako bikulu
8-what 8AGR-A-give-APPL-FV 2-that 2-woman
mwami mu-mwilo
1-chief 18-3-village
What did those women give the chief in the village?

If XP is not the subject as in (44b), the subject immediately follows the verbal complex thereby preceding all other dependents of the verb as well as adjuncts.

A similar pattern occurs in complex tenses in which a string of verbal elements is found (much like a string of English aspectual auxiliaries). In this case, we find the order $\mathrm{XP} \mathrm{V}_{1} \mathrm{~V}_{2} \ldots$ with all the verbs in the sequence but the main verb agreeing with $X P$. 
(45)(a) Nzogu zi-kili z-a-twag-a maswa. 10-elephants 10AGR-be still 10AGR-A-stampede-FV 6-farm.

The elephants are still stampeding over the farm.

(b) Buni bu-kili bu-a-twag-a

14-how come 14AGR-be still 14AGR-A-stampede-FV nzogu maswa.

10-elephants 6-farm.

How come the elephants are still stampeding over the farm?

(c) Biki bi-ete bi-ku-tenda

8-what 8AGR-ASP 8AGR-PROG-say

bána bi-tw-a-kit-ele.

2-children 8AGR-1PL-PT-do-PERF.

What are the children saying we had done?

As Kinyalolo (1991) and Carstens and Kinyalolo (1989) show, the sequence of verbs is a sequence Infl V V V, with Infl carrying the tense morpheme, the last verb being the main verb, and all the intermediate verbs being aspectual auxiliaries projecting to ASPP:

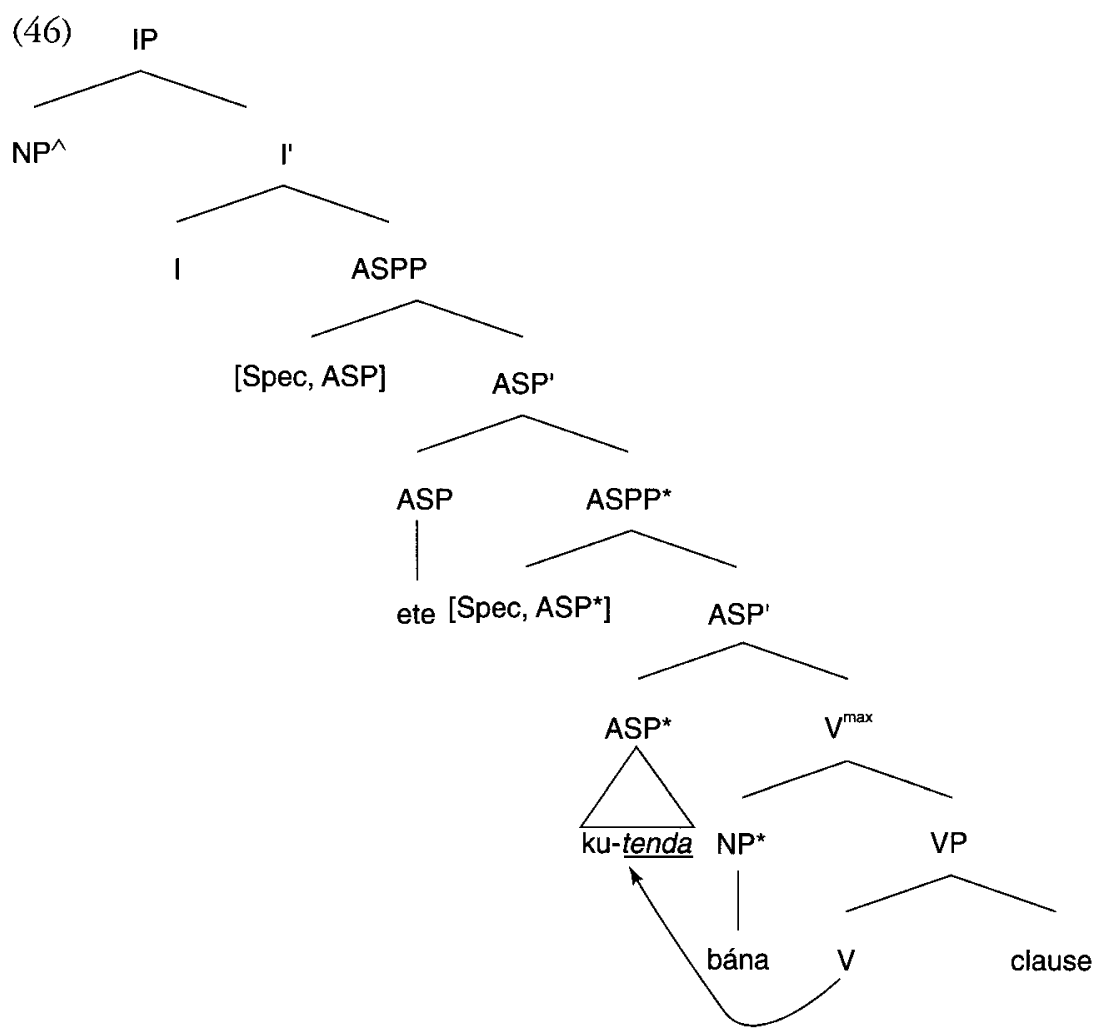


In particular, they very convincingly show that no clausal boundary (CP) intervenes between I and ASPP or between the two ASPP.

Note incidentally that the distribution of the subject in (45c) provides one further argument for the ISH since we find the order XP Infl ASP ASP VSO. The argument is similar to that based on the Celtic languages in Section 1.3.1. The argument here is in fact stronger since there is no possibility, as in the Celtic case, of arguing that the initial V is actually in C. Even if it is in Kilega, the other verbs are not. Note also a further difference with Welsh: the subject actually seems to occur in NP*, not in a position governed by the highest INFL.

The crucial example is $(45 \mathrm{c})$. It shows that a $w h$-phrase coming from an embedded clause triggers agreement on all the aspectual verbs of the main clause. In order to escape its own clause, this $w h$-phrase must transit through some intermediate A-bar position, namely the embedded [Spec, CP]. This is what allows wh Movement out of C-headed clauses and prevents NP Movement out of $\mathrm{C}$-headed clauses. In order to trigger agreement on the intermediate aspectual verb ASP*, the $w h$-phrase must transit through [Spec, ASPP*]. Consequently, this position cannot be an A-position. Otherwise, we would have a case of improper movement. Of course, the validity of this argument rests on the assumption that agreement on ASP* cannot be triggered in any other way, and in particular, that it cannot be triggered by the adjunction of the $w h$-phrase to ASPP*. Participle agreement in French shows that agreement cannot be triggered by adjunction, as Sportiche (1990) shows. As mentioned above in Section 2.4, participles can agree with objects preposed under $W b$ Movement:

(47)(a) L'écharpe que tu as offertE t.

The scarf(FEM) that you have given(FEM) t. The scarf that you have given.

(b) L'écharpe que tu as dit $\left({ }^{*} \mathrm{E}\right)$ que tu as offertE. The scarf(FEM) that have said that you have given(FEM) $t$. The scarf that you said you gave.

(c) La femme que tu as ditE [ $t$ belle].

The woman(FEM) that you have said(FEM) pretty. The woman that you said was pretty.

(47b,c) shows that agreement is not possible if the object triggering agreement comes from an embedded tensed clause, but is possible with the subject of an embedded small clause. If agreement could be triggered by adjunction, we would expect adjunction of the moved object in (47b) to the phrasal projection of the participle (which we take to be [Spec, VP], or of the associated AGRP) to licitly trigger agreement. Sportiche (1990) shows that the pattern in (47) follows from the fact that the position triggering participle agreement is an A-position. 
The same kind of questions arise in Kilega as in English. Is the clause initial constituent $\mathrm{XP}$ in [Spec, CP] or is it in [Spec, IP] with possibly no CP at all? Because of this problem, agreement of the first verb with a $w h$-phrase does not straightforwardly lead to a similar conclusion concerning $\mathrm{NP}^{\wedge}$. This issue is discussed in Carstens and Kinyalolo (1989) and Kinyalolo (1991).

[Spec, ASPP] can also be an A-position. This is the case in English (or French). Consider the structure of the English sentence They will have visited Paris, where ASPP is the projection of the aspectual auxiliary bave:

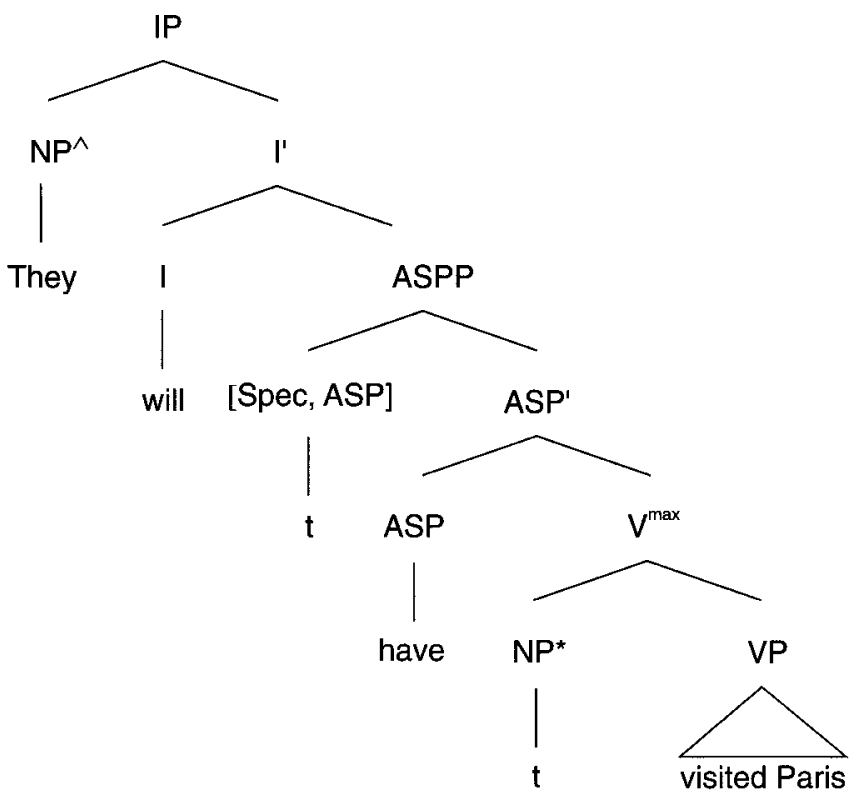

Suppose the derivation is as indicated, with a trace in [Spec, ASPP]. Then, [Spec, ASPP] must be an A-position, since movement to $\mathrm{NP}^{\wedge}$ of they must count as $A$-movement as discussed above. There are two reasons to support the existence of the intermediate trace. The first one is based on Sportiche's (1988b; 1990) theory of NP Movement which explains all the constraints on NP Movement by requiring that it be Spec to Spec movement with no possibility of skipping any intermediate specifier position. The second is based on the possibility of having floated Qs between will and bave as in They will all bave visited Paris. This possibility follows from the possibility of moving stepwise to $\mathrm{NP}^{\wedge}$.

\subsubsection{A-positions and A-bar positions}

The above discussion leads to the following conclusions concerning the way in which NPs in Spec distribute as A and A-bar positions: 
THE POSITION OF SUBJECTS

Adjoined positions and $[\mathrm{Spec}, \mathrm{CP}]$ are $\mathrm{A}$-bar positions

[Spec, VP] and/or [Spec, $\left.\mathrm{V}^{\max }\right]$ are A-positions

[Spec, IP] can be an A-position (English, French, Arabic) and, possibly, an

A-bar position (English, Yiddish, Malayo-Polynesian)

[Spec, ASPP] can be an A-position (English, French) or an A-bar position (Kilega).

From this, we can conclude that Chomsky's (1981) definitions need to be revised. How to do this is unclear at this moment.

\section{Notes}

* The first section of this article has been circulated as part of Koopman and Sportiche (1988) and is a written version of talks given in vatious places. It was given in March 1985 at the GLOW conference in Brussels as Koopman and Sportiche (1985), at the June 1985 CLA meeting in Montreal, at MIT and Umass Amherst in the winter of 1986 , and presented at UCLA and USC since. The contributions of these audiences are gratefully acknowledged. The second section is almost completely new.

1 For related ideas on what we call the canonical position of subjects, see Contreras (1987), Kitagawa (1986), Kuroda (1988), Speas (1986), Zagona (1982).

2 Throughout the paper, we will use $\mathrm{NP}^{\wedge}$ and $\mathrm{NP}^{*}$ to refer either to these particular positions or to the content of these positions. The context will make it clear what is meant in each particular case. We also need to distinguish between the various projections of $\mathrm{V}$. We call VP the phrasal projection of $\mathrm{V}$, while $\mathrm{V}^{\max }$ is its maximal projection.

3 The claim appears to be different from the case of raising verbs because raising is a property of individual verbs, not of a grammatical category. We want to claim that I never has a deep subject and that when it does have a surface subject, it is by raising.

4 The implication could plausibly be attributed to something other than thematic properties. Suppose the correct generalization is that all verbs involving a change of state can be put in the progressive. Since agentive verbs are always change-ofstate verbs they can be put in the progressive: reference to thematic structure is only indirect.

5 It is clear, however, that our proposal is not essentially tied to this issue and a ternary structure in (2) or in small clauses would be acceptable.

6 Further supporting arguments can be constructed on the basis of the head complement relations found in categories other than $\mathrm{V}$, for example.

7 We follow Chomsky (1986) in assuming that the $\mathrm{C}$ projection conforms to all others, with $\mathrm{CP}=\mathrm{S}$-bar. We will use Comp informally to refer to $\mathrm{C}$ or specifier of $C P$.

8 If it is Infl that preposes around S, then it cannot be to C, as already discussed. (We do not consider the possibility that I adjoins to an overt C.) If there is some intermediate head to which Infl moves, it means that $S$ is not the highest specifier of the complement of $\mathrm{C}$. But this would mean that the situation is unlike that of English, assuming the standard clause structure. 


\section{PARTITIONS AND ATOMS}

9 We disregard here gender agreement, cf. Mohammad (1989) for more discussion.

10 We differ here from Sportiche's (1988a) exact formulation of this process, and follow Koopman's (forthcoming) (for English and French) and Shlonsky (1991) (for Hebrew), who argue that the $Q$ actually is the head of the constituent [all [the people], taking an NP complement. NP moves through [Spec, QP] leaving $Q$ stranded.

11 (10) seems necessary (and sufficient for our present purpose), but it is not the strongest possible condition that idioms must meet: a plausible strengthening would extend it to all the intermediate constituents: if XP contains idiomatic material, $\mathrm{X}^{\mathrm{o}}$ is fixed. Thanks to Richard Carter for useful discussion on this topic.

12 The term external might need to be slightly redefined. The distinction external/ internal is still necessary, of course.

13 By specifier of $\mathrm{X}^{\mathrm{n}}$ (resp. $\mathrm{X}^{0}$ ), we mean no more than a ZP (left - in English or French) daughter of $\mathrm{X}^{\mathrm{n}}$ (resp. of some projection of $\mathrm{X}^{0}$ ). A category can have several specifiers: for example, in the $\mathrm{V}$ projection, the position NP* and the left daughter of VP are both V specifiers. When we do not specify, we always mean the daughter of the phrasal projection, i.e. of VP.

14 We ignore here the complex cases of nominative assignment to postverbal NPs found in Italian and other languages discussed in Borer (1986).

15 Note that we do not mean that lack of agreement between a head $\mathrm{H}$ and an NP getting Case from it is always an indication that governed case rather than Case by agreement is involved. We want to restrict this conclusion to situations in which the head $\mathrm{H}$ can show agreement. Therefore, this conclusion would not follow for heads never exhibiting agreement: we take lack of agreement with an agreeing head to be an indication that this head has not assigned Case by agreement. However, we agree with Borer (1986) that the converse is not true. The Kilega facts discussed in Section 2.5 show this as well. Presence of agreement does not indicate that Case by agreement has been assigned.

16 Recently, various proposals have suggested that Tense and Agreement should each have their own projections: Pollock (1989). If Agreement is what is responsible for nominative Case assignment the text must be amended accordingly throughout. Given the text, we would have to assume, in agreement with Chomsky and unlike Pollock, that the AGRP projection corresponding to subject agreement must be higher than the projection of Tense, rather than the opposite, so as to force raising to its Case assigning position (specifier of AGR) at the beginning of the clause. Note that this assumption is supported by the respective placement of tense and agreement affixes: it seems that agreement morphology on verbs is external to tense morphology, as expected.

17 More needs to be said about the distribution of pronominals in Welsh, cf. Koopman (forthcoming).

18 Both the copula and the aspectual markers might actually project to maximal projections; this will not affect the argument here. Crucial for our argument is the assumption that the aspectual categories are raising categories.

19 Note that $C$ is never a governor. In Chomsky's (1986) terminology, it does not L-mark its complement IP so that IP is always a barrier to government. This is further discussed in Sportiche (1988b; 1990).

20 In embedded clauses, one would expect this PRO to receive a control reading, 


\section{THE POSITION OF SUBJECTS}

contrary to the facts. Stenson (1989) attributes this to the fact that only nonfinite complements are c-selected by control verbs.

21 We have not really established that objects are theta-marked under $V^{\prime}$; it could be that objects are theta-marked in [Spec, VP]. For arguments against this view, see Koopman (forthcoming).

22 Note that we cannot link $\mathrm{AGR}_{\mathrm{S}}$ with external argumenthood, as shown by the case of subjects derived from underlying objects.

23 Note that in the case of NPs, the parallelism is not with NP small clauses of the type $I$ consider this a good picture for the NP this does not and cannot correspond to the external argument of the noun as noted in Campbell (1989). This is shown by the grammaticality of I consider this picture John's picture and by the impossibility of *I consider the engineer('s) survey of the valley. Rather the subject of an NP small clause is an additional argument.

24 There might be exceptional counterexamples to (3) and (4), e.g. the French constructions discussed in Kayne (1984: Chapter 5).

25 Note that this is much worse than a Weak Crossover Violation and that WCO effects are absent here anyway: These boys, their friends like.

26 Note however that although main verbs in Dutch clearly occur in C, past tensed main verbs (which would correspond to (42)) cannot raise to $C$ in counterfactuals. This suggests a different analysis. It is not the case that do does not occur in $\mathrm{C}$; rather the simple past tense cannot substitute for the particular $\mathrm{C}$ if.

27 The questions of why the agreement relation is retained despite the movement of Infl to $C$, and why $C$ (containing $\operatorname{Infl}$ ) does not agree with its own specifier need to be answered, of course (cf. Sportiche (1990)).

$28 \mathrm{nAGR}$ is agreement with class n; PROG is the progressive affix; APPL is the applicative suffix; PERF is the perfective affix; ASP is an aspectual verb; $f v$ is the final vowel.

\section{References}

Aoun, J. and Li, A. (1989) "Scope and constituency," Linguistic Inquiry 20(2): $141-72$.

Aoun, J. and Sportiche, D. (1983) "On the formal theory of government," The Linguistic Review 2: 211-36.

den H. Besten (1983) "On the interaction of root transformations and lexical deletive rules," in W. Abraham (ed.) On the Formal Syntax of West-Germania, 47-131, Amsterdam: Benjamins.

Borer, H. (1986) "I-subjects," Linguistic Inquiry 17(3).

Campbell, R. (1989) "The grammatical structure of verbal predicates," unpublished Ph.D. dissertation, UCLA.

Carstens, V. and Kinyalolo, K. (1989) "On IP structure: tense, aspect and agreement," unpublished manuscript, Cornell University and UCLA.

Chomsky, N. (1981) Lectures on Government and Binding. Dordrecht: Foris. (1986) Barriers. Cambridge, Mass.: MIT Press.

(1989) "Some notes on economy of derivation and representation," in I. Laka and A. Mahajan (eds), "Functional heads and clause structure," MIT Working Papers in Linguistics 10: 43-74. 


\section{PARTITIONS AND ATOMS}

Chung, S. and McCloskey, J. (1987) "Government, barriers and small clauses in modern Irish," Linguistic Inquiry 18(2): 173-237.

Contreras, H. (1987) "Small clauses in Spanish and English," Natural Language and Linguistic Theory 5(2): 225-44.

Diesing, M. (1990) "Verb movement and the subject position in Yiddish," Natural Language and Linguistic Theory 8(1).

Emonds, J. (1978) "The verbal complex V'-V in French," Linguistic Inquiry 9: $151-75$.

- (1981) "Word order in generative grammar," Journal of Linguistic Research 1: 33-54.

Fillmore, C. (1968) "The case for case," in E. Bach, R. T. Harms (eds) Universals of Linguistic Theory, New York: Holt, Rinehart and Winston.

Guilfoyle, E., Hung, H. and Travis, L. (1990) "Spec of IP and Spec of VP: two subjects in Malayo-Polynesian languages," unpublished manuscript, McGill University and Brandeis University.

Harlow, S. (1981) "Government and relativization in Celtic," in F. Heny (ed.) Binding and Filtering, Cambridge, Mass.: MIT Press.

Hornstein, N. (1977) "S and the X-bar convention," Linguistic Analysis 3(2).

Jones, M. and Thomas, A. (1977) The Welsh Language: Studies in Syntax and Semantics, Cardiff: University of Wales Press.

Kayne, R. S. (1984) Connectedness and Binary Branching. Dordrecht: Foris.

- (1985) "L'accord du participe passé en français et en italien," Modeles Linguistiques 7: 73-80.

Kitagawa, Y. (1986) "Subject in Japanese and English," unpublished doctoral dissertation, University of Massachusetts at Amherst.

Kinyalolo, K. (1991) "Syntactic dependencies and the spec/head agreement hypothesis in Kilega," doctoral dissertation, UCLA.

Kinyalolo, K. and Carstens, V. (1988) "Compound tenses in Bantu: a case for T" and $\mathrm{ASP}^{\prime \prime}$ in UG," paper presented at the 19th Conference on African Linguistics, Boston University.

Koopman, H. (1984) The Syntax of Verbs: From Verb Movement Rules in the Kru Languages to Universal Grammar, Dordrecht: Foris.

— (1987) "On the absence of case chains in Bambara," unpublished manuscript, UCLA.

(forthcoming) "Restrictions on SPEC positions."

Koopman, H. and Sportiche, D. (1985) "Theta theory and extraction," in GLOW Newsletter 14: 57-58, reprinted in this volume as Chapter 8. (1988) "Subjects," unpublished manuscript, UCLA.

Kuroda, Y. (1988) "Whether we agree or not: a comparative syntax of English and Japanese," Linguisticae Investigationes 12: 1-47.

Larson, R. (1988) "On the double object construction," Linguistic Inquiry 19: 335-92.

Manzini, R. (1983) "Restructuring and reanalysis," unpublished doctoral dissertation, MIT Press.

(1979) “Assessing the X-bar convention," unpublished manuscript, MIT.

- (1984) "On the nature of grammatical relations," Cambridge, Mass.: MIT Press.

May, R. (1977) “The grammar of quantification,” doctoral dissertation, MIT. 


\section{THE POSITION OF SUBJECTS}

(1985) Logical form. Cambridge, Mass.: MIT Press.

McCloskey, J. and Hale, K. (1984) "On the syntax of person-number inflection in modern Irish," Natural Language and Linguistic Theory 1: 487-533.

Mohammad, M. (1989) "The problem of subject verb in Arabic: towards a solution," in Mushira Eid (ed.) Perspectives in Arabic Linguistics, Amsterdam: Benjamins.

Pesetsky, D. (1990) "Language particular processes and the earliness principle," unpublished manuscript, MIT.

Pollock, J.-Y. (1989) "Verb movement, UG and the structure of IP," Linguistic Inquiry 20(3): 365-424.

Shlonsky, U. (1991) "Quantifiers as functional heads: a study of quantifier float in Hebrew," Lingua 84(2/3) 159-80.

Speas, M. (1986) "Adjunctions and projections in syntax," unpublished doctoral dissertation, MIT.

Sportiche, D. (1983) "Structural invariance and symmetry in syntax," MIT dissertation, to be published by Foris, Dordrecht.

(1988a) "A theory of floating quantifiers and its corollaries for constituent structure," Linguistic Inquiry 19(3): 425-49 reprinted as Chapter 1 in this volume. (1988b) "Conditions on silent categories," unpublished manuscript, UCLA.

_ (1990) "Movement, agreement, constituent structure and case" (provisional title), unpublished manuscript, UCLA, reprinted as Chapter 3 in this volume.

Sproat, R. (1985) "Welsh syntax and VSO structure," Natural Language and Linguistic Theory 3(2): 173-216.

Stenson, N. (1989) "Irish autononous impersonals," Natural Language and Linguistic Theory 7(3): 379-407.

Stowell, T. (1981) "The origins of phrase structure," unpublished Ph.D. dissertation, MIT.

(1983) "Subjects across categories," The Linguistic Review 2: 285-312.

Van Riemsdijk, H. (1978) A Case Study in Syntactic Markedness: The Binding Nature of prepositional pbrases, Dordrecht: Foris.

Zagona, K. (1982) "Government and proper government of verbal projections," unpublished doctoral dissertation, University of Washington at Seattle. 


\section{MOVEMENT, AGREEMENT AND CASE}

\section{Introduction}

How much do the Theory of Movement and the Theory of Government have in common? The Theory of Movement deals basically with superficially unbounded syntactic dependencies between identical categories, two NPs as in NP movement, two identical phrasal projections as in Wb Movement or two identical word level categories as in head movement. By contrast, the Theory of Government deals with extremely local syntactic relations between categories of different levels, most of the time a word level category and an NP as in Case assignment, theta role assignment or agreement relations.

Nevertheless, there have been several attempts (e.g. Chomsky (1986a), Kayne (1984)) to unify the Theories of Government and Movement usually by reducing movement properties to government properties. This has been done by construing constraints on movement as violations of required government. One of the most suggestive generalizations pointing in this direction is Huang's (1982) Constraint on Extraction Domain (CED) which states a constraint on movement explicitly in terms of government.

Here, I will pursue the goal of trying to reduce the constraints on movement processes to the Theory of Government by developing the idea that movement is so strictly local that each movement step is a movement from a governed position to a governing position. I will present a very specific version of this idea. Its central intuition is illustrated by the contrast between the two examples in (1):

(1)(a) Who did you see $\left[_{\mathrm{NP}}\right.$ pictures of $\left.t\right]$ ?

(b)* Who did you see $\left[_{\mathrm{NP}}\right.$ John's $\left[_{\mathrm{N}^{\prime}}\right.$ pictures of $\left.\left.\mathrm{t}\right]\right]$ ?

This contrast has been taken to suggest that $w h$-traces are anaphors because of their sensitivity to the presence of subjects (as e.g. in Chomsky (1976), Aoun (1984)). But why should intervening subjects matter?

There is a traditional way to handle the difference between the first two examples which requires no particular elaboration, and which is illustrated by the usual treatment of $w h$-islands, the ungrammaticality of the indicated movement \#1 in (2): 
This is due to the facts that (1) unbounded movement \#1 is prohibited, and (2) the necessary intermediate step \#2 is impossible because the intermediate landing site is already filled (by where). The analogy between (1) and (2) is immediate, if the (Spec, NP) containing John in (1b) is also a necessary intermediate step. We could construe this idea as requiring that specifier positions are necessary intermediate steps for movement. Subjects block movement because they are specifiers. However, this assumption is superficially contradicted by the underlined intervening subjects in cases such as:

(3)(a) I wonder [who [you think you saw t]].

(b) I wonder [what [you consider [these pictures pictures of $\mathrm{t}]]$ ].

The specifier of a CP or the specifier of an NP block Wb Movement. The intervening subject of a small clause or the subject of a clause does not block Wh Movement. Furthermore, for NP Movement, all subjects or specifiers of CP block movement. This raises the questions: what is the set of intervening specifiers (or subjects) which block movement and why is this set dependent on the kind of movement involved?

The Theory of Movement Processes I will develop here was first outlined in Sportiche (1987) and further developed in Sportiche (1988b). It takes this analogy between (1) and (2) as fundamental in that it will require as an intermediate landing site for a moving XP all the (Spec, YP) positions of the YPs out of which XP is extracted, except if adjunction to YP is permitted, as illustrated in the diagram below: movement of XP out of YP must proceed either as in \#1 or as \#2:

(4)

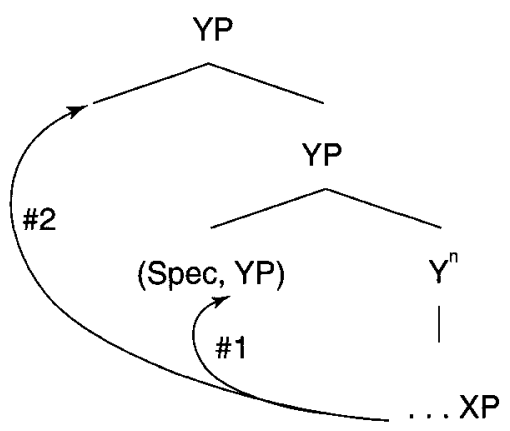

Essentially, I will adopt many of the background assumptions and general approach of Chomsky (1986), with a number of significant differences some of which I list here (returning to them in more detail in the next section). 
First, I suppose that every $\mathrm{XP}=\mathrm{X}^{\mathrm{p}}$ can have as immediate daughter an NP position which I call its specifier or (Spec, XP). In other words, every XP can be of the form $\left[{ }_{\mathrm{XP}} \mathrm{NP} \mathrm{X}^{\mathrm{p}-1}\right]$. Furthermore, [Spec, XP] is an A-position (i.e. a possible landing site for NP Movement) for every $\mathrm{X}$ except $\mathrm{X}=\mathrm{C}$. Second, I adopt the assumption that movement is substitution or adjunction of $\mathrm{X}^{\mathrm{n}}$ into or to $\mathrm{X}^{\mathrm{n}}$ and I suppose that adjunction of XP is only possible in the case of $\mathrm{A}$-bar movement (Wh Movement) and not in the case of A-movement (NP Movement). I will assume, unlike Chomsky (1986a), that VP, IP and AP are possible adjunction sites, while NP, CP and PP are not.

The consequences of these assumptions are clear. In the case of NP Movement, since movement as \#1 is not possible, movement as in \#2 is forced. Every NP specifier (i.e. every subject) will block NP Movement. In the case of $W b$ Movement, a specifier will block movement just in case option \#2 is excluded, i.e. when adjunction is forbidden: subjects or specifiers of CP, NP and PP will block Wh Movement, while those of IP, AP or VP will not.

The link between the Theory of Movement and the Theory of Government is established by requiring that traces meet the following general condition:

Condition on chain links

$t$ must be governed by an antecedent

where, as in Chomsky (1986a), government is defined in terms of barrier.

Section 2 describes and justifies some of the background assumptions we adopt. One of the most significant assumptions is our adoption of a modified version of Larson's (1988) construal of the VP-Internal Subject Hypothesis and the concomitant modifications of theta theory and the projection rules from the lexicon onto syntactic structure.

In Section $3,{ }^{1}$ I will show how this general condition, by forcing movement to take place as in (6), has the following effects:

1 It covers the facts above.

2 It subsumes the Subjacency Condition and the Condition on Extraction Domain (i.e. the $w$-island constraint, the sentential subject constraint, the subject condition, the adjunct condition, the condition on P-stranding, the complex NP constraint).

3 It subsumes the Head Movement Constraint.

4 It subsumes Principle A of the Binding Theory as it applies to traces and consequently it handles the locality of passive and raising NP Movement, the impossibility of the super raising constructions and the problematic facts discussed in Lasnik (1985).

This will establish that movement through a specifier position plays a fundamental role in explaining the properties of movement dependencies. 
In Section 4, I will apply this theory to the treatment of French clitic constructions. I will discuss how clitic movement should be analyzed as a combination of NP Movement, Wh Movement and head movement and why this explains both why it seems to display Specified Subject Condition effects and also seems to be able to move over subjects. I will then apply this analysis to past participle agreement in French and discuss how (most of) the properties of past participle agreement in French in clitic constructions and passive constructions simply follow from the system presented here.

In Section 5, I will discuss asymmetries between objects under agreement. This will lead me to propose substantial modifications of Case Theory and Agreement Theory which will further motivate the modified Larsonian approach to the constituent structure adopted in Section 2. I will argue that there is a substantial link between structural Case and agreement in languages similar to French. Among other things, I will discuss the notion that accusative objects are subjects of VP, that "Raising to Object" exists and is reducible to raising to subject, and that Burzio's Generalization can be derived.

In Section 6, I will show that an extension of Larsonian structures to NPs, coupled with this revised Case Theory, explains a substantial number of properties of extraction from NP and NP internal syntax previously left unexplained.

\section{Background assumptions}

In this section, I will outline some background assumptions on the various submodules of grammar. As I mentioned earlier, I will be driven to modify some of them in radical ways.

\section{$2.1 X$-bar Theory}

Following and elaborating on Chomsky (1986a), I assume that the X-bar schema strongly constrains every syntactic category. By this, I mean that in core grammar:

(6)(a) There is no difference of internal structure across categories in a given language: all its XPs are structurally identical.

and also possibly:

(b) There is no difference in internal structure of XPs across languages.

The consequences outlined in Chomsky (1986a) concerning C, I, A, N, V, P (or possibly, TP and AgrP if we split I into Tense and AGR as in Pollock (1989), and possibly more: Neg, Asp as in Carstens and Kinyalolo (1989), 
Koopman (1988)) thus hold, in particular for non-lexical categories (that is C, inflectional categories that I will note (annotated) I).

However, I also adopt the idea expressed by (a) above. Concretely, it means that the following reasoning is acceptable: assuming English allows a full NP as a specifier of NP (e.g. John's book), we may conclude that every XP in English allows an NP specifier. Following (b), we should conclude that this is true crosslinguistically. ${ }^{2}$ Accordingly, every category is of the form: ${ }^{3}$

(7) $\quad\left[\mathrm{XP}_{\mathrm{XP}} \operatorname{spec}\left[\mathrm{X}^{\mathrm{n}} \ldots\right]\right]$

Note that I define the specifier of $\mathrm{X}^{\mathrm{p}}$ as the YP (left-) daughter of $\mathrm{X}^{\mathrm{p}}$. I will also refer to the specifier of YP as the specifier of $Y \mathrm{P}$ or of $\mathrm{Y}^{0}$. Examining $\mathrm{CP}$, IP and NP, we see that the categorial status of the specifier varies according to that of its host: AP, PP, NP and possibly VP (and also possibly CP) can occur as specifier of C. Only NP seems to be able to occur as specifier of I; NP can occur as specifier of $\mathrm{N}$, along perhaps with QP. $\mathrm{C}$ on the one hand and I and $\mathrm{N}$ on the other differ in that the specifier of $\mathrm{C}$ is basically unrestricted, while the specifier of the others seems not to be (with some further questions concerning I). I suggest the break is $C$ versus A, N, P, I and V. Generalizing from the properties of $\mathrm{I}$, assume that (except for the effect of other principles):

(8)(a) Specifiers of $C$ can be any XP.

(b) Specifiers of I, N, V, A, and P can only be NP.

I return to a possible reason for the restriction in (8b) below. ${ }^{4}$ According to standard analyses of English or French, NPs do not surface as specifiers of $\mathrm{A}, \mathrm{P}$ or V. There are in fact good reasons to believe that in some languages, these positions do allow NPs to surface as their specifier. Koopman (1992) provides evidence for this in Bambara VPs, Koopman (1988) and Koopman and Sportiche (1988) for Dutch VPs and PPs and Koopman (1990) for English in particle constructions. By the assumption (6b), this alone is sufficient to suppose that NP specifiers of these categories should be allowed in English, French... I will therefore assume that these specifier positions are always available.

Next, I assume provisionally that an element YP getting an internal theta role from $\mathrm{X}$ occurs as a sister to $\mathrm{X}$. In particular, the head of a phrase $\mathrm{X}^{0}$ forms a constituent $\mathrm{X}^{1}$ with its theta marked complement(s):

$$
\text { (9) } \left.\quad \mathrm{I}_{\mathrm{X}} \mathrm{X}^{0} \mathrm{YP}\right]
$$

Except perhaps for the lowest $\mathrm{X}^{1}$ level, I follow Kayne (1984) in assuming strict binary branching. Essentially then, XP dominates its specifier and a lower projection of $\mathrm{X}$. While the head and its (theta-marked) complement(s) form a constituent, intermediate projections of $\mathrm{X}$ form constituents with modifiers or adjuncts of various sorts. 


\subsection{Theta Theory}

\subsubsection{Theta assignment and the A/A-bar distinction}

Theta relations, it is commonly assumed, are always realized as sisterhood relations. If correct, this means in particular that the external argument of XP must be realized as a sister to XP, thus deriving Koopman and Sportiche's $(1985 ; 1988)$ proposal according to which the structure of clauses is as in $(10)$ :

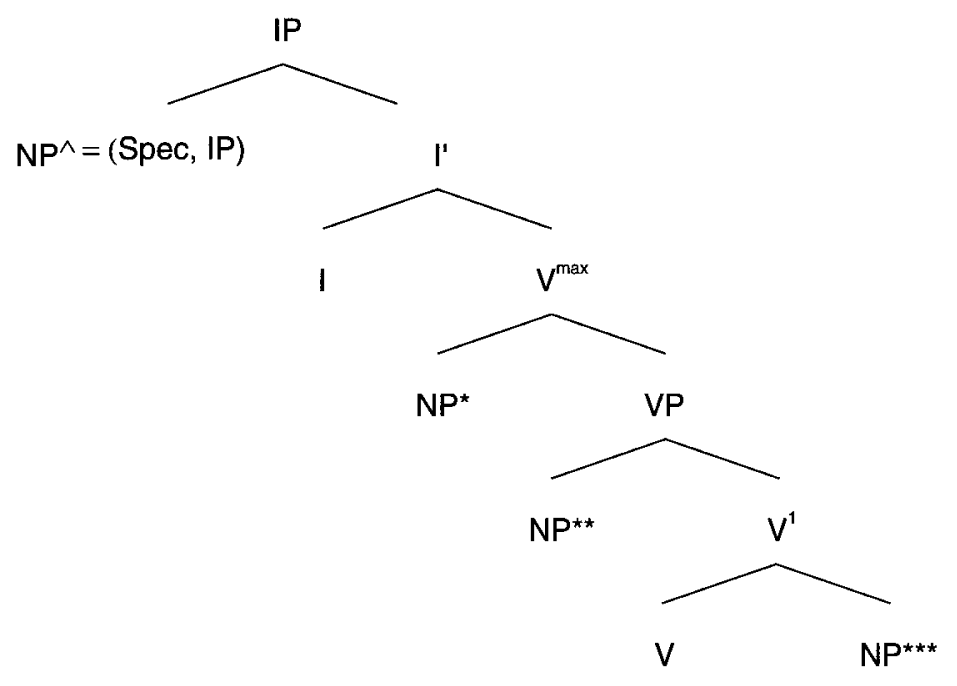

where NP* is the canonical or D-structure position of the subject (and is also the specifier of $\left.\mathrm{V}^{\max }\right), \mathrm{NP}^{\wedge}=(\mathrm{Spec}, \mathrm{IP})$ is its S-Structure position in simple declarative clauses in English, and $V^{\max }$ is a small clause whose predicate is VP. NP** is the specifier of VP. We return to the question of the precise structure of small clauses directly in Section 2.2.2.

Suppose provisionally that I, N, V, P and A all theta-mark their complements while $\mathrm{C}$ does not. This assumption leads to the following considerations:

First, notice that $[\mathrm{Spec}, \mathrm{IP}]$ never is a theta position. The question arises as to whether it is obligatory or not, i.e. as to the correct interpretation of the Extended Projection Principle in this framework. Koopman and Sportiche (1988) suggest that the Extended Projection Principle effects might be derived by forcing the existence of the position $\mathrm{NP}^{*}$ in (10), not of $\mathrm{NP}^{\wedge}$. ${ }^{5}$ This assumption would say nothing about [Spec, IP]. It might also be obligatory. The data discussed in Koopman and Sportiche (1988) or in Rouveret (1988) on Welsh suggest a negative answer. However, I want to maintain that this position is always available, if not always present.

Second, we need to address the question of what counts as an A-position. Chomsky's (1981) definitions would entail that [Spec, IP] must be an A-bar 
position. This is far from clear as discussed by Koopman and Sportiche (1991). Restricting ourselves to NP positions, it seems fairly clear that thetamarked positions must count as A-positions. Non-theta-marked NP positions are either adjoined positions or specifier positions. Adjoined positions must count as A-bar positions. The situation with specifiers is much less clear. It is fairly clear that $[\mathrm{Spec}, \mathrm{CP}]$ can count as an A-bar position.

One significant indication is the observed restrictions on the possible categories occupying a specifier position: is it exclusively an NP position or does it tolerate other categories? [Spec, CP] is unrestricted and an A-bar position. An internal argument NP is a severely restricted position and an A-position. Using this as a guideline we could say that $A$-positions are restricted NP positions, $A$-bar positions are unrestricted positions. Many questions then arise: (1) What does a survey of what can occur in specifier position show? (2) How do we define the A/A-bar distinction to get the right result? (3) Do positions partition in $\mathrm{A}$ and $\mathrm{A}$-bar positions?

Ignoring adjoined positions which always are A-bar positions, I will assume the following:

(11)(a) All NP positions within the projection of a lexical category must be A-positions.

(b) All NP positions within the projections of a contentive functional category can be A-positions.

(c) All positions within the projections of a functional category without content must be A-bar positions.

By this definition, we see that in (10), $\mathrm{NP}^{\wedge}, \mathrm{NP} *, \mathrm{NP}^{* *}$ and $\mathrm{NP} * * *$ can all be A-positions, while the specifier of $\mathrm{C}$ is not, since $\mathrm{C}$ is a contentless functional category.

More generally, NP specifiers and complements of $A, V, N$ and $P$ are A-positions (11a). [Spec, CP] is an A-bar position (11c). Specifiers of functional categories, i.e. of I, ASP, D, T can be A-positions. Must they? The answer is probably negative although it seems that the A/A-bar status is determined by the properties of the particular feature structure they have (e.g. if Case is assigned to their specifier, the specifier is an A-position). This is discussed to a certain extent in Koopman and Sportiche (1991). Here, we will assume that they always are A-positions, unless otherwise indicated.

These definitions are obviously different from the previously assumed characterization of A-positions - positions which may receive a theta role because of the position $\mathrm{NP}^{\wedge}$, for example. However, it tries to capture the same idea: A-positions are positions in which arguments may in principle freely occur.

The A/A-bar status of a position should correlate with other properties crucially involving the notion A-position. One such property states that landing sites for NP movement (i.e. movement subject to Principle A of the 
Binding Theory) are A-positions. This is consistent with our assumptions (see discussion in Koopman and Sportiche (1988), (1991) and in Sportiche (1988b)) that [Spec, IP] and [Spec, NP], and [Spec, $\left.X^{\max }\right]$ are A-positions. This is shown by structures like:

(12)(a) John will be seen $t$.

(b) The city's destruction $t /$ its destruction $t$.

(c) With [John beaten $t$ by his enemies].

In conjunction with the earlier assumption that specifier positions are always available, and the assumption that [Spec, IP] is not an obligatory position, we see that $[\mathrm{Spec}, \mathrm{XP}]$ does not obey the (Extended) Projection Principle throughout: its possible existence is independent of the lexical properties of the head $\mathrm{X}^{0}$.

\subsubsection{The Structure of VP and of small Clauses}

Consider again the clausal structure (10):

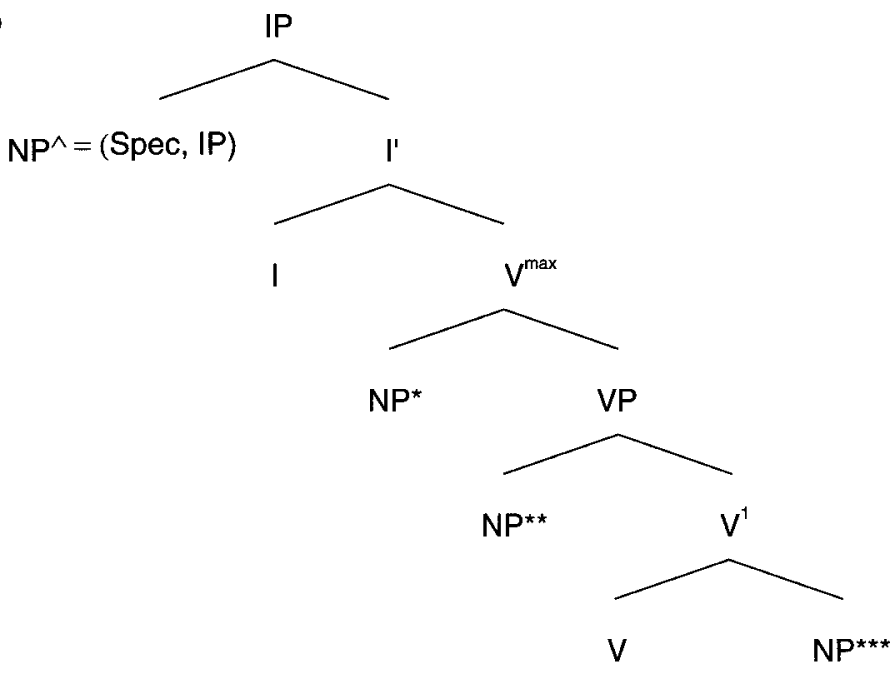

Notice first that the subject position of a small clause with head $X^{0}$, i.e. the NP* sister to XP is different from the specifier position of XP. Next, notice that we are now led to distinguish between what we note as the phrasal projection of V, i.e. VP, and its maximal projection, i.e. $V^{\max }$ (similarly for other categories). In fact, this is no innovation. This distinction is already implicit in the theory of small clauses as expounded in Stowell (1981). Indeed, in structures like: 
(13) I consider $\left[_{A}\left[_{E}\right.\right.$ this book $]\left[_{B}\right.$ my book $\left.]\right]$. I consider $\left[_{A}\left[{ }_{E}\right.\right.$ John $] L_{B}$ very sick $\left.]\right]$.

the constituent $A$ is larger than the constituent $B$. The constituent $B$ is subject to movement, but not the constituent $A$ (or at least not in the same way). Corresponding to (13), we can form (14a) but not (14b), even if they are all embedded under $I$ wonder:

(14)(a) Which book do you consider your book?/How sick do you consider John?

(b) Which book your book do you consider?/John how sick do you consider?

We now turn to the question of how to interpret $V^{\max }$ properly.

\subsubsection{VP SMALL CLAUSE}

We have supposed that NP* is external to VP and is therefore distinct from [Spec, VP]. This is by no means obvious. In exactly what relationship does it stand with respect to VP? Whatever the correct answer may be to this question, it should be consistent with the idea that the structural relationship between the external argument of a predicate and this predicate is identical across categories. Thus all the following phrases should have basically the same underlying internal structure:

(15)(a) (consider) $E_{W}$ John $\left[_{Z}\right.$ very sick $\left.]\right]$

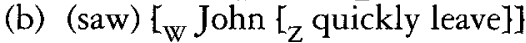

(c) (John will) $E_{\mathrm{W}} \mathrm{NP} * \mathrm{E}_{\mathrm{Z}}$ quickly destroy his toys $\left.]\right]$.

(d) (witness) $\left[_{W} \text { John's }\left[_{Z} \text { quick destruction of his toys }\right]\right]^{6}$

Extrapolating on the adjectival case (15a), it is clear that the constituent $\mathrm{Z}$ must be an XP, since it is not a head and is subject to movement as exemplified by How proud of Bill do you consider Jobn? What then is W? There are three alternatives:

(16)(1) $\mathrm{W}=\mathrm{XP}$ as suggested by Manzini (1983). In this case NP* is not configurationally distinguishable from an adjunct to XP, except for the fact that it gets an external theta role from $\mathrm{X}$. Presumably, it does not qualify as an adjunct.

(2) $\mathrm{W}=\mathrm{X}^{\max }$ as suggested in Koopman and Sportiche (1985; 1988). In this case, every $\mathrm{X}$ projects to XP and possibly to one further level.

(3) $\mathrm{W}=\mathrm{XP}$ as suggested by Larson (1988) for VP. 
In the last case, the projection rules from the lexicon to syntax are altered so that the underlying structure of $\mathrm{W}$ in $(15 \mathrm{c})$ for example would be:

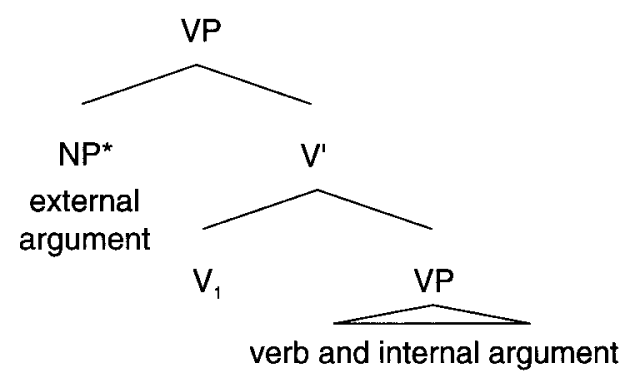

In derived structure, the verb raises to $\mathrm{V}_{1}$. In this structure, NP* is in [Spec, VP], but not of the minimal VP containing the verb in underlying structure.

Huang (1993) provides an argument bearing on this question. He argues in favor of the idea that NP* is really [Spec, VP] and specifically against alternative (2). Note first that there are many reasons why the external argument cannot be [Spec, VP] of the smallest VP containing V: Koopman and Sportiche (1991) present evidence that this position must be available for direct objects in Bambara and in Dutch. The same point will be argued for extensively here in Section 4. However, there is also a sense in which Huang's elegant argument is convincing. In what follows, I will show how the two can be reconciled.

This argument is based on a difference in behavior between the $\mathrm{W}$ constituent in (15a) and (15c). An AP small clause as in (15a) cannot be preposed by movement:

(18)(a) You consider [John very sick].

(b) How sick do you consider John?

(c)* [John how sick] do you consider.

If it can be shown that the $W$ constituent in (15c) can be preposed by movement, it would suggest that it is not a small clause. The argument that $\mathrm{W}$ in (15c) can be preposed is based on the contrast between $\mathrm{Wh}$ Movement and VP-preposing with respect to reconstruction illustrated below:

(19)(a) Which paintings of each other do the girls say the boys like?

(b) Listen to each other, the girls say the boys do.

In (19a), the antecedent of the reciprocal can either be the main subject or the embedded subject. In other words, the binding theory can be satisfied either by the S-Structure or by the reconstructed structure (i.e. as if the preposed 
phrases were still in their base position). In (19b), only one reading is possible, namely with the reciprocal taking the embedded subject as antecedent. Why is there a contrast? If VP preposing carries NP* along, we can derive this observation. Then the preposed VP is really [NP* listen to each other]. With NP* counting as a subject for the binding theory, the reciprocal can only take it as antecedent to explain the lack of ambiguity of (18b). The subject of an AP small clause cannot be preposed but the subject of a VP must be: the conclusion then is that NP* and VP do not form a small clause in the same way the subject of an AP small clause and the small clause do. The first thing to notice is that the contrast in (19) and its analysis provide an argument for the VP-Internal Subject Hypothesis, regardless of the way in which it is construed. The conclusion that NP* is internal to VP rather than a daughter to $\mathrm{V}^{\max }$ holds only if the difference can only be accounted for by a difference in constituent structure. Other possibilities come to mind. The two problems to solve are the following:

1 Why is pied-piping of NP* with VPs obligatory (if it were not, (53b) should be ambiguous), ${ }^{7}$ therefore necessarily carrying along NP*? The argument should still carry in the case of bare verbs.

2 Why cannot small clauses with overt subjects be moved as in (18c)?

Begin with (1). Under alternative (3) above (and perhaps under (1), although it is less clear), NP* is obligatorily carried along because movement moves $\mathrm{V}$, which is under the higher VP. Under (2), we would need some additional principle. For example, we would assume:

Move the highest projection of an $\mathrm{X}^{0}$ possible.

where possibilities are determined by the movement involved (to a head position, or to an XP position or to an unrestricted position) and the principles of grammar (only heads, XPs and possibly $\mathrm{X}^{\max _{\mathrm{s}}}$ are visible to Movement or Case Theory). The fact that (2) needs what appears to be an otherwise unnecessary principle would seem to argue against it.

Turning to (2), we can attribute the ungrammaticality of (18c) to Case Theory. Indeed, in (18c) neither Jobn nor one of its traces is in a Case position. We get a Case filter violation. This contrasts with $W b$ Movement in which either a $w$-trace is in a Case position, or (as in the case of pied-piping) the $w h$ phrase itself is in a Case position. This also contrasts with objects pied-piped under VP preposing in which the Case assigner $\mathrm{V}$ is moved along the object. Extending the range of data complicates the matter. Consider reciprocal binding under $W b$ Movement of APs:

(21)(a) John considers [them proud of each other].

(b) How proud of each other does John consider them? 
(c)* They consider [John proud of each other].

(d)*How proud of each other do they consider John?

(e)* They say I am considered proud of each other.

(f)* How proud of each other do they say I am considered?

We get the same effects in AP preposing as we do in VP preposing. If we give up Huang's account entirely, we lose the explanation for the VP preposing facts. If we accept it, in order to account for the ungrammaticality of (21d,f), we are led to assume that the AP preposed by $W b$ Movement also contains the subject of the AP small clause. This means that Jobn, the subject of the AP small clause, has raised out of the small clause: 8

(22)(a)* They consider $\mathrm{John}_{\mathrm{i}}\left[_{\mathrm{W}} \mathrm{t}_{\mathrm{i}}\right.$ [proud of each other]].

(b) $*\left[_{\mathbb{W}} t_{i}[\right.$ How proud of each other $\left.]\right]$ do they consider John ${ }_{i}$.

Two consequences would follow. First, it would show that small clauses, that is the constituent $\mathrm{W}$, can be preposed by movement. If we can explain why Jobn cannot be pied-piped under W preposing, we cancel a long standing argument against the idea that there are small clause constituents because they cannot move. Second, it undermines Huang's argument presented above against the $\mathrm{V}^{\max }$ hypothesis: it is still possible that all $\mathrm{W}$ are $\mathrm{X}^{\max }$, but the subject raises out both in VP small clauses and in AP small clauses.

Where does the subject of the small clause raise out to and why? This raising is local, suggesting NP Movement, hence movement to an A-position. NP Movement is usually forced by Case Theory: perhaps it must raise in order to receive the accusative Case (we present further evidence for this in Section 5.2.1.2). Where does it raise to?

$$
\text { will [ consider John } n_{j}\left[\mathbf{t}_{j} \text { proud }\right]
$$

The only plausible position is [Spec, VP]. However, the verb consider bears no morphology, hence plausibly has not raised anywhere for morphological reasons (that is, to attach to some affix, cf. Section 2.3). If it has not raised at all, it should follow Jobn, not precede it. This suggests that the verb has raised after all, but not for morphological reasons. A landing position for $\mathrm{V}$ must be provided. This is precisely what Larson's (1988) proposal provides. The structure of (23) would then be as in (24) on p. 100.

This is what we will adopt from now on. ${ }^{9}$ Why cannot $\mathrm{W}$ preposing piedpipe Jobn? The most appealing possibility is to invoke a Case Filter violation as mentioned above: if John is pied-piped, it cannot get Case. Alternatively but less desirably, we could appeal to some version of (19): the two $\mathrm{VP}_{j}$ s are the projections of the same $\mathrm{V}$ head and therefore movement will always try to move the top one. 
(24)
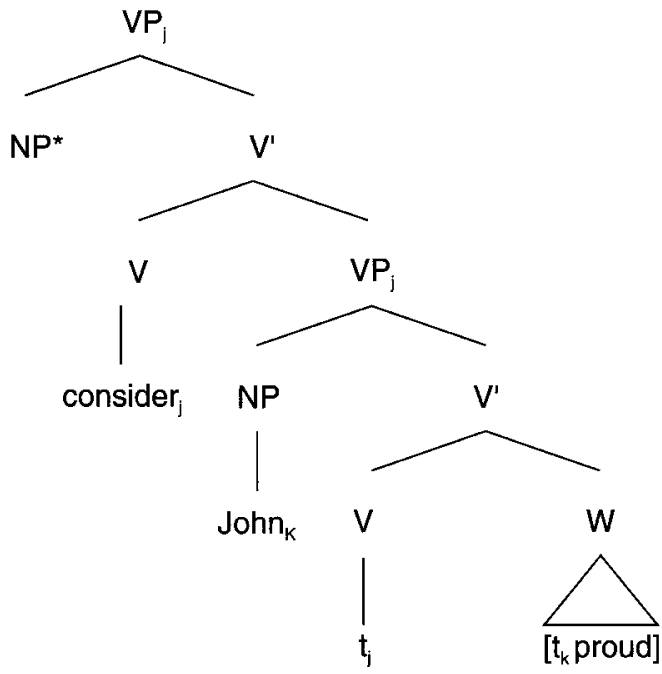

2.2.2.2 EXTERNAL ARGUMENTS AND SMALL CLAUSE STRUCTURE

We now adopt the VP structure (17), inspired by Larson (1988) (and which is quite close to what Kayne (1984) suggested).

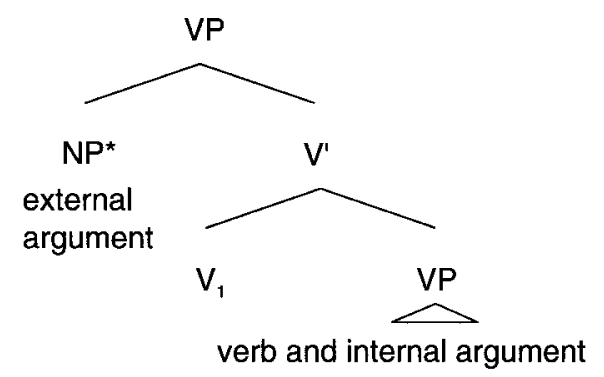

My assumptions will differ from Larson's (1988) in a number of ways. First, I will for the moment continue assuming a standard structure for the lower VP (returning to a discussion of this issue in Section 5.2.3.1). Furthermore, I assume that the projection rule for external argument is as in (17). The external argument of a category $\mathrm{K}$ is generated as the specifier of a $\mathrm{KP}$ containing none of the internal arguments. If $\mathrm{K}$ does not have an external argument, there is only the lower VP. ${ }^{10}$ Note that the distinction between VP and $\mathrm{V}^{\max }$ still exists: all the verbal projections in (17) are projections of one verb. Consequently, the highest VP is the maximal projection of that V, i.e. $\mathrm{V}^{\max }$. This distinction will become important in Section 5. 
In keeping with the idea that all W constituents in (15) have an identical hierarchical organization, I will assume the following underlying structures for an $\mathrm{A}$ with all its arguments, i.e. an AP small clause or an $\mathrm{N}$ with all its arguments, i.e. an NP small clause (which is just an NP):
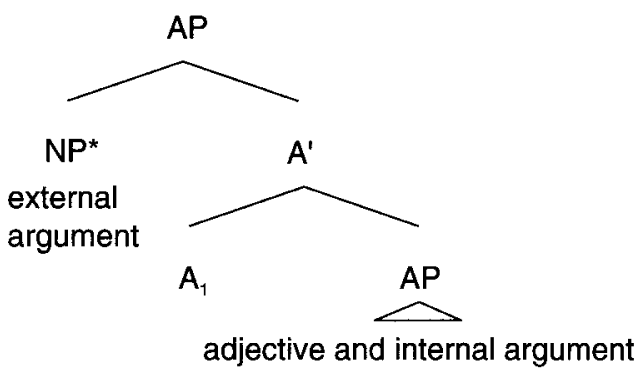

(26)

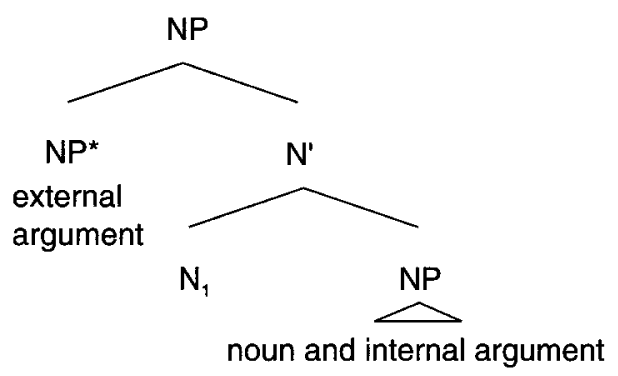

Earlier, I supposed that Vs and As theta-marked their complements. What does it mean in this new context? Does $\mathrm{V}^{1}$ in (17) theta-mark its VP complement or $A^{1}$ in (25) its AP complement? This looks implausible, just as implausible as our assumption that I theta-marks its complement (which theta role?). The right notion is more likely to be one distinguishing category with content from categories without. Basically, every category but $\mathrm{C}$ has content and therefore the right notion then should be close to Chomsky's (1986a) L-marking. We are now assuming:

\section{(27) All $\mathrm{X}^{0}$ but C L-mark their complements.}

In other words, a head different from C L-marks its sisters. ${ }^{11}$ Clearly, thetamarking a complement entails L-marking it. We can revert to the more natural assumption that lexical categories theta-mark their complements, while functional categories do not. As a final remark, note that there is a correlation between the L-marking status of a category and the A/A-bar status of its specifier, i.e. between (11) and (27): 


\subsection{Inflection and agreement}

Inflectional affixes appearing on some element usually modify the properties of the element (thematic, selectional). Furthermore, inflectional affixes might have selectional properties of their own apart from selecting the type of head to which they attach (e.g. co-occurence restrictions between particular tenses and temporal adverbs). Accordingly, I suppose, following much recent work (Baker (1988a), Pollock (1989)), that usually an inflectional affix A is a syntactic head, taking as complement the projection of the word $\mathrm{W}$ that it affixes onto, and that the affixation process itself is nothing else but the head movement of $\mathrm{W}$ to the affix position A (or possibly, but preferably not, lowering).

Let us turn now to agreement between a head and an XP. If the agreement affix in fact does display the properties of a head, it is natural to extend to it the general view of inflectional affixes described above. In this case, an agreement affix is just like any other projection of an inflectional affix and projects from an AGR to AGR' to AGRP as in Chomsky (1989), Mahajan (1990) and Pollock (1989) for example. The shape of the agreement affix in this case is determined by what occupies the specifier position of AGRP. It is quite unclear however whether agreement inflection displays any property of a syntactic head. In this case, following Koopman (1988), we would take the agreement inflectional affix to be the morphological spell out of a relation between a head (say an adjective) and its specifier. Under both of these views, agreement is determined by the relation between a specifier and a head. In what follows, I will assume without argument that Koopman's view is correct (see Koopman and Sportiche (1991) for discussion). AGR projections could be added throughout with only a few changes to the text.

\subsection{Case Theory}

I assume the standard version of Case Theory including the Case Filter or perhaps - cf. Sportiche (1983) - its reduction to theta assignment through the visibility requirement as it applies to non-silent NPs and wh-traces and perhaps certain other silent categories as well. The major modification I introduce concerns Case assignment. Current theories distinguish between two kinds of Case assignment, structural Case and inherent Case. Structural Case is a configurational property: a tensed I assigns Case to its specifier in English regardless of the semantic or thematic relation of this specifier to the rest of the sentence. Nominative Case is typically a structural Case. Inherent Case on the other hand is lexically dependent or thematically dependent. Its assignment depends both on the lexical choice of the Case assigner and on the themaric relation between the Case assigner and the category receiving Case. 
There is however another difference. The structural configuration in which nominative Case assignment occurs is different from the configuration in which an object is inherently Case-marked. Nominative Case assignment is a relation between a head, namely $\mathrm{I}$, and its specifier. Inherent Case assignment is a relation between a head and a complement. This seems to be a priori ground for distinguishing the two. Furthermore, Koopman and Sportiche (1988) argue that Infl varies in its Case assigning properties across languages. Consider again the structure of a clause:

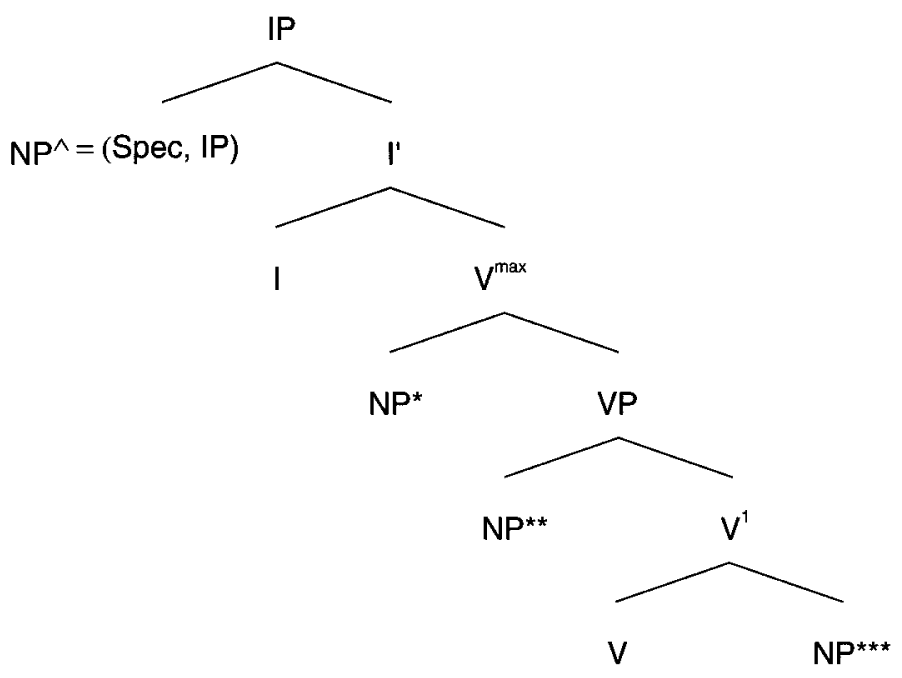

In languages in which Inf cannot Case-mark the position NP* (e.g. French, or English or Vata), an NP in this position must raise to the position $\mathrm{NP}^{\wedge}$ to get Case from Infl. In languages where Infl can Case-mark NP*, raising is not necessary (and perhaps impossible): this gives rise to AuxSVO languages or, after $\mathrm{V}$ to $\mathrm{I}$, VSO languages for example. Now, consider a language like English. If Infl can Case-mark NP ${ }^{\wedge}$, why can it not Case-mark NP* as well? It seems again that we need to distinguish between a head assigning Case to a complement or the specifier of a complement and a head assigning Case to its own specifier. We can meet these desiderata if we postulate that Case is assigned to an NP either under government by a Case assigner or by agreement of the NP with a Case assigning head. To summarize, we distinguish between structural Case assignment and inherent Case assignment. Furthermore, we distinguish between Case by agreement and governed Case. The nominative is both structural Case and Case by agreement, while inherent Case is always governed Case. Where does objective Case fit in this picture? Objective Case is a structural Case as shown by the fact that it occurs in Exceptional Case Marking structures or on the subject of small clauses, when it bears no thematic relation to its Case assigner. Is objective Case a governed 
Case or is it a Case by agreement? Given the assumption we made about $\mathrm{X}$-bar theory and the distribution of direct objects of $\mathrm{X}^{0}$ as sister to $\mathrm{X}^{0}$ inside $\mathrm{X}^{1}$, it looks as if objective Case is a governed Case. This is what I will assume until we return to this issue in Section 5 .

Governed Case is assigned under government where government is provisionally defined in terms of $\mathrm{X}$-command and in terms of barrier in the spirit of Chomsky (1986a):

(29)(a) Government

A governs $\mathrm{B}$ if $\mathrm{A} \mathrm{X}$-commands $\mathrm{B}$ and no barrier for $\mathrm{B}$ intervenes between $\mathrm{A}$ and $\mathrm{B}$.

(b) X-Command

A X-Commands $\mathrm{B}$ if the minimal constituent meeting property $\mathrm{X}$ containing A contains $\mathrm{B}$.

We return below to what barrier and intervene mean; we can provisionally take $\mathrm{X}$-command to be $\mathbf{M}$-command, i.e. where property $\mathrm{X}$ is the property of being a phrasal (or maximal) projection. We return to a discussion of which command notion is relevant, i.e. how X should be understood.

The second option is Case assignment by agreement. Let $\mathrm{H}$ be a Case assigning head. Then, if it is a Case assigner by agreement, it may assign Case to an NP in its specifier position, as a reflex of the general process of specifier head agreement that was discussed in the previous section.

Which of these two Case assigning options is realized depends on the particular category HP, the lexical content of the head $\mathrm{H}$ it contains, and the language L. A priori, we want to minimize possible inter and intralanguage variations. Given that there is crosslinguistic variation, the strongest position would be one asserting that languages choose once and for all one and/or the other option, regardless of the head and its content. A priori, this seems too strong. In English, I only assigns Case by agreement, while it seems that V only assigns governed Case (structurally or inherently). Koopman (1988) shows that in Dutch, certain Ps are governed Case assigners, while some others are agreement Case assigners. Koopman (1990) also presents an analysis of particle constructions in English discussed by Kayne (1986) based on the idea that particles can be agreement Case assigners. We will return to this issue in general terms when we discuss structural Case again in Section 5.2. Finally, given the existence of the Case Filter or the visibility requirement, I suppose that Case assignment (or checking) is optional.

\subsection{Movement}

Following Chomsky (1986a) we allow movement of lowest projections $\left(\mathrm{X}^{0}\right)$ and phrasal projections (XP). ${ }^{12}$ Only substitution and adjunction are available. Adopting Chomsky's formulation, itself derived from a proposal in 
Baltin (1981), we postulate that substitution or adjunction of $\mathrm{X}^{\mathrm{p}}$ can only be into or to some $\mathrm{Y}^{\mathrm{p}} \cdot{ }^{1.3}$ A given projection can be substituted only in an empty slot of the same projection level; a given projection can only be adjoined to a projection of the same level. There are some further restrictions on adjunctions. We stipulate that adjunction is restricted to VP, AP and IP, and impossible to other phrasal projections.

It is noted that we cannot "justify" this stipulation the way Chomsky does: he uses the idea that adjunction can only be to non-argument types. Since NP, $\mathrm{PP}$ and $\mathrm{CP}$ can count as canonical arguments (i.e. they can get theta-marked in D-structure), adjunction to NP, PP or CP is prohibited. As VP, AP and IP are not canonical arguments, they qualify as targets of adjunction. The reason is that VP or AP both count as arguments (say a small AP clause can be the theta-marked complement of a verb) but also allow adjunction. The situation of ECM IP complements is similar.

\subsection{Barrierbood}

We now define barrierhood and intervention. The crucial ingredient is of course the characterization of barrierhood. The basic idea is the following: when an $\mathrm{X}^{\mathrm{n}}$ is inside a projection $\mathrm{Y}^{\mathrm{P}}$ which lacks some proper relationship

with an outside head (e.g. is not a theta- or L-marked position) movement of $\mathrm{X}^{\mathrm{n}}$ is confined to within YP, except of course if $\mathrm{X}$ is also a projection of this particular Y. Formally, this will be expressed in the following way (we will modify this definition later in Section 3.4.5 and Section 5.4.3.2):

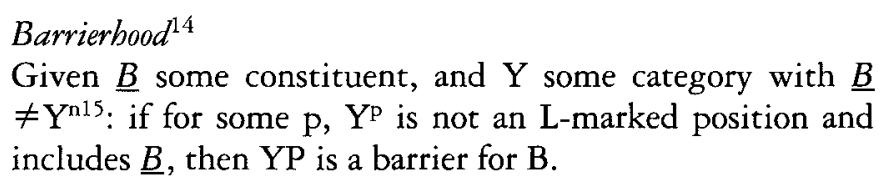
$\neq \mathrm{Y}^{\mathrm{n} 15}$ : if for some $\mathrm{p}, \mathrm{Y}^{\mathrm{p}}$ is not an $\mathrm{L}$-marked position and includes $\underline{B}$, then $\mathrm{YP}$ is a barrier for $\mathrm{B}$.

The intuitive idea here is that core cases of extraction can only be out of complements. When looking outside from inside some projection $\mathbf{P}$ which is not a complement, the highest projection of $\mathrm{P}$ is opaque. So in a sense, the exterior can be reached only when mediated through a strong binary relation with something in the vicinity outside such as a theta marker or an L-marker. Notice that L-marking is a property of positions, not a content of positions. The exclusion clause stating that $\underline{B} \neq \mathrm{Y}^{\mathrm{n}}$ will allow the head of $\mathrm{P}$ to look outside. This is basically to permit the existence of head movement. This clause means that the projections of a particular head can never count as barrier for that head.

The notion of barrier is relevant only when a barrier intervenes between two items, thus blocking some relation between the two. e.g. a movement relation. The notion intervention is defined following Chomsky's (1986a) and May's (1985) notions of inclusion and exclusion. It can be understood 
basically as domination in the usual sense. If $\mathrm{A}$ dominates $\mathrm{B}$ but not $\mathrm{C}$, $A$ intervenes between $B$ and $C$. The only exception arises in adjunction structures. What we want to achieve is the following. In a structure where some $\mathrm{ZP}$ is adjoined to XP, with WP inside (i.e. not adjoined to) XP:

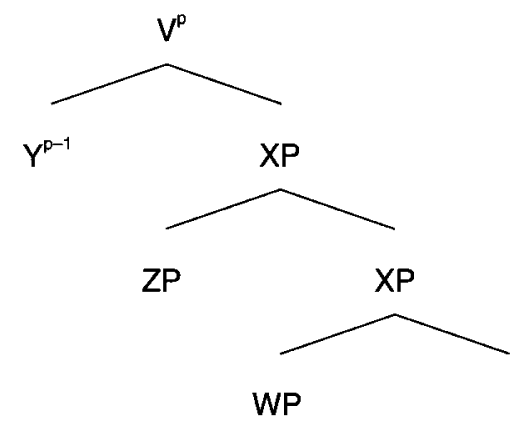

we want to say that $\mathrm{XP}$ intervenes between $\mathrm{Y}^{\mathrm{p}-1}$ and WP. But XP does not intervene between $\mathrm{Y}^{\mathrm{p}-1}$ and $\mathrm{ZP}$, nor does it between $\mathrm{ZP}$ and WP. In a sense, then, ZP is neither dominated nor not dominated by XP. Chomsky (1986a) or May (1985) achieve this result by introducing the notions of segment, inclusion and exclusion. ${ }^{16}$ More specifically, we define intervention as below:

\section{Intervention}

A projection $\mathrm{Y}^{\mathrm{p}} \underline{\text { intervenes }}$ between $\underline{a}$ and $\underline{b}$ if $\underline{a}$ is excluded by $\mathrm{Y}^{\mathrm{P}}$ and $\underline{b}$ is included in $\mathrm{Y}^{\mathrm{P}}$.

Let us apply these definitions to governed Case assignment assuming as mentioned earlier that both inherent Case and structural objective Case are assigned under government. Under what circumstances are such cases assigned? There are two main configurations:

1 NP to be assigned Case by $\mathrm{X}^{0}$ is an immediate complement of $\mathrm{X}^{0}$ (this is the normal case of a direct object or an indirect object).

2 NP to be assigned Case by $\mathrm{X}^{0}$ is the specifier of an immediate complement of $\mathrm{X}^{0}$ (this configuration arises in ECM constructions or small clause constructions).

Let us then check that our definition of barrierhood allows Case to be assigned properly in these instances as in (33). Considering the structure in (33), we can reason as follows.

$\mathrm{Y}^{\mathrm{p}-1}$ can Case-mark XP as long as it is a governed Case assigner, since no barrier for XP intervenes between $\mathrm{XP}$ and $\mathrm{Y}^{\mathrm{p}-1}$. Can $\mathrm{Y}^{\mathrm{p}-1}$ Case mark $\mathrm{ZP}$ ? If $\mathrm{XP}$ is not an L-marked position, it will count as a barrier for ZP since it includes $Z P$. Furthermore this barrier will intervene since $X P$ excludes $Y^{\mathrm{p}-1}$. Now if $\mathrm{XP}$ is an L-marked position, it means that $\mathrm{Y}^{\mathrm{p}-1}$ is a $\mathrm{Y}^{0}$ and $\mathrm{L}$-marks 


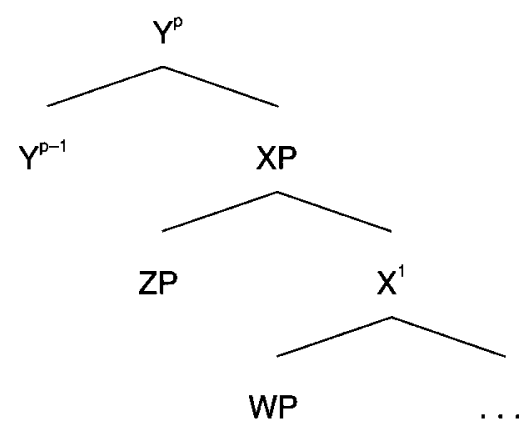

$\mathrm{XP}$ (which means that $\mathrm{YP}^{\mathrm{P}}$ is $\mathrm{Y}^{1}$ ). In this case, $\mathrm{XP}$ is the only projection of $\mathrm{X}$ containing $\mathrm{ZP}$ and is a theta position so it is not a barrier to government by $\mathrm{Y}^{\mathrm{p}-1}=\mathrm{Y}^{0}$. Now it can be noted that $\mathrm{Y}^{\mathrm{P}-1}$ cannot case-mark WP: WP is included in $\mathrm{X}^{1}$, which is not an L-marked position. $\mathrm{XP}$ is therefore a barrier for WP and it intervenes since it includes WP but excludes $\mathrm{Y}^{\mathrm{P}-1}$.

Generalizing somewhat, we see that the core prediction of this notion of government is that a head can govern its complement, and the specifier of its complement. It can also govern the head of its complement since no projection of the head of a complement can count as barrier for that head by definition (the clause I called the exclusion clause above). ${ }^{17}$ In the following section, we turn to a somewhat more exhaustive discussion.

\section{Syntactic movement}

\subsection{The general case of movement}

In this section, I am going to show that the notion of government introduced earlier plays a crucial role in explaining the constraints on movement, thus reducing Movement Theory or unifying Movement Theory with Government Theory. The unification is twofold: first, it unifies the constraints on various kinds of movement (head movement, NP Movement, $W b$ Movement) to the single condition below. Second, it formulates this condition in terms of the notion government, thereby reducing it to Government Theory. Beginning with syntactic movement (as opposed to LF movement), suppose that we require traces to obey the following condition that we call the Condition on Chain Links (henceforth CCL):

Condition on Chain Links (CCL)

Traces must be governed by an antecedent at S-Structure.

Before examining concrete consequences of the CCL on movement, let us reason on some hypothetical cases. We are trying to move some A out of some $\mathrm{XP}=\mathrm{X}^{2}$. 
First subcase: Suppose first that A is only included in XP. This can mean one of three possibilities:

$1 \quad A$ is the specifier of XP.

$2 \quad \mathrm{~A}$ is $\mathrm{X}^{1}$.

$3 \mathrm{~A}$ is dominated by $\mathrm{X}^{1}$ but not included in it (which means $\mathrm{A}$ is adjoined to $\left.\mathrm{X}^{1}\right)$.

Case (2) is irrelevant since A, as an intermediate projection, cannot be subject to movement. Case (3) should be excluded in principle as an adjoined structure to a non-phrasal projection. ${ }^{18}$ We are left with case (1) A is YP.

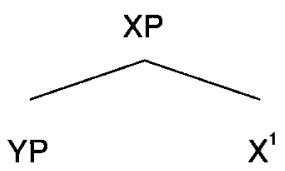

Two situations can occur:

1 If XP is L-marked, it is transparent and A can move out of XP without further assumptions: even though XP intervenes between $A$ and the outside, its L-marked character makes it crossable; this is for example what happens with VP (always L-marked by I), or with complement NPs or CPs. For $A=Y P$, this means that extraction of their specifiers is always permitted.

2 If XP is not L-marked, it counts as a barrier for A since, by assumption, it includes $A$. The only way A can move out is by moving out in such a way that XP does not intervene between $A$ and the outside position it moves to. The only way this can be achieved is by adjoining $A$ to XP if possible, and then escape. In order to be able to adjoin to XP (1) A must be a YP itself, and (2) XP must be a possible adjunction site. In other words, extraction out of XP will be possible only if XP is neither NP, $\mathrm{CP}$ nor PP, since adjunction to NP, CP and PP is barred. In particular extraction out of NP, CP and PP in this way is impossible when they are not in an L-marked position. In order to permit extraction out of them, they must be in an L-marked position.

Second subcase: Suppose now that $A$ is included in $\mathrm{X}^{\mathrm{n}}$ with $\mathrm{n}$ not a phrasal projection of $\mathrm{X}$ in that particular structure. We are going to deduce two consequences. Except for movement of $\mathrm{X}^{0}$, movement directly to a position excluded by XP is impossible. Second, it is (sometimes) possible to move from A's position to a position included in only one projection of $\mathrm{X}$, namely $\mathrm{XP}$ (that is, it is possible to reduce this case to the previous subcase). 


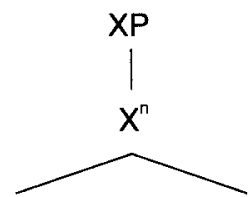

A

$\mathrm{X}^{\mathrm{n}}$ with $\mathrm{n}$ not maximal is never $\mathrm{L}-$-marked. Again, two situations may occur:

- If $A=X^{0}$, it can escape XP by stipulation: a head can always escape its maximal projection.

- If $A \neq X^{0}$, then $X P$ always counts as a barrier intervening between it and the outside of XP: by assumption, some non-phrasal $\mathrm{X}^{\mathrm{n}}$ includes A. So XP includes $A$ and is a barrier for $A$ since $X^{n}$ is not an L-marked position. Then, $A$ cannot move to a position such that XP excludes it without violating the CCL. If A did, XP, a barrier for A, would intervene.

There are at most two ways out:

- The first is to move to a position neither included nor excluded by XP. This means adjoin A to XP. This is a way to move up the tree to a position not excluded by XP. Again, this is possible only if A is a YP and XP is a possible adjunction site.

- The second is to move to a position within XP included in only one projection of X, namely XP. This brings us back to the first subcase discussed above because the only option is to move to the specifier position of XP. This will be possible if this specifier position can be a landing site for movement, e.g. it is not a theta position, and if $A$ is the kind of category that can appear in the specifier position of XP.

Summary of conclusions Let us summarize the implications of this discussion. When computing extraction out of some XP, we need to know the following parameters:

Is adjunction to XP allowed?

Is $\mathrm{XP}$ in an L-marked position?

What kind of movement are we dealing with (A, A-bar, head)?

Generally, extraction from inside some XP is either through its specifier if it is $\mathrm{L}$-marked or through adjunction (or a combination of both). If the kind of movement permits adjunction, (i.e. A-bar movement) it is always going to be possible to escape categories that can be adjoined to, i.e. AP, VP and IP, by adjoining to them. In other words, A-bar movement out of IP, AP, or VP and 
possibly others is free. A-bar movement out of categories that cannot be adjoined to must be through their specifiers. A-bar movement out of NP, CP and PP and possibly other categories must proceed through their specifier and these categories must be in L-marked positions.

If we are dealing with $A$-movement, escape through adjunction is impossible. By definition, A-movement is movement from an initial Aposition to a final A-position. Intermediate positions cannot be A-bar positions (or improper movement would result). Thus NP movement behaves like $\mathrm{Wb}$ Movement out of NP and other such categories: movement can only be through successive specifiers of categories in L-marked positions. We see that we derive the description of possible extraction paths given in the introduction. In the following sections, we go through a case-by-case analysis of possible movements.

\subsection{Head movement}

\subsubsection{The general case of bead movement}

We begin with head movement. Let $Y$ head of YP be the head that is under consideration. Suppose YP is immediately dominated by some projection of a head $\mathrm{X}$. There are basically two kind of cases to consider:

1 Can $\mathrm{Y}$ be moved to some head position $\mathrm{Z}$ different from $\mathrm{X}$ ?

2 Can $Y$ be moved to $X$ ? In the following configuration:

$$
Z \ldots\left[_{X P} \ldots\left[_{X}{ }^{n} \ldots X \ldots\left[_{Y P} \ldots Y[\ldots]\right]\right]\right]
$$

$\mathrm{Y}$ is the head of YP. XP is the first phrasal projection containing YP, $\mathrm{X}$ its head and $\mathrm{X}^{\mathrm{n}}$, some projection containing YP.

The principle we want to derive is the Head Movement Constraint (HMC) (Travis (1984)). We basically want to reduce to general constraints on movement very much like Koopman (1984) did (who did not name the constraint but ascribed its effects to the Empty Category Principle). The HMC basically states that a head can only move to the next head up the tree, where we understand the next head up the tree as the closest m-commanding head (or i-commanding head, see Section 3.2.2).

Consider the first question first. The constraint on moving $\mathrm{Y}$ out of $\mathrm{XP}$ is in fact identical to the constraints on moving YP out of XP: the reason is that $\mathrm{Y}$ is included in all the same projections YP is, except for the projections of $Y$ itself. Given that the projections of $Y$ cannot count as barriers for Y's movement, the set of barriers for $Y$ is identical to the set of barriers for $Y P$. In other words, just as for YP, Y cannot be moved beyond the specifier position of XP or a position adjoined to XP. In particular Y cannot be moved directly to the $Z$ position. Basically, this derives the effects of the HMC, except for the 
following case in which the above reasoning fails: if the only projection of $X$ including YP is XP, that is if in fact YP already is the specifier of XP. In other words if we are dealing with the following configuration:

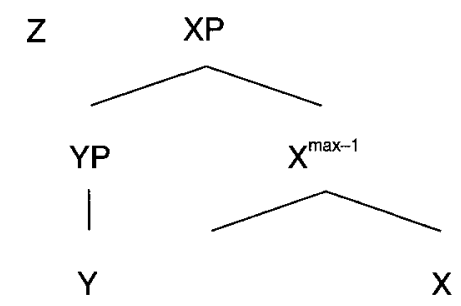

In this case, $\mathrm{Y}$ can move to $\mathrm{Z}$ directly if $\mathrm{XP}$ is in an L-marked position. Alternatively, if adjunction to XP is allowed, YP can adjoin to XP, from where $\mathrm{Y}$ can be moved to the $\mathrm{Z}$ position.

These are the predictions then: movement in one step of a head $Y$ out of the XP containing YP (more precisely: movement in one step of the head Y of YP contained in XP to a position excluded by XP) is possible if:

$1 \mathrm{XP}$ is in an L-marked position and YP is the specifier of $\mathrm{YP}$, or

$2 \mathrm{XP}$ is not an L-marked position, adjunction to $\mathrm{XP}$ is allowed and $\mathrm{YP}$ is adjoined to $\mathrm{XP}$.

For example, this allows incorporation into the verb of the head of the specifier of a direct object of this verb. It also allows the incorporation of the subject of a clause embedded under an ECM verb. This also allows I to $\mathrm{C}$ movement, or incorporation of a VP level adjunct into I.

Let us now turn to the second question: in the configuration (37), can $Y$ move to $X$ ? So far, nothing prevents the incorporation of $Y$ into $X$ (head movement) regardless of the relation between $\mathrm{X}$ and $\mathrm{Y}$. For example, it permits incorporation of the head of an adjunct to $X$ into $X$. Let us further examine these two questions, beginning with the latter.

\subsubsection{Incorporation of adjuncts and complements: i-command}

If indeed the head of an adjunct can in principle incorporate into the head that it is an adjunct of, then the notion of government that we use in the CCL is adequate. If, on the other hand, there are restrictions, e.g. only heads of a complement and of its specifier can incorporate, then we have to devise a more restrictive theory of possible movement. The relevant structures for the problem look like (39(a and b)) below.

Both situations are meant to illustrate potential cases of head movement of an adjunct to some superordinate head: here $\mathrm{Y}$ and $\mathrm{X}$ respectively. We have tentatively supposed that all the $\mathrm{L}$-marked complements of $\mathrm{X}$ are under $\mathrm{X}^{\mathrm{I}}$. 
$(39)(a)$

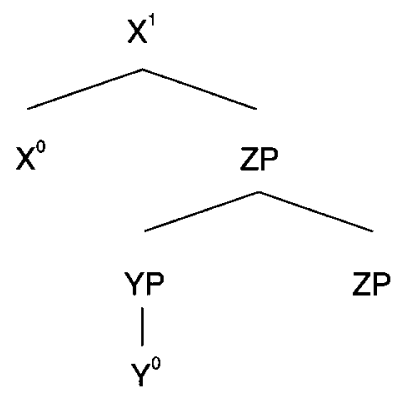

$(39)(b)$

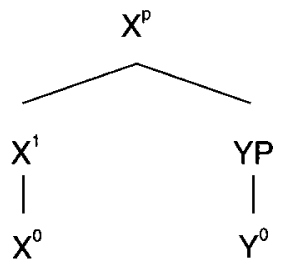

Adjuncts to X, e.g. YP, could be higher up as indicated in the (b) structure. Alternatively, they are generated as inner complements as Larson (1988) suggests. The (a) structure illustrates the case of a YP adjoined to a complement ZP of X.

The first question is whether $\mathrm{Y}$ can be incorporated into $\mathrm{X}$. For the (a) structure, the answer seems to be positive. This is what happens with clitics as we will show in Section 4.1. For the (b) case, it is more difficult to decide. Although there are cases that look like such cases (say manner adverbs incorporating into their selecting $\mathrm{V}$ ) this could also arise as a result of $\mathrm{X}$ raising up to the next head up, YP adjoining to XP and incorporating into the $\mathrm{X}$ from there, that is as in the (a) case (e.g. aspectual adverbs incorporating into ASP that has raised to T). So we need to look at cases of heads which do not move at all and see whether they can incorporate some adjunct they select. One such case might be temporal adverbs like yesterday, tomorrow, which are selected by Tense. If Tense does not raise, we can get an answer from whether or not these adverbs can incorporate into T. Here, I will tentatively assume that this is not possible.

In order to exclude incorporation of the head of an adjunct into its selecting head as in the (b) case, we need to restrict movement possibilities. One possibility is to modify the notion of barrier so that the phrasal projection $\mathrm{HP}$ of a head $\mathrm{H}$ is a barrier for movement of $\mathrm{H}$ out of HP if HP is not in an L-marked position. The alternative is to make the notion of government more restrictive by making the notion of an $\mathrm{X}$-command more restrictive. At the moment, government is defined in terms of an m-command. If this is too lax, the possibility to explore is to replace the $\mathrm{m}$-command by a $c$-command or rather an $i$-command. These notions are defined as follows:

c-command

A c-commands B if $A$ m-commands $B$ and the minimal constituent containing $\mathrm{A}$ and something other than $\mathrm{A}$ contains B.

i-command

$\mathrm{A}$ i-commands (immediately commands) $\mathrm{B}$ if the first constituent (distinct from $A$ and) containing $A$ contains $B$. 
Clearly, the c-command would give the wrong result because it would allow incorporation of the head of an adjunct into its selecting head $\mathrm{X}$, if this head does not have any complement, i.e. if $\mathrm{X}^{1}$ does not branch. Aoun and Sportiche (1981) provide further reasons why this conception of c-command is both empirically inadequate and conceptually undesirable. $i$-command on the other hand, coupled with the assumption that L-marked complements of $\mathrm{X}$ appear under $\mathrm{X}^{1}$ will have the desired effects. This will basically have the effect of restricting head movement toward some head $\mathrm{H}$ to the heads contained in the constituents that are sisters to $\mathrm{H}$. Given the assumption that $\mathrm{L}$-marked complements of a head $\mathrm{H}$ are under $\mathrm{H}^{1}$, these two possibilities are almost equivalent: if $\mathrm{HP}$ is in an L-marked position L-marked by another head $\mathrm{K}^{0}$, it will be $\mathrm{i}$-commanded by $\mathrm{K}^{0}$. The converse however is not true. At least in the case of $\mathrm{C}$, the complement of $\mathrm{C}$, IP, is i-commanded by $\mathrm{C}$ but not L-marked by it: the first alternative seems to be predicting the impossibility of I to $\mathrm{C}$. The second predicts that it is possible as we know it is. Cases like (39a) can help us decide: the i-command alternative straightforwardly allows incorporation of $\mathrm{Y}$ into $\mathrm{X}$. Making the non-L-marked boundary of a constituent a barrier even for the head of this constituent seems to be predicting that such incorporation is impossible since YP in (39a) is not $\mathrm{L}$-marked by $\mathrm{X} .{ }^{19}$ Let us therefore adopt the i-command alternative. From now on we adopt the following definition of government:

\section{(41)(a) Government}

A governs $\mathrm{B}$ iff $\mathrm{A}$ i-commands $\mathrm{B}$ and no barrier for $\mathrm{B}$ intervenes between $\mathrm{A}$ and $\mathrm{B}$.

(b) i-command

A i-commands B iff a sister of A contains B.

Naturally, we want this modified notion of government to be used everywhere government is relevant. This move will have far reaching consequences in all areas of grammar using government (e.g. Case, Binding). We will not pursue the questions concerning Binding here. We merely note that this conclusion accords with the conclusions reached by Saito (1984) concerning Binding Theory. ${ }^{20}$ Furthermore, no adverse effects seem to arise concerning Case Theory: as we have seen, only governed Case assignment relies on government, and governed Case assignment by some head $\mathrm{H}$ does seem to be restricted to complements of $\mathrm{H}$ and to specifiers of complements of $\mathrm{H}$ (as in small clause, or ECM, structures): previous cases of Case assignment to specifiers of some XP by its head X must now be uniformly analyzed as cases of agreement Case assignment. One positive consequence follows: a head assigning governed Case to some complement will not be able to assign it to its specifier, since a head does not govern its own specifier. 


\subsubsection{Incorporation of specifiers of complements}

Let us now turn to the former question. In the following configuration:

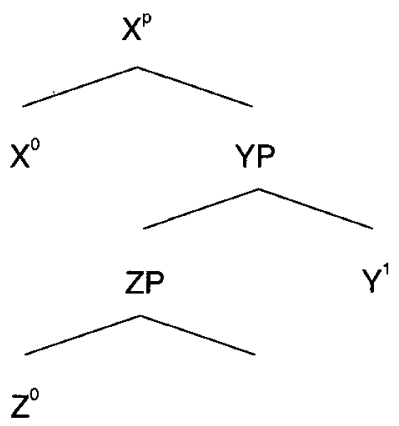

Is it possible to incorporate $Z^{0}$ into $X^{0}$ if $Y P$ is $L$-marked by $X^{0}$, i.e. if $X^{p}$ is $\mathrm{X}^{1}$ ? Is it impossible to incorporate $Z^{0}$ otherwise? Examples of structures (42) with $p=1$ are found in small clause constructions:

(43)(a) de considérer [Pierre malade]/to consider Peter sick

(b) de le considérer [t malade]/him-consider sick

Again, if clitic placement is analyzed as head movement, the second sentence above shows that head movement in such contexts is possible in principle. This is simply a consequence of the fact that the subject of a small clause is governed from the outside (for purposes of structural Case assignment) by the verb. ${ }^{21}$ The same kind of structure is illustrated by a verb taking a direct object which has a nominal specifier (i.e. $\left.V L_{N P} N P N^{1}\right]$ ). We predict that the head of the specifier should, in principle be incorporable into the verb. We will see that this is indeed the case when we discuss the syntax of $e n$ and of dont. The following generalization then seems to emerge from our discussion: given a head $\mathrm{H}$, a head can be incorporated into $\mathrm{H}$ only if it is the head of a complement of $\mathrm{H}$ or the head of the specifier of a complement of $\mathrm{H}$. As a last remark, notice that the illusion that this principle can be violated is found in the following scenario. Suppose $\mathrm{H}$ takes a YP complement and that $\mathrm{Y}$ takes a $\mathrm{ZP}$ complement. Can $\mathrm{Z}$ be incorporated into $\mathrm{H}$ ? According to the above conclusion, the answer should be negative. Suppose however that this incorporation takes place in two steps: this two-step derivation can occur in two different ways. The first is summarized in the structure in (44) opposite.

First, $\mathrm{ZP}$ moves to spec of $\mathrm{YP}$, then $\mathrm{Z}$ is incorporated into $\mathrm{H}$. Each step can be allowed, given the right circumstances, resulting in an apparent violation of our conclusion. We will show in Section 4.1.3 that such apparent counterexamples do exist but there are always good grounds for assuming the existence of the necessary intermediate step through a specifier position. 
(44)

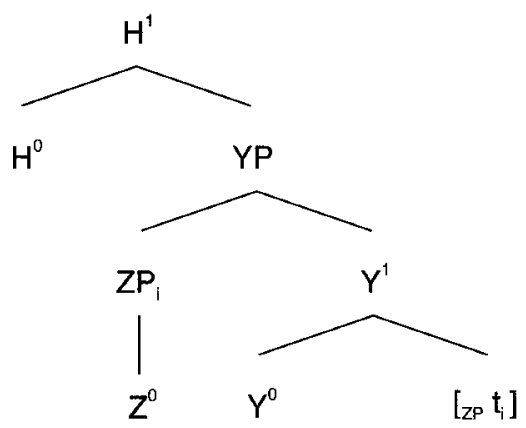

The second way is summarized by the following structure:

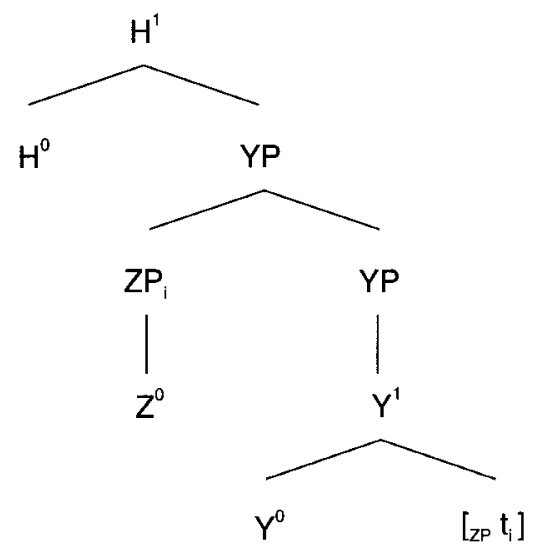

Here, $\mathrm{ZP}$ first adjoins to $\mathrm{YP}$, then movement of $\mathrm{Z}$ to $\mathrm{H}$ takes place. Note again that no barrier is crossed. Naturally, YP must be the kind of category that allows adjunction. Again, we will show in Section 4.1.3 that such derivations exist.

\subsubsection{Successive head movement: excorporation}

Recall that one principle we want to derive is the Head Movement Constraint principle. Limiting movement of a head to the next head up will not be sufficient to derive this result, in particular if successive movement of the same head is allowed. Thus, see the structure in (46).

If $\mathrm{Z}^{0}$ is allowed to move to $\mathrm{Y}^{0}$, and then to $\mathrm{H}^{0}$, movement is always only one head up but becomes essentially unbounded. This type of successive cyclic head adjunction is ruled out by our formulation. Thus moving $Z^{0}$ to $\mathrm{Y}^{0}$ creates the structure $\left[_{\mathrm{Y} 0} Z^{0}+Y^{0}\right]$. Since $Y^{1}$ is not a projection of $Z^{0}$, it does count as a non-L-marked projection containing $Z^{0}$ and thus induces $\mathrm{YP}$ to be a barrier for $Z^{0}$. Moving $Z^{0}$ our of YP is excluded. 
(46)

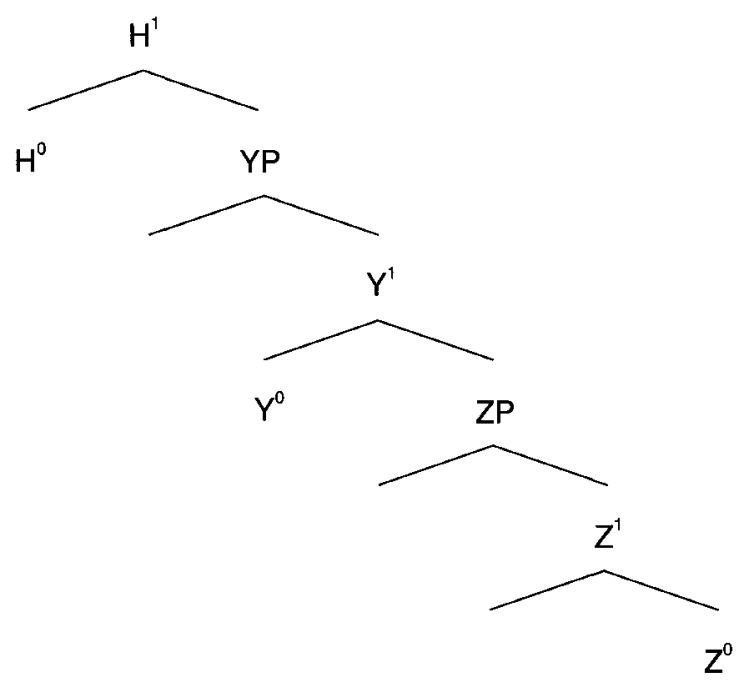

Once the structure has been formed, two types of further movement can take place. Since $\mathrm{Y}^{0}$ is the head of $\left[_{\mathrm{Y} 0} \mathrm{Z}^{0}+\mathrm{Y}^{0}\right]$, it is possible to move $\left[_{\mathrm{Y} 0} \mathrm{Z}^{0}+\right.$ $\left.\mathrm{Y}^{0}\right]$ freely out of YP. Note however the existence of a second option, that we might call excorporation. None of the projections of $Y^{0}$ can induce YP to be a barrier for movement of $Y^{0}$. Since $Y^{0}$ is the head of $\left.{ }_{Y_{0}} Z^{0}+Y^{0}\right]$, this includes $\left.\mathrm{L}_{\mathrm{Y} 0} \mathrm{Z}^{0}+\mathrm{Y}^{0}\right]$ itself. As a result, movement of $\mathrm{Y}^{0}$ from inside $\mathrm{L}_{\mathrm{Y} 0} \mathrm{Z}^{0}+$ $\left.\mathrm{Y}^{0}\right]$ to $\mathrm{H}^{0}$ is possible, creating the following structure:

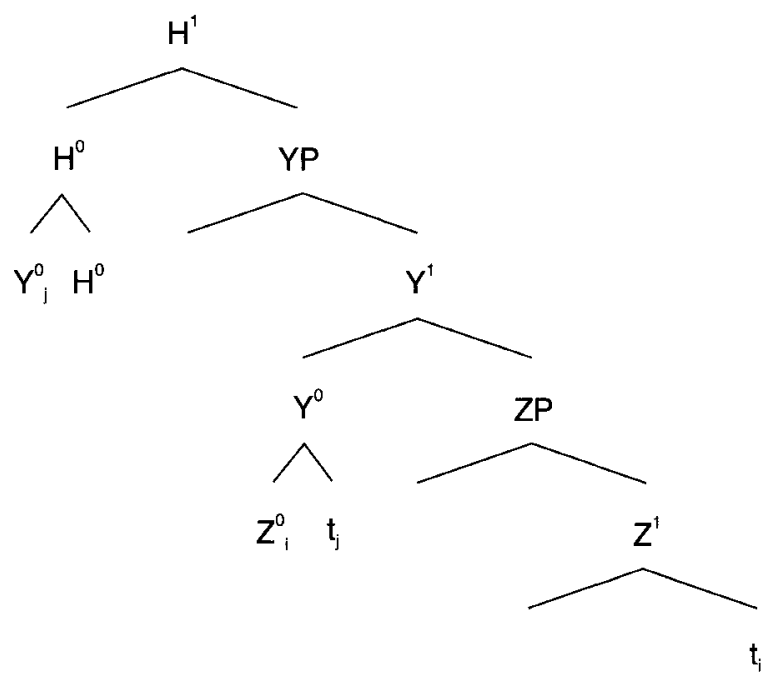

This is the only type of excorporation that is allowed. 


\subsection{Wh Movement}

We consider next the properties of syntactic Wh Movement. In subsequent sections we will turn to NP Movement.

\subsubsection{The grammatical cases}

We begin by making sure that the $\mathrm{CCL}$ as it stands allows the grammatical cases of $W$-extraction, that is, extraction in simple clauses or from embedded declaratives. Consider the following sentences (remember we adopt modified Larsonian VPs):

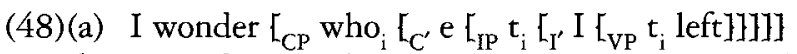

(b) I wonder $\left[_{C P}\right.$ who ${ }_{i} L_{C^{\prime}}$ e $f_{I P}$ you $_{j}\left[_{I^{\prime}} I L_{V P} t_{j}\right.$ saw $\left.\left.\left.\left.L_{V P} t_{i}\right] 1\right]\right]\right]$

Consider first $W h$-extraction from subject position (48a). The subject presumably originates in the most deeply embedded $t_{i}$ position and then raises to a specifier of IP. This is NP Movement and need not concern us here. The $W h$-phrase is inside IP. IP is not L-marked. The only escape is through adjunction to IP. From there, the $W h$-phrase is no longer inside IP in the relevant sense, but is inside $C^{\prime}$. $C^{\prime}$ is not $\mathrm{L}$-marked: so $C P$ is a barrier. But the $W h$-phrase moves to the specifier of $C P$ position and thus crosses no barrier. Consider next the case of (48b) which exemplifies extraction out of VP. The $W b$-phrase NP originates in the position $t_{\mathrm{i}}$. It is inside $\mathrm{V}^{\prime}$, which is not L-marked, so VP is a barrier. But the NP can adjoin to VP. There, it is no longer inside VP. Looking up the tree, we see that it is also inside IP. IP not being L-marked, it must escape by adjunction and so on: we find ourselves in the previous case. So we see that these acceptable cases are predicted as grammatical.

Clearly, we could also extract some VP or clausal adjunct instead of a complement of $\mathrm{V}$ without problems. Consider the next cases involving two (or more) clauses:

(49) I wonder who you think that John saw $t_{i}$.

Obviously, movement from the position $t_{i}$ to the specifier position of its $\mathrm{CP}$ is exactly as in the previous case. From the specifier position of $\mathrm{CP}$, the $\mathrm{Wb}$ phrase can adjoin to the next VP up: CP is L-marked and therefore no barrier. VP is a barrier (since extraction is from inside $V^{\prime}$ ) but can be gotten around by adjunction to VP. Again, we find ourselves in the previous case.

\subsubsection{Subjacency and the CED}

We now turn to ungrammatical cases. As we have shown, extraction out of $\mathrm{AP}, \mathrm{IP}$ and VP is always possible. However, since adjunction to NP, CP and 
PP is barred, the only way a phrase can escape these categories is through their specifier, if it is possible. However this will not be sufficient if they are not L-marked. If they are not L-marked, they will be a barrier to movement. From this we derive the usual constraints on syntactic movement. Let us examine them in turn.

\subsubsection{THE ADJUNCT CONDITION}

Let us begin with the adjunct condition. It states that no extraction is possible out of adjunct clauses. Adjunct clauses are usually PPs. By definition the adjunct boundary is not L-marked. It is always going to count as a barrier for extraction. As an example, consider the case of French. In French, adjunct adverbial clauses are PPs introduced by prepositions such as avant, après and sans taking clausal complements: avant de partirlavant qu'il (ne) parte, après être partilaprès qu'il est parti, sans partir/sans qu'il (ne) parte. Suppose we try to extract some complement XP out of such an adjunct as indicated:

$$
\complement_{P P} L_{P^{\prime}} \text { sans }\left[_{C P}\left[L_{C^{\prime}} \text { que }\left[_{I P} \ldots X P \ldots\right]\right]\right.
$$

Because adjunction to CP and adjunction to PP are excluded, the only way to escape is to move successively through the specifiers of CP and of PP. Suppose that this is possible. Then XP is in the specifier position of PP. Applying the definition of barrier, we see that because we are trying to extract out of a projection of $\mathrm{P}$, namely PP, and because this projection is, by assumption not in an L-marked position, it counts as a barrier. Extraction is therefore impossible: the adjunct clause condition follows.

\section{3 .2 .2 P-STRANDING}

Exactly the same reasoning applies to the core cases of P-stranding. The central generalization states that it not possible to strand non-subcategorized Ps. A non-subcategorized PP is not in an L-marked position. Accordingly, the PP boundary will count as a barrier and stranding is prohibited:

$$
\left[_{N P *} \text { Which meeting }\right]_{i} \text { did you sleep }\left[_{P P} t_{i}\left[_{P^{\prime}} \text { during } t_{i}\right]\right. \text { ? }
$$

In the best case, extraction of NP* will proceed through the [Spec, PP] position. From there the next possible landing site is out of the PP. The PP boundary will intervene between the two successive positions and it is not L-marked: it is a barrier. ${ }^{22}$ 


\subsubsection{THE SENTENTIAL SUBJECT CONDITION AND THE \\ SUBJECT CONDITION}

The subject condition and the sentential subject condition state the impossibility of extracting out of NP subjects or clausal subjects. In languages like English, the subject does not occur in an L-marked position. NP subjects occur in [Spec, IP]. Clausal subjects seem to occur higher up in the tree, as suggested by Koster (1978), perhaps adjoined to IP. Consequently the NP or the CP boundary of these subjects is always a barrier and will prevent extraction, since the adjunction option (to NP or CP) is excluded. We will return to languages different from French or English concerning the position of subject NPs.

\subsubsection{THE COMPLEX NP CONSTRAINT}

The complex NP constraint comprises two Cases: the relative clause Case and the adnominal clause Case. Let us begin with the prohibition of extraction out of a relative clause. Again the crucial property of a relative clause is that it is an adjunct $\mathrm{CP}$, adjoined to some nominal projection (which one exactly does not matter).

$$
\hbar_{\mathrm{NP}} \ldots \mathrm{L}_{\mathrm{N}}^{\mathrm{p}} \ldots \mathrm{N}^{1} \ldots \mathrm{L}_{\mathrm{CP}} \text { wh } \mathrm{E}_{\mathrm{C}}{ }^{1} \mathrm{C} \mathrm{L}_{\mathrm{IP}} \ldots \mathrm{XP} \ldots
$$

Trying to extract XP out of $\mathrm{CP}$, we see that the $\mathrm{CP}$ boundary will again always count as a barrier. Furthermore, extraction is actually from inside $\mathrm{C}^{1}$ since the specifier of $\mathrm{CP}$ is not available, being filled by the relative pronoun. So at least one barrier is crossed, namely $\mathrm{CP}$.

Consider next the other complex NP case, e.g. the assumption that. Here, the structure is that of a CP complement of a head noun:

$$
\tau_{N P} \ldots\left[N^{1} N^{0} E_{C P} \sum_{C}^{1} C E_{I P} \ldots X P \ldots\right.
$$

The specifier of $\mathrm{CP}$ is available and it is not clear that $\mathrm{CP}$ is not in an L-marked position. If it is not (as Stowell (1981) argues, in which case the CP is not under $\mathrm{N}^{\mathrm{l}}$ ), $\mathrm{CP}$ is a barrier and the constraint follows, just as in the relative clause Case, from the adjunct island condition. Suppose on the other hand that it is, i.e. that the structure (53) is correct. Then moving out of $\mathrm{CP}$ from [Spec, CP] is allowed. It must be that the next moving step is out, i.e. getting out of the NP. In order to escape from NP, XP must go through [Spec, $\mathrm{NP}$ s since by assumption, the $\mathrm{CP}$ is dominated by $\mathrm{N}^{1}$. But this is an instance of improper movement: according to (10a), the specifier of an L-marking lexical category such as $\mathrm{N}$ is always an A-position, while the specifier of a non-L-marking category is not: $[S p e c, C P]$ is an $A^{\prime}$-position, then. It follows that this escape hatch is closed. If extraction takes place, at least one barrier is crossed, namely NP. 
Let us now compare the two cases of complex NP violation and begin with the relative clause case. Trying to minimize the number of barriers crossed, we would be extracting XP from a position adjoined to IP (otherwise, IP not being L-marked, another barrier, IP, would be crossed). From there, as seen earlier, we cannot move to [Spec, CP]. CP, a barrier, will be crossed. Next, we need to extract out of the NP containing the CP. Again, [Spec, NP], the obligatory intermediate site, is unavailable as an A-position. Therefore, either we violate improper movement restrictions, or extraction crosses yet another barrier. In sum, this movement either crosses three barriers, or crosses two and violates improper movement.

Let us turn now to the other complex NP case, as in (53). Here the reasoning is exactly the same except that the CP boundary does not count as a barrier. So fewer barriers are crossed than in the previous case. Judgments on complex NP violations usually put the relative clause case as worse than the adnominal clause case. We have a simple explanation for this if we hypothesize, as is plausible, that compounding violations have an additive detrimental effect on judgments. In other words, assume:

(54) Everything else equal, crossing $\mathrm{n}+1$ barriers is worse than crossing n barriers.

\subsubsection{THE WH-ISLAND CONDITION}

Finally, consider the $w h$-island condition, which prohibits $w b$-extraction out of indirect questions, and more generally, out of CPs headed by a wh-element. Here the situation is slightly different:

$$
\ldots \hbar_{\mathrm{CP}} \text { wh } \mathrm{E}_{\mathrm{C}}{ }^{1} \mathrm{C} \mathrm{E}_{\mathrm{IP}} \ldots \mathrm{XP}
$$

Extraction out of CP must take place, except perhaps in whether wh-islands, its specifier position is unavailable: it is already filled by a $w h$-phrase. Since extraction is from inside $C^{\prime}$ to outside $\mathrm{CP}, \mathrm{CP}$ is a barrier. If we are dealing with whether-islands, the conclusion depends on the position of whether. Although it is more commonly assumed to be a complementizer, the evidence presented in Larson (1985) and in Kayne (1990) suggests that whether is a wh-phrase in the specifier of CP position corresponding to either. If whether is in the specifier position, the prediction is that violations of whether-islands should be on a par with other $w h$-island violations.

Violations of whether-islands are sometimes considered better than other $w b$-island violations but worse than regular extraction out of complement clauses. One possibility is that whether is in C, but disallows movement of other wh-elements to or through [Spec, $\mathrm{CP}$ ] of the $\mathrm{CP}$ it heads due to some Spec/head agreement in $C$ that would be violated. The violation then, would be a weak agreement violation, rather than a stronger violation of the CCL. 
If we take into account linguistic variation, we face the inverse problem from that of Chomsky's (1986a) book: our treatment is too strong for Italian or French or more generally for languages allowing wh-island violations, rather than too weak for English. This proposal disallows $w$ b-island violations throughout, Chomsky's (1986a) proposal allows them throughout. Our problem a priori seems more manageable from a learnability point of view, granting the absence of negative evidence. I have no useful proposal at this point. It is perhaps worth noticing that, if there is indeed linguistic variation, it occurs in a case of extraction out of a complement structure.

\subsection{Some further remarks on extraction}

From the above discussions, we see that the CCL derives the effects of the CED and of the Subjacency condition. In other words, the CCL subsumes the Subjacency condition and the CED for syntactic movement. Let us now consider some particular cases of extraction. Remember that wh-extraction out of VPs, IPs and APs is always possible because these are phrases that can be adjoined to. Such is not the case for CPs, NPs and PPs. We have already discussed extraction out of CP. Let us now consider the others.

As we have shown, it follows from the CCL that extraction of $\mathrm{X}^{\mathrm{n}}$ out of NP or PP must go through the specifier position of NP. Furthermore, the NP or the PP must itself be in an L-marked position. From this, it follows that extraction requires at least three conditions to be met:

$1 \mathrm{X}^{\mathrm{n}}$ must be allowed in principle to appear as [Spec, NP] or [Spec, PP].

2 The position [Spec, NP] or [Spec, PP] must be available.

3 The NP or the PP must be in an L-marked position.

Clause (3) is simply the CED already derived. Concerning clause (1), remember, as discussed in (8) and in (21), that only NPs can appear as specifiers of NP or of PP. From this it follows that only NPs can be successfully extracted out of NPs or PPs.

\subsubsection{Extraction out of $P P$ in Dutch}

Let us examine extraction out of PPs. As we have seen, the way barriers are defined combined with the requirement that traces be governed by an antecedent forces movement out of PPs to go through the [Spec, PP] position, and furthermore that this PP be in an L-marked position. In what follows, we limit ourselves to complement PPs throughout. Direct evidence supporting this conclusion comes from the analysis of extraction out of PPs in Dutch. In fact, this is basically the insight of van Riemsdijk's very detailed and convincing (1978) analysis of P-stranding. We now summarize it. Dutch allows preposition stranding of certain prepositions. These prepositions all have the following properties: 
1 They take an NP complement.

2 This NP can be pronominalized.

3 When this NP is pronominalized, if it is er, it appears in front of P; otherwise, it appears in the same position as the full NP.

Some examples are: op de tafeller op $/ *$ op er/op Jan/op bem/*bem op (on the table/on it/on John/on him). Van Riemsdijk's crucial observation is the following:

4 When and only when an NP complement of a preposition can be pronominalized as er can it be extracted out of the PP, thus stranding the $P$.

In that case, the $\mathrm{P}$ can be stranded either by movement of this pronoun, which is a clitic, out of the PP, or by Wh Movement. In the Wh Movement case, the $w h$-word is the $w$-er word, namely waer. This paradigm is illustrated below: ${ }^{23}$

(56)(a) Jan heeft de krant met zijn hand op de tafel gelegd. John has the paper with his hand on the table put. John put the paper on the table with his hand.

(b) Jan heeft de krant er mee op de tafel gelegd.

(c) Jan heeft er de krant mee op de tafel gelegd.

(d) Waer heeft Jan de krant mee op de tafel gelegd.

Of course, the main question is, why does the correlation stated in (4) hold? Van Riemsdijk's analysis states that there is a $[+R]$ position PP initially and that P-stranding either under clitic movement or under $W b$ Movement must transit through this $[+\mathrm{R}]$ position. Naturally, this claim is extremely strongly supported by the fact that the intermediate step is actually directly observable in Dutch (we will see a similar pattern in French extraction out of NP below) at least in the case of clitic (er-)movement. It is immediately apparent that the network of assumptions we have developed provides a general theoretical reason for why van Riemsdijk's analysis should be correct; it derives from van Riemsdijk's analysis, provided we take van Riemsdijk's [+R] position to be the $[\mathrm{Spec}, \mathrm{PP}]$ position:
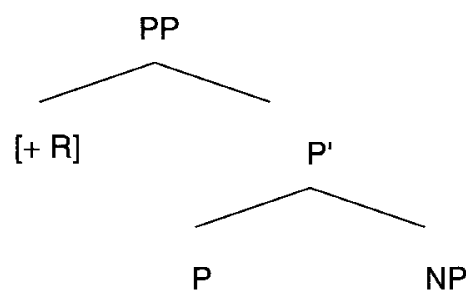

Stranding of a $\mathrm{P}$ by extraction of its complement NP is limited to NPs 
pronominalizable by er because only this kind of NP is allowed to occur in [Spec, PP], and [Spec, PP] as an obligatory intermediate step.

Koopman (forthcoming) shows that this analysis can be extended even further. Dutch does not only have prepositions. It also has postpositions (usually locative prepositions with a directional reading) e.g. de stad in/"into the city." It turns out that these postpositions can always be stranded. ${ }^{24}$ Two questions arise that can be answered in one stroke. Why does Dutch have both prepositions and postpositions? Why do prepositions and postpositions differ regarding stranding possibilities? Koopman argues that postpositions are in fact prepositions that, for Case theoretic reasons (postpositions are agreement Case assigners, prepositions are governed Case assigners), force their complement to move to their specifier positions. In other words, the object of a postposition is forced to occur in the position in which er occurs with prepositions:

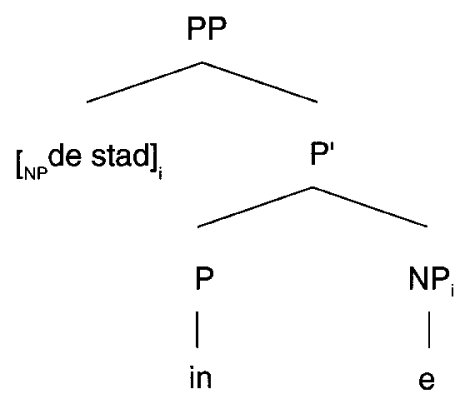

If this is so, it follows that stranding of postpositions should be allowed, since the only restriction operating on P-stranding of complement Ps is due to whether or not the specifier position of the complement of the PP is accessible. Obviously, with postpositions, this specifier position is always accessible. Note that we have not addressed the question of what permits or forces movement of the NP complement of a $\mathbf{P}$ to the specifier of that $\mathrm{P}$. The generalization we have explained is the following: if, and only if movement to specifier is allowed can movement out of PP occur.

\subsubsection{Extraction out of PP in English}

The analysis of extraction out of PPs in Dutch essentially carries over to English with two differences: first, movement of an L-marked NP complement of a $\mathrm{P}$ to $[\mathrm{Spec}, \mathrm{PP}]$ is unrestricted; second, no phrase can overtly appear in [Spec,PP] with prepositions. ${ }^{25} \mathrm{We}$ predict the following paradigm:

(59)(a) Who did you talk to $t$ ?

(b)* What did you sleep during $t$ ? 
(c) What did he pull a rabbit from under $t$ ?

(d) Where did he pull a rabbit from $t$ ?

(e)* Under what did he pull a rabbit from?

The difference between (a) and (b) illustrates the CED: extraction out of a complement is permitted, extraction out of an adjunct is not. ${ }^{26}$ This pattern is certainly the core fact. In (c), an NP has been extracted from inside a PP (under what) itself complement of a P from, itself complement of the verb pull. This is simply a case of iterated movement to specifier:

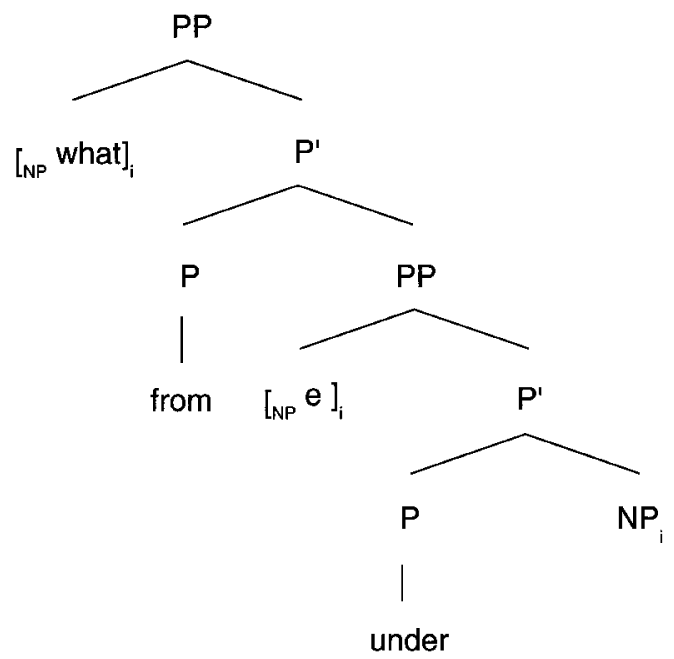

Movement of the $w h$-NP is to the specifier of the first PP complement of from, followed by movement to the specifier of the second PP, followed by movement out of this higher PP. In the (e) sentence, the extraction is excluded because a PP cannot appear as specifier of a P. In the (d) sentence, the NP where ( $=w b$-there) has been extracted, corresponding to the sentence He pulled a rabbit from there. This case is similar to (59a). The predictions are clear: extraction of the NP complement of a $\mathrm{P}$ is possible if the PP is in an L-marked position. This prediction extends to the structure of multiple embedded PPs [P[P[P ... [NP]]1], as long as each PP is L-marked by the preceding one.

One question raised is why is P-stranding not possible in all languages. Obviously, there is an arbitrary property distinguishing P-stranding languages from non-P-stranding languages. One possibility is the following. We qualify the statements made about what can appear in specifier position by the following markedness principle:

(61) Specifier positions are available only if forced by principles of grammar or by the data available to the language learner. 
The existence of these specifier positions does not follow from the Projection Principle: by assumption, they are A-bar positions since they serve as an escape hatch. In English, Dutch or Vata (which also allows P-stranding, cf. Koopman (1984)), learners postulate the possibility of the specifier position in PPs upon hearing P-stranding or upon hearing both NP P and P NP order as in Dutch. In French, no such data is available and P-stranding is impossible. Note further that in English (or presumably Dutch or Vata), there is another kind of direct evidence that specifiers of $\mathrm{P}$ can be used, namely particle constructions. As mentioned earlier, Koopman (1990) shows that particle constructions involve filled specifiers of $\mathrm{P}$. In put Jobn up or put it up, she argues that the NP is in the specifier position of the $\mathrm{P}$ (article). The fact that these constructions are extremely frequent, and Emonds' (1976) showing that particles and prepositions are the same category, provides the evidence needed by the language learner. ${ }^{27}$

\subsubsection{Extraction out of NP in English}

Let us now turn to NPs. ${ }^{28}$ The same considerations apply and the same general conclusions follow: movement out of an NP must go through its specifier, and the NP itself must be in an L-marked position. ${ }^{29}$

Consider English first. Clearly, an NP position as specifier is available as shown by Jobn's book. Consequently, extraction out of NPs is possible:

(62)(a) Who did you see a picture of $t$ ?

(b) ?Of whom did you see a picture?

(c) ?*Who did you see a picture of a portrait of $t$ ?

(d) Who did you talk about $t$ ?

(e) ?*Who did you talk about a picture of $t$ ?

First remember that we do not analyze articles as being in the position specifier of NP. Only NPs can be. The grammaticality of the (a) and the (b) sentences suggests that [whom] and [of whom] can be NPs, i.e. that of can either be analyzed as a P or marginally, as a Case marker on an NP. In the (a) sentence the stranding of the $\mathrm{P}$ of takes place first by movement through its specifier position. From there, movement proceeds to the specifier of NP and then out of the NP. The (b) sentence is derived the same way by successive movement of the NP [of whom]. The (c) sentence is an iteration of the same derivation found in the (a) sentence, and is predicted as grammatical, although it is judged worse than the (a) sentence. The (d) sentence is a case of P-stranding. The (e) sentence is an iteration of a different sort: first Pstranding, then extraction out of NP, then P-stranding again. Again, this is predicted as grammatical even though it is judged worse than the (d) sentence. If for some reason, the specifier is unavailable, extraction should be blocked. One reason why the specifier could be unavailable is if it is lexically filled. ${ }^{30}$ This prediction is correct: 
(63)(a)* Who did you see John's picture of $t$ ?

(b)* Who did you see my pictures of $t$ ?

(c)* Who did you see these pictures of $t$ ?

In each the specifier position is already occupied, blocking extraction. In the (a) sentence, it is occupied by a proper name, in the (b) sentence by a pronominal NP, in the (c) sentence by a demonstrative NP. The fact that demonstratives block extraction suggests that they should be analyzed as NPs, which they essentially have the distribution of. This is consistent with the fact that they cannot co-occur with full NP specifiers or possessive pronouns in English. ${ }^{31}$ The fact that demonstratives can co-occur with numerals (these three) coupled with the fact that there is only one specifier of NP per NP shows that numerals do not occur in the specifier of NP, ${ }^{32}$ they should not prevent extraction from NP. The contrast noted in Chomsky (1986a) between examples (63) and the examples below follows:

(64)(a) Who did you see three pictures of?

(b) Who did you see more pictures of (John or Bill)?

Movement of APs in general can only be through successive adjunction as in How angry did Jobn make his friends? (adjunction to VP, then to IP, then movement to [Spec, CP], since movement through specifier is prohibited, except of course for [Spec, CP]). Extraction of APs out of NPs should be impossible: from I met (a man proud of Bill) we cannot derive How proud of Bill did you see (a man t)?

Finally, we predict again that PP extraction out of NPs should not be possible. Roughly this seems correct (see the discussions in Huang (1982), Chomsky (1986a: 80, examples (181))). One case of PP extraction that seems allowed is the pied-piping of of. As we mentioned above, we can propose that of is ambiguously analyzed as a P or marginally as a Case-marking affix on the following NP. In the latter case, it is an NP that is in fact extracted. We are left with the problem illustrated by examples (62c and e). They are predicted as fully grammatical but appear deviant - it looks as if extraction from an NP inside an NP is deviant (example (62c)) (Chomsky (1973) discusses this paradigm and some possible counterexamples) as well as extraction from an NP inside a PP (example (62e)). However, extraction from a PP inside a PP is possible (example (59c) discussed earlier), as well as extraction from a PP inside an NP (example (62a)) or similar examples e.g. who did you read a book about? Clearly, this system must somehow be tightened. Call this Problem 1. We leave it unresolved until Section 6.

\subsubsection{Extraction out of NP in French}

Let us now turn to French. In French the facts concerning the availability of [Spec, NP] are more complicated and more interesting. The basic facts are 
well known. They have been discussed in Ruwet (1972), Milner (1978) and Zubizarreta (1979). Preanalytically, they can be described as follows:

1 Only pronominal or demonstrative NPs can occur overtly as specifier of $\mathrm{NP}^{33}$

2 A pronominal specifier must correspond to a de NP.

3 This de NP is either a possessive, an "external" argument or an "internal" argument of the $\mathrm{N}$. Correspondingly we can have:

(65)(a) Le portrait de ce collectionneur/son portrait (=appartenant à).

(b) Le portrait de Rembrandt/son portrait (=par Rembrandt).

(c) Le portrait d'Aristote/son portrait (=le dépeignant).

4 This correspondence obeys the following rule. The possessive determiner corresponding to the de NP can be a possessive if there is one, then an "external" argument if there is no possessive, then an internal argument if there is neither possessive nor external argument. Thus although son portrait/bis portrait is ambiguous in three ways: the portrait I own, the portrait by me and the portrait of me, son portrait de Jean is only ambiguous in three ways instead of the a priori possible six ways: the portrait I own by Jean, the portrait I own of Jean, the portrait by me of Jean. Given a thematic hierarchy ordered possessor > external argument $>$ internal argument, we have the following generalization:

(66) In a structure: $\complement_{\mathrm{NP}}$ specifier $\mathrm{L}_{\mathrm{N}}{ }^{1} \mathrm{~N}$ [de-NP]] the role of the specifier must always be higher than that of the [de-NP].

We offer no account for observation (66) until Section 6. Let us call the problem of accounting for it Problem 2. Instead, let us concentrate on the following observations:

(67)(1) The possible interpretations of nominal elements extracted out of an NP faithfully reflect that of nominal specifiers, and consequently

(2) the presence of a nominal specifier blocks extraction.

This second observation is similar to that found in English: the presence of an overt specifier blocks extraction. The first observation states that in a structure like:

$$
N P_{i} \ldots \quad t_{N P}\left[N[\text { de-NP }] t_{i} .\right]
$$

the thematic role of $\mathrm{NP}_{\mathrm{i}}$ must be higher on the hierarchy than the [de-NP]. 
This is exemplified by two sorts of extraction: Wh Movement and clitic placement. Concerning $\mathrm{Wb}$ Movement, we observe that the (a) sentence below is ambiguous in three ways while the (b) sentence is only ambiguous in three ways exactly as above, instead of the a priori possible six.

(69)(a) L'homme dont $t_{i}$ j'ai vu le portrait $t_{i}$. The man of whom I saw the porrtait.

(b) L'homme dont $t_{i}$ 'ai vu le portrait de Jean $t_{i}$. The man of whom I saw the portrait of John.

An explanation of this correlation follows if wh-extraction out of NP is through the specifier of the NP: movement to specifier is, for some reason, restricted in the way it is and influences the interpretation of whatever has moved to the specifier position. Naturally, if movement to specifier is blocked, say by the presence of a demonstrative NP, wh-extraction out of NP is ruled out. As Longobardi (1987) and especially Giorgi and Longobardi (1991) discuss, the same reasoning applies to Italian.

This account of wh-extraction out of NPs extends to the extraction of genitive en out of NPs. The facts are exactly the same as in the $w b$-extraction case:

(70)(a) Pierre en $\mathrm{i}_{\mathrm{i}}$ a vu le portrait $\mathrm{t}_{\mathrm{i}}$. Peter of him saw the portrait.

(b) Pierre en $n_{i}$ a vu le portrait de Jean $t_{\text {. }}$. Peter of him saw the portrait of John.

The (a) sentence is ambiguous in three ways. The (b) sentence is also ambiguous in three ways instead of the a priori possible six. Granting the property (66), the observations in (67) are exactly what we predict: extraction out of NP must proceed through its specifier position, mirroring the restrictions found on movement to specifier NP internally.

There are however a number of questions that are left unresolved by this account. Why is movement to specifier NP internally incompatible with certain determiners (e.g. a possessive or demonstrative determiner cannot co-occur with it), suggesting they occur in the same position, but movement of the clitic en or of a $w b$-phrase allows the article to appear as in (70)? If extraction is through the article position, it should not be able to appear. How does movement of the clitic en exactly take place? If en extraction proceeds as we claim it does, what kind of movement is the movement from its basegenerated position to the specifier of the NP? We have claimed that this specifier position, as specifier of a lexical category, is an A-position but we will see later on that this conclusion is due to an oversimplification. We will return to these questions in Section 6. 


\subsubsection{Extraction out of subjects}

Consider now the special case of extraction out of subjects. Subjects can be NPs or clausal. Clausal subjects can never occur in an A-position, since A-positions can only be NPs. In particular a clausal subject can never occur in the position specifier of VP, i.e. NP* of (10) below:

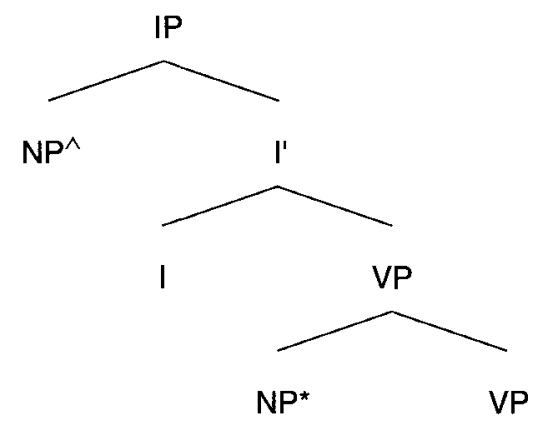

Consequently, a clausal subject is never in an L-marked position and its CP boundary is always a barrier. The sentential subject constraint should be an absolute constraint, i.e. should not be subject to crosslinguistic variation. Consider next the case of NP subject. In English, an NP subject must raise to $\mathrm{NP}^{\wedge}$ of (10). Call languages requiring such movement Class 1 languages. The same reasoning applies here as the one deriving the subject condition. Since the subject NP is always in a non-L-marked position, the NP boundary will act as a barrier blocking extraction. Consider however languages in which raising from $\mathrm{NP}^{*}$ to $\mathrm{NP}^{\wedge}$ is not obligatory. Let us call them Class 2 languages. Consider further a sentence in which the external argument appears in NP* (or partly raised out of $\mathrm{NP}^{*}$ but not to $\mathrm{NP}^{\wedge}$ ). Extraction from inside NP* is not permitted by the system of principles developed thus far; (see (71) on page 130 ).

Some complement $\mathrm{NP}_{\mathrm{i}}$ of the head noun of $\mathrm{NP}^{*}$ can move to [Spec, NP*], then adjoin to IP. However the NP* boundary is a barrier since NP* is not an $\mathrm{L}$-marked position. The prediction then is that this class of languages should obey the subject condition. Extraction from subjects is ruled out in all cases.

For English, these conclusions seem correct. For French, another Class 1 language, they are not correct since extraction out of subjects is possible. Conversely, for Class 2 languages, these conclusions seem incorrect regarding the subject condition. Extraction from subject in a VSO language like Welsh is possible (with the indirect strategy). Extraction from subject in Chinese, plausibly a Class 2 language, seems possible (cf. Huang (1982)). Extraction from preverbal subject in Italian is marginally possible, while extraction from postverbal subject (a Class 2 situation) is possible (Cinque (1980)).

In order to accommodate these facts we need to revise our notion of barrier 
(71)

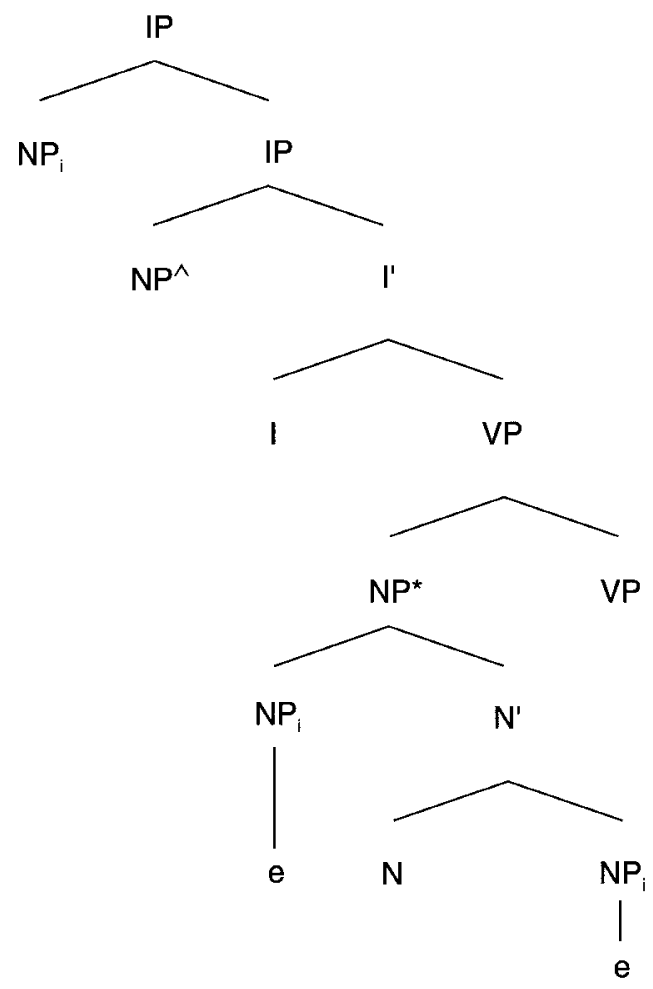

to permit the specifier of an L-marked category to sometimes count as Lmarked. This would remove the barrier status of NP* in (71) but would not affect that of $\mathrm{NP}^{\wedge}$, since IP is not L-marked. ${ }^{34}$ To accommodate the Welsh/ Chinese-like facts, we modify barrierhood:

\section{Barrierbood}

Given $\underline{B}$ some constituent, and $\mathrm{Y}$ some category with $\underline{B}$ $\neq Y^{\mathrm{n}}$ : if for some $\mathrm{p}, \mathrm{Y}^{\mathrm{p}}$ is not an $L$-dependent position and includes $\underline{B}$, then $\mathrm{YP}$ is a barrier for $\mathrm{B}$.

where we tentatively define $\mathbf{L}$-dependence as:

\section{(73) $L$-dependence}

An XP is L-dependent if it is L-marked, or if it is

$[\mathrm{Spec}, \mathrm{XP}]_{\mathrm{P}}$ of an L-marked $\mathrm{XP}^{35}$, where a $\left[\right.$ specifier $_{\mathrm{P}}$ is a specifier with property $\mathrm{P}$.

The need for requiring an additional property $\mathrm{P}$ arises from the following examples. Consider P-stranding in $[\mathrm{Spec}, \mathrm{CP}]$ as in:

$(74)^{*} \quad \mathrm{Who}_{\mathrm{j}}$ do you think $\mathrm{L}_{\mathrm{CP}}\left[_{\mathrm{PP}} \text { with } \mathrm{t}_{\mathrm{j}}\right]_{\mathrm{k}}\left[\right.$ John talked $\left.\left.\mathrm{t}_{\mathrm{k}}\right]\right]$ 
The PP is preposed in the embedded clause. There, as a specifier of CP, it is L-dependent. P should be strandable. It might be argued that some sort of necessary reanalysis is involved in P-stranding, which is possible with talk with but not with think with. However, the same point can be made with the clitic en in French:

(75)(a) Tu as vu quel film d'Almodovar? You saw which movie by Almodovar?

(b) Tu en as vu [quel film $t_{j}$ ]? You by-him-saw which movie?

(c)* Tu en ${ }_{\mathrm{j}}$ demandes [ [quel film $\left.t_{j}\right]_{\mathrm{k}}\left[\right.$ ill a vu $\left.\left.t_{\mathrm{k}}\right]\right]$. You by-him-ask which movie he saw. (= You ask which movie by him he saw.)

The object NP is preposed by $W b$-movement in the embedded clause. The NP is L-dependent, so no barrier intervenes between $t_{j}$ and the verb, and clitic placement should be possible, contrary to fact. The same point can be made in NPs.

(76)(a) Who did you visit [friends of $t$ ]? (b)* Who did you visit [ [friends of $t$ ] parents $]$ ?

If any specifier of an L-marked specifier can count as L-dependent, unacceptable extractions will be allowed. Some qualification then must be added that restricts the kind of specifiers that can become L-dependent. Assuming that NP* in (71) has property P, the Welsh facts are accommodated. So we need to distinguish such cases as NP* in (71) from [Spec, CP $]$ as in (74) and (75c) and [Spec, NP] as in (76). One obvious candidate is the A/A-bar distinction. If we take $\mathrm{P}$ to mean that the position must be an $A$-position, we draw the required distinction. Of course, this means that $[\mathrm{Spec}, \mathrm{NP}]$ has to count as an A-bar position. ${ }^{36}$ This is what we will assume now so that we can define Ldependence as:

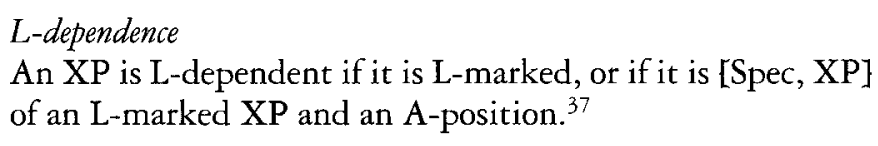

The prediction that the subject condition should hold in languages in which $\mathrm{NP}^{*}$ must raise to $\mathrm{NP}^{\wedge}$ holds in English. In French, the subject condition should hold as well. But it seems it does not:

(78)(a) La ville dont le général a ordonné [la destruction t]. The city of which the general ordered the destruction.

(b) La ville dont [la destruction $t\}$ serait entreprise. The city of which the destruction would be undertaken. 
The relative pronoun dont is interpreted as a genitive complement of the head noun destruction. In the (a) sentence, this relative pronoun has been extracted from inside a direct object NP. In the (b) sentence, it has been successfully extracted from a subject NP, thus violating the subject condition. Dont can only be used as a relative pronoun. In direct or indirect questions, the extraction of the genitive complement of the noun can take place by using de $w h-N P$, i.e. a $w b-\mathrm{NP}$ preceded by the Case marker $d e$. The surprising fact, however, is that the subject condition cannot be violated in that situation:

(79)(a) La ville de laquelle le général avait ordonné la destruction. The city of which the general had ordered the destruction.

(b)* La ville de laquelle la destruction serait entreprise. The city of which the destruction would be undertaken.

The second example is deviant. We now have a double problem. Why can the subject condition be violated in French and what is the difference between (78b) and (79b)? The unacceptability of the latter follows if the subject condition does hold in French as expected, because the subject condition is intended to prohibit phrasal movement only from inside a subject. It can be remarked that in (78b), the extracted $w b$-element is monomorphemic. I want to suggest that $W h$-movement of dont involves movement of dont to $\mathrm{C}$, instead of (or in addition to) movement of a $w$-phrase to $[\mathrm{Spec}, \mathrm{CP}]$. Suppose that dont is a clitic and as such must cliticize locally on a superordinate functional category, $\mathrm{C}$. The derivation would proceed as:

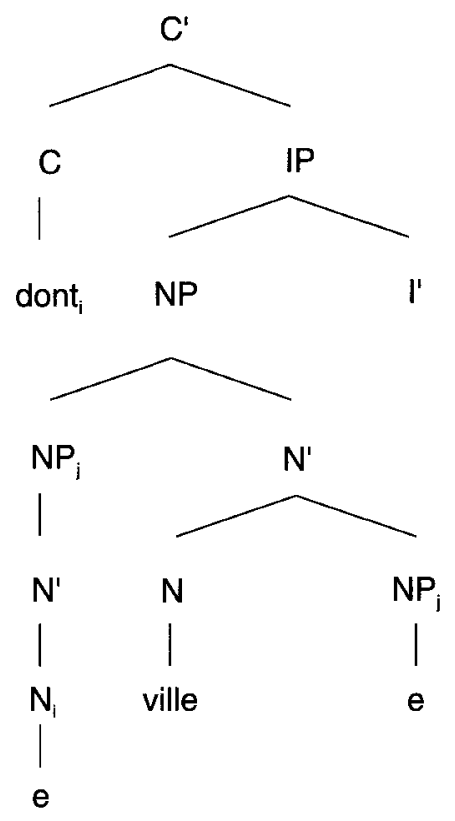


The genitive object $\mathrm{NP}_{\mathrm{i}}$ moves to the specifier of the subject NP. There, the head $N_{i}$ of this derived specifier cliticizes to $C$ (alternatively NP first adjoins to IP, crossing a barrier, whence dont cliticizes to $\mathrm{C}$ ). This should be blocked however because IP and the subject NP are barriers. Dont, being an N, now governs IP. If this counts as L-marking, it will remove the barrier status of IP, and by L-dependence, of the subject NP. A posteriori, this will license the cliticization (or the adjunction to IP). This analysis predicts that dont constructions should be excluded from contexts in which a wh-operator is actually selected, e.g. in direct or indirect questions, since dont constructions involve none. It is permitted in relative clauses precisely because they are modifiers. However, it is also predicted that dont constructions can license violations of the subject condition only locally:

(81) Un homme dont on croit que $\left[_{\mathrm{NP} *}\right.$ le fils $]$ réussira. A man of whom we believe that the son will succeed.

Dont raises to the upper $C$ first by $W b$ Movement ending in adjunction to the upper IP, followed by cliticization. The lower IP, hence the lower subject, is not L-dependent and should not be able to contain a trace. Extraction in (81) should not be from NP*. This is precisely what is shown by Tellier (1990), who argues for a similar idea of dont extraction. A more serious problem arises in English sentences like (76a). It must be assumed that I to $C$ of $d o$ or any other auxiliary verb is not able to induce L-dependency of the subject of IP in the same way dont does. Otherwise, (76a) should be good.

\subsection{NP Movement}

We now examine NP Movement. By definition, NP Movement is movement from an $\mathrm{A}$-position to an $\mathrm{A}$-position. Consequently, intermediate adjunctions are excluded. Recall the characterizations of barrierhood and intervention we have adopted:

Barrierbood

Given $\underline{B}$ some constituent, and $\mathrm{Y}$ some category with $\underline{B}$ $\neq \mathrm{Y}^{\mathrm{n}}$ : if for some $\mathrm{p}, \mathrm{YP}$ is not an L-dependent and includes $\underline{B}$ then $\mathrm{YP}$ is a barrier for $\mathrm{B}$.

Intervention

A projection $\mathrm{Y}^{\mathrm{p}}$ intervenes between $\underline{a}$ and $\underline{b}$ if $\underline{a}$ is excluded by $\mathrm{Y}^{\mathrm{P}}$ and $\underline{b}$ is included in $\mathrm{Y}^{\mathrm{P}}$.

This implies that the only way to escape an XP by NP Movement is to move through its specifier position and to have XP L-dependent. For example, in order to escape VP, movement must proceed through its specifier, i.e. through NP*. The general rule we derive for NP Movement, then, is this: 
(83) NP movement out of the highest projection of X present in a structure must transit through the specifier of this highest projection.

Naturally there are other conditions of NP Movement (distribution of the Case positions and theta positions in the chain - cf. Sportiche (1983) for example, perhaps the Uniformity Condition of Chomsky, (1986b)). Let us examine the consequences of (83) for passive and raising constructions.

\subsubsection{Passive}

Assume $\mathrm{X}$ is an $\mathrm{NP}^{38}$ governed by a verb $\mathrm{V}$ with passive morphology. $\mathrm{X}$ must move for reasons of Case. Where can it move to? In order to get Case, it must escape the $\mathrm{V}$ projection. The only way it can do so is by moving through all the specifiers of the phrasal projections containing it:

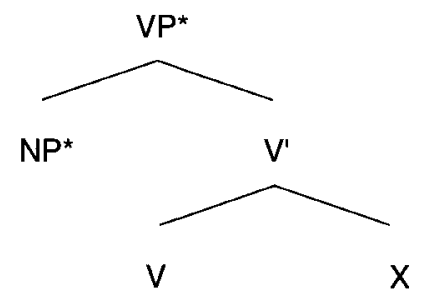

Since $\mathrm{V}$ is passive, (I will assume here that) it projects no external argument so that VP* is the maximal projection of $\mathrm{V}$ and $\mathrm{X}$ must move through NP*. If this position receives Case, nothing further need take place. Otherwise, raising must proceed to the specifier of IP. ${ }^{39} \mathrm{Can}$ an object NP be passivized over a subject? If VP had an external argument the structure in (84) would be changed to contain the substructure:

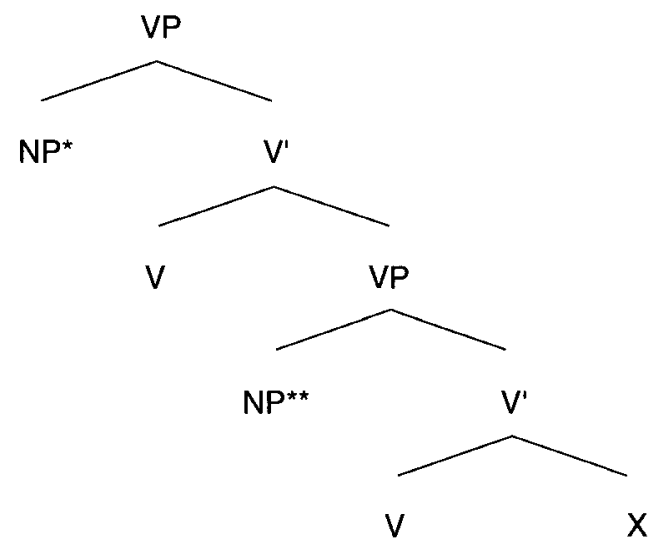


As above, movement must proceed through NP*. However, NP* is a theta position and NP Movement to a theta position is excluded (by the projection principle and the theta criterion). Basically, this derives from the effects of Principle A of the Binding Theory as it applies to NP Movement traces created by passive (and similar constructions: unaccusatives). In effect, it reduces these Binding theoretic effects to theta theoretic effects. There is one particular case to examine in more detail: NP Movement with ECM verbs. Consider the following sentence:

(86)(a) I believe [John to have left].

(b) John is believed [t to have left].

(c) Who do you believe [John to have seen $t$ ].

$J o b n$ in [Spec, IP] is somehow governed by believe since it receives the accusative Case like a direct object. This means that IP is governed by the ECM verb (either because the ECM verb Case-marks John under government, or because John raises out of IP to get Case higher in the structure so that IP cannot be a barrier). One way to construe this is to claim that ECM verbs exceptionally take IP complements. In this case, IP is not a barrier for a category moving out of its specifier, as required by the (b) sentence, since NP Movement does not allow intermediate adjunctions. With $\mathrm{Wb}$ Movement out of this IP being possible (the (c) sentence), it proceeds through adjunction to IP. This is necessary because extraction out of this IP comes in fact from within I'. IP should count as a barrier. The possibility of adjoining to IP makes the absence of a CP unproblematic. One alternative is to assume that the exceptional property of ECM verbs is: The projections of $C$ of a CP complement of an ECM verb are not barriers. This will have all the desired effects. ${ }^{40}$ From now on, we assume one or the other alternative for the class of "S-bar deletion" verbs.

\subsubsection{Raising}

Basically, the derivation is the same as in the passive case. Movement proceeds from the embedded $\mathrm{NP}^{*}$ to the embedded $\mathrm{NP}^{\wedge}$, that is from the highest specifier of VP to the specifier of IP. From there, it must move to the matrix NP*, which is possible only if the matrix VP does not have an external argument and the embedded CP boundary is transparent or absent. The case of raising adjectives is very similar:

(87)(a) I consider [John likely to leave].

(b) John is likely to leave.

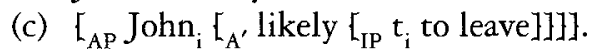

Inside the embedded IP, John raises as usual. From there it escapes IP in a way 
similar to that of ECM verbs and must move to [Spec, AP]. This is the derivation of the (a) sentence. For the (b) sentence, the last step above might be replaced by movement to [Spec, AP] (if AP does not necessarily have a subject). From there movement proceeds in the same way as in the passive case. Note that it is crucial to assume that the verb be L-marks its complement like Infl does.

\subsubsection{Super raising and Lasnik's examples}

The impossibility of super raising, or of Lasnik's (1985) examples illustrated in (88) minimally falls out from the CCL:

(88)(a) John $n_{i}$ seems [that it was killed $t_{i}$ ].

(b) John $n_{i}$ seems [that it appeared [ $t_{i}$ to be intelligent].

(c) A man seems [there to be killed $t_{\mathrm{i}}$ ].

(d) John ${ }_{i}$ is believed [that he likes $_{\mathrm{i}}$ ].

The first three examples are examples of super raising (the last two discussed in Chomsky (1986a)). In each case, movement is blocked by CCL. In the (a) and (b) sentences, the presence of the $\mathrm{C}$ projection forces movement through [Spec, CP], an A-bar position, and they reduce to a case of improper movement. In the (c) sentence, movement is blocked by the presence of there in [Spec, IP], which is an obligatory intermediate position.

Example (d) is due to Lasnik (1985). As formulated so far, the CCL does not require that the antecedent governor of a trace is actually the moved phrase or one of its traces. This example requires some modification. One possibility is to strictly restrict antecedent government satisfaction to the members of a single chain. As he in (d) does not belong to the same chain as $t_{i}$, it does qualify. Another possibility is to allow be to act as antecedent governor but to require that it too be somehow antecedent governed. Since the only possible antecedent governor for be would be Jobn, sentence (d) would be excluded. At this point, either alternative would work. The former is conceptually simpler and more natural. We return to further discussion in Section 5.4.3.

The solution explored by Lasnik in terms of a condition requiring adjacent elements in a chain to be in a local binding relation (discussed in Chomsky (1981), Rizzi (1986a) and Sportiche (1983)) is stronger than the CCL. The CCL construed as a condition on chain formation, does not require anything beyond what is necessary to handle the usual constraints on movement. Second, it does not require the relation of antecedent government to be a relation of local binding. As noted by Lasnik, the local binding condition creates problems precisely when an intervening local binder is not a subject (the seem cases, possibly the strike cases, cf. Lasnik, (ibid.)). Our approach does not have these problems. Given the result of the text, chains can be formed freely subject to the CCL. ${ }^{41}$ 


\section{Specifiers, clitics and agreement}

We now turn to the syntax of clitics in French and its relation to participle agreement. The basic investigation tool we use is the theory of movement rules we have developed and justified so far. This will force an analysis of clitic placement in terms of a combination of NP Movement, $\mathrm{Wb}$ Movement and head movement. This view of movement interacts with participle agreement, in that it basically derives the analysis of clitic-participle agreement that Kayne (1985; 1989a) originally suggested and that we adopt. The Theory of Movement outlined so far thus provides a reason why Kayne's approach is correct. Not all of the participle agreement pattern is immediately derived. We extend this analysis to cases of subject/participle agreement found in passive, unaccusative and se constructions, leaving a number of problems unsolved which we address in the next section.

\subsection{Movement of clitics}

\subsubsection{Background}

There are three fundamental assumptions I make about the analysis of the clitic element I discuss here. ${ }^{42}$ The first is that a clitic element in French syntactically binds a silent category in argument position. The main reason is that constructions in which clitics appear are also constructions in which a lexical NP can appear instead of the clitic. But the clitic appears in a category that may have no lexical relation with the category assigning the full NP its semantic function. There are two clear cases: (1) clitic climbing in causatives: a clitic may appear on the causative verb while it is an argument of some embedded verb. (2) small clause constructions: a clitic may appear on a verb even though it is the argument of a predicate embedded under that verb. The most obvious and the least controversial case is a third case: in a simple clause with (aspectual auxiliaries) a clitic argument of some verb always appears on the highest auxiliary verb in that clause. Consequently, the distribution of clitics could not be construed solely as the result of some intransitivization process.

The second assumption I am making is that this relation between the clitic and its associated silent category is a movement relation. Here, reasons are more complex. The strongest empirical argument $\mathrm{I}$ know in favor of this position is based on the facts of agreement we will discuss later.

The third assumption I am making, which I hinted at earlier on, is that, following Kayne (1989b), clitics are heads adjoined to their hosts. I find the reasons Kayne gives compelling (and the analysis of clitics as moved heads is the only one making sense of the otherwise desirable movement analysis of clitic placement). Let me repeat these reasons:

(89)(1) A clitic is one word long.

(2) A clitic never bears stress. 
(3) A clitic cannot be coordinated.

(4) Nothing can intervene between a clitic and its host (except other clitics).

(5) Syntactic processes affecting heads (movement) can affect the host and the clitic as well.

For example, this last point is illustrated by the complex inversion construction. The complex inversion construction illustrated below in (90) is only available in root clauses, and has therefore been analyzed by Kayne (1984) and Rizzi and Roberts (1989) as involving verb movement to Comp. The (a) example illustrates this construction without object clitic and the (b) example with object clitic:

(90)(a) $\left[_{C P} \operatorname{Jean}_{i}\left[\right.\right.$ aurait $-i l\left[_{I P} t_{i}\left[t_{i}\left[_{V P}\right.\right.\right.$ connu Pierre $\left.\left.\left.\left.]\right]\right\}\right]\right\}$ ? Would John have known Peter?

(b) $\mathrm{L}_{\mathrm{CP}} \operatorname{Jean}_{\mathrm{i}}\left[\mathrm{l}_{\mathrm{k}}\right.$ aurait $_{\mathrm{i}}$-il $\mathrm{L}_{\mathrm{IP}} \mathrm{t}_{\mathrm{i}}\left[\mathrm{t}_{\mathrm{j}} \mathrm{L}_{\mathrm{VP}}\right.$ connu $\left.\left.\left.\left.\left.\mathrm{t}_{\mathrm{k}}\right]\right]\right\}\right]\right\}$ ? Would John have known him?

In the (a) sentence, the aspectual verb has raised from I to $C$. In the (b) sentence, the derivation is similar except for the fact that the direct object clitic must appear in $\mathrm{C}$.

As a final preliminary note on clitics, I will follow Kayne (1991) in assuming that clitics in French must adjoin to some functional category. As far as we are concerned this category will always be I (inflection) (i.e. either Tense, or some other inflectional affix the verb has raised to).

\subsubsection{Clitic objects of verbs}

Let us begin with objects of verbs. An object of a verb can cliticize onto Infl. What kind of derivation is permitted by the theory of movement presented so far?

Consider the following examples:

(91)(a) avoir donné le chapeau à la femme have given the hat to the woman

(b) $\mathrm{l}_{\mathrm{i}}$ ' avoir donné $\mathrm{t}_{\mathrm{i}}$ à la femme it-have given to the woman

(c) lui avoir donné le chapeau $t_{j}$ her have given the hat

(d) le lui $i_{i}$ avoir donné $t_{i} t_{i}$ it-her-have given

They exemplify the case of a direct object (accusative) pronoun and of an indirect object (dative) pronoun cliticizing on the aspectual auxiliary. Consider the derived structure of (91b) for example: $:^{43}$ 
(92)

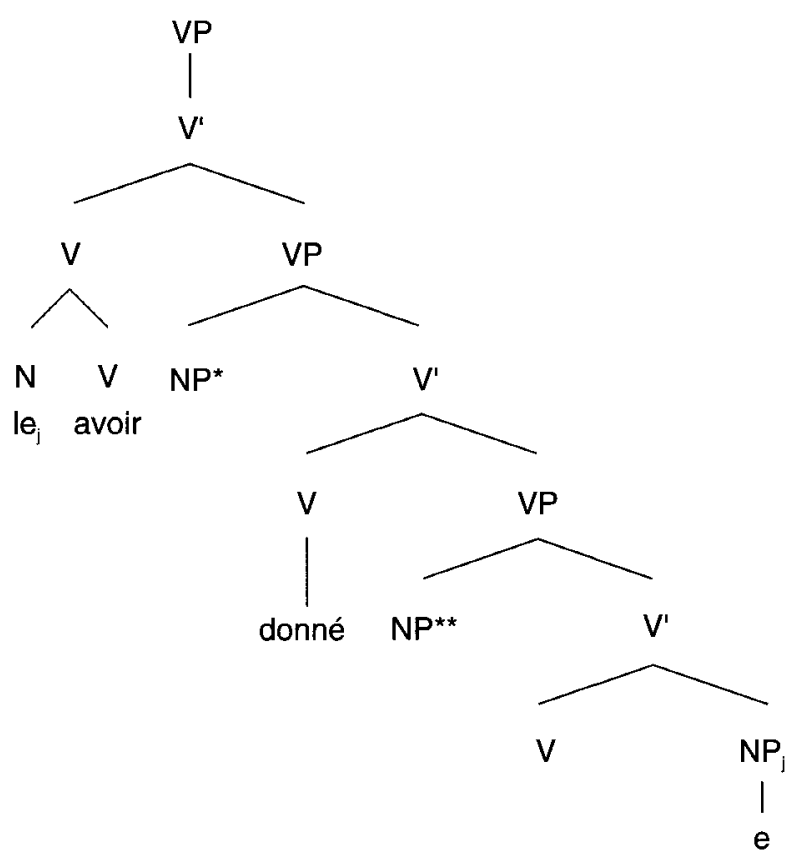

The clitic ends up adjoined to the aspectual V, coming from the object position of the main $\mathrm{V}$. We are not trying to derive why the clitic ends up there but rather how the derivation proceeds given that it does. Recall that there are exactly three ways to escape an XP:

1 by being the head of XP,

2 by adjoining to $\mathrm{XP}$ if possible,

3 by moving to $[\mathrm{Spec}, \mathrm{XP}]$ if $\mathrm{XP}$ is not a barrier.

We observe that the clitic escapes VP. Can the clitic count as the head of VP? One possibility might be to adjoin first the clitic $\mathrm{N}$ to the main $\mathrm{V}$, and then to adjoin it to the aspectual $V$, i.e. proceed by successive head adjunction. This is ruled out. After having adjoined to the main $\mathrm{V}$, the clitic is part of the structure $\left.\left[_{V}\left[_{N} C L\right] L_{V} V\right]\right]$. The clitic $N$ cannot count as the head of VP. The CCL rules out this kind of derivation. ${ }^{44}$ We are led to conclude that the clitic therefore escapes either by adjunction to VP or through $[\mathrm{Spec}, \mathrm{VP}]$ as shown below in (93).

In such a structure, the object either raises to NP** or/and adjoins to $\mathrm{VP}_{\mathrm{k}}$. Then it must adjoin to $\mathrm{VP}_{\mathrm{h}}$ since $\mathrm{NP} *$, being a theta position, is not available. From there the clitic $\mathrm{N}$ adjoins to the aspectual V. Thus Clitic Placement is seen as a combination of head movement, $W b$ Movement (adjunction to VP) and NP Movement (movement to [Spec,VP]). This kind of derivation is of course available for any kind of clitic dependent on a verb originating in VP: accusative objects, dative indirect objects and locatives. 
(93)

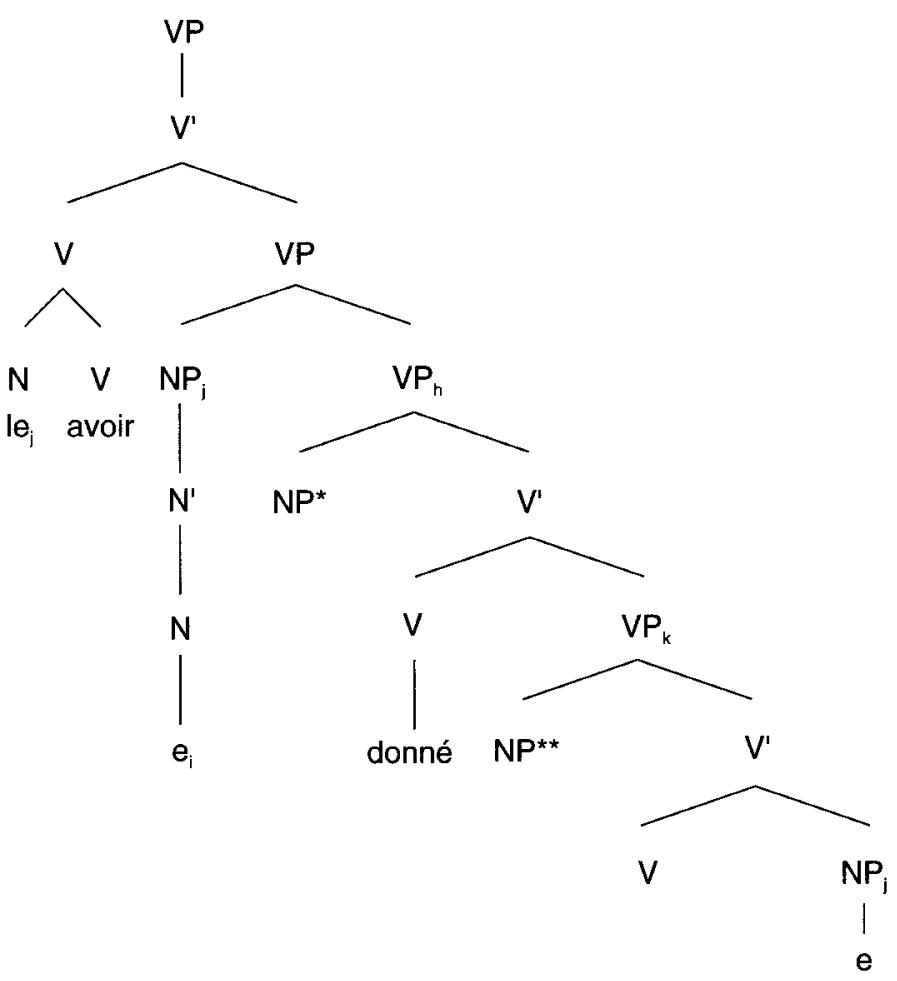

4.1.3 Clitic object of a noun

\subsubsection{GENERAL CONSIDERATIONS}

Nouns can take the whole range of categories as complements, and as far as NPs are concerned, NPs with varying Cases. Clitics can only correspond to a genitive dependent on the head noun and take the form of $e n .{ }^{45}$ As we have seen in Sections 3.4.3 and 3.4.4, extraction out of an NP must proceed through $[\mathrm{Spec}, \mathrm{NP}]$. The fact that only genitive dependents can cliticize outside will follow from:

(94) Only genitives can move to [Spec, NP].

Let us call the problem of accounting for it Problem 3 to which we will return in Section 6. We shall turn now to the properties of clitic en. As pointed out, the fact that en extractions out of NPs follow the pattern of $w b$-extraction out of NPs confirms that extraction of en out of NP must proceed through the specifier of this NP. We get the pattern:

(95)(a) Pierre en $\mathrm{i}_{\mathrm{i}}$ a vu le portrait $\mathrm{t}_{\mathrm{i}}$. Peter of him saw the portrait. 
(b) Pierre en $n_{i}$ a vu le portrait de Jean $t_{i}$. Peter of him saw the portrait of John.

(c)* Pierre en $n_{i}$ a vu mon portrait $t_{i}$.

The (a) sentence is ambiguous in three ways. The (b) sentence is also ambiguous in three ways instead of the a priori possible six. The (c) sentence is impossible. Just as in the case of $W h$ Movement out of NP, the only extractable item is the one that can be possessivized, i.e. moved to specifier position. Remember that a noun can take as complement several de-NPs. If it takes only one, this de-NP can be possessivized. If it takes several, only one of them can, according to the hierarchy discussed in Section 3.4.4.

\section{1 .3 .2 GENITIVE $E N$}

Since $e n$ is a clitic, cliticizing on a verb, we are forced to analyze it as a head. This means that, at some point, movement of $e n$ is head movement. Consider first the concrete case of $e n$ clitic on a verb:

(96)(a) voir [l'auteur de ce livre] see the author of this book

(b) en voir [l'auteur $t_{i}$ ]

it-see the author (of)

We see that the only possible derivation is similar to the one we have for the extraction of dont out of subject position in Section 3.4.5 and similar to the one we discussed for clitics which are objects of verbs. Ignoring verb raising to its infinitive morphology, we have:

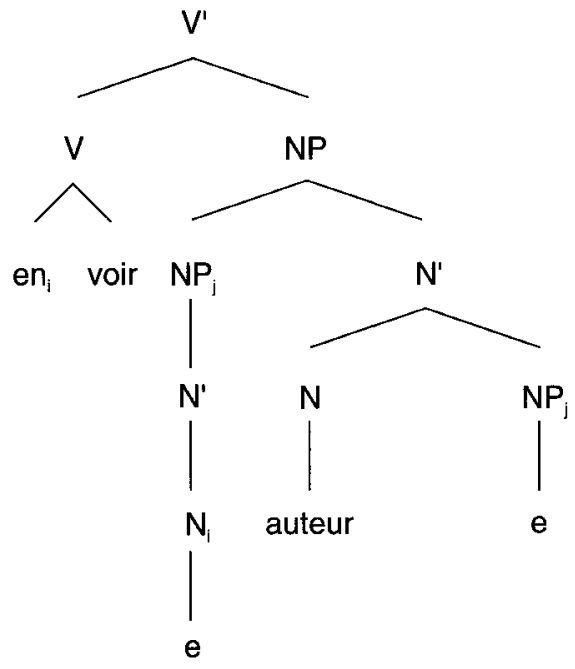


The pronominal object moves first to [Spec, NP]. From there, the head en of the derived specifier adjoins to the verb. If we take into account the raising of $V$ to its morphology ( $I_{\text {inf }}$ taking VP complement), an additional step would be involved moving $\mathrm{NP}_{\mathrm{i}}$ from $[\mathrm{Spec}, \mathrm{NP}]$ to a position adjoined to the $\mathrm{VP}$, where it would incorporate to $\mathrm{V}+\mathrm{I}_{\text {inf }}$.

Consider now a more complicated case:
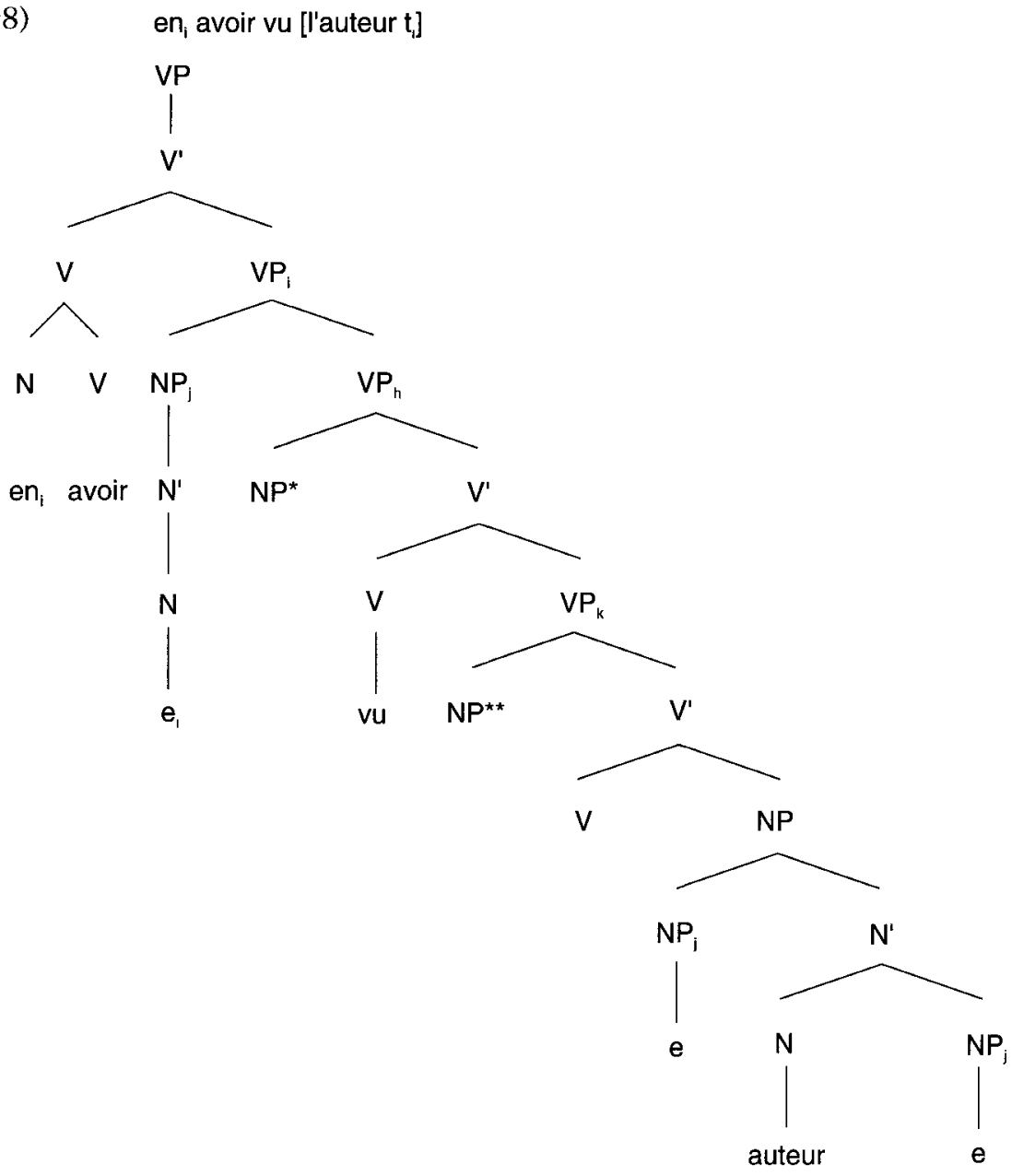

French clitics cliticize onto the highest verb of their clause. The derivation must proceed as follows. $\mathrm{NP}_{\mathrm{i}}$, the object of the $\mathrm{N}$, moves to [Spec, NP] just as above. From there, the derivation proceeds as in (93). Remark that this derivation, the only one possible, is possible only under the assumption that head movement of some head H is not blocked by HP if HP is not L-marked, 
since head movement in (98) is from the head position of an NP adjoined to VP, i.e. from an NP position which is not $\mathrm{L}$-marked. This goes back to the discussion about how to constrain properly head movement in Section 3.2: $\mathrm{i}$-command is, of course, compatible with this derivation. Here the noun incorporates from the position adjoined to $\mathrm{VP}_{1}$, which is not $\mathrm{L}$-marked. Notice incidentally that this will become relevant when we discuss agreement that $\mathrm{NP}_{\mathrm{j}}$ could in principle have moved through the $\left[\mathrm{Spec}, \mathrm{VP}_{\mathrm{k}}\right]$ position.

\subsection{Object agreement}

In this section, we discuss French object agreement. Basically, I adopt Kayne's $(1985 ; 1989 a)$ central insight that object/participle agreement obtains in the same structural configuration as, mutatis mutandis, subject/verb agreement. I show that Kayne's analysis follows from the assumptions made so far, lending further support for the Theory of Movement presented in earlier sections. Some facts, for which no explanatory account has ever been provided, will not however. In particular, we will propose an account of the restrictions on the kind of object that can trigger agreement, and will suggest that the consequent theoretical elaboration concerning the syntax of $\mathrm{VP}$ is totally paralleled to the internal syntax of NP and the syntax of extraction out of NP.

\subsubsection{Analysis: first pass}

\subsubsection{THE PATTERN OF AGREEMENT}

Consider the following examples of object/participle agreement in French:

(99)(a) ... avoir décrit/(*décrite) la robe à la femme to have described the dress to the woman

(b) $\ldots \mathrm{l}_{\mathrm{i}}$ 'avoir décrit/décrite $\mathrm{t}_{\mathrm{i}}$ à la femme

(c) $\ldots$ lui $_{j}$ avoir décrit $/(*$ décrite $)$ la robe $t_{j}$ (d) $\ldots \mathrm{la}_{\mathrm{i}}$ lui ${ }_{\mathrm{j}}$ avoir décritédécrit $\mathrm{t}_{\mathrm{i}} \mathrm{t}_{\mathrm{j}}$

The relevant facts are as follows. Objects following the participle do not trigger agreement. French spelling records gender and number agreement on the participle, but never person agreement. Here and after, we investigate only object agreement in gender with feminine objects. This agreement is indicated in the spelling with the feminine affix $\underline{e}$ and is audible only with well chosen participles. Both a direct object (accusative) pronoun and an indirect object (dative) pronoun can cliticize. Cliticization of the direct object can (but in my judgment, does not have to) trigger agreement on the past participle. The indirect object on the other hand cannot trigger agreement. The same facts hold with other object preposing processes such as Wb Movement with similar properties: 
(100) L'écharpe que tu as offert $(e) t \ldots$

The scarf(FEM) that you have offered(FEM) $t$...

These properties of participle agreement raise the following questions:

(101)(a) Why is participle agreement only possible with certain objects?

(b) Why must objects triggering agreement precede the participle?

(c) Why is agreement optional?

The answer to the last two questions is fairly straightforward as we will see below. The answer to the first one however, will require some substantial modification of our view of the internal structure of VPs, NPs and other argument taking heads.

\subsubsection{PRELIMINARY ANALYSIS}

We now go through a rough first analysis of object agreement, which we will refine later. We have discussed earlier how clitic placement must proceed. Consider for example the derivation of (99b).

(102)

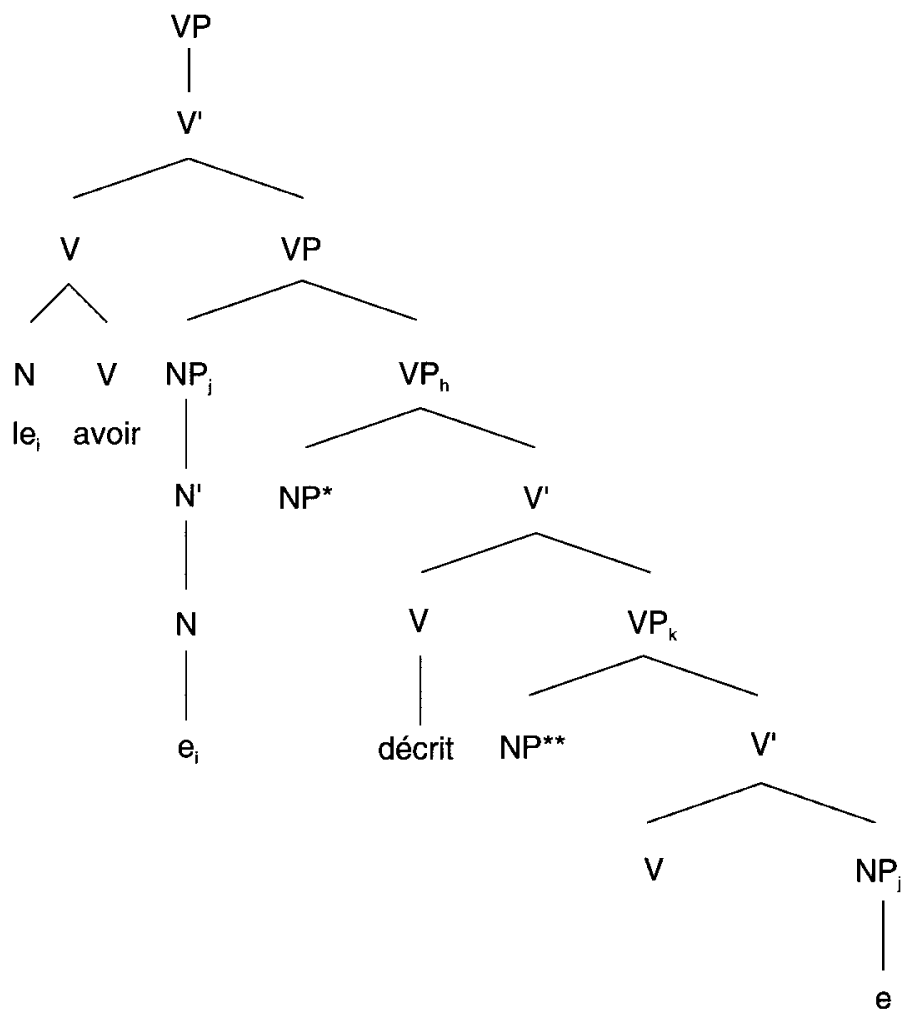


Consider the movement of the direct object $\mathrm{NP}_{j}$. There are two possible derivations in order to escape $\mathrm{VP}_{k}:(1)$ it can adjoin to $\mathrm{VP}_{\mathrm{k}}$ and then move out. (2) it can first move to the position $\left[\mathrm{Spec}, \mathrm{VP}_{k}\right]$ and then move out (perhaps by adjoining to $\mathrm{VP}_{\mathrm{k}}$ ).

This provides answers to the questions $(101 \mathrm{~b}, \mathrm{c})$. First, only objects preceding the participle can trigger agreement with it because they must have a chance to be in [Spec, VP] position in order to trigger agreement with V. This is the spirit, if not the letter of Kayne's (1989a) answer to question (101b). Second, agreement is optional because the $\left[\mathrm{Spec}, \mathrm{VP}_{\mathbf{k}}\right]$ position is not an obligatory intermediate position for object preposing. If the first derivation is chosen, no agreement will occur. Naturally the same account extends to the $\mathrm{Wb}$ Movement case exemplified in (100).

A number of further questions arise: first, the account of the optionality of agreement holds only if we can show that an adjunction to $\mathrm{VP}_{\mathrm{k}}$ cannot trigger agreement. Otherwise, given that agreement is obligatory in the structural configurations in which it can hold, we would lose our account. We show that the participle cannot agree with adjoined positions below in Section 4.2.1.3. Second, nothing so far prevents the same exact derivations being applied to the cliticization of the indirect object. If both derivations were available, we would expect them to be able to trigger agreement. This is the problem raised by question (101a). One way to answer it is to somehow prevent the indirect object from transiting through [Spec, VP]. Then, only the adjunction derivation is available, which, as we will see, does not trigger agreement.

4.2.1.3 THE A/A-BAR STATUS OF THE OBJECT AGREEMENT

POSITION

The following facts, attributed by Kayne (1989a) to Ruwet show that adjunction to VP cannot trigger agreement:

(103)(a) une femme ${ }_{i}$ qu'on a $\operatorname{dit}(\underline{e})\left[_{A P} t_{i}\right.$ belle $]$ a woman that people say beautiful

(b) une femme ${ }_{\mathrm{i}}$ qu'on a dit $\left({ }^{*} \underline{\underline{e}}\right) \mathrm{L}_{\mathrm{CP}}$ être belle a woman that people say to be beautiful

(c) une femme qu'on a dit $\left(*_{e}\right) \mathrm{L}_{\mathrm{CP}}$ que tu a vu(e) a woman that people say that you saw

In the (a) sentence, the subject of the AP receives the accusative Case from the verb dire. Consequently, it must count as governed by V. No barrier prevents it from moving to the specifier position of the VP headed by dire, thus triggering agreement. In the (b) sentence, the subject of the embedded infinitive can only get Case by moving to the specifier position of the embedded CP, as discussed by Kayne (1984). Consequently movement to 


\section{PARTITIONS AND ATOMS}

the specifier position of the VP headed by dire is prohibited, as would be movement from an A-bar position to an A-position, that is, improper movement. In the (c) sentence, extraction of the direct object from the embedded clause must proceed through the embedded [Spec, CP], an A-bar position. Subsequent movement to [Spec, VP] is excluded again as a case of improper movement. Of course, if adjunction to VP were able to trigger participle agreement, we would expect agreement to be possible in the (b) and (c) sentences. Contrary to fact, agreement cannot be with an adjoined position.

Note further that if the specifier position triggering agreement could be an A-bar position - contrary to what we have assumed - we would also expect agreement to be possible in the (b) and (c) sentences. Nothing would prevent the wh-phrase from moving from the embedded [Spec, CP] to [Spec, VP]. $(103 \mathrm{~b}, \mathrm{c})$ thus also show that $[\mathrm{Spec}, \mathrm{VP}]$ can only be an A-position, as we have assumed.

\subsubsection{Problems}

The analysis of participle agreement that we have presented in Section 4.2.1.3 derives the agreement facts for direct objects without any stipulation. The agreement facts follow from the combination of the Theory of Agreement (a Spec/head relation) and the Theory of Movement. However, it is far from being complete and without problems. ${ }^{46}$ There are two general complications:

First, participle agreement appears not always to be with a direct object. There are cases in which agreement is with a subject. Traditional grammars state that "in sentences where the auxiliary verb is être/be, participle agreement is with the subject and is obligatory."

Second, contrary to expectations, not all complement clitics can trigger agreement. We have already mentioned the case of dative objects which cannot trigger agreement. The problem is much more general - only a direct object of a V can trigger agreement on this V. Datives cannot, locatives cannot, genitives cannot even though we would expect them to. Furthermore not all direct objects can trigger agreement.

\subsection{Subject participle agreement}

Traditional grammars state that "in sentences where the auxiliary verb is être/'be,' participle agreement is with the subject and is obligatory." French, like other Romance languages (e.g. Italian) has two auxiliaries: être/"be" and avoir/"have." The auxiliary avoir always expresses perfective aspect. The auxiliary être also expresses perfective aspect except in passive clauses, where it is simply the passive auxiliary. The auxiliary be is used in passive constructions (104a), with unaccusative verbs (104b) and with verbs used with the clitic 
se, whatever the function of this clitic $(104 \mathrm{c}, \mathrm{d}, \mathrm{e}, \mathrm{f})$. Se can signal a reflexive construction (104c), a middle construction (104d), a neutral construction (104e) or an inherent construction (104f):

(104)(a) Jean a été vu/John has been seen.

(b) Jean est arrivé/John has arrived.

(c) Jean s'est rasé/John shaved himself.

(d) Ces maisons se sont construites rapidement/These houses were built quickly.

(e) Ils se sont réunis/They got together.

(f) Il s'est évanoui/He passed out.

The middle construction and the neutral construction differ from the inherent construction in that they have a causative counterpart without the morpheme se, e.g. here construirel"build" and réunir/"gather." There is no verb évanouir/"to cause to pass out." The middle construction and the neutral construction differ in a number of ways as discussed in Ruwet (1972) and Keyser and Roeper (1984). One prominent difference is that the middle has an implicit agent argument. The neutral does not. In what follows, we will analyze all these cases of apparent subject agreement as cases of covert object agreement, thus reducing them to the pattern already encountered with the object clitics.

\subsubsection{Agreement in passives and unaccusatives}

\subsubsection{AGREEMENT IN PASSIVES}

Let us begin with the passive construction. Agreement of the passive participle with a subject is obligatory in the case of passive:

(105) La robe est faite $/ *$ fait (par Cardin).

The dress was made (by Cardin).

The surface subject is an underlying object. If the passivized direct object must move through the position [Spec, VP] to reach [Spec, IP], agreement will be both obligatory and superficially with the subject. Passivization is NP Movement. In order to escape its VP, the object cannot adjoin to VP. It must move up by substitution. Whatever their correct structure, passive verbs end up behaving as if they were not projecting their external argument as an intervening specifier. To simplify, let us assume they simply do not project it. The object will be moving to [Spec, IP] by NP Movement in a clausal structure as in (10) on p. 148.

Where NP** is not a theta position the object will thus have to move from $\mathrm{NP} * * *$ through NP** $=[\mathrm{Spec}, \mathrm{VP}]$, triggering agreement as required. 
(10)

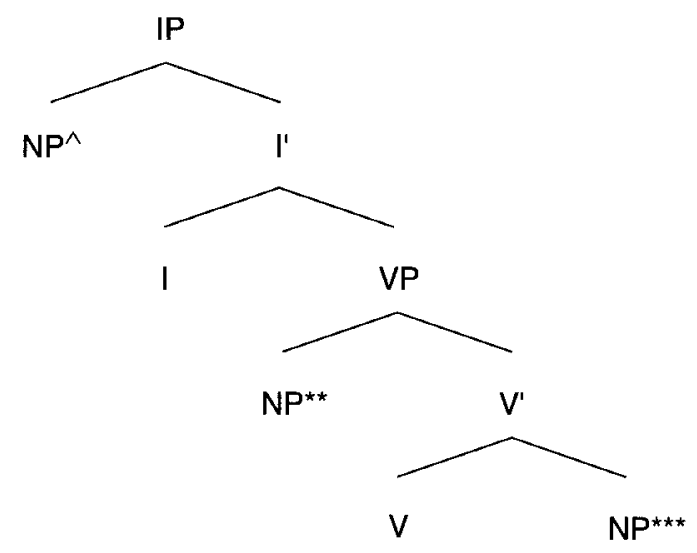

4.3.1.2 AGREEMENT IN UNACCUSATIVES

The case of participle agreement in unaccusative constructions can be treated in exactly the same fashion as we treated agreement in passive constructions. The superficial subject of an unaccusative verb is an underlying object moved to [Spec, IP] by NP Movement. It must move through the [Spec, VP] position triggering agreement. Not all verbs that may a priori qualify as unaccusative verbs are restricted to the auxiliary être. Take for example the verb cuire/"cook":

(106)(a) Ils ont cuit les tomates/They cooked the tomatoes.

(b) Les tomates ont été cuit*(es) par .../The tomatoes were cooked by...

(c) Les tomates ont cuit(*es)/The tomatoes (have) are cooked.

As the (b) sentence shows, the passive participle must show agreement with the subject. The (c) sentence shows that no agreement with the superficial subject is possible with the auxiliary avoir. If the verb in (c) is unaccusative, agreement on the participle should be obligatory.

Of course, we need to be able to determine unaccusativity independently of agreement. Surely, the strongest stance we could take on unaccusativity is one of maximal uniformity of theta assignment such as Baker's (1988b) Universal Theta Assignment Hypothesis, or similar approaches reviving assumptions from the mid 1960s. I adopt the strongest construal possible. If a particular theta role is demonstrably linked to a particular grammatical function in some case, it always is across constructions and across languages. Because the object in (106a) and the subjects in (106b and c) receive the same theta role, and given that this theta role is assigned to an object in (106a), it also is in the other sentences, so that their subjects are derived. This would force an unaccusative analysis of the (c) sentence with the associated 
agreement problem. There is direct evidence supporting this conclusion. Verbs like cuire/ "cook," couler/"sink" selecting the auxiliary verb avoir behave like unaccusative verbs and unlike intransitive verbs in the impersonal construction:

(107)(a) ?Il a dormi plusieurs enfants/*Il en a dormi plusieurs. There slept several children/There of-them slept several.

(b) Il est arrivé plusieurs enfants/Il en est arrivé plusieurs. There arrived several children/There of-them arrived several.

(c) Il a cuit beaucoup de tomates/Il en a cuit beaucoup. There cooked many tomatoes/There of-them have cooked many.

(107b) illustrates that an unaccusative verb like arriver allows its single argument to appear postverbally and allows cliticization of quantitative en from this postverbal position. This last property is a property characteristic of underlying objects. ${ }^{47}$ (107a) shows that an intransitive verb, even though it might marginally allow its argument to appear postverbally in an impersonal construction, disallows en-cliticization from the postverbal position. This suggests that this postverbal NP is not an object, but rather an inverted subject. (107c) shows that the class of verbs under consideration behaves like unaccusatives and unlike intransitives.

\subsubsection{Auxiliary selection and agreement}

The description of agreement given by traditional grammars is the following:

(108)(a) In sentences with the auxiliary verb être/"be," the participle agrees with the subject and agreement is obligatory.

(b) In sentences with the auxiliary verb avoir, the participle agrees with the direct object if the object precedes the participle. $^{48}$

In view of the conclusion of the previous section, (2) must be modified since unaccusative participles do not agree with their underlying object/derived subject when they select avoir. This observation seems to extend to other cases of derived subjects with the auxiliary verb avoir. The passive auxiliary verb être selects avoir as perfective auxiliary. When this aspectual auxiliary is present, être appears in its participial form and does not show agreement:

(109) La robe a été(*E) faite par Cardin. The dress was made by Cardin. 
This occurs despite the fact that the agreement configuration is met. Raising of the object to the subject position must transit through the [Spec, VP] position of the passive auxiliary as illustrated below:

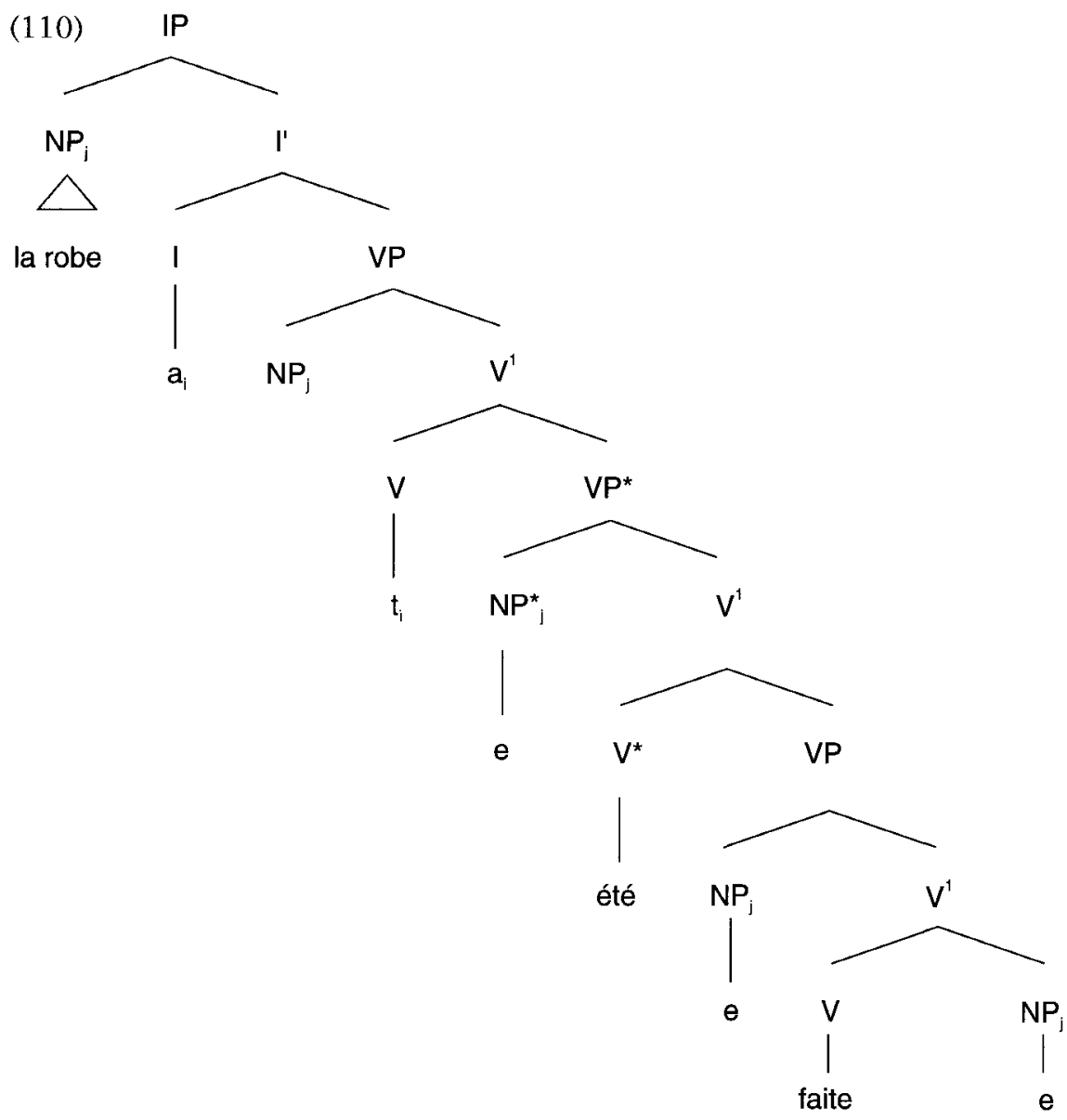

In order to escape $\mathrm{VP} *$ under A-movement, $\mathrm{NP} *$ must move through its specifier. We should expect obligatory agreement between $\mathrm{NP}_{j}^{*}$ and $\mathrm{V}^{*}$ but it seems we do not observe it. Another case is found with raising verbs. In a raising construction, the surface subject has raised from inside the main VP to subject position and thus has moved through the [Spec, VP] position. If the raising verb is in its participial form, we would expect agreement. Agreement does not seem to occur:

(111) Les filles ont semblé(*ES)/paru(*ES) malades. The girls seemed/appeared sick. 
Note that both for (110) and (111), the absence of agreement is based on spelling convention. This is why I write that it seems that agreement is lacking. There is no way to check otherwise for the passive auxiliary and I know of no raising verb whose masculine and feminine participle (or singular and plural) forms would display phonological differences. ${ }^{49}$ I will provisionally suppose that spelling is a reliable indicator of agreement possibilities because the emerging pattern is one in which subject/participle agreement must occur with auxiliary verb être, and cannot occur with auxiliary avoir and this configuration is found in Spanish and Catalan. Catalan is discussed below in Section 4.3.3.2. In Spanish, participles agree with their object only in cases where the auxiliary be is selected. This only occurs in passive constructions. In principle, this assumption could be directly checked in those dialects of French which realize phonetically the feminine agreement when it follows a vowel.

We need to reformulate the descriptive statements in (108). In traditional grammar terms, the subject of an unaccusative verb does not count as an object. No agreement is expected if the unaccusative verb takes auxiliary avoir. We claim however that participle agreement is always with the object. Let us replace (108) with the following descriptive statement:

(112)(a) With participles selected by the auxiliary verb être/"be," participle agreement is with the underlying direct object (when it precedes the participle) and is obligatory.

(b) With participles selected by the auxiliary verb avoir, participle agreement with the direct object is possible if the object precedes and if the participle has an external argument subject.

(112b) makes participle agreement with avoir contingent on the existence of an external argument for the verb. This is meant to permit agreement with object clitics when the verb has an external argument, and to exclude agreement with underlying objects/superficial subjects in unaccusative constructions, since the subject is not an external argument. When we discuss agreement in se constructions, we will see that (108) is not tenable. This data raises several questions: What governs auxiliary selection? Why is participle agreement with avoir possible with preposed direct objects unless they are preposed to subject by NP Movement?

I will not try to provide any answer to the first question. The fact that there is so much linguistic variation amongst closely related languages such as Spanish, French, Italian or Catalan suggests that no general answer can be given (see Kayne (1993) for recent discussion). I will briefly address the second question in Section 5.5.5. Given our treatment of participle agreement so far, agreement is determined purely on the basis of what happens in the XP projection of the participle. The influence of the auxiliary verb on agreement suggests that more than this is involved. 


\section{PARTITIONS AND ATOMS}

\subsubsection{Se constructions}

We now turn to constructions involving the clitic se. As mentioned earlier, the clitic se appears in a variety of constructions. Se can signal a reflexive construction (113a), a middle construction (113b), a neutral construction $(113 \mathrm{c})$ or an inherent construction (113d):

(113)(a) Ils se sont raséS/They shaved themselves.

(b) Ces maisons se sont construitES rapidement/These houses were built quickly.

(c) Ils se sont réuniS/They got together.

(d) Elle s'est évanouiE/She passed out.

All these constructions select the auxiliary être. The middle, the neutral and the inherent constructions behave exactly as expected under (108b) or (108a): the auxiliary verb being être, agreement with the subject is obligatory. The analysis we have provided for passives and unaccusatives will extend straightforwardly to these cases if we analyze them as unaccusatives: the superficial subject is an embedded object raising to subject through [Spec, VP], and thus triggering agreement. The en cliticization test discussed earlier in (107), applied to the impersonal construction, supports an unaccusative analysis:

(114)(a) Il se construit beaucoup de maisons/Il s'en construit beaucoup.

Many houses/of them are being built.

(b) Il s'est réuni plusieurs personnes/Il s'en est réuni plusieurs. Several persons/of them gathered.

(c) Il s'est évanoui trois touristes/Il s'en est évanoui trois.

Three tourists/of them passed out.

Many authors have already suggested an unaccusative analysis for these constructions (cf. Burzio (1986), Zubizarreta (1982)). Strictly speaking, the facts of (114) support the idea that the superficial subjects in (113) are underlying objects. It says nothing about whether or not the objects in (114) receive accusative Case or not. The two properties are usually linked as a consequence of Burzio's Generalization and also because the definiteness effect found in (114) - the objects must be indefinite - is taken to be an indication that the Case assigned to the object is not accusative (cf. Belletti (1988)).

The analysis of agreement and the agreement facts support the raising analysis. We have not discussed the status nor the role of the reflexive morpheme in these constructions. Let us delay this question until it becomes relevant in reflexive constructions. The reflexive construction is more complicated. Consider the following sentences: 
(115)(a) Marie a décrit la robe aux enfants. Mary(FEM) described the dress(FEM) to the children.

(b) Marie s'est décrit*(E) _ aux enfants. Mary described herself to the children.

(c) Marie s'est décrit $(* \mathrm{E})$ la robe. Mary described the dress to herself.

(d)* Marie s'est décrite _ _ (par Jean). Mary was described to herself (by John).

Informally speaking, the reflexive se stands for the direct object in (115b), and for the indirect object in (115c). Notice that the traditional description (108) is inconsistent with $(115 \mathrm{c})$. Even though the auxiliary verb is être, agreement with the subject is impossible.

\subsubsection{REFLEXIVE CONSTRUCTIONS}

The informal description given above corresponds closely to the standard analysis of the reflexive construction (Kayne (1975), Burzio (1986)). Let us call it the Theory of se as Internal Argument (henceforth TIA). It asserts that the reflexive clitic stands for a direct object and is subject to the same kind of clitic placement rules as non-reflexive clitics. If this were correct, we would, given our analysis of participle agreement, expect the agreement facts to be different. We would expect agreement in (115b) to be optional rather than obligatory.

Our description in (112) will give the right result if the superficial subject in (115b) is an underlying object. If this is the case, the presence of se somehow affects the expression of the external argument just like the passive. The external argument is no longer assigned to the subject, allowing the object to raise to subject position. This approach has been proposed by Marantz (1984), Bouchard (1984) and adopted by Kayne (1988). Call this the Theory of $s e$ as External Argument (henceforth TEA).

Before comparing the two analyses, let us list the properties of the reflexive construction that must be accounted for:

(116)(a) It uses the morpheme $S E$.

(b) The same morpheme is also used in the middle and the neutral.

(c) The reflexive interpretation arises.

(d) The NP being attributed a reflexive property must be a deep subject.

The first three properties are self-explanatory. The fourth one states first that "the antecedent of the reflexive" must be a subject. Second, it cannot be a derived subject, i.e. subject of a passivized verb or of a raising verb. This is illustrated below: 
(117)(a) Marie décrit la robe aux enfants. Mary(FEM) describes the dress(FEM) to the children.

(b) Marie se décrit $(* E)$ la robe. Mary describes the dress to herself.

(c)* Marie ${ }_{j} s_{k}$ 'est décrite $t_{j}\left[_{N P !}\right.$ e] (par Jean). Mary was described to herself (by John).

(d) Marie ${ }_{j}$ lui paraissait _ [ $\mathrm{t}_{\mathrm{i}}$ malade $]$. Mary appeared sick to him.

(e)* Marie $_{j}$ se $_{\mathrm{k}}$ paraissait $\left.\mathrm{L}_{\mathrm{NP} !} \mathrm{e}\right]\left[\mathrm{t}_{\mathrm{j}}\right.$ malade $]$. Mary appeared sick to herself.

(f) Marie ne parait malade qu'à elle-même. Mary appears sick only to herself.

(117c) shows that an indirect object reflexive is incompatible with a passivized object as antecedent. (117e) shows that an indirect object reflexive is incompatible with a raised subject. (117f) shows that this restriction is not due to the anaphoric interpretation of the dative object. If it is modified by only, it cannot cliticize and an emphatic reflexive is used instead.

For the TIA these facts are unexpected. If reflexives are like object clitics, they should be able to appear in raising or passive constructions the way other object clitics do. It is necessary to introduce some further assumptions to accommodate them. Burzio (1986) suggests that the reflexive clitic is an anaphor which must be bound at D-structure. Rizzi (1986a) suggests that the resulting configuration in the ungrammatical sentences violates a well formed Condition On Chains (COC) requiring the successive positions in a chain to be in a local binding relationship. In (117e), for example, NP is the trace of the clitic. Because of its anaphoric properties the clitic bears the same index as the subject so that we have two chains: $\left(\right.$ Marie $\left._{i},{ }_{t j}\right)$ and $\left(\mathrm{se}_{j}, \mathrm{NP}_{1: j}\right)$. The local binder of $t_{j}$ should, according to this condition, be Marie $e_{i}$ It is not. The local binder of $t_{j}$ is NP! . The same reasoning excludes (117c).

Compare this with the TEA's treatment. As Bouchard (1984) noticed, the ungrammaticality of these examples falls out. If the reflexive is or affects the external argument, it will not occur with verbs lacking an external argument altogether, or with verbs whose external argument has been locked by another process. This is the case of the passive. As Jaeggli (1986) has shown, the characteristic property of the passive morphology is its absorption (or redirection) of the external theta role, which is thus no longer available for the reflexive morpheme. The COC also faces some problems already mentioned in Section 3.5.3 in connection with our discussion of Lasnik's (1985) examples. Consider the following examples: ${ }^{50}$

(118)(a)* It appeared to him ${ }_{k}$ that John $n_{k}$ is sick.

(b) ?John ${ }_{k}$ appeared to himself ${ }_{k}\left[t_{k}\right.$ to be sick].

(c)* $\mathrm{John}_{\mathrm{k}}$ struck him $\mathrm{j}_{\mathrm{j}}$ [as $\mathrm{t}_{\mathrm{k}}$ proud of Bill $]_{\mathrm{j}}$. 


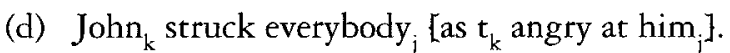

(e) John ${ }_{k}$ struck himself $f_{k}$ [as $t_{k}$ tired].

The ungrammaticality of the (a) and (c) sentences shows that the object can trigger a Principle $C$ violation, and thus can potentially bind something in the complement clause. ${ }^{51}$ The grammaticality of the (d) sentence shows the same thing, with the quantifier able to bind the pronoun in the embedded clause. The (b) and (d) sentences thus seem to violate the COC. In each sentence, the local binder of $t_{k}$ is bimself $f_{k}$, which belongs to a different chain. In conclusion, then, the TEA is clearly superior to the TIA concerning the treatment of property (116d). Let us turn now to (116c). In order to account for the reflexive interpretation, any approach will have to treat the theta role of the missing argument as anaphorically bound by the superficial subject. As usual, this process cannot be lexical, given that it may involve an NP which is not an argument of the reflexivized verb (as in small clauses constructions: Jean se considère fatiguél"John considers himself tired." For concreteness (we return to this question in Section 4.3.3), assume that the missing NP's theta role is assigned to se, which is also marked as anaphoric. By Principle A, it will have to be bound within the domain of the closest subject. This binder will have to be the subject of the reflexivized verb. This is neutral between the TIA and the TEA.

Turning now to (116b), what is the role of the se morpheme in the middle and neutral constructions? As we discussed earlier, a middle or neutral verb is unaccusative. It lacks an external argument subject and does not assign structural accusative Case to its object, forcing it to move to the subject position. ${ }^{52}$ But these verbs can also be used causatively with a subject external argument and an accusative object as internal argument. It looks then as if the central role of the morpheme se is to modify the normal expression of the external theta role and possibly to remove the verb's ability to assign structural accusative Case. ${ }^{53}$

If the TIA is correct, reflexive se has rather different properties. It would just be an affixal noun that can stand for an internal argument. If the TEA is correct, the function of reflexive se would be identical to that of middle or neutral se. This analysis would have the advantage of providing a reason why the same morpheme is used in all these constructions. ${ }^{54}$ Of course, there are also differences between the various constructions that must be explained (see Ruwet (1972), and Keyser and Roeper (1984)). In the neutral construction, the external theta role is semantically absent (e.g. in (113c) there is no understood agent of the gathering), hence it is syntactically inactive (cannot act as controller). In the middle construction, the external theta role is semantically present and syntactically active. For example, we have contrastive judgments between the inherent construction (119a) and middle construction (119b):

(119)(a)* L'eau s s'est renversée [pour $\mathrm{PRO}_{\mathrm{i}}$ mouiller le sol]. Water spilled to wet the floor. 
(b) Ces bains se prenaient [pour $\mathrm{PRO}_{\mathrm{i}}$ prendre soin de soi $\mathrm{i}_{\mathrm{i}}$. These baths took ( = were taken) to take care of oneself.

The reflexive construction resembles the middle in that the external role is semantically present, but it differs from it in that it has the added anaphoric property.

Ruwet (1972) argues that the middle construction is syntactically derived while the neutral construction is the result of lexical process. The main reason is the idiosyncratic composition of the class of verbs entering the neutral construction versus the predictability of a verb's ability to enter the middle construction.

I suggest the following. The central function of the morpheme se is to affect the normal expression of the external thematic role of a verb (and possibly, by Burzio's Generalization, affect the Case assigning possibilities of the verb). So in all cases, the superficial subject is an underlying object. The morpheme se is always nominal, in fact always pronominal. This pronominal element, like other pronouns, can freely be either $[+$ pronominal] (like a regular pronoun) or [+anaphoric] (like a reflexive pronoun). In the second case, we get the reflexive construction. Reflexive se is an argument and receives the external theta role. Because of its anaphoric nature, a reflexive se will require a local binder to satisfy the Binding Theory. In particular, it will not be able to occur in the impersonal construction, unlike the middle, the neutral or the inherent se:

Il s'en rasait beaucoup, de soldats.

Soldiers, many of them were being shaved.

* Soldiers, many of them shaved themselves.

The reflexive interpretation is ruled out because the antecedent of the anaphoric reflexive does not command it. ${ }^{55}$ In the middle this pronoun is interpreted as an indefinite pronoun and the external theta role is syntactically expressed and assigned to se. In the neutral (and the inherent) construction this pronominal is an expletive. The difference between the neutral and the middle construction concerns the way in which the external theta role is affected. In the neutral, it is suppressed. Taking Ruwet's conclusions into account, I further suggest that this option is lexically governed. Note that there must be an external theta role for the se to "absorb." Thus unaccusatives can never enter the neutral construction:

(121)* Jean s'est arrivé/Il s'est arrivé plusieurs enfants. John se-is arrived/It se-is arrived several children. 


\subsubsection{CATALAN AGREEMENT}

Participle agreement in Catalan provides independent support for the conclusions reached here, as Cortes (1992) shows and on which I rely for the following discussion. The agreement facts of Catalan are remarkably similar to the French, with one very useful difference. In Catalan, participle agreement is audible. Catalan uses two auxiliary verbs like French: baver/"have" and ser/"be." The passive auxiliary is ser. The perfective auxiliary is always haver, regardless of the choice of verb or construction: unaccusative, reflexive, transitive, intransitive. In this respect it resembles Spanish. In the passive, (with auxiliary verb ser), participle agreement with the subject is obligatory as in Spanish, Italian and French.

In every other construction, (with auxiliary verb haver), there is no participle agreement with a subject or with a postverbal object. If the direct object precedes the participle and is a $w$-phrase, agreement is impossible (in this respect, Catalan functions like Italian and Spanish and unlike French). If the direct object precedes the participle and is a pronominal clitic, agreement is optional (in this respect, Catalan functions like French, and unlike Spanish - no agreement possible - or Italian where agreement is obligatory with third person clitics). These facts are illustrated below:

(122) (a) Les nenas seran invitades/*invitat. the girls will-be invited[FEM-PL]/invited[MASC-SG]

(b) La Nuria ha arrivat. Nuria has arrived (no agreement).

(c) El Jordi les ha comprat/comprades. Jordi them[FEM-PL] has bought[MASC-SG]/bought [FEM-PL]

The crucial facts involve agreement in es constructions. Es is the Catalan counterpart of French se. It appears in all the same kinds of constructions as se and is disallowed in the same contexts:

(123)(a) La Nuria s'ha cremat (*cremada). Nuria burnt herself (REFLEXIVE).

(b) La Nuria s'ha donat (*donada) un llibre. Nuria gave herself a book (REFLEXIVE)

(c) La carta s'ha llegit (*llegida) facilment. The letter read easily (MIDDLE).

(d) L'aigua s'ha vessat (*vessada). The water spilled (NEUTRAL).

(e) Les nenes s'han desmaiat $(*$ desmaiades $)$. The girls fainted (INHERENT).

(f)* El Jordi es semblat enfadat. Jordi seemed angry to himself. 
First, es is incompatible with verbs lacking an external argument as example (f) shows. Second, examples (a) to (e) show that participle agreement is impossible with es. In other words, se constructions form patterns exactly like cases of derived subject constructions with auxiliary baver as in (122b) and unlike cases of object clitics with auxiliary baver as (122c). This is explained if $s e$ is an external argument, and remains mysterious otherwise.

\section{Agreement and Case}

In this section, I will address the problem of object/object asymmetries under agreement. I will suggest that these asymmetries follow from a central asymmetry in Case properties which links certain kinds of Case with agreement.

\subsection{Lack of participle agreement with objects}

Our account predicts that any NP that has access to [Spec, VP] should be able to trigger participle agreement. So far access to [Spec, VP] is determined solely on the basis of Movement Theory: if an NP can move to [Spec, VP] without crossing a barrier, it should be able to, and thus should be able to trigger agreement. However, participle agreement fails to occur in four different cases where it should: certain direct objects, dative objects, quantitative objects and genitive objects. Except for genitives, which we will discuss later in connection with our revised views of extraction out of NPs, we will begin by showing why agreement should be able to occur. Then, we will propose reasons why it does not.

\subsubsection{Why agreement is expected}

\subsubsection{DATIVES AND OTHER COMPLEMENTS}

Of all the complements governed by a participle, only direct objects can trigger agreement. Cliticizable complements that are usually not analyzed as NPs (e.g. locatives, de-complements) cannot trigger agreement when they cliticize (124b). This could follow from the more general stipulation that [Spec, VP] is restricted to NPs, a reflection of the more general stipulation that [Spec, XP] with X lexical is an A-position. Direct object NPs can trigger agreement, whether they are theta marked by the verb or not (as subjects of small clauses) (124c). Indirect (dative) objects cannot trigger agreement (124d):

(124)(a) ... avoir décrit/(*décrite) la robe à la femme à la ville to have described the dress to the woman in the city(FEM)

(b) ... y avoir décrit/(*décrite) la robe à la femme to there have described the dress to the woman 
(c) ... l. $\mathrm{l}_{\mathrm{i}}$ avoir décrit /décrite $\mathrm{t}_{\mathrm{i}}$ à la femme

(d) $\ldots$ lui $_{\mathrm{j}}$ avoir décrit $/\left(*\right.$ décrite) la robe $\mathrm{t}_{\mathrm{j}}$

Cliticizable complements that are not direct objects all look like PPs introduced by $a$ (datives, locatives) which cliticize as lui or $y$, or by de (genitives in NPs) which cliticize as en. We could treat indirect objects on a par with locatives (124b) by claiming that datives are PPs rather than NPs, thus unable to appear in [Spec, VP]. Surely, it would preferable to remove the stipulation that certain positions exclude PP and replace it by a system that derives it. I do not know how to achieve this in the general case. In the case of agreement however, I will later make a proposal that derives this property. This seems to be made even more necessary by the existence of some evidence suggesting the exact opposite, namely that all these cliticizable complements are NPs, rather than PPs. Some such evidence is due to Vergnaud (1974). It is based on the behavior of coordinated XPs modified by a restrictive relative requiring a plural antecedent. The data is as follows:

(125)(a) L'homme et la femme qui sont partis ensemble. The man and the woman who left together.

(b) Parler avec l'homme et (*avec) la femme qui sont partis ensemble.

Speak with the man and (with) the woman who left together.

(c) Voter contre l'homme et (*contre) la femme qui sont partis ensemble.

Vote against the man and (against) the woman who left together.

(d) Le donner à l'homme et ??(à) la femme qui sont partis ensemble.

Give it to the man and (to) the woman who left together.

(e) Parler de l'homme et ??(de) la femme qui sont partis ensemble.

Speak of the man and (of) the woman who left together.

(f) Aller à l'exposition et ??(à) la bibliothèque qui sont dans le même musée.

Go to the exhibition and (to) the library which are in the same museum.

(g) Ce vote contre l'homme et (*contre) la femme qui sont partis ensemble.

This vote against the man and (against) the woman who left together.

(h) Ce portrait de l'homme et ??(de) la femme qui sont partis ensemble.

This portrait of the man and of the woman who left together. 
(125b,c and g) show that a restrictive relative cannot modify a coordination of PPs. If the $\mathrm{P}$ is repeated, unacceptability results. We make sure that it is the coordination that is modified by choosing a relative clause requiring a plural antecedent and by making each NP of the coordination singular. The judgments are reversed in $(125 \mathrm{~d}, \mathrm{e}, \mathrm{f}$ and $\mathrm{h}$ ) (in which the head of the relative correspond to objects that cliticize respectively as $l u i$, en, $y$ and en): there, repetition of the "P" is possible. We conclude that the coordination is an NP coordination behaving like the NP in (125a) and unlike the PPs of $(125 \mathrm{~b}, \mathrm{c}$ and $\mathrm{g})$. The repetition is also much preferred, perhaps even required. Vergnaud concludes from this that these particles are really Case markers.

Further evidence for the NP status of locatives comes from the fact that they can pronominalize as $i c i$ "here" or $l a l$ l "there" which behave as NPs (they can head restrictive relatives (là où je vais/"there where I go"), can appear as [Spec, $\mathrm{PP}]$ in restricted environments (là-dessus/"thereon," là-dedans/ "therein"). Together with datives (126a-c) and de-complements (126c) and unlike NPs in PPs (126e), they trigger a Principle C violation: ${ }^{56}$

(126)(a)* C'est à lui $i_{j}$ que tu as dit que Jean ${ }_{j}$ était malade. It is to him that you said that John was sick.

(b)* ?C'est de lui ${ }_{j}$ que tu as appris que Jean était malade. It is from him that you learned that John was sick.

(c)* C'est là qu'il a décidé qu'il voulait vivre à l'ouest. It is there that he decided that he wanted to live in the west.

(d) C'est à l'ouest ${ }_{i}$ qu'il a décidé qu'il voulait vivre là. It is in the west that he decided he wanted to live there.

(e) C'est devant lui ${ }_{i}$ qu'il a déclaré que Jean ${ }_{j}$ avait tort. It is in front of him that he declared that John was wrong.

The problem we face then is more general. Not only datives but also subcategorized locatives and de-complements should be able to trigger participle agreement since they seem to be generated within VP in a position i-commanded by [Spec, VP], are not separated from it by any barrier and can move to it since they can be analyzed as NPs.

\subsubsection{LACK OF AGREEMENT WITH DIRECT OBJECTS}

Kayne (1989a) notes that not all direct objects trigger participial agreement:

(127)(a) Il a été repeint $\left({ }^{*} \mathrm{ES}\right)$ des portes. lit: It has been repainted doors. Doors were repainted.

(b) Des portes, il en a été repeint(*ES). 
lit: Doors, it has been repainted some.

Doors, some were repainted.

(c) Combien de portes a-t-il été repeint $\left({ }^{*} \mathrm{ES}\right)$ ?

lit: How many doors has there been repainted?

How many doors were repainted?

(127a) exemplifies an impersonal (passive) construction. The possibility of en cliticization from the postverbal NP shows that this NP is a direct object. The lack of agreement in $(127 \mathrm{c})$ is surprising. And, in view of the possibility of agreement with en described in Section 5.5.3, (127b) is also surprising. Kayne (1989a) suggests that the lack of agreement here is correlated to the fact that the subject of the clause is expletive. We will conclude that this correlation is correct, ${ }^{57}$ but this cannot be the whole story since it does not extend to all the other cases in which agreement is impossible whether or not the subject of the clause is expletive.

Guéron and Hoekstra (1988) suggest that lack of agreement is related to the obligatory indefiniteness of the object because non-generic indefinites cannot trigger agreement. They cite *une chaise est repeintel"a chair is repainted." But the data is not so clear. In my judgment, their example is fine. Furthermore, Dans ce pays, une chaise est repeinte toutes les beures/"In this country, a chair is repainted every hour" with non-generic non-specific indefinite reading is well formed, and so is Une chaise du Musée d'Orsay qui a été repeinte bier a été décrite dans les journaux/"a chair from the Orsay museum which was repainted yesterday was described in the newspaper" with non-generic specific indefinite reading. ${ }^{58}$ Moreover, just like Kayne's proposal, this one also does not account for the whole range of cases lacking agreement. ${ }^{59}$ Belletti (1988) remarks that impersonal constructions with a bare NP complement are possible only when this NP is theta-marked by the verb. In particular, impersonal constructions are impossible with NP subjects of small clauses:

(128)(a) Des étudiants ont été considérés [t malades]. Some students were considered sick.

(b)* Il a été considéré [des étudiants malades]. It has been considered some students sick.

(c)* Il en a été considéré [t malades]. It of-them has been considered some sick.

She concludes that the Case of the postverbal NP in (127a) cannot be structural, since it is sensitive to the presence of a direct semantic relation between the verb and the postverbal NP. Assume then that the postverbal NP does not bear accusative Case. ${ }^{60}$

On that basis, I want to suggest that the crucial factor determining the possibility of agreement is Case. The direct objects in (127) cannot trigger 
agreement because they do not receive structural accusative. More generally, I want to suggest that there is a causal link between getting structural Case and being able to trigger agreement. In particular, inherently Case-marked NPs will not trigger participle agreement. This approach will cover all the cases so far discussed, datives, locatives, etc. (and also genitive arguments of nouns, which we discuss in Section 6.1).

The idea that agreement and Case are closely linked is extremely rich in consequences. The rest of this section is devoted to the question of how to implement this idea and to an exploration of its intricate consequences for the theory of grammar.

\subsubsection{Structural Case and agreement}

I suggest that Case-marked complement NPs can trigger agreement only if they get structural Case. Nothing in the theory presented so far links these two properties. We now turn to the question of how they are related.

Agreement which we have assumed all along and argued for, is the reflection of a specifier/head relation. Case, as I have suggested in Section 2.4, is subdivided between agreement Case and governed Case on the one hand, and inherent Case and structural Case on the other. How do the two dimensions correlate? It looks as if inherent Case is always governed Case, while agreement Case is always structural Case. But governed Case seems not necessarily to be inherent Case. If direct objects are Case-marked in complement position under V', accusative will be an instance of governed Case. Similarly, nominative Case can be governed Case, for example, in some VSO languages, as Koopman and Sportiche (1991) argue.

It is tempting to try to correlate agreement Case and agreement. Both arise as a consequence of a Spec/head relation. What we want however is a correlation between agreement and structural accusative Case. This can be achieved by transitivity if we equate or somehow directly correlate structural accusative Case assignment with agreement Case. Equating accusative position with agreement position is a subcase of the more general $\mathrm{SCH}$, which equates structural Case positions and (potential) agreement throughout:

\section{Strong Correlation Hypotbesis ( $\mathrm{SCH}$ )}

Structural Case is identical to agreement Case.

Inherent Case is identical to governed Case.

Let us now explore whether this is feasible and how to implement it.

\subsubsection{THE SCH AND ACCUSATIVE}

Agreement arises from a Spec/head relation. We can hypothesize that when a NP triggers agreement, it does so because it gets structural Case in the very position in which it triggers agreement. This is what is usually assumed for 
nominative Case in French or English. Koopman and Sportiche (1991) claim that subject/verb agreement arises when the nominative Case assigning head shows agreement and assigns nominative to its specifier position. In the case of object agreement, the same approach would lead us to assume that an accusative object triggering agreement on the verb receives Case in the [Spec, VP] position:

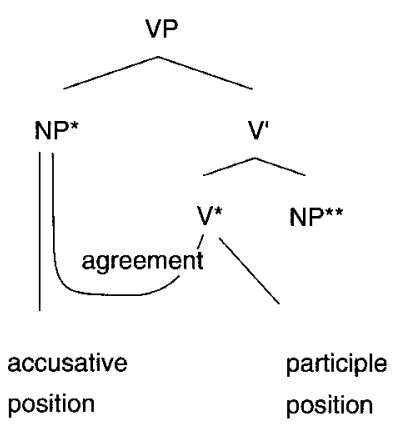

Several problems arise immediately:

1 If NPs must be Case-marked at S-structure, we might expect the order OV.

2 We expect all accusative NPs to trigger agreement. Contrary to facts, only preposed NPs trigger agreement.

3 Agreement can be triggered by caseless object NPs as e.g. in passive or unaccusative constructions. We must somehow allow Caseless NPs to appear in or go through [Spec, VP]. This shows that structural accusative is a necessary condition for agreement but not a sufficient condition.

4 We must prevent inherently Case-marked NPs to appear in or transit through $[\mathrm{Spec}, \mathrm{VP}]$.

Because of our modified Larsonian VPs, the projection of a particular V can actually be more complex than indicated in (130). We need to examine several cases depending on the properties of the V head of the VP: transitive verb and unaccusative verb each with or without morphology, transitive participle, unaccusative participle and passive participle in impersonal constructions or with the subject, etc. Before we do so, suppose that (130) is correct. Suppose further that if there is an accusative position, it is NP* and that somehow a direct object NP** can receive structural Case without having to be in the Case position NP*. This is going to solve problems (2) and (3). Let us see why. First, even though all objects somehow get Case from NP*, only preposed objects will have a chance to appear in it or transit through it in the course of the preposing. This solves (2). In case a participle assigns no accusative Case, a direct object raising to subject will have to transit through NP*, because of the theory of movement triggering obligatory agreement. This solves (3). 
Finally, suppose that some other complement or internal argument of $\mathrm{V}$ with inherent Case (or some Case other than the accusative assigned by V) is moved through NP*. NP* is either in the accusative position or a Caseless position but it is an A-position. Such a movement would be from a Case position (Inherent Case) to an A-position. This is independently excluded. Sportiche (1983) argues that Case-marked A-chains should be headed by the Case position (or almost equivalently, that a trace is a variable - i.e. locally A-bar bound - iff it is Case-marked in an A-position). Since Case assignment is optional, inherent Case does not have to be assigned. If an NP that receives inherent Case can receive Case in some other way compatible with moving to [Spec, VP], it should be able to trigger agreement. However, we want to block a situation that does not seem to happen. A verb with a syntactically realized external argument and a dative internal argument does not allow this dative to trigger agreement, i.e. to surface as accusative instead of dative. For example, we do not find systematic alternations Jean a parlé à PierrelJean a parlé Pierre/ "John spoke to Peter/John spoke Peter". ${ }^{61}$ In order to prevent this situation, I propose that accusative Case be considered an elsewhere Case:

Accusative as elsewhere Case

A verb assigns accusative Case to an NP only if it cannot assign it inherent Case.

(131) does not prevent a potential dative from receiving nominative, instead of dative, if this possible. Provided that we solve problem (1), the word order problem, or equivalently that we can explain how $\mathrm{NP} * *$ gets accusative Case without being in NP*, we will have a complete account of the lack of participle agreement with non-structurally accusative Case-marked NPs.

\subsubsection{PASSIVE AND UNACCUSATIVE STRUCTURES}

Abstracting away from unaccusative structures with auxiliary avoir mentioned earlier in Section 4.3.2 and further discussed in Section 5.5.5, passive participle structures and unaccusative participle structures are identical and behave in the same manner with respect to agreement:

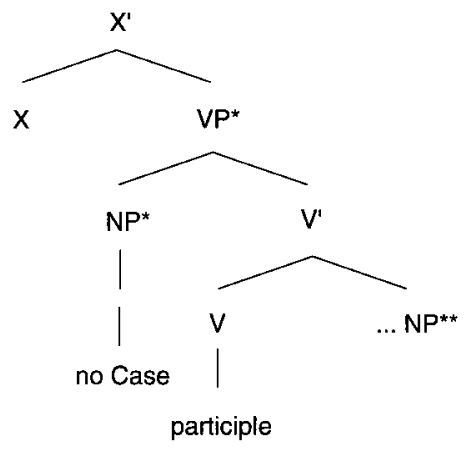


Since the participle $\mathrm{V}$ projects no external argument, there is only one VP structure, that is, $\mathrm{VP}^{*}$ is the maximal projection of $\mathrm{V}$. Furthermore, no accusative Case is assigned, perhaps because of Burzio's Generalization. If the object NP** preposes to subject position it will have to transit through NP*, triggering agreement on $\mathrm{V}$ (as in Trois portes ont été peintes/"Three doors(FEM.PL) were painted(FEM.PL)"). If the object NP** does not prepose the subject as in an impersonal construction or in an impersonal passive construction (e.g. Il est arrivé des hommes, il a été vu des bommes/"It is arrived men, it has been seen men"), the object is postverbal, as predicted, and receives some other Case than accusative. Note that since NP* is a caseless Aposition, nothing can surface there.

Passive constructions always involve passive participles in French, but unaccusative constructions do not. Consider what happens in a sentence like Ils arriveront/"They will come."

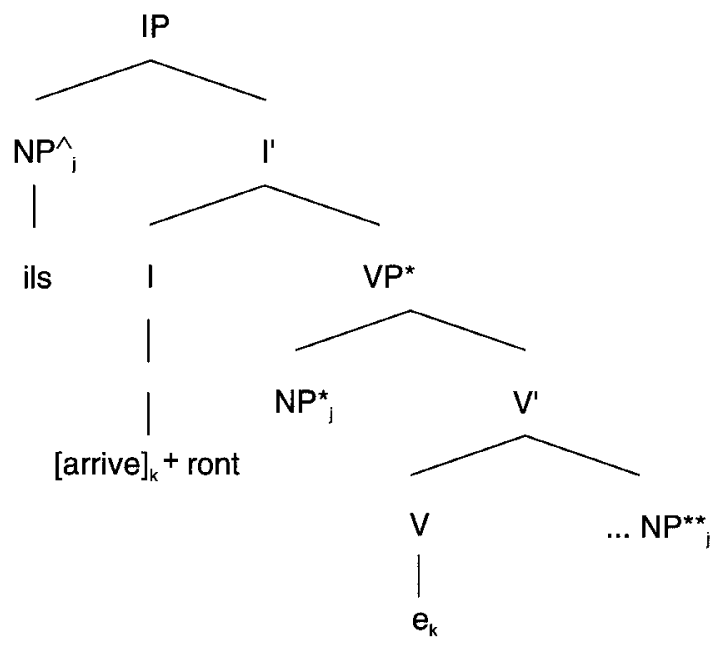

If the object does not prepose (Il arrivera des bommes) nothing special needs to be said. If the object preposes, it moves through $\mathrm{NP}^{*}$ to $\mathrm{NP}^{\wedge}$. The verb, on the other hand raises to the future tense morphology. There is no word order problem here but there is an agreement problem. Why does the verb+affix combination agree with $\mathrm{NP}^{\wedge}$, but the verb not show any agreement with NP*? We return to this problem in Section 5.3.

\section{1 .2 .3 TRANSITIVE VERBS}

The case of transitive verbs is slightly more complicated. Consider first the case of a transitive participle e.g. Jean a mangé la soupe/"John has eaten the soup," keeping in mind our modified Larsonian position (I am ignoring here the aspectual projection of avoir, which raises to Tense): 
(134)

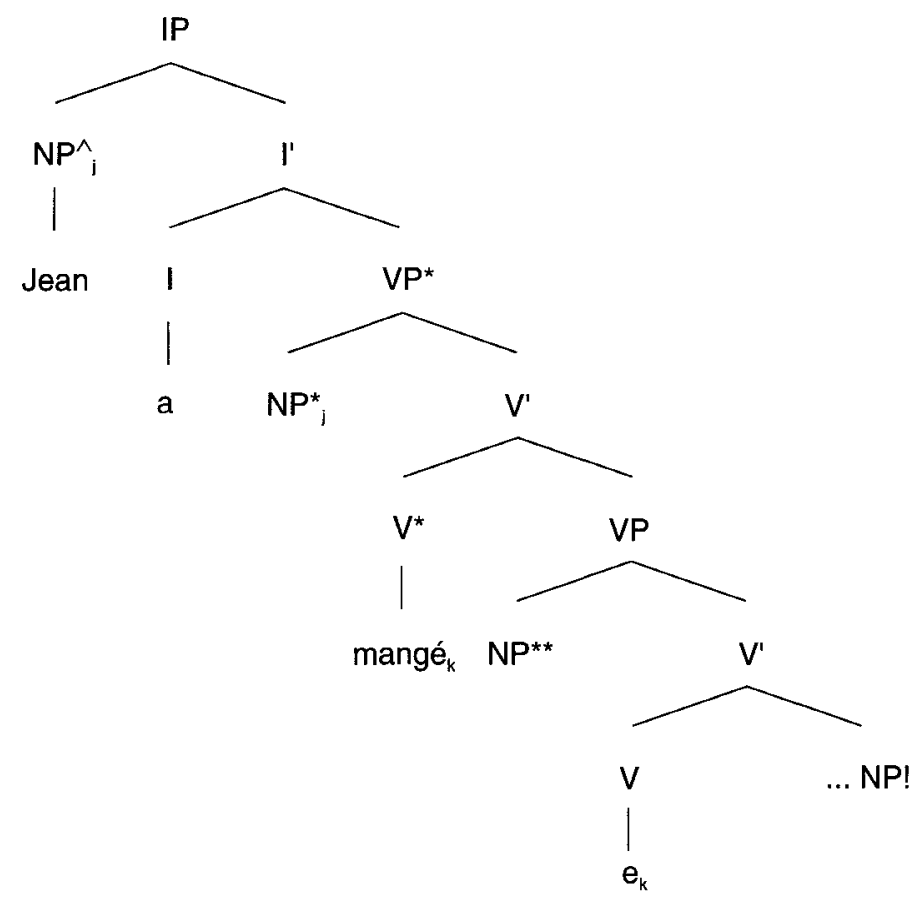

Because the $\mathrm{V}$ manger has an external argument, the lower VP is not its maximal projection. This external argument raises from $\mathrm{NP}^{*}$ to $\mathrm{NP}^{\wedge}$. The verb licenses the second VP projection by raising to $\mathrm{V}^{*}$. NP** is the accusative position. Even if the direct object NP is in NP** or has raised to NP** from NP!, there is no word order problem VO. Note again that an agreement problem appears. Since the participle appears in $\mathrm{V}^{*}$, why does it not agree with NP*? In other words, why does the participle not exhibit subject agreement rather than, or in addition to, object agreement if the object agreement conditions are met? We return to this question in Section 5.3. Finally consider the case of a transitive verb not in the participial form:

Jean mangeait la soupe.

John was eating the soup.

Its syntactic structure is as in (136) opposite.

There is no other IP in this case than the projection of Tense. The accusative position is NP**. Note that we have the same problem we had earlier concerning agreement. Why does the verb not show agreement with a preposed object in such cases if this object has been preposed? Why does the verb not pick up agreement on the way? We return to this question in Section 5.3. 
(136)

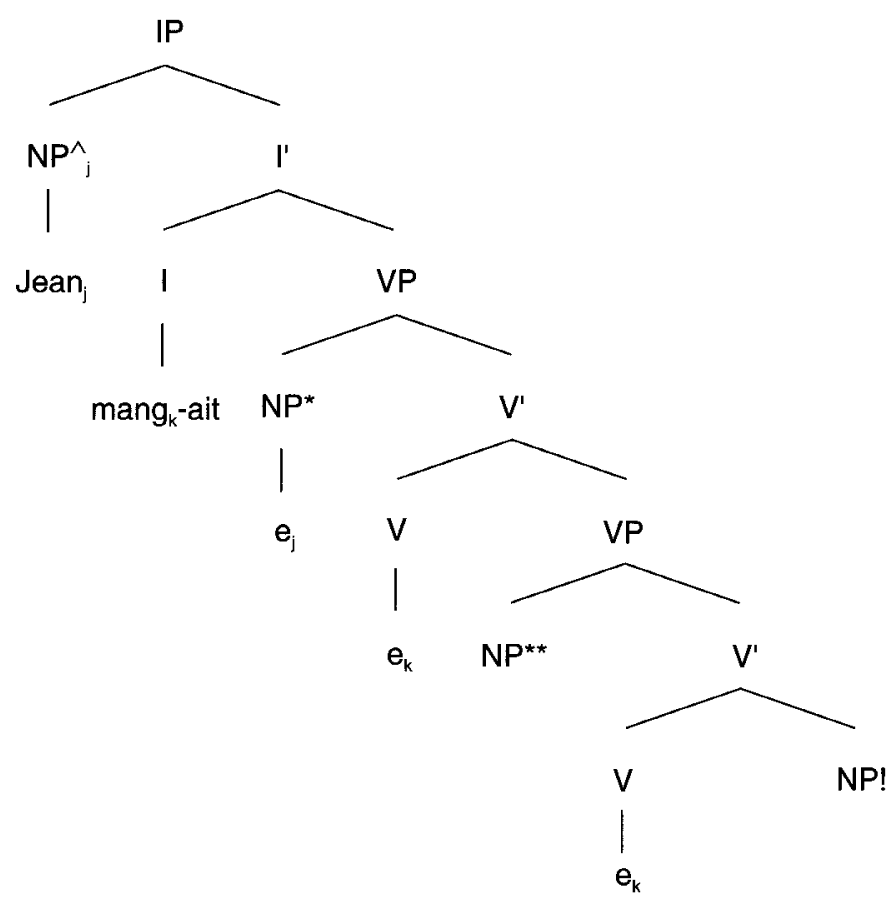

\subsubsection{AGREEMENT POSITION AS AN A-POSITION}

Identifying the accusative position with the object agreement position does not undermine our conclusion that the position triggering agreement is an A-position. In the case of object agreement with a superficial object, the conclusion does not hold any longer: only the potential accusative object will be able to move to the accusative position by definition. The A/A-bar distinction will play no role. However, the distinction will remain relevant in the case of a predicate not assigning accusative Case, e.g. a passive participle. In the French equivalent of It was said that John saw Mary/Which girl was it said that John saw? the preposed $w h$-phrase should be able to trigger agreement if the agreement position was an A-bar position, contrary to fact:

(137)(a) Il a été dit que Jean a vu Marie.

(b) Quelle fille a-t-il été dit(*E) que Jean a vu.

\subsection{Structural Case and the internal structure of VP}

In the preceding section, I proposed that agreement and structural Case assignment are linked in that they are properties of the same position. One problem with this idea is due to the fact that postverbal direct objects of 
participles in French do not trigger agreement. This means they have a way of acquiring Case without being in the accusative position. I will now discuss problems related to this proposal. Before listing some of the questions arising from this proposal, it is important to realize that the analysis I suggest for objects has been suggested by several authors for nominative subjects because, in some cases, it is quite easy to see that the external argument is not in the subject position of the clause. The questions arising for objects arise in exactly the same way for subjects:

(138)(1) If accusative objects are not necessarily in the accusative position, what is their surface position?

(2) If nominative subjects are not necessarily in the nominative position, what is their surface position?

(3) What is the base position of subjects (external arguments) and objects (internal arguments)?

(4) If in a given language and/or construction, objects or subjects must be in the accusative or nominative position, what forces it?

(5) If in a given language and/or construction, objects or subjects cannot be in the accusative or nominative position, what prevents it?

(6) In this last case, how is Case assigned?

\subsubsection{Surface position of nominative and accusative NPs}

Let us now examine what we can conclude about the position of subjects and objects from the syntactic structure, the theory of agreement and Case that we propose.

\subsubsection{SURFACE POSITION OF ACCUSATIVE OBJECTS IN \\ FRENCH}

Consider again the structure of the sentence Jean a peint la porte as in (139) opposite.

In French, the agreement facts show that the direct object cannot be in NP**. It must be lower in the tree; say NP! Otherwise, it would trigger object agreement. This means that NP! gets accusative Case without being in the accusative position. There must be a way for NP! to inherit Case from the position NP**. If our hypothesis that NP** is the accusative position is correct, $\mathrm{NP}^{* *}$ must be present. We cannot assume that this position is absent because it is not required by the Projection Principle. ${ }^{62} \mathrm{NP} * *$ must therefore be a silent expletive. The same conclusion will hold in Italian (dialects) or Catalan which allow or require participle agreement with preverbal accusative clitic objects, but do not with postverbal objects. There is independent 
(139)

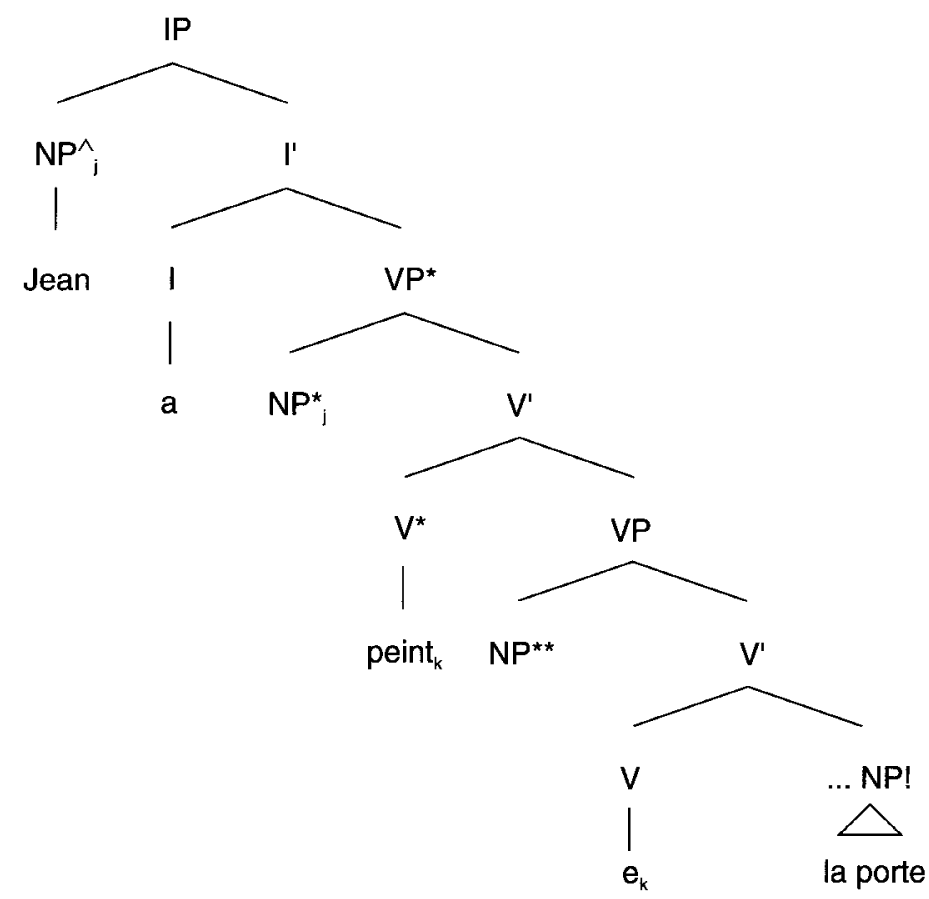

evidence suggesting that expletives are permitted in such a position. French (and Italian) differ from English in the following cases:

(140)(a)* These kinds of events persuade e [PRO to leave].

(b) I consider *(it) important [PRO to arrive on time].

(c) Ce genre d'évènements persuade e de [PRO partir].

(d) Je considère e important de [PRO venir à l'heure].

Rizzi (1986b) shows that the (a) sentence is out because some silent NP e is required to act as controller but is not allowed in this position, as opposed to the French equivalent in (c). Similarly, an expletive cannot be omitted in the (b) sentence for the same reason, while it may in French. In other words, silent objects are licensed in French but not in English, whether they are thematic or expletives. Plausibly, the grammaticality of the (b) and (d) sentences (and of their Italian equivalents) can be related to the existence of pronominal clitics in these languages. In both sentences, object pronouns must cliticize in these contexts, suggesting the presence of a silent clitic licensing e. It now becomes plausible to suppose that Case on NP! is somehow inherited from the expletive in $\mathrm{NP}^{* *}$ in these languages. This leaves open the question of why lexical objects cannot raise to NP** in French (or Italian) except in very restricted cases (cf. Section 5.5.5). 
5.2.1.2 THE SURFACE POSITION OF ACCUSATIVE OBJECTS IN

ENGLISH: RAISING TO OBJECT

A priori, in an English sentence John bad painted the door equivalent to (139), the object could be either in NP** or some NP! in a further down position since we cannot rely on agreement to assess the object position. In a sentence like Jobn will eat soup, the verb lacks any morphology suggesting it has (overtly) raised to the higher head position as below, where $V_{j}$ is raised to $V_{k}$ :

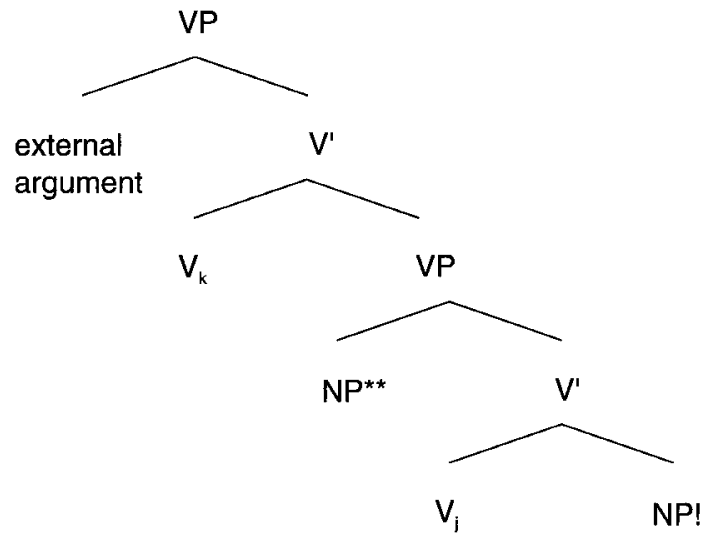

Accordingly, examples such as Jobn will eat soup are not conclusive for the question under discussion. The object could be either in NP** or NP! and still follow the verb. Verbs lacking external arguments do not have a $\mathrm{V}_{k} \mathrm{P}$ projection at all. Usually, such verbs (unaccusatives, passives) do not allow their direct objects in object position, so they are not conclusive either. Sentences such as There will arrive few men might be thought to decide the issue in favor of the NP! alternative if the postverbal NP is in object position:

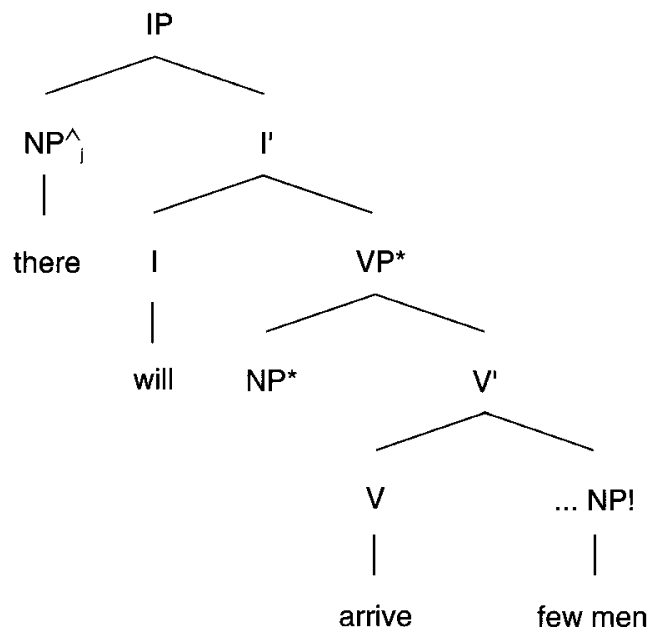


The verb is bare and therefore does not raise for morphological reasons. As it lacks an external argument it therefore lacks an external argument VP projection. It presumably is in its base position and is followed by its object. The only problem is that it is plausible that the object in such cases does not get accusative Case at all. The fact that silent expletives are not licensed in English suggests that accusative objects in English must surface in NP**. I conclude that English objects always raise to the accusative position. In fact, one argument to that effect has already been presented in the discussion of small clauses in Section 2.2.2 and based on (22b):

(22) (a)* They consider John ${ }_{j}\left[{ }_{W} t_{i}\right.$ [proud of each other]].

(b)* $\left[_{\mathrm{W}} t_{\mathrm{i}}\right.$ [How proud of each other $\left.]\right]$ do they consider John $n_{i}$.

The fact that the lexical anaphor cannot take the matrix subject as antecedent suggests that AP preposing carries along the trace of the subject of the $\mathrm{AP}$, which has therefore raised out of the small clause. We can now interpret this as meaning that the subject of the small clause has raised to the specifier position of the inner VP of consider in order to receive the accusative Case.

(143)

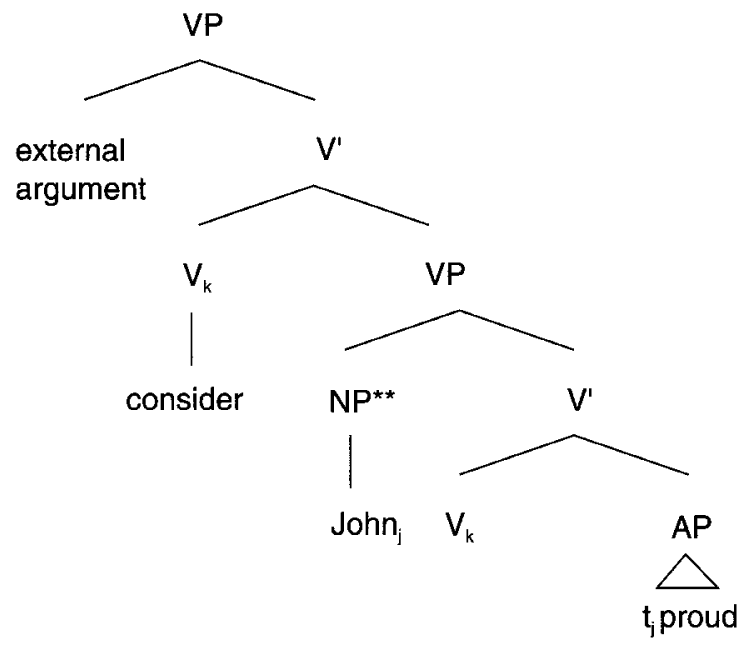

Naturally, raising to specifier will also take place in simple clauses as in I will see John: (see (144) on page 172).

The conclusion that raising to specifier can take place in English finds independent support in other constructions such as particle constructions discussed in Johnson (1990) and Koopman (1990). They show that in the (a) sentences:

(145)(a) John called Bill up/called him up.

(b) John called up Bill/*alled up him. 
(144)

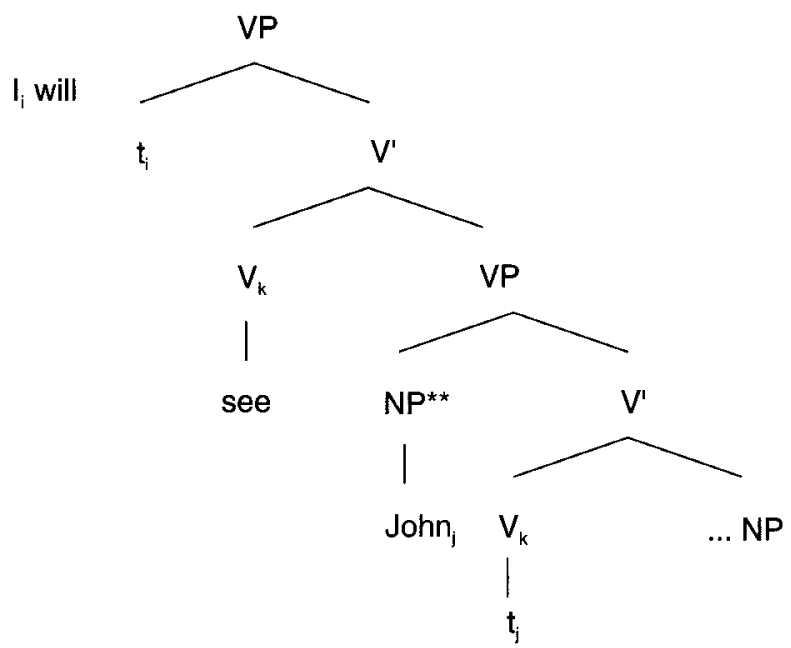

The NP has raised to specifier (of VP for Johnson, of PP for Koopman) to receive structural Case from V. Furthermore, Koopman argues that the impossibility of the second (b) sentence follows from a general process of attraction of pronouns to specifier positions also found in:

1 double object constructions: adopting something of Larson's (1988) analysis, the first object of a double object construction is in [Spec, VP] but the second is not: *give my friends it /give it to my friends.

2 "Q-movement" constructions: English $I$ saw all the children/*I saw all them/I saw (them all) (or French [eux tous], [nous tous]) which is now analyzed as $\left[_{Q P}\right.$ them ${ }_{j} L_{Q^{\prime}}$ all $\left.\left.t_{j}\right]\right], L_{Q_{P}} u s_{j} L_{Q^{\prime}}$ all $\left.t_{j} j\right]{ }^{63}$

\subsubsection{SURFACE POSITION OF NOMINATIVE SUBJECTS}

The mechanisms of Case inheritance necessary to handle the French facts described above are similar to the mechanisms necessary to handle nominative Case assignment to subjects. The situation for accusative Case is much less documented than that of nominative Case but has all the same properties. Koopman and Sportiche (1991) describe nominative Case assignment as follows. Calling NP* the subject in VP, $\mathrm{NP}_{\wedge}$ the subject of clauses and Infl the highest inflectional head of a clause, we see that in English or French, the subject cannot surface as NP*. This is construed as meaning that NP* receives no Case. Rather, the subject surfaces as $\mathrm{NP}_{\wedge}$ exhibiting agreement with Infl (in a fairly impoverished way in English). This means that when Infl is a Case assigner, it is a Case assigner by agreement only.

In Arabic, the external argument can surface in either position, NP* or $\mathrm{NP}^{\wedge}$ (examples from Mohammad (1989)): 
(146)(a) idda9a ar-rijaal-u ?anna

claimed 3SM the men-NOM that

(b) al-?awlaad-u katabuu risaalat-an.

the-boys-NOM wrote-3PM letter-ACC.

If the subject is in $\mathrm{NP}^{\wedge}$, the verb obligatorily agrees in person and number. Otherwise, the verb gets a default third person singular agreement which Mohammad (1989) analyzes as agreement with a null expletive. He provides evidence showing that when $\mathrm{NP}^{\wedge}$ must be phonetically realized, this expletive appears. Furthermore, only subjects may move to $\mathrm{NP}^{\wedge}$ suggesting that movement to $\mathrm{NP}^{\wedge}$ is A-movement (cf. Koopman and Sportiche (1991) for discussion). This is analyzed by Koopman and Sportiche (1991) as arising from Infl being able to assign either governed Case (in (146a)) or agreement Case (in (146b)). In Irish, only the order VSO is allowed and the subject never agrees with the verb or the auxiliary in Infl. The only apparent case of agreement is found with (covert) pronominal subjects but Hale and McCloskey (1984) have argued that this case is best analyzed as involving incorporation of a postverbal pronoun into Infl. A Case-marking Infl in Irish is analyzed as being a governed Case assigner and never an agreement Case assigner.

The parallelism with accusative Case is immediate. French, English and Arabic (with SVO order) nominative subjects behave like Bambara, Dutch (and possibly English) accusative objects. They are in the nominative position at S-Structure, receive nominative Case and trigger agreement. Welsh, Irish or Arabic subjects (with VSO order) on the other hand are not in the nominative position even though they receive nominative Case and do not trigger agreement. In this respect, they behave like French objects. Naturally, since we identify agreement Case and structural Case, this means that all these subjects somehow inherit nominative Case from $\mathrm{NP}^{\wedge}$. Some Case inheritance mechanism must be invoked here, as well as in the French object case. Note also that Welsh or Irish subjects further resemble French objects in that they cannot (except perhaps for Welsh pronouns) occur in the Case position $\mathrm{NP}^{\wedge}$.

\subsubsection{Structural Case, inberitance and agreement}

\subsubsection{ANTI-MOVEMENT CONSTRUCTIONS}

So far, we have discussed two kinds of situations with respect to nominative or accusative NPs summarized below in (147) as (1) and (4): (see (147) and table 3.1, page 174).

The cases in (2) are all the cases in which the head does not show agreement, as in English participles, and infinitives (except Portuguese). There are also many potential cases illustrating (3) that have been extensively discussed: English there insertion constructions, Italian inverted subjects, Italian impersonal constructions, postverbal direct objects in certain dialects of 
PARTITIONS AND ATOMS

(147) Table 3.1

\begin{tabular}{lcc}
\hline & In Case position & Not in Case position \\
\hline $\begin{array}{l}\text { Agreement with } \\
\text { Case assigner }\end{array}$ & $\begin{array}{c}\text { (1) French/English subjects } \\
\text { Arabic SVO subjects } \\
\text { Portuguese subjects } \\
\text { in infinitives }\end{array}$ & (3) Existential there (?) \\
\hline $\begin{array}{l}\text { No agreement with } \\
\text { Case assigner }\end{array}$ & (2) Bambara objects & (4) French objects \\
& English objects (?) & Irish/Welsh subjects \\
& Subjects of infinitives & Arabic VSO subjects \\
\hline
\end{tabular}

Italian, stylistic inversion in French. Similarly, further cases potentially illustrating (4) are impersonal unaccusative constructions and impersonal intransitive constructions in French.

Several relevant factors enter into play in the analyses of these constructions in which an NP, NP*, is not in the position $\mathrm{NP}^{\wedge}$ in which it receives structural Case from a head $\mathrm{K}$, and that I will call Anti-Movement Constructions. Some questions that arise are listed below:

$$
\left[\mathrm{KP}_{\mathrm{KP}} \mathrm{NP}^{\wedge} \quad \mathrm{K} \quad \mathrm{NP} *\right]
$$

(1) Is NP* in an A-position or not?

(2) If NP* is in an A-position, is it in its base position or not?

(3) Does NP* trigger agreement on the Case assigning head K or not?

(4) What are the properties of the expletive element appearing in $\mathrm{NP}^{\wedge}$ (the specifier position of the Case assigning head).

(5) Is there a definiteness effect on NP* or not?

(6) Is there a Case relation and what is the nature of this Case relation between $\mathrm{NP}^{\wedge}$ and $\mathrm{NP}^{*}$ ?

In Italian subject inversion constructions (149a), Italian impersonal constructions (149b), Italian postverbal object agreement (149c), ${ }^{64}$ English there insertion (149d), and French stylistic inversion constructions (149e), French $c e+\hat{e} t r e$ constructions (149f), there is agreement between NP* and $\mathrm{K}$.

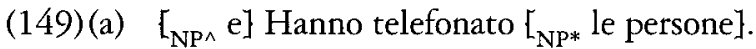

Have telephoned the people.

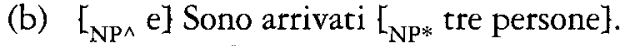

Are arrived three people.

(c) $\mathrm{Ha}\left[_{\mathrm{NP}^{\wedge}}\right.$ e] mangiato $\left[_{\mathrm{NP}^{*}}\right.$ le mele].

He has eaten the apples. 
(d) $\left[_{\mathrm{NP}^{\wedge}}\right.$ There $]$ are $\left[{ }_{\mathrm{NP} *}\right.$ three men] on the roof.

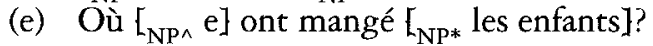

Where have eaten the children?

(f) $\mathfrak{L}_{\mathrm{NP} \wedge} \mathrm{Ce}$ s sont $\mathfrak{L}_{\mathrm{NP*}}$ mes amis]. ${ }^{65}$

This are my friends.

In French impersonal unaccusatives (150a), impersonal intransitives (150b), ${ }^{66}$ French (150c) and Italian (150d) postverbal objects, French ce+être constructions $(150 \mathrm{e})$, there is no agreement between $\mathrm{NP} *$ and $\mathrm{K}$ :

(150)(a) $\left[_{\mathrm{NP} \wedge}\right.$ Ill est arrivé $\complement_{\mathrm{NP} *}$ trois hommes].

It has arrived three men.

(b) $\left.\complement_{\mathrm{NP}^{*}} \mathrm{Il}\right]$ a dansé $\left[_{\mathrm{NP} *}\right.$ plusieurs lions $]$.

It has danced several lions.

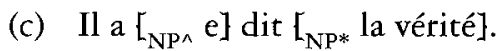

He has said the truth.

(d) $\mathrm{Ha}\left[_{\mathrm{NP}^{\wedge}}\right.$ e] mangiato $\mathrm{L}_{\mathrm{NP*}}$ le mele $]$.

He has eaten the apples.

(e) $\left[_{\mathrm{NP}^{\wedge}} \mathrm{C}^{\prime}\right]$ est $\left[_{\mathrm{NP*}}\right.$ mes amis $]$.

This is my friends.

In both French stylistic inversion and Italian inverted subject constructions, it could plausibly be argued that NP* is in an A-bar position, and therefore has exactly all the properties it would have if it were in $\mathrm{NP}^{\wedge}$ : it triggers agreement, displays no definiteness effect, and the expletive in $\mathrm{NP}^{\wedge}$ is silent. Otherwise, silent expletives co-occur with agreement $(149 \mathrm{a}, \mathrm{b}, \mathrm{c})$ or with lack of agreement $(150 c, d)$. Overt expletives co-occur with agreement $(149 \mathrm{~d}, \mathrm{f})$ or with lack of agreement $(150 \mathrm{a}, \mathrm{b})$. The definiteness effect can be defined as the impossibility for NP* to be a strong NP (where the class of strong NPs must be characterized independently), following Milsark's (1974) terminology. This effect co-occurs with silent expletives (149b) (cf. Belletti, (1988)) and agreement, but not necessarily (149c). Definiteness effects also co-occur with overt expletives and agreement (149d) but not necessarily (149f). Definiteness effects co-occur with overt expletives and lack of agreement $(150 \mathrm{a}, \mathrm{b})$ but not necessarily (150e). Finally, definiteness effects do not seem to co-occur with silent expletives and lack of agreement.

\subsubsection{CASE AND AGREEMENT INHERITANCE}

There is considerable literature on the properties of these constructions concerning Case assignment and agreement. How do we handle Case inheritance for the French (139), the Arabic (146a) or the Welsh or Irish cases? There are two ways to achieve this, Case checking or chain formation. Chomsky (1981) argued that NPs are not Case-marked but rather are Case checked. 
They are base generated with Case and Case-marking rules are replaced by Case checking rules. ${ }^{67}$ If Case is checked rather than assigned, we can handle accusative inheritance as Chomsky (personal communication) suggests:

(151) Structural Case is checked either at S-structure or at LF.

If structural Case is checked at S-structure, the Case-marked NP will have to be in the Case position at S-Structure. This is going to be the situation of Bambara direct objects, which Koopman $(1987 ; 1992)$ argues surface in [Spec, VP] at S-Structure, Dutch direct objects as argued in Koopman and Sportiche (1988a) and Koopman (1988), and English objects, which we concluded are in the accusative position at LF. If structural Case is checked at LF, the cased NP must be in a position to move to the Case position at LF in accordance with the constraints on movement rules. At LF, the cased NP is in the Case position and can be checked appropriately.

A second way is to assume that Case inheritance is done through chain formation as Burzio (1986) (or Borer (1986), albeit with a different execution) suggest. Under the standard view, NPs must be Case-marked/checked by S-Structure. This can be achieved through chain formation if Case is considered a property of chains and not of individual NPs. Suppose the accusative object NP! is not in the accusative position NP** or does not have a trace in the accusative position as in French. Chains can be formed freely, subject to the CCL and other principles governing chain formation (location of theta position or of Case position within the chain, etc.). Under this option, we can form a chain (NP**, NP!) as in (139). This chain will be Case-marked and well formed as its highest A-position is the Case position. Its lowest position is the theta position. This proposal becomes almost equivalent to the preceding proposal if expletives are eliminated at LF by expletive replacement: then at LF, NP! will have to move to NP** to eliminate the silent expletive NP**. As a final note, remark that it is crucial for our account of French agreement that such a chain should not transmit agreement with a head. Otherwise, all accusative objects would trigger agreement. This looks like a weakness of this approach as opposed to the LF Case checking approach.

A third way is to assume that NP* in an anti-moved construction receives Case in situ directly from some Case assigner, as Belletti (1988) or Pollock (1984) suggest. I propose in Section 5.2.2.3 that the first two approaches are correct.

\subsubsection{INHERITANCE IN ANTI-MOVEMENT CONSTRUCTIONS}

First expletive replacement must occur at LF. The reason is that elements devoid of content must be eliminated at the level at which interpretation is computed. The empirical support for it comes from the local relation that such expletives entertain with the categories (e.g. NPs) that overtly occur in the expletive position in the non-expletive counterpart construction. 
Thus, we have Il est arrivé trois bommes, and its non-expletive counterpart Trois bommes sont arrivés and $i l$ and trois bommes are in a possible NP Movement configuration.

Second, structural Case checking is done at S-Structure or at LF.

Third, an expletive $\mathrm{NP}^{\wedge}$ may form an S-Structure chain with its "replacing" NP*. If an S-Structure chain is formed, there will be agreement transmission from $\mathrm{NP}^{*}$ to $\mathrm{NP}^{\wedge}$ if we suppose, as is plausible, that the members of a chain must agree. Adapting a suggestion of Pollock (1984), I conclude that a chain can be formed between $\mathrm{NP}^{\wedge}$ and $\mathrm{NP}^{*}$ only if the expletive $\mathrm{NP}^{\wedge}$ is able to agree, i.e. lacks pronominal features of its own.

Fourth, if no S-Structure chain is formed, expletive replacement and Case checking both take place at LF.

In English, only overt expletives are allowed ${ }^{68}$ and there is the only one participating in chains. We expect agreement transmission to be possible, but not necessary in principle (nothing forces S-Structure chain formation viz. the colloquial There's several men in the room). The same applies to Dutch er constructions which also transmit agreement.

In French, the normal overt expletive il must be third person singular. Consequently, normal expletives, when allowed, must be third person singular and thus cannot enter into S-Structure chains: no agreement transmission takes place. The other one, $c e$, is featureless but restricted to appearing on the verb être/"be." This exceptional ce lacking feature allows both configurations: it may enter into a chain but does not have to. Stylistic inversion will have to be analyzed as not involving an expletive (perhaps a case of $\mathrm{NP}^{*}$ in an A-bar position or a case of predicate raising as Deprez (1988) has suggested).

In Italian, expletives are all silent. ${ }^{69}$ They can freely enter into chains and thus can transmit agreement but not necessarily. We expect dialect split $(149 \mathrm{c}) /(150 \mathrm{~d})$. In fact, we expect a more general dialect or construction split for (149b), although possibly not for inverted subjects for the same reasons as in French. This might be exemplified by the pairs si mangia le melelsi mangiano le mele/"one eats - 3rd PERS-PL the apples"/"people eat apples."

The same approach can be extended to the SVO/VSO Arabic examples discussed earlier in (146). Muhammad (1989) shows that in certain contexts (immediately following the complementizer ?anna) the subject expletive in [Spec, IP] in the VSO construction must appear. In this case, it must be third person singular. This predicts that Arabic is like French with respect to agreement transmission as there can be none. Note that in the cases of chain formation at S-Structure, it could be said that the nominative or accusative on NP* is transmitted via the chain. This makes sense only if it is necessary that Case be checked at S-Structure in these instances. Otherwise, it is no different from LF Case checking. ${ }^{70}$

Summarizing all cases, structural Case is checked at LF. In certain constructions, structural Case is also checked at S-Structure and overt 


\section{PARTITIONS AND ATOMS}

movement to the Case position is obligatory. In others, structural Case is checked only at LF as a consequence of expletive replacement. Expletive replacement by an NP takes place with or without the NP and the expletive in the same chain at S-Structure. Agreement transmission arises as a consequence of chain formation at S-Structure, which is only possible if expletives can agree. If non-restricted overt expletives cannot agree, such chains may not be formed. If expletives can agree, agreement is possible but not required.

Other mechanisms have been proposed to account for Case and/or agreement transmission in these constructions. An XP chain or movement account as we have adopted requires nothing that is not independently necessary. Existence and properties of NP Movement and/or chains, consistency of chain members, with respect to phi-features with Case transmission are a by-product. ${ }^{71}$ Downward feature percolation along successive heads not linked by movement, as suggested in many analyses, does not have the same independent support.

\subsubsection{GENERALIZED ANTI-MOVEMENT}

This analysis of anti-movement constructions could be extended much further than instances of covert NP Movement. For example, it could be extended to A-bar movement as e.g. cases of covert $W h$ Movement which seem to take place in French $w h$-in-situ constructions. Similarly, it could be extended to cases of head movement. We could view English affix hopping in verbs as the exact counterpart of head movement of what French impersonal constructions are to NP Movement or covert Wh Movement is to A-bar movement. This would require developing the analogues of Case checking, covert movement and expletive replacement for morphological affixation and incorporation. This seems to me in principle quite desirable and reasonably straightforward and rich in consequences (basically claiming identity of LF representations in a strong sense across languages), although I will not pursue this matter here.

\subsubsection{SPECULATIONS ON UNSOLVED PROBLEMS}

There are several questions left open. The previous discussion does not address the factors (148(2) and (4)). The question (148(2)) is raised by the following ungrammatical examples:

(152)(a) Il semble être arrivé trois hommes.

(b)* Il semble trois hommes être arrivés t.

(c) Sembrano essere arrivati tre ragazzi.

(d)* Sembrano tre ragazzi essere arrivati $t$.

(e) There seems to be a man on the roof. 
(f)* There seems a man to be $t$ on the roof.

In situations of LF expletive replacement, $\mathrm{NP}^{*}$ cannot in most cases have been subject to NP Movement in the syntax whether agreement is transmitted or not as shown by the French/Italian pairs. There constructions occur as a significant exception to this generalization. There were three men killed cannot be analyzed exclusively as an adjectival passive as shown by the grammaticality of There were three men given books or of There were three men considered stupid suggesting the existence of an NP-trace immediately following the participle. The impossibility (or marginal character) or the English There were killed three men as opposed to the French Il a été tué trois bommes (viz. *il a été trois bommes tués) suggests that additional factors are at play. The ungrammatical examples to explain also include:

(153) (a)* Il semble trois hommes être arrivés.

(b)* Il semble trois hommes dormir.

(c)* There were killed three men.

(d)* It was/were killed three men.

(e)* It was/were three men killed.

(f)* Il a été considéré trois hommes malades.

We can describe the facts as follows keeping the terminology of (148):

(154)(1) Movement takes place only if necessary.

(2) If NP* does not have to move, it must be governed by its theta assigner.

Property (2) is what led Belletti (1988) to propose that NP*, when it does not move, receives inherent Case, which excludes $(153 \mathrm{~b}, \mathrm{f})$. (1) is what led Belletti to suggest further that the Case positions in a chain must be the head or the coda, which would exclude $(153 \mathrm{a}, \mathrm{e})$. These assumptions seem to lead to problems in the there construction. The ungrammaticality of (152f) would force the assumption that be (inherently) Case-marks the following NP in (152f) and (153e), leaving the possibility of agreement and the possibility of partial movement in the there constructions unexplained. Pollock (1984) suggests that $\mathrm{NP} *$ must be governed by a Case assigner (leaving unexplained (153f) and the difference between French and English passive participles (152a) vs (153d)).

(1) accounts for the impossibility of (153a). The object has moved part way to the subject of the embedded clause, suggesting Case is checked at LF, but not all the way to the Case position. Movement then is unnecessary, therefore excluded. With there constructions, the situation is different; movement of NP* goes all the way to the position governed by be. I suggest that the classical approach to there insertion is correct. Insertion of there is allowed in the specifier of be when be governs an argument. Movement to the position 
governed by be is therefore necessary. No further movement is required (if there is present); therefore no further movement is possible. This excludes (152f) and $(153 \mathrm{c})$. In the case of $(153 \mathrm{~d}, \mathrm{e})$, we expect lack of chain formation since it is marked for phi-features. Without agreement, there is no S-Structure chain between the Case position and NP*. All the evidence so far suggests that English requires S-Structure Case checking consistently. Extending this requirement to these cases will properly exclude them. This leaves $(153 \mathrm{~b}, \mathrm{f})$ unexplained.

Putting all this together, I suggest the following. In English, structural Case checking is at S-Structure. S-Structure chains must be formed excluding expletive it from participating in anti-movement constructions. In there constructions, NP* must raise all the way to the position governed by be, the specifier of which permits there insertion. Lack of raising of NP*, or partial raising, is excluded.

In French and Italian, structural Case checking can apply at LF. In French, expletives have intrinsic phi-features, therefore cannot participate in chain formation. There is no agreement transmission. In Italian, expletives lack intrinsic features and are therefore free to enter in chain formation transmitting agreement. Part way movement is disallowed by (154(2)).

Let us turn to (148(4)). From the restricted survey presented in (149) and (150), the only correlation is the lack of definiteness effect when NP* co-occurs with a covert expletive not transmitting agreement, and this could be an accident due to the restricted set of data we survey. In French, or Italian, for example, where objects are linked to the accusative position, there does not seem to be any definiteness effect as the direct object can either be a weak or strong NP. A similar observation holds of subjects in VSO structures in Welsh or Irish which are not subject to definiteness effects.

Belletti's (1988) proposal that the inherent Case assigned is partitive and therefore incompatible with definites amounts to a different description of the facts (albeit one that links them to something known). The comparison of there constructions and impersonal constructions suggests that the definiteness effect in impersonal constructions might be due to the presence of a covert quantifier incompatible with strong NPs within the VP of the V assigning a theta role to $\mathrm{NP}^{*}$ as below:

$\begin{array}{lllll}(155) \mathrm{L}_{\mathrm{VP}} & \begin{array}{l}\text { external } \\ \text { argument }\end{array}\end{array} \quad\left[\mathrm{Q} \quad \mathrm{V} \quad \begin{array}{l}\text { internal }] \\ \text { argument }\end{array}\right.$

and within the scope of which NP* must be. This would prevent NP* from moving (it must stay in the scope of $Q$ ) and would prevent cases of external argument NP* (they are outside the scope of Q). In there constructions, the position of the quantifier is determined by the position in which there is licensed to appear, i.e. the VP of be and therefore, $\mathrm{NP}^{*}$ can (and must) raise up to $b e^{72}$ 
The covert quantification story would subsume (154(2)): NPs do not move because they must remain within the scope of a covert quantifier, and this $\mathrm{Q}$ is licensed in different positions in impersonal constructions and there constructions. This would exclude examples like $(152 \mathrm{~b}, \mathrm{~d}, \mathrm{f})$ and $(153 \mathrm{a}, \mathrm{b})$. $(153 \mathrm{c})$ is excluded because there is not licensed and (153d,e) are excluded as failure of S-Structure nominative Case checking. Taking into consideration the ungrammaticality of (153f), if the VP of considerer contains a $Q$, the NP* in this structure is in its scope. ${ }^{73}$

\subsubsection{The internal structure of $V P$}

Let us summarize what we have so far concluded about the internal structure of VP:

1 We have adopted the VP-Internal Subject Hypothesis.

2 We have adopted Larson's construal of it: the external argument of a verb is projected as the specifier of a VP.

3 We have argued that the accusative position is a specifier position and not a complement position.

\subsubsection{PARTICIPIAL MORPHOLOGY AND MORPHOLOGY TYPES}

In Section 2.3, I adopted the assumption that inflectional affixation is reducible to syntactic head movement. In the preceding discussions, I have ignored the morphological complexity of the participle and I have assimilated it to a verb assigning accusative Case, assigning external theta roles. If indeed, participial formation is a syntactic process, it is not obvious a priori that external theta-role assignment or accusative Case should be a property of participle projection, rather than of projection of the verb itself. Assume the head movement approach. The structure of a participial VP would be:

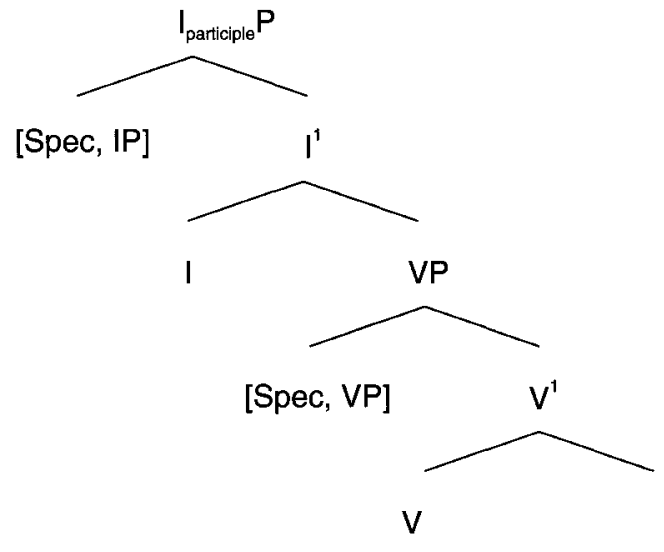


I am going to argue that Case properties and external thematic properties are not a property of the $\mathrm{V}$ but rather a property of the $\mathrm{V}+\mathrm{I}$ combination in the relevant cases. As a consequence, everything we said earlier remains unchanged except for the fact that there may be an additional VP projection complement of the participle head, which incorporates the $\mathrm{V}$ by movement.

Whether a verb assigns accusative Case or not is actually not always a property of the verb alone. Rather, it can be a combined property of the verb and the morphology carried by the verb. If the verb carries passive participle morphology, accusative Case may not be assigned. If it carries the identical past participle morphology or infinitive or tense morphology, accusative Case may be assigned. This seems to suggest that accusative Case should be a property represented in the projection of the morphology that attaches to the verb, rather than in the projection of the verb. This view is reinforced by a comparison of the Case assigning property of verbs and related nouns. Take a typical triplet verb/gerund/-ing-nominal.

(157)(a) John gave books to Bill.

(b) John's giving books to Bill.

(c) John's/the giving of books to Bill.

Their internal thematic structure is identical. They take exactly all the same internal arguments. Their Case assigning properties are also identical as far as inherent Case is concerned. They differ crucially in structural Case assignment. Where verbs and gerunds assign accusative, -ing nominals assign genitive. (I argue elsewhere e.g. in Sportiche (1984) that genitive is a structural Case.) Gerund formation or -ing nominal formation is a completely productive process. If participial inflection is a case of head movement, gerundive formation or nominal -ing affixation should also be (head movement to an -ing inflectional head). This again suggests that the kind of structural Case assigned is not solely a lexical property of the lexical head but rather a property of the word, i.e. part of the inflectional head. The fact that nonderived unaccusative verbs like arrive cannot assign accusative Case regardless of inflectional morphology shows the verbal root does play a role too. The same conclusion is suggested by intransitive verbs which cannot be used with cognate objects, if there are any. ${ }^{74}$ Consequently accusative, if assigned, is assigned as in:

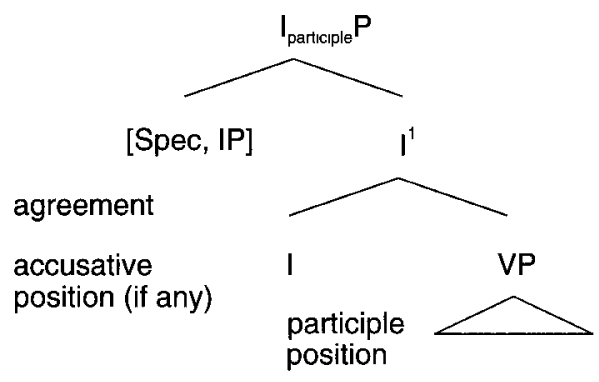


The same question arises concerning the underlying position of the external argument. Whether a verb can have an external role is sometimes determined by the verb alone, as in non-derived verbs e.g. sleep, dance, give, sometimes by the morphology alone as in causativization by affixation of an unaccusative verb. Whether or not and how this external role is syntactically expressed as an NP position may be determined by the verb alone if the verb is morphologically simple, but not if it is morphologically complex (in the relevant way). The ability to assign the external theta role of the verb to an NP in the normal way is suppressed with the passive participial morphology, and is suppressed completely in the neutral construction under se-affixation. The past participle morphology allows it. This suggests that it is the combination $\mathrm{V}+$ morphology that has an external argument. The same point can be made with the nominalizing affix -ing (gerundive nominals). Affixation of -ing to a verb affects the way in which the external theta role of this verb is syntactically expressed: the external argument does not have to be overt any longer viz. I saw (* (John) read a book\}/I resent (Jobn's reading of books\}/I resent (the reading of books\}. In effect, the affix locks or unlocks the external theta role of the head it selects.

More generally, we could claim that a certain class of affixes (call them Class $\mathrm{L}-\mathrm{L}$ for lexical) can syntactically project external roles of the head they attach to onto an NP. If a lexical head has an affix of class $\mathrm{L}$, it is always the affix on this verb that allows it. If the verb is bare and the VP looks like (141), we can interpret the upper $\mathrm{V}$ projection of the layered VP as some kind of null affix of class $\mathrm{L}$ that unlocks the external role. This is what I will tentatively assume. The distinction between class $\mathrm{L}$ affixes and others is meant to sort out tense affixes (which have no lexical content) from participial affixes or nominal -ing which do (category label, lexical properties). In Section 5.6, I discuss an alternative according to which external arguments are projected in VP regardless of the presence of affixes.

This external argument is going to be projected according to the usual layered predicate projection rules. For example, the sentence Jean a donné un livre/"John gave a book" will contain the substructure (159) with the external argument as [Spec, IP] ${ }^{*}$. The combination $\mathrm{V}+$ morphology raises from I*** to $I^{*}$. (See (159) on page 184.)

In conclusion, nothing of consequence is affected except for the possible presence of a lower VP projection. If this approach is correct, the lower VP now contains only the verb and its internal arguments. This raises a further question concerning the surface position of direct and other objects: the object could be either in the specifier or complement position of V. The simplest assumption takes it that positions within VP are only projected if they are thematic. This means that a verb with one object would have this object as its specifier and more generally that each object of a verb is projected as a specifier of a VP part of a VP shell. Consequently, the full structure of (159) will be as in (160) on page 184 . 
PARTITIONS AND ATOMS

(159)

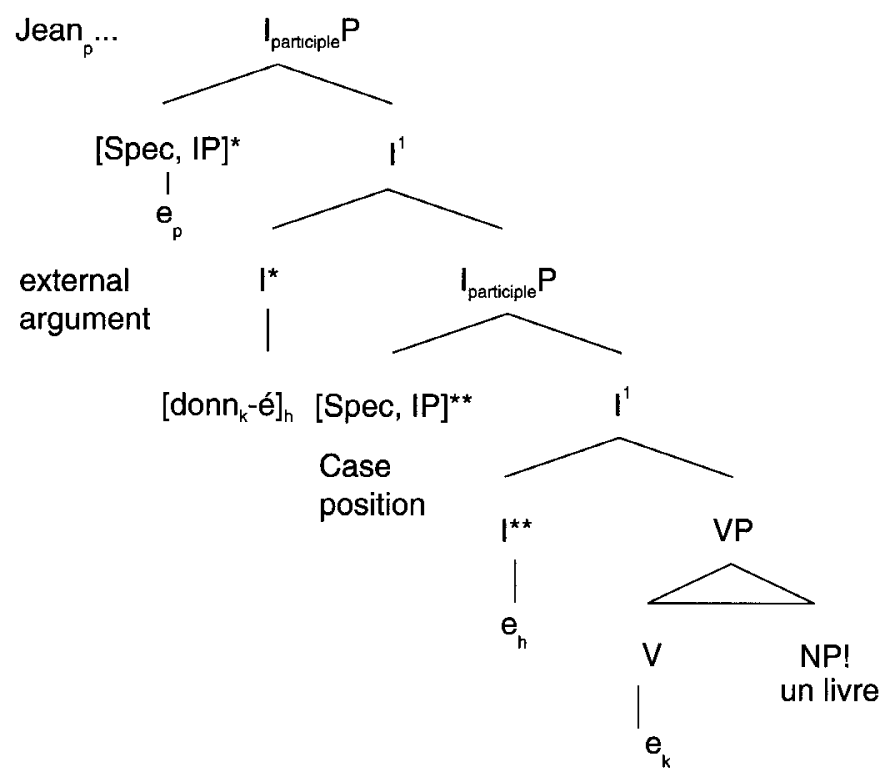

(160)

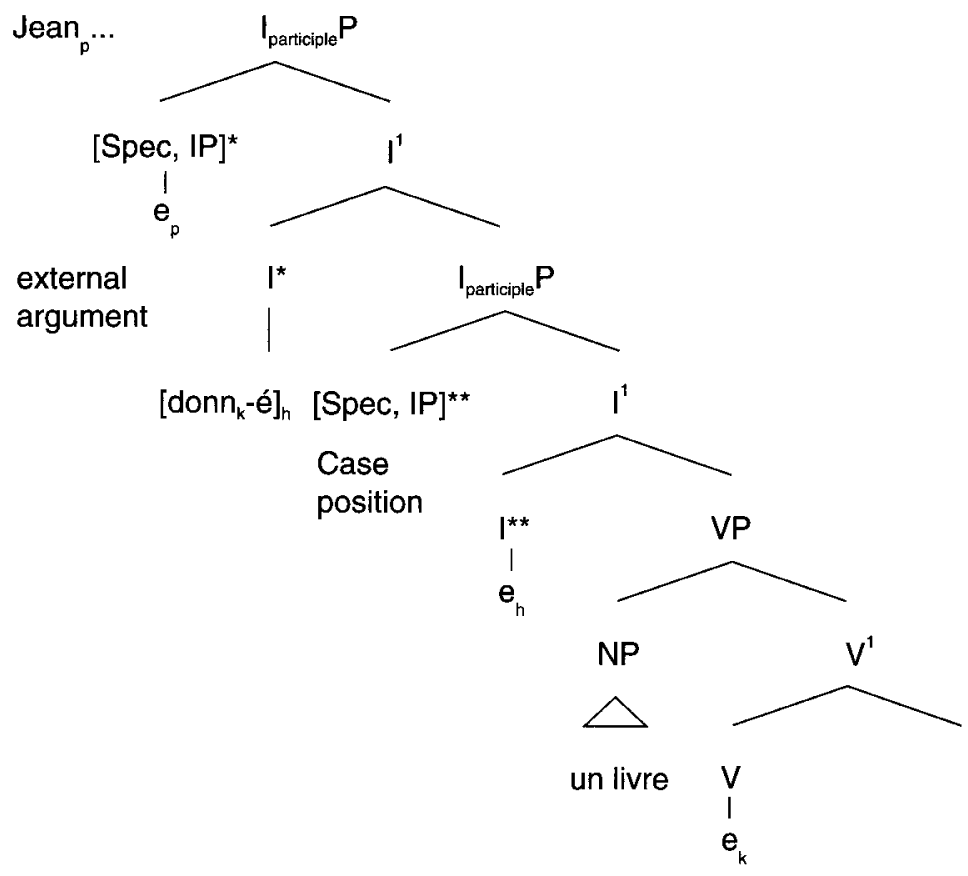


The question remains whether participial morphology affixation is a case of syntactic head movement. We may distinguish at least three types of verbal morphology represented by causative affixes, applicative affixes, passive participial affixes, past participle affixes or tense affixes.

Causatives and applicatives have a thematic structure of their own, and impose selectional restrictions on their complements, suggesting, as Baker (1988a) or Marantz (1990) propose, that they be considered affixal lexical categories projecting in syntax. Past participle affixes, verbal gerundive -ing, progressive -ing or tense affixes have aspectual or tense content but no thematic structure and impose selectional restrictions (they select a VP, and perhaps, as progressive -ing, subcategories of verbs). Tense is usually analyzed as a functional affixal head projecting in syntax. Finally passive participles affix only to verbs with external arguments as Jaeggli (1986) shows. If they project in syntax, this will be construed as a selectional property. Passive morphology selects a VP headed by a verb with an external argument.

Should all these affixes project to phrasal value in the syntax? Causatives and applicatives should as a consequence of the Theta Criterion and the Projection Principle. Tense should as shown by $d o$-support under VP preposing and other such processes where Tense shows up on auxiliary verbs rather than on main verbs. The treatment of the other is less clear. Verbal gerund -ing, past participles or progressive -ing also show up on whatever happens to follow them, a property suggesting syntactic affixation rather than lexical affixation. But they cannot appear on dummy auxiliaries like do under "VP" preposing:

(161)(a) John grew/grow, John did.

(b) John was growing/growing, John was/*grow, John was doing.

(c) John had grown/?*grown, John had/*grow, John had done.

(d) Tomatoes were grown $/ *$ grown, tomatoes were/*grow, tomatoes were done.

Lack of do-support with all these morphologies needs an explanation if do-support takes place simply to support stranded affixes. Passive morphology only appears on its verb and fails do-support as well. The first property comes from the fact that it is the most embedded of the verbal morphologies, but of course this last fact needs an explanation. In any case this means that passive morphology affixation could be done in the lexicon, as in Jaeggli (1986), or in the syntax as in Baker, Johnson and Roberts (1989) (cf. below Section 5.2.3.2 for further discussion).

The difference between participial-like affixes and Tense-like affixes overlaps with the distinction between Class $\mathrm{L}$ and other affixes we alluded to above. External argument is projected lower than the TP projection (this is the VP-Internal Subject Hypothesis) but possibly not lower than the participial 


\section{PARTITIONS AND ATOMS}

projection as discussed earlier. It might be identical depending on the properties of affixes with intermediate properties such as progressive -ing, which does not allow $d 0$-support, but leaves Case and theta properties unaffected.

\subsubsection{DERIVING BURZIO'S GENERALIZATION}

One way Burzio's Generalization can be formulated is:

A verb Case marks an NP it governs iff it externally thetamarks its subject.

This formulation appears too strong. First there are cases in which a verb does Case mark its object without assigning a theta role to its subject, for example, the impersonal construction or in cases like the verb falloir/ "to be necessary" as in Il faut une réponselil la faut/"an answer is necessary." When this happens, Case never seems to be assigned to an NP not thematically related to it. As Belletti (1988) suggests, this indicates that the Case assigned in these instances is inherent Case. Second, there are cases of verbs which assign an external role to their subject but assign no Case: all the intransitive verbs like dance. However, all these verbs can assign Case as is shown by the fact that they can all be used with cognate objects as in dance a dance. This suggests reformulating Burzio's Generalization as:

\section{Burzio's Generalization}

A verb can assign structural Case iff it externally thetamarks its subject.

If true, this generalization is a puzzle, because it links what appear to be two properties theoretically unrelated and not easy to relate. If external arguments are not generated inside VP (i.e. if the VP-Internal Subject Hypothesis is false), it links a non-lexical local property (Case assignment is a property of the $\mathrm{V}$ class, not of individual verbs) with a non-local lexical property (externally theta-marking the subject). The system of assumptions we have developed allows a possible account of this link.

Consider the VP of a morphologically simple verb (this can transpose easily to verb/affix combinations) illustrated in (164) opposite.

If there is an external argument, the projection $\mathrm{VP}^{*}$ exists and the verb raises from $\mathrm{V}^{* *}$ to $\mathrm{V}^{*}$. We have argued that in this case, the structural (accusative) position is NP**. If there is no external argument, the projection $\mathrm{V}^{*} / \mathrm{VP}^{*}$ does not exist at all. In this case, we want to say that $\mathrm{NP} * *$ is not a Case position. Burzio's Generalization follows if Case assignment is somehow related to verb raising from $\mathrm{V}^{* *}$ to $\mathrm{V}^{*}$.

Accusative is assigned to $\mathrm{NP} * *$ under a specifier head relation with NP**. However, because of $\mathrm{i}$-command, $\mathrm{V}^{* *}$ does not govern NP**. If the verb has 


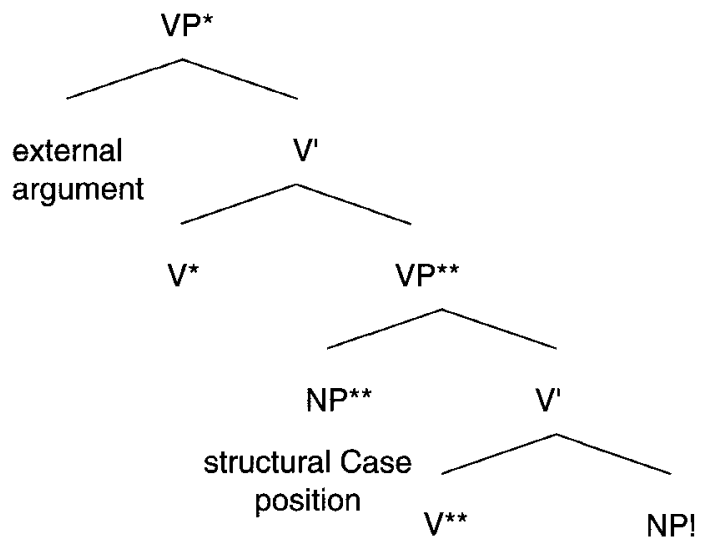

an external argument, $\mathrm{V}^{* *}$ will raised by substitution to $\mathrm{V}^{*}$. The effect of this raising is to make NP** governed by its agreement or structural Case assigner. Suppose we strengthen our view of structural Case by adopting:

(165) At LF, all Cases are governed.

(165) has no consequence for governed Cases (i.e. inherent Cases). However, it will require a structural Case position to be both in a Spec/head relation with its Case assigning head and governed by it. This is possible only if at LF, the Spec/head checking relationship is between the NP and the trace of the Case marking head, and the head has moved by substitution to a position where it governs the NP..$^{75}$ This is exactly what happens if $\mathrm{V}^{* *}$ moves into $\mathrm{V}^{*}$. If $\mathrm{V}^{*}$ was of a different category $\mathrm{H}$ to which $\mathrm{V}^{* *}$ adjoined, the result would be $[\mathrm{V}+\mathrm{H}]_{\mathrm{H}}: \mathrm{NP} * *$ would not be governed by its Case assigning head. In the case of English subjects, this means that I must substitute to $C$ at LF in order to license nominative Case. (Since $C$ is contentless, it can always be empty at LF, allowing substitution.)

Go back now to (164) and Burzio's Generalization. If a verb does not assign an external theta role to [Spec, $\mathrm{VP}^{*}$ ] in (164), the VP* projection is absent. $\mathrm{V} *$ is not there for $\mathrm{VP} * *$ to substitute into: (165) cannot be met if structural accusative cannot be assigned. Reciprocally, if structural accusative can be assigned, it means $\mathrm{V}^{* *}$ has a $\mathrm{V}^{*}$ to substitute into. But $\mathrm{V}^{*}$ exists (by the Projection Principle) only if there is an external theta role. If tenable and desirable, (165) entails Burzio's Generalization.

\subsection{Head movement, phrasal movement and agreement}

Consider again the structure of the VP of a sentence like Jean a donné un livre: 
PARTITIONS AND ATOMS

(159)

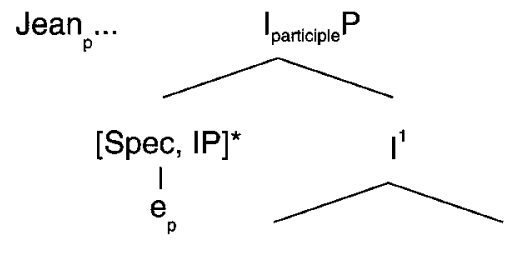

external

t

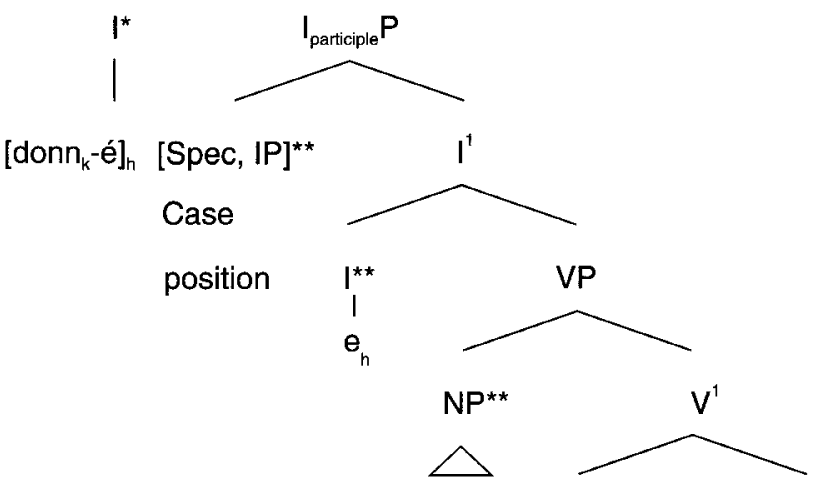

un livre $\quad \mathrm{V}$

l
$e_{k}$

Why does the participle not agree with [Spec, IP]* instead of/as well as [Spec, IP1**? This raises the general question of when agreement relations are established. Suppose that we have the following configuration with an $\mathrm{X}$ head able to manifest agreement:

(166)

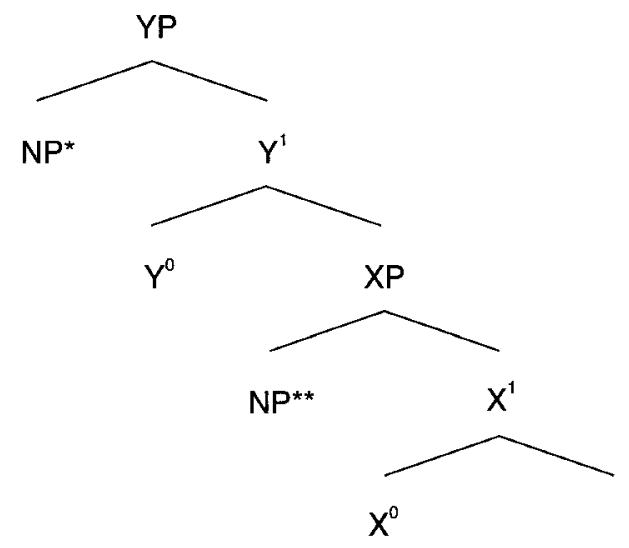


$\mathrm{NP**}$ can trigger agreement on $\mathrm{X}^{0}$ regardless of its derivational history. Obviously, it can if it has not moved at all. It also can if it raises to NP* (agreement with a trace) whether this movement is A or A-bar (cf. object agreement with NP-traces or subject agreement with $w b$-traces). It can also if it has been raised from some lower down position under NP Movement (subject agreement with derived subjects). Whether agreement with $\mathrm{X}^{0}$ can take place with $\mathrm{NP}^{* *}$ if $\mathrm{NP}^{* *}$ has moved to [Spec, XP] under A-bar movement is less clear. There do not seem to be overt examples in English or French.$^{76}$ More generally, there does not seem to be agreement between a head and its specifier if this specifier is an A-bar position.

We can ask the same questions about the head $\mathrm{X}^{0}$. Suppose it moves to $\mathrm{Y}^{0}$. Does it show agreement with NP**? Does it show agreement with NP*? Languages seem to differ here. We briefly examine French and English below, noting now that they seem to differ from languages with extensive agreement systems like the Bantu languages (cf. Kinyalolo (1990) on Kilega, the language of the Lega people of Zaire).

In English (or French), if $\mathrm{X}^{0}$ can agree with $\mathrm{NP}^{* *}$, it will then agree with $\mathrm{NP}^{* *}$ even if it is moved to $\mathrm{Y}^{0}$, and then, will not agree with NP*. There are two examples of this behavior. The first is the one we pointed out in connection with (159). The participle can agree with [Spec, IP]**. Therefore it will agree even if it raises to $I^{*}$ and it will fail to agree with [Spec, IP]*. A second example, identical in structure to the first one is illustrated by the following pair:

(167)(a) John is proud of the children.

(b) Which children is (*are) John proud of?

The verb be in (167) agrees with its specifier in the (a) sentence. In the (b) sentence, it raises to $\mathrm{C}$, failing to agree with $[\mathrm{Spec}, \mathrm{CP}]$ and instead continues agreeing with [Spec, IP]. Note that it is not sufficient to say that agreement is only with A-specifiers, as the case of the participle [Spec, IP]* in (159) shows. One can describe what is happening by stating that agreement takes place as soon as a complete word (apart from agreement) is formed in the course of the derivation. In the participle case, the word is formed after the $\mathrm{V}$ has raised to I**. It agrees exactly then, not before, not later (this is where Kilega differs for example, which seems to allow two agreement markers on the same head). The same is true in the case of (167). The word is formed only when it has raised to Tense. If it agrees, it agrees exactly then, neither prior to this raising, nor later. Perhaps one way to describe what happens here is that agreement must be external to any affix having semantic content.

This is most unclear. The observation that agreement, an inflectional property, only applies to "complete" words is reminiscent of the prohibition against interspersing inflectional and derivational affixes and in fact, this is the question that is raised here. Why do affixes occur where they do with 
respect to each other? Although it is possible to formulate an answer to this question, I know of none that is not essentially stipulative, building the answer into its framework without deriving it from deeper assumptions. I put in this category theories that are lexicalist to various degrees, e.g. some that put agreement in a different set than other affixes and in a different component as well (agreement in syntax and other relevant affixes in the lexicon). Although it is sometimes claimed that there are some empirical reasons to take syntax to operate on words (cf. Chomsky (1970)), these arguments are far from convincing and it remains to be shown what deeper reasons there are why the notion of a word (a non-obvious construct, whatever it may be) should be of any relevance to syntax.

For our purposes, we can stipulate some answer built on an analogy between agreement and Case. When movement of an NP takes place, say from some A-position to some A-bar position, the Case of this NP is determined by the highest $A$-position in the sequence of positions it occupies in the course of the derivation. Higher positions will all be A-bar positions. We have the observation:

The Case of an NP is determined by the highest Aposition of its chain.

Suppose that agreement for heads works the same way. Let us define the notion of $A$ and A-bar positions for heads. Meaning by complete word, a word stripped of its affixes devoid of semantic content, we can define:

(169)(a) A head position is an A-position if incomplete words may move to it.

(b) A head position is an A-bar position only if complete words may move to it.

We can now reformulate the above observation by saying that agreement on a head is determined by the highest A-position in the chain containing this head. Paralleling (168), we can state:

(170) Agreement on a head is determined by the highest Aposition of its chain.

Correctly, this will characterize the agreement relation as being established between $\mathrm{I}^{* *}$ and [Spec, IP]** in (159), or between $\mathrm{V}^{* *}$ and NP** in (164). This will also characterize properly tensed verb agreement as being between the tensed verb and the subject. 


\subsection{SSC effects of accusatives}

As it stands, Movement Theory predicts that an accusative object of $\mathrm{V}$ acts like a subject within its VP, i.e. it might produce SSC effects for NP Movement (although not for lexical anaphor binding):

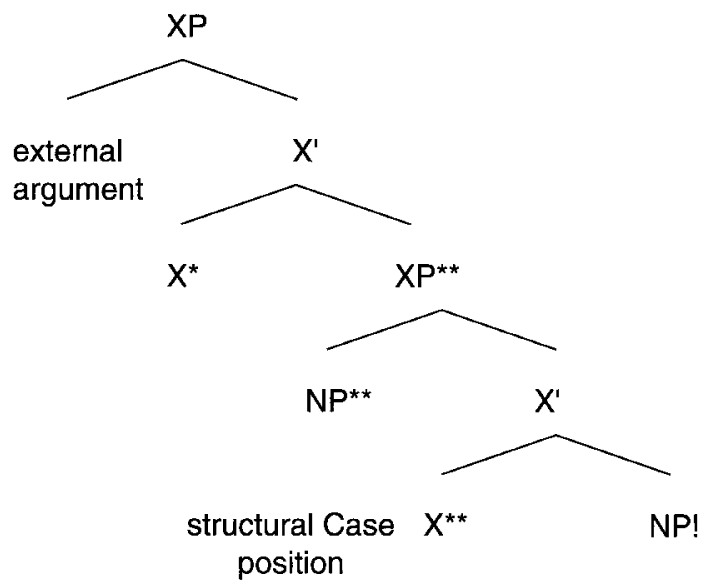

The accusative NP! makes the position NP** unavailable since it is in this position or is linked to this position for Case purposes. Since A-movement must be to the next specifier up, no A-movement is allowed out of XP**, i.e. over an accusative object. More precisely, it entails that no A-movement is allowed out of a VP containing an object triggering object agreement. A difference between the two formulations will arise if accusative can be inherent accusative rather than structural accusative (as has been suggested for English, cf. Section 5.4.1) ${ }^{77}$ This question has wide relevance. It might be relevant to the passivization possibilities of objects in double object constructions. It is relevant to the analysis of asymmetries between objects under object agreement and passivization in the Bantu languages as recently discussed in Baker (1988b), Marantz (1990) or Bresnan and Moshi (1990) for example. It is certainly relevant in Romance languages especially concerning the analysis of indirect objects as I will discuss in Section 5.4.3. As I will show, this prediction is too strong. There are cases of NP Movement over an accusative object. This means that barrierhood has to be relaxed. I begin by discussing some potentially problematic cases, then turn to violations of SSC.

\subsubsection{English double object constructions}

English double object constructions are possibly an example of accusative objects producing SSC effects. The relevant paradigm follows: 
(172)(a) John gave books to children.

(b) John gave children books.

(c) Children were given books.

(d)* Books were given children.

In the (a) sentence, the theme receives accusative and the goal dative. In the (b) sentence, the goal receives structural accusative, and the theme a morphologically bare Case, which Larson (1988) suggests is inherent accusative. The grammaticality of the (c) sentence reinforces this assumption since the goal can passivize. The ungrammaticality of the (d) sentence (apparently there is dialectal variation in certain dialects) might be taken to illustrate the SSC effect of the structural accusative goal. The problem of course is that the (d) sentence might be also excluded for Case reasons if Burzio's Generalization is correct, or if structural accusative is not assigned in the passive there would be no Case for the goal.

\subsubsection{Raising verbs}

Raising verbs should not be allowed to have direct objects as the raising of an NP over an intervening direct object should violate the SSC. However, English has verbs like strike or impress.

(173)(a) That John looked sick/This fact struck Bill.

(b) Bill was struck by this fact.

(c) It struck Bill that John looked sick.

(d) John struck Bill as sick.

(e)* Bill was struck as sick by John.

The $(\mathrm{a} / \mathrm{b})$ pair shows that strike may assign an external theta role. Otherwise the passive would be impossible. Nevertheless, it has been argued that in the (d) sentence, strike is a raising verb. This is based on the synonymy of the (c) and (d) examples, suggesting that John in the (d) example gets its theta role only from the embedded predicate sick. The usual correlates of raising are not systematically found. However, some idiom chunks may "raise" but there cannot (*there strikes as being good reasons to do this).

It is possible then that verbs like strike are not raising verbs in which case the SSC problem disappears. Alternatively, we could claim that there are two verbs strike. One would appear in the (c) and (d) sentences without external theta role, and one would appear in the (a) or (b) sentences with an external theta role. In this latter case, passivization would be possible because this (psych) verb has an external argument. In the former case, passivization would be impossible because this verb does not have an external argument. Suppose then that strike in (d) is a raising verb. If its object gets structural accusative, it violates Burzio's Generalization, and also violates the theory of NP 
Movement. This suggests that it is not getting structural accusative but rather inherent accusative. The fact that such verbs exist in English but not in French could then be linked to the non-existence of inherent accusative in French. This would correlate with the absence of double object constructions in French, as opposed to English, in which inherent accusative is used on the second object of double object constructions. For English, this assumption is hard to test. A language like English in all respects except for the existence of object agreement would make the difference. An inherently Case-marked object would not trigger agreement.

\subsubsection{The problem of indirect object reflexives}

\subsubsection{THE FRENCH CASE}

The most serious problem for the idea that accusative objects act as subjects of their VP for NP Movement comes from the interaction of two analyses: the analysis of object agreement and the analysis of reflexive clitics as extended to indirect object reflexives. The problem is illustrated by the following paradigm:

(174)(a) Jean a décrit les portes à Pierre. John described the doors to Peter.

(b) Jean n'a décrit les portes qu'à lui-même. John described the doors only to himself.

(c) Jean ne les a décrit(ES) qu'à lui-même. John only described them to himself.

(d) Marie s'est décrit $\left({ }^{*} \mathrm{E}\right)$ les chapeaux. Mary described the hats to herself.

(e) Jean se les est décrit(ES). John described them to himself.

The relevant facts are as follows. The (d) sentence shows that an indirect object reflexive in French does not trigger object agreement. Rather, as the (e) sentence shows, agreement, if present, is optional (in my dialect) and is triggered by the direct object. We see first that this paradigm contradicts the traditional description of agreement since we have a case of a construction using auxiliary be without obligatory agreement with the subject.

Recall the analysis we presented of reflexives. We showed that reflexives were best analyzed as external arguments, implying that the superficial subject is in fact an underlying internal argument. Applied to the (d) sentence, this means that se stands for the external argument in a sense to make precise, and that Marie is the underlying goal. This goal has raised to [Spec, IP], an A-position, triggering subject/verb agreement and therefore must have raised Spec to Spec exclusively through A-positions. ${ }^{78}$ Two problems arise. Consider the structure of the (d) or (e) sentences: 
PARTITIONS AND ATOMS

(175)

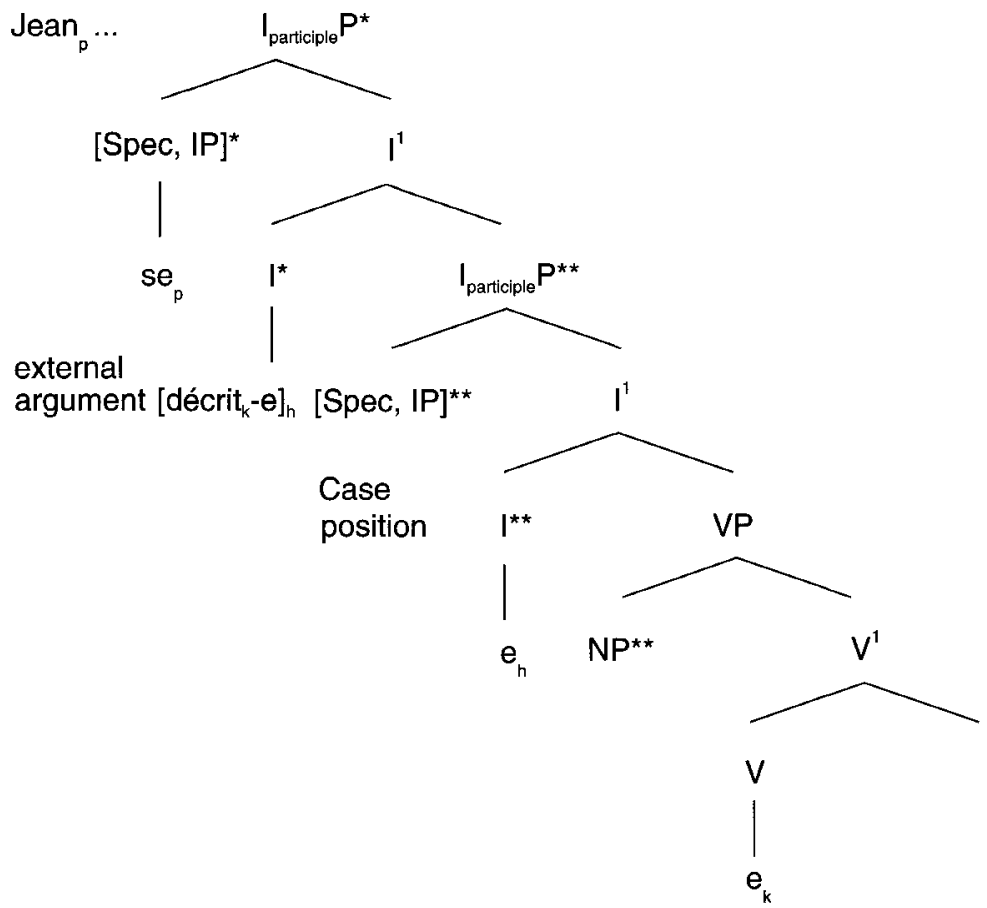

Both the goal and the theme originate within VP. Why does the goal not obligatorily trigger object agreement on the participle in the (d) sentence? Second, why is the direct object able to trigger agreement on this past participle? This last property of the direct object must be interpreted as meaning that the direct object can move to the accusative position, i.e. to [Spec, IP]**, a specifier that is higher than the base position of the goal NP. This means the goal NP is able to skip, and in fact must skip that specifier position. Worse, if the theme must move to [Spec, IP] ${ }^{* *}$ at S-Structure or at LF, this means neither $[\mathrm{Spec}, \mathrm{IP}] * *$ nor $[\mathrm{Spec}, \mathrm{IP}] *$ is available for the goal to move through, as these positions are needed for the theme and the external argument respectively. This means that the notion of barrierhood we operate under is too restrictive and should be amended.

Here then are the three problems we need to address:

1 The goal is able to skip the intervening specifier [Spec, IP]**.

2 The goal must skip the intervening [Spec, IP]**.

3 The goal must be able to skip [Spec, IP]*.

Problem (2) is solved by the fact that [Spec, IP]** is, by definition, the accusative Case position. This makes [Spec, IP]** unavailable for nonaccusative NPs to move to (whether there is a structural accusative direct object or not). Problems (1) and (3) are problems of locality of movement. 
So far, we have not said what the structural consequence of treating se as an external argument was. The question is whether or not there is a syntactic position in which se is generated or not. Notice that since the direct object in the (e) sentence is able to trigger agreement, it means it receives structural accusative. If our treatment of Burzio's Generalization in Section 5.2.3.2 is correct, this means that the main verb should have an external argument. For this reason, I want to suggest that reflexive se is in fact an affixal noun in the same way object clitics are, except that it occurs in the external argument slot of its predicate. This is consistent with the fact that reflexive se appears in Infl as do object clitics. As a consequence, the projection IP* exists and its specifier is occupied by se, so that movement of the indirect object to subject position must skip this specifier as well. Naturally, now direct object reflexives are reanalyzed too as involving movement of a direct object skipping over a se external argument.

\subsubsection{BARRIERHOOD REVISED}

In order to accommodate the facts above, barrierhood needs to be made more permissive. Recall the definition of barrierhood we have:

\section{Barrierbood}

Given $\underline{B}$ some constituent, and $\mathrm{Y}$ some category with $\underline{B}$ $\neq Y^{\mathrm{n}}$ : if for some $\mathrm{p}$, YP is not an $L$-dependent position and includes $\underline{B}$, then $\mathrm{YP}$ is a barrier for $\mathrm{B}$.

Consider the structure (175). We want to allow some NP originating in VP to move directly to a position outside IP*. The idea is to remove the barrier status of the projection XP of some head X just in case X has moved to some higher head position in a way to make it more precise. In the kind of Case we are interested in, the $\mathrm{V}$ originating in VP will raise first to $\mathrm{I}^{*}$ then to I**. Suppose then that we revise barrierhood so that neither VP nor IP** count as barrier but only IP*, as a result of the movement of $\mathrm{V}$ to I*.

\section{Barrierbood}

Given $\underline{B}$ some constituent, and $Y$ some category with $\underline{B}$ $\neq Y^{\mathrm{n}}$ : if for some $\mathrm{p}, \mathrm{YP}$ is not an $L$-dependent position and includes $\underline{B}$, then $\operatorname{clos}(\mathrm{Y})^{\max }$ is a barrier for $\mathrm{B}$.

where we define $\operatorname{clos}(\mathrm{Y})$ (closure of $\mathrm{Y})$ as:

$$
\mathrm{Clos}(\mathrm{Y}) \text { is the highest head position in the chain containing } \mathrm{Y} \text {. }
$$

$\operatorname{Clos}(\mathrm{Y})^{\max }$ is the maximal projection of $\operatorname{clos}(\mathrm{Y})$. Applied to (175), $\mathrm{Y}=\mathrm{V}$, $\operatorname{clos}(\mathrm{Y})$ is $\mathrm{I}^{*}$ and $\operatorname{clos}(\mathrm{Y})^{\max }$ is IP*.79 These modifications now permit direct 
NP Movement from within VP to [Spec, IP]*. This solves problem (1), but it is not sufficient to solve problem (3), for movement of the goal from within VP to outside IP*.

To solve problem (3), we should take the presence of the coindexed se to be crucial because, in general, the external argument blocks movement of any lower argument around it (this is the essence of the SSC). One way to achieve this result is to take this se coindexed with the raised goal to fulfill the antecedent government requirement of the trace of the goal. If se is not a member of the chain containing the goal, some NPs not members of a chain can qualify as antecedent governor for a trace in this chain. We would again face the problems raised by Lasnik's examples discussed in Section 3.5.3 unless we adopt the second alternative mentioned in Section 3.5.3 concerning the treatment of Lasnik's example. This alternative requires an antecedent governor intruding in a chain to be itself somehow antecedent governed. If we pursue this line, in order to avoid Lasnik's problems, we should state that CCL requires an antecedent governor intruding in a chain to be itself antecedent governed by a member of this chain.

Alternatively, reflexive se is a member of the chain containing the goal (or the theme, in the case of direct object reflexives) and we can adopt the first of the alternative construals mentioned in Section 3.5.3, namely that the CCL must hold chain internally. How can we reconcile this last assumption with our earlier contention that reflexive se is an argument absorbing the external role? This is discussed in Section 5.4.3.3.

There is one immediate advantage of this proposal. We do not need to stipulate the anaphoric character of reflexive se. Recall that we proposed in Section 4.3 .3 that reflexive se is simply a pronominal. If some object raises to subject the morpheme se will have to be coindexed with it to allow raising, so it has to be interpreted as anaphoric. If nothing raises to subject in overt syntax, the subject position contains an expletive and raising of some NP will have to take place at LF. The morpheme will also have to be coindexed with this NP to permit LF raising. In other words, coindexation of se with the raised (direct or indirect) object is necessary to allow the raising itself. Given that se "stands for" the external argument, coindexation is understood as referential dependency. The anaphoric relation follows.

There is also one immediate problem with this proposal. In the case of NP Movement it will preserve the treatment of agreement we have proposed in almost all cases since the agreement position is always going to be the highest position available within the projection containing the participle - call it $\mathrm{I}_{\mathrm{p}} \mathrm{P}$. If there is no external argument, the highest specifier position in I $\mathrm{P}$ is the agreement position. If there is an external argument, the only relevant cases of NP Movement are cases of movement over a coindexed se. In such cases, a problem arises. Precisely what allows lack of agreement in NP Movement of the indirect object over a se should allow lack of agreement in NP Movement of a direct object over a se. However agreement is obligatory in such cases: 
Marie s'est décrit* $(e)$ à Pierre. We can differentiate the two cases by noting that the agreement position is unavailable in the indirect object movement Case (either because there is a direct object absorbing accusative, or because accusative is an elsewhere (ase), but is available in the direct object movement Case. In order to account for the obligatoriness of agreement with object reflexives (and middles, inherent), we must require that movement be as short as possible. We can view this requirement as part of a broader requirement subsuming Chomsky's (1989) Principle of Least effort and reading:

\section{(178) Principle of Least Action (PLA)}

Movement only if necessary and if necessary as short as possible.

One consequence of the PLA is that we now may indeed derive the idea expressed in (131) that accusative (and in fact nominative) is an elsewhere Case. A DP that gets inherent Case gets it without movement. Since both accusative and nominative Case assignment involve movement, accusative or nominative will be used only as a last resort, i.e. as an elsewhere Case.

Note finally that the PLA is not redundant with the CCL. The PLA only imposes relative locality requirements - given several movement options, choose the shortest. The CCL imposes absolute locality requirements. It states that movement to a position lying outside a certain local domain is impossible, even if this position is the only landing site available.

5.4.3.3 THE NATURE OF $S E$ : REFLEXIVES, MIDDLES, NEUTRALS

AND INHERENT REVISED

The previous account of reflexive se raises new questions for the treatment of middle and other se. French se agrees in person and number with the subject of its clause, suggesting, on a par with our analysis of reflexives, that syntactic coindexation of some kind is involved and required:

(179) (a) Ces pommes se mangent/se sont mangées pendant longtemps (middle).

One eats these apples/ate these apples for a long time.

(b) Vous vous réunissez/vous êtes réunis (neutral). You gather/gathered.

(c) Tu t'évanouis/t'es évanoui (inherent). You lose consciousness/lost consciousness.

Given that agreement holds in specifier/head configurations and that se behaves as a head, this indicates that there should be a way to have the superficial subject and se in a specifier/head relationship in the course of the 
derivation to S-Structure. Consequently, I suggest that se is generated heading its own projection and that the NP it agrees with transits through its specifier $\mathrm{NP}^{\wedge}$ on its way to the subject position as follows (simplifying somewhat):

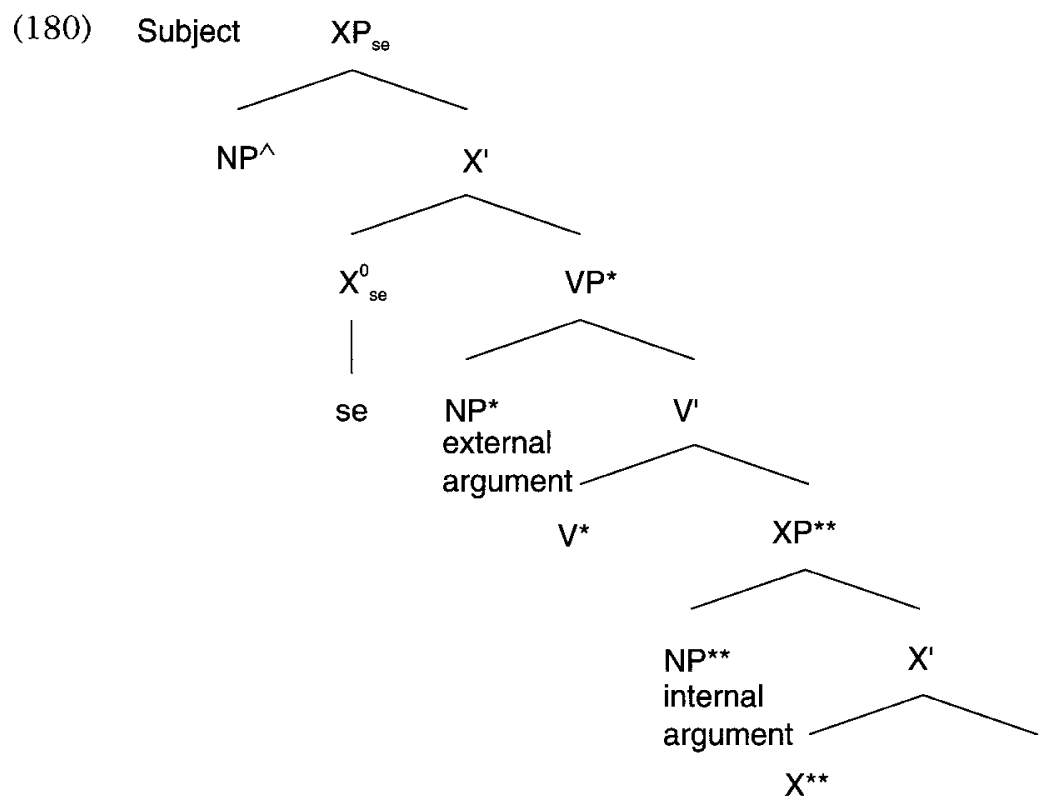

To preserve the analysis of "se as external argument," we replace the proposal that $s e$ is an external argument with the property that se selects as complement to a VP with an external argument slot. It might be thought that the second proposal is inferior to the first one but this would be incorrect. They are both descriptively adequate and explanatorily equivalent as long as we do not have an account of why se is limited to external argumenthood. If we could derive this limitation, the first proposal would clearly be superior.

The various constructions now differ as follows. In the neutral constructions, the external theta role is suppressed (a lexically governed option) and instead an expletive is projected in the external argument slot NP* (otherwise, we could not prevent unaccusatives from participating in this construction). The same thing happens in the inherent constructions. The inherent construction is a case of neutral where the causative counterpart never surfaces. In the middle construction, NP* is projected as an argument receiving the external theta role.

In the reflexive construction, NP* is also projected as an argument receiving the external theta role but is also coindexed with some internal 
argument. In this view, $\mathrm{NP}^{\wedge}$, the specifier of a functional category, is an expletive, and thus must be eliminated by LF. In all cases of movement to subject, i.e. to an A-position higher than $\mathrm{NP}^{\wedge}$, movement will have to transit through $\mathrm{NP}^{\wedge}$. This is how we derive the agreement of se with the superficial subject.

In the neutral and inherent constructions, both $\mathrm{NP}^{\wedge}$ and $\mathrm{NP}^{*}$ are expletives and must be eliminated. Overt raising of an internal argument, proceeding with steps as short as possible in accordance with the LAP (178) and compatible with the CCL will raise successively through $\mathrm{NP}^{*}$ and $\mathrm{NP}^{\wedge}$ to the subject position.

In the middle construction, $\mathrm{NP}^{*}$ is a thematic position. The result we want is for some internal argument, say $\mathrm{NP}^{* *}$, to be able to raise to $\mathrm{NP}^{\wedge}$ and beyond, skipping NP*, and to prevent NP* from raising to $\mathrm{NP}^{\wedge}$ (otherwise we lose the account of subject-se agreement). To achieve this, we must assume that VP* is not a barrier for the movement of NP**. Let us take this to be one defining property of se; its complement is never a barrier. The desired derivation follows. If we were trying to raise $\mathrm{NP}^{*}$ to $\mathrm{NP}^{\wedge}, \mathrm{NP}^{* *}$ will no longer be able to reach the subject position without violating the CCL. We would get an impersonal middle construction (il se mange beaucoup de pommes/"one eats many apples"). Left open here is how the subject expletive is eliminated. The simplest answer is by further raising $\mathrm{NP}^{*}$ from $\mathrm{NP}^{\wedge}$ to subject at LF. Since NP* is a pronominal anaphor, i.e. a PRO, this derivation might be impossible. This means that NP** must raise to subject instead. This will be possible only if NP* never raises to $\mathrm{NP}^{\wedge}$ but NP** does instead (as discussed below), but at LF. If $\mathrm{NP}^{*}$ does not raise to $\mathrm{NP}^{\wedge}, \mathrm{NP}^{* *}$ can raise directly to $\mathrm{NP}^{\wedge}$ (and beyond), eliminating the expletive $\mathrm{NP}^{\wedge}$, triggering agreement on se and raising further up to subject position. This derivation gives the (personal) middle.

Turning now to reflexives, NP* is by definition an argument and anaphoric. It will have to be coindexed with some object (say NP**). Since the derivations invoked in the previous case are also available in this case, we can examine them. NP* needs a local c-commanding antecedent. We will get proper binding of the anaphor only if NP** raises to subject. This excludes the first derivation $\left(\mathrm{NP}^{*}\right.$ to $\mathrm{NP}^{\wedge}$ ) and only permits the second derivation (NP** to $\mathrm{NP}^{\wedge}$ to subject overtly or covertly). The coindexing will follow. Note that we can now keep to the strongest form of the CCL (as described in Section 3.5.3), requiring the CCL to be satisfied strictly chain-internally. This assumption excludes other possible movement derivations (e.g. NP* to $\mathrm{NP}^{\wedge}$ together with NP** to subject).

In sum, we have concluded that:

1 The CCL should hold only chain-internally.

2 Se selects VPs with external argument slots.

3 Se removes the barrier status of its VP complement. 
From these assumptions, we have derived the agreement properties and movement patterns found in se constructions.

\subsubsection{BARRIERHOOD FURTHER REVISED AND SOME \\ CONSEQUENCES}

We derive the fact that se must always agree with its S-Structure subject. This subject is always a derived subject. Coindexation of se with this subject is necessary to allow the raising to subject position.

Consider the following sentence:

Nous nous regardions.

We were looking at ourselves.

In such a sentence, the verb raises to $T$ to get Tense as Emonds (1976) shows. The way barrierhood is now defined will permit some internal argument to raise directly to [Spec, TP] since $\operatorname{clos}(\mathrm{V})=\mathrm{T}$. In particular, a direct object should be able to raise over a se without transiting through its specifier. If we want to preserve the account given, we must prevent this derivation. In order to prevent it, it is necessary to limit the kind of head movement that is taken into account in the definition of $\operatorname{clos}(\mathrm{Y})$. Head movement to participial morphology extends the minimal barrierhood domain but head movement to $T$ does not. This distinction recalls the distinction previously introduced in Section 5.2.3.1 between affixes of Class I and others. Concretely, barrierhood is canceled by head movement only once, namely by moving a head to the next head up. I modify the definition of $\operatorname{clos}(\mathrm{Y})$ and replace it by:

$$
\mathrm{Clos}(\mathrm{Y}) \text { is the highest head lexically selecting } \mathrm{Y} \text { in } \mathrm{Y} \text { 's }
$$
chain.

Consequently, participial morphology will be within $\operatorname{clos}(\mathrm{V})$ but Tense, Comp or progressive -ing will not.

It should be clear that the relaxation of barrierhood resulting from (176) and (182) does not affect $W b$ Movement possibilities in VPs. Of course, it does affect NP Movement possibilities, by making it less free. Possibilities of head movement are radically altered however, since barrierhood is now defined in terms of the notion "closure", itself derived from head movement. Under (177), head movement became completely unconstrained by barrierhood and some other principle had to be responsible for Head Movement Constraint. Under (182), HMC remains derived from barrier theory except for the case of a head moving to another selecting it, even if this movement skips over some intermediate head. I think this consequence is actually highly desirable. It is at the root of the properties of restructuring constructions and reanalysis constructions. Furthermore it will allow 
incorporation of an $\mathrm{N}$ in a $\mathrm{V}$ that selects it even if intermediate projections such as $\mathrm{D} / \mathrm{DP}, \mathrm{Q} / \mathrm{QP}$ intervene. ${ }^{80}$

\subsubsection{SE CONSTRUCTIONS AND CASE}

Se is not an NP and thus needs no Case for visibility purposes. Because we derive Burzio's Generalization from verb raising, we might expect accusative Case to always be available in se constructions. As the structure (180) indicates, $\mathrm{X}^{* *}$ raises to $\mathrm{V}^{*}$, allowing accusative Case assignment. However, the same reasoning applies to passive constructions. It is thus possible that, even though accusative is assigned, it is unavailable because it is redirected (e.g. to NP*), as has been argued to happen in passives. ${ }^{81}$ Consider first the reflexive construction (reflexive interpretation intended throughout):

(183)(a) Beaucoup de gens se rasent. Many people shave themselves.

(b) Il se rase beaucoup de gens. It se-shave many people.

(c)* Il s'en rase beaucoup. It se-of-them shave many.

(d) Beaucoup de gens s'offrent des fleurs. Many people give themselves flowers.

(e)* Il s'offre des fleurs à beaucoup de gens. It se-give flowers to many people.

(f)* Il s'offre des fleurs beaucoup de gens. It se-give flowers many people.

In the direct object reflexive (183a), the accusative should be available, given the possibility of $(183 \mathrm{~d})$ with an overt direct object. This means that the postverbal NP in (183b) could be accusative, if it is in object position. The (b) sentence is acceptable with a reflexive reading, but the reflexive reading disappears in the (c) sentence with en cliticization. Since the possibility of en placement is a diagnostic property of direct objecthood, I interpret this as meaning that a postverbal NP in object position does not allow the reflexive reading. I ascribe the possibility of the reflexive reading in the (b) sentence to its structural ambiguity. The postverbal NP can also be an inverted subject as found in what I have called the impersonal intransitive construction illustrated by:

(184)(a) Il a dansé trop de gens ici. It has danced too many people here.

(b)* Il en a trop dansé ici, de gens.

It of-them has too much/many danced, of people.

(c)* Il en a dansé trop ici, de gens. 
It of-them has danced too many, of people.

(d)* Il a dansé des polkas trop de gens ici.

It has danced polkas too many people here.

This last construction does not allow en placement (184c), does allow the presence of a direct object (184d) and does not tolerate a preparticipial quantity adverb binding into the postverbal NP (184b). ${ }^{82}$ All this suggests that the postverbal NP is not an object. Expletive replacement at LF will force the postverbal NP to raise to subject position. If the external role is anaphoric, the reflexive reading arises, otherwise, we get an impersonal middle construction. ${ }^{83}$

We have seen in Section 5.4.3.3 that raising of $\mathrm{NP}^{* *}$ to subject position overtly or covertly should be able to provide an antecedent for an anaphoric external argument. What then blocks the reflexive interpretation when the postverbal NP is in object position as in (183c)? In (183c) the clitic se or its base position commands the object position. Coindexation then yields a Principle $\mathrm{C}$ violation if this principle is held at S-Structure, an assumption that seems reasonable. ${ }^{84}$ This reasoning does not extend to the impersonal intransitive construction. The impossibility of en placement in (184) shows that binding of the postverbal NP from the clitic position is not possible.

Returning to the question of Case, I will suppose on the basis of (183d) that accusative is in principle available in reflexive constructions. In (183a) optional Case assignment of accusative has not taken place, and the object has raised to subject, receiving nominative. In (183b), the accusative option is not exercised either (recall that under the relevant reading, the postverbal NP is not an object but an inverted subject). The postverbal NP gets nominative Case, and raises at LF to subject position, where nominative is checked. The situation of $(183 \mathrm{~d}, \mathrm{e}, \mathrm{f})$ is similar, except for the fact that the indirect object and inherent dative are involved, instead of the direct object and accusative. In the first one, the indirect object fails to receive inherent Case. Instead, it gets nominative checked by being moved to subject position overtly. The counterpart of $(183 \mathrm{~b})$ is (183f), without the $a$, which is ill formed like (184d) for unclear but general reasons (preventing subject inversion in the presence of a direct object). Finally, (183e) is ill formed for reasons similar to those ruling out (183c). Since the reflexivized argument is marked dative, it is an indirect object and thus in the c-command domain of the coindexed external argument, which leads to a Principle $C$ violation. Notice that because of the elsewhere character of accusative, indirect reflexive constructions cannot display participle agreement even in the absence of a direct object.

As we have seen (even, tentatively for inherent se constructions - cf. footnote 54) we have concluded that se can only occur on verbs which have an external argument and it expresses or absorbs this external argument. Reflexive se differs from the others in a couple of ways. First the reflexive se does not need a verb taking an internal argument realized as accusative in the 
absence of se. Unlike reflexive se, however, middle, neutral or inherent se needs to be affixed to a verb otherwise taking an accusative internal argument.

Second, reflexive se does not need to eliminate the normal expression of this argument, while middle, neutral and inherent se do. Thus they do not cooccur with an accusative direct object (assuming that the postverbal NP in the middle impersonal (185b) is not accusative), or enter in a construction with only an indirect object or a small clause subject assigned accusative. Thus middles, neutral and inherent se constructions differ from passives and reflexives. Passivization seems to involve structural accusative objects, regardless of thematic properties. ${ }^{85}$ Reflexivization can affect accusatives or dative NPs again regardless of thematic relations. Middle, neutral and inherent se constructions however only involve direct objects:

(185)(a) Ces gens se sont vendus./Il s'est vendu beaucoup de gens. These people were sold./Many people were sold.

These people sold themselves. $/$ Many people sold themselves.

(b) Ces gens se parlent./Il se parle beaucoup de gens.

*One speaks to these people./*One speaks to many people. These people speak to themselves./These people speak to themselves. ${ }^{86}$

(c) Ces gens se considèrent malades./* Il se considère beaucoup de gens malades.

*One considers these people sick./*One considers many people sick.

These people consider themselves sick./*These people consider themselves sick. ${ }^{87}$

(d) Je me décris facilement. ${ }^{88}$

One describes me easily.

I describe myself easily.

Apparent lack of co-occurrence with accusatives might suggest, given Burzio's Generalization, that these se are not projected as external arguments, a fact certainly consistent with the lack of external argument for neutral or inherent se constructions. But this is not consistent with the presence of the external argument of middles. This suggests instead that these constructions should be assimilated to passive constructions where accusative Case becomes unavailable because it is redirected to the external argument (cf. Baker, Johnson and Roberts (1989)). Furthermore inherent, neutral or middle se and reflexive se have the same surface distribution as reflexives and other clitics. It is external to all other clitics and internal to negation:

(186)(a) Jean ne se l'est pas donné (reflexive). John NEG-REFL-OBJ-be NEG given. 
John did not give it to himself.

(b) Jean ne s'y est pas évanoui (inherent).

John did not pass out there.

(c) L'eau ne s'y est pas renversée (neutral).

The water did not spill there.

(d) Les pommes ne s'y mettent pas facilement (middle).

One does not put apples there.

All the clitics appear adjoined to some functional category away from the verb, which suggests that they are positioned by syntactic processes. ${ }^{89}$

The ill-formedness of these constructions with indirect object or small clause subjects suggests that accusative per se is not at issue here. Now there are poorly understood semantic constraints on middle formation, involving interpretive properties of some internal argument. It is possible that the NPs with the right thematic relations to verbs (e.g. affected) must be realized as direct objects so that only direct objects can raise to subject in middle constructions. These constraints will prevent subjects of small clauses or indirect objects from raising to subject in middle constructions and perhaps prevent impersonal middle constructions lacking direct objects as well. If this last approach is correct, a postverbal $\mathrm{NP}$ in an impersonal middle construction such as (185a) could in principle be accusative. Our theory of participle agreement then predicts that participle agreement should be possible with a postverbal $\mathrm{NP}$ in an impersonal middle construction, contrasting with impersonal passive or unaccusative constructions. The facts are not as clear as one would wish:

(187)(a) Il a été construit des maisons récemment.

It has been built houses recently.

(b) Il s'est construit des maisons récemment. It se was built houses recently.

(c) ${ }^{* ?}$ Il en a été construites, des maisons, récemment. It some has been built-agr, houses, recently.

$(d)^{*}$ ? Il s'en est construites, des maisons, récemment. It se some was built-agr, houses, recently.

Both (187c) and (187d) strike me as unacceptable. ${ }^{90}$ This shows that the postverbal NP in $(187 \mathrm{a}, \mathrm{b})$ is not a structural accusative object, but rather nominative, in keeping with our treatment of impersonals.

Suppose then that the postverbal NP in middle and inherent impersonal constructions is not structural accusative. Even though accusative is possible in principle, it cannot surface. If there is only one internal argument NP, this NP must be nominative, because of overt movement or LF expletive replacement. Suppose there are two NP internal arguments, a direct object and an indirect object. Because indirect objects cannot be "middleized," 
expletive replacement will have to involve the direct object. This means it cannot be accusative. ${ }^{91}$

\subsection{Agreement with quantitativelpartitive en}

I begin with a note about the judgments on participle agreement in French. Participle agreement with accusative objects when they precede is a rule that is enforced by norm in school mostly for spelling reasons. The rule teaches that such agreement is obligatory. Most people's judgments disagree with the norm. If the preposed object is not a clitic (e.g. a wh-phrase), most people (I have interviewed) will agree that agreement is optional. Further, some will have obligatory agreement with accusative object clitics. My judgments (and many other speakers') is that agreement is optional even with accusative clitics. For most of these judgments, they are actually duplicated in neighboring Romance languages such as Catalan or Italian.

Judgments on agreement with (non-genitive) en vary more. Consulting French grammars such as Grevisse (1980) on this topic shows that the normative rule simply collapses in this case. I therefore take these judgments as highly significant as they reflect inner resources of the grammar. In everything that follows, the judgments are mine. Throughout this section, the diacritics indicate contrastive rather than absolute judgments on agreement facts.

\subsubsection{Basic facts}

So far, we have only discussed genitive en, a pronominal standing for a complement of a noun. Quantitative/partitive en appears superficially as complement of a bare Q. In Milner's (1978) terminology, Quantitative en is illustrated by the $(\mathrm{a} / \mathrm{b})$ pair (with the $\mathrm{Q}$ either followed by de or not, depending on the $\mathrm{Q}$ ), partitive $e n$ by the (c/d) pair, the difference between the two being the presence of the determiner in the partitive:

(188)(a) Il a vu beaucoup de photos./Il a vu trois photos. He saw many pictures./He saw three pictures.

(b) Il en a vu beaucoup, de photos./Il en a vu trois, de photos. He of-them saw many, pictures./He saw three of them.

(c) Il a vu beaucoup des photos. He saw many of the pictures.

(d) Il en a vu beaucoup, des photos. He of-them saw many, of the pictures.

Milner (1978) analyzes quantitative $e n$ as an N', and partitive $e n$ as an NP. Haik (1982) analyzes both $e n$ as the head of the NP object, as opposed to genitive $e n$ which stands for an NP. Clearly, if Milner is right for quantitative or Haik is, we might face a potential problem. Cliticization crucially involves 
an intermediate adjunction to VP of $e n$ that would not be available, if we were dealing with head movement or N' movement from the start. The alternative in the case of head movement would be to invoke successive adjunctions to the verbs (first to voir, then to avoir), which we showed earlier in Section 3.2.4 is ruled out. Now, it is clear that $e n$ is a head, since it is a clitic, but this does not imply that it must move as a head. Rather, a larger XP projection including the head can move first, followed by cliticization of en later in the derivation, exactly as in the case of accusative clitics discussed previously. Taking into account the DP hypothesis of Abney (1987), which we have not done so far, allows resolving the conflict between the two proposals and keeping the insight of each. With Haik, we must take $e n$ as a head (since it is a clitic), namely head of DP in all cases, with the advantage of a unified analysis for all ens (including genitive $e n$ ). With Milner, we take the object of beaucoup to differ depending on whether or not a definite article is present. An article is present in the partitive construction, but missing in the quantitative construction. This yields the following (simplified) structures: ${ }^{22}$

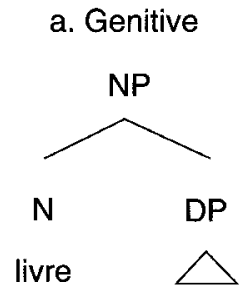

(de) mon père
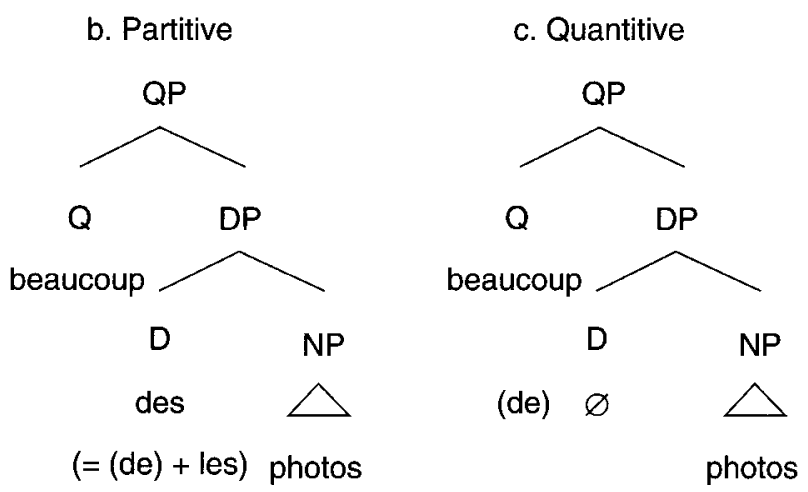

We can then take $e n$ to be a $\mathrm{D}$ (like other pronouns and clitics) agreeing with an NP. This gives a unitary analysis of $e n$ as (a) the head of a DP Case-marked genitive. $E n$ is a form incorporating the particle de and the D, i.e. partitive $e n=\mathrm{D}_{\text {definite }}+d e$, quantitative $e n=\mathrm{D}_{\text {null }}+d e$, genitive $e n=\mathrm{D}_{\text {pronominal }}+d e$. We give one additional reason to support the idea that quantitative (and partitive and genitive) en should also be treated as the head of an XP, i.e. as [Q $\left[_{\mathrm{XP}}\right.$ en ]]. Movement of quantitative en is sensitive to the presence of material in an intervening specifier exactly in the same way genitive $e n$ is:

(190) (a) Il a vu les trois premières photos. He saw the first three pictures. 
(b) Il en a vu les trois premières, de photos. He of-them-saw the first three, pictures.

(c) Il a vu mes trois premières photos. He saw my first three pictures.

(d)* Il en a vu mes trois premières, de photos. He of-them-saw my first three, pictures.

In the DP terminology, if the [Spec, DP] position of the higher NP is filled, here by a possessor, extraction of quantitative en is blocked, suggesting movement of en through this [Spec, DP], i.e. that this $e n$ is an XP. Note that head movement is not sensitive to the presence of material in specifier position. Infl to $C$ in English can take place in the presence of a subject in [Spec, IP].

(191) Will $\left[_{\mathrm{IP}}\right.$ John $\left[_{\mathrm{T}}, \mathrm{t}_{\mathrm{i}}[\right.$ like it $\left.\left.]\right]\right]$.

I conclude that in all cases, en stands for an XP and that it is the XP that moves out of the direct object, followed by incorporation of its head $e n$ in the same fashion as other clitics. This is not to say that I do not accept Milner's (1978) arguments. Milner convincingly argues for a structural difference between quantitative constructions and partitive constructions, which he analyzes respectively as $\left[_{N P} Q * N^{\prime}\right]$ and $\left[_{N P} Q\left[_{N^{\prime}} N^{\wedge} * N P\right]\right]$ with $N^{\wedge}$ silent. Milner suggests that the quantitative en stands for $* \mathrm{~N}^{\prime}$ while partitive en stands for $* \mathrm{NP}$. It seems to me that although structural differences between the two constructions are well motivated, it is quite unclear that we are dealing with two ens. We can reformulate his proposal here by adding to $(189 b)$ an additional DP projection in the partitive yielding $\mathrm{L}_{\mathrm{QP}} \mathrm{Q} \mathrm{L}_{\mathrm{DP}} \mathrm{D}_{\varnothing}$ $N P]\rfloor$ and $L_{Q P} Q\left[_{D P} D_{\varnothing}\left[{ }_{N P} N^{\wedge} D P\right]\right]$, with $N^{\wedge}$ silent, for quantitative and partitive respectively. This preserves his idea that the partitive is a structure including a quantitative subpart and is compatible with the text above. ${ }^{93}$

\subsubsection{Plural indefinite and partitive article}

A final type of en occurs in constructions containing what traditional grammarians have called the indefinite plural article des illustrated in (192a) and the partitive article $d e$ illustrated in (192b):

(192)(a) Il a repeint des portes./Il en a repeint(es). He repainted doors(FEM-PL)./He repainted some.

(b) Il a mangé de la soupe./Il en a mangé. $\mathrm{He}$ ate some soup./He (of it) ate some.

I will follow Milner's (1978) conclusions that both these constructions contain a silent quantifier and are of the form [Q $L_{D P}$ de art X]] (with rules 
$d e+l e s=d e s, d e+l e=d u)$, where the silent $\mathrm{Q}$ stands for an indeterminate quantity. I will further suppose that the so-called partitive article is simply the counterpart of the plural indefinite article when the head noun is or is used as a mass noun, rather than a count noun.

\subsubsection{Agreement with quantitative en}

Let us now turn to the agreement facts. First, none of these en can trigger agreement when they originate as part of the object in an impersonal unaccusative construction, an impersonal passive construction or an impersonal middle construction.

\section{(193) Impersonal unaccusative}

(a) Il est arrivé des femmes./Il en est arrivé(*ES).

It is arrived women./It some is arrived.

(b) Il est arrivé beaucoup de femmes./Il en est arrivé(*ES) beaucoup.

It is is arrived many women./It of-them is arrived many.

(c) Il est beaucoup arrivé de femmes./Il en est beaucoup arrivé(*ES).

It is is many arrived women./It of-them is many arrived.

Impersonal Passives

(d) Il a été peint des portes./Il en a été peint(*ES).

It has been painted doors./It some has been painted.

(e) Il a été peint beaucoup de portes./Il en a été peint(*ES) beaucoup.

It has been painted many doors./It of-them has been painted many.

(f) Il a été beaucoup peint de portes./Il en a beaucoup été peint $(* E S)$.

It has been many painted doors./It of-them has been many painted.

\section{Impersonal middle}

(g) Il s'est construit des maisons./Il s'en est construit $\left({ }^{*} \mathrm{ES}\right)$.

It se is built houses./It se some is built.

(h) Il s'est construit beaucoup de maisons./Il s'en est construit $(* E S)$ beaucoup.

It se is built many houses./It se of-them is built many.

(i) Il s'est beaucoup construit de maisons./Il s'en est beaucoup construit(*ES).

It se is many built houses./It se of-them is many built. 
MOVEMENT, AGREEMEN'T AND CASE

\section{Impersonal intransitive}

(j) Il a conduit des femmes./Il en a conduit(**ES). It has driven women./It some has driven.

(k) Il a conduit beaucoup de femmes./Il en a conduit(**ES) beaucoup. It has driven many women./It of-them has driven many.

(l) ? Il a beaucoup conduit de femmes./*Il en a beaucoup conduit(ES).

It has many driven women./It of-them has many driven.

This is as predicted. Since the object in all these cases does not get accusative Case, agreement is not possible. There is nevertheless a contrast between the first three constructions and the last one that can be attributed to the fact that the postverbal NP in the first three is nevertheless a complement of the verb, while it is an inverted subject in the last one (using inverted without prejudging how this should be analyzed - as inversion or failure of raising from VP internal position). As illustrated by the examples (c), (f), (i) and (l), the initial $Q$ of the postverbal object can appear separated from the rest of that object (if it is immediately followed by de as beaucoup but not say, numerals). Call these Qs separable Qs. Some analysis of this process is provided in Section 5.5.4.

In the transitive case, the facts are as follows.

(194)(a) Il a peint des portes./Il en a peint(ES). He has painted doors./He some has painted.

(b) Il a peint beaucoup de portes/Il en a peint(*ES) beaucoup. He has painted many doors./He of-them has painted many.

(c) Il a beaucoup peint de portes./Il en a beaucoup peint(ES). $\mathrm{He}$ has many painted doors./He of-them has many painted.

(d) Il a peint autant de maisons qu'il en a construit(ES). He has painted as many doors as he of-them has built.

In the (a) sentence, agreement is possible with a preposed clitic even though the clitic does not stand for the direct object itself but rather for a subpart of the direct object. This is also true in the (d) sentence. In both cases, the object is of the form $\left[_{\mathrm{OP}} \mathrm{Q} \mathrm{L}_{\mathrm{DP}}\right.$ de (art) X]] with Q silent or beaucoup. Why is agreement possible? In order to account for this data, I suggest that the derivation of the (a) and (d) sentences proceeds as follows. The constituent QP (which I had been calling an NP throughout) raises to the accusative position, triggering agreement. As a next step, DP raises out to [Spec, QP] position and then out of QP from which en (the head of DP) can be incorporated into Tense. The (silent or overt) Q from QP is left behind, possibly in the accusative position or possibly is subject to further movement rules (as in the (d) 
example of subdeletion). In the (c) sentence, the $Q$ overtly remains postverbal. $\mathrm{QP}$ has never raised to the accusative position, only $\mathrm{Y}$ has been extracted out of it without being able to transit through the accusative position. Agreement is impossible.

\subsubsection{Separable Q position}

The general account of agreement I have given argues that in French, agreement is triggered by the presence of the direct object in [Spec, $\mathrm{I}_{\text {participle }} \mathrm{P}$ ] in the course of the derivation. This leaves open the question of why the agreement position allows movement through it but does not appear to allow an overt filler as the (a) example shows:

(195)(a) *Jean a les portes peint(es). John has the doors painted.

(b) Jean a beaucoup/trop/peu peint de portes. John has many/too many/few painted doors.

The analysis of the data in (194) above might be taken to suggest that some non-silent filler might be allowed to appear overtly in that position as in the (b) sentence, if it can be shown that beaucoup actually occupies the agreement position. The possibility of

Il a trop/peu imprimé ses articles.

He printed his articles too many times/few times.

indicates that these Qs do not have to appear in the accusative position. Since this position is needed for the postverbal object, trop cannot occupy it. These Qs fit in the class of unselective quantifiers in Lewis's (1975) sense. The Qs in sentences like (195b) are ambiguous between the many/too many/few readings (quantifying over NPs) and the too much/not much reading (of frequency quantifiers over events). In (195b), they have the first reading, in (196) the second. The reason is clear. If the postverbal NP contains an empty Q, the preverbal $Q$ will have to act as antecedent for it and have its restriction defined by this postverbal NP. Otherwise, it is free to quantify over events or other open entities in the sentence. This shows that in each case we are dealing with only one Q (and not two homophonous Qs) as could be suspected from the systematic ambiguity. It also shows that the $Q$ does not have to be in the accusative position, since it is not in (196). This is corroborated by sentence (195b). Q by itself is a head. If it were in the accusative position, it would mean the whole QP has moved there with DP extraposed or moved out of QP prior to QP raising. But if the Q-headed QP were in the accusative position, we would expect agreement with the postverbal object. Agreement however is impossible. The same reasoning extends to lack of agreement in (193(c), (f), (i) and (l)) above. 
This reasoning is consistent with the stronger conclusion that the $Q$ in (195b) cannot be in the accusative position. Of course, this does not show that the $\mathrm{Q}$ is not in the accusative position when $e n$ movement has taken place as in Il en a beaucoup mangé, but it shows it does not have to be. These conclusions are consistent with our contention that the accusative position is in fact lower than the participle's at S-Structure. Indeed, remember that the relevant structure for a transitive sentence is: ${ }^{94}$

(197)

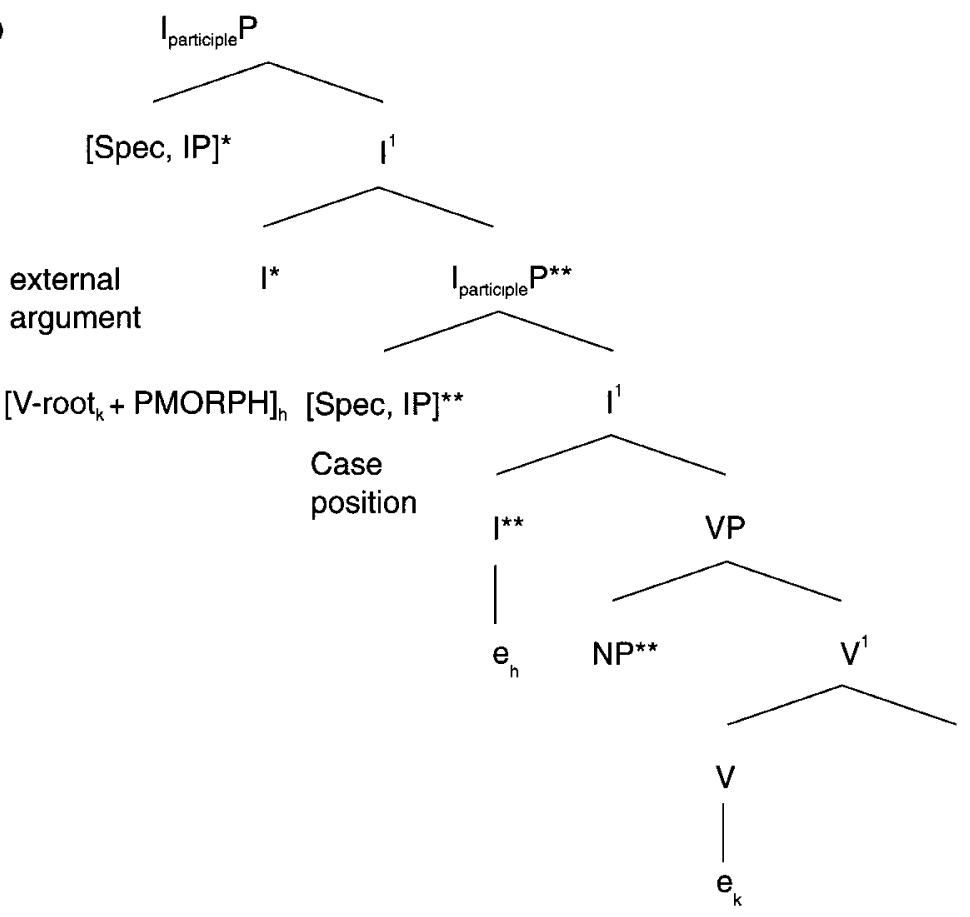

That these separated NP quantifiers cannot appear in the accusative position is supported by the following observations showing that the $\mathrm{Q}$ position is as close to the verb as possible:

(198)(a) J'ai donné beaucoup de bonbons à tous les enfants. I have given many sweets to all the children.

(b) J'ai beaucoup donné de bonbons à tous les enfants. I have many given sweets to all the children.

(c) Je leur ai tous donné beaucoup de bonbons. I to-them have all given many sweets.

(d) Je leur ai tous beaucoup donné de bonbons. I to-them have all many given sweets. 
(e) * Je leur ai beaucoup tous donné de bonbons. I to-them have many all given sweets.

Thus the adverbial $Q$ beaucoup cannot be separated from the verb by a floating quantifier (quantifying over the indirect object). ${ }^{95}$ Where then are these Qs appearing in (195b)? I suggest that they incorporate to the participle in $I^{*}$ from $[S p e c, I P]^{* *}$ : QP raises to [Spec, IP]** triggering agreement. DP is extracted from there, and ultimately cliticized and $Q$ incorporates to $I^{*} .{ }^{96}$ Returning to our original question, these facts indicate that the agreement/ accusative position must be empty at S-Structure.

\subsubsection{A puzzle about the partitive article}

There is a minimal and puzzling contrast with respect to agreement between an indefinite plural object and a partitive singular object illustrated below:

(199)(a) Il a pris des poires./?Il en a pris(es), des poires. He has taken pears./He some has taken, pears he.

(b) Il a pris de la glace./Il en a pris $\left.{ }^{*} \mathrm{E}\right)$, de la glace. He has taken ice./He some has taken, ice.

(c) Il a fait des soupes./?Il en a faitES, des soupes. He has made soups./He some has made, soups.

(d) Il a cuit de la soupe./Il en a cuit $(* \mathrm{E})$, de la soupe. He has cooked soup./He some has cooked, soup.

The impossibility of the (b) and (d) sentences appears mysterious. The derivation available for (a) and (c) should be available for (b) and (d). This different behavior can be related to structural differences between indefinite plural and singular partitive constructions, and/or to some semantic difference between the two such as the count/mass distinction or the nature of the covert quantifier.

This suggests imposing an (unexplained) restriction on the agreement/ accusative position, namely that it is restricted to certain types of quantified NPs that would include pronouns (analyzed as D heading a DP), wh-words and certain Qs such as beaucoup, trop and the silent $Q$ appearing in the indefinite plural but not la moitié or la plupart and the silent $Q$ appearing in the singular partitive. A potentially serious problem with this is raised by the possibility of agreement in de la soupe a été faitE ce matin. The agreement here is triggered by transit through the specifier of the participle, suggesting silent Qs that cannot move to agreement position in transitive sentences can do so in unaccusative structures.

Pushing this proposal further, we might distinguish the agreement position in transitive structure, which also is the accusative position AGR$A C C$, from the agreement position in unaccusative structures which is not a 
Case position AGR. Suppose that AGR-ACC is an A-bar position restricted to certain kinds of Q-headed NPs, while AGR is an A-position allowing any NP to move there (a possibility consistent with our conclusions in Section 5.1.2.4). In NP Movement structures, the AGR position will have to be empty for Case reasons (an NP Movement through it must move the NP higher to get its Case). In other structures, AGR-ACC must be empty for the same reason that an intermediate $[\mathrm{Spec}, \mathrm{CP}]$ must be in $\mathrm{Wb}$ Movement constructions.

\subsection{Appendix: remarks on AGR-O and an alternative theory}

Kayne's (1989a) analysis of participle agreement differs from that presented above in that he postulates an AGR projection to mediate agreement between the preposed object and the participle. Roughly there is an AGRP projection. The participle and the agreement affix merge and the object determines the shape of the agreement affix by transiting through [Spec, AGRP] or by adjoining to AGRP. Similarly, Chomsky (1989) proposes that objects actually move to the $\mathrm{Spec}$ of a functional projection, $\mathrm{AGR}_{\mathrm{O}}$, triggering object agreement. According this view, we would be dealing with a structure like (200) below.

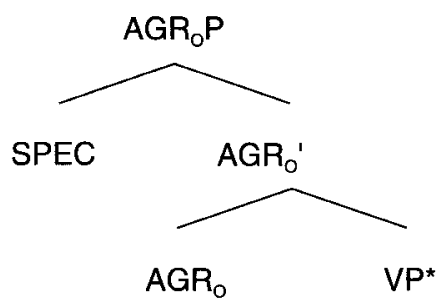

We basically propose the same analysis except for the fact that we take the AGR projection to be the syntactic projection of the affix that attaches to the verb. We have also argued that structural Case and external theta properties are properties of the affixal head. This causes the external theta role of the verb to be expressed as specifier of a projection outside the projection triggering agreement as in (159) for example. If the agreement causing projection is AGRP, there is no principal reason why AGRP should be lower than the external argument position, as we have argued, rather than higher (the external theta role would be assigned to [Spec, VP*] in (190)).

The existence of an agreement projection (possibly identified with the participial morphology projection) raises a crucial question. How do the existence of the agreement projection and the VP-Internal Subject Hypothesis combine? There could be a priori two possible structures: 
1 The external argument NP* could be lower than $\mathrm{AGR}_{\mathrm{O}}$ as in (201a), in [Spec, VP] or

2 it could be external to $A G R_{O}$ as in (201b).

$$
\begin{array}{r}
\text { (201)(a) } \ldots[\text { Spec, IP }] \ldots\left[\mathrm { AGR } _ { \mathrm { O } } \mathrm { E } _ { \mathrm { VP } } \mathrm { NP } * \left[_{\mathrm{V}}, \mathrm{V}\right.\right. \\
\text { (b) } \ldots[\text { Spec, IP }] \ldots\left[\mathrm { NP } * \ldots \left[\mathrm{AGR}_{\mathrm{O}} \mathrm{VP}\right.\right.
\end{array}
$$

(201b) is basically what we adopt. Let us discuss the alternative (201a). Translating our proposals concerning Case, we would claim that the external argument has to move (or get linked) to [Spec, IP] ([Spec, $\left.A_{G R} P\right]$ in Chomsky's terminology) (either for Case reasons or because it is PRO for government reasons), while the direct object has to move (or get linked) to $\left[\right.$ Spec, $\left.A G R_{\mathrm{O}}\right]$. The problem is how we can insure this result. How do we insure that the object moves to [Spec, $A_{G R} \mathrm{P}$ ], over the thematic subject or its trace, and the subject to $\left[\mathrm{Spec}, \mathrm{AGR}_{\mathrm{S}} \mathrm{P}\right.$ ] (i.e. [Spec, IP])?

If both the movements involved are A-bar movements, it is unclear how to force this derivation at all. Note that linking the position $\left[S p e c, A G R_{O} P\right.$ ] with accusative Case and $\left[\mathrm{Spec}, \mathrm{AGR}_{\mathrm{S}} \mathrm{P}\right]$ with nominative Case (linking which we have argued for) is not sufficient. Both nominative and accusative Cases are structural Cases, blind to thematic properties. How would we prevent a sentence like (i) John believes Bill to have left with two subjects from being interpreted with John the external argument of the embedded clause having raised to $\left[\mathrm{Spec}, \mathrm{AGR}_{\mathrm{S}} \mathrm{P}\right]$ and Bill the external argument of the main clause having moved to [Spec, $\left.A G_{\mathrm{O}} \mathrm{P}\right]$ ??7

Suppose therefore that one of the movements or both is an A-movement. If movement to [Spec, $A G R_{O} P$ ] is A-movement and movement to [Spec, $\left.\mathrm{AGR}_{\mathrm{S}} \mathrm{P}\right]$ is A-bar movement, we face the same problem. Nothing will prevent an internal argument from raising to $\left[\mathrm{Spec}, \mathrm{AGR}_{\mathrm{S}} \mathrm{P}\right]$ over an external argument that has raised to $\left[\mathrm{Spec}, \mathrm{AGR}_{\mathrm{O}} \mathrm{P}\right]$, since again $\mathrm{A}$-bar movement is not sensitive to the SSC.

I conclude that movement to [Spec, $\left.\mathrm{AGR}_{\mathrm{S}} \mathrm{P}\right]$ must count as A-movement or must contain a step which does (e.g. movement to [Spec, TP]). The status of movement to $\left[\mathrm{Spec}, \mathrm{AGR}_{\mathrm{O}} \mathrm{P}\right]$ is open. As should be clear from the discussion in Section 5.1.2.4, $\left[\mathrm{Spec}, \mathrm{AGR}_{\mathrm{O}} \mathrm{P}\right]$ must be an A-position in case the verb is passive, unaccusative etc. In this case there is presumably no external argument NP at all (except perhaps in reflexive constructions, as argued in Section 5.4.3). However, it may be an A-bar position or an Aposition otherwise. The configuration contains the following substructure:

$$
\left[\text { Spec, } A G R_{S} P\right] \ldots\left[\text { Spec, } A G R_{O} P\right] A G R_{O} \quad L_{V P} N P *\left[_{V^{\prime}}\right. \text { V NP** }
$$

The object NP** moves to [Spec, $\mathrm{AGR}_{\mathrm{O}} \mathrm{P}$ ] over NP*. Either this is A-bar movement, in which case it is licit, or it is A-movement, in which case we need to assume a theory of NP Movement where an intervening subject 
(or perhaps the trace of a subject) does not block NP Movement. Next, the external argument NP* moves to [Spec, IP]. In this case, it must be Amovement but is able to skip over $\left[\mathrm{Spec}, \mathrm{AGR}_{\mathrm{O}} \mathrm{P}\right]$. We need a theory of NP Movement that allows skipping over certain intermediate specifiers, basically what was needed under one of the alternatives previously discussed in connection with the movement of NP**. I quickly explore a couple of alternatives. If NP Movement possibilities are to be derived from antecedent government (as it is with the CCL), we would need to revise the notion of barrierhood so that no bartier is crossed.

One possibility would be to put together proposals by Aoun (1984) and Chomsky (1986b) and to count as barrier only constituents containing a complete functional complex closed under A-chain formation for their external argument. ${ }^{98}$ In (201a), this would make IP a barrier if the external argument has raised to IP, while it would make $A G R_{O} P$ the barrier if the external argument has raised to [Spec, $A G R_{O} P$ ]. This would have the desired effect. In the former case, the object would be free to associate with [Spec, $\mathrm{AGR}_{\mathrm{O}} \mathrm{P}$ ], crossing no barrier. In the latter case, the sentence would be ruled out for lack of Case on the object: the only way for it to get Case is to move to [Spec, IP], which lies outside the relevant complete functional complex, namely $\mathrm{AGR}_{\mathrm{O}} \mathrm{P}$. Clearly, under such an approach, NP Movement of the external argument must be determined by other means. If nothing further is said, no matter where it moves, the external argument will never cross any barrier. This means we lose an account of NP Movement possibilities of external argument on the same terms as NP Movement of other arguments. We would need a principle restricting NP Movement to certain domains (e.g. IP) within which the NP Movement possibilities would be restricted by antecedent government. This loss of generality strongly argues against such an alternative, I believe.

Another alternative could take advantage of the fact that the $\mathrm{V}$ moves to $A G R_{O}$. We could again need to modify our early notion of barrierhood and close it under head movement chain as we have suggested is necessary in Section 5.4.3.2. Then, an object moving to $\left[\mathrm{Spec}, \mathrm{AGR}_{\mathrm{O}} \mathrm{P}\right]$ over the trace of the subject would not cross any barrier. But then, movement of the external argument to $\left[\mathrm{Spec}, \mathrm{AGR}_{\mathrm{s}} \mathrm{P}\right.$ ] would. Unless we assume that the $\mathrm{V}$ raises even further than to $A G R_{O}$, making a higher specifier accessible to NP*. This last and necessary assumption seems to me to weaken the adoption of (201a) over (201b), although it is quite close to what we have assumed under (201b). The difference of course, is that our assumption concerning the existence of this extra projection comes from our general assumption about the projection of external theta position. A structure like (201a) would have some desirable features, for example that of making theta structure of verbs uniformly projected independently of agreement (certainly a desirable move if AGRP is not ParticipleP). I will leave this matter open at this point. 


\section{PARTITIONS AND ATOMS}

\section{Structure of NPs}

\subsection{NP structure}

\subsubsection{The DP Hypotbesis}

Recall first of all that we started with the assumption that [Spec, NP] should, by the definition we have given, be an A-position but we have noted in Section 3.4.5 that it was advantageous to take [Spec, NP] as an A-bar position. When looking at the properties of the various ens in Section 5.5, we further concluded that it was necessary to adopt explicitly the DP hypothesis of Abney (1987). Furthermore, as Szabolcsi (1983) argues, it is advantageous to suppose a certain parallelism between NPs and clauses in that NPs seem to have a Comp-like A-bar position through which extraction out of NP can proceed in Hungarian. I will thus adopt the idea that bare arguments of predicates have a structure roughly along the lines of (202) with $\mathrm{XP}$ an A-bar position.

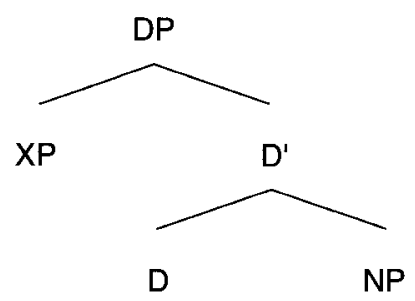

Naturally, when a DP is an object of say, a verb, the DP boundary will count as L-marked by this verb so that extraction out of DP can, in such cases proceed through XP.

\subsubsection{Larsonian NPs}

As Stowell (1988) has remarked, adopting the DP Hypothesis allows one to draw a simple parallel between NPs and VPs in that they can now be seen as both being predicative categories. A second and natural assumption I will adopt is the following: the projection rules of argument structure onto syntactic structure in NPs mirror those for VPs and other lexical categories. In particular, this means that NPs have a modified Larsonian internal structure, much like VPs. Putting together this proposal with the standard DP Hypothesis, we get the following (simplified) structure for an NP having a possessor, an external argument and an internal argument, e.g. La photo de lions de McPberson du musée de l'ouest./"The museum of the west's picture of lions by McPherson," parallel to what we have adopted for VPs in Section 5.2.3.1: 
(203)

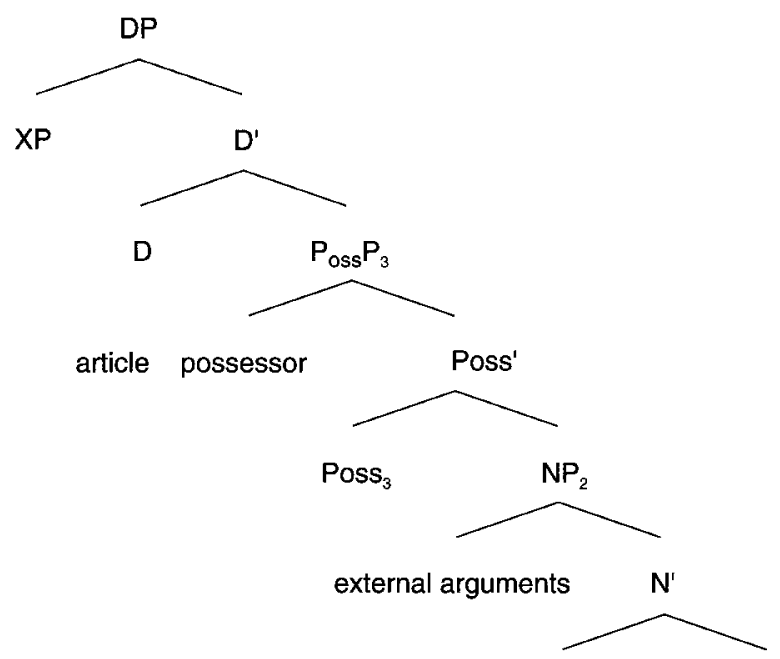

$\mathrm{N}_{2}$

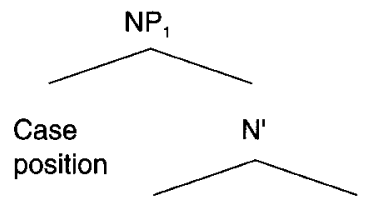

$\mathbf{N}_{1}$

DP internal arguments

The Poss $\mathrm{P}_{3}$ projection projects the possessor as its specifier and takes an NP complement (so, following what we said earlier, it should project the possessor on a higher projection than the one containing the head and the NP complement - this simplification is unimportant). The noun projects its internal arguments inside $\mathrm{NP}_{1}$, and its external argument as specifier of $\mathrm{NP}_{2}$. As in the case of VP, we suppose that the internal argument corresponding to the accusative NP raises to $\left[\mathrm{Spec}_{\mathrm{N}} \mathrm{NP}_{1}\right]$ to get Case under agreement with $\mathrm{N}_{1}$ and be governed by $\mathrm{N}_{2}$. As with VPs, the projection Poss $\mathrm{P}_{3}$ exists only if there is a possessor to project, and the projection $\mathrm{NP}_{2}$ only exists if the noun has an external argument. The head noun raises from $\mathrm{N}_{1}$ to $\mathrm{N}_{2}$ to Poss 3 . If this structure is correct, we expect all sorts of asymmetric command relations between the possessor, the external argument and the internal argument. They are indeed found as Giorgi and Longobardi (1991) and Valois (1991) show.

Note two aspects in which this structure is not rich enough. First, it provides no way for either the external argument or the possessor to get Case. This suggests additional structures must be present. To be consistent with our previous treatments, there should be an additional Case position above PossP 
(equivalent of AGRs in clauses) ${ }^{99}$ for the possessor DP and the possessor predicate should have a layered Larsonian structure. Putting all this together yields the following:

(204)

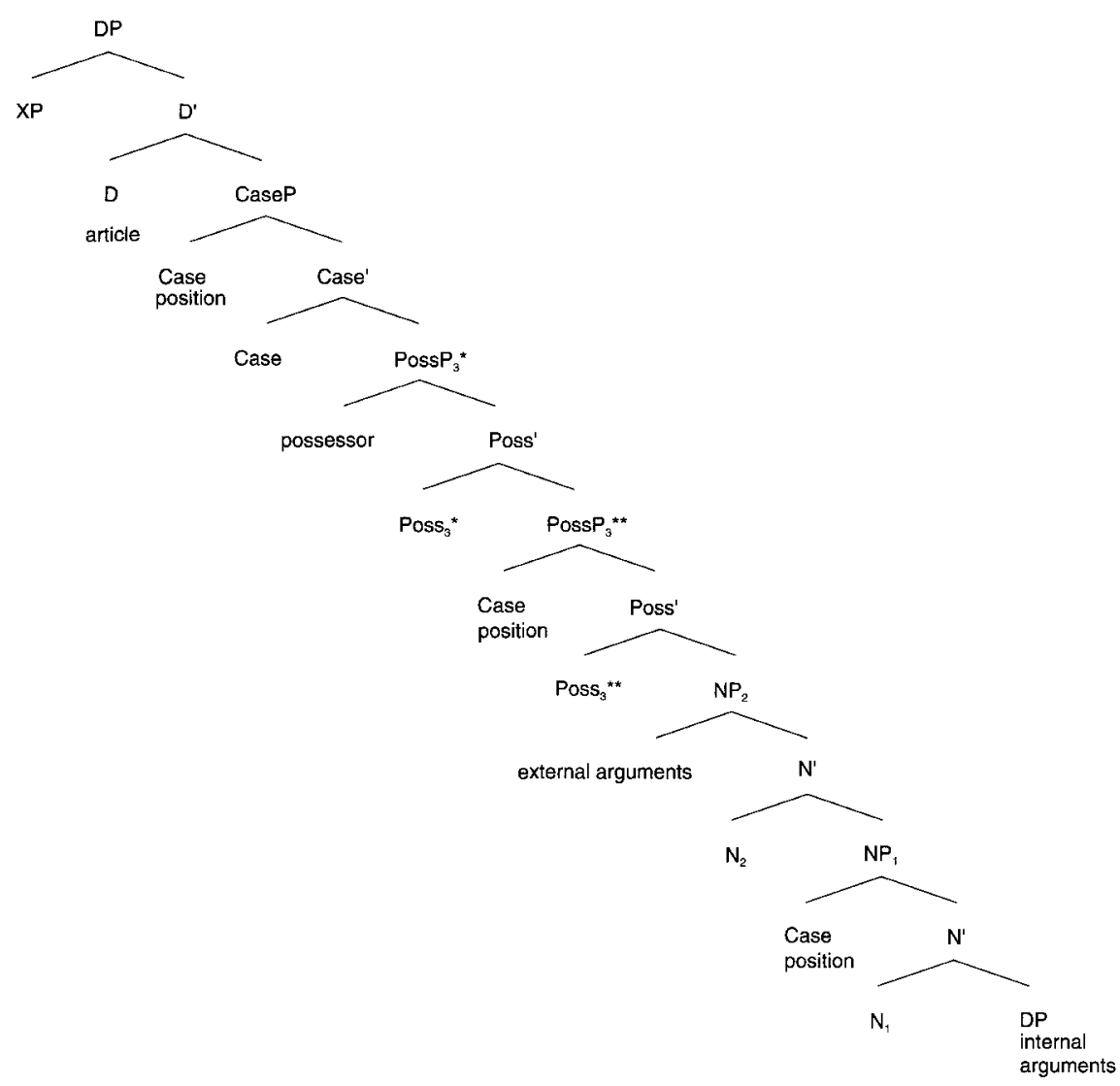

Second, in this view the Poss head is a predicative head expressing possession and thus should take two DPs as argument. Again this suggests that more structure is involved (in particular that there should be a DP projection intervening between Poss** and $\mathrm{NP}_{2}$ ).

Finally, notice that the modification of barrierhood introduced in Section 5.4.3.2 does not affect the results derived in Section 3.4.3 and Section 3.4.4 concerning extraction out of NP (or more precisely DP) since the only noun raising affecting barrierhood is from $\mathrm{N}^{1}$ to $\mathrm{N}^{2}$. 


\subsubsection{Case-marking}

Consider now Case-marking. These three arguments surface in French as de-NP. I will assume that de is simply the surface realization of genitive Case, which I take to be a structural Case assigned in the various Case positions given above in (204). This is a departure from standard assumptions about of/de. The only reason why nouns or adjectives are postulated to assign inherent Case instead of structural Case is the absence of exceptional Casemarking Structures complement of $\mathrm{N}$ (or A) as in the (a) and (b) phrases below.

$$
\begin{aligned}
& \text { (205) (a) * belief [John sick]/believe John sick } \\
& \text { (b)* belief [John to be sick]/believe John to be sick } \\
& \text { (c) *John's belief [t sick] } \\
& \text { (d) *John's certainty [t leave] (with the raising reading) } \\
& \text { (e) John's belief [PRO to have to leave] }
\end{aligned}
$$

An inherent Case proposal does not explain the deviance of the raising cases (c) and (d). Granting that the ungrammaticality of (e) is due to the PRO being governed by $\mathrm{N}$, we cannot rule out (a) through (d) simply by claiming that exceptional government (needed for (c) and (d), hence exceptional Casemarking) is absent in NP. We do not have an explanation for the (c) and (d) cases. Note however that the (a) and (b) cases reduce to the impossibility of raising. In order to get Case, John in (a) and (b) will have to raise to [Spec, NP] in a way similar to what happens with ECM verbs discussed in Section 5.2.1.2. That is, we will have to have "raising to object" in NPs in exceptional Case-marking structures in the same way we have raising to object in Exceptional Case-marking structures in VP. Consequently, there is no need for the assumption that $\mathrm{N}$ (or $\mathrm{A}$ ) assigns only inherent Case. Furthermore, there is substantial evidence that the conditions governing the appearance of of/de are not thematically related. First $d e$ is compatible with a range of (semantic) relations (this is atypical of inherent Case) as shown by the examples (206a) and appears in contexts where no thematic role at all is involved (206b):

(206)(a) frère de Jean, ville de Paris, table de bois, photo de Pierre, brother of John, city of Paris, table of wood, picture of Peter,

(b) drôle de type, beaucoup de mes amis, regard de pitié, strange (of) guy, many of my friends, look of pity

I conclude offde is the realization of the structural Case assigned in the nominal system. 


\subsection{The internal structure of NP and extraction}

Recall the basic proposal we adopted for extraction out of NPs in Section 3.4.3 and Section 3.4.4. Phrases extracted out of NPs must go through [Spec, $\mathrm{NP}$, now [Spec, DP], an A-bar position. Consequently, a phrase can be extracted out of a given DP only if it is allowed to move to the [Spec, DP] position. There are a number of problems that we have encountered and to which we return below. I now will argue that these problems can be traced back to a misconstrual of the internal structure of NPs. When the principles we have adopted or developed for the structure of VP are transposed to the analysis of NPs within the DP hypothesis together with modified Larsonian structures, many of these problems disappear.

\subsubsection{Agreement problems with genitive en}

Extraction of NP leads to problems with reference to agreement similar to those we had with datives and other non-accusative complements, and suggests the same kind of linking of the agreement position with the accusative position. To see this, consider the relevant part of the structure of Pierre en a décrit le portrait, de Marie./"Peter described her portrait, Mary."
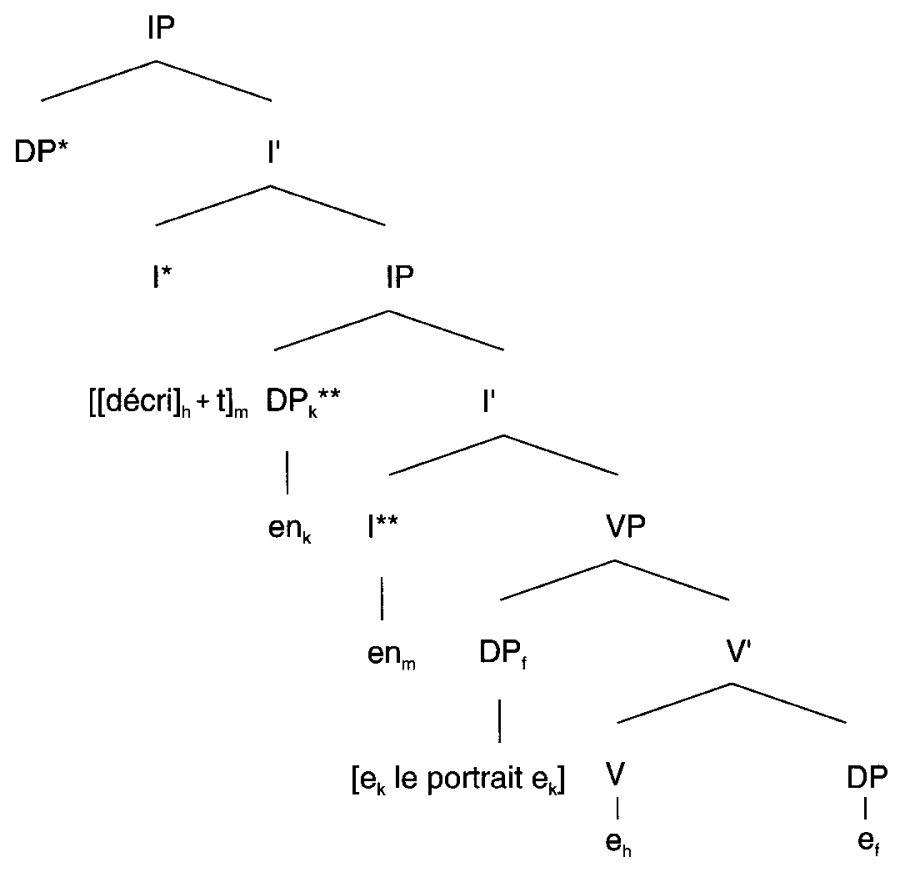
The verb is raised to $I^{* *}$ to pick up participle morphology. The participle moves to I* to license the external argument. The DP en raises to [Spec, DP] inside the direct object. The direct object raises to [Spec, VP] to be governed by the participle. Since escape of $e n$ from $\mathrm{DP}_{\mathrm{f}}$ is through an A-bar position, $e n$ movement from within DP is never going to trigger agreement regardless of circumstances since agreement triggering positions are A-positions. Nor will en ever be able to move to subject position.

\subsubsection{Problems of extraction from $D P$ and movement within $D P$}

\subsubsection{EXTRACTION FROM NP IN NP OR PP}

This is the problem we listed as Problem 1 in Section 3.4.3, and is illustrated below by a modified $(62 c, e)$ :

(62) (a) Who did you see a picture of $t$ ?

(b) ?Of whom did you see a picture?

(c)?*Who did you see $\left[_{\mathrm{XP}}\right.$ a picture of $\left[_{\mathrm{DP}}\right.$ a portrait of $\left.\left.t\right]\right\}$ ?

(d) Who did you talk about $t$ ?

(e)?*Who did you talk $\left[_{\mathrm{XP}}\right.$ about $\left[_{\mathrm{DP}}\right.$ a picture of $\left.t\right]$ ?

In these examples, nothing prevents movement from the $t$ position to [Spec, $D P]$ to [Spec, XP] and then out. Because DP is L-marked, moving through its specifier should be sufficient to escape it, if [Spec, DP], an A-bar position, ${ }^{100}$ is available.

We need to explain why extracting $E$ from $X P$ in $\left[_{X P} X\left[_{D P} E\right]\right]$, where $X$ is either $\mathbf{N}$ or $\mathbf{P}$, is deviant. The idea I will pursue attributes this impossibility to improper movement. With $\mathrm{X}=\mathrm{P}$, extraction out of $\mathrm{XP}=\mathrm{PP}$ will have to transit through [Spec, PP], which is an A-position. Extraction out of DP is through an A-bar position ([Spec, DP $]$ ). Movement from [Spec, DP] to [Spec, $\mathrm{XP}]$ is movement from an A-bar position to an A-position. It is a case of improper movement and as such excluded. In the case of $X=N$, this suggestion will not work if NP, like VP, allows adjunction to it. Indeed, recall that in VP, anything can extract by A-bar movement. The same derivation should allow adjunction to NP followed by movement to [Spec, DP]. In order to rule this out, I will stipulate that NPs and more generally N-related predicative categories (e.g. PossP) cannot be adjoined to. This will rule out the (c) example and thus solve Problem 1.

\subsubsection{DP INTERNAL MOVEMENT}

Problem 3 (from Section 4.1.3.1) is the problem of accounting for generalization (94).

(94) Only genitives can move to [Spec, DP]. 
Recall that in French, we can establish what can move to [Spec, NP] independently of extraction by looking at possessive "adjectives." (94) observes that only genitive complements (i.e. de-complements) can move to [Spec, NP]. In particular, datives cannot, locatives cannot etc., despite the fact that they seem to be NPs, as discussed in Section 5.1.1.1.

It is not sufficient to be a genitive NP to be able to appear as a possessive. Given a thematic hierarchy ordered possessor $>$ external argument $>$ internal argument among genitive complements, we have the following generalization, as yet unaccounted for, that we called Problem 2 in Section 3.4.4:

(66) In a structure: $\left[_{D P}\right.$ specifier $\left[{ }_{N^{1}} \mathrm{~N}\right.$ [de-NP] the role of the specifier must always be higher than that of the [de-NP].

Look again at the typical structure of a DP:

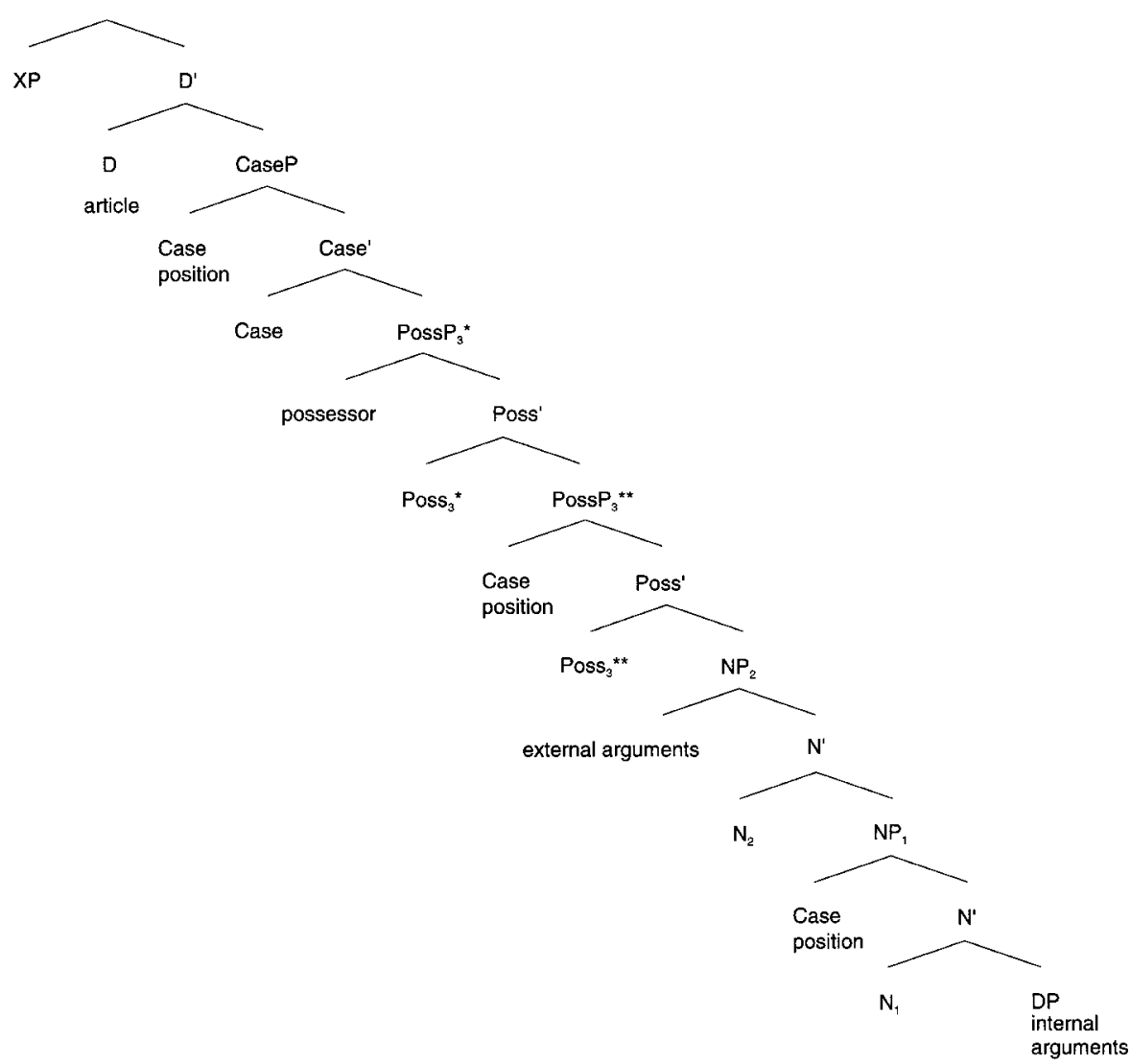


Clearly, nothing prevents the possessor DP from moving to XP and further out. In the presence of a possessor, the external argument (and, a fortiori, any internal argument) cannot reach the XP position due to the intervening barrier PossP which cannot be circumvented by adjunction.

In the absence of the PossP projection, the structure reduces to:

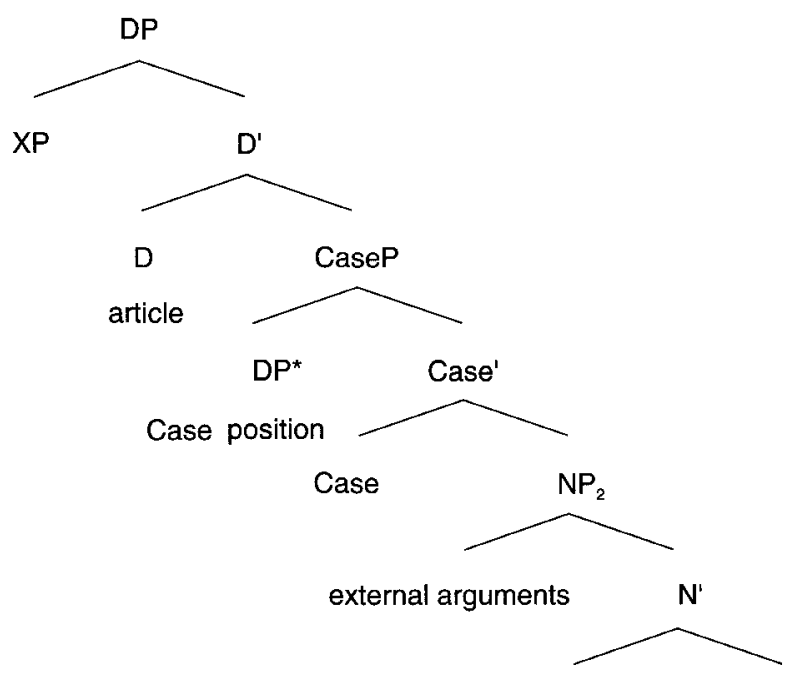

$\mathrm{N}_{2}$
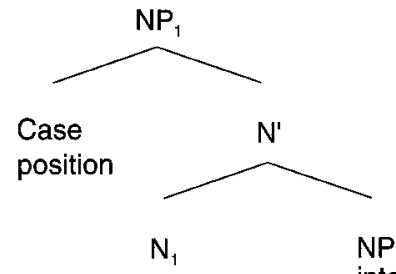

internal

arguments

In this case, the external argument may move to the higher Case position DP* then to XP, but any internal argument is blocked by the intervening barrier $\mathrm{NP}_{2}$. This provides a solution to Problem 2 in Section 3.4.4. Suppose now that the NP only has one non-oblique argument. The structure of the DP containing it further reduces to $(210)$ on page 224.

In order to reach XP, a DP must transit through the Case position DP*, where it is assigned structural Case. An oblique argument will be barred from reaching such a position for the general reason that movement to DP* is motivated by Case reasons. By definition, an oblique DP is assigned some other Case and thus is disallowed to move to DP*, hence to reach YP. It is the 
(210)

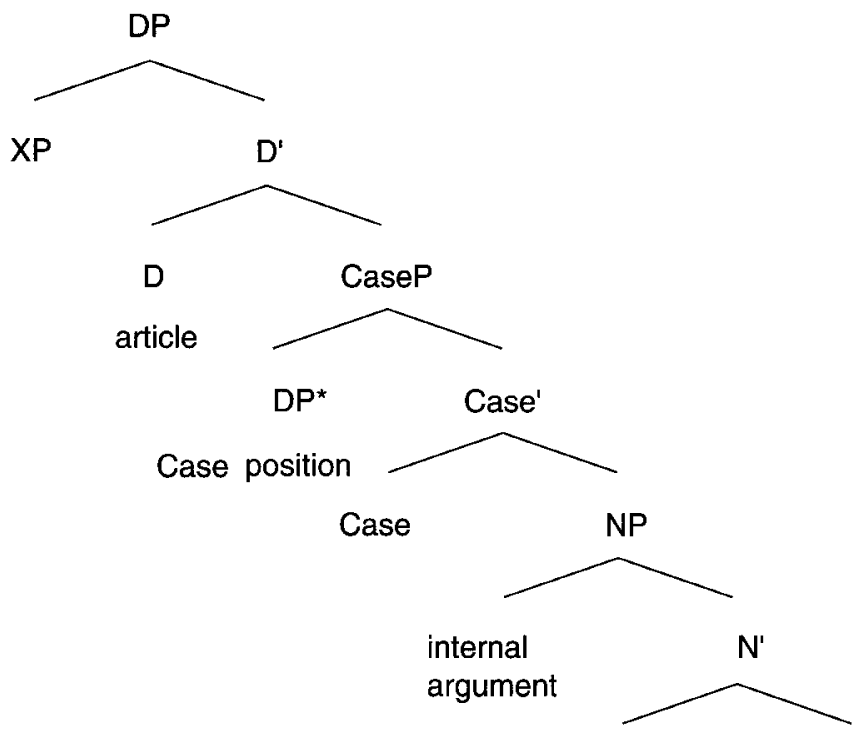

N

same generalization that prevents an oblique object from moving to an accusative position (or a nominative position), namely the Principle of Least Action (PLA) (178). This provides a solution to Problem 3.

\subsubsection{RESTRICTIONS ON SPECIFIERS: FURTHER PROBLEMS}

There are a number of further problems with our account of extraction that we now list. We have claimed that movement of en out of a DP transits though the [Spec, DP] position and that the restrictions on movement to [Spec, DP] are illustrated by the possibilities of possessivization. Yet, when a possessive moves to specifier position, it appears in a different form from en, namely as son, sa, ses. The possessive also agrees with the head noun in number and gender, just like an article. Furthermore it is in complementary distribution with articles. $E n$ on the other hand is invariable and its extraction co-occurs with the presence of the article. Why is there this morphological difference? If the possessive agrees with the head noun, and en transits through the possessive position, why does it not agree with the head noun? Presumably, en as a clitic must raise out of DP to some functional head in the clausal system. We thus do not expect it to surface in [Spec, DP]. However, we have claimed that extraction of phrases from DP proceeds through [Spec, DP] and that [Spec, DP] is an A-bar position. Why then are overt DPs excluded from this position? This is illustrated by the grammaticality of the following type of sentence: 
(211) De quel auteur ${ }_{i}$ as-tu suivi les recettes $t_{i}$ à la lettre? Of which author did you follow the recipes to the letter?

[Spec, DP] in French looks severely restricted. Possessives may appear there, it seems, and possibly en on its way out, but no phrase. Extraction in (211) is of a phrase, which, we should conclude on the basis of our analysis of extraction, transited through $[\mathrm{Spec}, \mathrm{DP}] .^{101}$ Why cannot phrases surface in this position?

The more articulated internal structure of DPs we have adopted allows the elimination of some of these problems, as we will now see. Because possessives block extraction, we assumed earlier in Section 3.4.4 that extraction proceeded though the position occupied by the possessives. However French possessives are not phrases; they are at most single words. I would like to suggest that possessives are the exact counterpart of the verbal pronominal clitics and as such, that they must cliticize onto a functional category in the nominal system, namely $\mathrm{D}$ :

(212) Possessive adjectives in French are clitics on D.

This would immediately explain why possessives agree with the head noun. A possessive like sa in sa soeur/"his sister" is the conflation of the definite article $l a$ which is $[+\mathrm{SG},+\mathrm{FEM}]$ agreeing with the head noun soeur and a $[+\mathrm{SG}$, third person] pronoun (i.e. a kind of D) determined in form by its antecedent. This cliticization operates from the Case position immediately following $\mathrm{D}$ as follows:

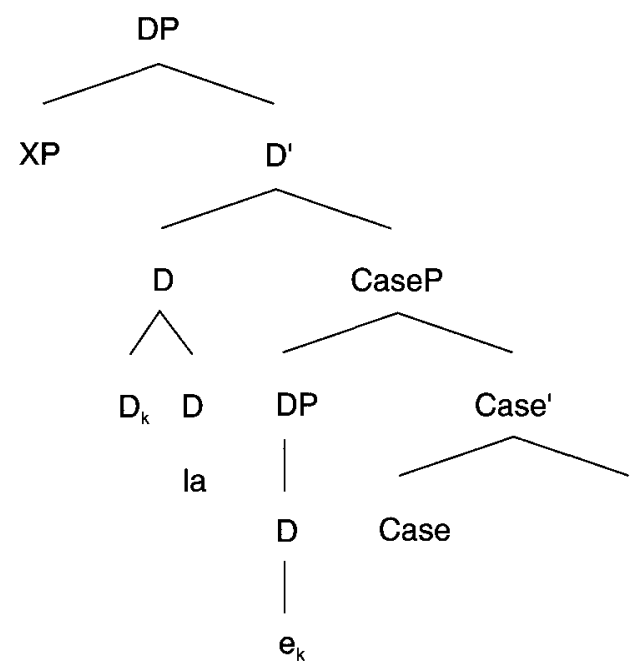

Naturally, only a DP able to appear in this Case position will be able to cliticize in this way. The parallelism between possessivization possibilities 
and extraction possibilities is established but not directly by saying that extraction proceeds through the possessive position. Rather, it is access to the specifier of the immediate complement of $\mathrm{D}$ that is crucial. The fact that this parallelism is not established directly permits an account of the differences between possessives and extracted phrases noted above. Because extraction is through [Spec, DP], we expect phrasal extraction as in (211) to be possible. No morphological similarities between possessives and extracted en is expected. It also means that the [Spec, DP] position, much like the accusative position, can never be overtly filled in French, a generalization for which we have no account.

\subsubsection{Word order in NP}

Adopting (204) raises word order questions. A DP like La pboto des lions de Barbaral "the picture of the lions by Barbara" will have the structure:

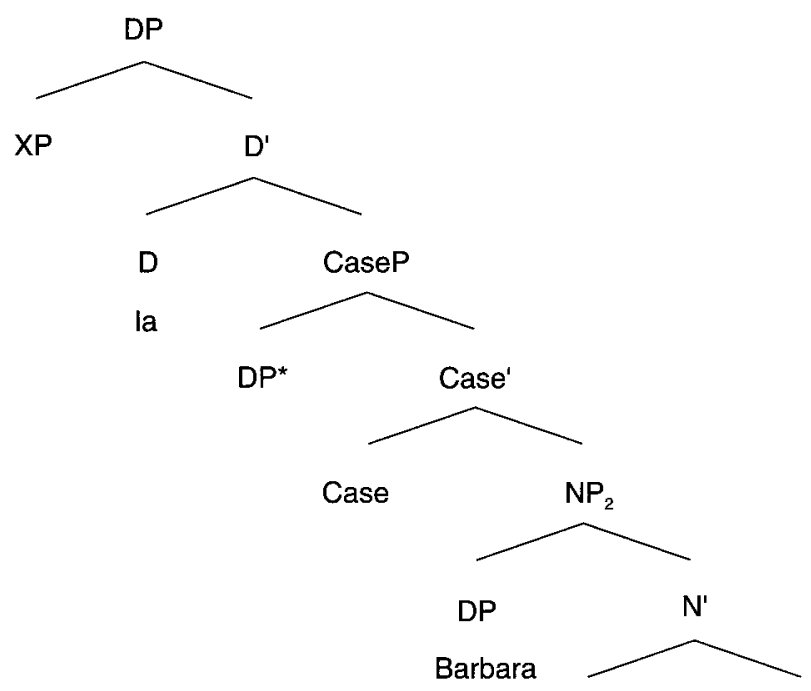

$\mathrm{N}_{2}$

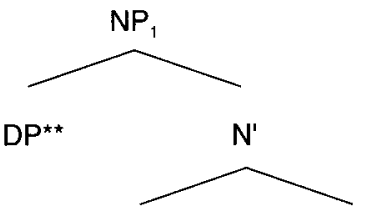

$\mathrm{N}_{1} \quad \mathrm{DP}$

photo des lions 
Given the surface word order, we have to suppose that the noun photo raises further than the external argument Barbara. This suggests that it raises to Case, but that Barbara raises to its Case position only at LF. Furthermore, if the conclusion (165) is correct, it should be governed by its Case assigner (at S-Structure or at LF). This means that Case raises to D overtly or covertly. ${ }^{102}$

Cinque (1992) provides a further argument for the existence of this raising of the noun based on the distribution of argument adjectives that $I$ adopt and adapt below. A detailed discussion of these issues can be found in Valois (1991). Cinque's argument is based on the observation that thematic roles assigned by an $\mathrm{N}$ appear to be not necessarily satisfied by NPs. Sometimes, they can be satisfied by adjectives.

(215)(a) l'invasion de l'ouest par les teutons the invasion of the west by the Teutons

(b) l'invasion teutonne de l'ouest the Teutonic invasion of the west

These argument adjectives function exactly as if they were NPs with respect to both possessivization and extraction:

(216)(a) l'invasion teutonne de l'ouest the Teutonic invasion of the west

(b) *son invasion teutonne its Teutonic invasion

(c) * dont $_{i}\left[l^{\prime}\right.$ invasion teutonne $\left.\mathbf{t}_{\mathbf{j}}\right]$ of which ... the Teutonic invasion

This suggests that there is in fact a DP (here I differ from Cinque). This is corroborated by the Theta Criterion. Two theta roles are assigned here, the agent role of invasion and the external role of the adjective Teuton/Teutonic. Consequently, I suggest the structure of (216) is as in (217) on page 228, with $\left[\mathrm{Spec}, \mathrm{NP}_{2}\right.$ ] getting the external theta role of invasion and the adjective teuton assigning its theta role to its subject PRO. The AP is adjoined to [Spec, $\mathrm{NP}_{2}$ ] and PRO (ungoverned here as it is in an adjunct) is controlled by it. The fact that the order in (215) is rigid provides Cinque's argument in favor of raising the head noun (he suggests it is raising to some intermediate projection XP which he takes to be AGRP, which is consistent with its being a structural Case position).

Although the distribution of "argument" adjectives is consistent with the predicted word order, some problems arise with genitive arguments, which I will simply allude to here. The problem concerns the respective order of the "arguments" of the noun, e.g. the possessor, the agent and the internal arguments. The facts are quite complex. We predict the order possessor > external arguments $>$ internal arguments. With genitives, the preferred order 
(217)

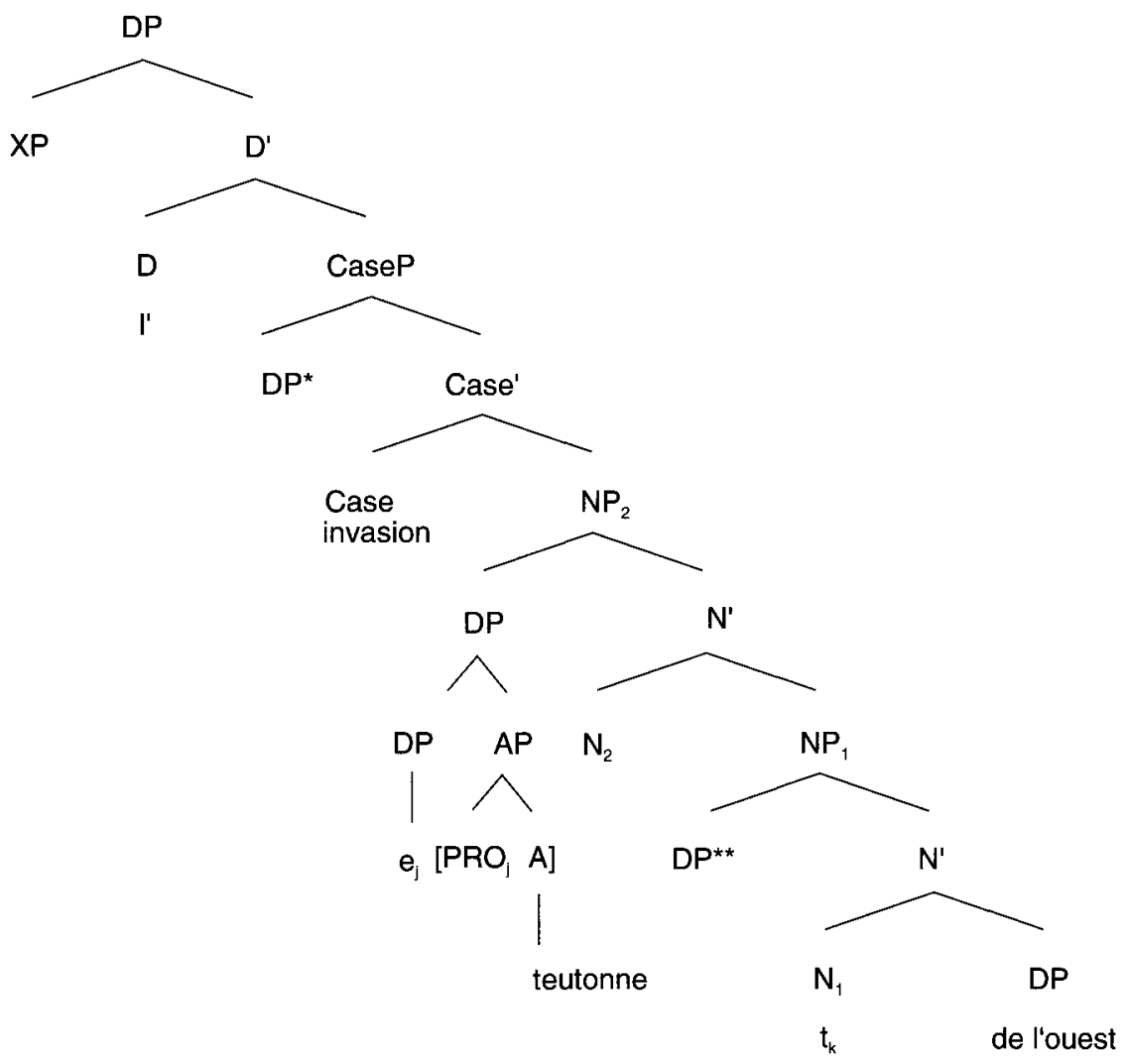

is the mirror image of the one predicted. If all three genitives are present (with nouns whose referent can be possessed, say concrete nouns, but see below), the noun phrase is awkward as in (218a), but the order indicated is much preferred to any other. If only two are present, with this kind of noun, either order is possible $(218 \mathrm{~b}, \mathrm{c}, \mathrm{d})$ (with a marked preference for the first one).

(218)(a) le portrait d'Aristote de Rembrandt du Musée d'Orsay the portrait of Aristotle of Rembrandt of the Orsay Museum

(b) le portrait d'Aristote de Rembrandt/de Rembrandt d'Aristote

the portrait of Aristotle of Rembrandt/of Rembrandt of Aristotle

(c) le portrait d'Aristote du Musée d'Orsay/du Musée d'Orsay d'Aristote 
the portrait of Aristotle of the Museum/of the Orsay Museum of Aristotle

(d) le portrait de Rembrandt du Musée d'Orsay/du Musée d'Orsay de Rembrandt the portrait of Rembrandt of the Museum/of Rembrandt of the Orsay Museum

With nominalizations, the situation is different. In my judgment, possessors are excluded, unless the result of the action denoted by the predicate can somehow be interpreted concretely, e.g. description referring to the book containing the description. With this concrete interpretation (incompatible with adjectives such as répétées/"repeated"), the facts are basically as above. Under the non-concrete interpretation, these nominals get either a process or a result interpretation and they do not get a possessor. In my judgment, they are quite awkward with two genitives (one internal and one external argument). It is much preferred to have the external argument as an adjective as above, or in a by-phrase:

(219)(a) les descriptions (??répétées) de Paris de La Bruyère the descriptions repeated of Paris of $\mathrm{La}$ Bruyère

(b) les invasions répétées de l'ouest ??des teutons/par les teutons the invasions repeated of the west of the Teutons/by the Teutons

The external argument can be a genitive though, if the internal arguments are not. In this case, the preferred order is external argument > internal argument. The same is true with abstract nouns derived from adjectives. All these judgments recall those of heavy NP shifted structures, the heavier the external argument the better:

(220)(a) le déferlement des teutons sur l'ouest/?sur l'ouest des teutons the advancing wave of the Teutons on the west/? on the west of the Teutons

(b) l'obéissance des troupes à leur chef/?à leur chef des troupes the obedience of the troups to their chief/to their chief of the troups

(c) la fidélité de Jean à ses amis/? à ses amis de Jean the faithfulness of John to his friends/to his friends of John

In other words, when word order is rigid, it supports the basic structure we propose. When it is not, an explanation is required as in (218). I will leave this question pending and refer to Valois' (1991) work arguing that the 


\section{PARTITIONS AND ATOMS}

variation in word order there is due to the existence in French (and in Italian but not in English) of subject inversion in NPs akin to what is found in the sentential system.

\section{Notes}

* Sections 1,2, 3 and 6 are substantially modified and considerably expanded versions of my 1987 Unifying Movement Theory and my 1988 Conditions on Silent Categories presented at McGill University in 1987, the 1989 GLOW Conference, the Catholic University of Tilburg in 1988, University of Paris 8 in 1988 and the University of Geneva in 1988 and possibly elsewhere. The central modifications include discussions of Case Theory, the Theory of Small Clauses and Constituent Structure adopted here, both cast in a Larsonian mode. Except for a small part of Section 4 on Conditions on Silent Categories, the essential ideas of Sections 4, 5 and 6 have been presented to various audiences since 1987 including my UCLA seminars, at the University of Washington at Seattle, University of Paris 8, the Catholic University of Tilburg, MIT and possibly elsewhere. Many thanks to all these audiences and to everybody I forget to mention.

1 Section 3.1 and Section 3.2.1 establish the properties of movement rules abstractly. They are technical and can be skipped. It is sufficient for the understanding of most of the rest of this chapter to read the summary of the conclusions of each section.

2 As Koopman (1988) shows, there is substantial crosslinguistic support for this position.

3 Throughout, linear order is irrelevant.

4 Note that there are some terminological differences in the usage of the term specifier. For example Emonds (1985) and earlier work defines as specifier elements like very in very sick. I do not. Perhaps a better terminology would reserve the term specifier for what Emonds calls specifier and subjects could be used for what I call specifier. One way to view the claim that every phrasal projection can have a subject is then similar to Stowell's (1983) claim. Note further that adverbs such as very do not have the same kind of relationship to $A$ that specifiers do. Rather, they enter into the same relation with $A$ as adjectives do with nouns, basically modifiers. The same holds of elements like right in relation to Ps (as in right on the nose), or $\mathrm{V}$ modifiers such as manner adverbials in relation to $\mathrm{V}$.

5 If true, it would seem minimal to suppose that this extends to all such cases, so that any NP, PP, VP and AP must have an NP* position regardless of whether it assigns an external theta role or not.

6 Note that in the case of NPs, the parallelism is not with NP small clauses of the type Consider this a good picture, for the NP this does not and cannot correspond to the external argument of the noun as noted in Campbell (1989). This is shown by the grammaticality of I consider this picture John's picture and by the impossibility of *I consider the engineer('s) survey of the valley. Rather, the subject of an NP small clause is an additional argument.

7 We disregard here the fact that VP preposing sometimes preposes the verb with whatever inflection it bears other than tense; for example, Criticizing John, 
Bill is/Criticize Jobn, Bill did. This suggests that VP preposing sometimes is a kind of IP preposing (with I the verbal morphology).

8 The position of $t_{i}$ in (22b) relative to bow is irrelevant to our point (it could be lower than how) as long as it is part of the preposed constituent.

9 This means that "object raising constructions" are "object raising" constructions, cf. Section 5.2.1.

10 This is not what Larson proposes. For him, the external argument will be external to the minimal VP containing the $\mathrm{V}$ only if some argument is projected as specifier of that VP.

11 Note that this is preserved under adjunction: if X L-marks YP, and something is adjoined to YP, the derived YP nodes all count as L-marked.

12 Or possibly maximal projection instead, to prevent a VP part of a layered VP à la Larson to move.

13 Possibly, as Chomsky suggests, because X-bar theory holds of derived structures.

14 Thanks to E. Stabler for his input here.

15 That is, B is not a projection of $Y$.

16 I will assume the following: A includes B iff every segment of A contains B, where contain is the usual inclusion relation between nodes. A excludes B iff no segment of A contains B. From now on I distinguish the notion of inclusion, or being inside of from the notion contain, which is the pre-barrier notion of inclusion or domination.

17 Note also that in (10), $\mathrm{Y}^{\mathrm{p}-1}$, which is head, should be able to govern an adjunct to $\mathrm{X}^{1}$ if such adjuncts exist. Note however that it should not be able to govern the specifier of ZP since it does not L-mark ZP, but it should be able to govern an adjunct to $Z P$ (if $Z P$ allows adjuncts to it).

18 Cases of base generated adjuncts to intermediate projections, if any, can now be reanalyzed as cases of adjunction to some intermediate XP in a layered XP à la Larson, or as inner complements, as he suggests.

19 Even if, as we do later, we extend L-marking to the specifier of an L-marked category, we do not want an adjunct to inherit L-marking in this way.

20 Saito defends c-command, but his arguments are consistent with adopting i-command instead.

21 I am glossing over an inconsequential complication here. It is natural to assume that infinitive morphology in French has its own projection, say InflP, with Infl taking a VP complement whose head $V$ raises to Inf. In this case, the pronominal subject of the small clause in (43) incorporates to Infl and not to $\mathrm{V}$ as shown by the morphological structure of the result (le + [consider[[er]]). The only way this could happen is if the NP subject of the small clause first adjoined to VP. Then its head - the clitic - incorporates in Infl, as allowed by the CCL. This derivation also illustrates a case of incorporation as in (42).

22 As is known, there is no perfect match between the property of being a PP in an L-marked position, and strandability of the P. What is at stake here is the correct characterization of the notion that makes a boundary create a barrier. Here we adopt "being in an L-marked position." The P-stranding pattern might suggest that this should be replaced by whatever the right structural characterization is, say $\mathrm{K}$. For consistency, it must be the case that $\mathrm{K}$ will entail "being in an L-marked position." This looks correct. We expect other 


\section{PARTITIONS AND ATOMS}

correlations, e.g. if a PP bears property $\mathrm{K}$ to some verb V, the $\mathrm{PP}$ will be strandable and the head $\mathrm{P}$ of this PP will be incorporable in the V. I will leave this question pending at this point.

23 Note that prepositions are not incorporated in the verb, as is shown by the fact that they cannot be pied-piped under verb raising.

24 Postpositions can be incorporated into the verb, but incorporation is not a precondition on strandability - see Koopman (ibid).

25 Except if we analyze sluicing constructions but I don't know who with the same way van Riemsdijk does, i.e. with the $w b$-phrase in the $[+\mathrm{R}]$ position. Furthermore, particles do allow a filled specifier as Koopman (1990) shows.

26 Notice incidentally that the CED, and consequently the CCL, predicts that stranding of $\mathrm{P}$ in $[\mathrm{Spec}, \mathrm{CP}]$ is impossible. If a PP has been moved to [Spec, $\mathrm{CP}$ ], the PP in Comp is not L-marked: the $\mathrm{P}$ cannot be stranded there. More on this in Section 3.4.5.

27 Note that this proposal must be supplemented by a diachronic story. How does P-stranding ever get in a language? We could conjecture that it can arise only in languages undergoing word order changes e.g. from head final to head initial, and in which an intermediate stage has NPs complement Ps appearing on either side of the P. One of the orders becomes analyzed as reflecting a specifier head order.

28 See Giorgi and Longobardi (1991) who have arrived at conclusions similar to those of this section concerning the fact that extraction from NP in English must proceed through its specifier position. We will argue later on in Section 6.2 that this position is not quite correct.

29 Everything we say could be translated straightforwardly in an analysis postulating the existence of DPs. If it were the case, we would need to assume (1) that D L-marks NP, (2) that everything we say is in either [Spec, NP] or in $[$ Spec, DP], See later Section 6.2.

30 There are other reasons to which we will return in some detail later.

31 We would probably want to relate the change of morphology of demonstratives to the same kind of considerations applied to NPs, i.e. Case.

32 Our reanalysis of the extraction out of NP in Section 6.2 will contradict this. The conclusion will stand, as numerals are not in the specifier position but rather heads taking NP complements.

33 This is an informal description: these elements are claimed to occur in the specifier position because they are in complementary distribution with articles.

34 This modification actually permits a slightly different account of the difference between Class 2 languages and Class 1 languages. We no longer need to attribute the different behavior of subjects in Class 2 languages to total lack of raising from the position NP*. There is mounting evidence that the structure of clauses is more complex than had been previously thought. There might be projections to phrasal level for C, Neg, AGR-S, Tense, Aspect, each taking the following as complement (for concreteness, let us say in this order). Assuming each L-marks its complement except for $C$, all the specifiers will count as $\mathrm{L}$ dependent, except the specifier of the category complement of $\mathrm{C}$. Call this position NP\&. We can now change the characterization of the difference between Class 1 and Class 2 languages from (1) to (2): 
1 Old version: in Class 2 languages raising from NP* is optional; in Class 1 languages, raising is obligatory.

2 Modified version: In Class 1 languages, raising to $\mathrm{NP}^{\wedge}$ is obligatory; in Class 2 languages, it is optional.

In other words, we are no longer committed to the view that Class 2 characteristics are found whenever NP* does not raise at all. It may raise, but not all the way to NP\&. Further discussion of these issues can be found in Koopman and Sportiche (1991).

35 Notice that this definition is not recursive, so that it rules out extraction from the specifier of the specifier of an L-marked category. Recursivity can be introduced, if necessary, by changing "specifier of XP" to "specifier of an L-dependent XP."

36 Szabolcsi (1983) suggests that nominal constituents have a [Spec, CP] escape hatch similar to that found in clauses which then makes sense of the idea that the highest specifier position within NP is an A-bar position. Probably this can be made sense of more readily if we were talking about DPs throughout. Compare Section 6. Note that this in general will not have adverse effects since A-movement from within NPs is usually impossible (unless some head movement takes place that makes the highest NP boundary transparent); cf. the discussion in Section 5.4.3.2.

37 Notice that this definition is not recursive, so that it rules out extraction from the specifier of the specifier of an L-marked category. Recursivity can be introduced, if necessary, by changing "specifier of XP" to "specifier of an L-dependent XP."

38 Assume $\mathrm{X}$ is distinct from PRO. PRO works the same way but for government reasons instead of Case reasons.

39 Probably, the internal structure of a passive VP is more complicated with $I_{e d}$ the passive morphology.

(1)

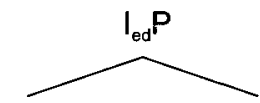

$\mathrm{NP}^{*}$

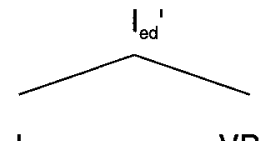

$I_{\text {ed }}$

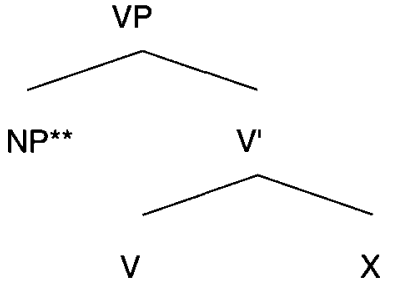

To simplify the exposition, I assume the verb V projects no external argument and raises to $I_{\text {ed }}$ (which absorbs the external theta role of $V$ as suggested by Jaeggli (1986) and Baker, Johnson and Roberts (1989)). 


\section{PARTITIONS AND ATOMS}

40 Note the difficulty of claiming that John is in fact the specifier of CP. If present, the specifier of $\mathrm{CP}$ must be available for $\mathrm{Wb}$ Movement.

41 The motivations provided by Rizzi (1982) for a locality condition on chain formation can probably be gotten around. Rizzi's central motivation is discussed later in Section 4.3.3, where we return to these issues.

42 Which excludes ethical dative clitic constructions and inalienable clitic constructions, cf. Kayne (1975).

43 I am ignoring here the fact that the aspectual auxiliary and the verb might each be raising to their affix, so that the derivation has the same properties but is in fact deeper than we represent it.

44 Clearly, the adjoined structure $\mathrm{N}+\mathrm{V}$ does count as the head of VP and should be able to move out. This is exemplified in $\mathrm{V}$ to I picking up Tense. The inflected $\mathrm{V}$ can then move onto $\mathrm{C}$. In the structure $\mathrm{N}+\mathrm{V}$, does $\mathrm{V}$ also count as the head of VP? The answer would seem to be positive. Given that heads can freely move out of their projections, it should be able to move out of VP, leaving $\mathbf{N}$ stranded behind. Of course, in the case of clitics, this derivation would not yield the required result. A complete theory should explain why this option is not exercised. Here we are trying to account for the path of movement, given the result. In other cases of $\mathrm{X}+\mathrm{V}$, it can be shown that $\mathrm{V}$ can move, leaving some head $\mathrm{X}$ that had adjoined to it behind. Such cases arise in the Dutch verbal complex (op te willen bellen), Koopman (1990).

45 A dative of inalienable possession is not placed by movement from within the NP as it does not obey the constraints on movement.

46 One minor problem that will disappear under the more exhaustive analysis presented in the next section is the fact that agreement is triggered on $V$ even though $\mathrm{V}$ raises up out of $\mathrm{VP}_{\mathrm{k}}$.

47 In fact, as Belletti (1988) shows, the impersonal construction with a postverbal NP is possible only if the postverbal NP is an argument of the verb. En cliticization in these cases shows something stronger, namely that the postverbal NP is a direct object and an internal argument of the verb.

48 Traditional grammars state that agreement is obligatory here too. I have already mentioned that other speakers and myself disagree with this statement. We feel that agreement is preferred with a preceding clitic, but not obligatory, and a little freer with a preceding wh-object. Italian, however, requires participle agreement with a preceding third person clitic object, but disallows it with a preceding $w h$-object.

49 Liaison can in principle reveal a final / $\mathbf{z} /$ plural morpheme. Unfortunately, it cannot be used conclusively in the context in which these participles appear.

50 It is possible that the verb strike is not a raising verb. See Section 5.4 .2 for discussion. In this case, only the (a) and (b) sentences are significant.

51 Same remark for the French example *il ne semble qu'a lui que Jean $_{j}$ est malade. It could not be argued that the emphatic reflexive does not command the trace of the raised subject in (117).

52 Although the object can surface in object position, as in the impersonal constructions (114), it does not bear structural accusative Case as shown in Belletti (1988).

53 If the two aspects are related as stated by Burzio's Generalization. A verb Case- 
marks an NP it governs iff it theta-marks its subject. We return to a discussion of this in Section 5.2.3.2.

54 Even the inherent construction could be similarly described assuming the causative version of the verb was lost. This would explain Kayne's (1975) observation that inherent se verbs never have both a direct object and an indirect object.

55 Cliticization of en shows that the postverbal NP is in object position and therefore not in a position to bind the reflexive se. Without cliticization, the position of the postverbal NP is not so clear. Impersonals are possible with intransitives such as in (107a) Il a dormi plusieurs enfants ici./*Il en a dormi plusieurs ici. The postverbal NP there cannot be an object. Rather it must be an inverted subject, perhaps similar to what is found in stylistic inversion L'endroit où ont dormi plusieurs enfants/L'endroit où en ont dormi plusieurs. My judgment is that without en cliticization, the reflexive interpretation is marginally possible in (120), that is $I l$ se rasait beaucoup de soldats may only marginally be interpreted as "many soldiers shaved themselves." I would analyze this case as a case of inverted subject being able to bind the reflexive, similarly to cases of stylistic inversion, L'endroit où se rasaient les soldats or impersonal constructions with intransitives as in $I l$ dormait beaucoup d'enfants. Note a problem here with the conjunction of expletive replacement (raising of the object to the expletive subject position at LF) and Belletti and Rizzi's (1988) proposal that Binding Principle A can be satisfied anywhere or with any proposal assuming that the Binding Theory can be satisfied at LF. Expletive replacement in (120) should be able to provide a binder.

56 The marginal character of (126b) suggests the de complement might be marginally analyzable as a PP.

57 We will later argue that (1) lack of agreement correlates with the fact that the postverbal NP does not get structural Case and (2) Burzio's Generalization should be stated as linking lack of external theta role to the subject to lack of structural accusative assignment as suggested in Belletti and Rizzi (1988). Putting the two together entails Kayne's correlation.

58 See also the discussion of agreement with indefinite plural $e n$ in section 5.5.3.

59 Furthermore, neither position can readily be extended to the different behavior of complements of NPs discussed in Section 6.2.

60 For reasons that will become clear in the discussion of genitive Case assignment in Section 6.1.3, I do not accept her specific conclusion that the Case assigned here is inherent. It could still be structural, e.g. nominative, as long as it is not accusative.

61 Note that despite the absence of direct object, Burzio's Generalization makes accusative available, given the presence of an external argument. One line of argumentation would make accusative unavailable because it is in fact always assigned either to an overt object when it is assigned, or to a covert object when it looks unassigned. Although this view is plausible with cases like speak *(to) $J o b n$ (which would then be speak $X_{\text {covert }}$ to Jobn), it appears hard to reconcile with alternating cases like goûter la soupelgoûter à la soupe "taste the soup/taste at the soup" where there is no plausible missing object in the second case.

62 Obviously, the Projection Principle must be amended to permit non-thematic specifiers in lexical projections. 


\section{PARTITIONS AND ATOMS}

63 On that basis, Koopman (1990) also suggests for English and French that Sportiche's (1988a) Q-Float analysis should be modified accordingly by assuming that NP* (or more precisely $\mathrm{DP}^{*}$ ) moved away from $\mathrm{Q}$ actually transits through the specifier position of the $\mathrm{Q}$.

$$
\left.\left[_{\mathrm{NP} *} \text { the children }\right]_{\mathrm{j}}\left[\text { will }\left[\mathrm{L}_{\mathrm{QP}} \mathrm{t}_{\mathrm{j}}\left[_{\mathrm{Q}} \text {, all } \mathrm{t}_{\mathrm{j}}\right]\right] \text { sleep }\right]\right]
$$

This modification is independently proposed in Schlonsky (1990) for Hebrew. All this of course strengthens the movement to specifier analysis.

64 It is not clear that the structure indicated for agreement with a postparticipial $\mathrm{NP}$ is correct. Another option is that NP* is in the accusative/agreement position and the participle has raised higher. However, I will nevertheless suppose that the structure indicated is correct.

65 This construction is also possible without agreement, cf. (150f) below. $C e$ is restricted to appear as a clitic on the verb être/"be": *C'ont été mes amis, ?ce seront mes amis, *'auront été mes amis/"this have been my friends, this will be my friends, this would have been my friends."

66 As mentioned earlier, the two are distinguished by the fact that the inverted NP is an internal argument in object position in the first one, and an external argument in the second one. They are distinguished by a number of syntactic properties, such as en cliticization possible in the first, but not in the second, Q-movement (Il est trop venu d'enfants/? *il a trop dansé de lions).

67 A necessary assumption if theta assignment requires visibility, and visibility requires Case. Otherwise, the theta criterion would be violated at D structure since no chain would have Case.

68 I assume there is no expletive PRO (see for example Sportiche (1983)).

69 Except presumably si, the Italian counterpart to French se, see the discussion in Section 5.4.3.3.

70 The conclusion that the Case of NP* is structural is at odds with Belletti's (1988) conclusions. We argue in Section 6.2 below that lack of Case-marking across a small clause boundary or in ECM contexts does not reduce to inherent Case assignment.

71 A little misleading if, as plausible, Case is a visible property of chains, not of individual NPs.

72 Note that Welsh subjects raise part way as the following sentences illustrate:

(1)(a) Dylai y plant fod wedi bod yn edrych ar y teledu.

Should-3S the children be PERF be at watch at the TV

The children should have been watching television.

(b) Agorodd y dynion ddim y drws.

Opened-3S the men not the door.

The men did not open the door.

In the (a) example, the modal in Infl does not agree with the plural subject which immediately follows it. The thematic subject surfaces in a position governed by Infl, preceding all aspects, not in its base generated position, sister to VP, following all aspects. It must therefore have undergone partial raising. The (b) example shows the same thing with sentence medial negation 


\section{MOVEMENT, AGREEMENT AND CASE}

intervening, instead of aspect. This suggests that Welsh only permits [Spec, IPI as silent expletive. In this respect, this expletive behaves like English there forcing raising of NP* to a position governed by I, with the difference that it does not form a chain, and therefore does not transmit agreement.

73 One last problem for which we have no account is the impossibility of total raising of non-pronominal overt $\mathrm{NPs}_{\mathrm{s}}$ from $\mathrm{NP}^{*}$ to $\mathrm{NP}^{\wedge}$ with French or Italian direct objects, Welsh or Irish subjects.

74 It is possible that there are none. Superficially intransitive verbs not allowing cognate objects might all be unaccusatives as a strong construal of Burzio's Generalization would suggest.

75 Head raising here does not perturb the agreement relationships; cf. Section 5.3 for discussion.

76 Rizzi has suggested that there may be agreement between a $w b$-phrase in [Spec, $\mathrm{CPl}$ and $\mathrm{C}$ (to account for restrictions on the que $\rightarrow$ qui alternation in French), but he argues this turns [Spec, CP] into an A-position.

77 If there is no accusative object, there are two possibilities. If some object needs to move out of VP for Case reasons, it will act as subject. Otherwise, all the specifiers are open and one NP can move out of VP by NP Movement.

78 Consistent with this analysis and with the facts reported in Note 55 is the observation that a reflexive reading is impossible in an "indirect object reflexive impersonal construction" such as Il s'offre des robes à des femmes tous les jours/"It is offered dresses to women every day." Note that in these examples, there is a definiteness effect on both objects (the direct and the indirect object) which casts doubt on Belletti's proposal that this effect is due to the assignment of partitive Case (since the indirect object gets dative here).

79 This implies that in layered Larsonian structures of say a V, no intermediate $\mathrm{VP}$ is ever going to count as a barrier because $\mathrm{V}$ raises to the highest $\mathrm{V}$ position in the layered structure. More generally, for any $\mathrm{X}, \mathrm{XP}$ is going to be a potential barrier only if it also is $\mathrm{X}^{\max }$. In (175), skipping over [Spec, IP]** is possible if I projects to one further IP like IP* because I** raises to I*.

80 Baker and Hale (1990) document such a case, attributing the possibility of the violation of the strict $\mathrm{HMC}$ to the fact that only a relativized minimality (in the sense of Rizzi (1990)) version of the HMC holds whereby intermediate functional projections do not count as intervening between two non-lexical projections. In our account, the RM effect is actually not attributable to a primitive RM condition, but rather derived from lexical selection. Note further that selection of $\mathrm{N}$ by $\mathrm{V}$ violates government in the absence of $\mathrm{N}$ movement to $\mathrm{V}$. If as is reasonable, selection is checked at LF, this suggests that LF incorporation is needed to license government.

81 Note that this proposal of redirecting accusative to NP* differs from that of Baker, Johnson and Roberts (1989) as accusative is assigned to an NP and not to a head. We would take the same approach with passives. How precisely this redirection is to be handled is unclear, given the way in which accusative Case assignment now proceeds.

82 The sentence (184b) improves with indefinite en as in Il en a trop dansé ici, de gens./"It of-them has too much/many danced, people" instead of quantitative en.

83 Left unexplained under this account is the impossibility of French $I /$ se danse as an impersonal middle, whose Dutch Er wordt gedanst and German Es wurde 


\section{PARTITIONS AND ATOMS}

getanzt passive equivalents are grammatical. Possibly, this is related to the necessity of an affected internal argument in the middle.

84 Note that the object cannot be definite. As a tesult, it cannot be a definite pronoun. If it is an indefinite pronoun, it would be instead a Principle B violation. An anaphor should be ruled out too. The case of anaphors however is more complex, due to the various possible interpretations of Principle A satisfaction and their consequences for the distribution of anaphors. Cf. Belletti and Rizzi (1988) for example.

85 The non-passivizability of objects like Bill in Jobn resembles Bill can be attributed to Bill receiving inherent accusative, another such instance in English contrasting with French, in which these NPs receive the dative.

86 The second member of the pair seems to me marginally acceptable with a reflexive reading.

87 Here the reflexive interpretation is totally ruled out, reinforcing the conclusion of Note 54.

88 The impersonal counterpart is only possible with the third person, due to the definiteness effect.

89 All the ses also behave similarly in causative constructions in disallowing climbing, differently from other clitics. Cf. Kayne (1975), Burzio (1986) and Zubizarreta (1982).

90 Although the second of these examples seems marginally less unacceptable.

91 Alternatively, the accusative is unavailable perhaps because it is redirected as in passives, an option that will have to be made compatible both with our treatment of structural accusative Case assignment and our treatment of Burzio's Generalization.

92 In particular, we totally ignore the presence of the particle de, which seems external to the necessary semantic relations realized here (reminiscent of Case) and whose presence raises additional issues (a structural Case treatment similar to accusative would make de more or less external to these "base" structures) that will have to be resolved elsewhere.

93 There is one important difference between genitive en and quantitative en. Cliticization of genitive $e n$ is possible from a derived subject, e.g. La cheminée en est pencbée/"The chimney of it is tilted" but cliticization of quantitative en is not possible. *Trois en sont penchées/"Three of them are tilted." Milner (1978) notes that even though these examples are not clear, it seems that the partitive $e n$ behaves more like ordinary $e n$ and not like quantitative en as in Ces pommes, beaucoup en sont gatées/"These apples, many of them are rotten." My own judgments are contrary and treat such sentences as on a par with quantitative en sentences.

94 The fact that both $I l$ a beaucoup été peint de portes./Il a été beaucoup peint de portes are possible, with incorporation to the participle in an unaccusative structure lacking participle raising supports the idea that the postverbal NP is not accusative, and therefore lower than either participle. Indeed, recall that the lack of external argument in these cases would imply, if Burzio's Generalization was incorrect, that the accusative position is the unique [Spec, IP] present in the participle projection which is higher than the participle and should disallow incorporation from it into the participle.

95 And so do the following in which the $Q$ is the totality of the QP constituent. 
(1)(a) J'ai donné tout à tous les enfants.

(b) J'ai tout donné à tous les enfants.

(c) Je leur ai tous donné tout.

(d) Je leur ai tous tout donné.

(e)* Je leur ai tout tous donné.

(2)(a) J'ai donné toute ma confiance à tous les enfants.

(b) Je l'ai toute donné à tous les enfants.

(c) Je leur ai tous donné toute ma confiance.

(d) Je la leur ai tous toute donné.

(e)* Je la leur ai toute tous donné.

96 Note that the distributional facts are quite complex with the frequency reading of beaucoup, trop. Thus, beaucoup, trop behave distributionally like NP quantifiers and unlike frequency adverbs. We find the following contrasts, suggesting that "pure" frequency adverbs systematically occur higher than the beaucoup type.

(1)(a) J'ai beaucoup/rarement parlé à tous les enfants.

(b)* Je leur *beaucoup/OK rarement tous parlé.

(c) Je leur ai tous beaucoup/*rarement parlé.

Furthermore, although the (a) sentence with beaucoup is ambiguous between speak often and speak a lot, the (c) sentence only has the latter reading.

97 Note that we cannot link $A_{G} R_{S}$ with external argumenthood either, as shown by the case of subjects derived from underlying objects.

98 That is a predicate. All its arguments and the entire A-chain contain its external argument. A-chain closure for all its arguments would not block the sentence (i) discussed above and similar sentences.

99 I will take this projection to be headed by the particle-s in English.

100 Although either the adjunction option (to DP), or the substitution (to [Spec, DP]) option can derive the results we want, I will adopt the substitution alternative, which Valois (1991) suggests (over my own previous view of extraction under adjunction to DP). There are several reasons for this as Valois (1991) points out. One is Szabolzsi's (1983) Hungarian evidence that noun phrases should contain a Comp-like position. Another is Tellier's (1990) analysis of double dont constructions in French to the same effect. A last one is Valois' (1991) argument that (an otherwise desirable) complete parallelism between the internal structure of clauses and the internal structure of noun phrases can explain many properties of the internal syntax of NPs/DPs.

101 Of course, in Engish, the question arose of why *Wbose did you see $t$ book is out or perhaps most tellingly - since who $+s$ is not a constituent - why the sentence Who did you see t's book is out.

102 Note that raising of $\mathrm{N}$ to $\mathrm{D}$ is what seems to happen in languages having the so-called construct state (Semitic languages), the associative construction (various African languages). This is present in earlier stages of French as in the place-name Bois-le duc = le bois du Duc/"The Duke's woods." 


\section{PARTITIONS AND ATOMS}

\section{References}

Abney, S. (1987) "The noun phrase in its sentential aspect," unpublished Ph.D. dissertation, MIT.

Aoun, J. (1984) The Logical Nature of Anapboric Relations, Cambridge, Mass.: MIT Press.

Aoun, J. and Sportiche D. (1983) "On the formal theory of government," The Linguistic Review 2: 211-36.

Baker, M. (1988a) Incorporation, Chicago, Ill.: University of Chicago Press.

- (1988b) "Theta theory and the syntax of applicatives in Chichewa," Natural Language and Linguistic Theory 6: 353-89.

Baker, M. and Hale K. (1990) "Relativized Minimality and Pronoun Incorporation," Linguistic Inquiry 21: 289-98.

Baker, M., Johnson K. and Roberts I. (1989) "Passive arguments raised," Linguistic Inquiry 20: 219-51.

Baltin, M. (1981) "A Landing Site Theory of Movement Rules," Linguistic Inquiry 13.1: $1-38$.

Belletti, A. (1988) "The case of unaccusatives," Linguistic Inquiry 19: 1-34.

Belletti, A. and Rizzi L. (1988) "Psych verbs and theta theory," Natural Language and Linguistic Theory 6: 291-352.

Bouchard, D. (1984) On the Content of Empty Categories, Dordrecht: Foris.

Bresnan, J. and Moshi L. (1990) "Object asymmetries in comparative Bantu syntax," 21: $147-85$

Burzio, L. (1986) Italian Syntax, Dordrecht: Reidel.

Campbell, R. (1989) "The grammatical structure of verbal predicates," unpublished $\mathrm{Ph} . \mathrm{D}$. dissertation, UCLA.

Carstens, V. and Kinyalolo K. (1989) "On IP structure: tense, aspect and agreement," unpublished manuscript, Cornell University and UCLA.

Chomsky, N. (1970) "Remarks on nominalizations," Studies in Semantics in Generative Grammar, The Hague: Mouton.

- (1973) "Conditions on Transformation," in S. Anderson and P. Kiparsky (eds) A Festschrift for Morris Halle, New York: Holt, Rinehart and Winston, 232-86. - (1976) "Conditions on rules of grammar," Linguistic Analysis 2: 303-51. (1981) Lectures on Government and Binding, Dordrecht: Foris Publications. (1986a) Barriers, Cambridge, Mass.: MIT Press.

- (1986b) Knowledge of Language: Its Nature, Origin, and Use, New York: Praeger.

(1989) "Some notes on economy of derivation and representation," in I. Laka, and A. Mahajan (eds) Functional Heads and Clause Structure, MIT Working Papers in Linguistics 10: 43-74.

Cinque, G. (1980) "On extraction from NP in Italian," Journal of Italian Linguistics 5: 47-99.

- (1992) "Functional projections and N Movement within the DP," paper presented at 15th GLOW Colloquium, GLOW Newsletter 28, 12-13.

Cortes, C. (1992) "Issues in Catalan Syntax," doctoral dissertation, UCLA.

Deprez, V. (1988) "Stylistic inversion and verb movement," unpublished manuscript, MIT.

Emonds, J. (1976) A Transformational Approach to English Syntax, New York: Academic Press. 
(1985) A Unified Theory of Syntactic Categories, Dordrecht: Foris.

Giorgi, A. and Longobardi, G. (1991) The Syntax of Noun Phrase Configurations: Parameters and Empty Categories, Cambridge: Cambridge University Press.

Grevisse, M. (1980) Le Bon Usage, 11ème édition, Paris-Gembloux: Éditions Duculot.

Guéron, J. and Hoekstra T. (1988) "T-chains and the constituent structure of auxiliaries," in A. Cardinaletti, G. Cinque and G. Giusti, (eds) Constituent Structure, Dordrecht: Foris.

Haik, I. (1982) "On clitic en in French," Journal of Linguistic Research 2.

Hale, K. and McCloskey J. (1984) "On the syntax of person-number inflection in Modern Irish," Natural Language and Linguistic Tbeory: 487-533.

Huang, C.-T. J. (1982) "Logical Relations in Chinese and the Theory of Grammar," doctoral dissertation, MIT.

- (1984) "On the distribution and reference of empty pronouns," Linguistic Inquiry 15.4: 531-75.

— (1993) "Reconstruction and the structure of VP," Linguistic Inquiry 24.1: $103-38$.

Jaeggli, O. (1986) "Passive," Linguistic Inquiry 17: 587-622.

Jaeggli, O. and Safir K. (1989) The Null Subject Parameter, Dordrecht: Kluwer.

Johnson, K. (1990) "Object positions," manuscript published as Johnson (1991). (1991) "Object positions," Natural Language and Linguistic Theory 9: 577-636.

Kayne, R. (1975) French Syntax: the Transformational Cycle, Cambridge, Mass.: MIT Press.

(1984) Connectedness and Binary Brancbing, Dordrecht: Foris Publications.

— (1985) "L'accord du participe passé en français et en italien," Modèles linguistiques 7: 73-80.

- (1986) "Principles of particle constructions," in J.-Y. Pollock and J. Guéron, (eds) Levels of Syntactic Representations, Dordrecht: Foris.

- (1988) "Romance se/si," paper given at GLOW conference, Budapest, GLOW Newsletter 20.

- (1989a) "Facets of Romance past participle agreement," in P. Beninca (ed.)

Dialect Variation and the Theory of Grammar, Dordrecht: Foris Publications. - (1989b) "Null subjects and clitic climbing," in O. Jaeggli and K. Safir, (eds) The Null Subject Parameter, Dordrecht: Kluwer.

(1991) "Romance clitics, verb movement and PRO," Linguistic Inquiry: 22.4.

(1993) "Toward a modular theory of auxiliary selection," Studia Linguistica 47 (1): $3-31$.

Koster, J. (1978) "Why subject sentences don't exist," in S. J. Keyser (ed.) Recent Transformational Studies in European Languages, Cambridge, Mass.: MIT Press 53-64.

Keyser, S. J. and Roeper T. (1984) "On the middle and ergative constructions in English," Linguistic Inquiry 15: 381-416.

Kinyalolo, K. (1990) "Syntactic dependencies and the spec/head agreement hypothesis in Kilega," unpublished doctoral dissertation, UCLA.

Koopman, H. (1984) The Syntax of Verbs: from Verb Movement rules in the Kru Languages to Universal Grammar, Dordrecht: Foris Publications.

(1987) "On the absence of case chains in Bambara," unpublished manuscript, UCLA, published as Koopman (1992). 


\section{PARTITIONS AND ATOMS}

(1988) "The Internal Structure of Dutch VPs," lecture(s) given at the University of Geneva, Tilburg Catholic University and MIT.

- (1990) "The verb particle construction and the syntax of PPs," unpublished manuscript, UCLA.

— (1992) "On the absence of case chains in Bambara," Natural Language and Linguistic Theory 10: 555-95.

- (1995) "The structure of Dutch PPs," unpublished manuscript, UCLA.

— (forthcoming) "The structure of Dutch PPs," now published as Koopman (1995).

Koopman, H. and Sportiche D. (1985) "Theta theory and extraction," in GLOW Newsletter 14: 57-88, reprinted in this volume as Chapter 8.

(1988) "Subjects," published as Koopman and Sportiche (1991).

(1991) "The position of subjects," Lingua 85(2/3): 211-85, reprinted in this volume as Chapter 2.

Larson, R. (1985) "On the syntax of disjunction scope," Natural Language and Linguistic Theory 3: 217-64.

_ (1985) "Bare-NP adverbs," Linguistic Inquiry 16: 595-621.

(1988) "On the double object construction," Linguistic Inquiry 19: 335-92.

Lasnik, H. (1985) "Illicit NP movement: locality conditions on Chains?," Linguistic Inquiry 16(3): 481-90.

Lewis, D. (1975) "Adverbs of Quantification," in E. Keenan (ed.) Formal Semantics of Natural Languages, Cambridge: Cambridge University Press.

Longobardi, P. (1987) "Extraction from NP and the proper notion of head government," unpublished manuscript, Scuola Normale Superiore, Pisa.

Mahajan, A. (1990) "The A/A-bar distinction and movement theory," unpublished Ph.D. dissertation, MIT.

Manzini, R. (1983) "Restructuring and Reanalysis," unpublished doctoral dissertation, MIT.

Marantz, A. (1984) On the Nature of Grammatical Relations, Cambridge, Mass.: MIT Press.

(1990) "Implications of asymmetries in double object constructions," published as Marantz (1993).

- (1993) "Implications of Asymmetries in Double Object Constructions," in S.A. Mchombo (ed.) Theoretical Aspects of Bantu Grammar, vol 1, Stanford: CSLI Publications, CSLI Lecture Notes No. 38, 113-50.

May, R. (1985) Logical Form, Cambridge, Mass.: MIT Press.

Milner, J.-C. (1978) De la syntaxe à l'interprétation, Paris: Le Seuil.

Mohammad, M. (1989) "The Sentence Structure of Arabic," unpublished doctoral dissertation, University of Southern California, Los Angeles.

Pollock, J.-Y. (1984) "Accord, chaines impersonnelles et variables," Linguisticae Investigationes 7: 131-81.

— (1989) "Verb movement, universal grammar and the structure of IP," published as Pollock (1990).

- (1990) "Verb movement, universal grammar and the structure of IP," Linguistic Inquiry 20: 365-424.

Riemsdijk, H. van. (1978) A Case Study in Syntactic Markedness; the Binding Nature of Prepositional Phrases, Dordrecht: Foris Publications.

Rizzi, L. (1982) Issues in Italian Syntax, Dordrecht: Foris Publications. 
(1986a) "On chain formation," in H. Borer (ed.) The Syntax of Pronominal Clitics, Syntax and Semantics 19, San Diego, CA: Academic Press.

(1986b) "Null objects in Italian and the theory of pro," Linguistic Inquiry 17: 501-58.

(1990) Relativized Minimality, Cambridge, Mass.: MIT Press.

Rizzi, L. and Roberts, I. (1989) "Complex inversion in French," Probus 1: 1-30.

Ruwet, N. (1972) Thérie syntaxique et syntaxe du français, Paris: Le Seuil.

Saito, M. (1984) "On the Definition of C-command and Government," in C. Jones and P. Sells (eds) Proceedings of the 14th NELS Conference, University of Massachusetts, Amherst.

Schlonsky, U. (1990) "Quantifiers as heads: a study of Quantifier float in Hebrew," unpublished manuscript, Haifa University.

Sportiche, D. (1981) "Bounding Nodes in French," The Linguistic Review 1(2): 219-46.

- (1983) "Structural invariance and symmetry in syntax," doctoral dissertation, MIT, distributed by the MIT Working Papers in Linguistics.

(1987) "Unifying movement theory," unpublished manuscript, UCLA.

(1988a) "A theory of floating quantifiers and its corollaries for constituent structure," Linguistic Inquiry 19(3): 425-49, reprinted in this volume as Chapter 1.

(1988b) "Conditions on silent Categories," unpublished manuscript, UCLA.

Stowell, T. (1981) "The origins of phrase structure," unpublished Ph.D. dissertation, MIT.

(1983) "Subjects across categories," The Linguistic Review 2: 285-312.

(1988) "Determiners in NP and DP," manuscript published as Stowell (1991).

- (1991) "Determiners in NP and DP," in K. Leffel and D. Bouchard (eds) Views on Pbrase Structure, Dordrecht: Kluwer, 37-56.

Szabolcsi, A. (1983) "The possessor that ran away from home," The Linguistic Review 3: 89-102.

Tellier, C. (1990) "Universal licensing," published as Tellier (1991). (1991) Licensing Theory and French Parasitic Gaps, Dordrecht: Kluwer.

Travis, L. (1984) "Parameters and effects of word order variations," unpublished doctoral dissertation, MIT.

Valois, D. (1991) "The Internal Syntax of DP," unpublished doctoral dissertation, UCLA.

Vergnaud, J.R. (1974) “French Relative Clauses," unpublished doctoral dissertation, MIT.

Zubizarreta, M.-L. (1979) "Extraction from NP and a reformulation of subjacency," unpublished manuscript, MIT.

— (1982) "On the relationship of the lexicon to syntax," unpublished doctoral dissertation, MIT. 


\title{
CLITIC CONSTRUCTIONS*
}

\begin{abstract}
An analysis of French and Romance clitic pronominal constructions in simple clauses is proposed, reconciling the two dominant approaches on this topic. This analysis treats them as involving both movement and base-generation and, as part of a larger research program, assimilates the syntax of clitics to that of other functional heads. Accordingly, a clitic is analyzed as heading its own projection and as licensing in its specifier a particular property of a designated argument agreeing with it in the relevant features (person, number, gender, Case, etc.). It is further argued that clitics subdivide into two types. The first type (such as French en or $l e$ ) assimilates to such functional heads as $[+w b]$ complementizers or $[+$ negative $]$ heads licensing certain operator-like properties (e.g., wh or negative quantifiers). We show that (some) Germanic Scrambling is fundamentally similar to these clitic constructions. This leads us to postulate that the operator-like property these clitics license is specificity in DPs as has often been proposed, but that reciprocally the specificity of some DPs must be licensed in similar clitic-like configurations. In essence, this comes down to assimilating pronouns to bare operators (like bare $w h$-phrases who, what, why), and specific DPs to non-bare operators with operator determiners (like which book, what reason, etc.). The second type of clitics, such as French lui, are not linked to specificity. We suggest that these clitics should be analyzed as pure agreement, analogous to $A G R_{0}$ or $A G R$, presumably responsible for Case assignment (here dative, i.e., $A G R_{10}$ ).
\end{abstract}

\section{Introduction}

\subsection{Preamble}

The existence of pronominal clitics in languages like French and other (Romance) languages raises two questions, one of descriptive adequacy and one of explanatory adequacy. The question of descriptive adequacy asks what 


\section{CLITIC CONSTRUCTIONS}

the correct structural analysis of constructions with these pronominal clitics is. How to structurally analyze clitics has been the subject of very substantial discussion ever since the earliest studies of the late 1960s, and much disagreement remains. I want to suggest an analysis that reconciles two main, but conflicting, views on this problem.

The question of explanatory adequacy asks why language learners choose the analysis of pronominal clitics they do. This kind of question is the most central theoretical question generative linguists such as myself can ask about the analyses they propose; their field is defined by the fact that they ask these questions. It is unlikely that learners go through the kind of deductive reasoning that linguists go through; years of clever debate have not yet produced a consensus on the matter of clitics. How is it that learners reach such uniform conclusions on such complex systems on the basis of much more limited evidence than is available to linguists? It appears plausible to assume that the range of analyses that they can entertain at all is so restricted to start with that the choice is easy to make on the basis of the data available to them. In fact, in the extreme, it is conceivable that this range is a singleton. This most extreme position is the one I am going to suggest. Although I hope to substantiate this claim empirically, I independently believe that some such extreme narrowing of a priori possibilities is necessary; even the kind of extremely restrictive theories of syntactic structures proposed today allow so many possible syntactic analyses (possibly infinitely many) of any finite set of data as to make learning untractable (see Sportiche (1995)).

At the most general level, I want to suggest that the kind of structural analysis that can be ascribed to clitic constructions is identical to those that can be ascribed to $w b$-constructions, negative quantifier constructions, focus constructions, and almost every other construction that can reasonably be argued to involve XP-movement (NP or DP movement constructions, lexical anaphor constructions, quantifier scope constructions, etc.), because there is only one kind of structural analysis available for these constructions in principle, namely movement demanded by specifier/head licensing. Substantiating this approach requires establishing its plausibility and accomplishments for each individual construction. This is what I will do for clitic constructions in this paper. The general theoretical approach this is a part of is discussed in more detail in Sportiche (1995) and some consequences for subject clitics are more specifically discussed in Sportiche (1993).

\subsection{Boundary condition on analyses}

Every analysis of clitics must account for the fact that the items treated as clitics by French are treated as clitics by French. This is another side of the question of explanatory adequacy previously discussed This very strongly suggests a fundamentally uniform analysis of clitics. For if universal grammar allows pronominal clitics to be analyzed in one of several ways the question of 
converging on the right analysis for each individual case arises again together with the problem of underdetermination of the theory by the evidence. Although a uniform approach to the analysis of clitics is not forced, it is clearly more desirable a priori and I will adopt it as a working hypothesis. As we will see, this seems largely justified a posteriori. As will be clear, I also take as point of departure the idea that this uniformity also holds true crosslinguistically, at least as far as the most analyzed Romance languages such as Italian and Spanish are concerned. Work on these languages has revealed enough similarity of functioning with each other and French clitic constructions to warrant this assumption. How uniform can these analyses be? Surely they cannot be uniform to the point of identity (since pronominal clitics do behave differently). Minimally, we may assume that clitics are uniformly analyzed up to lexical differences. One important consequence of this assumption is the following: it is reasonable to argue for or against particular proposals concerning the analyses of pronominal clitic constructions by bringing any pronominal clitic to bear on the question (again up to lexical differences).

\subsection{Outline of the central proposal}

There are strong arguments in favor of each of the two widely held, and apparently incompatible, types of analysis of Romance clitics found in the literature. Illustrating with a French accusative clitic, clitics are argued to be either base-generated in their surface position (Strozer (1976), Rivas (1977), Jaeggli (1982), Borer (1983), Sportiche (1983)) or moved from the underlying XP* position (Kayne (1975), Kayne (1989a), Sportiche (1990)):

(1) Marie les aura présentés $\mathrm{XP}_{i}$ * à Louis.

Mary them will-have introduced-AGR ${ }_{\mathrm{i}}$ to Louis.

Mary will have introduced them to Louis.

Proponents of movement analyses that have held that movement, but not base-generation, can explain the condition of locality holding between the clitic and $\mathrm{XP}^{*}$ and are reminiscent of conditions on A-movement (traditionally attributed to the Binding Theory). Base-generation has been argued to be unlike movement in being compatible with the lack of complementarity between clitic and a full XP* (clitic doubling constructions). Although I do not consider the argumentation based on this observation convincing, as I discuss below, I will offer some reasons to support its conclusions.

These properties suggest that both movement and base-generation are correct and this is what I will propose. Specifically: (1) All clitics are always base-generated in preexisting slots, namely as $\mathrm{X}^{0}$ heading their own projection and (2) clitic constructions may also involve movement. Illustrating the basic idea with (1), I suggest that the accusative clitic is a base-generated 


\section{CLITIC CONSTRUCTIONS}

head, call it Acc, selecting as its specifier an accusative DP*. This selection must be satisfied by LF by moving $\mathrm{DP}^{*}$ to $\mathrm{Spec}, \mathrm{AccP}=\mathrm{DP} \wedge$, as an instance of Spec/head licensing:

$$
\left[_{\text {AccP }} D^{\wedge}\left[\left[_{\text {Acc }} \text { les }\left[\ldots \text { aura présentés } D P^{*}{ }_{+ \text {acc }} \ldots\right]\right]\right]\right.
$$

I will show how this approach reconciles the properties listed above and many others and can provide a unified analysis of clitics. This will lead to related proposals concerning clitic doubling, the treatment of participle agreement and the distribution of stranded quantifiers. I will also discuss why this approach is superior to recent alternatives such as Kayne's (1989a) (clitic movement as successive head movement) and supports a version of Sportiche's (1989) or (1990) approach (clitic movement as XP movement followed by incorporation of X). Finally, we will propose that overt clitic morphemes split into two classes. Members of the first class, which include accusative clitics, are similar to $[+w b]$ complementizers or the head of $\mathrm{NegP}$ and characterize constructions resembling operator/variable configurations, which license parasitic gaps. We will propose that the presence of these clitics is a necessary and sufficient condition to license specificity in their associated argument phrase. We analyze accusative Scrambling (in Dutch - and that should extend to German) as an instance of a clitic construction as in (2) with $\mathrm{XP}^{\wedge}$ filled and the head silent. Members of the second class, like dative clitics, behave like Agreement Phrases. The projections they head are thus $A_{G} \mathrm{GR}_{\mathrm{IO}}$ responsible for dative Case, much in the same way that $A_{G} R_{S}$ and $A G R_{O}$ are responsible for nominative and accusative Case properties.

\section{Some preliminaries about French clitics and clitics in general}

French (representative of many Romance languages) has a rich system of preverbal clitics. Their surface distribution is summarized by the template below:

$$
\begin{aligned}
& \text { French clitic template } \\
& \text { nom neg 1st/2nd refl 3rdacc 3rddat loc gen } \\
& \text { il ne me/te/se/nous le/la/les lui/leur y en }
\end{aligned}
$$

As Perlmutter (1972) describes it, for each column of the template, no more than one clitic is allowed (so for example, it is not possible to have a first person accusative object and a second person dative object at the same time). Furthermore, the third and fifth columns cannot be filled at the same time (e.g. * Je me lui montrel"I show myself to him"). Why there are these vertical cooccurrence restrictions (only one clitic per slot), these horizontal co-occurrence 
restrictions (not both third and fifth) and why the clitics are ordered this way is not understood, and I have no light to shed on these questions.

Syntactic clitics are so called because they are claimed not to constitute autonomous syntactic units. Rather, they seem to form a unit with some host. Kayne (1975) argues that pronominal clitics adjoin to V in French (a conclusion with substantial validity across Romance that Kayne (1991a) refines by claiming that clitics adjoin to a functional category to which a verb may move). This is exemplified by the fact that they cannot be conjoined independently of their host nor can their host be conjoined independently of them:

(4)(a)* Jean le et les connaît. John him and them know. John knows him and them.

(b)* Jean le connaît et/ou respecte. John him knows and/or respects. John knows and/or respects him.

These properties are not true for all clitics. Subject clitics (except for on, ce "one," "it/that/this") alone allow the equivalent of (4b), and other clitics which in principle may tolerate conjunction (because there are several different instances occurring in the same clitic slot) allow disjunction:

(5)(a) Il connaît et respecte Marie. He knows and respects Mary. He knows and respects Mary.

(b) Il ou elle connaît Marie. He or she knows Mary. He or she knows Mary.

(c) ?Pierre le ou les renverra. Peter him or them will-see. Peter will see him or them.

As Rizzi (1986b) points out, facts like (5a) as opposed to (4b) indicate that the cluster of clitics is not homogeneous (and unstructured), and that there is a break, which he places after the negative clitic ne. In particular, this seems to indicate that subject clitics do not have to be syntactic clitics at all (although they may become syntactic clitics under certain circumstances, such as in Complex Inversion constructions, cf. Sportiche (1993), and they look like phonological clitics, being devoid of possible stress). The significance of examples of the (5c) type in parallel with (4b) is less clear. We will continue assuming that non-subject pronominal clitics are syntactically cliticized, noting the potential problem raised by (5c) (that is discussed in Sportiche (1993)). 


\section{CLITIC CONSTRUCTIONS}

In non-imperative clauses, the string of clitics immediately precedes the highest verb of its clause. In particular, it will always precede the highest auxiliary of its clause. This is illustrated below:

(6)(a) Il le lui donnera.

$\mathrm{He}$ it to-him will-give.

He will give it to him.

(b) Il le lui a donné.

He it to-him has given.

He gave it to him.

(c) Ils lui ont été donnés.

They to-him have been given.

They were given to him.

(d) Il ne le lui donnera pas.

He neg it to-him will-give not.

He will not give it to him.

(e) Ils ne lui ont pas été donnés.

They neg to-him have not been given

They were not given to him.

In infinitives, we find more evidence of the split in the clitic cluster noted by Rizzi (1986b) and mentioned above. The negative clitic may be separated from subsequent clitics and precede the negative marker pas, all other clitics will immediately precede the verb and follow pas.

(7)(a) ne pas le lui donner/*ne le lui pas donner neg not it to-him giveINF to not give it to him

(b) ne pas l'avoir donné/ ne l'avoir pas donné neg not it have given to not have given it to him

(c) ne pas leur en avoir parlél??ne leur en avoir pas parlé neg not to-them of-it have talked to not have talked to them about it

(d) ne pas m'en avoir parlé neg not to-me of-it have talked to not have talked about it to me

\section{Types of previous analyses}

Previous analyses of the distribution of clitics can be broadly organized in two subclasses: lexical analyses and syntactic analyses. Lexical analyses in effect claim that a clitic is a derivational affix modifying the lexical entry of a predicate. To illustrate, the alternation between lire un livre "to read a book" 
and le lire "to read it" would be one between a transitive verb lire and an intransitive le+lire. Initially, there is what appears to be a technical problem due to the fact that this derivational affix does not actually always show up on the verb that it intransitivizes (cf. Section 2 above). In other words, a lexical analysis claims that adding a clitic in a clause is an operation on the lexical entry of some lexical item. However, since there are many cases in which a clitic appears on a verb to which it bears no lexical relation, this will lead to considerations strongly disfavoring this option. By the principle of uniformity of analysis discussed in Section 1.2 above, this dismisses these analyses altogether. The examples below illustrate this point:

(8)(a) Jean croit Pierre malade/ le croit malade. John believes Peter sick/ him believes sick. John believes that Peter/he is sick.

(b) Jean est semblable à sa mère/ lui est semblable. John is similar to his mother/ to-her is similar. John is similar to his mother/her.

(c) Jean croit Pierre friand de tout/en croit Pierre friand. John believes Peter fond of all/of-it believes Peter fond. John believes Peter fond of everything/it.

(d) Jean veut manger la pomme/ la veut manger. John wants to-eat the apple/ it wants to-eat. John wants to eat the apple/it.

(e) Jean a peint la cheminée de l'usine/en a peint la cheminée. John has painted the chimney of the factory. John painted the chimney of the factory/its chimney.

In example (8a), the clitic stands for an argument DP subject of a small clause bearing no thematic relation, and hence no lexical relation, with the main verb. Although it is possible to devise theories in which a DP is subcategorized by a predicate without being theta marked by it - hence bearing a lexical relation with it - such theories make it a mystery where subcategorization comes from and would require far stronger evidence than has ever been adduced in their favor to make them reasonable initial candidates. At any rate, none of the other examples could be so fixed. In examples $(8 b, c)$, the clitic is an argument of the adjective and bears no lexical relationship to the main verbs. In (8d), the so-called restructuring constructions (an example which is ungrammatical in standard French but well formed in middle French and its counterparts in many (all?) varieties of Italian or Spanish), the clitic bears no lexical relationship to the verb it is affixed to. Instead, if anything, it might be argued to be lexically related to the embedded verb. In (8e), the clitic stands for a dependent of the head of the object DP. All these cases duplicate the central observation made earlier regarding the basic distribution of clitics: clitics appear on the highest verb 


\section{CLITIC CONSTRUCTIONS}

of their clause (with provisions made for restructuring constructions). This essentially correct generalization is syntactic in nature and thus seems incompatible with a strictly lexical approach. At best, the detransitivization property would have to be coupled with a syntactic device ensuring that the clitic morpheme shows up in its proper place. Of course, this is not to deny that there may be a lexical component to the distribution of clitics, such as modification of Case properties of the affected predicate, but we must conclude that the $\mathrm{CL}+\mathrm{V}$ sequence is not an intransitive verb.

In fact, turning now to syntactic analyses found in the literature, they all more or less have a lexical component to them. Syntactic analyses have typically assumed that the presence of a pronominal clitic, say CL, standing for some argument, say $A$, does not affect the thematic properties of the predicate $\mathrm{Y}$ taking $\mathrm{A}$ as argument. Consequently, by the Projection Principle, $A$ is syntactically represented as some phrase $\mathrm{XP}^{*}$ generated in its usual position. This conclusion seems warranted: a transitive verb as we just saw remains transitive even if there is a clitic "standing for" its direct object. The question is to make explicit what "stand for" means in structural terms and this is what the Projection Principle provides in part: clitic constructions obey the general schema (from now on, we will use XP* to refer to the argument position associated with a clitic):

$$
\ldots \mathrm{CL}_{\mathrm{i}} \ldots\left[\mathrm{Y} \ldots \mathrm{XP}{ }_{\mathrm{i}} \ldots\right] \ldots
$$

For these syntactic analyses, the questions raised by clitic constructions have mostly concerned the nature of XP* and the properties of the relation between $\mathrm{CL}$ and XP*. Again, broadly speaking, past syntactic analyses can be grouped in two subsets: base-generation analyses and movement analyses. According to the base-generation analyses, the clitic is essentially basegenerated in its surface position and $\mathrm{XP}^{*}$ is to be analyzed as a pro or a PRO somehow related to the clitic. According to movement analyses, $\mathrm{XP}^{*}$ is to be analyzed as a trace. The second position is usually correlated to the assumption that $\mathrm{XP} *$ is literally the trace of $\mathrm{CL}$, a non-necessary assumption (as discussed in Sportiche (1990) and below). Let us survey these two proposals.

\section{Movement and base-generation}

The movement analysis is probably the oldest generative analysis and one that is adopted in Kayne $(1975 ;$ 1989a) and Sportiche $(1989$; 1990). Basegeneration analyses are probably most widely held today in one form or another. They are advocated by Strozer (1976), Rivas (1977), Jaeggli (1982), Borer (1983, Bouchard (1982), Burzio (1986) or Roberge (1990) (see Borer (1986) for a survey and references) for essentially the reasons discussed below in Section 4.1 and in Sportiche (1983) for somewhat different reasons not reviewed here. ${ }^{1}$ We now briefly survey the motivations for each approach. 


\subsection{Complementary distribution}

One simple motivation for Kayne's (1975) movement analysis is the apparent complementary distribution between clitics and their associated $\mathrm{XP}^{*}$ found in French illustrated below, where the (c) sentence contains one intonational phrase (i.e., no dislocation intonation, pause, etc.):
(10)(a) Marie connaît Louis.
Mary knows Louis.
Mary knows Louis.
(b) Marie le connaît.
Mary him knows.
Mary knows him.
(c)* Marie le connaît (à) Louis.
Mary him knows to Louis.
Mary him knows (to) Louis

Kayne (1975) suggests that clitics are base-generated in the position XP* and are Chomsky-adjoined by a movement operation to an appropriate verb up the tree. Almost all the authors challenging Kayne's proposal have done so on the basis of the existence of clitic doubling constructions found in varieties of French (Roberge (1990)), Spanish (Strozer (1976), Rivas (1977), Jaeggli (1982)), Romanian, Hebrew, Arabic (Aoun (1981), Borer (1983)) and exemplified below for Romanian and River Plate Spanish:

\section{(11)(a) L-am vazut pe Popescu. Him-have-I seen OM Popescu. I have see Popescu. \\ (b) Lo vimos a Juan. \\ Him saw-we to John. We saw John.}

In these constructions, both a clitic and a full phrase - the doubled phrase seem to compete for the same grammatical function. In itself, this observation does not pose any particular challenge to Kayne's proposal. However, an analysis of these facts claiming that the doubled DP occupies XP* would appear fundamentally incompatible with a movement analysis. It is, however, quite difficult to conclusively establish this point. The position of the doubled $\mathrm{XP}^{*}$ has been the subject of substantial debate, because of its assumed implications for the movement analysis. In general, however, these discussions have concentrated on the external distribution of the doubling element. In order to preserve the movement analysis, one may try to claim that the doubled element is not $\mathrm{XP}^{*}$ but rather an adjunct related to $\mathrm{XP} *$. For example, Hurtado (1984) defends the idea that the doubled XP in (11b) occupies the 


\section{CIITIC CONSTRUCTIONS}

position of right dislocated phrases. Aoun (1981) defends a variant of this position. Jaeggli (1986) convincingly argues against these proposals, although he does not generally settle the wider question of whether or not the doubled XP is in an adjunct position (he only argues that they are not in dislocated position). However, as Pat Schneider (personal communication) remarks for Greek, doubled elements seem to occur in positions in which adjuncts are simply not tolerated, e.g., as subject of small clauses or ECM subjects:

o Yiorghos tin-perimene [[tin Maria] na paraponiete] the George $\mathrm{CL}_{\text {acc }}$ expected the Mary ${ }_{\text {acc }}$ subj complain George expected Mary to complain.

This evidence concurs with Jaeggli's conclusions that the doubled element is not an adjunct and in fact has the same external distribution as XP*. From this it is quite reasonable to conclude that the doubling element is indeed $\mathrm{XP} *$. The fact that the doubled phrase has the same external distribution as the missing argument does not suffice, however, to establish the point. Indeed, it is conceivable that the doubled element may be in fact an adjunct as in (1) or a YP specifier as in (2) to a silent $\mathrm{X}^{0}$, head of XP* as illustrated below which would in effect further specify the intended content of the clitic element:

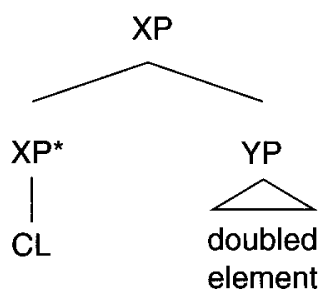

(2)

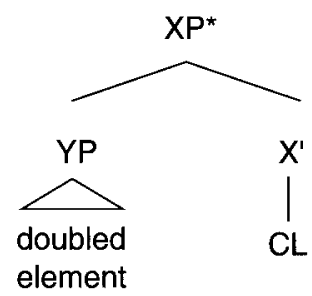

If this were the case, we would expect this doubled element to have the same external distribution as XP*. Until this question is settled (a non-trivial matter), the movement approach is not challenged by the existence of clitic doubling constructions. Nor is it particularly supported by the appearance of complementarity in some cases. Although complementarity of distribution between clitics and $\mathrm{XP} *$ might have provided an argument for a movement analysis, there may be other ways of handling this complementarity inspired by the treatment of clitic doubling constructions, particularly, as many authors have done, by capitalizing on "Kayne's generalization." Kayne observes that, in some languages and in some constructions at least, doubled elements must appear affixed by a morpheme (dative-looking in Spanish, the object marker pe in Romanian) that does not appear in the absence of the clitic. One idea pursued by the proponents of base-generation analyses 
attributes the presence of this morpheme on $\mathrm{XP}^{*}$ to the fact that the Case which XP* would normally receive is lost ("to the clitic"). Requiring Case for clitics will yield complementarity effects in languages lacking these rescuing devices, such as insertion of a special morpheme.

\subsection{Movement properties}

There are however much stronger arguments adduced in favor of a movement analysis. The strongest such arguments are based on the observation that the clitic/XP* relation displays defining distance properties of movement. We document these properties below in non-doubled cases, strongly suggesting that movement is involved.

\subsubsection{Specified subject condition (SSC) effects}

One type of evidence Kayne (1975) used to corroborate his conclusions capitalized on the blocking effects of intervening subjects on (some type of) clitic placement (we will use clitic placement preanalytically, i.e., to refer to the surface distribution of a clitic, without taking a stand on the mechanism responsible for it). One simple case is illustrated below:

(14)(a) Jean a laissé Pierre parler à Marie. John has let Peter to-speak to Mary. John let Peter speak to Mary.

(b) Jean l'a laissé lui parler. John him has let to-her to-speak John let him speak to her.

(c)* Jean lui a laissé Pierre parler. John to-her has let Peter to-speak John let him speak to her.

(d)*Jean le lui a laissé parler. John him to-her has let to-speak. John let him speak to her.

Placement of the dative clitic cannot reach the main clause over the subject of the embedded clause, suggesting an SSC effect. Whether an SSC effect in turn is a diagnostic of movement is less clear. One construal of the binding theory (e.g., Chomsky (1981)) takes it as a condition on representations and not necessarily on movement relationships per se, so that it can straightforwardly extend to antecedent/lexical anaphor dependencies. Expanding on Lebeaux (1983), antecedent/lexical anaphor relations have also been treated in terms of movement (cf. Chomsky (1986b)). If DP/trace or antecedent/anaphor anaphoric relations are indeed movement relations, then obeying Binding Principle $A$ is a diagnostic property of movement (although not necessarily an 


\section{CLITIC CONSTRUCTIONS}

independent diagnostic property, as it might be, and very plausibly is, derived from the ECP antecedent government requirement).

\subsubsection{Condition on extraction domain (CED) and ECP effects}

Much more direct evidence in favor of a movement analysis comes from examining the kind of constituents out of which clitics can be extracted. Such data consistently suggest that movement is involved.

\subsubsection{EXTRACTION FROM PP}

P-stranding under clear cases of movement is disallowed in French. For example, $W h$ Movement of the object of the preposition is ill-formed (unless the $\mathbf{P}$ is pied-piped):

(15)(a) Jean a voté pour Maastricht. John has voted for Maastricht. John voted for Maastricht.

(b)*Quel traité Jean a-t-il voté pour $\mathrm{t}$ ? Which treaty John has he voted for? Which treaty did John vote for?

(c) Marie est partie avec la valise. Mary is left with the suitcase. Mary left with the suitcase.

(d)*Quelle valise est-elle partie avec t? Which suitcase is she left with? Which suitcase did she leave with?

The same observations hold for stranding under clitic placement ("sc" stands for silent category):

(16)(a) Jean a voté pour lui/*Jean lui a voté pour [sc]. John has voted for him/John him has voted for. John voted for him.

(b) Marie est partie avec elle/*Marie lui est partie avec [sc]. Mary is left with it/Mary her is left with. Mary left with it.

It is not true, however, that Ps cannot be stranded at all. As Zribi-Hertz (1984) discusses, the equivalents of (16) are perfectly well formed without the clitic:

(17) Jean a voté pour/ Marie est partie avec. John has voted for/ Mary left with.

John voted in favor of it. /Mary left with it/him/her. 
In these cases, the missing object is interpreted as referring to some discourseprominent entity, exactly as an overt pronoun object of the $P$ is in (16b and d). In fact, Zribi-Hertz (1984) argues that this missing element is best analyzed as a silent pronoun pro. If the [sc] in (16) is a trace, the paradigm follows: (16) patterns like (15) and unlike (17) because the relevant part of the ECP only applies to traces. ${ }^{2}$

\subsubsection{EXTRACTION OUT OF DP}

Patterns of extractability out of DPs make the same point: clitic placement behaves exactly like a movement process. Extraction out of direct object DPs in French obeys a simple generalization (cf. Giorgi and Longobardi (1991), Sportiche (1989), Valois (1991) for recent discussion):

(18) Extractability requirement

An XP may be extracted out of a DP in a given structure iff $\mathrm{XP}$ may otherwise appear as the possessor of this $\mathrm{DP} .^{3}$

This generalization is illustrated by the paradigm below:

(19)(a) Jean a vu une/la photo de qui? John has seen a/the picture of whom? John saw a/the picture of whom?

(b) La personne dont Jean a vu [une/la/*ma/*cette photo $\left.t_{j}\right]$. The person of-whom John has seen a/the/my/this picture. The person of whom John saw a/the picture.

(c) Jean a lu une dépêche de Paris. John has read a dispatch from Paris. John read a dispatch from Paris.

(d)* 'D'où jean a-t-il lu [une dépêche $t_{j}$ ] ? From where John has he read a dispatch? From where did John read a dispatch?

Only when the extracted phrase could otherwise be a possessor can it be extracted, though this is blocked by the presence of another possessor like ma or a demonstrative like cette as in (19b), or because only DPs and not locative PPs may be possessivized as in (19d). Sportiche (1990) and Valois (1991) propose that this pattern is explained by appealing to antecedent government, a property required of traces but not of silent categories: extraction of a postnominal XP must proceed through the $[\mathrm{Spec}, \mathrm{DP}]$ position guaranteeing antecedent government and deriving the generalization in (18). (20) illustrates this for the sentence in $(19 \mathrm{~b})$ :

$$
\text { La personne dont }{ }_{j} \text { Jean a vu }\left[_{D P} t_{j}\left[\text { la }\left[_{N P} \text { photo } t_{j}\right]\right]\right. \text {. }
$$




\section{CLITIC CONSTRUCTIONS}

The same pattern holds of clitic extraction:

(21)(a) Jean a vu une/la photo de qui? John has seen a/the picture of whom? John saw a/the picture of whom?

(b) Jean en $n_{\mathrm{j}}$ a vu [une/la/*ma/*cette photo $\left.t_{\mathrm{j}}\right]$. John of-him has seen a/the/*my $/ *$ this picture. John saw a/the/my/this picture of him.

(c) Jean a lu une dépêche de Paris. John has read a dispatch from Paris. John read a dispatch from Paris.

(d)* Jean en a lu [une dépêche $t_{i}$. John therefrom has read a dispatch. John read a dispatch from there.

(e) Jean vient de Paris/Jean en, vient $t_{\text {. }}$ John comes from Paris/John therefrom comes. John comes from Paris/John comes from there.

Note in particular that cliticization of the locative of origin is disallowed in the sentence (21d), even though it may in principle be cliticized as en as in the sentence (21e). Note also that the [sc] following stranded prepositions is allowed in these contexts, further supporting the contention that we are dealing with different kinds of gaps:

(22) $\mathrm{le} / \mathrm{mon} / \mathrm{ce}$ vote pour (cela)/ mon départ avec (cela) the/my/this vote for it my departure with it the/my/this vote for (it)/my leaving with (it)

\subsubsection{Participle agreement}

Participle agreement in French provides further reasons to adopt the movement analysis. A participle may (or must depending on the variety of French) agree with its accusative direct object when it precedes the participle. Agreement is excluded when the participle follows.

(23)(a) Jean a peint $\left(*^{*}\right)$ la porte. John has painted $(*$ FEM) the door. John painted the door.

(b) La porte $e_{i}$ que Jean a peint(e) $t_{i}$. The door that John has painted(FEM). The door that John painted.

(c) Jean l'a peint(e) [sc]. John it has painted(FEM). John painted it. 
(23b) illustrates this with a relativized object, (23c) with a cliticized object. Under a movement analysis, these data make sense if, as Kayne (1989b) suggests:

1 There is only one way to get agreement. Participle/object agreement is similar to subject/tense agreement in being the reflex of a relation between a head and its specifier.

2 There is an intermediate specifier (of the participial morphology) through which the moved object may (or must) transit.

Of course, only assumption (2) is linked to a movement analysis. Assumption (1) is just a particular (and quite convincing) construal of how agreement arises. As Sportiche's (1989) or (1990) extensive discussion shows, there is substantial independent evidence for the existence of this intermediate position in French. At any rate, this account would attribute to $(23 \mathrm{c})$ the following analysis:

$$
\mathrm{le}_{\mathrm{j}} \mathrm{a}\left[\mathrm{t}_{\mathrm{j}}\left[\text { peinte } \ldots \mathrm{t}_{\mathrm{j}}\right]\right]
$$

Under a base-generation analysis, i.e., one in which the [sc] in (23c) is pro or PRO, this configuration appears arbitrary. Assuming the object is pro is essentially saying the object remains postparticipial. Since the agreement in this case would have to be treated differently than in the subject/verb agreement Case, there is no reason why the facts turn out this way rather than the other way around (i.e., agreement only with a postverbal (lexical) object).

Corroborating evidence for the movement plus Spec-head approach is found when a fuller picture of agreement is presented. As mentioned, in some varieties of French (like mine), participle agreement is optional with direct object clitics. Now, in all varieties of French, the participle agrees with the subject (instead of the object) if the verb uses the auxiliary etre "to be" (including in passive constructions), and this agreement is obligatory.

(25) La porte a été peint*(e)

The door(FEM) has been painted*(FEM).

The door was painted.

Verbs using the auxiliary être are all constructions with derived subjects; in all these cases (which include passive and reflexive constructions (cf Sportiche (1990) and Cortés (1992)), the superficial subject is an underlying object. Now there are two observations to account for: (1) first, why does NP Movement pattern with clitics in being able to trigger agreement; (2) secondly, why is NP Movement more restricted than clitics in that it must trigger participial agreement.

Both of these facts follow if clitic placement is analyzed as movement 


\section{CLITIC CONSTRUCTIONS}

plus agreement under Spec/head. First, clitic placement and NP Movement both trigger agreement because they both involve movement (in fact XP Movement, as we will see later). Furthermore, it comes as no surprise either that $\mathrm{Wb}$ Movement may (optionally) trigger agreement. Agreement on this account arises as a result of movement.

Second, a movement analysis of clitic placement can account for the second observation as follows. If both clitic placement and raising to subject involve movement, we expect that this movement may pass through the specifier position of the participial phrase. We may suggest that the intermediate step through the specifier of the participial phrase is optional in the case of clitic placement but not in the case of NP Movement: only in the course of the movement involved in clitic placement may this position be skipped. This is consistent with what we know of NP Movement, namely that it is the most restricted kind of movement and the one subject to the strictest locality conditions, i.e., quite possibly more restricted than clitic movement. ${ }^{4}$ If any difference is found between NP Movement and clitic movement, it is reasonable to expect more latitude for clitic movement. In particular, if movement through the participial specifier is available in principle for clitic movement and raising to subject (and it is, as agreement demonstrates), we expect skipping this position in the course of movement to be at least as available for clitic movement as it is for NP Movement and possibly more so. In other words, the French agreement pattern comes as no surprise. Note in particular that the opposite pattern is predicted as impossible (requiring participle agreement with object clitics and allowing but not requiring participle agreement with derived subjects). This seems correct: no variety of French (or, as far as I know, of Romance, for that matter) patterns in the opposite way as the French just described. It is less clear how to get the same results in a base-generation approach. There is no good reason why NP Movement or $W h$ Movement should pattern in any way like clitics in being able to trigger agreement.

We will now review reasons in favor of adopting a base-generation analysis. Basically they are either lack-of-source arguments (there is no possible source from which the clitic could have moved) or impossibility-of-movement arguments (the distance between the clitic and its source are not allowed by movement).

\subsubsection{Etbical datives and inberent clitics}

Two lack of source cases are illustrated by the ethical dative construction in (26a) and the inherent clitic construction in (26b):

(26)(a) Je t'achèterais un cadeau à Pierre. I you ${ }_{C L}$-buy-would a present for Peter. I tell you I would buy Peter a present. 
(b) Pierre en a bavé.

Peter of-it has drooled.

Peter suffered.

In the first, the clitic te (also possible with some other dative clitics but probably most natural with second person singular) simply cannot be replaced by any full XP. In fact, the function of the clitic is not to link to any argument. This would seem to argue against a movement analysis for these clitic structures, and by uniformity, against movement analyses in general, for lack of a possible source. Example (26b) illustrates the same point. The clitic en does not correspond to any XP slot. It would seem to be in some way part of the lexical entry of the verb en-baver but nevertheless behaves positionally like a regular clitic in preceding the auxiliary verb avoir. By uniformity, we would then expect all clitics to be part of the lexical entries of their predicate. As we have discussed before, this is clearly an unacceptable conclusion, which might cast doubt on the requirement that clitics be uniformly analyzed.

However, as Kayne (personal communication) points out, the lack of source argument is seriously weakened by the existence of such English examples as Jobn perjured bimself, John kicked the bucket. In these examples, we have direct objects which, as part of an idiom, must somehow be listed as part of a lexical entry including the verb and at the same time be realized as independent DP objects, even though they do not seem to receive any "real" theta role. If having a "real" theta role is not a precondition for DPs to occur as objects, the lack of source argument disappears. French inherent clitic verbs could just as well list a theta-less clitic object, which would then be subject to the normal rules of clitic placement. Likewise, for ethical dative constructions, in which the clitic is not obviously related to the verb, we would have to allow the generation of a theta-less XP headed by the dative clitic which would then be subject to the normal rules of clitic placement.

Note finally that a base-generation analysis faces somewhat the same complication as a movement analysis. Since clitics usually are linked to an argument position, inherent clitics and ethical datives would constitute an exceptional class of clitics. However, the base-generation analysis does not entail the existence of an $\mathrm{XP}^{*}$ corresponding to the clitic, and, in that, it appears slightly simpler than the movement analysis. In sum, we conclude that these constructions, although they pose a problem, do not really help to decide the issue and in particular do not invalidate a movement analysis.

\subsubsection{Stranded quantifiers in L-tous cases}

The distribution of stranded or floating quantifiers seems difficult to reconcile with a movement analysis of clitics. Stranded quantifiers are illustrated in the following examples: 


\section{CLITIC CONSTRUCTIONS}

(27)(a) Les enfants ont tous mangé.

The children have all eaten.

The children all ate.

(b) Je les ai vus tous.

I them have seen all.

I have seen them all.

Following Sportiche's (1988) proposal, we take it that the distribution of these quantifiers is determined in part by the position of intermediate traces of their antecedents. These quantifiers are in fact adjacent to a silent DP (trace, pro or PRO) bound by another (usually overt) DP they quantify over. Thus, in (27a), the presence of the quantifier tous reveals that the subject of the sentence has raised from a lower position (yielding the VP-Internal Subject Hypothesis). Koopman (1991) and Schlonsky (1990) have convincingly argued that the mechanism of Q-stranding under DP movement involves raising the DP through Spec, QP as revealed by the fact that the $\mathrm{Q}$ agrees with the DP only under stranding in Hebrew (Schlonsky) and by the existence of phrases like eux tous "them all" in French and them all in English (Koopman) (in effect, a modern version of Kayne's (1975) Q-post analysis). ${ }^{5}$

There are actually two distinct ways of implementing Sportiche's (1988) basic idea in a way consistent with Koopman's and Schlonsky's proposals. The first one is to assume, as did Sportiche (1988), that a strandable Q is always generated with a DP complement. Stranding under this view is always under syntactic movement of this DP through the specifier position of the QP. An alternative is to postulate that this kind of $Q$ may be generated in a wider variety of environments, e.g., as a $Q$ taking a VP complement, and is understood to quantify over some DP moving through its specifier position (and thereby triggering agreement): $\mathrm{L}_{\mathrm{QP}} \mathrm{DP}_{\mathrm{i}}\left[\right.$ tous $\left.\left.\left.\mathrm{L}_{\mathrm{VP}} \mathrm{V} \ldots \mathrm{t}_{\mathrm{i}}\right]\right]\right]$. This last analysis of tous would be akin to that of other quantifiers appearing to be both determiners and adverbials (e.g., Il a vu beaucoup d'enfants, il a beaucoup vu d'enfants "He saw a lot of children"). Either approach straightforwardly extends to case (28a) but not to $(28 \mathrm{~b}-\mathrm{d}):^{6}$

(28)(a) Marie les a tous pris.

Mary them has all taken.

Mary took them all.

(b) Marie a toutes voulu [les manger].

Mary has all wanted them eat ${ }_{\mathrm{INF}}$.

Mary wanted to eat them all.

(c) Il a tous fallu [qu' ils parlent].

It has all necessitated that they speak.

It was necessary that they all speak.

(d) Il a tous fallu [que Louis les lise].

It has all necessitated that Louis them read.

It was necessary that Louis read them all. 


\section{PARTITIONS AND ATOMS}

In the last three examples, which we shall call L-tous cases (from Kayne's 1975 Leftward-tous Rule) the stranded Q is higher than the clitic it modifies. In fact, it is outside the clitic's clause altogether. ${ }^{7}$ The fact that an intervening head, the complementizer que, does not block this construction indicates that, if the $\mathrm{Q}$ is placed by movement in its overt position, it does not move out of its clause by head movement but rather by XP movement. This movement is most plausibly of the A-bar type, given that NP Movement out of a tensed clause is strictly forbidden in French. Sportiche (1988) attributed the possibility of $(28 \mathrm{c}, \mathrm{d})$ to the syntactic application of Quantifier Raising (QR).

Suppose we adopt both of the analyses of floating quantifiers described above and a movement analysis of clitics. This might be more precisely construed as follows: (1) the clitic is the head of the DP modified by or quantified over by tous, (2) the clitic itself moves to its surface position, (3) tous quantifying over this DP is licensed by this DP moving through the specifier position of the $Q$. Consider now what this would mean for a sentence like (28d). The following configuration will need to be arrived at:

$$
L_{Q P}\left[\text { tous V } \ldots\left[_{C P} \ldots\left[_{D} \operatorname{les}_{i}\right] \ldots\left[\left[_{D P} t_{i}\right]\right]\right]\right]
$$

The clitic, i.e., the D head of the object DP, has incorporated to some host. If the stranded $Q$ gets to its surface position by movement, and gets stranded by movement of its complement DP (or the head D of such a DP) out of QP, the raised QP will contain the unbound trace of this D or this DP. If, alternatively, the stranded $Q$ is base-generated in the top clause, its licensing will require the following scenario: by LF, this DP will have to raise to the position Spec, $\mathrm{QP}$ even though it would contain a trace of the incorporated clitic. ${ }^{8}$ This violates the requirement that traces be properly bound. This problem of course does not arise if the clitic is generated independently of the DP argument it is related to, a basic property of base-generation analyses. One potential way to remove this offending trace would require reconstruction of this DP into the lower clause at LF. It is clear however that these leftward moved Qs have matrix scope, not embedded scope. This is illustrated by the following pair:

(30)(a) Il aurait tous fallu que tu ne les It would-have all necessitated that you NEG them aies pas vus.

have not seen.

It would have been necessary that you see none of them.

(b) Il aurait fallu que tu ne les It would-have necessitated that you NEG them aies pas tous vus. have not all seen. It would have been necessary that you do not see all of them. 


\section{CLITIC CONSTRUCTIONS}

As the paraphrases indicated, in the first sentence the $Q$ must have scope wider than the embedded negation. If reconstruction was required, we would expect at the very least the $\mathrm{Q}$ to be able to take narrower scope than the embedded negation as in the second sentence.

\subsubsection{Dative constructions and stranded quantifiers}

There is a second class of clitic constructions which superficially also seem to lack a plausible movement source for their clitics. The movement analysis makes the claim that the relation between the clitic and its associated $\mathrm{XP}^{*}$ is a possible movement relation. The base-generation makes no such claim (although there is a need under the base-generation analysis to elucidate the nature of the locality relation holding between the clitic and its associated $\left.\mathrm{XP}^{*}\right)$. The central paradigm I want to discuss, representative of all the constructions involving a dative clitic, is illustrated below. ${ }^{9}$

(31)(a) Je (*leur) ai offert un cadeau à tous les enfants. I (to-them) have given a present to all the children. I gave a present to all of the children.

(b) Je (*à) leur ai (tous) offert un cadeau *(à) tous. I to them have all given a present to all. I gave them all a present.

(c) Ces garçons, *(à) qui j' ai (??tous) offert

These boys, to whom I have (all) given un cadeau ((à) tous).

a present (to all).

These boys, all of whom I gave a present.

These examples show that a dative clitic may appear in conjunction with a stranded quantifier, but not with a full phrase. If this $Q$ appears stranded by clitic placement in the normal dative position, this $Q$ must be preceded by the preposition (or the dative marker) $\grave{a}$. Otherwise, it must be bare. These dative Qs cannot be stranded under $W b$ movement however. They may be very marginally stranded as bare Qs in preparticiple position. This behavior is in marked contrast with that of accusative objects, which allow stranding of Qs both under clitic placement and under Wh movement Je les ai tous lus "I read them all." Ces livres que j'ai tous lus/"These books, which I have all read."

These examples constitute a serious problem for a movement analysis of clitics in the dative case. The complementarity of the clitic and full DP suggests that the two positions are related by movement. But the difference between clitic placement and Wh Movement with postverbal stranded Qs preceded by $\grave{a}$ suggests otherwise: if clitics actually moved, why should they pattern any differently from $w h$-phrases? One possibility is that they do not move as phrases - unlike wh-phrases - but rather as heads throughout, 
an option consistent with some of Kayne's recent proposals and also with Kayne's (1975) handling of this problem. ${ }^{10}$ He suggests that dative à may be transformationally inserted on a dative DP. The derivation of (31c) would include the following steps: $\ldots$ offert $\left[_{\text {Dative }}\right.$ [tous $\left[_{D P}\right.$ quil] $] \rightarrow$ DP-to-Spec $\rightarrow$ offert $\mathrm{E}_{\mathrm{DativeP}}\left[\mathrm{LP}_{\mathrm{DP}}\right.$ qui] [tous $\left.\mathrm{t}\right]$ ]. At this point $\mathrm{A}$-insertion on the DP qui (cf. J'ai parle a eux tous/"I spoke to them all") and DP movement (Wh Movement) may apply in either order. This forces à to be carried along with the moved phrase as desired. The problem is to force the opposite with clitic placement in (31b). From the structure ... offert [ ${ }_{\text {DativeQP }} L_{D P}$ leur] [tous t]], pied-piping of an inserted $\grave{a}$ or insertion of a moved leur must be prevented. This behavior is surprising, if clitic placement is DP movement, but would follow if leur moves as a $\mathrm{D}$ rather than as a DP, construing $\grave{a}$ as a property of the DP rather than of the D. If, however, we can show that clitic movement cannot be construed as head movement throughout, as we will in Section 5.3, it suggests that clitic placement in these structures is not the result of movement.

\section{Analysis}

Throughout, we restrict ourselves to cases in which the clitic is associated with an argument $\mathrm{XP}^{*}$. Other cases, as discussed earlier, can be similarly treated.

\subsection{Clitic voices}

The previous discussion suggests we have a contradiction. On the one hand, the search for explanatory adequacy strongly supports a uniform analysis of clitic constructions. On the other, different clitic constructions seem compatible with different analyses. As mentioned in Section 1.3, this contradiction may be resolved. Movement analyses have typically assumed that movement is movement of the clitic. None of the arguments supporting a movement analysis actually shows this. The first proposal we make is to abandon the assumption made by movement theories, namely that the movement involved in clitic constructions is movement of the clitic. Basegeneration analyses, on the other hand, seem to be faced with a fundamental problem. It is particularly striking that the movement properties holding of the relation between a clitic and its XP* hold regardless of whether or not $\mathrm{XP}^{*}$ is silent. In clitic doubling constructions as well, the clitic and the doubled phrase are in a possible movement relationship, in fact the exact same as in non-doubled constructions. The second proposal we make is to abandon one assumption made by base-generation theories, namely that no movement is involved.

If the clitic is not the element moved from the position $\mathrm{XP}^{*}$, we need to account for the fact that the clitic agrees with $\mathrm{XP} *$. Thus, if the missing 


\section{CLITIC CONSTRUCTIONS}

argument $\mathrm{XP} *$ is understood to be what is denoted by a third-person singular pronoun direct object, the clitic appearing in the structure is a third-person singular accusative clitic. Furthermore, as we have discussed in Section 2, clitics are so called because they do not form autonomous syntactic units. Rather, they seem to form a unit with some host. As Kayne (1989a) points out, there is a natural interpretation of this clitic property in contemporary syntactic theory: the clitics are heads whose close syntactic connection with a host can be analyzed as a case of head-to-head movement incorporating the clitic head to the host head (or vice versa). ${ }^{11}$ This immediately suggests that the agreement between a clitic and its corresponding $\mathrm{XP} *$ be viewed as an instance of agreement between a phrasal constituent and a head, a kind of agreement naturally handled as a reflex of a relation between a head and its specifier and is consistent with the general idea that agreement is always a phrase/head relation between a specifier and a head.

In order to implement this agreement requirement as the result of a $\mathrm{Spec} / \mathrm{head}$ relationship, we must provide a projection whose head is the clitic and whose specifier is a landing site for movement of the corresponding $\mathrm{XP}^{*}$. This is, of course, the proposal outlined in Section 1. Without getting into much structural detail for the moment, remember that a typical clitic construction (such as the one exemplified in (1), repeated below) would receive the analysis indicated in (2) (repeated below), given that XP* is in this case a DP: ${ }^{12}$

(1) Marie les aura présentés $\mathrm{XP}_{\mathrm{i}}$ * à Louis.

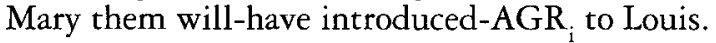

Mary would have introduced them to Louis.

(2) $\leftarrow_{\text {Accp }} \operatorname{DP}^{\wedge}\left[\left[_{\text {Acc }}\right.\right.$ les $]\left[\ldots\right.$ aura présentés $\left.\left.\left.D P *\left[_{\text {Acc }}\right] \ldots\right]\right]\right]$

In order to achieve or sanction agreement, $\mathrm{DP}^{*}$ will have to move to $\mathrm{DP}^{\wedge}$ at some point. More generally, with each clitic slot of the clitic template given in (3), there is a corresponding projection available. Call these projections Clitic Voices, and individual instances of them nominative voice, accusative voice, dative voice, and so on. ${ }^{13}$ Thus, a sentence such as (32a) will be associated with the structure in (32b) (linear order irrelevant at this point): (see page 266).

(32)(a) Il le lui donnera.

$\mathrm{He}$ it to-him/her will-give.

He will give it to him/her.

We must now answer the following questions:

(33)(1) How does this analysis reconcile movement and basegeneration? 
(2) How is it compatible with the properties of individual clitic constructions?

(3) What kind of movement is involved, what causes movement (linked to the question of the function of these clitic projections)?

(4) What is the nature of XP* (when it is silent)?

(5) What is the nature of the position $\mathrm{XP}^{\wedge}$ ?

(6) Where are these clitic projections located structurally?

(32) (b)

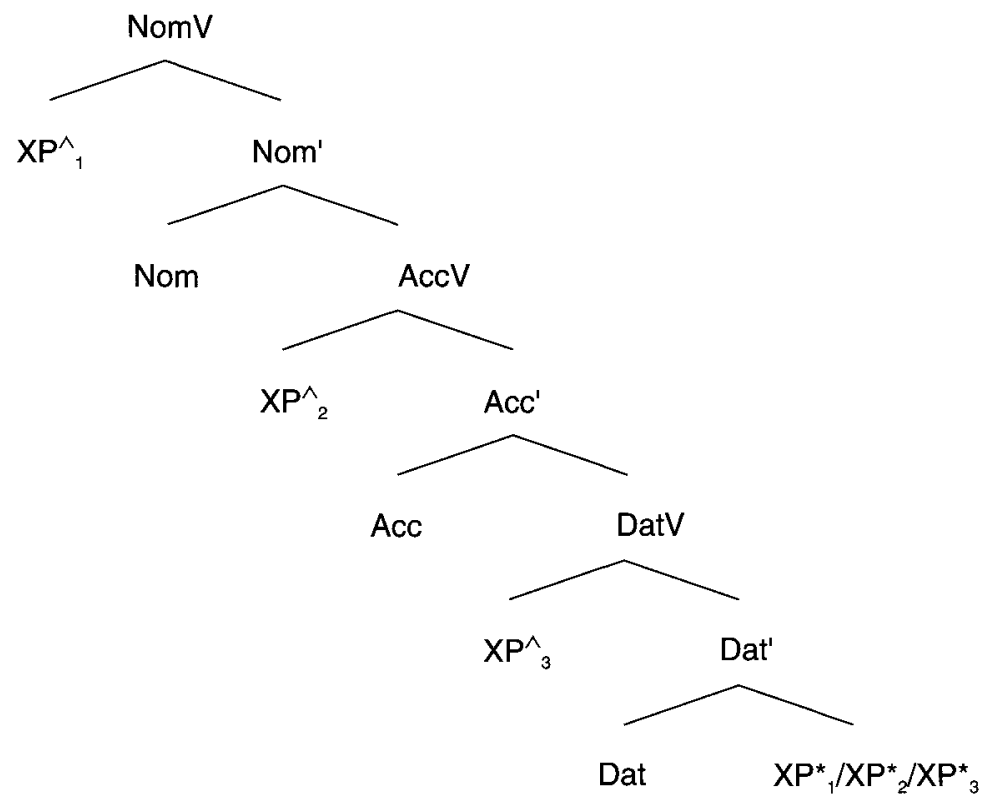

Of these, (33(4)) can be partially answered immediately. Reflexive (or rather se clitic constructions) behave differently from the others (see Sportiche (1990) and the references cited therein). In a non-reflexive clitic construction, a silent XP* is interpreted exactly as a pronoun would be. We therefore postulate that XP* is pro (as did Sportiche (1983)) for exactly the same reason; see also Bouchard (1982). XP* moves to $\mathrm{XP}^{\wedge}$ where it does not have to be bound (ignoring the possibility of further movement). PRO would not give rise to the right interpretation (of definite pronoun) in the absence of any antecedent (and none is necessary in clitic constructions). ${ }^{14}$ As pro, XP* needs to be properly identified, which it is after movement to $\mathrm{XP}^{\wedge}$, by the coindexed clitic head. In effect, we are treating non-reflexive clitic constructions (i.e., accusative voice, dative voice, genitive voice, locative voice and nominative voice) as pro-drop constructions, again very much along the lines of Sportiche (1983) (see also Roberge (1990), Bouchard (1982)). Sportiche (1993) explores ways of extending this approach to pro-drop throughout, 


\section{CIITIC CONSTRUC'TIONS}

claiming in effect that subject agreement is not what licenses pro drop, but that subject clitics are.

\subsubsection{The Clitic Criterion}

We may now give a preliminary answer to question (33(3)) in relation to what causes movement. We have already noted the similar behavior of $W b$ Movement and clitics with respect to agreement. Let us pursue the analogy with Wh Movement one step further. We know that wh-phrases typically occur overtly only clause-initially in specific clauses bearing the property $[+\mathrm{Q}]$ or $[+$ wh]. Rizzi (1990) captures this generalization by updating May's (1985) Wb Criterion and by taking into account Chomsky's (1986a) generalized X-bar proposal extending the X-bar schema to the complementizer system:

\section{(34) Wh Criterion or $Q$ Criterion}

1 A $w h$-head must be in a Spec/head relationship with a $w h-\mathrm{XP}$ at LF.

2 A $w b$-XP must be in a Spec/head relationship with a $w h$-head at LF.

Satisfaction of this criterion will guarantee the proper distribution of wh-phrases at LF. We can treat clitics along similar lines. Assume that clitics license in XPs a particular property or feature $[+F]$ (about which we will speculate in Section 7; for concreteness' sake, consider it in French to be that of being a null pronoun). Assume further that this property may be licensed at LF only in an appropriate agreement relationship. By analogy with (34), we may state ${ }^{15}$

\section{(35) Clitic criterion}

1 A clitic must be in a Spec/head relationship with a $[+\mathrm{F}] \mathrm{XP}$ at LF.

$2 \mathrm{~A}[+\mathrm{F}] \mathrm{XP}$ must be in a Spec/head relationship with a clitic at $\mathrm{LF}^{16}$

where the agreement reflex of the Spec/head relationship is expressed by Case, number, gender and person agreement (at least in French). Thus, if a clitic is related to an $\mathrm{XP}^{*}$, this $\mathrm{XP}^{*}$ will have to move in order to satisfy the Clitic Criterion (35).

Looking at this analysis in full generality, we see that a clitic construction is a (non-local) movement relationship between two XPs, $\mathrm{XP}^{*}$ and $\mathrm{XP}^{\wedge}$ mediated by a (clitic) head $\mathrm{H}$, such that $\mathrm{XP}^{\wedge}=\mathrm{Spec}, \mathrm{HP}$, so that $\mathrm{XP}^{*}$ agrees with $\mathrm{H}$. By LF, $\mathrm{XP} *$ must have moved to $\mathrm{XP}^{\wedge}$. Some of the parameters of the constructions are then as follows: 


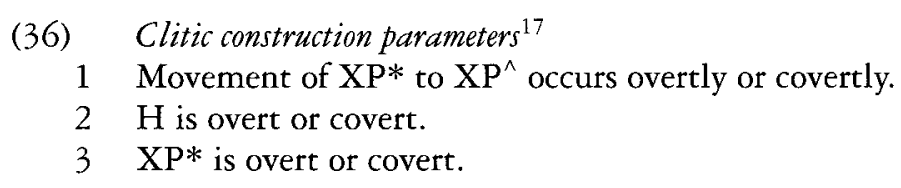

All clitic constructions (involving a $\mathrm{CI} / \mathrm{XP}^{*}$ dependency), we claim, involve movement. The movement of XP* is to the specifier position of the projection headed by the clitic, where agreement between $\mathrm{CI}$ and $\mathrm{XP} *$ is sanctioned. This approach treats clitics as complex agreement morphemes, deriving the agreement relation $\mathrm{XP} * / \mathrm{CI}$ as an instance of a Spec/head relation, and it determines the locality relation between the clitic and $\mathrm{XP} *$ as in effect being characterized by the necessary movement relation between $\mathrm{XP}^{\wedge}=$ Spec, CIP and XP*. ${ }^{18}$ This provides a general way of handling true clitic doubling constructions, i.e., clitic doubling constructions not analyzable as in (13). Superficially, (true) clitic doubling constructions differ from non-doubled constructions in that XP* is overt in the first and covert in the second. So far, we have not specified whether movement should be overt or covert. When $\mathrm{XP}^{*}$ is overtly realized in the $\mathrm{XP} *$ position, as might be the case in Spanish clitic doubling constructions, the analysis will postulate covert movement of $\mathrm{XP}^{*}$ to $\mathrm{XP}^{\wedge}$. This does not mean that movement of an overt $\mathrm{XP} *$ may not be overt as well. I suggest later that this happens in Dutch Scrambling constructions. Similarly, when XP* is covert, movement may a priori be overt or covert. Settling this question will require checking the properties of the $\mathrm{XP}^{\wedge} / \mathrm{XP} *$ relation in individual constructions.

If the parameters given in (36) are independent, it is easy to see the kind of constructions each combination of the options in (36) will give rise to. A covert $\mathrm{XP}^{*}$ moving overtly or covertly to $\mathrm{XP}^{\wedge}$ with $\mathrm{H}$ overt gives rise to undoubled clitic constructions as in French, or Italian or Dutch. An overt $\mathrm{XP}^{*}$ moving covertly with an overt $\mathrm{H}$ gives rise to clitic doubling constructions as might be illustrated by Spanish or Romanian. An overt XP* moving overtly with $\mathrm{H}$ overt will give some object agreement constructions such as might be found in Lebanese Arabic. The clitic left-dislocation construction of Cinque (1991) may also be such a case, or a case of overt movement of XP* to $\mathrm{XP}^{\wedge}$ and then beyond, as is found in Romanian or Spanish wh-questions. An overt XP* moving overtly with a covert $\mathrm{H}$ will have the appearance of simple phrasal movement. We argue that Scrambling of specific DPs in Dutch is such an instance.

\subsubsection{The position of clitic voices}

We now briefly address question (33(6)). It subdivides into two: (1) where are the clitic projections generated within the clause? (2) how are the clitic projections ordered with respect to each other? I will limit myself to the first question here and leave the second entirely open, as its understanding 


\section{CLITIC CONSTRUCTIONS}

requires resolving many issues about the internal structure of strings of clitics about which little is understood. Relevant to this determination are the Head Movement Constraint, which prohibits movement of heads to skip intermediate heads and Baker's Mirror Principle, which claims that morphological constituent structure reflects syntactic constituent structure. Here we are only concerned with non-nominative clitics which obey the basic generalization given in Section 2: ${ }^{19}$

(37) Clitics occur adjoined to the highest verbal element of the clause containing XP*.

Consider again the sentences given in Section 2:

(6) (a) Il le lui donnera.

$\mathrm{He}$ it to-him will-give.

He will give it to him.

(b) Il le lui a donné.

He it to-him has given.

He gave it to him.

(c) Ils lui ont été donnés.

They to-him have been given.

They were given to him.

(d) Il ne le lui donnera pas.

He NEG it to-him will-give not.

He will not give it to him.

(e) Ils ne lui ont pas été donnés.

They NEG to-him have not been given.

They were not given to him.

(7) (a) Ne pas le lui donner / *ne le lui pas donner.

NEG not it to-him give INF $_{\text {. }}$

To not give it to him.

(b) Ne pas l'avoir donné/ ne l'avoir pas donné.

NEG not it have given.

To not have given it to him.

(c) Ne pas leur en avoir parlé/??ne leur en avoir pas parlé.

NEG not to-them of-it have talked.

To not have talked to them about it.

(d) Ne pas m'en avoir parlé.

NEG not to-me of-it have talked.

To not have talked about it to me.

Pollock (1989) demonstrates, mostly on the basis of adverbial/verb ordering possibilities, the existence of two different head positions (which he takes to be $T$ and AGR). He shows that French tensed verbs must raise from $V$ to 
the highest of the two and French infinitival verbs optionally raise from $\mathrm{V}$ to the lowest of the two (except for auxiliaries which may marginally raise to the higher slot). Suppose initially the following construal of his results: Infl is split between $A G R_{s}$ and $T$ with $A G R_{s}$ the higher head. The order of projections is thus $A G R_{s} T$, with short $V$ movement (of infinitivals) being to $\mathrm{T}$ and long $\mathrm{V}$-movement (obligatory for tensed verbs, optional for infinitival auxiliaries) being to $\mathrm{AGR}_{\mathrm{s}}{ }^{20}$ Generally, in clauses with one or two auxiliary verbs, we have the following relevant structures:

(38)(a) Tensed clauses
$\begin{array}{lllll}\text { (ne) } \mathrm{Cl} & \text { AUX1+Tense (pas) } & \text { (AUX2) } & \text { Verb XP* } \\ \text { ne lui } & \text { aura } & \text { pas } & \text { été } & \text { rapporté } \\ \text { NEG } & \text { to-him will-have } & \mathrm{NEG}_{2} & \text { been } & \text { brought-back } \\ \text { Will not have been brought back to him. } & \\ & & & \\ \text { (b) Tenseless } \text { clauses }^{21} & & & \\ \text { (ne) } & \text { (pas) } \mathrm{Cl} & \mathrm{AUX} 1+\mathrm{Inf} & \text { (AUX2) } & \text { Verb XP* } \\ \text { ne } & \text { pas lui avoir } & \text { été } & \text { rapporté } \\ \mathrm{NEG}_{1} & \mathrm{NEG}_{2} & \text { to-him to-have } & \text { been } & \text { brought-back }\end{array}$

If there is no auxiliary verb, the position of AUX1 above is occupied by the main verb. Note the two negative particles ne $\left(=\mathrm{Neg}_{1}\right)$ and pas $\left(=\mathrm{Neg}_{2}\right)$. Clearly, the fact that the highest verbal element is on different sides of $\mathrm{Neg}_{2}$ is consistent with Pollock's conclusions and might indicate that we should have the order $\mathrm{NEG}_{1}>\mathrm{H}_{1}>\mathrm{NEG}_{2}>\mathrm{H}_{2}$ with $\mathrm{H}_{1}=\mathrm{AGR}_{\mathrm{s}}$ and $\mathrm{H}_{2}=\mathrm{T}$.

How should clitic projections be ordered with respect to these? Let us begin with tensed clauses (38a). Given the possibility of $(5 c)$ ? Pierre le ou les renverral"Peter will send him or them back" and the imperative order of $\mathrm{V}+$ Tense $+\mathrm{AGR}_{\mathrm{s}}+\mathrm{CI}$ (renvoyons-les "let us send them back"), the morphological structure of the sequence $\mathrm{CI}+\mathrm{Tense}+\mathrm{AGR}_{\mathrm{S}}$ seems to be $\mathrm{CI}+[\mathrm{V}+$ Tense $+\mathrm{AGR}]$. By the Mirror Principle, the corresponding projections should be ordered $\mathrm{CIP}>\mathrm{AGR}_{\mathrm{S}} \mathrm{P}>\mathrm{TP}>\mathrm{VP}$, with AUX raising to $\mathrm{T}$, (then to $A G R_{S}$ ) then to CI. Now, given the infinitive case, and in particular the fact that the whole verbal complex follows $\mathrm{NEG}_{2}$, the order will have to be: $\mathrm{NEG}_{1}>\mathrm{H}_{1}>\mathrm{NEG}_{2}>\mathrm{CIP}>\mathrm{AGR}_{\mathrm{S}} \mathrm{P}>\mathrm{TP}>\mathrm{VP}$. This shows that it is incorrect to identify $\mathrm{H}_{1}$ with $\mathrm{AGR}_{\mathrm{S}}$ with $\mathrm{H}_{2}$ with $\mathrm{T}$. Rather, it is more plausible to take $\mathrm{H}_{1}$ to be an additional projection. Furthermore, given that clitics in standard French always end up on $\mathrm{V}$, even in infinitives, we must assume that all verbs eventually raise at least to $A_{G} R_{S}$.

If, following Chomsky's (1992) proposal of morphological checking, words are inserted in the syntax fully formed and morpheme properties and ordering are checked under successive head movement to the relevant projections, we may assume that a clitic is base-generated on its verbal host and we may 


\section{CLITIC CONSTRUCTIONS}

construe Pollock's split between tenseless verbs and tensed verbs as a failure for tenseless verbs to raise overtly to $\mathrm{AGR}_{\mathrm{S}}$. If we limit Chomsky's proposal to words (excluding clitics from being base-generated lower than the projection in which they are checked, and thus deriving the difference between clitics and inflectional bound morphemes), this split must be handled differently than we have assumed above, since raising to CIP, and hence to $A G R_{S}$ will have to be overt. We are led to assume that all verbs raise to $A_{G R}$ and to $C I$. To handle Pollock's split, we must postulate that there is an additional head above CIP but below $\mathrm{NEG}_{2}$, say $\mathrm{H}_{2}$, to which tensed verbs obligatorily raise (and thus obligatorily preceding adverbs like complètement/"completely") and tenseless auxiliaries optionally raise but to which tenseless main verbs cannot. In other words, we are led to construe Pollock's observations as evidence for two projections, as he does, but to take these projections $\mathrm{H}_{1}$ and $\mathrm{H}_{2}$ to be higher than $A_{G R}$. Taking morphemes to head their own projections (with $\mathbf{P M}=$ participial morphology), we will have the following template for French, with each verbal element raising to the next head (pas does not count as an intervening head for purposes of the Head Movement Constraint, just like other adverbials; cf. Pollock (1989)):

$$
\text { ne } \mathrm{H}_{1} \text { pas } \mathrm{H}_{2} \text { CIP AGR } \mathrm{t}_{\mathrm{l}+1-\text { tense }\}}\left(\text { avoir } \mathrm{PM}_{1}\right)\left(\text { être } \mathrm{PM}_{2}\right) \mathrm{V}
$$

In all cases, the highest verb raises to $\mathrm{AGR}_{\mathrm{S}}$ and $\mathrm{CI}$ if necessary. ${ }^{22}$ Tenseless verbs may then raise to $\mathrm{H}_{2}$. Tensed verbs must raise to $\mathrm{H}_{1}$ presumably via $\mathrm{H}_{2}$.

\subsection{A second look at the various arguments}

We now go back to each of the arguments for or against movement discussed earlier. Clearly, arguments for movement (i.e., those involving SSC, CED or ECP effects, as well as the pattern of participle agreement) pose no particular problems, since we adopt a movement analysis. Left to handle properly are questions of complementary distribution and clitic doubling in the case of L-tous stranding of quantifiers or quantifier doubling in dative constructions.

\subsubsection{Clitic doubling}

So far, we have concentrated mostly on Standard French. Standard French appears not to allow clitic doubling, except for the cases of Complex Inversion (cf. Jean est-il malade?/"Is John sick?") which Sportiche (1993) analyses as clitic doubling. ${ }^{23}$ This is correct for object clitics, if it is understood as requiring the lexical head of $\mathrm{XP} *$ to be silent. However, some other varieties of French do allow true clitic climbing and many Romance languages (and in fact even non-Romance languages) do as well. Recall that clitic doubling is the co-occurrence of a clitic and an overt $\mathrm{XP}^{*}$, and that we have analyzed it as postponement until LF of the necessary movement of $\mathrm{XP}^{*}$ to $\mathrm{XP}^{\wedge}$. In fact, 
there is nothing in the analysis presented so far that really bears on the overt/covert character of XP*: whether overt or covert, $\mathrm{XP} *$ will have to raise by LF to the right specifier so that the Clitic Criterion is met. Clitic doubling constructions and non-doubled clitic constructions are analyzed exactly the same way: the problem is not to account for the possibility of clitic doubling. This central dilemma faced by earlier accounts disappears. Rather, the problem is to account for the distribution of clitic doubling, and for the differences between clitic doubling and non-doubled clitics.

We may preanalytically subdivide the problem of distribution in two subproblems, whose exact boundaries are of course unknown as yet: necessary distributional properties and (apparently) accidental distributional properties. If clitic doubling exhibits necessary properties, they should follow from its analysis. One such necessary property seems to be the following:

(40) If clitic doubling is allowed, it is allowed with (stressed) pronouns. ${ }^{24}$

This gives us clitic doubling Problem 1. Why is (40) true?

In the second category, we find questions such as why does French disallow it while Spanish allows it? Why do different dialects of Spanish allow it in different circumstances, e.g., only with indirect objects, or only with indirect objects and pronominal direct objects? This is clitic doubling Problem 2: What accounts for the inter- and intralinguistic distribution of clitic doubling?

Another kind of variable property is Kayne's Generalization, already mentioned earlier (which does not appear to hold of all clitic doubling languages): it claims that bare objects may not be doubled, but must be affixed by a dummy marker (typically a preposition, often the one otherwise inducing dative Case). This is clitic doubling Problem 3. What accounts for Kayne's Generalization?

Unfortunately, apart from Problem 1, I do not have good answers to provide to these problems. Past proposals can be incorporated within the present account, as I will do, but none of them is really satisfactory. Let us postpone consideration of Problem 1 until the discussion of the function of clitic projections in Section 7.2. There, we will suggest a possible answer to the first problem.

As for Problems 2 and 3, I can suggest some speculations along the lines of similar problems arising in other constructions. We need to answer the following questions: (1) why do certain languages (or constructions) allow the co-occurrence of overt clitics and overt XP* and others do not? (2) why do certain languages (or constructions) allowing overt $\mathrm{XP} *$ disallow its overt raising to $\mathrm{XP}^{\wedge}$ while others do not? Adapting the line that the proponents of base-generating clitics have proposed, we might rule out clitic doubling by appealing to Case Theory. More specifically, we might argue that languages 


\section{CLITIC CONSTRUCTIONS}

disallow realizing the same Case on two different nominal elements. Because Romance clitics are nominal and encode Case morphology, they cannot cooccur at the same time as a full case-marked XP* (unless an extra option exists to case-mark this XP*, cf. Kayne's Generalization). One way of implementing this idea pursues the analogy with $W b$ Movement entertained earlier. The output of syntactic $\mathrm{Wb}$ Movement is subject to the doubly filled Comp filter. Suppose that just as the Clitic Criterion suggests a generalization of the Wh-Criterion to a more general principle of licensing (a general line pursued in Sportiche 1995), the doubly filled Comp filter generalizes in such a way that it covers clitic projections or voices as well. The general idea might be that functional heads such as certain Cs or certain clitics cannot be simultaneously filled as their specifier if they encode a property overtly realized on this specifier (here this might be Case). This would be a sort of principle of economy minimizing use of unnecessary morphophonological overtness (similar in a sense to the Avoid Pronoun Principle): ${ }^{25}$

$$
\begin{aligned}
& \text { Doubly filled voice filter } \\
& { }_{L_{\mathrm{HP}}} \mathrm{XP}[\mathrm{H} \ldots] \\
& \text { where } \mathrm{H} \text { is a functional head and both XP and } \mathrm{H} \text { overtly } \\
& \text { encode the same property } \mathrm{P} \text {. }
\end{aligned}
$$

Clitic doubling may arise in a language if the clitic encodes no (relevant) property that the doubling phrase expresses; this might be the case in languages such as Lebanese Arabic that have a morphologically poorer clitic system than Romance or Spanish, where the insertion of a preposition embeds $\mathrm{XP} *$ under a $\mathrm{P}$ that makes the property expressed by $\mathrm{XP} *$ inaccessible to (41). Another possibility, which we will ultimately reject, would be to limit the scope of (41) to overt movement. XP* raising would behave in a way similar to what is assumed of LF Wh Movement (possibly because (41) is not operative on LF or because its effects can be trivially voided by deleting a semantically empty $\mathrm{C}$ ). Delaying XP* movement until LF would then provide a way of preventing a violation of $(41)$ with an overt $\mathrm{XP}^{*}$ in the presence of an overt clitic.

\subsubsection{Participle agreement}

Let us now return to participle agreement within the approach of clitic constructions proposed here. The basic premise we adopt is that put forth in Kayne (1989b): participle agreement (and agreement in general) is an $\mathrm{XP} /$ head relation that should be handled in a fashion similar to subject/tense agreement. Given that the latter is analyzed as a Spec/head relation, the former should be, too. Pursuing the analogy, given that the subject agrees with $T$ and that this is analyzed as $T$ raising to an agreement head, $A G R_{s}$, we postulate that participle agreement is triggered by the moving of a phrase 
through the specifier position, which we call $\mathrm{Spec}_{\text {AGR-O }}$, of an agreement head $\mathrm{AGR}_{\mathrm{O}}$ to which the participle raises. ${ }^{26}$

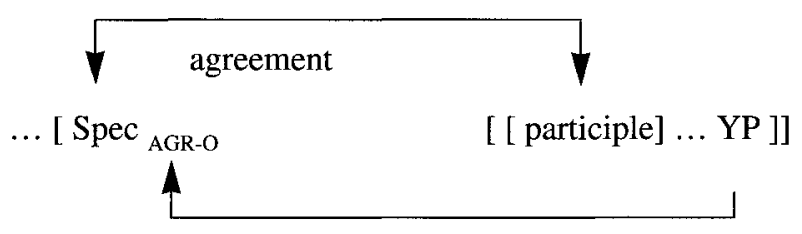

Here, we limit ourselves to agreement with accusative DPs. As mentioned earlier, such agreement may not take place if the accusative DP overtly follows the participle. When the DP precedes the participle, as in clitic constructions, agreement is possible, but not necessary, except in more formal registers. This is illustrated below:

(43)(a) Jean a peint(*e) la porte. John has painted(*FEM) the door. John painted the door.

(b) Jean l' a peint(e).

John it has painted(FEM). John painted it.

This optionality is actually not an isolated fact of French participle agreement. The same is true of Catalan participle agreement in clitic constructions, as discussed in Cortés (1992). It is also true of Italian participle agreement in clitic constructions when the object clitic is not a third person clitic.

(44)(a) Giovanni la ha accusata/ *accusato.

Giovanni her has accused $\mathrm{FEM} /$ *accused $_{\text {MASC }}$ Giovanni has accused her.

(b) Maria e Paola, vi ho visto/ viste.

Mary and Paula you ${ }_{\text {FEM.PL }}$ I-have seen ${ }_{\text {MASC.SG }} /$ seen $_{\text {FEM.PL }}$. Mary and Paula, I have seen you.

How should we handle this optionality? First, subject/tense agreement suggests that agreement is not optional. If the agreement configuration is met, agreement takes place. In movement theories moving the clitic from the position $\mathrm{XP} *$ (essentially) to its surface position, the only way to get the right result is to provide two possible movement paths, one of which may skip the position Spec AGR-O . This is the proposal put forth in Sportiche (1990) (and also in an earlier draft of the present article). Sportiche (1990) adopts Kayne's proposal that if a clitic moves, the movement must involve head movement at some point, since the clitic does end up incorporated to the verb as only a head 


\section{CLITIC CONSTRUCTIONS}

may do. To reconcile this view of clitic movement as head movement with Kayne's analysis of agreement (which assumes XP movement) as well as with the Head Movement Constraint (Head movement from XP* to its surface position would violate this condition), Sportiche (1990) proposed that clitic movement is decomposed into two different steps. The first step is a phrasal movement up to a position from which the clitic may incorporate as a head to its hosts without violating the HMC. Now consider a case of an accusative clitic not triggering agreement:

$$
\text { l'avoir }\left[_{A G R P} S_{\text {Ppec }}{ }_{A G R-O}{ }_{A G R} \text {, [construit }\right] \ldots X P \text {. }
$$

The clitic head of XP must move out of VP and skip Spec AGR-O $_{\text {to a position }}$ from which it may incorporate to the auxiliary. Since incorporation cannot be done from within AGR', and there is no A-position available into which XP could move, we are led to assume that XP moves to some A-bar position XP', governed by the auxiliary from where the clitic may incorporate to avoir. That is, we are forced to assume that clitic movement involves A-bar movement when participle agreement fails. This predicts that accusative clitics should be able to license parasitic gaps from this position XP'. This consequence appears to contradict Chomsky's (1982) or Sportiche's (1983) conclusions, according to which these clitics do not license parasitic gaps. We return to this problem in Section 6.2.2.

Consider now the optionality problem within the framework of the analysis of clitics proposed here.

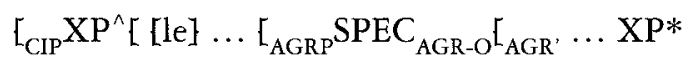

The clitic does not move. Only its associated XP* does. ${ }^{27}$ Under a skipping approach to agreement failure, we may claim that $\mathrm{XP}^{*}$ moves to $\mathrm{XP}^{\wedge}$ without moving through $\operatorname{Spec}_{A G R-O}$. We do not face the potential parasitic gap problem if $\mathrm{XP}^{\wedge}$ is an A-position. If $\mathrm{XP}^{\wedge}$ is an A-bar position we do. We may also propose that agreement fails because movement of XP* does not occur early enough to feed the phonology, i.e., it is delayed until LF. In other words, agreement is triggered if XP* movement takes place overtly in the syntax. Agreement is not triggered if movement of XP* takes place covertly at LF.

Either option seems compatible with the function of $\mathrm{Spec}_{\mathrm{AGR}-\mathrm{O}}$, although the second one seems simpler: as mentioned, only accusatively Case-marked NPs have access to Spec $_{\text {AGR-O }}$. This restriction can be explained if object agreement and accusative Case are two sides of the same coin. Adopting Chomsky's (1991) or Sportiche's (1990) views on the subject, we take Spec $_{\text {AGR-O }}$ to be the position in which accusative Case is obligatorily sanctioned at LF: accusative NPs must in the end move to (or through) Spec $_{\text {AGR-O }}$. If agreement is present, this means that $\mathrm{XP}^{*}$ movement to $\mathrm{XP}^{\wedge}$ through $\mathrm{Spec}_{\text {AGR-O }}$ takes place overtly. If agreement is absent, the delaying view 
assumes that this exact same movement is delayed until LF. The skipping view is forced to assume that $\mathrm{XP}^{*}$ moves to $\mathrm{XP}^{\wedge}$ skipping $\mathrm{Spec}_{\mathrm{AGR}-\mathrm{O}}$, but accusative checking later needs to incorporate $S_{\text {Pec }}{ }_{A G R-O}$ in the movement chain (e.g. by moving the trace of XP* to $\mathrm{Spec}_{\mathrm{AGR}_{-} \text {). }}$.

The delaying approach (movement of $\mathrm{XP}^{*}$ to $\mathrm{XP}^{\wedge}$ is $\mathrm{LF}$ movement - in Chomsky's (1992) terms, movement is procrastinated) seems more straightforward than the skipping approach and I will tentatively adopt it, at least for accusatives, for two reasons: (1) There are good grounds for believing that $\mathrm{XP}^{\wedge}$ should count as an A-bar position (see Section 7 on Dutch Scrambling), and (2) accusative clitics do not license parasitic gaps (see Section 6.3). There are however a number of problems requiring mention. First, Wh Movement may also optionally trigger participle agreement in a clause containing an accusative $w$-trace. ${ }^{28}$

$$
\begin{aligned}
& \text { Quelle maison as-tu construit(e)? } \\
& \text { Which(FEM) house have-you built(FEM). } \\
& \text { Which house did you build? }
\end{aligned}
$$

In this case, movement is obviously overt. The delaying approach is thus not an option. Only the skipping option is. If the skipping option must be appealed to for $W b$ Movement, it comes as no cost for clitic constructions. Second, if XP* movement to $\mathrm{XP}^{\wedge}$ may be delayed until LF, we might expect clitic doubling to be allowed if the doubly filled voice filter is an S-Structure filter. Consequently, clitic doubling should surface in all French dialects allowing optional participial agreement with accusative clitics, a prediction contrary to what is observed. This indicates, in a way consistent with Chomsky's (1992) Minimalist assumptions, that this filter is specifically not an S-Structure filter but rather an LF filter, and this is what I will assume.

Third, we have seen in Section 4.2.2 that lui object of prepositions displays CED effects. This used to be taken as a diagnostic property of overt syntactic

movement. Again, to be consistent, we must deny the validity of this conclusion, which raises very general problems about the apparent lack of CED effects for covert movement. We leave this general problem, which also arises as a consequence of Chomsky's (1992) proposals, unaddressed here.

\subsubsection{Stranded quantifiers}

We can now return to the properties of stranded quantifiers discussed earlier in Sections 4.3.2 and 4.3.3. One type of problem is illustrated in (28) repeated here:

(28)(a) Marie les a tous pris. Mary them has all taken. Mary took them all. 


\section{CLITIC CONSTRUCTIONS}

(b) Marie a toutes voulu [les manger]. Mary has all wanted them eat ${ }_{\text {INF }}$. Mary wanted to eat them all.

(c) Il a tous fallu [qu' ils parlent]. It has all necessitated that they speak. It was necessary that they all speak.

(d) Il a tous fallu [que Louis les lise]. It has all necessitated that Louis them read. It was necessary that Louis read them all.

The analysis of clitics we propose allows a simple treatment of these facts: in all cases, $\mathrm{XP}^{*}$ will be analyzed as moving to the specifier position of the $\mathrm{Q}$ tous. If tous is actually moved to its surface position, we may analyze $\mathrm{XP}^{*}$ as $\left[\right.$ pro $_{\mathrm{i}}\left[\right.$ tous $\left.\left[\mathrm{DP}_{\mathrm{DP}} \mathrm{t}_{\mathrm{i}}\right]\right]$. This $\mathrm{QP}$ raises to $\mathrm{XP}^{\wedge}$ whence it moves to its surface position by A-bar movement: ${ }^{29}$

$$
\left.{ }_{\mathrm{QP}} \text { pro [tous }\left[\mathrm{DP}_{\mathrm{DP}} \mathrm{t}\right]\right]_{\mathrm{j}} \text { ] fallu [que Louis }\left[\mathrm{t}_{\mathrm{j}} \text { les lise } \mathrm{t}_{\mathrm{j}}\right] \text { ] }
$$

This approach to (28b-d) extends straightforwardly to the sentences below, which do not involve clitics modified by Qs but bare Q DPs:

(49)(a) Marie a tout voulu manger.

Mary has all wanted eat ${ }_{\mathrm{INF}}$.

Mary wanted to eat everything.

(b) Il a tout/rien fallu [que Louis lise t].

It has all/nothing necessitated that Louis read.

It was necessary that Louis read everything/nothing.

(=There is nothing that it was necessary for Louis to read.)

Alternatively, we may generate tous in the matrix clause and raise a pro XP* to its specifier (be it overtly or covertly, with different predictions concerning agreement, parasitic gaps, etc.).

A similar approach extends to the problematic cases of (31) (repeated here):

(31)(b) Je (*à) leur ai (tous) offert un cadeau $*(\grave{a})$ tous. I to them have all given a present to all.

I gave them all a present.

(c) Ces garçons *(à) qui j'ai (??tous) offert

These boys to whom I have (all) given

un cadeau ((à) tous) ...

a present (to all).

These boys, all of whom I gave a present.

The obligatory versus impossible pied-piping of the dative marker can now be attributed to a difference between overt and covert movement: $\mathrm{Wh}$ Movement 
of the DP [à qui] must pied-pipe the dative case marker. In the clitic case, we may claim that overt movement is prevented by the insertion of the dative marker $a$. The structure would include leur ...XP* with an unmoved XP* of the form $\left[\grave{a}+\left[_{\mathrm{DP}}\right.\right.$ pro $][$ tous $\left.\mathrm{t}]\right]$.

\subsection{Head movement and clitic placement}

Our analysis of clitics imposes boundary conditions on the analysis of clitic climbing (and of restructuring constructions). In what follows, we explore this issue within the context of a proposal made in Kayne (1989a) as to the treatment of clitic movement and of its critique.

Clitic movement is typically clause bound, a property we have not yet discussed. In the short generative tradition, this property has most often been taken to follow from assimilating clitic placement to some kind of NP Movement. This was always a problem, the locality of NP Movement used to be handled by assimilating NP-traces to anaphors, a reasonable assumption if the trace of the clitic is phrasal. However, the movement analyses typically assume that clitic placement was movement of the clitic itself, which seemed to share no other property with NP Movement than locality (which led Sportiche (1983) to conclude that clitic placement did not involve movement).

Theories reducing locality constraints of NP Movement rules to the ECP, such as Chomsky (1986a; 1986b) and, more precisely, to antecedent government do not face this problem. The apparent similarity between NP Movement and clitic placement can be derived even if clitic placement is not phrasal movement. In particular, if clitic placement is head movement, locality effects will be found, too, since traces of heads are subject to antecedent government as well. This is in fact the view taken in Kayne (1989a). He proposes to treat clitic movement as head movement throughout: in our terms, the head of XP* moves as a head from its base position to adjoin to its host head. This view is at odds with what we are suggesting. We do treat clitics as heads. As such, they may move as heads do, and in fact they do. ${ }^{30}$ But we claim the fundamental relation between CI and XP* is not one of head movement. Kayne's (1989a) analysis of clitic placement as head movement, throughout seems to contradict Kayne's (1989b) proposal about participle agreement that we have adopted here. If clitic placement is head movement agreement of the object with the participle can no longer be seen as an XP/Y $\mathrm{Y}^{0}$ specifier/head relation. Furthermore, Kayne's proposal makes it surprising that participle agreement may be triggered by clitic placement, NP Movement (as in passives, etc.) and $W b$ Movement, the last two being clear cases of phrasal movement (cf. Section 4.2.3). 


\section{CLITIC CONSTRUCTIONS}

\subsubsection{Restructuring and clitic climbing}

Kayne wants to correlate the possibility for a clitic to leave its VP, the possibility of clitic climbing in restructuring constructions (see below) and the licensing of null subjects (pro-drop) and trace them all to the same property, namely whether or not Infl is strong enough to make a VP boundary transparent to movement. What actually matters for Kayne's analysis is not so much that clitic movement is head movement from XP* on. Most of his proposals can be preserved if a clitic can escape its minimal VP only by head movement. What happens inside the VP is not really relevant.

Our position and his can be reconciled by altering Kayne's (1989a) proposal along the lines of Sportiche (1990). As previously discussed, clitic movement could be XP movement VP-internally or more precisely within AGR- ${ }_{\mathrm{O}} \mathrm{P}$ - triggering agreement - followed by head movement. ${ }^{31}$ Although this would preserve most of Kayne's (1989a) analysis, it appears incompatible with the data discussed below in (54). The main type of evidence Kayne (1989a) presents to independently support the idea that clitic movement is head movement involves showing that intervening heads intercept clitic movement, an expected Minimality effect. Below, we discuss one such case. The others could be discussed in a similar way.

There are apparent violations of the clause boundedness effect on clitic placement in restructuring constructions, present in earlier stages of French and in contemporary Italian and Spanish, among others.

(50)(a) Pierre le voulait [lire XP*]. (*in Modern French) Peter it wanted to-read. Peter wanted to read it.

(b) Gianni li vuole vedere. (Italian)

John them wants to-see.

John wants to see them.

(c) Lo quiero ver. (Spanish)

Him/it I-want to-see.

I want to see him/it.

These constructions raise many more questions than we can answer here. Here we ask what the process is by which the clitic appears in the matrix clause. The most common view is that the process of clitic placement in these restructuring constructions is identical to that found in simple clauses. The appearance of clitic climbing derives from the prior application of another process. This restructuring process affects the syntactic structure in such a way that normal application of clitic placement will have the clitic climbing effect. Since this scenario is independent of the precise characterization of clitic climbing, it is perfectly compatible with our analysis. ${ }^{32}$

Kayne (1989a) suggests a different view illustrated in the diagram below: 


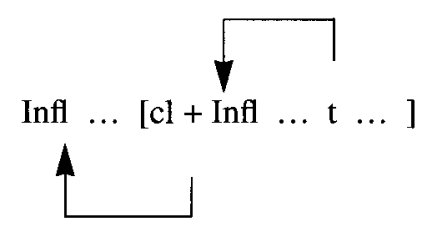

He suggests that clitic placement in restructuring constructions is different from normal clitic placement. Clitic climbing arises because these constructions involve raising of a lower Infl to a higher Infl. The clitics climb because, being adjoined to the lower Infl, they get a ride up to the higher Infl, so to speak. The central data Kayne cites supporting this proposal are due to Rizzi (1982). They show that, in a restructuring construction of colloquial Italian, an intervening head in C blocks clitic climbing, while an intervening $\mathrm{XP}$ in Spec, CP does not, strongly suggesting a Head Movement Constraint effect of some sort. ${ }^{33}$ In this variety of Italian, we find clitic climbing out of infinitival indirect questions:

(52)(a) ?Mario, non lo saprei [a chi affidare $\left.\mathbf{t}_{j}\right]$.

Mario not him I-would-know to whom entrust ${ }_{\mathrm{INF}}$. Mario, I would not know to whom to entrust him.

(b)*Su questo problema, non lo $_{j}$ saprei [se consigliare $t_{j}$ ].

On this problem, not him I-would-know whether advise.

On this problem, I would not know whether to advise him.

The significant observation is this: the complementizer se blocks clitic climbing while the $w b$-phrase $a c b i$ does not. ${ }^{34}$ This is a clear indication that head movement is somehow involved, and is interfered with by the intervening head se.

For us, these data might appear at first unexpected, if we always fundamentally treat clitic placement as a case of phrasal movement rather than as head movement. We would expect an intervening phrase to block clitic placement and an intervening head not to interfere. As heads, object clitics can incorporate to some head, say Infl. Thus we may in principle adopt Kayne's treatment of restructuring constructions. It would be extremely suspicious however, if the surface distribution of clitics in a restructured clause were identical to that found in a comparable simple clause even though the processes involved are fundamentally different.

Further data provide interesting clues. First of all, Rizzi (1982) reports that the data in (52) with climbed clitics are mirrored by object preposing (see Burzio (1986), Rizzi (1978) for discussion and description of object preposing):

(53)(a) ?Certe riposte non si sanno mai come dare. 


\section{CLITIC CONSTRUCTIONS}

Certain answers not si know never how give ${ }_{\mathrm{INF}}$

One never knows how to give certain answers.

(b)*Certe riposte non si sanno mai se dare.

Certain answers not si know never whether give INF .

One never knows whether to give certain answers.

Object preposing of this sort behaves like clitic placement: it is usually clause bound, except in clitic climbing contexts. In restructuring contexts allowing a clitic to climb out of its clause, an object may also be preposed out of its clause. Following Burzio (1986), let us call this Long Object Preposing (LOP). This preposing is an instance of movement to subject position of the main clause (that may occur in the presence of the clitic si), as evidenced by the agreement reflex on the matrix verb. Since object preposing is clearly a case of phrasal (DP) movement, why is it blocked by an intervening head and not by an intervening phrase?

From the earliest approaches to restructuring (such as Evers (1975) for Dutch, or Rizzi (1978) for Italian) to recent ones (such as Kayne 1989a), restructuring has been postulated to involve incorporation of some head from the lower clause ( $\mathrm{V}$ or Infl) to a head in the higher clause ( $\mathrm{V}$ or $\mathrm{Infl})$. We expect that process to be sensitive to intervening heads, and thus to be blocked by a $\mathrm{C}$ but not by a Spec, CP. Taking the possibility of LOP to be contingent on restructuring being available predicts the data: in $(53 \mathrm{~b})$, se blocks restructuring. The context for LOP is not met.

The same line of explanation can now be applied to the cases of clitic climbing in (52). Clitic climbing itself is not blocked by se, but it may not apply if restructuring cannot. There is no need to suppose that clitic placement itself is head movement. Furthermore our analysis can straightforwardly capture the coextensiveness of clitic climbing and LOP, since both are phrasal movement. In this connection, the facts of (53) might appear surprising from the point of view of Kayne's proposal, or from the point of ours since we see that the intervening $w h$-phrase does not block phrasal movement over it. Since LOP is an instance of A-movement, this is less surprising. In the spirit of minimality effects, we would not expect an intervening A-bar position such as Spec, $\mathrm{CP}$ to interfere with A-movement. ${ }^{35}$

\subsubsection{Agreement and restructuring}

Not only is there no need to take clitic placement to be head movement, but there is also some evidence directly supporting the idea that clitic placement must involve some phrasal movement beyond the smallest VP containing XP*. Our reinterpretation of Rizzi's data, together with the analysis of clitic climbing as another instance of XP* movement, predicts the clustering of clitic, wh, or NP movement constructions with respect to participle agreement. Furthermore, it predicts that participle agreement should be 
available on either main clause or embedded clause participle in restructuring constructions, which is correct:

(54)(a) $\mathrm{Li}_{\mathrm{j}}$ vorrei [aver gia $\left[\mathrm{e}_{\mathrm{j}}\right\}_{\mathrm{XP}}$ letti $/ *$ letto $\left.\left[\mathrm{e}_{\mathrm{j}}\right]_{\mathrm{XP} *}\right\}$. Them I-would-want have ${ }_{\mathrm{INF}}$ already read $\mathrm{PL}_{\mathrm{PL}} / \mathrm{read}_{\mathrm{SG}}$. I would want to have already read them.

(b) $\mathrm{Li}_{\mathrm{j}}$ ho $\left[\mathrm{e}_{\mathrm{j}}\right]_{\mathrm{XP}}$ voluti $/ *$ voluto leggere $\left[\mathrm{e}_{\mathrm{j}}\right]_{\mathrm{XP}}$. Them I-have wanted ${ }_{\mathrm{PL}} /$ wanted $_{\mathrm{SG}}$ read $_{\mathrm{INF}}$. I have wanted to read them.

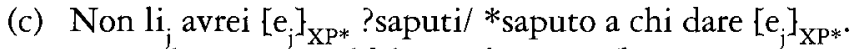

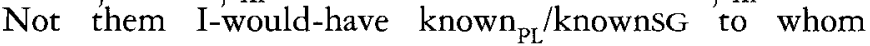
given $_{\text {INF: }}$.

I would not have known to whom to give them.

In all these cases, the participle must agree with the preposed clitic (remember that participle agreement is obligatory in standard Italian with third person clitics), thereby demonstrating the presence of a phrasal element related to the clitic, namely XP*, in the bottom clause of a restructuring construction as in (54a), or in the top clause, be it a regular restructuring construction as in $(54 \mathrm{~b})$, or an exceptional $w h$-island restructuring construction as in (54c). This last case is particularly significant since we are clearly dealing with a biclausal structure. This means that clitic placement must involve moving an XP* out of a clause and a fortiori out of a VP.

\section{Clitics binding parasitic gaps and the nature of $\mathrm{XP}^{\wedge}$}

We now turn to a discussion of the properties of individual clitics in French. We essentially limit ourselves to some cased clitics, i.e., genitives, accusatives, datives, ignoring the clitic se and predicate $l e$. Nominative clitics are discussed Sportiche (1995). ${ }^{36}$

\subsection{Clitics and Binding theory}

In this section we explore how clitics interact referentially with other nominals. Non-reflexive clitics have often been treated as pronominal elements of some sort. The main reason, which has led us to analyze XP* as pro, is that they are understood the way pronouns would be. Another, more directly relevant reason is based on their behavior with respect to coreference or binding with other nominals. Just like a pronoun, a clitic must be disjoint in reference from a nominal c-commanding it in its clause or its governing category, but may be coreferential or bound by a more remote nominal:

(55)(a) Marie l'a vue.

Mary her has seen. 


\section{CLITIC CONSTRUCTIONS}

Mary saw her.

(b) Marie dit que le lion l'a vue.

Mary says that the lion her has seen.

Mary says that the lion saw her ${ }_{\text {jor }}$.

(c) Personne ne dit que le lion l'a vue.

No-one NEG says that the lion her has seen.

No one ${ }_{j}$ says that the lion saw her ${ }_{j}$ or $k$.

These data do not establish whether it is the clitic itself that counts for the interaction. It may be the position of the clitic itself, it may be the position of $\mathrm{XP}^{*}$, or it may be the position $\mathrm{XP}^{\wedge}$ (or it may be some other, presumably intermediate, position, a possibility we will not consider here). Only the present approach postulates the existence of $\mathrm{XP}^{\wedge}$. If it turned out that it is $\mathrm{XP}^{\wedge}$, it would strongly support our approach.

First of all, note that the c-command domain of the clitic and that of $\mathrm{XP}^{\wedge}$ are (almost) identical. If it turns out that it is one of the two that is relevant for binding effects, we will not be able to determine on empirical grounds which one is actually relevant. However, simplicity considerations favor $\mathrm{XP}^{\wedge}$ : referential dependencies are minimally found between potentially denoting elements, i.e., DPs. If the clitic itself mattered, we would have to extend this to include heads. If, on the other hand, it is $\mathrm{DP}^{\wedge}$, nothing further needs to be added.

Second, to distinguish between $\mathrm{XP}^{\wedge}$ and $\mathrm{XP}^{*}$, we need to find one of two configurations (given that $\mathrm{XP}^{\wedge}$ or the clitic must c-command $\mathrm{XP} *$ ): either a $\mathrm{DP}$ c-commanding $\mathrm{XP}^{*}$ but not $\mathrm{XP}^{\wedge}$, or a $\mathrm{DP}$ that $\mathrm{XP}^{\wedge}$, but not $\mathrm{XP}^{*}$ $c$-commands. It is difficult to find convincing cases of the first configuration. The second one however can be constructed in French:

(56)(a) Jean a présenté l'auteur de ce livre à

John has introduced the author of this book to l'éditeur de ce livre. the publisher of this book.

John introduced the author of this book to the publisher of this book.

(b) Jean a présenté son ${ }_{j}$ auteur à l'éditeur de ce livre. John has introduced its author to the publisher of this book. John introduced its author to the publisher of this book.

(c)* Jean en a présenté l'auteur à l'éditeur de ce livre. John of-it has introduced the author to the publisher of this book.

John introduced its author to the publisher of this book.

Coreference between the two DPs ce livre is possible in (the admittedly awkward) (56a), even if the first one is pronominalized to son (presumably a D 
coindexed with a pro in Spec, DP), as in (56b)). Cliticizing this first DP yields an unacceptable form. Superficially, it is the clitic that matters. We conclude that it is the position $\mathrm{XP}^{\wedge}$ that matters for binding effects. ${ }^{37}$ This has to do with the status of the position $\mathrm{XP}^{\wedge}$ associated with the clitic en. Movement to $\mathrm{XP}^{\wedge}$ from $\mathrm{XP}^{*}$ is from within a $\mathrm{DP}$ and thus must be A-bar movement (see Sportiche (1990) or Valois (1991) for discussion). Binding Principle C must therefore be formulated so as to require names to be A-free as well as A-bar free. The conclusion that Spec, en is an A-bar position in these cases is strengthened by the inability of $\mathrm{XP}^{\wedge}$ to provide a possible binder for an anaphoric element, since they require A-binders:

(56)(d) Jean ${ }_{i}$ a présenté [le frère de $\left.[\text { Pierre }]_{k}\right]_{1}$ à John has introduced the brother of Peter to [son propre $]_{\mathrm{j},{ }^{*}, \mathrm{l}}$ père. his own father. John introduced the brother of Peter to his own father.

(e) $\operatorname{Jean}_{\mathrm{j}} \mathrm{en}_{\mathrm{k}}$ a présenté [le frère $]_{1}$ John of-him has introduced the brother à [son propre $]_{\mathrm{j},{ }^{*} \mathrm{k}, 1}$ père. to his own father. John introduced the brother of Peter to his own father.

To show that we are not merely dealing with a linearity effect, we should be able to reverse the judgment of (56c) by removing the offending DP from the c-command domain of the clitic, while keeping the same relative ordering of the two, despite the right branching structure of French. The following paradigm illustrates this possibility:

(57)(a) Jean n'a pas critiqué l'auteur de ce livre John NEG has not criticized the author of this book [à cause de ce livre]. because of this book. John did not criticize the author of this book because of this book.

(b)*Jean n'en a pas critiqué l' auteur John NEG of-it has not criticized the author [à cause de ce livre.]. because of this book John did not criticize the author of it because of this book.

In (57a), coreference between the two DPs ce livre is possible. The sentence is ambiguous as to whether the causal adjunct is in the scope of the negation or not. We get the two readings: (1) It is not because of this book that John criticized its author, (2) It is because of this book that John did not criticize 


\section{CLITIC CONSTRUCTIONS}

its author. In (57b), with the first DP cliticized as $e n$ the first reading becomes more difficult, if at all available, but the second remains. We may interpret this state of affairs as follows. Under the second reading, the causal adjunct is outside the scope of negation, hence outside the c-command domain of $e n$ (or its associated $\mathrm{XP}^{\wedge}$ ). Under the first one, the causal adjunct is within the c-command domain of $e n$, hence within the scope of the negation, but the corresponding reading is excluded as a Principle $\mathrm{C}$ effect.

This line of reasoning, based on French, does not depend on the level at which the Binding Theory applies since movement of $\mathrm{XP}^{*}$ to $\mathrm{XP}^{\wedge}$ is overt in French. However, there are theoretical and empirical reasons for wanting the Binding Theory to hold at LF. The empirical reasons are well known (reconstruction effects, etc.). The theoretical reasons are twofold. First, Binding Theory deals with matters of (possible) referential interpretation. It should therefore operate on and only operate on the level(s) relevant for referential interpretation, namely LF. Second, as Chomsky (1992) discusses, there are reasons to doubt the existence of a well defined level of S-Structure. Since the Binding Theory cannot be assumed to hold exclusively at Dstructure, there are only two options: Binding Theory holds everywhere, hence at LF, or it only holds at LF. So it holds at LF. What this means for prohibition principles such as Principles $B$ or $C$, as opposed to prescriptive principles such as Principle $A$, is that the prohibition must either be met at LF, or everywhere, hence at LF. In particular, the conclusion we reached for French should also hold in cases of clitic doubling, where raising of XP* to $\mathrm{XP}^{\wedge}$ is delayed until LF. That this is the case is shown in Varela (1988).

\subsection{Clitics and parasitic gaps}

\subsubsection{Genitive en}

We mentioned earlier a conclusion reached in Chomsky (1982) based on Italian examples and discussed in Sportiche (1983), to the effect that clitics do not license parasitic gaps. Descriptively, parasitic gaps are licensed in S-structure configurations like (58) (linear order irrelevant),

$$
\text { ... A-bar binder ... RG .. [ . . PG ... }]_{K}
$$

where the A-bar binder binds both gaps, and there is no c-command between the real gap (RG) and the parasitic gap (PG). If $K$ is the most inclusive constituent containing PG but not RG, overt movement from the position PG must be possible to a position immediately dominated by $\mathrm{K}$ (See Aoun and Clark's (1985) A-bar anaphor treatment, or Chomsky's (1986a) 0-subjacency treatment for discussion). This last condition was not recognized at the time of Chomsky (1982). Some clitics do license parasitic gaps. Using again the genitive clitic en, we have grammatical forms such as in (59): 
(59)(a) Marie en a présenté [le frère e $e_{j}$ à [la sœur e. $]$.

Mary of-him has introduced the brother to the sister.

Mary introduced his brother to the sister.

(b)* Marie en a présenté [le frère $e_{j}$ ] à [cette sœur $e_{j}$ ].

Mary of-him has introduced the brother to this sister.

Mary introduced his brother to this sister.

(c) La personne dont ${ }_{j}$ Marie a présenté [le frère $\left.e_{j}\right]$ The person of-whom Mary has introduced the brother à [la sœur $\mathrm{e}_{\mathrm{j}}$ ]. to the sister.

The person of whom Mary introduced the brother to the sister.

In (59a), en is interpreted as linked both to the argument of frère and the argument of soeur. The second gap is inaccessible to movement of $e n$, being in a PP. But movement within the PP must be unimpeded, as exemplified by the unacceptability of $(59 \mathrm{~b})$ under the relevant reading, in which the demonstrative cette blocks DP internal movement (see previous discussion in Section 4.2.2). This kind of sentence is very closely reminiscent of sentences of the type (59c) with $\mathrm{Wb}$ Movement, extensively studied in Tellier (1991), and convincingly analyzed there as involving parasitic gaps. They essentially behave in identical ways, apart from the differences due to the position of the A-bar binder, i.e., wh- in Spec, $\mathrm{CP}$ versus en.

A number of important conclusions follow from these observations. First, just as in the case of the binding theoretic argument of the previous section, we may simply attribute this licensing of parasitic gaps to the presence of the appropriate A-bar binder $\mathrm{XP}^{\wedge}$. Alternatively, we may envision extending the theory of parasitic gap licensers to include (certain kinds of) heads. But again, this last move appears otherwise unmotivated. We conclude that the existence of parasitic gaps with en shows that: (1) en clitic placement is actually phrasal movement, and (2) [Spec, en] is an A-bar position. Because of uniformity we certainly want to extend these two conclusions to all clitics. Extending the first is what we have been proposing here. Extending the second raises some difficulties concerning parasitic gaps with accusative clitics which we now discuss.

\subsubsection{Accusatives and parasitic gaps}

For uniformity, we would like to treat all Spec, CIP as A-bar positions, not A-positions. This conclusion is probably incorrect for clitics not linked to Case such as reflexive se, which we will ignore here. As for cased clitics, they do not seem to license parasitic gaps, except, of course, en. With respect to third person datives, we will conclude that $\mathrm{XP}^{\wedge}$ is an A-position. At any rate, the question only arises for accusatives, because, for various reasons, the relevant configurations are difficult or impossible to construct with other clitics. ${ }^{38}$ 


\section{CLITIC CONSTRUCTIONS}

An illustration of this failure for accusatives to license parasitic gaps is found in Chomsky (1982), based on some Italian data of Luigi Rizzi's given below $(R G=t, P G=e)$, and is equally applicable for French:

(60)(a) I libri che li dobbiamo far mettere $t$ nello The books that him we-must make put on-the scaffale [invece di lasciare $e$ sul tavolo].

shelf instead of leave on-the table.

The books that we must make him put on the shelf instead of leaving on the table.

(b)* Glieli dobbiamo far mettere $t$ nello scaffale

Them-him we-must make put on-the shelf

[invece di lasciare $e$ sul tavolo].

instead of leave on-the table.

We must make him put them on the shelf instead of leaving them on the table.

(60a) is acceptable, $(60 \mathrm{~b})$ is not. In its essentials, the relevant structure is (61):

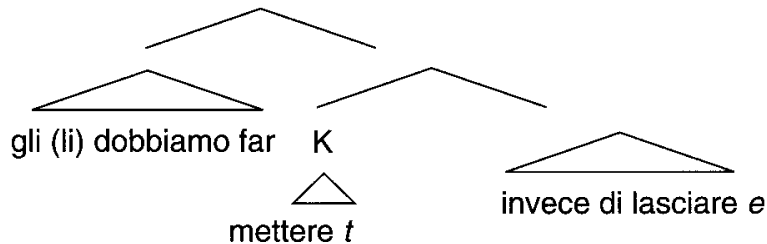

In (60a), $t$ is bound by the relative operator, which also c-commands $e$. Neither $e$ nor $t$ c-commands the other, and $e$ is free to move up within $\mathrm{K} .{ }^{39}$ Let us consider the clausal structure more carefully. Four positions are relevant: $\mathrm{XP}^{\wedge}$, XP*, PG and Spec, AGR ${ }_{0}$.

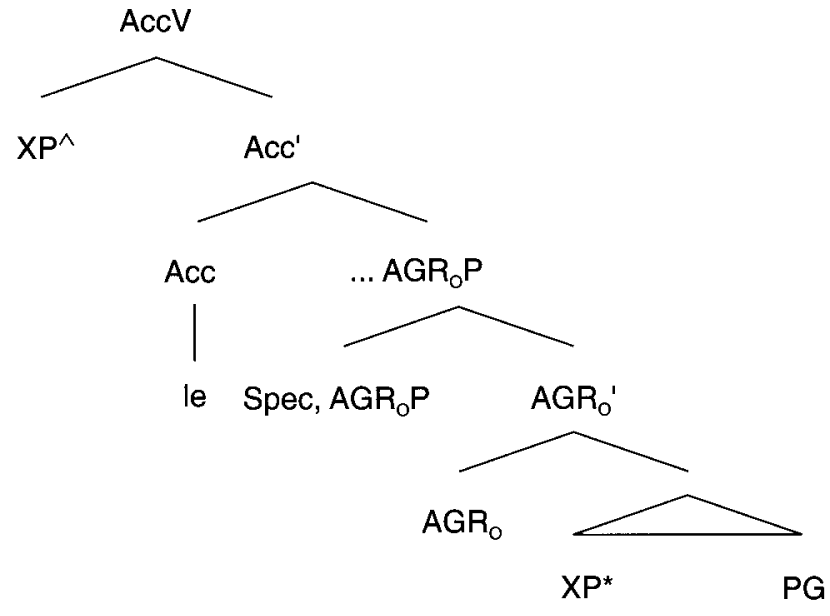


We know that Spec, $A G R_{O} P$ is an A-position (as it may be an intermediate landing site for raising to subject). At $\mathrm{LF}$, we must have a chain $\left(\mathrm{XP}^{\wedge}, \mathrm{Spec}\right.$, $\left.\mathrm{AGR}_{\mathrm{O}} \mathrm{P}, \mathrm{XP}^{*}\right)$ to license both accusative Case and the clitic. If movement of $\mathrm{XP}^{*}$ to $\mathrm{XP}^{\wedge}$ is overt and if $\mathrm{XP}^{\wedge}$ is an A-bar position, we should expect parasitic gaps to be licensed, even in simple clauses, contrary to fact, viz. ${ }^{*}$ Louis les a offensé(s) en insultant/"Louis offended them by insulting (them)."

In order to circumvent this problem, we may try either to argue that $\mathrm{XP}^{\wedge}$ is not an A-bar position or that raising of $\mathrm{XP}^{*}$ to $\mathrm{XP}^{\wedge}$ must be delayed until LF. As mentioned earlier, I will argue that the accusative $\mathrm{XP}^{\wedge}$ must be an $\mathrm{A}$ bar position; I thus suggest adopting the second option. That raising of an accusative XP* may be delayed until LF is independently motivated by the optionality of participle agreement. However we must now argue that raising of $\mathrm{XP}^{*}$ to $\mathrm{XP}^{\wedge}$ must be delayed until LF in French (otherwise PG should be licensed when movement is overt) even when participle agreement obtains. This is because participle agreement does not seem to interfere with PG licensing: parasitic gaps may be licensed whether agreement obtains or not, viz. Wh Movement of a direct object $\mathrm{XP} *$ to Spec, $\mathrm{CP}$ as in ?Quelles maisons as-tu construit(es) sans babiter?/"Which houses have you built(FEM) without inhabiting?" This means that (1) movement of $\mathrm{XP}^{*}$ to Spec, $\mathrm{AGR}_{\mathrm{O}} \mathrm{P}$ may take place overtly, (2) it does not license parasitic gaps (this is consistent with its A-position status), and (3) it does not force movement to $\mathrm{XP}^{\wedge}$.

In sum, French (or Italian) accusative clitics do not license PGs because they involve LF raising to $\mathrm{XP}^{\wedge}$. This means that there is no principled bar against accusative clitics licensing PGs. They should do so in a language or in a construction where overt movement of $\mathrm{XP}^{*}$ to $\mathrm{XP}^{\wedge}$ is possible. This is, for example, the case of French genitive clitics. It is also the case with accusative Scrambling in Dutch which we later argue can be viewed as overt movement of $\mathrm{XP}^{*}$ to $\mathrm{XP}^{\wedge}=$ Spec, $\mathrm{Acc} \mathrm{P}$ (via Spec, $\mathrm{AGR}_{\mathrm{O}} \mathrm{P}$ ), where $\mathrm{XP}^{\wedge}$ is the specifier of a silent clitic head. The relevant fact here is that accusative Scrambling does license $\mathrm{PGs}$, i.e., $\mathrm{XP}^{\wedge}$ is an A-bar position.

\subsection{The nature of $X P^{\wedge}$}

We may now turn to question (33(5)). Let us therefore first list a number of properties distinguishing $A$ and A-bar positions, as in (63):

(63)(1) Movement to an A-position obeys the SSC, not movement to an A-bar position.

(2) Movement to an A-position must be from an A-position; movement to an A-bar position may be from either kind.

(3) Movement to an A-position must be from a Caseless position; movement to an A-bar position can be from a Case position (and must be, if it is from an A-position).

(4) An A-position can be a Case position, an A-bar position cannot. 


\section{CLITIC CONSTRUCTIONS}

(5) Movement to an A-bar position but not to an A-position licenses parasitic gaps.

(6) Movement to an A-position does not create Weak Crossover effects, movement to an A-bar position may (if the moved element is not "referential"). ${ }^{40}$

(7) A-positions qualify as binders for lexical anaphors, A-bar positions may not.

\subsubsection{Genitives}

The conclusion that $\mathrm{XP}^{\wedge}{ }_{\text {en }}=\mathrm{SPEC}$, en is an A-bar position and only an A-bar position is consistent with all these properties. Corresponding to the list in (63), we find:

1 It does not obey the SSC (Marie en ${ }_{i}$ croit (Louis capable t) / "Marie believes Louis capable of it").

2 Movement to it is from an A-bar position: extraction out of DP must be through Spec, DP, an A-bar position (cf. Sportiche (1990), Valois (1991)).

3 Genitive Case is assigned DP-internally: movement to $\mathrm{XP}^{\wedge}{ }_{n}$ is from a DP-internal Case position.

4 Same as above.

5 Parasitic gaps: as discussed above

6 Irrelevant: $\mathrm{XP}^{\wedge}$ is pro, hence referential.

7 Movement to $\mathrm{XP}^{\wedge}{ }_{e n}$ does not provide an antecedent for lexical anaphors, as noted in (56 d, e) above.

\subsubsection{Accusatives}

The behavior of accusative clitics suggests this conclusion should be extended to them. Accusative Case is assigned independently of and lower than the specifier of its clitic voices. Movement to these specifiers is thus movement from (or through) Cased positions, indicating that these specifiers are A-bar positions. It is at best difficult to construct relevant examples to test criterion (7). ${ }^{41}$ With respect to criterion (5), we have already discussed parasitic gaps, which provide evidence for accusative clitics only. For criterion (6), Weak Crossover is irrelevant as in the case of $e n$. The only criterion that might favor the idea that the specifier of these clitics is an A-position is (1). We may distinguish between violations of the SSC and intervention effects of Infl or T. The SSC cases discussed in Section 4.2.1 do not distinguish between the two since the Infl of the embedded clause might be rich enough to block clitic placement, since an intervening Infl system (e.g., the presence of T or similar elements) seems to block all clitic movement. Given the other criteria, we conclude that $\mathrm{XP}^{\wedge}$ of accusatives is an A-bar position. 


\subsubsection{Locatives}

The case of locatives would seem to lead to the same conclusion, if the question makes sense. There is no principled reason why the specifiers of clitic voices should be DPs or should be allowed to contain DPs, but the properties of all other clitics we have so far discussed do suggest that $\mathrm{XP}^{\wedge} \mathrm{s}$ in these cases are DPs. If locatives are also DPs, the specifier of locative $y$ would seem to be best analyzed as an A-bar position, since $y$ placement violates the SSC. Examples similar to those for $e n$ which violate the SSC can be constructed for the clitic $y$, with the locative extracted from inside the small clause:

(63)(1) Louis croit [Marie fidèle à ses idées].

Louis believes Mary faithful to her ideas.

Louis believes Mary faithful to her ideas.

Louis y croit [Marie fidèle].

Louis to-them believes Mary faithful.

Louis believes Mary faithful to them.

\subsubsection{Datives}

Turning now to datives, a different picture emerges. The dative object of an adjective can cliticize in certain cases:

(64)(a) Marie lui est [ $t_{\text {j }}$ toute dévouée].

Mary to- $3 \mathrm{~S}$ is all devoted.

Mary is entirely devoted to him/her.

(b) Marie *lui/*me croir [Louis tout dévoué\}.

Mary to-3S/me believes Louis all devoted.

Mary believes Louis entirely devoted to him/me.

(c) A qui Marie croit-elle [Louis tout dévoué].

To whom Mary believe she Louis all devoted.

Who does Mary think Louis is entirely devoted to?

The unacceptability of (64b) suggests that dative clitics are subject to the SSC. The minimal contrast of dative clitics in (64b) with dative $w$ h-phrases in (64c) further supports singling out dative clitic placement as A-movement, since it demonstrably diverges from $W h$ Movement with respect to SSC configurations. ${ }^{42}$ Although this might seem inconsistent with the facts of (64a), where a dative clitic has moved over (the trace of) a subject, Rizzi (1986a) has argued that these are restructuring constructions, thus resolving the inconsistency (cf. also Stowell, (1991)). Taking the other criteria in (63) seriously, we have to conclude that movement of a dative XP* to Spec, lui must be from a caseless position. To accommodate this consequence, I suggest treating the dative voice not as a clitic voice but rather as the dative 


\section{CLITIC CONSTRUCTIONS}

equivalent of $A G R_{O}$ or $A G R_{S}$, i.e., as the locus of assignment (or checking) of dative Case; in effect, then it is $\mathrm{AGR}_{\mathrm{IO}}$ (indirect object agreement). ${ }^{43}$

\subsubsection{Summary}

Overall, we have the following picture in French: different clitics differ as to the A/A-bar status of the specifier of their voice, and whether or not they allow (or require) overt raising of their associated $\mathrm{XP} *$ to their $\mathrm{XP}^{\wedge}$. Both genitive and accusative clitic voices (as well as nominative, if Sportiche (1993) is correct) have A-bar specifiers, while datives have A-specifiers. Furthermore, movement of a genitive XP* to the specifier of $e n$ may (must?) take place overtly, while movement is necessarily covert for accusative XP*. In the case of datives, we have seen some evidence in Section 5.2.3 that movement may be delayed until LF. As we will see, the discussion of the function of clitic voices provides independent reasons to treat the specifiers under discussion as A-bar positions, as does the analysis of Scrambling in Dutch (Section 7), since Scrambling does license PGs.

Two issues remain unresolved that have much larger implications than the analysis of clitics proper. First, the issue of the locality of clitic placement to an A-bar $\mathrm{XP}^{\wedge}$, sensitive to what we have called intervening Infl material, remains unsettled. It should be noted that the very same question arises for other processes, most notably here for the locality of Scrambling in Dutch, but in general for some cases of long distance anaphora (cf. Koster and Reuland (1991)) and such dependencies as that between the negative head ne and negative quantifiers like personne/"no one." Second, the treatment of apparent differences in properties between overt and covert movement, be they their sensitivity to the CED, licensing of parasitic gaps, etc., is left open.

\section{Scrambling in Dutch, the clitic criterion and specificity}

\subsection{Dutch Scrambling}

In this section, we briefly discuss Dutch Scrambling, and show why it can be analyzed exactly like clitic constructions in French (possibly all the same conclusions would hold of German, given Moltmann (1990)). Let us begin by summarizing the findings of Koopman (1988) on direct objects which provides a crucial basis for the following discussion.

\subsubsection{Accusative scrambling}

Throughout this section, we limit the discussion to direct objects. Dutch has clitic objects. Specific full DP objects, non-specific objects and clitic objects 
(pronominal and specific) all have a different distribution. Non-clitic specific objects must occur higher than the negative marker niet and may occur lower or higher than adverbs such as waarschijnlijk/"probably." Clitics must occur higher than all of these different elements. We illustrate these observations now. (All examples in this section are drawn from Koopman (1988)). ${ }^{44}$

(65)(a) Hij heeft 't waarschijnlijk (*'t) niet (*'t) gezien.

He has it probably not seen.

He probably has not seen it.

(b) Hij heeft Jan waarschijnlijk (Jan) niet (*Jan) gezien.

He has John probably (John) not (John) seen.

He probably has not seen John.

Non-specific objects must occur essentially in what appears to be VP initial position. Furthermore, they must follow VP external particles, such as niet, waarschijnlijk or particles such as maar/"but," toch, nu/"yet," al/"already," eens/"once."

(66)(a) Pak 't maar/*maar' $t$.

Take it but/but it.

Go ahead and take it.

(b) Pak dat boekje maar/?*maar dat boekje.

Take that book but/but that book.

Go ahead and take that book.

(c) Pak maar een boekje/*een boekje maar.

Take but a book/a book but.

Go ahead and take a book.

Koopman concludes that besides the base position for objects, three positions are needed: one VP initially (for case assignment reasons, she claims) where non-specific objects occur, one for specific objects (which she claims is high enough to be governed by a - sometimes covert - inflectional head) and one for the clitics (which she claims are incorporated to this inflectional head). ${ }^{45}$ Compare Koopman's analysis for Dutch (67a) with our analysis for French (67b):

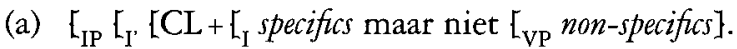

(b) F CL avoir pas $A G R_{O}$ PM verb.

The analysis of Romance clitics we have pursued so far, as well as the structural analysis of the middle field of a French clause, provides an interpretation of the data consistent with Koopman's conclusions. The VP initial position to which non-specific DPs raise from their VP internal base positions can be taken to be Spec, $A G R_{0} P$, where accusative Case is assigned/sanctioned (Dutch differs in that respect from French in requiring 


\section{CLITIC CONSTRUCTIONS}

S-Structure movement to this position). The position of specifics is nothing else but $\mathrm{XP}^{\wedge}=\mathrm{Spec}$, het, i.e., the specifier of the accusative voice. In Dutch, as in French, the doubly filled voice filter is operative, preventing simultaneous appearance of the clitic and the accusative specific object. If the object is null, the clitic head of the accusative voice reappears, but just like in French, it must incorporate to a higher functional projection. The fact that the adverb waarschijnlijk may either precede or follow $\mathrm{XP}^{\wedge}$ simply indicates that it does not interfere with the government by this functional head of the clitic voice and its head.

Note finally that this clitic voice in Dutch must appear quite high in the structure, and in particular higher than the position of the negative niet. If Sportiche (1995) is correct in analyzing niet as a negative adverb corresponding to French pas and not to French ne, we have some justification for taking the position of the clitic voices in French to be where we assumed they were in Section 5.1.2.

\subsubsection{Dative Scrambling}

Dutch has dative clitic pronouns appearing in the same position as other clitics, as shown in (68a). ${ }^{46}$ Datives can also scramble, much like accusatives when they are specific, they must move out of the VP to a position adjacent to that hosting scrambled accusatives. Datives exhibit a number of differences from accusatives. First, datives must move in front of such particles as maar even if they are not specific, and they must always precede the direct object, if there is one. In particular, if a specific direct object has been scrambled, the indirect object, whether specific or not must precede it. These observations are illustrated in $(68 \mathrm{~b}, \mathrm{c})$. Finally, datives do not license parasitic gaps, whether scrambled or not.

(68)(a) Hij heeft ' $m$ waarschijnlijk $\left(*^{\prime} \mathrm{m}\right)$ deze brief He has to-him probably (to-him) this letter niet (*'m) geschreven. not (to-him) written.

He probably has not written this letter to him.

(b) Hij heeft Jan deze brief waarschijnlijk niet geschreven. Hij heeft Jan waarschijnlijk deze brief niet geschreven. ?*Hij heeft deze brief waarschijnlijk Jan niet geschreven. ?*Hij heeft waarschijnlijk deze brief Jan niet geschreven. He probably has not written this letter to John.

(c) Verzoek iemand maar om uit te stappen. ?*Verzoek maar iemand om uit te stappen. Ask someone but/ to out to step but someone.

Just ask someone to get off. 
Aside from the failure to license parasitic gaps (which cannot be made much of in the present context since datives seem to fail to license PGs quite generally), these facts can get a straightforward interpretation within the system of assumptions we have developed here. We analyze Scrambling of datives as movement to the specifier, $\mathrm{XP}^{\wedge}$ of the dative voice, headed by a (covert) dative clitic. Suppose, as we have discussed for French, that the dative clitic is in fact heading $\mathrm{AGR}_{\mathrm{IO}}$. We expect the dative object, when nonspecific, to move overtly to $S p e c, A G R_{I O} P$. Indeed the fact that such direct objects must occur VP initially (in fact in SPEC, $A G R_{O}$ as discussed above) indicates that Case licensing in Dutch must be satisfied at S-Structure. If the $\mathrm{AGR}_{\mathrm{IO}}$ projection is higher than the accusative voice in Dutch, an indirect object will have to scramble out of VP to a position preceding that of specific direct objects.

\subsubsection{Scrambling, parasitic gaps and locality}

If accusative Scrambling is nothing else than movement to $\mathrm{XP}^{\wedge}$, the fact that it licenses parasitic gaps, as noted by Bennis and Hoekstra (1984), supports the conclusion that $\mathrm{XP}^{\wedge}$ may be an $\mathrm{A}$-bar position:

Dat ik deze boeken [zonder $\mathbf{t}$ in te kijken] aan Jan doorverkoop.

That I these books [without in to look] to Jan sell.

That I sold these books without looking into (them).

Furthermore, the fact that Scrambling is local despite being movement to an A-bar position, and the fact that it is local in the same way as clitic placement makes our conclusions concerning the locality of clitic placement and the need to appeal to some additional locality principle more plausible (although unfortunately not explained). ${ }^{47}$

\subsection{The clitic criterion and the function of clitic projections}

\subsubsection{Licensing specificity}

So far, we have only assumed clause (1) of the Clitic Criterion:

\section{(35) Clitic Criterion}

1 A clitic must be in a Spec/head relationship with a $[+\mathrm{F}] \mathrm{XP}$ at LF

$2 \mathrm{~A}[+\mathrm{F}] \mathrm{XP}$ must be in a Spec/head relationship with a clitic at LF.

There is one property of Dutch Scrambling that we have left unaccounted 


\section{CLITIC CONSTRUCTIONS}

for, namely the fact that accusative or dative specific DPs must raise to $\mathrm{XP}^{\wedge}$. I suggest that we derive this property by enforcing clause (2) of the Clitic Criterion above, choosing the property $[+\mathrm{F}]$ to be specificity. This means that at LF, specific DPs will have to raise to their corresponding $\mathrm{XP}^{\wedge} .{ }^{48} \mathrm{In}$ other words, as Sportiche (1995) proposes, some clitics (in French, those with structural Case - nominative, accusative, genitive) license specificity in DPs. In this respect, clitics are like $[+w h] C s$, which license $w b$-phrases, $[+$ neg] heads, which license negative quantifiers and polarity items, and [+focus] heads, which license focalized items and are overtly realized as particles (cf. Chinese shi (Chiu 1992)), or phonologically realized in intonational contours (English or French). This provides an answer to question (33(3)).

If this approach is on the right track, we need to reformulate the Clitic Criterion somewhat. In Dutch, specificity is licensed within particular projections the heads of which are not necessarily clitics. They may in fact be silent. Upon reflection, it is clear that the fact that the heads of these projections are clitics in French is accidental. Anticipating Sportiche (1995), we replace (35) with (70). There is a set of properties $P_{1} \ldots P_{n}$, that phrasal categories may have or lack (such as specificity, $W h$, neg, focus, scope, etc.) which we denote by assigning these categories the features $\left[+/-\mathbf{F}_{1}\right], \ldots$, $\left[+/-F_{n}\right.$. We can then further generalize the Clitic Criterion:

\section{(70) Generalized Licensing Criterion}

$1 \quad \mathrm{~A}[+\mathrm{F}]$ head must be in a Spec/head relationship with a $[+\mathrm{F}]$ $\mathrm{XP}$ at LF.

$2 \mathrm{~A}[+\mathrm{F}] \mathrm{XP}$ must be in a Spec/head relationship with a $[+\mathrm{F}]$ head at LF.

If $[+F]$ denotes specificity, the corresponding heads in French will either be clitics or possibly silent (see Section 7.2.2). In Dutch, the corresponding heads will be clitics in case the specific element is pro. If the specific phrase to be licensed is lexical, the corresponding head is silent. From this perspective, we can return to clitic doubling Problem 1 from Section 5.2.1. Since pronouns are quintessentially specific DPs, we expect that if anything is doubled (i.e., singled out as specific by a clitic marking specificity), it will at least include the archetype of specific DPs, i.e., pronouns.

Generalizing the Clitic Criterion so that it and Rizzi's $W b$ criterion (and Haegeman and Zanuttini's (1991) Neg Criterion, etc.) are special cases of a more general licensing requirement fits well with our conclusions that $\mathrm{XP}^{\wedge}$ is an A-bar position. All the other cases involve operators of some sort which, because of the respective criteria, will have to be in a Spec/head relationship with their licensing head. As operators, we expect them to end up at LF in an A-bar position (as is certainly the case for both wh-phrases and negative quantifiers). If the analogy is indeed to be complete, we expect $\mathrm{XP}^{\wedge}$ to be an A-bar position. ${ }^{49}$ 


\subsubsection{Datives, clitics and agreement}

Not all elements classified as object clitics license specificity. Although all object clitics in French do, this is not telling since the only doubled elements are (silent) pronouns anyway. ${ }^{50}$ Across Romance languages, the data consistently suggest that dative clitics, as opposed to others, are not bijectively linked to specificity (see Suñer $(1988$; 1992) for Spanish or Dobrovie-Sorin (1990) for Romanian). This suggests that we should extend to other Romance languages the conclusion we reached for French (and Dutch), that is, that a dative clitic is not like other clitics, which head specificity licensing projections with A-bar specifiers. Rather a dative clitic is the head of an agreement projection devoid of interpretive consequences, and assigning or licensing dative Case in its A-position specifier. ${ }^{51}$

Of course, this does not exclude the existence of a projection licensing specificity in datives. In fact, because of the GLC (70), such a projection is required. This means that in the functional system of a clause, two systems of projections coexist: Case-licensing projections and specificity-licensing projections. This complicates considerably the question of explanatory adequacy raised in the introduction. Limiting ourselves to nominatives, accusatives and datives, we have as many as six functional projections. This raises a number of new questions which we will not address here. Which ones overtly occur? On what basis does the language learner decide the answer to the previous question? Why is specificity licensing Case driven?

From a broader perspective, it is the question of the synchronic and diachronic relationship between agreement and clitics that is raised. Fundamentally, our answer is that they are identical, both being heads agreeing with phrasal specifiers. They also differ. Agreement, as is clear from French subject/verb agreement or participial agreement, imposes no particular interpretative requirement on the agreeing DP, while some clitics obviously do, namely specificity. Another related aspect is that clitic voices have A-bar specifiers, while AGRPs have A-specifiers. Finally, a clitic linked to a DP is typically unique, while a same DP can trigger agreement on several different categories (as e.g., passivized objects trigger agreement on both $\mathrm{T}$ and on participles). ${ }^{52}$ This is consistent with the many traditional proposals treating agreement as weakened pronouns.

\section{Concluding remarks}

\subsection{On related questions and proposals}

The ideas presented here concerning clitic constructions have consequences or suggest research directions for a number of open questions which we will not pursue in detail here. ${ }^{53}$ Three of the most lively research questions relating to

clitic constructions noted are the interaction of clitic doubling with Weak Crossover discussed for example in Suñer $(1988 ; 1992)$ and Dobrovie-Sorin 


\section{CIITIC CONSTRUCTIONS}

(1990), the clitic left-dislocation constructions (discussed by Cinque (1990)) which we have briefly alluded to, and the general problem of Scrambling crosslinguistically.

\subsubsection{Clitic left-dislocation}

From the present perspective, it is tempting to take clitic left-dislocation (in Italian or Greek) to be similar to the kind of clitic doubling found in French with quantifiers to the left of the clitic, i.e., as obligatory movement of the doubled element to or beyond the specifier of the clitic it is associated with, because "true" clitic doubling is not allowed. The specifier of the clitic voice would provide the position postulated by Iatridou (1990) from which the dislocated constituent is moved in her analysis of this construction in Greek.

\subsubsection{Weak Crossover (WCO)}

It should be clear how the present proposals affect WCO. Clitics may have either free A-positions or specific A-bar positions as specifiers and are quite high in the structure of the clause: depending on what kind of specifiers a particular clitic has, the effects of the existence of this position on Weak Crossover will be radically different. Roughly, we expect specificity inducing clitics to remove WCO effects entirely, probably because the doubled element simply does not qualify as a WCO inducer (see Dobrovie-Sorin (1990) or Lasnik and Stowell (1991) for discussion). This is what is reported to take place by Suñer (1988), and the same is true of Romanian accusative doubling: ${ }^{54}$

(71)(a) ¿[A cuáles de ellos $]_{i}$ no los aguanta ni su ${ }_{i}$ madre? To which of them not them stand not-even their mother? Which ones of them cannot even their mother stand?

(b) ¿[A cuáles de ellos $]_{j}$ dijo $\mathrm{su}_{j}$ madre que no los aguanta? To which if them said their mother that not them stand? Which ones of them did their mother say that she cannot stand?

On the other hand, we expect non-specificity inducing clitic doubling such as dative doubling in Spanish to possibly remove WCO within its clause but not beyond (much as discussed in Mahajan (1990) in a slightly different setting). This appears to be found in Spanish and (for some speakers of) Romanian, though the example $(72 b)$ is less acceptable than the example (72a):

(72)(a) ¿A quién $n_{j} e_{j}$ habló su madre? To whom to-him $_{j}$ spoke his mother? $^{\text {? }}$ To whom did his mother speak? 
(b) ¿A quién ${ }_{j}$ dice $s_{j}$ madre que le dieron un premio? To whom ${ }_{j}$ says his ${ }_{j}$ mother that to-him ${ }_{j}$ they-gave a prize? To whom did his mother say that they gave (him) a prize?

The straightforward interpretation is that the pronoun $s u$ in (72a) is bound from an A-position, namely the specifier of the dative clitic voice. No WCO effect arises. In the sentence (72b), there is no such available A-position (the relevant specifier is in the embedded clause), and the WCO effect reappears.

\subsubsection{Scrambling and agreement}

It should be apparent that some of the ideas that we have presented here have been anticipated in different forms. Approaches to clitic doubling emphasizing the agreement relation between the clitic and the doubled element (Strozer (1976), Rivas (1977), Sportiche (1983) or Suñer (1988) would naturally lead to our proposal, if the contemporary construal of agreement is taken seriously.

On the question of the analysis of Scrambling, the work of Koopman (1988) on the internal structure of VP in Dutch or that of Mahajan (1990) on Scrambling and Case assignment in Hindi are prominent precursors. Expressed in our terms, Mahajan's analysis of Hindi assimilates $A_{G} R_{O} P$ and accusative voice, leading to the view that accusative Case is assigned differently to specific DPs and non-specific DPs. If we are correct, this view is inadequate for Romance languages, as there is no longer a specificity requirement for accusative objects triggering object agreement (see Sportiche (1990) for examples) then there is a specificity requirement for nominative subjects triggering agreement on verbs. More generally, we want to keep accusative Case assignment and accusative voice separate. Given the existence in Hindi of participial agreement similar to that found in Romance, we will want to take $\mathrm{AGR}_{\mathrm{O}}$ to materialize as participial agreement in Hindi, in the same way as in French. We then might reinterpret Mahajan's work as providing substantial and independent support for the existence of an accusative voice. Mahajan (1990) also postulates a relationship between the landing site of Scrambling (his argument shift) and some agreement projection (his $A G R_{O}$ which is a conflation of our $A G R_{O}$ and clitic), and suggests a typology of Scrambling roughly distinguishing between clausebound Scrambling (his argument shift) and non-clausebound Scrambling (his adjunction to XP Scrambling). As Dutch illustrates, there seem to be two types of (clausebound) Scrambling: one for Case-assignment purposes, to an AGR projection and one for specificity reasons, to a clitic voice. It is tempting to recast the results of recent work on Scrambling in the Germanic languages and the dichotomy found there (see Vikner (1990)) in terms of whether movement is to an agreement projection (A-movement) or to a voice projection (A-bar 
movement), and to distinguish both from the kind of unbounded Scrambling more akin to English topicalization.

\subsubsection{Summary and problems}

First of all, we analyze clitics as agreement heads or morphemes. Each clitic heads its own projection, which we call a clitic voice and agrees with its specifier in a way similar to the general treatment of agreement between a head and a phrase. This specifier is in turn linked by a movement dependency with the argument position the clitic agrees with.

Beyond this fundamental uniformity, clitics split into two classes depending on whether they have interpretive import or not. When they do not (datives in Romance languages) we observe a number of correlated properties. Their associated argument $\left(\mathrm{XP}^{*}\right)$ is not required to display any particular interpretive property. Their specifier $\left(\mathrm{XP}^{\wedge}\right)$ is an A-position. The $\mathrm{XP}^{\wedge} / \mathrm{XP}^{*}$ relation is subject to the SSC. The movement from $\mathrm{XP}^{*}$ to $\mathrm{XP}^{\wedge}$ does not license parasitic gaps and may relieve Weak Crossover effects only in restricted configurations. When they do have interpretive import (cf. accusatives in Romance languages), their associated argument is required to be specific. Their specifier $\left(\mathrm{XP}^{\wedge}\right)$ is an $\mathrm{A}$-bar position. The $\mathrm{XP}^{\wedge} / \mathrm{XP}^{*}$ relation is not subject to the SSC (although it is not unbounded). The movement from $\mathrm{XP}^{*}$ to $\mathrm{XP}^{\wedge}$ licenses parasitic gaps and relieves Weak Crossover effects throughout.

We leave a number of questions open and some observations unaccounted for. We offer no reason as to why there is such a remarkable uniformity across the Romance languages in treating datives as agreement and accusatives as a specificity licenser. This is particularly striking given that, for example, doubling of direct objects in River Plate Spanish starts behaving like doubling of indirect objects if the doubling clitic is dative like (the so-called leismo). Similarly, we claim that specificity of DPs is always licensed through movement to the specifier of an appropriate head This was partly motivated by the obligatoriness of Scrambling in Dutch. Yet not all specific DPs overtly (must) scramble. We have no account of this disparity (although here an analogy with $W b$ Movement with or without pied-piping is obviously promising).

\subsection{Conclusion: modularity and uniformity of licensing}

Beyond the particular problem of clitics, this proposal instantiates the general approach presented in Sportiche (1995) according to which all dependencies which are not strictly local are of the same structural type. Taking phrases (such as DPs) to be a matrix of properties, some lexical and some "scopal" in a general sense, the fundamental idea can be formulated as requiring that every single scopal property of every phrase must be satisfied in a Spec/head relationship with an appropriate head whose unique function is the licensing 


\section{PARTITIONS AND ATOMS}

of this particular property, very much in the way $W b$ Movement is thought of. The centerpiece of this conception is the Generalized Licensing Criterion given in (70), which is motivated by a fundamentally uniform approach to property licensing. At the same time, this conception yields a highly modular view of constituent structure in which the organization of a clause can be seen as made of a succession of groups of projections, each meant to license a particular property type. Thus for French, we have roughly the following clausal organization:

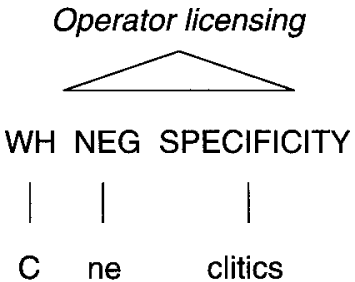

C ne clitics
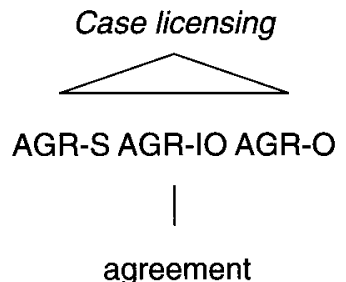

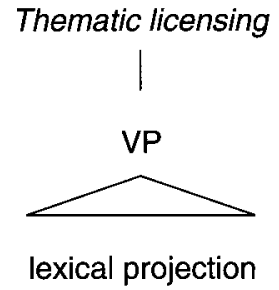

In this representation, the projections for operator-like property licensing are the highest, and projections licensing thematic properties the lowest.

\section{Notes}

* This is in part a written version of work presented at various universities in the USA and Europe. I would like to thank their audiences for their input and Hilda Koopman and Guglielmo Cinque for very useful discussion. This work was supported in part by a UCLA Academic Senate Grant.

1 Note that Sportiche alone, it seems, has argued for both positions, so some burden is on him to reconcile the two positions.

2 The base generation analysis could be salvaged if the [sc] in (16) were a PRO: it could be ruled out by the PRO Theorem. Because Ps are governors (if not proper governors), this appears dubious, unless as Jaeggli (1982) has argued, a clitic may absorb the government property of the predicate selecting $\mathrm{XP}^{*}$, here of the $\mathrm{P}$. This view is criticized in Sportiche (1983) on the grounds that government is a configurational property, not a lexical property. Furthermore, the evidence Jaeggli bases his case on is inconclusive, as shown by Suñer (1988) (cf. also Borer (1983)).

3 More precisely, if the XP could bind Spec, DP.

4 We have not yet discussed what kind of movement clitic movement could be. See Section 6.3.

5 This proposal might find direct support even in French as non-stranded Qs are pronounced tous [tu]'“all-masc.", toutes [tut]/“all-fem.", but stranded Qs are tous [tus], toutes [tut]. The difference in form for the masculine form can be taken to indicate that number agreement between the $\mathrm{Q}$ and the DP only occurs under stranding, in a way reminiscent of the Hebrew data. Note that gender agreement 


\section{CLITIC CONSTRUCTIONS}

seems to obtain regardless of stranding, a surprising fact under this view but not unlike subject-verb agreement in standard Arabic in the SV and VS orders (cf. Aoun, Benmamoun and Sportiche (1994) for discussion).

6 The distinction corresponds to the distinction made in Kayne (1984, Chapter 4) between Qs as anaphors and Qs as quantifiers. Our discussion in this section can be seen as an update of this distinction in the framework of Sportiche (1988).

7 The examples $(28 \mathrm{c}, \mathrm{d})$ are perfectly acceptable for me. They are sometimes judged as ?. Movement out of a clause is only possible from infinitivals or subjunctives.

8 At the latest. If the analysis of the [tu]/[tus] alternation is correct, this configuration will have to have been reached by S-Structure.

9 I report my own judgments here, in the standard register. As far as I can tell, they hold for all cases involving dative clitics e.g., indirect objects, affected objects of locative prepositions (Je leur ai tiré dessus/"I shot at them"), and inalienable possession constructions (Les livres lui sont tombés des mains/"The books fell from his hands," see Vergnaud and Zubizaretta (1991). In that, I differ from what is reported in Kayne (1975) (e.g., he gives Cela leur est tous arrivél"That happened to all of them" as deviant, while this sentence is fine for me). This suggests a much more complicated and much more dialectally detailed situation.

10 We update his proposals, essentially in the way we construe his Q-post.

11 Kayne also proposes that the clitic/XP* relation is head movement, a view to which we return below in Section 5.3.1.

12 This analysis is a contemporary instantiation of Sportiche's (1983) analysis, which argues that (1) the clitic is a morpheme not occupying a phrasal position (i.e., a head, in contemporary terms) and (2) its relation with its associated argument is one of agreement (which is now instantiated as Spec/head). Sportiche's (1983) analysis of clitics conformed almost exactly to the schema adopted here, in a way reminiscent of Kayne's (1972) analysis of French subject clitic constructions.

13 By extending a usage adopted in some French traditional grammars for reflexive clitics and in some Spanish traditional grammars for accusative clitics as well (cf. the references in Suñer (1988)). There is also a theoretical rationale to lump together active, passive, reflexive and other clitic voices discussed in Sportiche (1993).

14 This is the part that does not necessarily apply to se constructions.

15 Sportiche (1993) argues that this analogy is far from accidental and extends to all other types of non-local head/phrase or phrase/phrase relations.

16 We add this second clause for symmetry at this point. It will be discussed in Section 7.

17 I use "overt movement" to mean syntactic movement, i.e., feeding the phonology, as opposed to LF movement, which does not even if the moved category is covert.

18 But not identical to agreement morphemes: clitics possess properties other than regular subject or object agreement morphemes that make them akin to pronouns.

19 Given the discussions of Kayne (1975; 1990) and Rizzi (1986), it seems that nominative clitics are not syntactic clitics (except the indefinite on or in certain constructions like Complex Inversion). Their distribution is discussed in Sportiche (1993). 


\section{PARTITIONS AND ATOMS}

20 Thus $\mathrm{I}$ assume that $\mathrm{AGR}_{\mathrm{o}}$ is not involved at all, based on the fact that it must be available for participle agreement.

21 The order (ne)-C1 $+\mathrm{AUX}_{1}+\mathrm{Inf}-$ (pas)- $\left(\mathrm{AUX}_{2}\right)-$ Verb is also marginally possible (cf. Pollock 1989), as noted in the text.

22 Marginally, a tenseless verb only raises to $A G R_{S}$ and the clitics cliticize to a higher host, viz. n'en pas parler/"not to speak about it," where the head of NegP is ne. See Sportiche (1993) for arguments to the effect that ne, the head of NegP, is higher than $\mathrm{AGR}_{\mathrm{s}}$.

23 This standard description is somewhat incorrect if it is taken to mean that the XP* must be null, as is shown by doubling of clitics by stranded quantifiers.

24 Another property, which seems true of Romance languages and is related to Problem 2 in the text, appears to be the following: if clitic doubling is allowed of accusatives, it is allowed of datives (but not vice versa).

25 The idea that $\mathrm{XP}^{*} / \mathrm{XP}^{\wedge}$ coexists with a clitic and that only one of them may surface is not unlike Kayne's (1972) analysis of French subject clitic constructions. A similar idea with Wh Movement is developed in Cheng (1991). At this point the formulation in (41) is tentative in particular concerning the set of relevant properties that $(41)$ is sensitive to.

26 This view is essentially Kayne's (1989b). Many questions relating to object agreement will not be addressed here. A detailed look at this proposal and its implications is found in Sportiche (1990). There and in Chomsky (1991) (see also Mahajan (1990) for closely related although somewhat different views), it is argued that participle agreement with Case-marked DPs is limited to accusative DPs, the two properties being different reflexes of the same underlying configuration. Detailed discussions of many complex cases are also examined there.

27 Let us note that this analysis of clitic placement is consistent with that of Sportiche $(1989 ; 1990)$. The present analysis specifies that the position XP' of the text about (45) is $\mathrm{XP}^{\wedge}$, or is reached after movement through $\mathrm{XP}^{\wedge}$. We conclude later that it is indeed $\mathrm{XP}^{\wedge}$.

28 Though there exists an agreement asymmetry between $\mathrm{Wb}$ Movement and clitic placement. We use the fact that skipping is necessary for $\mathrm{Wb}$ Movement to reinforce the plausiblity of this approach for the optionality of agreement under clitic placement. The facts are more complex: agreement with $\mathrm{Wb}$ Movement, when it takes place, belongs to a more formal register than agreement with clitic placement (Burzio (1986) reports the same observation for Italian). Furthermore, as Kayne (1989b) discusses, there are varieties of Romance language which have agreement with clitics and lack agreement with wh-phrases (cf. standard Italian). The reverse situation is not found.

29 See Kayne (1984) for a discussion of the differences and similarities with $\mathrm{Wh}$ Movement as far as the ECP, etc. is concerned.

30 Rightward incorporation of the subject clitic il in Complex Inversion constructions or subject clitic inversion are such instances (Louis) est-il fatigué?/"Is Louis/he tired?" See Sportiche (1993) for further discussion.

31 This is not the only option. Another option is to revise the theory of agreement to make all species of agreement related to head movement, as attempted in a talk given by Taraldsen at the 1992 GLOW conference.

32 Kayne expresses doubts as to the correctness of a restructuring approach to clitic climbing. The general issue here is one of domain extension: clitic placement or 


\section{CLITIC CONSTRUCTIONS}

object preposing (see below (53)) can operate over a larger domain (two clauses) than otherwise possible. The earlier approaches made restructuring collapse two clauses into one, by incorporating the bottom verb into the top one. These approaches are no longer compatible with basic theoretical principles but their effect can be mimicked by head movement of some head in the lower clause to the main clause with a concomitant domain extension - see Sportiche (1990). Kayne in fact adopts such an approach as we see below: his restructuring is raising of the bottom Infl to the top one.

33 This (somewhat marginal) case of restructuring requires the higher verb to be in a subjunctive mood. Apparently, the same facts hold of Catalan.

34 See Kayne (1991a) for arguments that se is a complementizer.

35 We conclude later that clitic placement is also A-bar movement of a different sort than Wh Movement. Clitic climbing over a wh-phrase is nevertheless allowed, either because of this difference (reflected in the fact that Spec, CP is not a possible intermediate site for $\mathrm{XP}^{*}$ ) or because Italian allows $w h$-island violations.

36 Throughout this section $e n$ is meant to be the genitive complement of nominals and adjectives and not the en of quantity, unless otherwise indicated.

37 One significant consequence that we elaborate on later in Section 6.3.

38 Dative or locative XPs do not license PGs, quite generally and for poorly understood reasons. In the case of datives, it might be related to the specifier of dative voice being an A-position, especially if Mahajan's (1991) approach concerning the analysis of parasitic gaps is correct. Only structurally Case-marked NPs appear to license parasitic gaps, i.e., nominatives, accusatives and genitives. Relevant configurations cannot be constructed for nominative clitics.

39 The reason why Rizzi uses complex causative constructions to illustrate his point is that, in a simple clause, it is difficult to guarantee that $\mathrm{XP} *$ in our terms does not c-command the PG while the clitic does (especially given that at the time, the clitic was thought to be more or less inside VP). Sentences like (60b) circumvent this problem.

40 See Sportiche (1983) and Lasnik and Stowell (1991) for a more comprehensive discussion.

41 Since Marie is a possible antecedent for ses propres in

1 Louis a cru [Marie fière de ses propres idées]. Louis has believed Mary proud of her own ideas. Louis believed Mary proud of her own ideas.

a possible example might be the following, if the indicated structure is correct

2 *Louis $l_{j}$ 'a fait [[croire $t_{j}$ épuisé à son propre ${ }_{j}$ auteur]. Louis him has made to-believe run-out to its own author. Louis made its own author believe it out of print.

42 See Kayne's (1975) detailed discussions, and also Sportiche (1990).

43 Given that dative clitics have an A-position $\mathrm{XP}^{\wedge}$, they should never license PGs. We expect that dative Scrambling in Dutch should not license PGs, as appears to be the case. 


\section{PARTITIONS AND ATOMS}

44 In embedded clauses, we would get:

1 Omdat hij 't waarschijnlijk (*'t) niet (*'t) gezien heeft. Because he it probably not seen has.

Because he probably has not seen it.

2 Omdat hij Jan waarschijnlijk (Jan) niet (*Jan) gezien heeft.

Because he John probably not seen has.

Because he probably has not see John.

Koopman notes that care should be taken to avoid contrastive or focal stress on the objects.

45 Koopman offers many other arguments not reproduced here; e.g., pied-piping of non-specific DPs is obligatory under VP-preposing, but not for specific DPs. In addition, clitics exhibit clitic climbing.

46 One potential difference with Romance clitics is whether the order of clitics is fixed or not. Dutch or German may substandardly allow for alternative clitic orderings.

47 In other words, it is apparently blocked by a certain type of Infl material accusative Scrambling may violate the SSC but may not violate the Tensed S Condition.

48 In particular, the reasoning we appealed to in Section 6.3.

49 We intend the analogy with Wh Movement to be pushed as far as possible. Thus Specific DPs in PPs or complements to Ns, adjectives, etc., will be licensed under movement to the specifier of a specificity-licensing head (situated in the structure presumably where Romance clitics are) very much in the way $w$ bphrases in situ are licensed in the framework of the $W b$ Criterion, i.e., by covert movement, pied-piping, etc.

50 Sportiche (1993) shows that this conclusion for objects extends to French nominatives as well except for the indefinite on "one." Just as in the object cases, there might be crosslinguistic variation here: Trentino subject clitics have been argued not to license specificity and thus constitute a nominative counterpart to our analysis of datives.

51 We will not elaborate on this last proposal here but I would conjecture that a number of facts can be linked to this proposal: (1) the obligatory presence of the dative clitic le in dialects of Spanish in $w b$-extraction of an indirect object (e.g., ¿A quién *(le) regalaron un auto? "Who did they give a car to?" (2) participle agreement with superficial subject in indirect object reflexive constructions with non-clitic direct objects (e.g., Maria si è compratal?? comprati libri/Maria se li è *comprata/comprati/"Mary bought books/them for herself," and similar (dialectal) facts in French, e.g., Marie s'est construit(e) un abri/"Mary built a shelter for herself."

52 I would conjecture that the cases of doubled clitics such as Je le veux le voir/ "I want to see him" found in certain Romance dialects or in child speech reflect a stage or a grammar in which the clitic $l e$ is treated as a pure agreement marker.

53 Some phenomena we do not discuss here at all are the so-called accusative Case markers in languages like Hebrew or Turkish. Given that they are linked to specificity, they should be viewed as the analogue of clitic doubling particles rather than Case markers. 


\section{CLITIC CONSTRUC'TIONS}

54 Thanks to A. Comorovski, C. Dobrovie Sorin, D. Steriade, M. Suñer and M.-L. Zubizarreta for their help with the judgments of Romanian and Spanish in this section.

\section{References}

Aoun, J. and Clark, R. (1985) "On non-overt operators," Southern California Occasional Papers in Linguistics 10, University of Southern California.

Aoun, J., Benmamoun, E. and Sportiche, D. (1994) "Agreement, word order and conjunction with several varieties of Arabic," Linguistic Inquiry 25: 195-221.

Bennis, H. and Hoekstra, T. (1984) "Gaps and parasitic gaps," The Linguistic Review $429-87$.

Borer, H. (1983) Parametric Syntax, Dordrecht: Foris.

- (1986) "The syntax of pronominal clitics," Syntax and Semantics 19, Orlando: Academic Press.

Bouchard, D. (1982) "On the content of empty categories," unpublished Ph.D. dissertation, MIT.

Burzio, L. (1986) Italian Syntax, Dordrecht: D. Reidel.

Cheng, L. (1991) "On the typology of Wh-questions," unpublished Ph.D. dissertation, MIT.

Chiu, B. (1992) "The syntax of functional words in Chinese," unpublished Ph.D. dissertation, UCLA.

Chomsky, N. (1981) Lectures on Government and Binding, Dordrecht: Foris. (1982) Some Concepts and Consequences of the Theory of Government and Binding, Cambridge, Mass.: MIT Press.

- (1986a) Barriers, Cambridge, Mass.: MIT Press.

- (1986b) Knowledge of Language: its Nature, Origin and Use, New York: Praeger. (1991) "Some Notes on Economy of Derivation and Representation," in Robert Freidin (ed.) Principles and Parameters in Comparative Grammar, Cambridge, Mass.: MIT Press, 417-54.

(1992) "A Minimalist Program for Linguistic Theory," MIT Occasional Papers in Linguistics 1, Cambridge, Mass.

Cinque, G. (1991) Types of A-bar Dependencies, Cambridge, Mass.: MIT Press.

Cortés, C. (1992) "Issues in Catalan syntax," unpublished Ph.D. dissertation, UCLA.

Dobrovie-Sorin, C. (1990) "Clitic doubling, Wh movement and quantification in Romanian," Linguistic Inquiry 21: 351-97.

Evers, A. (1975) "The transformational cycle in Dutch," unpublished Ph.D. dissertation, University of Utrecht.

Giorgi, A. and Longobardi, G. (1991) The Syntax of Noun Phrases: Configurations, Parameters and Empty Categories, Cambridge: Cambridge University Press.

Haegeman, L. and Zanuttini, R. (1991) "Negative Heads and the Neg-criterion," The Linguistic Review 8: 233-51.

Hurtado, A. (1984) "On the Properties of LF," Cornell Working Papers in Linguistics 5: Ithaca, NY.

Iatridou, S. (1990) "Clitics and Island Effects," unpublished manuscript, MIT.

Jaeggli, O. (1982) Topics in Romance Syntax, Dordrecht: Foris. (1986) "Three issues in the theory of clitics," in H. Borer (ed.) The Syntax of Pronominal Clitics, New York: Academic Press, 15-42. 


\section{PARTITIONS AND ATOMS}

Kayne, R. (1972) "Subject Inversion in French Interrogatives," in J. Casagrande and B. Saciuk (eds) Generative Studies in Romance Languages, Rowley, Mass.: Newbury House, 70-126.

(1975) French Syntax: The Transformational Cycle, Cambridge, Mass.: MIT Press. (1984) Connectedness and Binary Branching, Dordrecht: Foris.

(1988) "Romance se/si," paper presented at the GLOW conference, Budapest, GLOW Newsletter 20.

(1989a) "Null Subjects and clitic climbing," in O. Jaeggli and K. Safir (eds) The Null Subject Parameter, Dordrecht: Kluwer 239-61.

(1989b) "Facets of Romance past Participle agreement," in P. Beninca (ed) Dialect Variation and the Theory of Grammar, Dordrecht: Foris, 85-103.

—_ (1991a) "Romance clitics, verb movement and PRO," Linguistic Inquiry 22: 647-86.

(1991b) "Italian negative infinitival imperatives and clitic climbing," unpublished manuscript, CUNY.

Koopman, H. (1988) "The internal structure of Dutch VPs," Notes to lectures given at Geneva University and MIT.

(1991) "The verb particle construction and the syntax of PPs," Tilborg Catholic University, unpublished manuscript.

(1993) "Licensing Heads," in N. Hornstein and D. Lightfoot (eds) Verb Movement, Cambridge: Cambridge University Press, 261-97.

Koopman, H. and Sportiche, D. (1991) "The Position of Subjects," Lingua 85: 211-85 reprinted as Chapter 2 in this volume.

Koster, J. and Reuland, E. (1991) Long Distance Anaphora, Cambridge: Cambridge University Press.

Lasnik, H. and Stowell, T. (1991) "Weakest crossover," Linguistic Inquiry 22: $687-720$.

Lebeaux, D. (1983) "A distributional difference between reciprocals and Reflexives," Linguistic Inquiry 14: 723-30.

Mahajan, A. (1990) "The A/A-bar distinction and movement theory," unpublished Ph.D. dissertation, MIT.

- (1991) "Operator movement, agreement and referentiality," MIT Working Papers 15 Cambridge, Mass.

May, R. (1985) Logical Form, Cambridge, Mass.: MIT Press.

Perlmutter, D. (1972) Deep and Surface Structure Constraints in Syntax, New York: Holt, Rinehart and Winston.

Pollock, J.-Y. (1989) "Verb movement, universal grammar and the structure of IP," Linguistic Inquiry 20: 365-424.

Rivas, A. (1977) "Theory of clitics," unpublished Ph.D. dissertation, MIT.

Rizzi, L. (1978) “A Restructuring Rule in Italian Syntax," in S.J. Keyser (ed.) Recent Transformational Studies in European Languages, Cambridge, Mass.: MIT Press 113-59. (1982) Issues in Italian Syntax, Dordrecht: Foris.

(1986a) "On Chain Formation," in H. Borer (ed.) The Syntax of Pronominal Clitics, Orlando: Academic Press 15-42.

(1986b) "On the Status of Subject Clitics in Romance," in O. Jaeggli and C. Silva Corvalán (eds), Studies in Romance Linguistics, Dordrecht: Foris 391-421.

(1990) Relativized Minimality, Cambridge, Mass.: MIT Press. 


\section{CLITIC CONSTRUC'TIONS}

Rizzi, L. and Roberts, I. (1989) "Complex Inversion in French," Probus 1, 1-30.

Roberge, Y. (1990) The Syntactic Recoverability of Null Arguments, Montreal: McGillQueen's University Press.

Ruwet, N. (1972) Théorie syntaxique et syntaxe du français, Paris: Le Seuil.

- (1969) "A propos des prépositions de lieu en français," in C. Hyart (ed.) Mélanges Fohalle, Liège University, pp. 115-37.

Schlonsky, U. (1990) "Quantifiers as Heads: a Study of Quantifier Float in Hebrew," unpublished manuscript Haifa University.

Sportiche, D. (1983) "Structural Invariance and Symmetry in Syntax," unpublished Ph.D. dissertation, MIT, distributed by MIT Working Papers in Linguistics.

- (1988) "A Theory of floating quantifiers and its corollaries for constituent structure," Linguistic Inquiry 19(3): 425-49, reprinted in this volume as Chapter 1. (1989) "Le mouvement syntaxique: contraintes et paramètres," Langages 95: 35-80.

- (1990) “Movement, Agreement and Case," unpublished manuscript, UCLA, reprinted as Chapter 3 in this volume.

- (1993) "Subject clitics in French and Romance, complex inversion and clitic doubling," unpublished manuscript UCLA, reprinted in this volume as Chapter 5. (1995) "Sketch of a reductionist approach to syntactic variation and dependencies," in H. Campos and P. Kempchinsky (eds) Evolution and Revolution in Romance Syntax, Washington, D.C.: Georgetown University Press.

Stowell, T. (1991) "Small clause restructuring," in R. Freidin (ed.) Principles and Parameters in Comparative Grammar, Cambridge, Mass.: MIT Press.

Strozer, J. (1976) "Clitics in Spanish," unpublished Ph.D. dissertation, UCLA.

Suñer, M. (1988) "The role of agreement in clitic doubled constructions," National Language and Linguistic Theory 6: 391-434.

- (1992) "Two properties of clitics in clitic doubled constructions," in J. Huang and R. May (eds) Logical Structure and Linguistic Structure: Cross Linguistic Perspectives, Dordrecht: D. Reidel, pp. 233-53.

Tellier, C. (1991) Licensing Theory and French Parasitic Gaps, Dordrecht: Kluwer.

Valois, D. (1991) "The internal syntax of DP," unpublished Ph.D. dissertation, UCLA.

Varela, A. (1988) "Binding in Spanish: a theoretical and experimental study," unpublished Ph.D. dissertation, University of Connecticut.

Vikner, S. (1990) "Verb movement and the licensing of NP-position," unpublished Ph.D. dissertation, University of Geneva.

Vergnaud, J.-R. and Zubizaretta, M.-L. (1991) "The definite determiner and inalienable construction in English and French," unpublished manuscript, University of Southern California.

Zribi-Hertz, A. (1984) "Prépositions orphelines and pronoms nuls," Recherches Linguistiques 12: 46-91. 


\title{
SUBJECT CLITICS IN FRENCH AND ROMANCE
}

\author{
Complex Inversion and clitic doubling
}

The analysis of the French construction of Complex Inversion illustrated in (1) below raises difficulties with respect to current conceptions of available phrase structure:

(1)(a) Jean est-il malade? Is John sick?

(b) Depuis quand Jean est-il malade? Since when is John sick?

The problem arises from the conjunction of the following several factors. First, Complex Inversion is a root phenomenon. It is consequently reasonable, as suggested in general by den Besten (1983) extrapolating from the classic V2 effect and adopted ever since, to suppose that it involves raising of $I$ to $C$. Second, Wb Movement is to [Spec, CP]. Third, it appears that the subject DP intervenes between the $w$-phrase and the highest verb (as in (1)). Where then is this DP subject? Finally, and to compound the problem, it appears that a pronominal copy of the subject may co-occur with a full DP subject, making Complex Inversion a construction with two subjects.

In this paper, I will propose an analysis of this construction (as well as of the simpler Subject Clitic Inversion - est-il malade/ "is he sick") that builds on and complements two theoretical developments. First, the theory of clitic constructions developed in Sportiche (1995a, c and forthcoming) which focused primarily on object clitics. I will show that this theory naturally extends to subject clitics (SCL). I will also show that it leads to simple analyses of Subject Clitic Inversion (SCI) and of Complex Inversion (CI), making Complex Inversion much less exceptional than other proposals have made it, in that no structure building is required. I will further suggest it entails a much greater deal of underlying similarity across Romance languages than most analyses have assumed.

Second, the conception of morphology alluded to in Chomsky (1992) is meant to provide a principled account of the cases of affix lowering, whereby 
morphological concatenation of an inflectional morpheme with some other unit is not a syntactic operation, but checking of the properties of this inflectional morpheme is done through the syntactic operation of head movement.

\section{Some background}

The approach to clitic constructions suggested in Sportiche (1995a) can be summarized as follows. In a Romance clitic construction, there is an inflectional head $\mathrm{H}^{0}$ heading a projection within the inflectional system, call it CIP in the general case. I will take this $\mathrm{H}^{0}$ to be the clitic itself generally. ${ }^{1}$

Thus, a sentence like (2) receives the analysis in (3):

Marie les ${ }_{\mathrm{i}}$ aura présentés $\mathrm{XP}_{\mathrm{i}}{ }^{*}$ à Nick.

Them would-have introduced-AGR $\mathrm{A}_{\mathrm{i}}$ to Nick. Mary would have introduced them to Nick.

$$
\left[_{\text {AccP }} \mathrm{DP}^{\wedge}\left[\left[_{\text {Acc }} \text { les }\right]\left[\ldots \text { aura présentés } \mathrm{DP}^{*}{ }_{+ \text {acc }} \ldots\right]\right]\right]
$$

The XP associated with this clitic, that we will refer to as XP* throughout (here a $\mathrm{DP} *$ analyzed as pro) must raise to the specifier position (here $\mathrm{DP}^{\wedge}$ ) of CIP (here accusative marked or AccP) by LF, thereby guaranteeing agreement between the two and explaining why the clitic/XP* relation (in fact the $\mathrm{XP}^{\wedge}$ / $\mathrm{XP*}$ relation) can only occur between two entities over a syntactic context transparent to movement. In French, raising of $\mathrm{XP}^{*}$ to $\mathrm{XP}^{\wedge}$ takes place overtly (as shown by the triggering of object agreement - see Sportiche (1995a) for detailed discussion). This XP* may be required to be covert (pro) as in French, or may be overt, yielding various instances of clitic doubling. If it is overt, movement to $\mathrm{XP}^{\wedge}$ may be overt to yielding various cases of Scrambling (in the Germanic languages) or involved in Clitic Left Dislocation constructions.

If there are several clitics, there are as many CIPs. The function of these CIPs is not necessarily uniform. Although clitics share a number of constructional properties, they may diverge on others as e.g. the interpretive consequences of their presence. Thus typical accusative clitics induce specificity of their associated XP while dative clitics do not. In Sportiche (1995a), I have suggested that the function of accusative CIP in Romance languages is to license specificity and in this is a projection different from AGRoP the function of which is to license Case assignment, when available. The function of the dative CIP does seem akin to AGRoP in licensing dative Case and would thus best be analyzed as AGRioP. Correlated with these differences is the A-bar status of the specifier position of the accusative CIP, making it analogous to an operator projection in keeping with its interpretive function. The specifier of AGRioP on the other hand is an A-position, as we would expect by analogy with the specifier of the agreement projections licensing accusative or nominative. 


\section{Questions about subject pronoun clitics}

The set of elements referred to as pronominal clitics found in French is illustrated by the following template:

$$
\begin{array}{lllll}
\begin{array}{l}
\text { French clitic template } \\
\text { nom }
\end{array} \text { 1st/2nd/refl } & \begin{array}{l}
\text { 3rdacc } \\
\text { predicate }
\end{array} & \text { 3rddat } & \text { loc } & \text { gen } \\
\text { il me/te/se/nous } & \begin{array}{l}
\text { le/la/les } \\
\text { il lui/leur }
\end{array} & \text { y } & \text { en }
\end{array}
$$

As Perlmutter (1972) describes it, for each column of the template, no more than one clitic is allowed (so for example, it is not possible to have a first person accusative object and a second person dative object at the same time). Furthermore, the second and fourth column cannot be filled at the same time (e.g. *Je me lui montre/"I show myself to him"). These restrictions are discussed to a limited extent in Sportiche (1995a). For the most part, their existence and properties are not understood.

Clitics are so called because they are claimed not to constitute autonomous syntactic units. Rather, they seem to form a unit with some host either syntactically (syntactic clitics) or phonologically (phonological clitics). ${ }^{2}$ Henceforth, clitic without further specification always means syntactic clitic. Kayne (1975) argues that object pronominal clitics adjoin to V in French (a conclusion with substantial cross Romance validity). This is exemplified by the fact that they cannot be conjoined independently of their $\mathrm{V}$ host nor can their $\mathrm{V}$ host be conjoined independently of them. Disjunction is however more acceptable at least for third person items of the same column in the template.

(5)(a)* Jean le et les présentera a Pierre. John will introduce him and them to Peter.

(b)* Jean le présente a Pierre et/ou envoie a Paris. John presents him to Peter and/or sends him to Paris.

(c) ?Pierre le ou les (le ou la) verra au concert. Peter him or them (him or her) will see at the concert.

Other properties typical of object clitics are their lack of stress and the impossibility of modifying them by an adjacent modifier.

The subject "pronouns" je, tu, il, ils, on/"I, you-sg, he, they-mpl, one" only occur as nominative. The other pronominal subjects elle, elles, nous, vous/"she, they-fpl, we, you-pl" are not exclusively nominative. They may occur for example as the object of prepositions (e.g. avec $*_{i l l} *$ tulelle/nous/"with he/you/she/we." Like object clitics, subject clitics disallow being conjoined, i.e. the equivalent of (5a) is unacceptable as shown in (6a). Surprisingly, definite subject proforms allow the equivalent of $(5 \mathrm{~b})$, i.e. their verbal host may be conjoined independently of them. The indefinite subject proform on 
(which we imperfectly gloss as "one" here - see Cinque (1988), for relevant discussion), as well as the proform $c e,{ }^{3}$ disallow this option. These facts are illustrated in (6b). Disjunction as in (6c) seems more acceptable for subject forms than for object forms :

(6)(a)* Il/tu/je et tu/je/elle connaiss-ont/ez/ent Marie. $\mathrm{He}$ and she know Mary.

(b) Il/tu/*on mange du pain et boit du vin. $\mathrm{He} / \mathrm{you} / \mathrm{one}$ eats bread and drinks wine.

(c) Il ou elle connaît Marie. He or she knows Mary.

Weak object pronouns clearly are clitics. If it is correct that they head an inflectional projection as described above, they are not pronominal in the sense that they do not stand for a regular DP. The same questions arise for subject clitic pronouns:

What is their categorial status?

Where in the structure are they generated?

Are they syntactic clitics and/or phonological clitics?

Furthermore, since subject clitics may either appear preverbally (e.g. in statements) or postverbally (in SCI or CI constructions), the last question arises for both cases.

To anticipate the conclusions I will reach, I will argue that preverbal subject forms conform to the general picture of clitics argued for in Sportiche (1995a, c, and forthcoming) and described above. They are generated as heads of an inflectional-like category, call it NomP and not of a DP. They are located above AGRsP in the structure of a clause; they are not necessarily, although they may be, clitics (except for on, ce which are always clitics). Postverbal clitics on the other hand are always clitics and not DPs. I will suggest that they be treated as morphological affixes base-generated on the verb itself and checked in the head position of NomP (much the way tense affixes are generated on the verb in English and checked in T).

\section{Extending the analysis to subject clitics (SCL)}

Suppose we extend the analysis offered in Sportiche (1995a) for accusative clitics to nominative clitics. What then would we expect? Consider a sentence with an object clitic:

(7) Jean l'a repeinte. John has repainted it(FEM).

We analyze its relevant aspects as follows (AGRs omitted, see Sportiche (1995a) for details): 


$$
\text { Jean } \mathrm{T} \_\underset{\text { ACC }}{\mathrm{Cl} \text { avoir }}-\text { AGRo peint pro }
$$

The function of AGRo is to license accusative Case. Because in French, pro raises to $[\mathrm{Spec}, \mathrm{CIP}]$ in the syntax, (optionally) transiting through [Spec, AGRo], object agreement is (optionally) triggered on the participle (see Sportiche (1989)). The head of CIP, le, ends up cliticized to avoir which has moved to T. For a nominative clitic, we would expect two different projections, one responsible for subject agreement and sanctioning nominative Case, and one for the clitic. Furthermore, given that the clitic is the head of the nominative CIP, it should a priori be able to co-occur with an overt DP* in its specifier or with a covert one.

\section{Trentino Italian}

The former expectation is actually fulfilled as (a slight modification ${ }^{4}$ of) the analysis of subject clitics in some northern Italian dialects presented in Rizzi (1986) or illustrated in Brandi and Cordin (1989). Consider for example Trentino as a representative of such dialects (which include at the very least Fiorentino):

(9) El Gianni/O el magna. John/he eats.

As Rizzi (1986) or Brandi and Cordin (1989) discuss in a pre-split-Infl framework, the subject clitic occupies the head of an inflectional projection (namely AGR in Infl for them) and the DP subject the regular subject position as follows:

(10) [El Gianni [Infl el] [VP magna]]. John (he) eat-PRES-3SG.

This analysis is inadmissible within the more restrictive current theoretical framework. This Infl projection cannot stand for the usual subject agreement. The property of (unsplit) Infl includes both Tense specification and subject/ verb agreement. These properties do show up morphologically on the verb independently of the subject clitic exactly as they do in standard Italian or French. This suggests that this inflectional projection headed by $e l$ is an additional inflectional projection. We can immediately make sense of its existence within our general proposal for clitics by identifying this Infl node with nominative CIP. Taking into account subject/verb agreement (and ignoring Tense, which would, if represented within the split Infl paradigm, require an additional TP projection lower than AGRs), we are led to enrich the structure as follows: 
(11) [NomP El Gianni ${ }_{i} /$ pro $_{i}$ [Nom' [Nom el] [AGRs $t_{i} \ldots$ magna ... ] ] ]

The DP subject has raised through [Spec, AGRs] getting nominative Case, and in [Spec, NomP] (where NomP is CIP with CI in the nominative), the clitic heads NomP and the verb is in or is connected to AGRs thereby agreeing with the subject. This result is the exact analogy for nominative clitics of our conclusions for accusative clitics. In our view, then, the syntactic structure of Trentino subject clitics fits the general mold of the analysis of "pronominal" clitics (as in, and in the form argued in, Sportiche (1983) or in Roberge (1990)). ${ }^{5}$

\section{French preverbal subject clitics}

Can French preverbal subject clitics be analyzed along similar lines as Trentino's? If the analysis of Romance clitics is fully general, we expect a positive answer. Prior to Rizzi's or Brandi and Cordin's work on the northern Italian dialects, this was actually a dominant approach to the analysis of preverbal subject clitics. For example, Jaeggli (1982), Sportiche (1983) (or more recently Roberge (1990)) argue that they are generated as part of a complex Infl node. However, both Rizzi and Brandi and Cordin argue against this suggestion. Rather, they argue that French subject clitics head DPs, i.e. are generated in the same position as full DP subjects. I will now argue that a modernized version of Jaeggli's analysis along the lines of the general analysis of clitics I offer is superior, i.e. that French can and should be analyzed like Trentino above. In this, our analysis is extremely close to Safir's (1982) or Roberge's (1990).

Rizzi (1986), and Brandi and Cordin (1989), henceforth RBC, propose the following analysis for French and Trentino respectively (where $\mathrm{CI}$ is the underlying position of the SCL):

Trentino
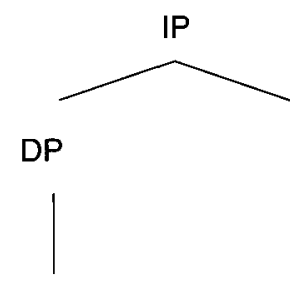

pro
I'

1

I

$\mathrm{Cl}$
French

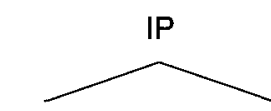

DP

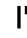

I 
The logic of their reasoning is as follows: there are systematic differences (mostly distributional, e.g. with respect to Complex Inversion) between northern Italian subject clitics and French subject clitics: since northern Italian clitics sit in Infl (i.e. are subject agreement for them), French subject clitics cannot, and must thus be heads of the subject DP (which may eventually cliticize, as Kayne (1972), (1975) or (1984) has suggested). I will show that their correct observations do not entail their conclusions and are completely compatible with an analysis of French along the lines of that of Trentino.

They consider three differences between Trentino and French:

1 Standard French SCLs, unlike Trentino's, cannot co-occur with a full DP subject.

2 Some but not all French SCLs may co-occur with a conjoined VP. Trentino SCL may not.

3 French subject clitic paradigms are regular, unlike those of the northern Italian dialects.

Let us discuss them in turn.

\subsection{Complementarity of distribution}

Let us begin with the first difference. Standard French SCLs cannot co-occur with a full DP subject. Some dialects of French allow co-occurrence but never with the full range of DPs that Trentino SCLs allow. Furthermore, Trentino SCLs are obligatorily present if possible; French SCLs are not:

(13)(a)*/OK Jean il mange/Jean mange/il mange. John he eats/John eats/he eats.

(b) El Gianni el magna/El Gianni magna/el magna. John he eats/John eats/he eats.

(c)* Personne il n'a rien dit. Nobody he said anything.

(d) Nessuno l'ha detto nulla.

Nobody he said anything.

Note first, as indicated, that (13a) is acceptable - without dislocation intonation - for certain French speakers (e.g. from Quebec). ${ }^{6}$ As noted by $\mathrm{RBC}$, this distributional complementarity found in standard French is accounted for if the French SCL is the head of the DP subject. The clitic and the full subject would then be competing for the same position. Although this kind of explanation is plausible for cases in which two identical elements - such as e.g., two heads, or two phrasal categories - are in complementary distribution, it is considerably weakened by the fact that complementarity is 


\section{SUBJECT CLITICS IN FRENCH AND ROMANCE}

between a clear phrasal category, the subject, and a single word, the clitic. Indeed, it is reminiscent of other cases in which competition for the same position is less plausible: consider for example the doubly filled CP filter. ${ }^{7}$ This is a case of complementarity between a word (the head C of CP) and a phrase (a wh-phrase in [Spec, CP]). There is no a priori reason to believe that the case of complementarity under discussion is of the first type (competition for the same position) rather than of this second type (a doubly filled phrase prohibition). This second type is of course immediately compatible with our proposal. If the French SCL heads a nominarive CIP, i.e. NomP with the subject DP in [Spec, NomP], the configuration would be ruled out by a doubly filled NomP filter prohibiting the head and the specifier of the nominative CIP from being lexically filled simultaneously in Standard French. This filter would not operate in Trentino nor in certain dialects of French. Additionally, note that the complementarity found for SCL and its account exactly parallels the data for object Clitics and the account offered in Sportiche (1995a). French object clitics (OCL) are also in complementary distribution with their associated $\mathrm{XP} *$. It turns out then that this doubly filled CIP prohibition is fully general in Standard French. Furthermore, handling those French dialects in which this prohibition does not hold for SCL is straightforward, and does not require analyzing SCLs any differently from the way they are analyzed in Standard French.

Of course, the preceding discussion only shows that the account we just outlined is a reasonable alternative to the one proposed in Rizzi (1986). We now turn to some independent evidence in its favor. As in the case of $W b$ Movement to [Spec, CP], the doubly filled CIP (resp. CP), prohibition does not hold if the specifier of the CIP (resp. CP) contains a trace or a pro instead a lexical element.

Who do you think $\mathrm{t}$ that John saw?

This situation with CIP is illustrated by Q-float structures. Consider the following sentences (read with continuous non-dislocated intonation):

(15)(a) Il a tous fallu qu'il les achète.

It is all necessary that he buys them.

(b) Il a tous fallu qu'ils partent.

It is all necessary that they leave.

The stranded $Q$ tous appears in the main clauses but relates to the object or the subject of the embedded clause. A straightforward account of these data can be constructed if the SCL and OCL are analyzed as heading CIP and not the DP subject or object:

$$
\text { [tous pro }]_{j} \ldots\left[\text { [CIP } t_{j}\left[L_{\mathrm{Cl}} \text { ils/les }\right] \ldots\right]
$$


The presence in the main clause of the leftward moved quantifier, an operation only possible via A-bar movement of a phrase, suggests that this $Q$ is part of an XP moved out of its clause. This XP is most simply and most plausibly analyzed as the subject (or the object) DP itself. Its structure may be [tous pro $],{ }^{8}$ which is a possible DP as shown by Tous sont venus (see Sportiche (1983; 1988) for discussion). Of course, this implies that the SCL cannot be part of this subject DP.

Alternatively this XP may be (tous $t$ ), $t$ the trace of the SCL cliticized prior to the leftward extra sentential movement of the Q. In this case, the "underlying" structure (tous $i l s$ ) would have to be postulated, ils heading the DP modified by tous. This would leave unexplained the ungrammaticality of:

(17)* Tous ils partent/Ils tous sont partis. All they left/They all have gone.

Furthermore, in this case the preposed (tous t) in (15b) would contain an unbound trace. This potentially offending trace would require reconstruction of this DP into the lower clause at LF. It is clear however that these leftward moved Qs have matrix scope, not embedded scope. This is illustrated by the following pair:

(18)(a) Il aurait tous fallu que tu ne les aies pas vu.

It would have been necessary that you see none of them.

(b) Il aurait fallu que tu ne les aies pas tous vu.

It would have been necessary that you do not see all of them.

As the paraphrases indicate, in the first sentence the $Q$ must have scope wider than the embedded negation. If reconstruction was required, we would expect the $\mathrm{Q}$ to be able to take narrower scope than the embedded negation as in the second sentence. We conclude that the preposed DP cannot be of the form (tous t), hence that the SCL (or the OCL) cannot be part of the subject (resp. object) DP.

Another potential source of problems for the SCL as subject assumption is the existence of clitic doubling structures in questions: complex inversion does allow what appears to be a full subject and a SCL: Jean est-il malade?, which we will discuss later in Section 7.

\subsection{SCLs and conjunction}

Let us turn now to the second difference: some but not all French SCLs may co-occur with a conjoined VP. Trentino SCLs may not.

(19)(a) $\mathrm{Il} / *_{\text {on }}$ chante et danse. $\mathrm{He} /$ one sings and dances. 
(b) Il $/ *$ on mange de la viande et boit du vin. $\mathrm{He} / \mathrm{one}$ eats meat and drinks wine.

(c) La canta $\mathrm{e}^{*}$ (la) balla. She sings and (she) dances.

There is obviously a difference between il on the one hand and Trentino la on the other. Is this difference an indication of different categorial status? Rizzi (1986) concludes so. Let us summarize briefly his treatment. He suggests that all these cases of conjunction are ambiguous between clausal conjunction with a null SCL in the second conjunct and VP conjunction.

\section{(20)(a) [SCL VP] and $\left[\left[_{S C L}\right.\right.$ e] VP]}

(b) SCL VP and VP

In Trentino, repetition of the SCL is forced because (1) either it is VP conjunction; then, the clitic character of the SCL forces it to cliticize in PF to the first member of the conjunction, violating the Coordinate Structure Constraint (CSC), or (2) it is a clausal conjunction but a general principle prohibits a zero pro form (here the null SCL) from surviving if it is a minor category, but not if it is a major one.

In French, conjunction of VPs is disallowed, because SCLs are always PF clitics and cliticization would violate the Coordinate Structure Constraint. Clausal conjunction on the other hand is possible because the null SCL in the second conjunct is a major category, a DP in modern terms. Left to explain is the behavior of French on, akin to Trentino la and unlike French il. Here, Rizzi suggests that it is due to the inability of French on to act as a possible antecedent for the null SCL in the second conjunct. This contrast pointed out in Kayne (1972) between these two French pronouns is illustrated below:

(21)(a) $\mathrm{Il}_{\mathrm{i}}$ a dit que je $\mathrm{l}_{\mathrm{i}}^{\prime}$ ai insulté. He said I insulted him.

(b) $* \mathrm{On}_{\mathrm{i}}$ a dit que je $\mathrm{l}_{\mathrm{i}}$ 'ai insulté. One said I insulted him.

(c) $\left[\mathrm{on}_{\mathrm{i}} \mathrm{VP}\right]$ and $\left[\mathrm{e}_{\mathrm{i}} \mathrm{VP}\right]$.

These differences may not be not fully conclusive: we can plausibly argue that the anaphoric pronoun in the second conjunct always receives a specific interpretation. We can expect non-specific interpretation on not to be able to act as antecedent for it. If on is interpreted as nous (we) - see Cinque (1988) for discussion - as it may, then it is possible that it may corefer with a pronoun (On a dit que Jean nous a insultél "We said that John insulted us") without any relevant changes to its syntactic properties. Rizzi's point concerning (21c) would remain if there were principled reasons why the null pronoun could not be indefinite. However, the same question arises for the impossibility of (5b): 
* Jean le présente a Pierre et envoie à Paris. Ruling out a VP type conjunction ... Le (tprésente à Pierre\} et tenvoie à Paris\}) would follow from the Coordinate Structure Constraint. Ruling out ... (le plésente à Pierre) et $\left\{(e)_{\mathrm{i}}\right.$ envoie à Paris\}) could not be attributed to the impossibility for $l e$ to act as antecedent (recall $l e$ is assumed to be a major category similar to that of French $i l)$.

I would like to suggest an analytically more uniform analysis for this array of data. We know that the syntactic realization of a particular property as head is subject to a degree of lexical arbitrariness crosslinguistically. Thus the same function can be realized as a covert head or an overt head (e.g. French zero $\mathrm{Q}$ morpheme versus Vata la $\mathrm{Q}$-morpheme). If overt, it may be realized as an autonomous word, or as a bound morpheme (cf. future of present in English will-vs. French -er). ${ }^{9}$ Taking this into account, we can construct an account of these differences that does not appeal to categorial differences but to an independently motivated diacritic, namely $[+/$ - syntactically bound], i.e. [ $+/-$ clitic]. This parameter in the SCL systems is of a theoretically acceptable kind since it falls within the range of attested lexical variation. On this approach all subject clitics are consistently analyzed as head of NomP. The difference in behavior is due to the fact that French subject clitics like il do not have to be syntactic clitics at all, while Trentino la (see Safir (1985) for discussion) or French on are, both fairly uncontroversial assumptions. ${ }^{10}$ The possibilities for conjunction discussed in (19) follow (or rather they follow the same way they do for object clitics - see (5) - however that may be precisely). The verbal host of a clitic cannot be conjoined independently of this clitic. This conclusion is corroborated for French by differential judgments of the following sort between (22a) and (22c). ${ }^{11}$

(22)(a) ?*Il, à mon sens, a besoin d'une bouffée d'air. $\mathrm{He}$, in my opinion, needs fresh air.

(b) Elle, à mon sens, a besoin d'une bouffée d'air. She, in my opinion, needs fresh air.

(c) **Jean le, à mon sens, respecte profondément. John it, in my opinion, respects deeply. John in my opinion respects it deeply.

We mentioned earlier that subject "pronouns" can be further subdivided between exclusively nominative pronouns like $i l, o n$, $j e$ and others like elle, nous. As the pair $(22 \mathrm{a}, \mathrm{b})$ illustrate, this distinction seems reflected in their clitic behavior. This second class of SCL are indeed clitics in some constructions at least (e.g. SCI or CI), and like other SCLs disallow modification. However, except for these constructions, they may be stressed. We can accommodate their properties by stipulating that unlike exclusively nominative SCLs, they do not require phonological cliticization (although phonological cliticization is certainly preferred), because they are ambiguous between head of NomP and head of DPs. As head of NomP, they are 
phonological clitics and belong to the weak nominative paradigm je, $t u, i l$, elle, nous, vous, ils, elles, on, ce. As head of DPs, they are not clitics at all and may be stressed, i.e. they belong to the paradigm of strong pronominal forms moi, toi, lui, elle, nous, vous, eux, elles, ça which are not clitics. This structural ambiguity would explain why non-exclusively nominative pronouns are optional phonological clitics. ${ }^{12}$ We end up with a three way distinction: exclusively nominative SCL are always phonological clitics. Some of them -on and $c e$, which we have not discussed - must also be syntactic clitics. Finally, some of them do not have to be syntactic clitics.

\subsection{SCLs, paradigmatic regularity and null subjects}

A third difference concerns the compared regularity of the clitic paradigms in French and in the northern Italian dialects. Rizzi (1986) citing work by Renzi and Vanelli (1982) on 27 northern Italian dialects with "uncontroversial subject clitics," reports that in 21 of those, some clitic form is lacking for at least one element of the pronominal paradigm and even in the last 6 , native speakers "often seem to consider the specification of first person singular and first person plural and second person plural clitics as a matter of strong preference rather than one of strict obligatoriness." This situation contrasts markedly with the standard French situation in which the presence of a subject "clitic" if possible at all, is obligatory. Rizzi, correctly I believe, relates this observation to the difference between Standard Italian and French that allows the first, but not the second to have silent subjects without any other morphological indication of their presence than the subject/verb agreement on the verb: "a gap in the clitic paradigm would amount to an S-Structure representation with a null subject position in a non-null Subject Language ..."

This difference then, can be attributed to the usual difference between French and Italian regarding silent subjects without any implication regarding the categorial status of SCL. We take standard Italian or the northern dialects to be silent subject languages in the following sense: nothing beyond the content of AGRs - i.e. the agreement properties showing up on the verb $^{13}$ - is required to recover these silent subjects. SCLs are in effect superfluous from this point of view. The phonetic gaps in the SCL paradigm do not interfere. French subject/verb agreement, on the other hand is not strong enough: silent subjects are disallowed unless recoverable in some other way. It is not that silent subjects are disallowed in French. Their licensing conditions are different. Indeed, our analysis of French SCLs makes French a silent subject language as well (see Bouchard (1982), Safir (1985), Sportiche (1983)). As we have argued, in the presence of an SCL in Nom, the DP is [Spec, NomP] silent and pro, in other words, a standard silent subject. Unlike in Italian, the SCL is phonetically required to license the appearance of this pro. Pushing one step further, we may adopt Safir's (1985) proposal that Italian 
(Spanish, etc.) and French differ precisely in that Italian SCLs may be silent (possibly due to the strength of AGRs), so that from the present perspective, all these languages have a full set of SCLs (i.e. NomP projections). They only differ as to whether or not the head of NomP is or must be phonetically realized, again an acceptable type of parametric variation.

\section{Postverbal nominative clitics: SCI}

We now turn to postverbal SCL. They occur in SCI and CI. Ever since Kayne (1972), it has been clear that CI is a supercase of SCI and we thus begin with SCI.

\subsection{General properties of SCI}

There are three properties of SCI that need to be accounted for:

1 the position of the SCL

2 the root character of the construction

3 the triggered character of the construction.

Properties (1) and (2) are illustrated below:

\section{(23)(a) Mange-t-il?}

Eats-t-he (does he eat?)

(b)* Je me demande si mange-t-il.

I wonder if does he eat.

Property (3) encodes the fact that either there is a particular overt trigger for SCL or SCL triggers a particular interpretation, which we may also describe as involving a covert trigger with particular interpretive properties. The first example below illustrates the trigger case with peut-être/"perhaps," a class of adverbials that allows SCI. The other examples illustrate the second kind of interpretive trigger (respectively hortative, conditional and yes/no questions):

(24)(a) Peut-être mange-t-il de la soupe.

Maybe he eats soup.

(b) Puisse-t-il résoudre le problème à temps.

May he solve the problem on time.

(c) Aurait-il résolu le problème à temps, nous aurions ...

Would he have solved the problem on time, we would ...

(d) A-t-il résolu le problème à temps?

Has he solved the problem on time? 


\section{SUBJECT CLITICS IN FRENCH AND ROMANCE}

\subsection{The standard account}

If we adopt the standard account of the second property, as have Kayne (1984) or Rizzi and Roberts (1989) which elaborates on den Besten's proposal for the treatment of V2 in Germanic, a root phenomenon par excellence, in terms of movement of I to $\mathrm{C}$, we derive the correlation between the impossibility of SCI and V2 and a filled C, i.e. the root character of the phenomenon.

(25)(a) Peut-être qu' il mange (*que mange-t-il) de la soupe.

(b) Qu'il puisse (*que puisse-t-il) résoudre le problème à temps.

(c) Si il avait (*si aurait-il) résolu le problème à temps, nous aurions ...

(d) Je me demande si il a (*si a-t-il) résolu le problème à temps.

Accordingly, the highest verb of the clause, say V, will have to raise to $\mathrm{C}$. In order to do so and given our structural analysis, $\mathrm{V}$ will have to raise to I (or $\mathrm{T}$ or AGRs) and then to Nom in order to reach $C$ without violating the Head Movement Constraint. The step through Nom will cause the head of NomP, the SCL, and the V to form an incorporated structure.

\subsection{Clitic-bost proximity problems and the syntax-morphology interaction}

\subsubsection{The proximity problem}

This analysis, although quite appealing, is not fully satisfactory. Consider the following differences (cf. (5c)): $:^{14}$

(26)(a) Il ou elle connait bien le problème. He or she knows well the problem.

(b) (?) Jean le ou les connait bien. John knows him or them well.

(c)* Mange-t-il ou ( $\mathrm{t}-$ )elle?

Eats he or she?

We can readily handle the difference between (26a) and (26c): a preverbal SCL like $i l$ is not a syntactic clitic according to us: we can describe this fact by having $\mathrm{V}$ not raise to Nom. By contrast, the postverbal SCL in (26c) is syntactically cliticized due to the intermediate movement of the Verb through Nom. However, the contrast between (26b) and (26c) is unexplained:

(27)(1) If both object clitics and postverbal clitics in SCI are cliticized, why are some preverbal and other postverbal, i.e. what accounts for the difference in positions? 
(2) Why is it that postverbal SCL appear more strongly connected to the verb than preverbal object clitics (an observation with substantial cross-Romance validity)?

Kayne (1991) offers a proposal potentially bearing on the first problem. He suggests that clitic-verb sequences are to be analyzed essentially as ${ }_{F} \mathrm{CL}$ $\left.{ }_{\mathrm{F}} \mathrm{V}\right] 1$, while verb-clitic sequences involve the movement of the verb (adjunction to $\mathrm{F}^{\prime}$ ) around a functional projection $\mathrm{F}$ to which the clitic is adjoined: $\left[_{\mathrm{F}^{\prime}}, \mathrm{V}\left[_{\mathrm{F}^{\prime}},\left[\left[_{\mathrm{F}} \mathrm{CL}\left[_{\mathrm{F}}\right.\right.\right.\right.\right.$ e]\}. Taking the position of the clitic as fixed in the clausal structure, Kayne (1991) proposes that a verb to the right of a clitic is actually higher in the tree than when it is to its left, ceteris paribus, a valid observation as he shows in some of the cases he discusses. Ignoring the theoretical problem of allowing adjunction of a head to a non-head (which could be fixed by assuming that the verb actually raises to a higher head position) it is hard to see how this mechanism (adjunction to F') accounts for the closer proximity we are observing. If anything, this mechanism would lead us to expect the reverse given the greater "distance" between the morphemes involved. Postulating a further incorporation of the clitic to the right of the preposed V does not answer the question either, since nothing would account for why incorporation of a clitic to the left of its host would be of a looser kind than incorporation to the right of its host.

\subsubsection{Syntax-morphology interaction}

How then should this closer proximity be handled? Recall Chomsky's (1992) proposal concerning affix hopping in English. In English, it seems clear that the morphological material licensed in T or AGRs may appear on the verb even though the verb does not overtly raise out of the VP to T or AGR (unlike what happens in say, French). Consequently, the traditional analysis (Chomsky (1957)) postulates a process of affix lowering to V. The problem is that this lowering type of head movement creates an illicit configuration (the unbound trace of the lowered affix) which now must be rectified by LF by raising the verbal complex back to T (and AGRs). Chomsky (1992) proposes a simpler and more principled approach to this problem by treating morphological concatenation independently of the process of checking the form and ordering of affixes (I simplify his proposal here). He suggests that the verb is drawn from the lexicon fully inflected, say $[[\mathrm{V}+\mathrm{T}]+\mathrm{AGRs}]$, and that the verbal complex must raise by LF to $T$ and AGRs in order to check (or cancel out) the properties of the inflectional affixes. English can then be seen as a language which delays raising of $\mathrm{V}$ to LF (i.e. raises covertly: it is a procrastinating language) while French raises the verb overtly. Let us adopt for purposes of exposition the more precise machinery postulated by Chomsky (nothing here turns on precisely what the mechanism is). A morphologically complex form $[[A+\operatorname{aff} 1]+\operatorname{aff} 2]$ is a pair $([A+a f f 1+a f f 2],(a f f 1, a f f 2)$,$) . The string of affixes$ 
affn must be canceled or checked in a left to right order (aff1 first, then aff2) under head movement up the tree of this complex form. One immediate advantage of this approach is the treatment of suppletion, i.e. of forms such as eat + past $=$ ate. In a system in which concatenation of morphemes is syntactic, the syntax must be allowed to check back into the lexicon after having performed the concatenation. This is a dubious property of the system since there does not seem to be any syntactic property dependent on whether or not checking of suppletive forms in the lexicon has indeed taken place. Suppletive forms have exactly the same syntax as non-suppletive forms. This intermediate recourse to lexical information must therefore be eliminated, as Chomsky's proposal implies. Note as a consequence of this proposal, we can now hold that there are no lowering rules at all in syntax, surely a desirable result.

This is not to say that morpheme concatenation is always morphological rather than syntactic. We in fact expect to find both, but with different properties:

Syntactic incorporation (SI) The concatenation of morphemes is the result of the application of head movement. Because it takes place in the syntactic component as a case of a (head-)movement rule, we expect this kind of concatenation to (1) always involve upward movement, never lowering and (2) the resulting morphology to be strictly compositional (since input from the lexicon is unavailable).

Morphological incorporation (MI) The concatenation of morphemes is a morphological (or lexical) operation. In this case, we expect to find (1) apparent cases of lowering (since a word may be generated with an affix whose licensing position is higher in the tree) and (2) non-compositionality of the concatenation (lexical exception, i.e. suppletion, etc).

\subsubsection{Typology of cliticization}

The proposal I would like to entertain capitalizes on the difference between MI and SI in order to explain the properties in (27). I would like to suggest that cliticization (i.e. the concatenation itself, not the licensing of particular morphemes which, if required, as for example, inflectional morphemes, always involves syntactic movement upward to some licensing position) is either morphological or syntactic.

Syntactic cliticization instantiates the way cliticization is usually thought of. It involves head movement of the clitic or the host upward, is morphologically strictly compositional and, in French and some other Romance languages, derives configurations in which the clitic is to the left of its host. In particular, this is the case of preverbal object clitics.

Morphological cliticization is a morphological operation (subject of course to syntactic checking under head movement). The clitic in this case is affixed 
prior to lexical insertion. As discussed above, it may involve apparent cases of lowering, and we should observe morphological compositionality violations. This is the case, I would like to suggest, of postverbal SCLs. Return now to properties (27). In French, because inflectional affixation is suffixal, we expect morphologically affixed clitics to follow their host and to be as closely integrated to their host as inflectional affixes, since we are in effect dealing with one word. On the other hand, with syntactic concatenation, there is a priori neither ordering expectation (it turns out to be mostly perhaps exclusively - prefixal in French) nor one-word formed: we are dealing with two words, one adjoined to the other e.g. $\left[_{V} C L\left[_{V} V\right]\right]$, much the way compounds are formed..$^{15}$

Furthermore, as expected we find compositionality violations with postverbal SCLs, not with preverbal clitics. In French such a violation is illustrated by the epenthetic $/ \mathrm{t} /:^{16}$

(28)(a) Il a téléphoné/A-t-il téléphoné?

He has called/Has he called?

(b) Elle aura fini/Aura-t-elle fini?

She will have finished/Will-have she finished?

The same conclusion extends to the northern Italian dialects such as Trentino or Fiorentino discussed earlier. Recall that in these languages, preverbal SCLs unlike French SCL are indeed clitics syntactically. They also allow SCI constructions (examples from Brandi and Cordin (1989)):

(29)(a) Quand es-tu arrivé?

(b) Quando se'-tu arrivato (Fiorentino)?

(c) Quando set riva (Trentino)?

When did you arrive?

Not only do postverbal clitics exhibit allomorphy (or morphologically conditioned epenthesis):

\begin{tabular}{llll}
\multicolumn{2}{l}{ Preverbal SCL } & \multicolumn{2}{l}{ Postverbal SCL } \\
IIs & tu & tu & Fiorentino \\
& te & t & Trentino \\
IIIsM & e/gli & egli & Fiorentino \\
& el/l & lo & Trentino \\
IIIsF & la & ella & Fiorentino \\
& la & la & Trentino
\end{tabular}

But Fiorentino exhibits cases in which the postverbal SCL actually occurs between the verbal root and AGRs: 
(31)(a) Icche gl' hanno fatto?

(b) Icche ha(e)-gli-no fatto?

What have they done?

As Brandi and Cordin note, examples such as $(31 \mathrm{~b})$ are not expected if the ordering of the morphemes resulting from the morphological merger is entirely determined by the linear order of syntactic categories in a tree. In effect, we even find cases of violation of the Mirror Principle if what was involved was concatenation under head movement. Under an MI analysis, there may be (although it certainly appears to be exceptional) cases of mismatch between linear ordering of morphemes and ordering of the syntactic projection in which they are checked because the two are dissociated: there may be exceptional complex forms ( $[A+\operatorname{aff} 2+\operatorname{aff} 1]$, (aff 1 ,aff 2,$)$ ). We would treat this as a case of complex allomorphy (hence exceptional, but allowed in principle) swapping the order of affixes in MI. ${ }^{17}$

\subsubsection{SCI and V movement}

Because of the existence of the phenomenon called affix lowering, we know that the presence of a particular morpheme on some head says nothing of the syntactic position of that head in the overt syntax. Applied to the case of SCI, the fact that Nom - the SCL - appears adjoined to the right of the verb says nothing about whether verb raising has applied or not. The fact that $\mathrm{SCI}$ only occurs in possible V2 contexts makes overt raising to $\mathrm{C}$ a plausible analysis. We now turn to $\mathrm{CI}$. As we will see, the properties of $\mathrm{CI}$ challenge this conclusion and I will propose an analysis of the root character of SCI or CI not involving overt $\mathrm{V}$ to $\mathrm{C}$.

\section{Postverbal Nominative clitics: CI}

Recall the basic examples and the basic problems raised by $\mathrm{CI}$ :

(1) (a) Jean est-il malade?

(b) Depuis quand Jean est-il malade?

Just like SCl, Complex Inversion is a root phenomenon. It is consequently reasonable to suppose that it involves raising of I to $C$, the classic V2 effect. Given that $\mathrm{Wb}$ Movement is to [Spec, $\mathrm{CP}]$ and the subject DP intervenes between the $w h$-phrase and the highest verb (as in (1b)), where is this DP subject? This is what Rizzi and Roberts call the "landing site problem." Finally, why may a pronominal copy of the subject co-occur with a full DP subject, making Complex Inversion a construction with two subjects? In the words of Rizzi and Roberts, the existence of two apparent subjects in CI pose the Case problem - how each subject is assigned Case - and the source problem - where these subjects originate. 


\subsection{The landing site problem: I to $C$ and root phenomena}

\subsubsection{Some remarks on the availability of I to $C$}

The landing site problem arises primarily because of the assumption that $\mathrm{CI}$ involves I to $\mathrm{C}$ in the overt syntax. It is tempting to drop this assumption, as is suggested in Morin (1979) or Noonan (1989; 1992), provided that a plausible account of the root property can be constructed. If there was no overt I to $C$, there would not be such problems: the tensed verb could either be in AGRs with the subject in [Spec, AGRs] or [Spec, NomP] or have raised to Nom with the subject in [Spec, NomP]. The only obstacle to assuming that there is no I to C in CI, it seems, is the account of the root character of CI. Let us examine it in more detail.

Initial accounts of CI (and V2 and related phenomena) are built on the observed correlation between $\mathrm{CI}$ and the presence of an overt complementizer. Postulating I to $\mathrm{C}$ accounts for the correlation because an overt complementizer fills the $\mathrm{C}$ slot, making it unavailable for a raised I. Surely however, as Rizzi and Roberts discuss, this account is insufficient, and at the very least needs a condition along the lines of (1) below to supplement (2). ${ }^{18}$

(32)(a) The CP is not lexically selected (restricting $I$ to $\mathrm{C}$ to main clauses, adjuncts).

(b) The $\mathrm{C}$ position is not filled.

The necessity of some additional condition with the effect of (32a) is supported by the instances Rizzi and Roberts point out of selected "complementizer-less" clauses which do not allow I to $\mathrm{C}$ even if the language has I to $\mathrm{C}$ - infinitive clauses on a widespread scale, that-less tensed complement clauses in English. Even if the cases of silent Cs could somehow be dealt with, there are also cases of overt clitic complementizer in Hebrew or Irish in which a clitic C lowers to I (see Shlonsky (1988), McCloskey (1992)), or very possibly French "interrogative" que (as in e.g. Que fais-tu? "What do you do?" in which que and fais are cliticized to each other) that do not show the complex of properties associated with I to C.

The necessity of (32b) is supported, according to Rizzi and Roberts, by cases of unselected clauses with filled Cs disallowing CI:

(33)(a) Peut-être Jean est-il parti/Peut-être que Jean est $\left({ }^{*}\right.$-il) parti? Maybe John is gone/Maybe that. ...

(b) Aurait-il mangé/Si il avait mangé (*aurait-il). Would he have eaten/If he had eaten (*had he eaten).

Some questions arise however. The first clause appears somewhat too strong. Certain varieties of English, for example, allow I to $C$ in embedded interrogatives (as e.g. John wonders should be go to the store). The second clause, if it 
is indeed a primitive, is suspicious; it seems to condition the availability of a syntactic process (I to C) on a phonological one ("filled" or not); it introduces a degree of arbitrariness (if it is a primitive, the distribution of unfilled $\mathrm{Cs}$ is arbitrary). Most important, however, is the following difficulty: French CI or English $\mathbf{I}$ to $\mathrm{C}$ is impossible in statements in main clauses lacking an overt complementizer.

This strongly suggests that $I$ to $C$ is a triggered rule. It applies only if some specific property (question or doubt) triggers it. This points in the following general direction that I will not elaborate on in detail here (but see Sportiche (1995b)). I to C (or more broadly head movement) is fundamentally a triggered process. $C$ sometimes contains a property, say, $[+Q]$, that must be realized. In selected environments, this licensing does not require the raising of a lower I to $\mathrm{C}$ because insertion of a subordinator - e.g. the complementizer - typically (but not always, cf. the varieties of English alluded to above) is available. ${ }^{19}$ Although in principle, this licensing could arise from raising of a lower I to this C, this is prohibited by Chomsky's (1992) Principle of Greed. Movement is motivated only to license a property of the moved item. Here I has no property of itself to license.

If the $[+Q]$ property appears on an unselected $C$, the insertion of a subordinator may be excluded (in main clauses, by definition). The only way to licitly license this property is to raise $I$ to $C$. This means that we must interpret the Principle of Greed as an Economy Principle and not as an absolute prohibition. ${ }^{20}$

This type of account is probably rendered necessary as soon as we adopt Chomsky's view of the morphology/syntax interaction described earlier. It would indeed be extremely surprising not to find cases of covert V2 - with the same distribution of properties as overt $\mathrm{V} 2$ - analogous to cases of covert $\mathrm{V}$ to $\mathrm{T}$ raising analogous to French overt $\mathrm{V}$ to $\mathrm{T}$ raising. But more generally, the fundamentally identical properties (in terms of locality, etc.) of cases of head raising ( $\mathrm{V}$ to $\mathrm{T}$ ) and head lowering (affix lowering) demand treatments of head movement that are neutral between raising and lowering - i.e. between overt and covert raising. This naturally applies to V2 effects.

Consequently, and most importantly for our purposes, there is no variation in the configurations allowing and thus requiring I to $C$. As usual, this raising may take place either in the overt syntax or in the covert syntax, depending on the language and the particular construction. But this is the only type of variation allowed.

\subsubsection{Covert I to $C$ in French}

Nothing then really turns on whether I to $C$ movement is overt movement or covert movement. We have to make sure that a complementizer that counts as filled at S-Structure also counts as filled at LF. If this is ensured, in principle the cases of permissible overt I to $C$ and covert I to $C$ are going to be identical. 
This can be achieved by the null assumption: if a complementizer position counts as "filled" at S-Structure, it means that its licensing does not require I to $C$, hence does not require I to $C$ at LF. Consequently, we are free to assume that $\mathrm{CI}$ involves in fact covert $\mathrm{I}$ to $\mathrm{C}$ without affecting the account of the overall distribution of CI. This is what I am going to assume now. We should expect never to find really free alternations between I to $C$ and lack thereof. Let us now return to the cases in (33) which look like such cases. ${ }^{21}$

(33)(a) Peut-être Jean est-il parti/Peut-être que Jean est(*-il) parti. Maybe John is gone/May be that.

(b) Aurait-il mangé?/Si il avait mangé (*aurait-il).

Would he have eaten/If he had eaten $(*$ had he eaten).

Rizzi and Roberts assume that the que headed clause in (33a) is unselected. ${ }^{22}$ It seems at least as plausible, extrapolating from Morin's (1985) work on voilà clauses, that the que clause in peut-être que is actually lexically selected as a complement by the higher adverbial predicate peut-être, a reasonable assumption given the semantics of these adverbials reminiscent of corresponding adjectives possible, probable, certain, diffuile, rare which do take a clausal argument. The independence of the two structures is supported by the existence of adverbs which allow I to $C$ but not a que clause as e.g. $\grave{a}$ peine/"as soon as," encore (untranslatable discourse particle) viz. À peine est-il arrivél*À peine qu'il est arrivé, encore aurait-il fallu/*encore qu'il aurait fallu and which, correlatively, are not predicates at all. Furthermore, the following lack of alternation is unexpected if indeed, all that is at stake is the filled/ unfilled alternation in otherwise identical structures or the selected/non-selected distinction:

(34)(a) Je crois que peut-être qu'il est parti. I believe that maybe that he left. (b)*Je crois que peut-être est-il parti.

In our terms, the impossibility of SCL inversion (which incidentally is always optional in Standard French, i.e. freely overt or covert if we are right) in (33a), $(34 a, b)$ must be attributed to the Principle of Greed - i.e. to the existence of an alternative to I to $\mathrm{C}$ (roughly, insertion of que, a subordinator, which is not available in main clauses).

The cases of $(33 \mathrm{~b})$ are different since we are dealing throughout with nonmain clauses. Here too, the structures differ. In inverted structures, the verbal mood inflection is conditional. In non-inverted structures the inflection on the verb is indicative. Thus, no real optionality is found here either. ${ }^{23}$ 


\section{SUBJECT CLITICS IN FRENCH AND ROMANCE}

\subsubsection{The distribution of $-\mathrm{il}$ and the Principle of Greed}

I propose that there is no overt verb movement to $\mathrm{C}$ in Complex Inversion constructions nor of course in SCI constructions. I also propose that SCLs are licensed in the position head of NomP. In the case of postverbal SCLs, which I assume are morphologically incorporated to the verbal complex, licensing of the SCL requires movement of the verbal complex to Nom. However, as we saw, postverbal SCLs are licensed only in questions (or other triggered environments), i.e. only in cases in which $I$ to $C$ is otherwise required. How then is the licensing of a postverbal SCL prevented in other contexts? To put it differently, what excludes the raising of the verbal complex to Nom in environments lacking an appropriate trigger in $\mathrm{C}$ ?

We need a three way distinction:

1 Type 1 heads such as Tense or AGR, which may be generated on V, must be able to force $\mathrm{V}$-raising to $\mathrm{T}$ or AGR (see below) to get themselves licensed in a way consistent with the Principle of Greed. Thus, V + T raises to $T$ to license $T$.

2 Type 2 heads such as [ $+\mathrm{Q}] \mathrm{Cs}$ must be able to trigger raising of the verb to $\mathrm{C}$ (more precisely of $\mathrm{I}$ to $\mathrm{C}$, as $\mathrm{V}$ to $\mathrm{I}$ is independently motivated), in violation of the (economical interpretation of) the Principle of Greed, if no more economical option is available, to get themselves licensed. Thus, $\mathrm{I}$ raises to $[+\mathrm{Q}] \mathrm{C}$ only in unselected contexts. ${ }^{24}$

3 Type 3 heads such as $i l$ which cannot trigger raising at all in order to get themselves licensed and are therefore (1) either generated where they are licensed (preverbal SCLs), or (2) are generated on some other head $\mathrm{H}$ and can only be licensed if $\mathrm{H}$ has to raise to or through their licensing position for independent reasons (postverbal SCLs licensed though the independent raising of $\mathrm{I}$ to $\mathrm{C}$ ).

The distinction Type $1 /$ Type 2 is necessary as a consequence of Chomsky's proposal concerning morphology and lowering. The distinction between Type 3 and the others, the innovation we introduce, appears necessary as well. Recall that complex forms are assumed to be generated fully inflected and that the affixes are licensed through head movement either overtly or covertly. This proposal raises the following question. How complex can complex forms get? Thus, we would in principle expect to find cases of (35a) with (35b) reading:

(35)(a) I [e] [ e UJohn left-that-believe]].

(b) I believe [that John left].

in which the complex embedded form would be properly licensed by successive movements from its $\mathrm{V}$ position to $\mathrm{I}$ to $\mathrm{C}$ to $\mathrm{V}$ to $\mathrm{I}$ - all possible instances of head movement. Such cases appear rare. In order to account for 
this restriction we in effect need a notion of "natural complex word." The distinction between Type 3 heads and the two others would be of this nature. In effect a Type 3 head, when attached to another head, does not form a natural complex word with it. Its licensing requires some exceptional rescuing device (here raising to $\mathrm{C}$ ) which will allow the parasitic licensing of this Type 3 head.

Leaving Type 2 heads aside, I suggest relating the distinction between Type 1 heads (AGR, ASP) - call them X-strong heads - on the one hand, and Type 3 heads (Nom) - call them X-weak heads - on the other to whether or not the property expressed by the head is lexically related to X. AGR is a Case related property, and $T$ (enses) can lexically differ as to their Case assigning properties. AGR is hence a T-strong head forcing the raising of $T+A G R$ to AGR (hence of $\mathrm{V}+\mathrm{T}+\mathrm{AGR}$ to AGR). ${ }^{25}$

Nom however is not an X-strong head for any head in the verbal system. Nom cannot trigger raising on its own or some other verb-related head to Nom. ${ }^{26}$ Consequently, a postverbal SCL is never licensed unless the verb raises to a position higher than Nom for independent reasons, licensing the postverbal SCL on the way. In other words, it may licitly be affixed to a verb only in V2 triggering contexts.

\subsubsection{Excursus on other varieties of French}

Yes/no questions in two varieties of French can be quite naturally analyzed very much along the same lines. In each, postverbal SCIs have been replaced by an invariable suffix, namely $-t u$ in varieties of Quebec French and $-t i$ in varieties of popular French documented in Morin (1979):

Jean/Je peux-ti ajouter quelque chose. Can John/I add something.

Here, the post verbal particle may co-occur with a preverbal SCL, something impossible in the standard variety. Otherwise this construction has a similar distribution and allows the same subject DPs as the standard CI or SCI. The analysis is straightforward. The postverbal affix is a morphologically concatenated question morpheme (as Noonan (1989) suggests) that must eventually be licensed by the verbal complex raising to $C$ at LF. Raising to $C$ however does not take place overtly for the same reasons as in the standard variety.

\subsection{The double subject problems}

For Rizzi and Roberts (1989) or Kayne (1984), the postverbal pronominal form is essentially a DP subject cliticized, hence the Case problem, the source problem and, as an additional question, the difference between pronominal and non-pronominal DPs in regard to their potential ability to appear 
postverbally. Since we do not treat these postverbal elements as DPs at any level, it is clear that none of these problems arise.

\subsubsection{The Case problem}

Rizzi and Roberts or Kayne suppose that SCI or CI involve I to C. Postverbal subjects are then expected to be possible (just like in English yes/no questions or German V2 structures).

(37)(a) A Jean parlé? (Has John spoken?)

(b) A-t-il parlé? (Has he spoken)?

The impossibility of (37a) needs to be accounted for and in a way that allows (37b). Rizzi and Roberts propose, following Szabolcsi's (1983) suggestion, that I to $C$ in French, contrary to English, destroys the context for nominative Case assignment. Rizzi and Roberts postulate that nominative Case assignment in French is only right to left: raising of $I$ to $C$ yields a Case filter violation in (37a): Jean has no Case. In (37b), the pronominal DP subject can satisfy its Case requirement by incorporating into its Case assigner, as Baker, Johnson and Roberts (1989) have suggested. The first part of this proposal is difficult to reconcile with the current conception of (structural) Case assignment. Structural Case assignment is the reflex of a Spec/head relationship. This raises two problems: first we expect English and French to be treated exactly alike from the point of view of Case theory - which is incompatible with Rizzi and Roberts's proposal; second nominative and accusative Case assignment should function exactly alike. Nominative arises from a Spec/head relationship between $T$ in AGRs and the latter's specifier position. Accusative arises from a Spec/head relationship between $V$ in AGRo and the specifier of $A G R o$. If $T$ to $C$ destroys the configuration for nominative Case assignment, why does $\mathrm{V}$ to $\mathrm{T}$, which occurs systematically and is parallel to I to $\mathrm{C}$, not destroy the configuration for accusative Case assignment?

$$
\text { C DP AGRs T T DP AGRo } V
$$

If pre or postverbal SCL are not analyzed as DPs, this problem does not arise at all. (37a) is excluded simply because French lacks overt I to C. Kayne (1984) conjectured that CI was possible in French only because postverbal SCI were clitics (and more generally that a language may have CI-like constructions only if the equivalent of postverbal SCL were clitics, thus partially explaining its rarity). Rizzi and Roberts' account has the property of theoretically substantiating this conjecture: for them, incorporation in (37b) must take place motivated by Case theoretic considerations: the postverbal SCL is a DP, hence Case is needed. It is not in a Case receiving configuration, and thus is incorporated. ${ }^{27}$ Kayne's conjecture is also derived within the 
present account. CI involves inversion of some sort by definition but not verb/ subject. Instead, it is inversion of two normally adjacent heads $\mathrm{H}_{1}$ and $\mathrm{H}_{2}$ normally occurring in this order. If the inverse order cum adjacency is found, because of the Head Movement Constraint, this can only occur if one incorporates to the other in one way or another. Hence, in a sense each of them must be a clitic on the other.

\subsubsection{The source problem}

Clearly, the source problem is a problem of potential undergeneration and does not arise under the present proposal. In $\mathrm{Cl}$ constructions, there is only one subject. However, converse problems arise, i.e. problems of overgeneration: so far we have assumed that there is nothing in particular about the availability of the NomP projection (except that it may only occur in clauses having nominative, i.e. tensed clauses in French). Consider first the case of preverbal SCL, e.g. Il a mangél"He ate." Here, we have the following relevant substructure:

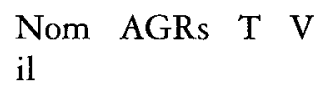

The SCL il is not (necessarily) a syntactic clitic. The verb does not (have to) raise all the way to Nom but must raise at least to AGRs (the standard analysis, cf. Pollock (1990)). Let us now look at the DP subject. Sportiche (1995a) shows that, in French and when it is possible to tell, a silent DP, i.e. pro, doubling a clitic head of CIP must raise to [Spec, CIP], at least. It is difficult to show directly that this is the case in the present situation, but it is not unreasonable to extend this conclusion here.

With a preverbal SCL, this DP cannot be overt in Standard French. Provided that this DP is required to raise to [Spec, NomP] overtly, ${ }^{28}$ surely the null hypothesis given the behavior of silent DPs, we may, as mentioned earlier, encode this impossibility in a version of the "doubly filled Comp filter" extended to the Nom system. Nom and [Spec, NomP] cannot be simultaneously filled (in the relevant sense) at S-Structure. ${ }^{29}$

Let us turn now to postverbal SCLs.

$$
\text { Nom AGRs T V }
$$

The difference here is that both overt and silent DP subjects are allowed. The latter case raises no particular question. It raises just as in the previous case to [Spec, NomP]. But the former does. Why are overt DPs suddenly allowed? By uniformity, an overt DP should be considered to have raised to [Spec, NomP $]$ as well. Furthermore, we have good independent evidence that tensed verbs always raise to AGRs overtly (Pollock(1990)). The only conclusion 
compatible with the doubly filled projection approach is that the combination V-SCL raises no further than AGRs. It does not raise to Nom, hence does not create a doubly filled projection. This means that the affixation of the postverbal SCL could not have resulted from the V raising to (or past) Nom. Rather, concatenation V+SCL must have occurred independently of head movement of $\mathrm{V}$ to Nom. This is what we had concluded earlier. It is precisely the fact that postverbal SCLs are subject to MI that allows CI to exist in French.

It is worth pointing out the logical relations between the various proposals we make at this point. The fundamental proposal we make, i.e. that SCLs are the head of Nom, and that there is no head movement to C in SCI and $\mathrm{Cl}$ constructions is independent from the analysis of postverbal clitics as involving MI. This proposal could be maintained even if the surface position of the postverbal SCL was due to movement (of the verb or of the SCL). It would probably indicate that tensed verbs in French routinely raise to Nom (or that there is syntactic lowering of the SCL).

\subsection{Some direct evidence for lack of overt I to C}

\subsubsection{Distributional evidence}

If $\mathrm{I}$ to $\mathrm{C}$ does not take place overtly in French, nothing special need be said about the position of the DP subject in CI: it occurs in [Spec, NomP]. The $\mathrm{V}+$ SCL combination is in AGRs. This is illustrated in (41a).

In Kayne's or Rizzi and Roberts' analysis, I to $C$ does take place overtly: $\mathrm{V}+\mathrm{SCL}$ is in $\mathrm{C}$ and the DP subject in some intermediate position between $\mathrm{C}$ and [Spec, CP], where $w h$-phrases occur. This is illustrated in (41b).

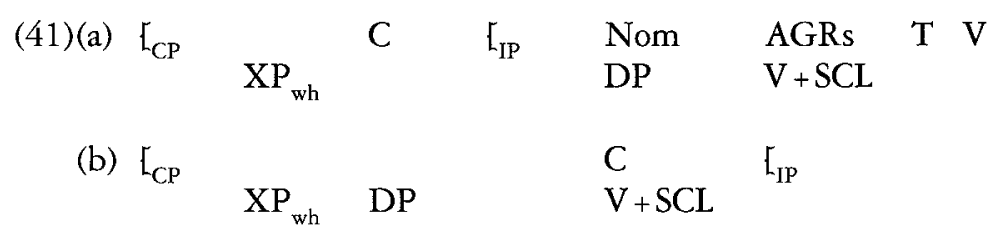

The two analyses make quite different predictions concerning "IP" initial material. In CI or SCI, this material is predicted to occur to the right of a wh-phrase and

1 to the left of the V + SCL complex or to the left of an overt subject if any, according to the first analysis

2 to the right of the $\mathrm{V}+\mathrm{SCL}$ complex, according to the second analysis.

The following examples illustrate a possible case. Consider the temporal clausal adjunct (42a). Elements of its distribution are illustrated in (42b) (the 
symbol $*^{\wedge}$ indicates that the adjunct clause in (42a) cannot appear in the position of ${ }^{\wedge}$ in (42b), a bare ${ }^{\wedge}$ indicates that it may):

(42)(a) Quand le vote a eu lieu. When the vote took place.

(b) ${ }^{\wedge}$ Les électeurs sont ?? ${ }^{\wedge}$ allés à la pêche. The voters went fishing.

(c) Dans quelle ville ${ }^{\wedge} 1$ (les électeurs) sont-ils ??^ 2 allés à la pêche. In which town (the voters) went fishing.

The judgments seem preserved in (42c): although Position 1 is perceived as a natural position for the intervening temporal clause, Position 2, although not impossible, is perceived as less natural, but stylistically more marked. This observation is explained if the subject DP and the verbal complex occupy respectively the same positions as in ( $42 \mathrm{~b})$, and mysterious otherwise. The postverbal Position 2 in (42c) is particularly significant. Both Kayne (1984) and Rizzi and Roberts (1989) consider that because of I to C, the pre-C position hosting the preposed subject counts as a [Spec, IP] so that there are in effect two IPs: the original IP and a derived IP. Position 1 is thus a pre-IP position.

\subsubsection{Binding evidence}

The position of the overt subject in CI must be construed the way a (possibly derived) A-position usually is (however this should be defined). This is shown by the possibility of sentences like De qui $i_{j}$ (le père $t_{\mathrm{j}}$ \} est-il venu?/"Of whom did the father come": extraction from elements preposed in A-bar position is not possible (cf. *De qui te demandes-tu quel frère est venu/De qui ${ }_{\mathrm{j}}$ (le père $t_{\mathrm{j}}$ \} Jean l'a vu/"Of whom do you wonder which brother came, Of whom the father John saw him." This is as expected under both Rizzi and Roberts and the present analyses (but is the opposite conclusion to Kayne's (1984)). In the present analysis, the subject is in the normal subject position. In Rizzi and Roberts' analysis, it is the specifier of a derived IP (so that raising to $\mathrm{C}$ is in effect a structure building rule). SCI, est-il venu, is treated differently by each analysis:

(43)(a) $C E_{\text {Nom }} P$ pro $\left[e E_{A R G s P}\right.$ Lest-il

(b) $L_{I P} e[\text { est-[il }]_{j} L_{I P} t_{j}[$

Consider now the following sentences:

(44)(a) Si Jean était venu, $\mathrm{il}_{\mathrm{j}}$ aurait decidé.

If John had come, he would have decided. 
(b) $* \mathrm{Il}_{\mathrm{j}}$ aurait, si Jean était venu, decidé. He would have, if John had come, decided.

(c) *Aurait-il, si Jean était venu. Would he, if John had come, decided.

(44b) illustrates a Principle C effect: the pronoun il or the proc-commands the name Jean. Why is this effect also found in (44c)? According to structure (43a), it is for an identical reason: the if clause is lower than AGRs and is thus c-commanded by pro. According to structure (43b), the reason is less clear. The if clause can appear, say in an adjoined position, IP initially (an observation corroborated, within Rizzi and Roberts' proposal, by the possibility of Quand, si Marie est malade, Jean pourra-t-il lui rendre visitel "When, if Mary is sick, will John be able to visit her." Adjoined to the lower IP in (43b), it should not trigger any Principle $C$ effect as it is not within the c-command domain of any coindexed DP. There is also no reason to count the incorporated head $i l$ of the subject DP as relevant for the Binding Theory. ${ }^{30}$

\section{Subject extraction and the role of NomP}

There are two interrelated questions that we have not addressed yet. The first is the function of the NomP projection. The second concerns the deviance of simultaneous subject extraction and SCI illustrated in (45):

$$
\text { Qui }{ }_{i}{ }_{i} a-t-i l_{i} \text { mangé de la soupe? }
$$

Who has-he eaten soup?

The deviance of (45) is actually not fully representative of the data. Thus, as noted in Grevisse (1980), subject extraction and SCI, i.e. Wh Movement of the subject in $\mathrm{CI}$ constructions, are compatible for example in questions and in exclamatives: ${ }^{31}$

(46)(a) [Combien d'entre nous $\}_{\mathrm{i}} \mathrm{t}_{\mathrm{i}}$ auraient-ils droit?

How many among us would-have-they the right?

(b) [Quel féroce magicien $]_{i} t_{i}$ a-t-il ${ }_{i}$ enfermé ton secret?

Which fierce magician has-he locked up your secret?

Specificity of the subject seems to be a determining factor allowing cooccurrence of SCI and subject extraction. The correct notion, say specificity, however it should be characterized (see Enç (1991) and references therein), is strongly reminiscent of that relevant for accusative clitic doubling (in River Plate Spanish, see Jaeggli (1982), in Porteño Spanish, see Suñer's work, e.g. Suñer (1991), Romanian, etc.) or "accusative case-marking" in Turkish (see Enç (1991)). Sportiche (1995a) suggests that the function of AccP, the accusative counterpart of NomP whose head hosts accusative clitics, is 
precisely that of a "specificity" licenser. Extending this idea to NomP not only provides an answer to the first question, but explains the difference between (45) and (46). The examples in (46) are acceptable precisely because it is natural to interpret the variable bound by the wh-quantifier as ranging over a set previously introduced in the discourse. After all, this conclusion is not surprising as the cases under discussion are cases of clitic doubling involving nominative instead of accusative.

If this approach is correct, the specificity requirement imposed by the presence of the SCL, i.e. of NomP, should be observed in non-wh-structures as well. Non-wh DPs incompatible with a specific interpretation should not be able to occur as subjects in CI constructions. This prediction is difficult to test if specificity is correctly characterized in terms of prior introduction of some (restricted) set of possible referents for it might rely not so much on the internal structure of DPs as on the discourse conditions on use of DPs (e.g. why could qui in (45) not be interpreted as specific in particular discourse conditions). Fortunately, there is a class of DPs which cannot receive a specific interpretation, namely indefinite DPs modified by a relative clause in the subjunctive mood (they cannot appear with a definite determiner). These DPs cannot appear on the CI construction. Indefinite DPs modified by a relative clause in the indicative mood are ambiguous between a specific and a nonspecific interpretation. They may appear in CI constructions. When they do, however, they can only be interpreted as specific:

(47)(a) Je cherche un homme qui sache/sait cuisiner. I am looking for a man who could-SUBJ/can-IND know how to cook. specific reading only/ambiguous

(b) Un homme qui *sache/sait cuisiner chercherait-il un tel poste?

A man who could-SUBJ/can-IND cook would-seek-he such a position?

Would a man who can cook seek such a position? (specific reading only)

Given the similar treatment we propose for French and northern Italian SCLs, we expect that similar conclusions should hold of the northern Italian dialects. Suñer (1991) suggests that they do despite prima facie evidence to the contrary, such as the possibility of doubling quantifiers such as nobody with SCLs. She points out that in Porteño Spanish, doubling of a direct object ninguno/"nobody" is possible and suggests that this negative quantifier in all these cases is interpreted as partitive. ${ }^{32}$ Whether this is correct is unclear. As I mentioned earlier, specificity as I have been using it may not be the right notion (see Sportiche (forthcoming) for a different suggestion). It seems that the right set of DPs should at least include specific DPs. The 
crucial factor here is that we find parallel behavior in subject doubling and object doubling.

\section{Concluding remarks}

The analysis of $\mathrm{SCl}$ clitics we advocate for French and, more generally, Romance languages, is in line with that suggested in Sportiche (1995a) for object clitics. It also marks a return to one of the earliest analyses of SCLs, namely that of Kayne (1972). Kayne suggested that NPs be generated in the form [NP-SCL] (e.g. [Jean -il], or [lui -il]), where either the strong pronoun lui or the SCL il be deleted, except in special contexts such as CI. The idea that the SCL and the DP are distinct entities is the fundamental idea we have pursued. Essentially, we have departed from Kayne's proposal only in requiring that the configuration DP-SCL is not base-generated as such but has to hold at LF.

\section{Notes}

* This chapter is dedicated to the memory of Osvaldo Jaeggli. Its content, I hope, is no mere "bourdonnement de mouches à ses oreilles" ("buzzing of flies in his ears").

1 Although this is not necessary for the conceptual basis of my proposals, however, it seems quite plausible for subject clitics as we will see later, and for object clitics for the reasons discussed in Sportiche (1995a). Alternatively, in certain cases, the clitic heads the XP (XP* or the head of the specifier of XP*, see Koopman (1993)) referred to below and the head $\mathrm{H}^{0}$ is silent. Correlated changes to the text below would be necessary if this alternative were correct.

2 Syntactic clitics cannot be separated from their host by any syntactic process and are necessarily phonological clitics. Phonological clitics are syntactically autonomous if they are not syntactic clitics as well.

$3 \mathrm{On}, \mathrm{ce}$ are subject clitics which we do not discuss here; see Kayne (1972), Jaeggli, (1982) and Rizzi (1986).

4 The difference with the analyses mentioned has to do with the modernized treatment of subject/verb agreement that we adopt here.

5 One immediate and somewhat surprising consequence of this, which we do not discuss here, is the apparent position of the subject. The subject appears not to be in what is usually deemed to be the normal subject position, i.e. [Spec, AGRs]. Rather, it appears higher in the structure in [Spec, NomP].

$6(13 c)$ is not ungrammatical throughout. Speakers who routinely accept (13a), as e.g. certain Quebec French speakers, also allow a quantifier. The basic point remains however for Standard French (although it may be not for other varieties).

7 Also known as the doubly filled Comp filter. Of course, why the doubly filled $\mathrm{CP}$ filter exists needs to be explained. The idea of competing for one particular entity could be preserved if the competition is for a particular property instead of a position.

8 Or more precisely $\left[\right.$ pro $_{\mathrm{j}}$ tous $\left.t_{\mathrm{j}}\right]$, given $[$ eux tous $]$ and $*[$ tous eux $]$, cf. Sportiche (1995a) for discussion. 


\section{PARTITIONS AND ATOMS}

9 I will discuss later a further subdivision between lexically bound morphemes, regular inflectional morphemes, and syntactically bound morphemes, i.e. morphemes which become bound as a result of a syntactic operation of head movement and are therefore not as closely integrated in their host as the others. I would call these clitics.

10 Note that Rizzi's (1986) discussion of reordering phenomena between SCL and negative clitics in northern Italian only shows that SCLs belong to different clitic clusters than object clitics. It does not show that they are not syntactically cliticized.

11 Kayne (1984) attributes to Hirschbuhler the report that some dialects accept (22a) as well formed. Another contrast is noted in Kayne (1984): Ils sont partis. Qui, ils?""They left. Who, they?"; *Pierre les a vus. Qui, les? "Peter them saw. Who, them?" Les obligatorily being a clitic, it may not appear stranded. On the other hand, $i l s$ is not. There is more to say about this topic, suggesting further distinctions: on behaves like a syntactic clitic as suggested by the data in (6) but allows On a mangé mon gâteau. Qui, on?/ "Someone ate my cake. Who, someone?"; the ungrammaticality of (6b) with on is less sharp than that of (5b). This suggests a more refined distinction amongst syntactic clitics, making clitic SCLs not as closely tied to their verbal host as OCLs.

12 Left open is the deviance of CI Elle est-elle partiel "She is-she gone" under normal stress and intonation, as compared with the acceptable Marie et elle sont-elles parties/ "Marie and her are-they gone."

13 We differ here from Rizzi's understanding, who takes the SCL to be the realization of subject agreement.

14 These judgments I believe are duplicated although all have shifted toward the worse with et (and) instead of oul "or."

15 This distinction has sometimes been implemented differently by distinguishing two types of head movement: substitution (into a subcategorized slot) and adjunction, which would correspond to the MI/SI distinction. In effect, we are claiming that head movement as substitution is morphological incorporation subject to syntactic checking under head movement. The converse seems false however. There are, if we are right, cases of MI not involving subcategorized slots as loci of substitution in any reasonable sense, postverbal SCL for example.

16 Its appearance is morphophonologically conditioned (between a verb with appropriate phonological ending and an SCL cliticized to it beginning with a vowel). It cannot be analyzed as third person agreement being sometimes deleted, as it never shows up in any other context where it should, e.g., in liaison contexts.

17 I will not discuss other cases of postverbal clitics, i.e. object postverbal clitics found in positive imperatives: Mange le, donne le luil"Eat it, give it to him." Extending the present proposal to these cases is suggested by the relative lack of parallelism between what is found in preverbal position and what is found in postverbal position: Jean m'en donne, Donne m'en; *Jean moi (z-)en donne, donne moi $z$-en (note the epenthetic -z- intervening in the clitic string); *Jean en moi donne, donne z'en moil "John to me of it gives, give (some) of it to me," or Pierre l'y déposera, ?* dépose l'y/"Peter will drop him there, drop him there."

18 They also suggest deriving the first condition from the Projection Principle. See Sportiche (1995b) for further discussion. 


\section{SUBJECT CIITICS IN FRENCH AND ROMANCE}

19 Selection also must be licensed. Sportiche (1995b), expanding on Koopman (1994), suggests that this is done by incorporating this $\mathrm{C}$ into its selector.

20 This approach naturally extends to other cases. I would interpret negative inversion as in (Mary said that) under no circumstances would she agree to that exactly as a case of licensing by I to Neg of the head Neg of NegP, whose specifier position is occupied by the negative adverbial phrase, i.e. exactly as cases of unselected $\mathrm{C}$ discussed below.

21 Rizzi and Roberts also cite other types of examples which raise the same kind of questions or are less convincing for a variety of reasons: for example the popular French pairs: Qui que tu as vulqui qu'as-tu vu/"Who (that) did you see." Besides the questions of the text, an additional problem here is that the register allowing doubly filled comp simply lacks the constructions SCI or CI even, of course, in contexts lacking overt complementizers.

22 They also assume that the small class of items (peut-être, probablement, sans doute, difficilement, rarement) allowing these alternations appear in [Spec, CP]. This seems incompatible with the acceptability of adjunct extraction like: Pourquoi as-tu dit que peut-être que Jean a démissionné t?/"Why did you say that maybe John resigned?" If they did, this should be on a par with adjunct extraction out of wh-islands.

23 Si clauses and their inverted counterpart differ in interpretation as well, I believe. The former may be interpreted either as (past) hypothetical or (present) counterfactual, the latter only as counterfactual.

24 It is clear that Type $1 /$ Type 2 distinction is for expository purposes only here. It is an accidental fact where a particular head is generated. What matters is that it transits through or ends up in its licensing position by $\mathrm{LF}$.

25 This might be extended to Type 2 heads as well with an extended notion of lexical property: $[+Q]$ would be an I-strong head because of the kind of selectional restrictions between $\mathrm{Cs}$ and $\mathrm{T}$ or $\mathrm{T}+\mathrm{AGRs}$.

26 If anything, the function of Nom is to license specificity of the associated $\mathrm{XP} *$, hence could only license the raising of the head of XP* to Nom; see Section 9.

27 Although the link between Case and incorporation seems to me theoretically highly desirable, the motivation that has been given for it seems to me unconvincing. For example, English passive $-e n$, which motivates Baker, Johnson and Roberts' (1989) analysis leading to the view of the relation Case/incorporation can, I believe, be reanalyzed as heading a projection, the passive voice, in a way very similar to my current analysis of clitics heading clitic projections, and not as a nominal at all (see Sportiche (1995b) for further discussion).

28 In Chomsky's (1992) terms, the property licensed on this DP by the raising, to a discussion of which we return below, is strong, and must thus be licensed prior to spelling out.

29 Not all varieties of French exhibit this prohibition. Thus Quebec French has been argued to allow an overt, non dislocated, subject DP doubling as SCL (see Bouchard (1982) for example). Interestingly, Quebec French also appears to lack the doubly filled Comp filter.

30 See Sportiche (1995a) for discussion of comparable cases with object clitics.

31 It should be remembered that $\mathrm{CI}$ belongs to a fairly formal register. Intuitions in complex cases are not as secure as in more spoken varieties of French. This 
PARTITIONS AND ATOMS

caveat given, I may add that, although the $w$-question examples given below are acceptable, such cases are most natural with exclamatives.

32 My judgments on French are not straightforward. Thus, while personne in simple CI is impossible (*Personne n'a-t-il mangél"Nobody has-he eaten = Has anybody eaten," it improves in why questions (Pourquoi personne n'a-t-il mangél "Why has nobody eaten."

\section{References}

Baker, M., Johnson, K. and Roberts, I. (1989) "Passive arguments raised," in Linguistic Inquiry 20: 219-51.

Besten, H. den (1983) "On the interaction of root transformations and lexical deletive rules," in W. Abraham (ed.) On the Formal Syntax of West-Germanian, Amsterdam: John Benjamins, 47-131.

Brandi, 1. and Cordin, P. (1989) "Two Italian dialects and the null subject parameter," in O. Jaeggli and K. Safir, (eds) The Null Subject Parameter, Dordrecht: Kluwer.

Bouchard, D. (1982) "On the content of empty categories," unpublished Ph.D. dissertation, MIT.

Chomsky, N. (1957) Syntactic Structures, The Hague: Mouton.

- (1992) "A minimalist program for linguistic theory," MIT Occasional Papers in Linguistics 1, Cambridge, Mass.

Cinque, G. (1988) "On si constructions and the theory of arb," in Linguistic Inquiry 19: $521-81$.

Enç, M. (1991) "The semantics of specificity," Linguistic Inquiry 20: 1-25.

Jaeggli, O. (1982) Topics in Romance Syntax, Dordrecht: Foris Publications.

Jaeggli, O. and Silva Corvalan, C. (1986) Studies in Romance Linguistics, Dordrecht: Foris.

Jaeggli, O. and Safir, K. (1989) The Null Subject Parameter, Dordrecht: Kluwer.

Kayne, R. (1972) "Subject inversion in French interrogatives," in J. Casagrande and B. Saciuk, (eds) Generative Studies in Romance Languages, Rowley, Mass.: Newbury House.

(1975) French Syntax: The Transformational Cycle, Cambridge, Mass.: MIT Press. (1984) Connectedness and Binary Branching, Dordrecht: Foris.

(1991) "Romance clitics, verb movement and PRO," Linguistic Inquiry 22 (4): 647-86.

Koopman, H. (1993) "The internal and external distribution of pronominal DPs," to appear in K. Johnson and I. Roberts (eds) Essays in Honor of Osvaldo Jaeggli, Dordrecht: Kluwer.

(1994) "Licensing heads," in N. Hornstein and D. Lightfoot, (eds) Verb Movement, Cambridge: Cambridge University Press.

McCloskey, J. (1992) "On the scope of verb movement in Irish," unpublished manuscript, University of California Santa Cruz.

Morin, Y-C. (1979) "There is No Inversion of Subject Clitics in Modern French," manuscript, Montreal University.

_- (1985) "On two French subjectless verbs voici and voilà," Language 61 (4): $777-820$. 


\section{SUBJECT CLITICS IN FRENCH AND ROMANCE}

Noonan, M. (1989) "Operator licensing and the case of French interrogatives," in Proceedings of the Eighth West Coast Conference on Formal Linguistics, vol. 8, 315-30.

(1992) "Case and syntactic geometry," unpublished Ph.D. dissertation, McGill University, Montreal.

Perlmutter, D. (1972) Deep and Surface Structure Constraints in Syntax, New York: Holt, Rinehart and Winston.

Pollock, J-Y. (1990) "Verb movement, universal grammar and the structure of IP," in Linguistic Inquiry 20: 365-424.

Renzi, L. and Vanelli, L. (1982) "I pronomi sogetto in alcune varieta romanze, in Studi Linguistici in Onore di G.B. Pellegrini, Pisa: Pacini.

Rizzi, L. (1986) "On the status of subject clitics in Romance," in O. Jaeggli and C. Silva Corvalan (eds) Studies in Romance Linguistics, Dordrecht: Foris Publications.

Rizzi, L. and Roberts, I. (1989) "Complex inversion in French," Probus 1: 1-30.

Roberge, Y. (1990) The Syntactic Recoverability of Null Arguments, Montreal: McGill-Queen's University Press.

Safir, K. (1985) Syntactic Chains, Cambridge: Cambridge University Press.

Shlonsky, U. (1988) "Complementizer cliticization in Hebrew and the empty category Principle," Natural Language and Linguistic Theory 6: 191-205.

Sportiche, D. (1983) "Structural invariance and symmetry in syntax," doctoral dissertation, MIT, distributed by MIT Working Papers in Linguistics.

— (1988) "A theory of floating quantifiers and its corollaries for constituent structure," Linguistic Inquiry 19(3): 425-49, reprinted in this volume as Chapter 1.

(1989) "Le mouvement syntaxique: constraintes et paramètres," in Langages 95: $35-80$.

(1995a) "Clitic Constructions," in L. Zaring and J. Rooryck (eds) Pbrase Structure and the Lexicon, Dordrecht: Kluwer, reprinted in this volume as Chapter 4.

(1995b) "Sketch of a reductionist approach to syntactic variation," in $\mathrm{H}$. Campos and P. Kempchinsky (eds) Evolution and Revolution in Linguistic Theory: Essays in Honor of Carlos Otero, Washington D.C.: Georgetown University Press, reprinted as Chapter 7 in this volume.

- (1995c) "French predicate clitics and clause structure," in A. Cardinaletti and M.T. Guasti (eds) Syntax and Semantics, vol. 28, New York: Academic Press, reprinted as Chapter 6 in this volume.

- (forthcoming) "Pronominal clitic dependencies," in Henk van Riemsdijk (ed.) Language Typology: Clitics in the European Languages, Berlin: Mouton de Gruyter.

Suñer, M. (1991) "Two properties of clitics in clitic-doubled constructions," in J. Huang and R. May (eds) Logical Structure and Linguistic: Cross Linguistic Perspectives, Dordrecht: Reidel.

Szabolcsi, A. (1983) "On the non-unitary nature of verb-second," manuscript, Max Planck Institute for Psycholinguistics, Nijmegen. 


\section{FRENCH PREDICATE CLITICS AND CLAUSE STRUCTURE}

\section{Introduction}

Although in general we have a reasonably good idea of constituency, we do not have as good an idea of the internal organization of constituents. A substantial amount of recent research has led to the conclusion that in many cases, a much more elaborate internal structure than previously assumed is necessary. Consider for example the internal structure of the NP the destruction of the boat. It would have been analyzed as in (1) until the mid 1980s but could reasonably be attributed the structure in (2) today (incorporating a combination of various ideas by Szabolcsi, (1987), Abney (1987), Ritter (1991), Valois (1991), Koopman (1993), and simplifying somewhat).

$$
\begin{aligned}
& \left.\left[_{N P}\left[_{\text {Det }} \text { the }\right]\left[{ }_{N^{\prime}} \text { destruction }\left[\text {, of }\left[_{N P} \text { the [boat }\right]\right]\right]\right]\right] \\
& \left.\mathrm{f}_{\mathrm{DP}} \mathrm{L}_{\mathrm{D}^{\prime}} \text { the } \mathrm{f}_{\mathrm{Num}} \mathrm{P} \mathrm{L}_{\mathrm{Num}} \mathrm{L}_{\mathrm{Num}} \mathrm{e}\right] \mathrm{L}_{\mathrm{NP}} \mathrm{L}_{\mathrm{N}^{\prime}}[\text { destruct }]_{\mathrm{i}}+\text { ion } \mathrm{L}_{\mathrm{VP}} \mathrm{e} \mathrm{L}_{\mathrm{V}^{\prime}} \mathrm{e}_{\mathrm{i}} \\
& \left.\left.L_{V P}\left[_{D P} \text { the boat }\left[V_{V}, e_{i}\right] 1\right] 1\right]\right]
\end{aligned}
$$

Every substring forming a constituent in (1) also forms a constituent in (2), but their internal organization is quite different. There are several sources of this kind of development. One undoubtedly is an ever greater scrutiny of detailed data simply ignored previously, which has led to the one (inflectional) morpheme/one head widely assumed now, as a result of the work on head movement (Koopman (1984), Travis (1984), Baker (1988), Pollock (1990)) or the developing consensus in favor of greater crosscategorial uniformity in internal syntactic organization. In this context, what makes syntactic analysis particularly challenging is the fundamental indeterminacy consequential to the existence of silent morphemes (hence, by the one morpheme/one head principle, of silent categories). Arguments (based on paradigmatic regularity) for the existence of silent morphemes (e.g., English present Tense) are as strong as linguistic arguments can get. Once the existence of silent morphemes is accepted, the general question is raised of their content and distribution. Absence of overt morphemes is no guarantee of their structural absence. To illustrate, consider the French small clause in (3). 
(3) Jean considère $\complement_{\mathrm{W}}$ Pierre malade $\}$. John considers Peter sick.

We face the question of whether W is indeed a constituent - Stowell's (1981) proposal. Granting that it is, we face the question of whether Pierre is generated within the AP projection of the adjective and whether it stays there. We also face the question of whether the head of W is $A$ or not; and if not, the question of the nature of $\mathrm{W}$.

In this chapter I primarily examine some syntactic properties of small clauses. At the most general level, I do this in the context of some general and restrictive assumptions about the nature of linguistic variation further discussed in Sportiche (1993), a sort of methodological null hypothesis to which I try to adhere as strictly as possible.

At a more concrete level, I do so in French, on the basis of the properties of the predicate clitic le. This leads to non-standard conclusions about the syntactic analysis of clauses and of VP small clauses in particular. The general conclusion is that there are no small clauses, only (possibly restructured) clauses. Given the ubiquitous nature of small clauses due to the introduction of VP shells (Larson (1988)), the furthest consequences of this conclusion would entail the following.

1 A one predicate/one clause principle: each (elementary) predicate projects a full clause, containing at least the projection of this predicate with possibly its extension (modifiers and adjuncts), an agreement projection, and a complementizer projection.

2 Lexical decomposition: complex predicates are made up of as many elementary subpredicates as they take arguments. Each subpredicate is syntactically represented by its own projection with its argument as specifier and is part of a clausal structure as in (1).

\section{Initial considerations on the structure of small clauses}

\subsection{Some background on small clauses and VP small clauses}

The dominant current view on small clauses is in its essentials shaped by the work of Stowell (1981). Stowell suggested then that a predicate of category $\mathrm{X}$ allows the projection of its subject as specifier of the phrasal projection XP of $\mathrm{X}$. At the same time, Stowell suggested that these possibilities were illustrated by the "small clause" constructions in (4).

(4)(a) Louis considère $\left[_{A P}\right.$ Marie $\left[_{A}\right.$ drôle $\left.]\right\}$. Louis considers Mary funny. 
(b) Marie voulait $\left[_{\mathrm{PP}}\right.$ Louis $\left[_{p^{\prime}}\right.$ dans son bureau $]$.

Mary wanted Louis in her office.

(c) Louis voyait $\left[_{\mathrm{VP}}\right.$ Marie $\left[_{\mathrm{v}^{\prime}}\right.$ jouer de la cornemuse $\left.]\right]$.

Louis saw Mary play the bagpipes.

Putting aside for the moment the question of the exact constituent structure of the small clause, the proposal that the subject of the predicate and this predicate form a syntactic constituent met with some skepticism primarily, although not exclusively, ${ }^{1}$ on the basis of the contention that rules affecting constituents (e.g., movement rules) cannot affect small clauses. Whether this contention is true is open to question. One of the most robust tests of constituency, constituent coordination, ${ }^{2}$ does treat small clauses as constituents [(1) Louis considère Marie drôle et Pierre stupidel "Louis considers Mary funny and Peter stupid", but it also treats as constituents strings that were believed not to be [(2) Louis a donné un livre à Marie bier et une peinture à Barbara le jour d'avant/ "Louis gave a book to Mary yesterday and a painting to Barbara the day before"]. Two opposite conclusions are possible from these data: either constituent coordination is not a good test for constituency (and then some alternative theory must be put forth to explain how conjunction works), or the strings in italic do form constituents (and a theory of constituent structure must be developed to accommodate them). Consistent with the second conclusion are Stowell's theory of small clauses (for the facts in (1) above) and Larson's (1988) Theory of VP Shells and Adverbial Modifications which motivates the right kind of constituent structure for the facts in (2).

As for the failure of small clauses to undergo movement rules (preposing, etc.) as an argument against small clause analyses, it is not convincing: these tests - unlike, say, constituent coordination, are not necessary and sufficient conditions for constituency, but only sufficient conditions: not all constituents, say, prepose, an observation that surely needs to be explained but makes inferences of this kind suspicious. Thus VPs prepose in English (eaten bis soup, John has), in Italian (trovata, non l'bo ancora, "found her, I have not yet"), but not in French (*mangé sa soupe, Jean a) despite the good grounds for taking VPs to be constituents in French as well. Without a comprehensive theory of such failings, the validity of these inferences of non-constituency is in doubt.

Koopman and Sportiche's (1991) VP-Internal Subject Hypothesis contributes to these questions their conclusion that a predicate of category $X$ does not merely allow the projection of its subject (i.e., its "external argument") as specifier of the phrasal projection $\mathrm{XP}$ of $\mathrm{X}$, it requires it. In other words, explicitly on analogy with small clauses, they in effect advocate a Predicate-Internal Subject Hypothesis (PISH) on the basis of a variety of distributional arguments. In most cases it is impossible to show directly that some particular structural analyses are impossible: instead, they are ruled out 
by Occam's razor, i.e., because they are unnecessary. In the present situation, the reasoning goes as follows. The VP-Internal Hypothesis shows that subjects of (non-"raising") clauses can sometimes be shown to raise from some VP-internal position. Hence, subjects always raise from VP-internal position. But the relation of the subject of a clause to its VP was the major case - apart from the controversial small clause case - in which a predicate $\mathrm{X}$ did not project all of its arguments within XP. Lack of support for such instances leads to the PISH.

The PISH puts the problem of small clauses in a different light: small clauses become the norm; a small clause in Stowell's sense is the canonical syntactic expression of the thematic relations holding between a predicate and its arguments. It should be noted, however, that, although Koopman and Sportiche's work provides a number of reasons for believing that the subject of a clause is generated lower than where it appears, it is extremely difficult to provide a direct empirical argument for the proposition that the lowest such position is within the projection of the head (in general a verb) taking it as argument. It is thus possible that all the arguments they present are correct, but the PISH is not strictly speaking correct. The only consideration directly in favor of the PISH is the following: if some arguments of a predicate $\mathrm{X}$ are projected within XP and immediately dominated by a projection of X, e.g., objects, then all of them are. I will, having noted its empirical vulnerability, continue adopting the PISH for the time being, despite the conclusions reached in Section 5 that cast doubt on this last argument.

\subsection{The internal structure of small clauses}

\subsubsection{Subjects of small clauses move out}

There are a number of reasons to believe that the structures of the strings given in (4) represent a substantial oversimplification. First, there are reasons to believe that the subject of small clauses always moves out. The possibility of movement is based on the distribution of stranded quantifiers. One of the arguments in favor of the PISH is developed at length in Sportiche (1988): ${ }^{3}$ the distribution of rightward-stranded quantifiers. The idea is simply that the distribution of these stranded quantifiers reveals that of adjacent traces linked to their antecedents. A stranded quantifier like tous, Sportiche (1988) claims, appears (preceded and) followed by the trace of the DP denoting its restriction. Thus the structure of Les enfants ont tous mangél "The children have all eaten" must be Les enfants ont (tous t mangéf/"The children have [all t eaten]."

(5)(a) Louis considère $\left[_{D P *}\right.$ ces immeubles] tous monumentaux. Louis considers these buildings all monumental $(\mathrm{pl})$.

(b) Marie voulait ces enfants tous dans son bureau. 
Mary wanted these children all in her office.

(c) Louis voyait les musiciens tous jouer de la cornemuse.

Louis saw the musicians all play the bagpipes.

The sentences in (5) show the presence of a trace of the subject of the small clause following the $\mathrm{Q}$ (to the left of=) lower than the position in which the subject actually appears. This suggests at the very least that all these structures can, and thus possibly must, be movement structures, as in (6).

$$
\left.\mathrm{DP} * \ldots \mathrm{IXP}_{\mathrm{XP}} t^{*} \ldots \text { Predicate } \ldots\right]
$$

Obligatory overt movement in the case of adjectival small clauses is corroborated by two observations. The first is the agreement on the adjective in French. ${ }^{4}$ A uniform theory of agreement leads to postulating the presence of an $\mathrm{AGR}_{\mathrm{a}}$ (for agreement-adjective) phrase in which the agreement is established or checked, as in (7).

$$
\left.\left.\mathfrak{f}_{\mathrm{AGR}_{\mathrm{a}} \mathrm{P}} \mathrm{DP} * \mathrm{AGR}_{\mathrm{a}} \mathrm{L}_{\mathrm{AP}} t^{*} \mathrm{~A} \ldots\right]\right\}
$$

Since agreement is obligatory [e.g., in (5a)], this suggests, for French, that $\mathrm{DP}^{*}$ has overtly moved at least to $\left[\mathrm{Spec}, \mathrm{AGR}_{\mathrm{a}}\right]$ (and possibly that $\mathrm{A}$ has raised to AGR). Movement is corroborated in English by a second observation discussed in Huang (1993) based on the contrast between Wh Movement and VP-preposing with respect to reconstruction illustrated in (8) (this argument is discussed in Sportiche (1990)).

(8)(a) Which paintings of each other do the girls say the boys like?

(b) [Listen to each other], the girls say the boys do.

In (8a), the antecedent of the reciprocal can either be the main subject or the embedded subject. In other words, the binding theory can be satisfied either as if the preposed phrase were still in its base position or as if it were higher than the embedded subject c-commanded by the main subject. In (8b), only one reading is possible, namely with the reciprocal taking the embedded subject as antecedent. Why is there a contrast? If VP-preposing carries the trace of its subject along, Huang reasons, we can derive this observation. Then the preposed VP is really [DP* listen to each other]. DP* counting as a subject for the binding theory, the reciprocal can take only it as antecedent, explaining the lack of ambiguity of (8b). The contrast in (8) and its analysis provides an argument for the VP-Internal Subject Hypothesis, hence for the Predicate-Internal Subject Hypothesis, i.e., for Stowell's theory of small clauses. Turn now to AP small clauses, which cannot be preposed by movement, as noted previously; see (9). 
(9)(a) You consider [John very sick].

(b) How sick do you consider John?

(c)* [John how sick] do you consider?

However, consider reciprocal binding under Wh Movement of APs, as in (10).

(10)(a) John considers [them proud of each other].

(b) How proud of each other does John consider them?

(c)* They consider [John proud of each other].

(d)* How proud of each other do they consider John?

(e)* They say I am considered proud of each other.

(f)* How proud of each other do they say I am considered?

In order to account for the ungrammaticality of (10d,f), we are led to assume that the constituent preposed by $W h$ Movement also contains the subject of the AP small clause, and we are thus led to the assumption that Jobn, the subject of the AP small clause, has raised out of it (position of $t$ within W irrelevant), as in (11).

(11)(a) They consider the children/*John $\left[_{W} t\right.$ [proud of each other]]

(b) $L_{\mathbb{W}} t$ [How proud of each other $\left.]\right]$ do they consider the children/*John?

\subsubsection{Where do subjects of small clauses move to?}

Since the subject DP* of (some) small clauses raises out of the small clause, we may ask where the DP* of (6) raised to, say in the case of adjectival small clauses such as $(5 \mathrm{a})$.

Consider the case of French: DP* gets accusative, so that [Spec, $A_{G} R_{\mathrm{O}}$ ] of the main clause would appear to be a reasonable candidate. But this is implausible because when this happens in the presence of a past participle, participle agreement should be able to be triggered. ${ }^{5}$ However, DP* remains postparticipial - Louis a considéré ces immeubles monumentaux - and participle agreement is impossible with postverbal accusatives. Although DP* could have raised to [Spec, $A G R_{O}$ ] and the participle to $A G R_{O}$ and then beyond it, recall, as illustrated by English $D o / *$ does the children know this?, that once established, the agreement relation is preserved under further movement of the agreeing head (see Aoun, Benmamoun and Sportiche (1994)). This seems to rule this alternative out. Now as things stand, the only plausible alternative is [Spec, AGR P]. We need an intermediate $A$-position (since ultimate raising to [Spec, $\left.A \mathrm{GR}_{\mathrm{O}}\right]$, required for accusative Case checking, is to an A-position). The facts in (12) suggest that even more structure might be necessary (cf. Cardinaletti and Guasti (1995)). 
(12)(a) J'ai considéré ces enfants tous très fiers de toi.

I considered these children all very proud of you.

(b)*J'ai considéré ces enfants très tous fiers de toi.

I considered these children very all proud of you.

(c) All how proud of you do you consider the children?

(d)*How all proud of you do you consider the children?

If the degree modifier is outside AP much as adverbs are outside VP - see Corver (1990) - the contrast between (12a) and (12b) suggests that additional positions are necessary: presumably $t^{*}$, trace of the DP* the children, is in [Spec, AP]. The Q all can only immediately precede the degree modifier and is stranded: there is another $t^{*}$ adjacent to it. For the same reason as before lack of participial agreement - DP* cannot be in $\left[\mathrm{Spec}, \mathrm{AGR}_{\mathrm{O}}\right]$ associated with the superordinate verb, as in (13). ${ }^{6}$

$$
\left[\mathrm{DP} * \ldots \text { all } \mathrm{t}^{*}\left[\ldots \text { very } \ldots\left[\left[_{\mathrm{AP}} t^{*} \ldots \text { adjective } \ldots\right]\right]\right]\right.
$$

Because the structural relationship between the external argument of a predicate and this predicate should be identical across categories, all the examples in (14) raise the same question (note the lack of participle agreement throughout).

(14)(a) (avoir considéré) [les enfants [tous [trés t malades]]] have considered the children all very sick

(b) (avoir vu [les enfants [tous [rapidement lancer leurs ballons] ]

have seen the children all quickly throw their balloons

(c) (avoir voulu) [les enfants [tous [exactement [au milieu de la pièce]l]

have wanted the children all right in-the middle of the room

In Section 3, I develop a proposal concerning the syntax of the French predicate clitic le and argue on that basis that projections of predicates are dominated by a CP projection. Coupled with the conclusion that they are dominated by an AGR projection, it means that small clauses are clauses. ${ }^{7}$

\section{Predicate clitic le}

\subsection{The problem}

I now turn to the syntax of the predicate clitic le. We start from a number of observations made in Kayne (1975). The clitic le appears corresponding to predicates in sentences such as (15). 
FRENCH PREDICATE CLITICS AND CLAUSE STRUCTURE

(15)(a) Louis est fou/Louis l'est//Louis reste fou/Louis le reste. Louis is crazy/Louis it-is//Louis remains crazy/Louis itremains.

(b) Louis semble fou/Louis le semble//Louis devient fou/Louis le devient.

Louis seems crazy/Louis it- seems//Louis becomes crazy/ Louis it- becomes.

This $l e$ can be found corresponding to syntactically diverse predicates, as in (16).

(16) Louis l'a été, en colère/à plaindre/professeur/fidèle à ses amis/adoré de ses enfants/trahi par ses amis.

Louis it-has been in a rage/to pity/professor/faithful to his friends/adored by his children/betrayed by his friends.

The clitic seems to be able to stand for a PP, an infinitival CP, an NP, an AP or an adjectival participial phrase, or a verbal participial phrase. ${ }^{8}$ As we will see, it also exhibits a number of puzzling properties: it may only appear in certain kinds of constructions; it cannot co-occur with certain other clitics; it shows selective sensitivity to the specified subject constraint (its placement is sometimes blocked by subjects, sometimes not). The rest of the chapter is devoted to trying to explain this behavior.

\subsection{Adjectives with be}

I begin by looking at constructions in which an adjective (A) is in the complement structure of the verb etre/"be" or related verbs such as rester/ "remain" (=keep on being) or devenir/"become" (=come to be) which behave identically. I suppose that constructions of the form [DP belbecome/remain adj] involve raising of the DP subject of the adjective, in agreement with Stowell's (1981) proposal and the PISH. In the case of adjectives, le must be analyzed as pronominalizing a constituent larger than an adjective. This conclusion is suggested by the paradigm in (17).

(17)(a) Louis l'est/le devient/le reste très, fidèle. Louis is it/becomes it/remains it very, faithful.

(b) Louis l'est/le devient/le reste, très fidele. Louis is it/becomes it/remains it, very faithful.

(c) Louis est si fiable qu'il viendra/*Louis l'est si qu'il viendra. Louis is so trustworthy that he will come.

(17a) simply suggests that the clitic must pronominalize a category containing at least the degree phrase, especially given the well-formedness of such discourse as: Est-tu fatigué? Très, showing that the degree does not have 
to be a clitic itself requiring a host to its right. The same point is made by the deviance of (17c): it is impossible to leave the degree behind, even if it is followed by some material. (17b) shows that the string "pronominalized" by le can indeed be understood to include a degree. The examples in (18) are more puzzling.

(18)(a) Louis est aussi fidèle que Marie était infidèle. Louis was as faithful as Mary was unfaithful.

(b) Louis est aussi fidèle que Marie l'était. Louis was as faithful as Mary was (it).

The puzzle is raised by the well-formed (18a), an example of the subcomparative construction, a degree comparison exemplified also by (18b), but one in which the compared adjective has been pronominalized. This construction of degree comparison is parallel in every respect to the quantity comparison found in such sentences as J'ai mangé autant de pommes que tu as acheté de poires/"I ate as many apples as you bought pears" and is most plausibly analyzed as an instance of $W b$ Movement of a covert equivalent of combien/ "how many/how much," as it obeys all the diagnostic properties of $W b$ Movement, e.g., ability to trigger stylistic inversion (Kayne and Pollock (1978)), apparent unboundedness, and sensitivity to islands (cf. Chomsky, (1977)); see (19).

(19) Louis est aussi fidèle.

Louis is as faithful.

(a) Que tu dis que Marie l'était/Marie était infidéle. As you say that Mary was/Mary was unfaithful.

(b) Que tu dis que l'était Marie/?qu'était infidèle Marie. (stylistic inversion)

As you say that was Mary/that was unfaithful Mary.

(c) Que tu dis que Pierre pense que Marie l'était/était infidèle. (unboundedness) As you say that Peter thinks that Mary was/was unfaithful. (d)*Que tu sais quand Marie l'était/était infidèle.

As you say when Mary was/was unfaithful.

(wh-island)

(e)*Que tu connais l'endroit où Marie l'était/était infidèle. (complex noun phrase) As you know the place where Mary was/was unfaithful.

In (18a), then, there has to be a covert combien operator (glossed as OP) overtly (because of stylistic inversion) moving to $[\mathrm{Spec}, \mathrm{CP}]$ of the comparative clause, as in (20).

Louis est aussi fidèle [OP ${ }_{\mathrm{i}}$ que [Marie était [t,infidèle $]$. 
The difficulty is apparent: if $l e$ is a pronoun that pronominalizes a category including the AP and the DegreeP (DegP), there is no source for the needed quantity operator: $(18 \mathrm{~b})$ should be deviant the same way *Whose did you see it (it = picture) or *l'bomme dont je le connais (=l'bomme dont je connais le frèrel"the man whose brother I know") are, in which the original trace of the wh-phrase ought to be included in the pronominalized constituent. I conclude that $l e$ does not pronominalize an AP (or more precisely a DegreeP containing an AP).

The idea I will pursue is that (18b) is analogous to its English translation. The English equivalent, an instance of the comparative construction, must be analyzed as involving $\mathrm{Wb}$ Movement for the same reasons as subcomparative (cf. Chomsky (1977)). ${ }^{9}$ The analysis assumed that there was one involving $W b$ Movement of [how faithful] followed by deletion in Comp. Updating it in current terms without deletion operations, we are led to assume that the adjective starts as silent, hence as a pro, hence as an AP complement of a silent equivalent of how (realized in some dialects as what), heading or in the specifier of a DegP.

(21) Louis was as faithful as $\left[_{C P}\left[_{D e g P} \text { (what) } L_{A P} \text { e }\right]\right]_{i} L_{I P}$ Mary was $t_{j}$ ]

The only difference between English and French, I would contend, is that French must identify these silent elements by clitics. This can be done by adopting the proposal made in Sportiche (1992) concerning the syntax of pronominal clitics. There, I proposed that a pronominal clitic was the head, with some designated property $[+\mathrm{P}]$, of a projection part of the inflectional system of a clause. The element $\mathrm{XP}^{*}$ with property $[+\mathrm{P}]$ that the clitic pronominalizes must move by $\mathrm{LF}$ to the specifier $\mathrm{XP}^{\wedge}$ of this projection in order to have its $[+\mathrm{P}]$ property properly licensed, as in (22).

$$
\left.\left[\mathrm{CIP} \mathrm{XP}^{\wedge}\left[\mathrm{E}_{\mathrm{Cl}} l e_{[+\mathrm{P}]}\right]\left[\ldots \mathrm{XP}^{*}{ }_{[+\mathrm{P}]} \ldots\right]\right]\right]
$$

(I use the notation $\mathrm{XP}^{\wedge} / \mathrm{XP}^{*}$ to designate respectively the constituent pronominalized by le and its base position throughout.) Combining (21) and (22), we get the representation in (23) (with subsequent LF movement when the clitic is present and the adjective absent). ${ }^{10}$

(23)

Louis était aussi fidèle

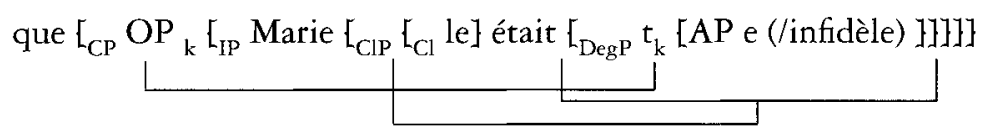


The constituent "pronominalized" by the clitic must be in [Spec, CIP] at LF. It must contain the trace of the $w$ h-operator that has (overtly) moved to [Spec, $\mathrm{CP}$ ]. The presence of the overt clitic element in French versus its absence in English leads to one difference: the missing AP must be understood as identical to the compared AP in English but not in French: although the interpretation of (23) is most naturally that Louis is as faithful as Marie was faithful, any adjective, rendered pragmatically prominent in some way, can qualify (so (23) could mean, say, that Louis is as faithful as Marie was unfaithful). This is to be expected, as in effect, the clitic construction is interpreted as a pronominal. In effect, this analysis treats French comparatives and subcomparatives in extremely similar ways.

The necessity to take $\mathrm{XP} *$ in the predicate clitic construction to be at least a DegP is corroborated by the paradigm in (24).

(24)(a) Louis l'est, fidèle à ses amis/friand de gourmandises. Louis is-it, faithful to his friends/fond of these sweets.

(b) Louis l'est à ses amis, fidèle/Louis l'est de ce genre de gourmandises, friand.

Louis is it to his friends, faithful/Louis is it of these sweets, fond.

(c) Louis leur est fidèle/Louis en est friand. Louis to them is faithful/Louis of-it is fond.

(d) Louis le leur est/Louis l'en est.

Louis to them is it/Louis of-it is it.

Although (24a) might seem to suggest that the clitic can correspond to the entire AP, (24b) suggests that it may correspond to the bare adjective. This second alternative is disconfirmed by the last two examples. The object of the adjective may cliticize (as dative leur or genitive en) but only if the predicate has not been cliticized. This is unexpected if $l e$ could stand for the bare A. If, however, le stands for at least A-bar, hence AP (given the general prohibition against affecting intermediate projections, cf. Chomsky (1986)), the ungrammaticality of the last example follows from the clitic having already pronominalized the entire AP. The possibility of (24b) can then be attributed to the PP (à ses amis or de ce genre de gourmandises) being understood as "extraposed," a conclusion that squares well with its being perceived as a topic or even contrasted. Two related questions remain: first, that of how this $\mathrm{PP}$ is ultimately licensed; second, the source of the ungrammaticality of ( $24 \mathrm{~d})$. One might think that (24d) would be ruled out by the following considerations: the source of the clitics leur and en being the extraposed PPs, they may not be cliticized because extraposed elements generally do not cliticize. But this is insufficient. To see why, turn to the first question, i.e., consider the grammaticality of (24b). How are the objects of the adjective licensed when the AP has been pronominalized? We cannot argue that le 
ambiguously pronominalizes A or A-bar: that would leave us with no explanation for either (17a) or (24d). We must conclude that a sentence containing the predicate clitic le is well-formed if we are in principle able to construct an XP* with an internal structure that meets all the demands imposed by the various other elements present in that sentence. In this respect, (24b) is well-formed because we are able to construct an XP* providing a source for these extraposed PPs. It is possible to have Il l'est à ses amis because I can construct an XP*, [fidèle t], to "replace" le and provide a source for ses amis. The ungrammaticality of (24d) is no longer derived: we can reconstruct an $\mathrm{XP*}$ containing an adjective with a silent object that would provide a source for the dative or the genitive clitic. The explanation for the ungrammaticality of (24d) must be found elsewhere.

The kind of explanation I would like to give is one that I will invoke several times throughout this chapter: the idea is that the various licensing conditions that have to be met at LF lead to ill-formed representations because the antecedent of a trace must end up lower than this trace at LF. To see how this would come about consider the kind of representation we would have to construct in order to license a sentence like (24d). Consider Louis le leur est, for example. To license the predicate clitic we need to postulate an appropriate $\mathrm{XP}^{\wedge}$ appearing in $[\mathrm{Spec}, l e]$ at LF. To license the dative clitic leur, we must make sure that this $\mathrm{XP}^{\wedge}$ can contain a silent category object of an adjective that will act as source for the dative clitic. Of course, (22) is meant to apply to all French clitics. This means that there should be a clitic projection associared with the dative clitic as well, in the specifier of which, call it $\mathrm{YP}^{\wedge}$, the phrase licensed by the dative clitic, say $\mathrm{YP}^{*}$, must appear at LF. Now notice the order of the clitics: the predicate clitic precedes the dative clitic (or the genitive clitic). If this order reflects the order of clitic projections, we have conflicting requirements: $\mathrm{XP}^{\wedge}$ must contain a trace of $\mathrm{YP}^{*}$ at $\mathrm{LF}$ which is bound by a $\mathrm{YP}^{\wedge}$ lower than $\mathrm{YP}^{*}$ at $\mathrm{LF}$, as in (25).

$$
\left.\mathrm{L}_{\mathrm{CIP}}\left[_{\mathrm{XP} \wedge} \ldots \mathrm{L}_{\mathrm{YP} *} \mathrm{t}\right] \ldots\right]\left[\mathrm{E}_{\mathrm{Cl}} l e\right] \mathrm{E}_{\mathrm{CIP}} \mathrm{YP}^{\wedge}\left[\mathrm{I}_{\mathrm{Cl}} \text { leur }\right][\ldots \mathrm{XP} *
$$

This approach makes the prediction that, were the clitic originating within the predicate higher than the predicate clitic, the result should be wellformed. It turns out that this prediction is correct, and it makes sense of a very odd fact noted in Kayne (1975). He remarks the contrast between (26b) and (26c). ${ }^{11}$

(26)(a) Louis est dévoué à Pierre. Louis is devoted to Peter.

(b)*Louis le lui est, dévoué, à Pierre/*Louis le lui est fidèle. Louis it to-him is, devoted, to Peter/Louis ie to-him is faithful.

(c) ?Louis me l'est, dévoué/?Louis me l'est, fidèle. Louis to-me it is, devoted/Louis to-me it is, faithful. 
First and second person dative clitics, unlike third person, appear higher than the predicate clitic le. The resulting configuration is consequently wellformed as the order and thus the c-command relations of $\mathrm{YP}^{\wedge}$ and $\mathrm{XP}^{\wedge}$ are reversed, inducing c-command of YP* by its antecedent.

\subsection{Adjectival small clauses are CPs}

We have shown that the constituent pronominalized by the clitic is at least DegP, but there is no bar to its being a larger constituent containing a DegP.

There are several advantages to assuming that the small clause complement of the verb be is at least a CP. A richer small clause structure explains why le appears to pronominalize the array of constituents that it does which is described in part in (16), namely CP, AP, PP, NP, VP. If the small clause can contain structure over and above the (extended) projection of its predicate, we can explain this property by postulating that all small clauses contain, say, a $\mathrm{CP}$ and that non-CPs apparently pronominalized by $l e$ are actually included in this CP. Besides the example in (16), there are several instances in which le seems clearly to pronominalize a CP. Thus the verbs devoir/"must," pouvoir/ "may/can" or se demander/"wonder" in French only take clausal complements and allow sentences such as Marie le doit/Marie le pourrait/Marie se le demandel "Mary must it/Mary can it/Mary wonders it." $\mathrm{CP}$ is in fact the minimal choice that will allow all these various constituents as subparts, ${ }^{12}$ and I will from now on assume it is the correct choice.

As a second consequence of this proposal, it allows analyzing some apparent exceptions to the general principle that only phrases of like categories can be conjoined, as most recently noted in Bowers (1993). Such cases of conjunctions as (27) can be analyzed as involving the $\mathrm{CP}$ conjunction $\mathrm{L}_{\mathrm{CP}} \ldots$ $\left[_{\mathrm{AP}}\right.$ triste $\left.]\right]$ et $\left[_{\mathrm{CP}} \ldots\left[_{\mathrm{PP}}\right.\right.$ en colère $\left.]\right\}$ (an approach which of course carries over to other kinds of small clauses such as I consider Jobn crazy and a good doctor).

$$
\text { Louis est [triste et en colère]. }
$$

Louis is sad and in a rage.

Finally, looking at small clause complements of non-raising verbs, we have seen in (13) and (14) that the subject of the small clause must be outside DegP. This also provides evidence distinguishing between option (23) and the option under consideration. If the small clause is a $\mathrm{CP}$, we can assume that the subject occurs where it normally does in a clause, namely [Spec, $A_{G R}$ ] (obviously, we will need to assume that $T$ in such CPs does not turn $A_{G R}$ into a nominative Case assigner). This also allows accommodating the further requirement exemplified in (12) that the subject be at least one projection removed from the DegP projection (because of intervening, rightward- 
stranded Qs). Under such a view, (14a) will have representation (28) similar to what we would expect a clause to be.

$$
\text { (avoir considéré) } \hbar_{C P} \mathrm{e}\left[_{\mathrm{IP}} \text { les enfants ... [très } t\right. \text { malades]]] }
$$

This provides an (A-)position for the subject lower than the object agreement position of the participle, as required, and makes it unsurptising that stranded Qs in small clauses distribute similarly to stranded Qs in clauses. Thus, parallel to the examples in (14), we find (29).

(29)(a) avoir consideré/have considered)

[Les enfants tous très $\mathbf{t}$ malades].

Les enfants sont tous très $t$ malades.

The children (are) all very sick.

(b) (avoir vu/have seen)

[Les enfants tous rapidement lancer leurs ballons].

Les enfants ont tous rapidement lancé leurs ballons.

The children (have) all quickly throw(n) their balloons.

(c) (avoir voulu/have wanted)

[Les enfants tous exactement au milieu de la pièce].

Les enfants sont tous exactement au milieu de la pièce.

The children (are) all right in the middle of the room.

The distribution of degrees and stranded quantifiers with respect to the subject of the predicate adjective of a small clause, as well as the failure of participle agreement, indicate that this subject occupies an intermediate position, which we just took to be subject of the clause. The distribution of predicate le can in fact provide an argument that this subject and the rest of the small clause form a constituent (which we will ultimately conclude is indeed a CP). This argument is based on the paradigm in (30).

(30)(a) Louis est fou/Louis l'est. Louis is crazy/Louis it-is.

(b) Marie considère [Louis fou].

* Marie le considère Louis.

Mary considers Louis crazy.

Mary it-considers Louis.

(c) Louis a longtemps été considéré fou.

?Louis l'a longtemps été considéré. ${ }^{13}$

Louis has long been considered crazy.

Louis it has long been considered.

(d) Marie croit Louis dans sa chambre.

?Marie y croit Louis.

Mary believes Louis in his bedroom.

Mary there believes Louis. 
The puzzle is raised by the difference between (30a,c) and (30b). Clitic placement of le appears to be blocked by an overt subject of the predicate as in (30b) but not by a subject trace, cf. (31).

$$
\begin{array}{lll}
\text { Subject }_{j} & \text { Verb } & \mathbf{t}_{\mathrm{j}} \mathrm{A} \\
\text { Louis }_{j} & \text { est } & \mathbf{t}_{\mathrm{j}} \text { fou. } \\
\text { Louis }_{j} & \text { a été considéré } t_{j} \text { fou. }
\end{array}
$$

(30d) illustrates that overt subjects of small clauses do not block the movement of all clitics: the locative $y$ replacing the locative predicate may cliticize over it. This shows that the small clause itself is not a general opacity domain for clitic placement. How, then, can we account for the different effects of lexical subjects and traces on the behavior of predicate le? A simple explanation can be constructed under the assumption that predicate le actually stands for the entire small clause. If it does the LF representation of sentences (30a) and (30b) should, according to the proposal in (22), include raising of the small clause to $[\mathrm{Spec}, l e]$. Furthermore, if the subject of the small clause is an accusative DP, it should raise to $\left[S p e c, A G R{ }_{0} P\right.$ ] to sanction accusative Case. Given that the object agreement projection is lower than the clitic projections, we get the respective representations in (32) for (30a) and (30b) at LF.

$$
\begin{aligned}
& \text { (32)(a) Louis }{ }_{j} \ldots\left[\left[_{\text {small clause }} t_{j} \text { fou }\right]_{k}\left[[\text { le }] \text { est ... } t_{k}\right]\right.
\end{aligned}
$$

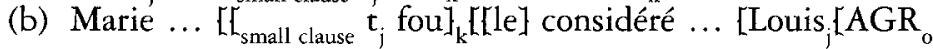

$$
\begin{aligned}
& \ldots \mathrm{t}_{\mathrm{k}}
\end{aligned}
$$

The raised small clause will in all cases contain the trace of its subject. In the second case, the small clause must at LF raise higher than the (highest A-)position that its subject must raise to. We thus have a failure of proper binding. Not so in the first case, since the subject of the small clause raises to an even higher A-position (Spec, AGR ).

\section{Participial small clauses}

\subsection{Avoir, être, and predicate le}

We have noted that the possibility of pronominalizing a variety of constituents by predicate le suggests a common categorial analysis for all small clauses. We also have seen some evidence, in the case of adjectival small clauses, that this constituent was reasonably taken to be CP. I now turn to evidence suggesting that (passive and past) participial small clauses should also be analyzed as full clauses and that $l e$ pronominalizes a $C P$.

In the most natural cases of predicate cliticization, the main clause contains the verb be (or the related devenir/"become," rester/"remain"). This is illustrated 
in (33a) for an adjective case. As expected, passive participles can also enter this construction, as in (33b). That verbs like be play a special role in licensing the possibility of predicate clitics would appear confirmed by the impossibility of (33c).

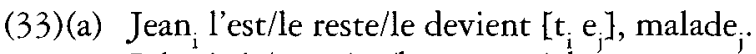
John it is/remains/becomes sick.

(b) Jean $\mathrm{l}_{\mathrm{i}}$ 'a souvent été $\left[\mathrm{t}_{\mathrm{i}} \mathrm{e}_{\mathrm{j}}\right]$, [arrêté par la police $]_{\mathrm{j}}$. John it has often been arrested by the police.

(c)* Jean l'a mangé sa soupe. John has it eaten his soup.

Although there are a priori many possible approaches to the difference (that might be based on Case properties or invoke differences between passive and past participles), I will take the central factor to be the nature of the auxiliary involved, i.e., the difference between have and be. This approach will crucially be based on Kayne's (1993a) proposal concerning the havelbe alternation and on the clausal character of participial (small) clauses, which I find extremely convincing (in general outlook, with some disagreement in analytical details), and which I briefly summarize.

Kayne starts with two points. First, clausal possessive constructions alternate crosslinguistically roughly between $I$ bave this book and $a$ book is with melto me/mine. Second, aspectual auxiliary choice of have or be for a verb V in Romance languages (and beyond) systematically depends on the internal structure of the VP and correlates with the various possibilities of agreement (subject or object) on the participle. Kayne constructs the following account of this rule-governed behavior: he suggests that have and be are variants of each other. He motivates the proposal that have $=b e+\mathrm{X}^{0}$, i.e., that have is the verb be incorporating some head (which he takes to be like a hybrid P/D category). The relevant part of his proposal deals with aspectual auxiliary selection by a verb in its participial form as it interacts with (a) whether the superficial subject is an external argument of the verb and (b) participle agreement. $\mathrm{He}$ suggests that participle phrases are clauses containing at least the participial projection $V P$, an $A G R_{O}$, a $T$, and an $A_{G} R_{s}$ projection, as well as an additional projection of $\mathrm{D} / \mathrm{P}$ whose specifier is an $\mathrm{A}$-bar position (which I will simply note $\mathrm{D}^{*}$ and $\mathrm{DP} *$ its projection); and that such clauses are complement of the verb be, as in (34).

$$
\underset{\left.\left.\mathrm{DP}_{\text {object }}\right]\right]}{\ldots B L_{\mathrm{DP} *}} \mathrm{D}^{*}\left[\mathrm { AGR } _ { \mathrm { S } } \left[\mathrm { T } \left[\mathrm{AGR}_{\mathrm{O}} I_{\mathrm{VP}} \mathrm{DP}_{\text {subject }}[\mathrm{V}\right.\right.\right.
$$

The AGR ${ }_{0}$ projection is present to handle object agreement on the participial $\mathrm{V}$; the DP projection is initially motivated by properties of the possessive constructions. The general idea - as it applies to French - is the following. If 
the $\mathrm{V}$ has an external argument, namely $\mathrm{DP}_{\text {subject }}$ here, this $\mathrm{DP}$ should not be allowed to raise outside DP* by A-movement. The blocking is due to the intervening [Spec, DP*], an obligatory A-bar step on the way, which must be neutralized. This can be done either by incorporating $\mathrm{D}^{*}$ to $\mathrm{BE}$ (yielding HAVE, and, he takes it, making [Spec, DP*] an A-position, but I would take it, alternatively, to extend the local domain of $\mathrm{A}$-movement so that [Spec, $\mathrm{DP} *$ ] may be skipped); or by raising a strong - i.e. equipped with relevant features $-A G R_{S}$ to $D$, turning [Spec, DP] into an $A$-position. If this $A G R_{S^{-}}$ to-D*, or $\mathrm{D}^{*}$-to-be, takes place, $\mathrm{DP}_{\text {subject }}$ may then raise out of $\mathrm{DP} *$ through $\left[\mathrm{Spec}, \mathrm{AGR}_{\mathrm{S}} \mathrm{P}\right]$. We know that this raising does not proceed through $[\mathrm{Spec}$, $\left.A G R_{O} P\right]$ because it does not trigger participle agreement.

The motivation for having an $\mathrm{AGR}_{\mathrm{s}}$ projection comes from Kayne's observation that auxiliary selection is sensitive, in a variety of cases found in Italian dialects to the pronominal features of the subject (of subject pronouns in transitive and unergative sentences, of the reflexive clitic ${ }^{14}$ in reflexive constructions). The motivation for $\mathrm{T}$ is similarly based on the sensitivity to tense exhibited by certain Italian dialects in selecting an auxiliary. The general way in which this sensitivity is worked into auxiliary selection is by letting $T$ or $A_{G R}$ incorporate to $D^{*}$, prior to $D^{*}$ incorporating into $B E$. The raising of an internal argument, $\mathrm{DP}_{\text {object }}$, does not usually require any of this to happen (although it may nevertheless happen). In general, however, a raising $\mathrm{DP}_{\text {object }}$ transits through $\left[S_{p e c}, A G R\right.$ P $]$, triggering agreement. Finally, for reasons that we discuss in the next section, reflexives always involve raising out of a subject (and of an object as well, usually with participle agreement). Here, the $\mathrm{DP}^{*}$ projection is neutralized by $\mathrm{AGR}_{\mathrm{S}}$ incorporating into $\mathrm{D} *$.

Applied to the French situation, this derives the fact that transitives and unergatives (which, agreeing with Chomsky (1992), and Kayne, we take to be covert transitives) select auxiliary have and never trigger participle agreement, while unaccusative constructions (including passive constructions, arriver type verbs) select be and do trigger participle agreement obligatorily. With some unaccusatives (la viande a cuit (*e)/"the meat has cooked"), the auxiliary have is selected. It must then be that escaping through $\left[\mathrm{Spec}, \mathrm{AGR}_{\mathrm{O}} \mathrm{P}\right]$ is disallowed; and it indeed is, as the impossibility of participle agreement shows. Finally, reflexives involve both raising of a subject, and selection of be with participle agreement (when object raising is of a direct object).

It is worth noting that the mechanisms invoked by Kayne mirror exactly what we know happens in clauses: raising to subject (or exceptional case marking, now seen as involving raising to $A G R_{O}$ ) requires $S$-bar/CP deletion or some kind of S-bar/CP transparency (this corresponds to $\mathrm{D}^{*}$ incorporating to $\mathrm{BE}$ ). Mirroring the reflexive case are the constructions in (35).

(35)(a) Marie voit Louis qui vient. Marie voit Louis que/qui Suzanne embrasse. Mary sees Louis (who is) coming. 
Mary sees Louis who Susan is kissing.

(b) Marie les a vus qui venaient.

Marie les a vus que/qui Suzanne embrassait.

Mary them saw who came.

Mary them saw who Susan kissed.

(c) Qui crois-tu qui est venu?

Qui crois-tu que/*qui Marie embrasse?

Who do you believe has come?

Who do you believe Mary is kissing?

Rizzi (1991) argues that the availability of Kayne's (1972) quelqui rule can be derived from qui being analyzed as an agreeing form of the complementizer que turning $[\mathrm{Spec}, \mathrm{CP}]$ into an A-position. Movement of anything but the subject immediately following this $\mathrm{C}$ into $[\mathrm{Spec}, \mathrm{CP}]$ would violate locality conditions (that we can think of as A-movement skipping over a subject), as is exemplified in $(35 \mathrm{a}, \mathrm{c})$. Turning $[\mathrm{Spec}, \mathrm{CP}]$ into an $\mathrm{A}$-position would then allow A-movement of the subject of a tensed clause to an A-position outside it, an occurrence of which is presumably found in $(35 \mathrm{~b})$, where the subject of the embedded clause has raised to [Spec, CP] and on to [Spec, $A_{G R}$ ], triggering participle agreement on voir.

Return now to (33c). Since the embedded DP* in (34) has most of the properties of a clause, I will take it to be a CP, the null hypothesis. In order to keep Kayne's generalization across possessive and participial constructions, I will continue taking participial clauses to be DPs, that is, with $\mathrm{D}^{*}$ taking a $\mathrm{CP}$ complement. Putting together our various assumptions, we now attribute it the underlying structure in (36) $\left(\mathrm{IP}=\mathrm{AGR}_{\mathrm{S}} \mathrm{P}\right)$.

$$
\begin{aligned}
& \text { le ÊTRE } \digamma_{\mathrm{DP}} \mathrm{D}^{*} \mathrm{I}_{\mathrm{CP}} \mathrm{C} \mathrm{I}_{\mathrm{IP}} \mathrm{AGR}_{\mathrm{S}}\left[\mathrm{AGR} \mathrm{O}_{\mathrm{O}} \mathrm{I}_{\mathrm{vP}}\right. \text { Jean [mangé sa } \\
& \text { soupel] }
\end{aligned}
$$

To license the raising of the subject Jean to subject of the main clause, the $\mathrm{DP}_{\text {subject }}$ Jean can raise to Spec $\mathrm{AGR}_{\mathrm{s}}$ but must be able to skip the CP and DP boundaries. In such cases, Kayne argues (we slightly modify his proposal to take into account the additional $C$ projection) $A G R_{S}$ lacks the required properties to turn $C$ (and $D$ ) into heads with A-specifiers (obviously, this must be allowed in tensed clauses, if the paradigm in (35) is any indication, as well as in reflexive constructions, to which we return). The other option is to raise $\mathrm{C}$ to $\mathrm{D}$ and to $\mathrm{BE}$, thereby extending the local domain within which Jean can move (by Baker's (1988) government transparency corollary). This allows movement of this subject to the main clause and triggers ÊTRE $\rightarrow$ AVOIR $\left(\hat{E} T R E+D+\right.$ irrelevantly, C). ${ }^{15}$

Suppose now that le pronominalizes $\mathrm{CP}$, as we have assumed without argument so far. By LF, this CP pronominalized by le will have to raise to $[S p e c, l e]$ in the main clause containing the trace of $C$. The antecedent of this 
trace now appears lower in the structure than this trace, as it is incorporated to $D^{*}$, see (37) (with I+AGR). ${ }^{16}$

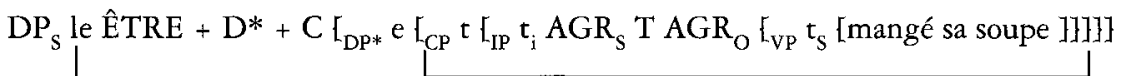

As a consequence, the structure is ill-formed. Note that the preceding account does not rule out predicate cliticization on the verb avoir in general. It only does so if the heads incorporating to $\hat{e}$ tre, turning it into avoir, originate within the CP that le pronominalizes. Thus, interessé, Jean l'a étél "interested, Jean it has been" is perfectly well formed. There are two participial clauses here, one with interessé and one with été. It is the first one that is the CP pronominalized by $l e$. It is $\mathrm{C}+\mathrm{D}$ of the second one that yields avoir under incorporation.

Here, then, is the general form of the argumentation: le should be seen as always pronominalizing the same category. We have grounds to believe, in the adjectival small clause case, that this category is the whole small clause. Kayne provides independent evidence that participial small clauses are CPs. Postulating that le pronominalizes the whole participial CP explains a number of restrictions on the distribution of $l e$. From this we conclude that le sometimes stands for a CP. Consequently it always does. The following sections provide more reasons to assume that le pronominalizes participial clauses.

\subsection{Reflexives}

Having auxiliary être in participial constructions turns out not to be a sufficient condition for predicate cliticization of the participle. Cliticization is impossible in reflexive constructions, whether the reflexivized argument is an object or an indirect object, (38a) and (38b) respectively.

$$
\begin{aligned}
& \text { (38)(a)* Jean se l'est, présenté à Marie. } \\
& \text { ( } \leftarrow \text { Jean s'est presenté à Marie.) } \\
& \text { John himself it is, introduced to Mary. } \\
& \text { (John has introduced himself to Mary.) } \\
& \text { (b)* Jean se l'est, offert un cadeau. } \\
& \text { ( } \leftarrow \text { Jean s'est offert un cadeau.) } \\
& \text { John to himself it is, given a present. } \\
& \text { (John has given a present to himself.) }
\end{aligned}
$$

This is utterly unexpected, since (a) participial phrases are able to cliticize, (b) the auxiliary is the verb be, and (c) there appears to be an additional clitic 
- the reflexive - originating in the participial clause but it cliticizes higher than predicate clitic and thus should create no problem, given the discussion of examples in (26). However, this is exactly what we expect when we conjoin Kayne's analysis of the participial clause structure/bave-be alternation with the idea that le pronominalizes CP.

To see why, we first need to understand the syntax of reflexives. Reflexive constructions use auxiliary be and they show obligatory subject/participle agreement. ${ }^{17}$ There are strong grounds (see Sportiche (1990) and references therein for assuming that reflexive clitics (in Romance languages) correspond to $\mathrm{DP}_{\mathrm{S}}$ in (37); and consequently superficial subjects in reflexive constructions are underlying objects raising to subject under A-movement much as in passive constructions. Consider now (39), the structure of (38a), incorporating our general thesis about clitics (22) ( $\mathrm{I}=$ AGR).

$$
\begin{aligned}
& \text { se le ÊTRE } \ldots \Sigma_{\mathrm{DP} *} \mathrm{D}^{*} \tau_{\mathrm{CP}} C \mathrm{I}_{\mathrm{IP}} \mathrm{I}_{\mathrm{S}} \mathrm{T} \mathrm{I}_{\mathrm{O}} \mathrm{t}_{\mathrm{VP}} \mathrm{DP} \text { sub } \text { [présenté } \\
& \text { Jean]l1]] }
\end{aligned}
$$

To license the raising of the object Jean to subject of the main clause, $\mathrm{DP}_{\text {sub }}$ (i.e., the pro that se pronominalizes which we assume matches se's features) must raise at least to $A G R_{s}$, Kayne argues, which $I$ will take to mean to [Spec, IP] making $A G R_{S}$ strong. ${ }^{18}{ }^{A G R}$ subsequently incorporates to $C$ and $\mathrm{D}$ to allow A-movement out of DP*. The clitic le pronominalizes $\mathrm{CP}$. This $\mathrm{CP}$, containing the trace of $\mathrm{C}$ incorporated in $\mathrm{D}$, will have to raise to [Spec, $l e]$ in the main clause. We find again the usual violation: this trace is now lower in the structure than its antecedent, ruling out the LF representation.

\subsection{Raising structures and non-reflexive se}

An account along the same lines can be constructed for cases involving the se morpheme that are not reflexive (see Ruwet (1972)). Consider the paradigm in $(40)$.

(40)(a) Ce livre s'est bien vendu $\rightarrow *$ Ce livre se l'est bien. This book sold well.

(b) L'eau s'est renversée $\rightarrow$ *L'eau se l'est. The water spilled.

(c) Jean s'est avéré fou $\rightarrow$ *Jean se l'est avéré, fou. Jean turned out it, crazy.

$S e$ 's function is not restricted to indicating reflexive constructions. It may also be used to indicate middle constructions (40a), appear in a lexically determined class of verbs $(40 \mathrm{~b})$ with causative counterparts (renverser, disperser, réunir/"spill, disperse, gather") or be inherent, i.e., appear on a lexically determined class of verbs without any apparent semantic or grammatical 
correlations (s'évanouir, s'averer/ "lose consciousness, turn out"). All these constructions have the same characteristics as reflexive constructions: they use auxiliary be, show obligatory subject/participle agreement, and are naturally analyzed in the same way. On the same grounds (see Sportiche (1990)), we assume that all French se clitics, whether reflexive, middle, neutral, or inherent (a) correspond to $\mathrm{DP}_{\mathrm{S}}$ and consequently (b) superficial subjects in $s e$ constructions are underlying objects. The ill-formedness of (40a) follows: $s e$ pronominalizes the thematic subject of vendre, and the account proceeds as in the reflexive case (the only difference is that the subject is interpreted as existentially bound tather than bound to the object, as in reflexive constructions).

The cases of (40b) and (40c) cannot be treated exactly alike because the verbs involved lack thematic subjects altogether (this is one of the major differences between inherent and middle constructions). What then does se pronominalize? To understand what happens here, it is necessary to review the reasons leading to the adopted analysis of Romance se/si.

We have already noted that se constructions use auxiliary être (a fact especially significant in languages like Standard Italian in which this is a diagnostic property of unaccusativity) and trigger participle agreement (which is always object agreement in French) with the superficial subject. Furthermore, Bouchard (1982) notes that reflexives are impossible with verbs lacking external argument; thus we have the contrast in (41).

(41) Jean lui semble être pâle/*Jean se semble être pâle. John seems to him(*self) to be pale.

These facts point to the same conclusion: reflexive constructions are unaccusatives, with the superficial subject being the underlying object (this is why be is used, and obligatory participle, i.e., object, agreement is triggered exactly like passive constructions). Then (41) follows if we assume that se always pronominalizes the external argument of the verb: since seem lacks one, there is no source for the reflexive. This analysis is compatible with the properties of middle constructions (middle verbs always have an implicit external argument), but not with the existence of neutral se verbs or inherent se verbs, particularly when they are raising verbs like s'averer, which all lack external arguments. The essence of the problem is that we want se to be able to be an expletive, a proposition incompatible with its being an (external) argument. Instead, this suggests that se should be linked to a position "subject of a clause," but lower than say, Tense of the main clause. This is exactly what the full clausal structure of participial constituents provides: suppose that the morpheme $s e$ is not inherently linked to the external argument of a predicate but rather, as stated in (42), to [Spec, $A \mathrm{GR}_{\mathrm{S}}$ ], and in the case of participial clauses to that of the participial clause (as a consequence we now lose the account for (41)). 
(42) The morpheme se/si is inherently linked to $\left[\mathrm{Spec}, \mathrm{AGR}_{\mathrm{S}}\right]^{19}$

By the extended projection principle, this is a position that exists regardless of whether the participle has an external argument. Hence there is no bar to having it in the neutral or inherent cases. The facts in $(40 \mathrm{~b}, \mathrm{c})$ follow now, exactly as in the reflexive case, (43).

$$
\begin{aligned}
& \ldots \text { se le } \hat{E} T R E \ldots I_{D P *} D * L_{C P} C I_{I P} \text { pro } A G R_{S}\left[T A A_{O}\right. \\
& \mathrm{E}_{\mathrm{VP}} \text { [avéré/renversé ... DP**]1]1]\}] }
\end{aligned}
$$

An expletive pro must be sitting in $\left[\mathrm{Spec}, \mathrm{AGR}_{\mathrm{S}}\right]$ which will have to raise to [Spec, se] at LF (a chain must be formed). This raising of pro out of DP* requires transparency of $C P$ and $D P^{*}$, i.e., raising of $A G R_{S}$ to $C$ and $D$. Raising of CP to $[\mathrm{Spec}, l e]$ at $\mathrm{LF}$ will then create the offending configuration.

One question we have not addressed is how objects in passives, reflexives, or arriver-type unaccusative structures escape DP*. The unaccusative case is different from the reflexive case because the verb only has one argument (passive is like either unaccusative or reflexive depending on whether the external argument of the predicate is represented or not). For unaccusatives, Kayne suggests the DP* projection is absent (it would be absent too for adjectives, explaining why they never take bave). In our terms, even if $A_{G} R_{S}-$ to-C takes place, CP raising to [Spec, le] would not create an offending configuration. In the present case, DP** raising out of DP* and IP takes place (which participle agreement indicates is through $\left[S p e c, A G R_{O}\right]$ ). There are two ways of allowing this to happen: if pro is an expletive, as in the present case, it would be enough to move DP** through $[S$ pec. AGR $]$. Since it must move to [Spec, se], DP** will have to move to $[\mathrm{Spec}, \mathrm{se}]$ on its way to the subject position of the main clause: we end up with expletive se agreeing with the superficial subject (Je me suis avéré malade, Nous nous réunissons). Kayne (1993a), however, provides reasons to believe that movement from [Spec, $\mathrm{AGR}_{\mathrm{O}}$ ] to [Spec, $\mathrm{AGR}_{\mathrm{s}}$ ] is never possible in these participial clauses. Let us then adopt a second option which will work even if pro is an argument, as in reflexive or middle constructions. Assume incorporation of $A G R_{O}$ into $A G R_{S}$ prior to $A G R_{S}$ raising higher. Since the resulting category $A G R_{S}+A G R_{O}$ is able to have only one set of pronominal features, this will derive subject/ object pronominal agreement; that is, the same agreement facts as previously (Je me suis avéré malade, Nous nous réunissons. It might also be the source of the surprising agreement found in middle constructions in French between the se morpheme and the superficial subject (viz. a talking book: je me vend bien). ${ }^{20}$ 


\section{Clausal structure}

\subsection{The complement structure of tense}

We still need to account for the facts of (41) under assumption (42). Before we do so, it is worth pointing out that the facts about the possibility of having predicate $l e$ are in fact independent of the presence of the aspectual auxiliaries. Thus the full paradigm is duplicated, as in (44).

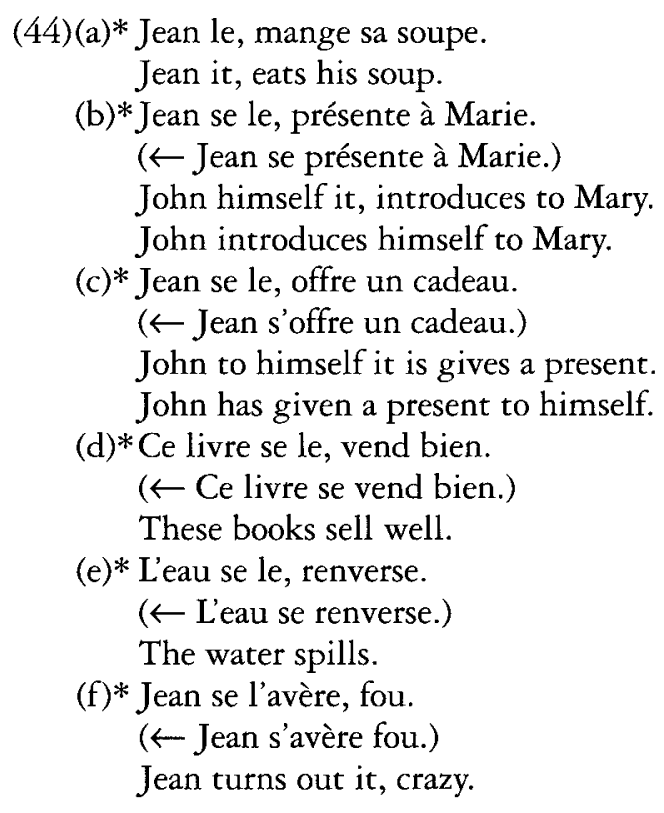

It might appear that all but (44f) would be ruled out independently by the necessity for le to cliticize, but this is far from obvious given Kayne's (1991) proposal that clitics may attach to (silent) functional heads, and thus may appear to be left stranded in front of adverbials (cf. the archaic sounding le bien connaître/"know him well," orders of magnitude better than $(44 a-e)$ or the comparable **connaître Jean, le bien/"know Jean, it well" with le standing for the string connaître Jean). It might also appear that (44f) could be ruled out in a way parallel to the slightly deviant (45).

(45)(a) ?Jean le semble, fatigué. John it-seems tired.

(b) ?Jean le paraissait, fou. John it appears crazy.

But again the magnitude of deviance is quite different from that found in (44f). ${ }^{21}$ The facts of (41) strongly suggest that we find under Tense a structure 
similar in the relevant respects to that of participial clauses. Suppose $T$ takes a $\mathrm{CP}$ complement with the main verb in it, as in (46).

$$
\ldots \mathrm{T} \ldots\left[_{\mathrm{CP}} \ldots\left[\left[_{\mathrm{vP}} \mathrm{DP}_{\text {subject }}\left[\mathrm{V} \mathrm{DP}_{\text {object }}\right]\right]\right]\right.
$$

Attempting to pronominalize this $\mathrm{CP}$ is incompatible with the requirement that $\mathrm{V}$ incorporate to $\mathrm{T}$ at LF (in fact overtly in French). All the facts of (44) follow immediately.

We can now return to (41), the underlying structure of which will be as in (47).

$$
\begin{aligned}
& \text { se } \ldots \mathrm{T} \ldots \mathrm{L}_{\mathrm{CP}} \mathrm{C} \mathrm{L}_{\mathrm{IP}} \mathrm{pro}_{\text {expl }} A_{\mathrm{GGR}}\left[\ldots \mathrm{AGR}_{\mathrm{O}} \mathrm{L}_{\mathrm{VP}}\right. \text { sembleDP* } \\
& \text { [ DP** être pâle]1]]] }
\end{aligned}
$$

In essence the account here is that both $\mathrm{DP}^{*}$ and $\mathrm{DP} * *$ compete for the same subject position: $\mathrm{DP}^{*}$ because of the presence of the reflexive, $\mathrm{DP}^{* *}$ because we are dealing with a raising-to-subject predicate. In the absence of the reflexive construction, sembler selects auxiliary avoir and DP** raising does not trigger participle agreement. This is an indication that the raising of DP** cannot go through [Spec, $A G R_{0}$ ], and hence must go through [Spec, $A G R_{\mathrm{s}}$ ]. The same can be said of the raising of DP*. As an indirect object in French, it does not trigger participle agreement and hence does not raise through [Spec, $\mathrm{AGR}_{\mathrm{O}}$ ] either. Both DPs must therefore transit through the same position, [Spec, $\mathrm{AGR}_{\mathrm{s}}$ \}, which is impossible. ${ }^{22}$

\subsection{Some general consequences about clause structure}

I have suggested that the predicate clitic le pronominalizes a $\mathrm{CP}$. This hypothesis, taken in conjunction with Kayne's analysis of participial clauses can derive a number of complex distributional properties of this clitic. One consequence is that it confirms the clausal character of small clauses. In particular, we have concluded above in (47) that the VP small clause complement of $\mathrm{T}$ is in fact a full-fledged $\mathrm{CP}$. The same conclusion is reached on independent grounds by Sportiche (1994), who proposes to eliminate adjunction and adjunction structures altogether from the grammar. There I argued that there was some benefit in taking each VP to be a CP, as it allowed strict locality conditions on $W h$ Movement to be kept wirhout any recourse to adjunction structures (intermediate or not), with the consequence that $W b$ Movement is exclusively to [Spec, CP] (because landing sites for movement to specifier are target-specific and because adjunction structures do not exist). I further argued that, if we take seriously Stowell's (1993) view of Tense as temporal ordering predicates and the idea that crosslinguistic variation should be kept to a minimum, the reason $\mathrm{T}$ takes a $\mathrm{CP}$ complement is that it is of the category $\mathrm{V}$ (or P) itself (I will pick V for concreteness). Putting all 
these results together, the structure of a simple clause changes from (48a) to (48b), with the first $A G R$ corresponding to $A G R_{s}$, and the second to $A G R_{O}$.

(48)(a) $\tau_{\mathrm{CP}} \mathrm{CAGR}$ T $\mathrm{AGR}_{\mathrm{O}}\left[_{\mathrm{VP}} \mathrm{V} \ldots\right.$

(b) $\mathrm{L}_{\mathrm{CP}} \mathrm{C}\left[\mathrm{AGR} \mathrm{I}_{\mathrm{VP}} \mathrm{V} \mathrm{L}_{\mathrm{CP}} \mathrm{C}\left[\mathrm{AGR} \mathrm{I}_{\mathrm{VP}} \mathrm{V} \ldots\right.\right.$

That is, that clausal structure is constructed by stacking verbs, each with its extended projection, i.e., an agreement projection and a complementizer projection, a basic architecture that should be extended to all lexical categories. Naturally, we expect the evidence for these intermediate structures to be quite complex and remote. I furthermore believe them to be obscured by the existence of the well-documented process of restructuring that is apparent in several Romance and Germanic languages (e.g., Italian, see Rizzi (1978), Burzio (1986), and Dutch, Evers (1974)). This process makes it appear that two clauses behave as one with respect to a number of phenomena (e.g., clitic placement, A-movement, etc.), and its existence is established on the basis of pairs of biclausal sentences, identical in all relevant respects except for the fact that one sentence behaves as monoclausal and the other does not. Granting the existence of such a process, it should not be surprising to find cases of biclausal structures that obligatorily restructure, and thus systematically obfuscate underlying syntactic organization. I would argue that this is what happens in French tensed clauses (explaining why tensed verbs raise to "T"), or with French sequence of auxiliaries (explaining why the pronominal argument of a main verb must appear on the highest auxiliary of its clause or why passive may skip all auxiliaries - recall that we would treat them as regular verbs, each with its own clause).

If, furthermore, we take seriously the idea of VP shells introduced by Larson (1988), a transitive VP such as [ John cook the food], reasonably composed of two VPs, each with one argument in its specifier, ${ }^{23}$ represents two small clauses, one on top of the other. By parity of reasoning, such a VP would have to be analyzed as two clauses, understood as above, i.e., as (49b) instead of (49a), so that the sentence John cooked the food would have the structure (49c).

(49)(a) [ [vp John V [ [ food cook]]

(b) $\tau_{\mathrm{CP}} \mathrm{C}\left[\mathrm{AGR} \mathrm{L}_{\mathrm{VP}}\right.$ John $\mathrm{V} \mathrm{L}_{\mathrm{CP}} \mathrm{C}\left[\mathrm{AGR} \mathrm{L}_{\mathrm{o}} \mathrm{L}_{\mathrm{VP}}\right.$ food cook $\ldots$

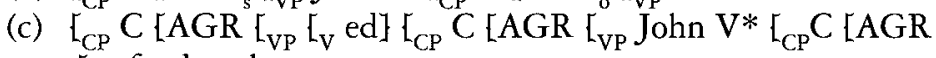
$\mathrm{L}_{\mathrm{VP}}$ food cook ...

We can now reasonably take $\mathrm{V}^{*}$ as essentially being CAUSE, i.e., adopt a lexical decomposition analysis to syntactic structure, a consequence already implicit in the work of Hale and Keyser (1991). Again here, we expect the evidence to be complex. But even so, some suggestive evidence exists. Consider a VP with a double object construction alternation $\mathrm{I}_{\mathrm{VP}}$ John give a

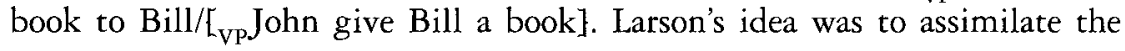


double object alternation to active/passive alternation. That there should be an essentially transformational approach (i.e., an exceptionless rule expressing redundancy) to this question is surely the null hypothesis. We should try to adopt it, but we can follow a different route than Larson's, conceptually similar to the one taken by Stowell (1981) to account for there insertion. His insight was to implement the idea that There is a man on the roof and $A$ man is on the roof are transformationally related by deriving them both from a common source (an underlying small clause structure), rather than trying to derive one directly from the other. A biclausal approach to the VP offers just that, as in (50).

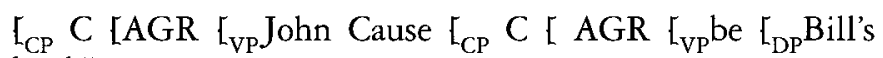
book]...

Under such a view we can paraphrase the structure of give Bill a book by cause there to be Bill's book, with the expletive (there) in [Spec, AGR*]. The double object alternation would then reduce to the two ways in which possessive constructions are realized (as construed by Kayne (1993)): give Bill a book would correspond to cause Bill to have a book, with Bill raising to [Spec, AGR*], while give a book to Bill would correspond to cause a book to be to Bill. This approach would provide an account of some striking similarities: e.g., give John this new kind of cold/ ${ }^{*}$ give this new kind of cold to John is mirrored by Jobn has this new kind of cold $/$ *this new kind of cold is John's.

I will not pursue this any further here, but its logic is clear.

\section{Further properties and residual problems}

\subsection{Idiomatic le and raising}

I now turn to other prohibitions on predicate pronominalization by le. First of all, idiomatic predicates cannot undergo it, as seen in (51).

(51)(a) Marie tombera malade/*Marie le tombera. Mary will fall ill.

(b) Louis voit juste/*Louis le voit.

Louis is right (lit. sees correct).

In the present case, the idea is the following: tomber-malade behaves syntactically like a $\mathrm{V}+\mathrm{A}$ pair. Each member can be modified (by adverbials or degrees), moved in a limited way (to $\mathrm{T}$ for $\mathrm{V}$ ), yet form a semantically non-compositional unit. Koopman (1994) shows that there are good grounds to assume that idiom chunks must incorporate (under head movement) to their highest member (basically to explain their restricted movement possibilities). Applied to the present case, this means that in order to get the 
idiomatic reading, malade incorporates into tomber. Suppose now we pronominalize malade with le. The possibly silent malade will have to be incorporated to tomber at $\mathrm{LF}$, while the constituent $\mathrm{XP} *$ containing the trace of this silent $\mathrm{A}$ will have to appear in $\mathrm{XP}^{\wedge}=[\mathrm{Spec}, \mathrm{CIP}]$ which is higher than the verb. The antecedent will thus end up lower than its trace; see (52).

$\left.\mathrm{L}_{\mathrm{CIP}} \mathrm{L}_{\mathrm{CP}} t_{\mathrm{k}} \ldots\right]_{\mathrm{i}}\left[\mathrm{L}_{\mathrm{C} 1}\right.$ le] [tombera $+\left[_{\mathrm{C}} \mathrm{C}_{\mathrm{k}} \ldots \mathrm{L}_{\mathrm{A}}\right.$ malade $\left.\left.\left.\left.\left.\left.\left.]\right]\right] \mathrm{L}_{\mathrm{CP}} t_{\mathrm{i}}\right]\right]\right]\right]\right]$

For this account to work, the possibility of reconstruction must be prevented. I have assumed throughout that when a phrase is targeted for movement, say $\mathrm{XP}, \mathrm{X}^{0}$ cannot reconstruct, only complements of $\mathrm{X}^{0}$, possibly adjuncts to $\mathrm{XP}$, and higher pied-piped material may (i.e., a $\mathrm{P}$ taking $\mathrm{XP}$ as complement). The clitic pronominalizes CP. Then $\mathrm{C}$ must be in $[\mathrm{Spec}, \mathrm{CIP}]$ at LF. In effect, the antecedent of this pro-CP must be understood as including an incorporated A (malade). Incorporation of this A being subject to the head movement constraint, all heads intervening (in the sense of c-command) between the $A$ and its ultimate incorporation site will have to incorporate too, and this includes C. Note that we cannot attribute the ungrammaticality of the examples under discussion to the impossibility of pronominalizing an idiom chunk as pronominalization of (some) idiom chunks appears to be possible: La justice, Saint Louis la rendait sous un chêne; il l'a cassée et bien cassée, sa pipe/"Justice, Saint Louis, dispensed it under an oak tree; he kicked it, his bucket."

In the case of voir juste or travailler fort, "work hard," le is ruled out because justelfort really are adverbials rather than adjectives (as their lack of agreement with the subject indicates: *elle travaille forte).

\subsection{Adjunct small clauses}

Adjunct predicates cannot be cliticized, an observation leading to substantial complications in various areas of syntactic analysis that I will not pursue here. I will limit myself to sketching the basic idea of an account; see (53).

(53)(a) Louis lit son journal allongé.

* Louis le lit son journal.

Louis reads his newspaper lying down.

Louis it reads his newspaper.

(b) Marie travaille ivre/*Marie le travaille.

Mary works drunk/Mary it works.

(c) Jean dort couvert $/ *$ Jean le dort. John sleeps covered/John it sleeps.

(d) Marie mange sa viande crue/Marie le mange sa viande. Mary eats her meat raw/Mary it eats her meat. 
(e) Henri est arrivé fatigué/*Henri l'est arrivé. Henry arrived tired/Henry it arrived.

(f) Jean mourra jeune/*Jean le mourra. John will die young/John it will die.

Why should this be? Intuitively, these deviant sentences feel wrong because the predicate is not a complement of the main verb. Since cliticization involves movement (see, e.g., Sportiche (1992)), it is tempting to capitalize on this intuition by attributing the deviance to an argument/adjunct distinction, i.e., to the Empty Category Principle (ECP). The simplest way to bring in the ECP is to postulate enough structure so that we get an adjunct island violation. Assuming that le pronominalizes $\mathrm{CPs}$, these adjunct constituents will have to be postulated to be larger constituents XP out of which a CP will be extracted, yielding a violation of the adjunct island condition.

This simple account, however, cannot be the whole story. First, it is not completely clear what this constituent XP could be. A sort of a while/during clause (en/pendant clauses in French), as in Jean travaille en sifflant/"Jean works while whistling" is plausible in certain cases (54) but seems not to give rise to the right interpretation. It seems to me that the correct semantic interpretation can be paraphrased by introducing an existential or universal frequency adverb (that may be redundant in case it is pragmatically clear that only one (potential) event is referred to) or a sort of generic when the sentence can be and is understood generically. For example, see (54).

(54) (for 53a): Sometimes (/Always), when John reads his paper, John is lying down.

or Typically, when John reads his paper, John is lying down.

Second, extraction facts out of this putative adjunct give the results in (55).

(55)(a) John works drunk/*how drunk does John work?

* John works drunker than Bill works/*John works as drunk as Bill works sober.

(b) John eats his meat rare/?how rare does John eat his meat?

*John works drunker than Bill works/*John works as drunk as Bill works sober.

?John eats his meat more cooked than Bill eats his chicken/?John eats his meat as rare as Bill eats his chicken cooked.

(c) John will die young/?how young will John die? ?John will die younger than Bill will die/?John will die as young as Bill will live long. 
(d) John considers Bill intelligent/how intelligent does John consider Bill?

John considers Mary more intelligent than he does Bill/ John considers Mary as intelligent as he considers Bill stupid.

These examples show the paradigm of $w b$-extraction of small clause adjectives respectively in wh-questions, comparatives, and subcomparatives. The judgments, although not secure, seem to be graded more or less as indicated: subject-controlled adjunct small clauses (55a) yield the least acceptable results. Object-controlled adjunct small clauses ((55b), and (55c) - an unaccusative case, i.e., a case of trace control by the subject) yield better results, perhaps almost as good as in the case of complement small clauses (55d) or the similar raising case John is $t$ sick ... . ..$^{24,25}$ My own judgments in French are more mixed, as in (56).

(56)(a) ??Louis travaille aussi ivre que Marie travaille sobre. Louis works as drunk as Mary works sober. ??Les couvertures dont Marie dort couverte sont chinoises. The blankets that Mary sleeps covered with are Chinese.

(b) Louis mange le boeuf aussi cru que Marie le mange cuit. Louis eats beef as raw as Mary eats it cooked. ??La confiture dont il mangeait son pain tartiné était faite maison.

The jam he ate his bread spread with was home-made.

(c) Pierre est arrivé aussi fatigué que Paul est arrivé ivre.

Peter arrived as tired as Paul arrived drunk.

??Les travaux dont Pierre est arrivé satisfait ne sont pas les siens.

The works Peter arrived satisfied with are not his.

(d) Pierre considère Marie aussi intelligente que tu la considères stupide.

Peter considers Mary as intelligent as you consider her stupid.

Les gourmandises dont je crois Marie friande viennent de Cambrai.

The sweets you believe Mary fond of come from Cambrai.

Under the account given here, the relative acceptability of the examples in $(56 a-c)$ as compared with the (totally unacceptable) cliticization case is problematic: we would expect extraction out of these adjunct small clauses to be unacceptable. The two questions we are faced with are the following, the first one being most crucial to us. (a) Do these facts affect the conclusion that the small clause is a CP? (b) What accounts for the better than expected status of these cases of extraction out of adjuncts? 
Let us address the impossibility of cliticization as it relates to the first question. There is another way of construing the intuition locating the problem in a distinction between the type of dependent that these small clauses instantiate. Trying to parse, say (53b), one wonders: Work what? In fact in all the grammatical cases of predicate cliticization, the pronominalized constituent can be replaced by quoi, "what," whereas in these bad cases, the question word would have to be comment, "how." Let us take this to suggest that the missing element is perceived as an accusative object of the verb. We could then attribute the ungrammaticality of the examples in (53) to the fact, reflected by the choice of question word, that they are not getting accusative Case. Some support for this idea comes from what happens with control structures. Subject control verbs allow pronominalization of their CP infinitival complements, viz., Partir, Jean l'a voululessayélespérélpromis à Pierre/"Leave, John it has wanted/tried/hoped/ promised," but they are never transitive. Object control predicates systematically disallow it: *De partir, Pierre l'a persuadé Henri "“To leave, Peter it has persuaded Henry," but indirect object control allows it: De partir, Pierre l'a ordonné à Henri/"To leave, Peter ordered it to Henry." The emerging generalization is that predicate cliticization is impossible if there is an accusative object that may also cliticize as $l e$. This would follow if $l e$ were accusative and could only pronominalize accusative-marked CPs. An additional advantage, noted previously, would be to explain the homophony between the predicate clitic le and the pronominal accusative clitic le (a homophony also found in Italian with $l o$ ). As I mentioned at the outset, this raises many questions that I will leave unaddressed (the Case-marking status of verbs like $b e$, the relationship between accusative Case and participle agreement, Burzio's Generalization, etc.).

Turn now briefly to the second question. If $l e$ must be accusative, these adjunct small clauses could be either adjuncts or non-accusative complements. The extraction facts are surprising either way. Additionally, if indeed we were treating these adjunct small clauses as CPs, we would expect that they would in principle be cliticizable with an appropriate kind of oblique clitic (locative adjuncts, for example, may cliticize as $y$ in French). I know of no language allowing this. If these small clauses were complements, the reverse problem would arise (why are extractions simply not perfect, and why do they not cliticize with oblique clitics, e.g., lui?). That they should not be complements is corroborated by Dutch facts involving overt incorporation, with the pair of subject/object-controlled small clauses (indicated by the choice of auxiliary bave vs. be) in (57).

(57)(a) ...*omdat heeft dronken gewerkt. because John has worked drunk.

(b) ...*Omdat Jan is dronken vertrokken. because John is gone drunk. 
The presence of the adjective drunk between the auxiliary and the participle indicates that this adjective has incorporated. Both sentences are out, as we would expect given the adjunct status of the adjective. So it would seem we want these small clauses to be neither complements nor adjuncts.

I have no solution to offer. Instead, I will offer a radical speculation (further explored in Sportiche (1994)) based on the paraphrase given in (54).

The idea is to make the adjunct predicate be the main predicate of the clause and include what appears to be the main clause as a subconstituent. The missing frequency adverb behaves semantically as a quantifier comparing the frequency of events (de Swart (1991)). In example (54), these events are denoted respectively by [John reading bis newspaper] and [John lying down], i.e., roughly speaking where the first one corresponds to the restriction of the quantifier and the second one to its nuclear scope. We might pursue the idea that these kinds of relations are always mapped syntactically the same way. On the model of $[[$ All [the children $]]$ came $]$, we would be led to postulate the equative substructure for (58a) with its intuitive paraphrase (58b) and a simplified derived structure as in (58c).

(58)(a) [Sometimes [John reads the paper]] BE [John lying down]

(b) Some occurrences of [John reading the paper]] are [occurrences of John lying down]

(c) $\left[\mathrm{John}_{\mathrm{i}}\right.$ Tense [sometimes $\left[\mathrm{t}_{\mathrm{i}}\right.$ read the paper] [be $\left[\mathrm{PRO}_{\mathrm{i}}\right.$ lying down] ]

We would then expect that predicate cliticization and extraction would work as in equative structures, disallowing predicate cliticization (the "object" is referential) and assimilating problems of extraction out of this object to difficulties in extracting out of specific DPs. It is easy, however, to imagine the obstacles that such a view encounters. ${ }^{26}$

\subsection{Some residual problems}

I now go through some further observations about properties of predicate cliticization, for some of which I have no account to offer. Predicate cliticization with raising verbs is not perfect: ?Jean le semble (for unknown reasons), but much less degraded than if an idiom chunk is subject, as in *Justice le semble, avoir été rendu. I take the latter as showing that infinitival complements to seem do not pronominalize. This correctly captures the contrast Malade, Jean le semble but not $* \hat{E}$ tre malade, Jean le semble. This impossibility might be related to "CP-deletion" in view of the better (although not perfect)?Que Jean soitlest malade, il le semble.

Second, cliticization is impossible in unaccusative and extraposition impersonal constructions, as in (59). 
(59)(a) Il est arrivé trois hommes $\rightarrow *$ *il l'est. There is arrived three men $\rightarrow$ there is it.

(b) Il est important que Jean parte $\rightarrow *$ *il l'est. It is important that John leave $\rightarrow$ it is it.

(c)* Important, il l'est que tu partes. Important it is it that you leave.

Comparing (59b) with C'est important que Jean parte $\rightarrow$ ca l'est, which differs minimally from it by having an argument rather than an expletive subject, ${ }^{27}$ suggests invoking the necessity of expletive replacement as a source of ungrammaticality. This does not seem compatible with the well-formedness of the previously mentioned $I l$ semble que Jean soit parti $\rightarrow$ ? Il le semble/"It seems that John left $\rightarrow$ It seems it," or with the impossibility of (59c) (compare (24b)): there is no generalization that expletive subjects prevent predicate clitics.

Start with sentence (59a). Recall that we assumed unergatives to be covert transitives. Unaccusatives then become monadic predicates essentially so that the underlying structure of (59a) would be as in (60).

$$
\ldots \complement_{\mathrm{VP}} \text { être } \complement_{\mathrm{CP} *} \mathrm{C}^{*} \ldots \mathrm{L}_{\mathrm{VP}} \text { trois hommes arrivé } \ldots
$$

How, then, is the verb/subject order reached? Postulating that this arises from the verb arriver raising beyond the CP boundary (where it incorporates to être, which may then excorporate (see Koopman (1994) for discussion of the relevant theory of head movement and an analysis of causatives along these lines), we derive both the word order and the impossibility of (59a) for the usual reason: the pronominalized $\mathrm{CP}$ will contain the unbound trace of $\mathrm{C}^{*}$ (since raising of the $\mathrm{V}$ will have to be through $\mathrm{C}^{*}$ ). Reformulating Belletti's (1988) analysis, let us make the raising of arriver responsible for allowing the subject to stay in postverbal position (by making the availability of Case contingent on this raising). We derive the impossibility of impersonal constructions with small clauses (which Belletti (1988) attributes to the inherent nature of the Case assigned by the participle), as in (61a).

$$
\begin{aligned}
& \text { (61)(a)* Il a été considéré [trois hommes malades]. } \\
& \text { It has been considered three men sick. }
\end{aligned}
$$

(b) Il a été considéré + malades ${ }_{\mathrm{i}}$ [trois hommes $\mathrm{t}_{\mathrm{i}}$ ]. It has been considered sick three men.

Case on the subject of the small clause is available only if the predicate of the small clause raises out of it. When this does not take place, the structure is ruled out, as in (61a). If this raising does take place, the result improves, as in the much improved (61b). 


\section{PARTITIONS AND ATOMS}

This account can be extended to (59b) in the following manner. Suppose that the adjective important incorporates to être as well.

$$
\left.\left.\left.\left.\ldots \complement_{\mathrm{VP}} \hat{e} \text { tre }+ \text { important }_{\mathrm{i}}\left[_{\mathrm{CP}} \mathrm{t}_{\mathrm{i}} \ldots \mathrm{L}_{\mathrm{IP}} \text { [que Jean parte }\right] \ldots \mathrm{t}_{\mathrm{i}}\right] 1\right]\right]\right]
$$

Kayne's (1994) general thesis implies the non-existence of rightward movement. The clause interpreted as subject of the adjective is, according to this thesis, not extraposed. Suppose instead it stands in the usual subject-predicate relation (e.g., [que Jean parte] est important), clearly the null hypothesis. To account for the surface word order, we now need to raise the adjective, as indicated in (62). This would derive both (59b) - for the same reason as (59a) - and (59c) because there is no available CP to pronominalize.

\section{Notes}

1 For example, Schein (1995), or Williams (1983) who disputes the small clause analysis of Someone seems $[t$ sick $]$ because someone takes scope necessarily wider than seem, unlike in the raising case of Someone seems $[t$ to be sick]. Some of these problems are discussed in Stowell (1991).

2 Another might be right node raising: Louis a emprunté et Marie a rendu un livre à Jean hier/"Louis borrowed and Marie returned a book to/from John yesterday."

3 Further discussion of some of these issues is found in Sportiche (1993), especially concerning the exact structural relationship between $t$ and the $\mathrm{Q}$ tous, argued to be $[t[Q[t]]]$.

4 The same argument can be constructed for English on the basis of bare nominals: I consider the children fool*(s).

5 This is true even if participle agreement and $A G R_{0}$ are two distinct projections, as long as AGR is higher, which is plausible in the event the two positions are not identical (participle agreement is always available while the availability of accusative depends on the larger context in which the participial complex is found, e.g., with auxiliary have but not with auxiliary be).

6 Also in need of an explanation is why (12c) is ill-formed (no pied-piping) and why $(12 \mathrm{~b}, \mathrm{~d})$ are ill-formed (not all $t^{*}$ are eligible stranding sites for $\mathrm{Q}$ ).

7 This conclusion is close to that of Bowers (1993). Bowers suggests that small clauses are instances of predicate phrases that can be thought of as a kind of VP layer similar to Larson's (1988), not as clauses as argued here and elsewhere. See also Starke, and references therein.

8 Respectively exemplified by the alternatives in (16). The distinction is made apparent by the choice of preposition introducing the agent (de vs par) and correlates with a stative/eventive difference in interpretation that can be corroborated by the kind of modifier they take ( $s i$ "so" vs. tant "so much," cf. Ruwet (1972)).

9 Grimshaw (1987) and Corver (1993) argue that subcomparatives in English and Dutch do not involve $W b$ Movement. Strictly speaking, my arguments are only concerned with French and at any rate with the location of the missing quantity quantifier. I remain convinced that movement is involved because it overtly 


\section{FRENCH PREDICATE CLITICS AND CLAUSE STRUCTURE}

occurs in French quantity wh-question questions with combien (thus violating the left branch condition and more generally undermining their most potent arguments against movement).

10 In this structure, $t_{k}$ must not interfere with the raising of the DP Marie out of the small clause.

11 The contrast between (26b) and (26c) going in the direction indicated seems to be shared by all speakers, but the absolute degree of acceptability of (26c) varies. The same is found in passive partiple cases and follows in the same way: Cette lettre a été envoyé aux enfants $/ *$ Cette lettre le leur a étél? Cette lettre me l'a étél "This letter has been sent to the children/This letter it to them has been/This letter to me it has been."

12 This might explain why the predicate clitic le has the same form as the accusative clitic le under the assumption that CPs need Case. This would mean that the verb be may assign accusative.

13 The relative unacceptability of (30c) needs to be explained. I suggest it might be related to the marginal availability of restructuring the considerer clause with the previous one in French to allow the clitic to climb. The same marginality is perceived in (1) ?Jean en a longtemps été considéré fierl? Il Iui a longtemps été considéré fidèle/"John of it has long been considered capable/He to him has long been considered faithful," for the same reason.

14 See Sportiche (1990) and references therein for extensive support that (a) all French se clitics, whether reflexive, middle, neutral, or inherent, correspond to DP subjects and consequently (b) superficial subjects in reflexive constructions are underlying objects. This conclusion seems to extend to other Romance languages (see, e.g., Cortés (1992) for Catalan).

15 These aspects of Kayne's proposal are crucial to what follows, namely that either (a) C (and D) must incorporate to allow A-movement out of the participial clause, or (b) AGR incorporates to $C$ (and D) for the same reason. Thus, essentially, what follows would be consistent with there being no $\mathrm{T}$ and only one AGR projection within the participial clause.

16 Recall that strictly speaking the structure is well-formed if a well-formed "antecedent" for le can in principle be constructed. I will ignore this in order to simplify exposition.

17 Except when the reflexive "is" an indirect object in Standard French (e.g., Marie s'est offert $\left(*_{e}\right)$ un cadeau/Marie s'est parlé $\left(*_{e}\right)$ "Mary gave herself a present/Mary spoke to herself." The Standard Italian situation is different with participle agreement with indirect reflexives, but only in the absence of a direct object clitic (if there is such a clitic, the participle agrees with it).

18 Kayne suggests that se actually adjoins to AGR to make it strong. This is incompatible with our treatment of clitics. I assume the relevant effect is triggered by the pro that se stands for raising to [Spec, AGR P]. My skepticism concerning clitic placement as successive incorporation is also based on considerations similar to those discussed in Sportiche (1992) in connection with restructuring and agreement.

19 There is another undesirable feature of the analysis stating that se is an external argument, namely that we have to stipulate external. Under this new formulation, it might be possible to drop specific reference to $A G R_{s}$ and simply state that $s e$ is linked to AGR. I will not pursue this question here. 


\section{PARTITIONS AND ATOMS}

20 How to handle the passive case is less clear, given the well-formedness of passive participle predicate cliticization. Kayne suggests that, just like adjectives and unaccusatives, they lack the DP* projection because they all lack Tense interpretation.

21 Kayne (1975) actually attributed the first one to the impossibility of pronominalizing a raising complement, before the theory of small clauses multiplied the existence of raising structures.

22 The same result would hold for Standard Italian, but for the opposite reason, as sembrare takes essere and its participle agrees with its derived subject. At the same time, reflexivizing an indirect object (without cliticizing or passivizing it) also triggers participle agreement. In Italian, the structure is thus ruled out because both DPs compete for [Spec, AGR ]. This account predicts that (41) should be well-formed in a language unlike French but like Italian in selecting be with seem, and like French but unlike Italian in not having participle agreement with indirect object reflexive.

23 This is slightly different from Larson's proposal but preserves its essential features.

24 Judgments vary somewhat on the intermediate case. Chomsky (1986) gives them as unacceptable. There seems to be agreement, however, on the intermediate status.

25 Absent from consideration here are subject control and object control complement small clauses, which seem not to exist (nothing like Jobn persuaded/promised Bill (PRO sick) - this gap is discussed in Schein, 1995 - nor certain raising-tosubject complement small clauses such as e.g., John strikes me [as $t$ intelligent]).

26 For example, how exactly to derive the surface structure? What is a root clause? One problem, namely how to account for the fact that PRO can only be controlled by subjects and objects - cf. Williams' (1980) contrast He ate the meat $\mathrm{raw} /{ }^{*} \mathrm{He}$ ate at the meat raw - could be linked to objects and subjects having to raise high enough in the structure (if, say, the relevant $A G R_{0}$ is above sometimes in $(58 \mathrm{c})$ )

27 The syntactic structures also differ as the latter is more akin to right dislocation in correlating the clause forming its own intonational phase, unlike what happens in (59b).

\section{Acknowledgments}

Many thanks to Hilda Koopman and Jean Roger Vergnaud. A grant from the UCLA Academic Senate partially funding this research is gratefully acknowledged.

\section{References}

Abney, S. (1987) "The noun phrase is in its sentential aspect," unpublished Ph.D. dissertation, MIT.

Aoun, J. Benmamoun, E. and Sportiche, D. (1994) "Agreement, word order and conjunction in several varieties of Arabic," Linguistic Inquiry 25: 95-221.

Baker, M. (1988) Incorporation. Chicago, University of Chicago Press. 
FRENCH PREDICATE CLITICS AND CLAUSE STRUCTURE

Belletti, A. (1988) "The case of unaccusatives," Linguistic Inquiry 19: 1-34.

Bouchard, D. (1982) "On the content of empty categories," unpublished Ph.D dissertation. MIT.

Bowers, J. (1993) "The syntax of predication," Linguistic Inquiry 24: 591-656.

Burzio, L. (1986) Italian Syntax. Dordrecht: Reidel.

Cardinaletti, A. and Guasti, M.T. (1995) Small Clauses, Syntax and Seventies, vol. 28, New York: Academic Press.

Chomsky, N. (1977) "On Wh-movement," in P. Culicover, T. Wasow, and A. Akmajian (eds) Formal Syntax, New York: Academic Press. (1986) Barriers, Cambridge, Mass.: MIT Press.

- (1992) "A minimalist program for linguistic theory," in K. Hale and S.J. Keyser (eds) The View from Building 20: essays in linguistics in honor of Sylvain Bromberger, Cambridge, Mass.: MIT Press, 1-52.

Cortés, C. (1992) "Issues in Catatan Syntax," unpublished Ph.D. dissertation, UCLA.

Corver, N. (1990) "The syntax of left branch extraction," unpublished Ph.D. dissertation, Tilburg Catholic University.

_ (1993) "A note on subcomparatives," Linguistic Inquiry 24: 773-81.

Evers, A. (1975) "The transformational cycle in Dutch," unpublished Ph.D. dissertation, University of Utrecht.

Grimshaw, J. (1987) "Subdeletion," Linguistic Inquiry 18: 659-69.

Hale, K. and Keyser, J. (1991) "On argument structure and the lexical expression of syntactic relations," unpublished manuscript, MIT.

Huang, C.-T.J. (1993) "Reconstruction and structure of VP," Linguistic Inquiry 24: 103-38.

Kayne, R. (1972) "French relative QUE," Recherches Linguistiques 2. University of Paris 8 .

(1975). French Syntax. Cambridge, Mass.: MIT Press.

(1991) "Romance clitics, verb movement and PRO," Linguistic Inquiry 22: $647-86$.

_ (1993) "Toward a modular theory of auxiliary selection," Studia Linguistica 47 (1): 3-31.

- (1994) "The Antisymmetry of Syntax," Cambridge, Mass.: MIT Press.

Kayne, R. and Pollock, J.-Y. (1978) "Stylistic inversion, successive cyclicity and move NP in French," Linguistic Inquiry 9: 595-621.

Koopman, H. (1984) The Syntax of Verbs: from Verb Movement Rules in the Kru languages to Universal Grammar, Dordrecht: Foris.

- (1993) "The internal and external distribution of pronominal DPs," unpublished manuscript. UCLA.

_ 1994) "Licensing heads," in N. Hornstein and D. Lightfoot (eds) Verb movement, Cambridge: Cambridge University Press.

Koopman, H. and Sportiche, D. (1991) "The position of subjects," Lingua 85 (2/3): 211-57, reprinted in this volume as Chapter 2.

Larson, R. (1988) "On the double object construction," Linguistic Inquiry 19: 333-92.

Ritter, E. (1991) "Two functional categories in noun phrases: evidence from modern Hebrew," unpublished manuscript, University of Quebec at Montreal.

Rizzi, L. (1978) "A restructuring rule in Italian syntax," in S.J. Keyser (ed.) Recent Transformational Studies in European languages, Cambridge, Mass.: MIT Press. 
- (1991) "Proper head government and the definition of A-positions," Paper presented at GLOW Conference, Leiden, The Netherlands.

Ruwet, N. (1972) Théorie syntaxique et syntaxe du français, Paris: Le Seuil.

Schein, B. (1995) "Small Clause and Predictions," in A. Cardinaletti and M.T. Guasti (eds) Small Clauses, Syntax and Semantics, vol. 28, New York: Academic Press.

Sportiche, D. (1988) "A theory of floating quantifiers and its corollaries for constituent structure," Linguistic Inquiry 19(3): 425-49, reprinted in this volume as Chapter 1.

-... (1990) "Movement, agreement and case," unpublished manuscript, UCLA. reprinted as Chapter 3 in this volume.

__ (1992) "Clitic constructions," to appear in J. Rooryck and L. Zaring (eds) Pbrase structure and the lexicon, Dordrech: Kluwer.

- (1993) "Subject clitics in French and Romance, complex inversion and clitic doubling," reprinted as Chapter 5 in this volume. (1994) "Adjuncts and Adjunctions," unpublished manuscript, UCLA.

Starke, M. (1995) "On the format for small clauses," in A. Cardinaletti and M.T. Guasti (eds) Small Clauses, Syntax and Semantics, New York: Academic Press.

Stowell, T. (1981) "The origins of phrase structure," Ph.D. dissertation, MIT.

— (1991) "Small clause restructuring," in R. Freidin (ed.) Principles and Parameters in Comparative Grammar, Cambridge, Mass.: MIT Press, 182-218. (1993) "Syntax of tense," unpublished manuscript, UCLA.

Swart, H. de (1991) "Adverbs of quantification: a generalized quantifier approach," Ph.D. dissertation, Groningen State University.

Szabolcsi, A. (1987) "Functional categories in the noun phrase," in I. Kenesei (ed.) Approaches to Hungarian, vol. 2, Szeged: JATE, 167-90.

Travis, L. (1984) "Parameters and effects of word order variations," unpublished Ph.D. dissertation, MIT.

Valois, D. (1991) “The internal syntax of DP," unpublished Ph.D. dissertation, UCLA.

Williams, E. (1980) "Predication," Linguistic Inquiry 11: 203-38.

— (1983) “Against Small Clauses," Linguistic Inquiry 14: 287-308. 


\section{SKETCH OF A REDUCTIONIST APPROACH TO SYNTACTIC VARIATION AND DEPENDENCIES*}

\section{Some restrictive boundary conditions}

I want to explore a strongly universalist and reductionist view of syntactic theory that seeks to radically restrict the inventory of (a) variations between different languages and (b) variations among different processes. The general proposal is that (surface) syntactic structure is crosslinguistically invariant, principles are not parametrized, and variation is essentially confined to the pairing between morphophonological properties and semantico-syntactic properties of morphemes.

Syntactic theory has so developed in recent years that some fairly radical hypotheses can be entertained concerning crosslinguistic variation. In the principles and parameters approach (see Chomsky (1981)), languages are seen as sharing a common core grammar of available principles. With some of these are associated parameters whose value may vary along finite discrete scales from language to language. What aspects of grammatical systems can be parametrized in this way? Borer (1984) suggests that parametric differences between languages are found only in the lexicon. Given that a certain amount of lexical variation among languages is incontrovertibly found, the idea of limiting linguistic variation to the lexicon appears to be the strongest initial hypothesis. I will therefore adopt it. Lexical variation itself is not unlimited. Thus, Borer (1984) suggests that variation might be restricted to the inflectional properties of different formatives and the inventory of inflectional rules. In modernized terms we might take this to mean that variation is limited to the inventory and properties of functional projections. This proposal does not state exactly how inflectional properties may vary. If indeed syntactic representations are projected to a substantial extent from lexical properties (as the Projection Principle, in whatever guise, entails), lexical differences could entail the existence of important differences in the structure of syntactic representations as well. For example, if inflectional heads may vary in their selectional or subcategorizing properties, possible 
structural variation that ensues could be quite substantial. I would like to suggest a stronger possibility, namely, that neither the inventory of inflectional processes nor the functioning of inflectional processes may vary. In terms of functional categories this means that languages differ neither in the functional categories they use in a given syntactic context nor in the inflectional properties correlated with the presence of a particular functional category. More generally, I would like to suggest that syntactic structures are crosslinguistically invariant.

Functional heads either instantiate grammatical properties (e.g., Case, agreement, subordinating functions (complementizers), etc.) or realize interpretive properties (Tense, clause typing, clause polarity, focus. definiteness). Clearly, languages differ in the way they exhibit Case, agreement, or Tense, if at all. Restricting variation to functional projections is thus quite natural. However, although their audible correlates vary, the properties expressed by functional heads that have a grammatical function are not obviously absent in any language for either normal or semantic reasons. For example, if (structural) Case is a necessary property of certain DPs, and if structural Case is assigned in [Spec, AgrP] (Chomsky (1991), Mahajan (1990), Sportiche (1990)), all languages will have to have Case and Agr even if they are not overtly realized. Postulating variations for functional heads expressing interpretive properties is dubious, as it appears plausible (are there languages without yes/no questions or $w h$-questions, without definiteness, or without negation?), the set of interpretive functions that have to be expressed and the conditions under which they are expressed are universal. Furthermore, every serious grammatical theory reasonably assumes the existence of phonologically or phonetically unrealized syntactic or interpretive properties, e.g., a non-past tense morpheme in English. This simple observation raises a very general problem concerning the availability of silent morphemes and in particular of silent functional heads (that may be equivalently viewed as features of particular heads) and more generally of silent categories. Because we know that they are not necessarily phonologically realized, their observed absence is not an indication of their structural absence.

To begin to accommodate the crosslinguistic or crossconstructional observed variations in this area, I will resort to a type of lexical difference that is incontrovertibly available, namely, the pairing between a signifie and a signifuant ('arbitraire du signe), that is, the pairing between morphophonological properties of a given item and its syntactic and semantic properties. Clearly, languages differ as to how the same head is pronounced. As an initial assumption, I want to suggest that this is, apart from lexical vocabulary differences (e.g., lack of a French word for shallow or the systematic absence of, say, adjectives in a given language) ${ }^{1}$ the only type of difference found in the lexicon. Thus, the sound associated with a particular referent or property varies. This is, of course, a substantial window of variation. However, if it is plausible that there are no syntactically relevant variations with respect 
to lexical categories, the only type of parameter will concern morphophonological properties of functional heads and in particular how functional heads may be realized.

There are two fundamental types of effects arising from the morphophonological space of variation, which I will address in turn.

\subsection{Affixation and conflation}

\subsubsection{Affixation}

Let us begin with the idea that the mapping from words or morphemes to syntactic heads can be one-to-many. Consider first surface structure words. A surface word or morpheme does not necessarily correspond to an atomic property. For example, neither of the words derives or derivation is atomic. However, they are usually treated differently depending on the version of the Lexicalist Hypothesis adopted. Derivation is usually considered to enter syntax as one nominal unit without further internal structure, as it is assumed that its internal structure has no bearing on and is not dependent on syntax. The internal structure of the third person singular verb derives is syntax dependent (on what the subject of its clause is and whether its distance to Tense is short enough). This means that, fundamentally, it is not atomic from the point of view of syntax. There are a variety of ways to handle this observation. One that has been dominant in recent years is to suppose that derives is syntactically two morphemes derive $+s$, each heading a different projection ( $\mathrm{V}$ and $\mathrm{Agr}_{\mathrm{S}}$ ) concatenated by a syntactic rule (of head movement relating $\mathrm{V}$ and $A g r_{\mathrm{S}}$ ). In this account some stipulation has to be introduced to the effect that the head of $\mathrm{Agr}_{\mathrm{S}}$ is both overt and affixal in English. This description of the realization of Agr, or more generally of functional heads, is not crosslinguistically stable. Thus English $\mathrm{Agr}_{\mathrm{O}}$ is always silent, whereas French $\mathrm{Agr}_{\mathrm{O}}$ may be overt. In English the future of the present morpheme may be an independent word (will), while in French it is a bound morpheme (-er-). The French future of the present is a morphologically affixed bound morpheme, while in English it may be a clitic (' $l l)$. Finally, the French preverbal definite third person clitic pronoun is a phonological clitic, while in Trentino Italian it is a syntactic clitic (see Sportiche (1993a)). More generally, we find the following kind of variation:

(1) Functional heads may be realized as silent (covert) or as

overt

if overt as independent words

or as bound morphemes

if as bound morphemes

as morphological affixes (with or

without segmental content) 


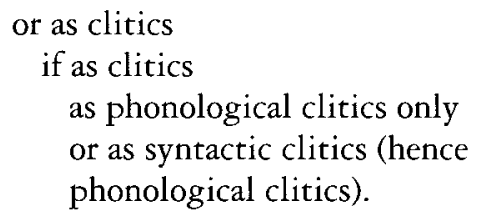

To take a clear case, the correspondence between the French word mangeront (will eat-3rdPL) and correlated syntactic heads is one to many (in fact, here, one to three: $\mathrm{Agr}_{\mathrm{S}}$, Tense, and V). However, there is a sense in which it is not a one-to-three mapping, as there are three clearly identifiable morphemes mang +er +ont, each corresponding to, and very possibly syntactically generated as, one head. Notice that the English case eats, eat (eat + Present +3 rdSG, eat + present non3rdSG) can be treated in similar way if, as is standard, appeal is made to silent morphemes, here non-past.

\subsubsection{Conflation}

Some cases of one-to-many correspondences cannot be handled in this fashion. Consider the English form ate. Here the word ate contains two morphemes in a sense (morphosyntactically: $\mathrm{V}+$ past) but is atomic in another (phonologically), and presumably this irregular spellout of the concatenation of two morphemes is stored in the lexicon. Suppose, thinking in derivational terms, ${ }^{2}$ that morphosyntactically complex inflectional forms of this sort are always formed by head movement. This forces the existence of lowering rules in syntax. In English, there is good evidence that a main verb inflected for Tense, say past, does not raise to $T$ in the syntax (since it follows all VP initial material). Allomorph checking will require concatenation of past and V, that is, lowering or affix hopping of $\mathrm{T}$ to $\mathrm{V}$. This raises problems because (a) lowering is extremely restricted (it seems to occur only with affixes): unlike raising, it does not occur with phrasal movement; and (b) lowering of an affix $\mathrm{A}$ to a head $\mathrm{H}$ is possible iff raising of $\mathrm{H}$ to $\mathrm{A}$ is possible in principle. One solution adopted in Chomsky (1991) to explain the second property is to require that, in case $A$ overtly lowers to $H, L F$ raising of $H+A$ to $A$ takes place in order to erase the effects of $\mathrm{A}$ to $\mathrm{H}$ lowering. Besides the unnaturalness of this proposal (why lower to raise later?), this says nothing of the first problem (why can DPs not lower overtly and taise back at LF?).

Chomsky (1993) proposes a simpler and more principled approach to this problem that explains both (a) and (b). He suggests dissociating the concatenation process itself from the process of checking the form and ordering of morphemes composing a complex word. According to this view call it the checking approach ${ }^{3}-a$ verb is drawn from the lexicon fully inflected, say $[[\mathrm{V}+\mathrm{T}]+\mathrm{AgrS}]$. This verbal complex must raise by $\mathrm{LF}$ to $\mathrm{T}$ and AgrS in order to check (or cancel out) the properties of the inflectional affixes, Tense and agreement features of the verb. One immediate advantage of this 
approach is that it does away with lowering entirely but keeps the idea that head movement is involved. Second, the treatment of suppletion, that is, of forms such as eat + past = ate, becomes identical from the point of view of the syntax to that of non-suppletive forms like $+e d=l i k e d$. Finally, it explains why lowering occurs only with affixes, that is, heads. It will occur only when a word made of several morphemes is involved.

This proposal does not eliminate incorporation as a syntactic process of concatenation. Incorporation could be the result of either syntactic movement or the morpholexical process subject to syntactic checking just discussed. In the first case incorporation takes place in the syntax proper as a result of overt movement, as is the case of, say, preverbal pronominal cliticization in Romance languages. Call it "syntactic incorporation" (SI). Because the concatenation of morphemes is the result of the application of head movement, we expect (a) that it will always involve upward movement, never lowering, and (b) that the properties of the compound will be strictly compositionally computed (since input from the lexicon is unavailable). When concatenation is not syntactic, call it "morpholexical incorporation" (Ml). ${ }^{4}$ Because the concatenation of morphemes is a lexical operation, we expect to find (a) apparent cases of lowering (since a word may be generated with an affix whose licensing position is higher in the tree) and (b) non-compositionality of the concatenation (lexical exception, suppletion, etc., or meaning idiosyncrasies, as in the case of derived nominals discussed in Chomsky (1970)). Both phonological and semantico-syntactic information about lexical items is stored in the lexicon. If the lexicon interfaces with the rest of the grammar at only one point, phonological features would be inserted at the same time as others: in this case phonological allomorphy and suppletion are indicative of MI. Alternatively, phonological properties of lexical items are not present at all in syntax and are accessed by morphophonological rules that map syntactic representations onto phonological representations (that is, the "phonological lexicon" interfaces with postsyntactic representations input to morphophonology only, unlike the "syntactic/semantic lexicon"). This has some conceptual and other advantages, which I will not discuss here, and the drawback that access to the lexicon occurs twice. If this view is correct, morphophonological suppletion has no bearing on whether MI or SI is involved in concatenation (unlike what is assumed in Sportiche (1993a)).

Cases of morpholexical incorporation or "conflation" are cases of one-tomany mappings from words to syntactic heads. I believe recent work shows this phenomenon to be extremely pervasive, both when functional categories are involved and when lexical categories are involved (thus considerably reducing the actual syntactic variation observed for lexical categories).

Extremely general instances of morpholexical incorporation for lexical categories are illustrated by (an interpretation of) the recent work of Hale and Keyser (1991) on lexical decomposition of verbs and other predicators. They demonstrate that an illuminating account of the existence of systematic gaps 
in the set of available verbs can be given by assuming that verbs have a sort of internal syntactic structure subject to syntactic well-formedness conditions (e.g., the ECP). For example, they postulate incorporation of the head of internal arguments in a great variety of cases (very roughly: the verb dance has the VP-internal structure of do a dance, with the noun dance incorporating into the verb $d o$; similarly, put the book, on shelf $\rightarrow$ shelve the book). I read them as assuming that these processes take place in the lexicon (their L-syntax level). However, because they are subject to constraints defining syntactic processes, operative in syntax, they should be viewed as belonging to syntax proper (thus explaining why syntactic constraints are relevant). In the perspective of Chomsky's checking approach we can analyze a $\mathrm{V}+\mathrm{N}$ category such as [dance] as generated syntactically in the $\mathrm{V}$ slot licensing its $\mathrm{V}$ part with its $\mathrm{N}$ part incorporated (whether by MI or SI) in it. ${ }^{5}$

We can modify slightly Larson's (1988) VP shell proposal so that it can be looked at from the same point of view. It is because kill is the "lexical" concatenation of CAUSE and DIE that kill projects two VPs, one with the agent as specifier and the other with the patient as specifier. Under this modification the lower VP has the same internal structure as that projected by DIE and the higher one as that projected by CAUSE. ${ }^{6}$ In effect, this is a contemporary version of lexical decomposition analyses. ${ }^{7}$ However, because there is a lexical component to it - the verb kill=cause-to-die is formed in the lexicon and is listed as such in the lexicon - it is immune to the arguments leveled against lexical decomposition, as nothing prevents these conflated words from displaying idiosyncratic properties beyond their basic "decomposed" meaning (indicating in the present instance that $\mathrm{MI}$ is involved). ${ }^{8}$

Other examples are found in the work of Kayne (1993), which justifies the derivation of the verb bave from $b e+F$, that is, from the verb be incorporating a category $\mathrm{F}$ (which Kayne takes to be a $\mathrm{P}$ or a $\mathrm{D}$ - another illustration involving functional categories), or in the work of Bhatt and Yoon (1991), which we discuss below.

Since conflation exists and is available in principle, we must inquire, any time we find linguistic variation, whether the observed variation is not reducible to conflation being used in one case but not in another. This is especially significant where variation is most obvious, the crosslinguistic distribution of functional properties, which involves closed classes.

\subsection{Universal constituent structure and recoverability}

Perhaps only because of the way in which functional properties differ crosslinguistically in their realization (or lack thereof), one central question concerns variability in the way functional heads are syntactically mapped. Essentially, because of the way we construe affixation and conflation and the possibility of invoking the existence of silent morphemes (as English present tense), it seems to me plausible to assume the most restrictive position from 
the point of view of syntax, namely, that languages simply differ neither in the stock of functional heads that they have, nor in the principles that govern their appearance in structure. Functional heads being associated with lexical categories, ${ }^{9}$ I will assume that every lexical category is uniquely and invariantly associated with a set of functional projections, all of which are always projected with the same hierarchical organization. ${ }^{10}$ In effect this is saying that from the point of view of syntactic structure, there is only one language, that is, that syntactic structure is invariant. The price to pay for syntactic invariance is analytic abstractness. Some of it is inevitable but I would like to limit it to a certain extent by requiring a degree of overtness.

The choice among the various modes of realization listed in (1) seems arbitrary from the point of view of synchronic grammars except for the overt/ covert distinction. It seems reasonable to require that the presence of a given property be somehow "recoverable." Let us distinguish between "necessary" and "contingent" properties of clauses and other constituents and between predictable and arbitrary properties of heads. Necessary properties need not be overt; their existence is required. Such are $\mathrm{Agr}_{\mathrm{O}}, \mathrm{Agr}_{\mathrm{S}}$, nominative, T, and so on. Among contingent properties of clauses, predictable properties of heads may be left covert. Thus, knowing what the verb of a clause is, we may infer how many arguments there are and their categories. These arguments may thus stay covert if there is some universal convention allowing the recovery of their content. There seem to be such principles. The content of a covert category may be recovered through some antecedent; or antecedentless covert DPs must be pronouns - pro. There seems to be no such general predictability for other lexical categories. As they by definition have idiosyncratic, hence non-recoverable, properties, they must be overt except, of course, when they have antecedents (e.g., VP deletion, gapping).

Among contingent properties of clauses we also find properties expressed by functional heads. Take clause type (the statement/question distinction) or polarity (the affirmation/negation distinction), for example. The clause type information must be recoverable. A clause is not necessarily a question or necessarily a non-question, but it is necessarily one or the other. This suggests that it is not the presence of the functional category that is contingent, but rather the value that its head assumes. A plausible construal of this recoverability requirement is that these values must in a given paradigm, say, of clause type, all be overt save one ${ }^{11}$ (thus, non-question clause typing may be left covert but questions must be overt; affirmation may be covert but negation must be overt, and so on). Whether this is tenable is unclear. I assume for the moment that it is. Making explicit this discussion, assume (2):

Recoverability Principle

Optional properties of heads must be recoverable.

Let us understand it as follows. If a head (or some property of this head) is 
present in a particular location in which its occurrence is paradigmatically optional, there must be a way to recover its presence. We will try to specify how this is possible as we go along, introducing modifications as we proceed with particular case studies.

It is possible that we also find truly contingent properties expressed by functional heads (although it is not completely obvious that they exist). Thus, we may argue that Focus is not necessarily present (some sentences may lack a focused constituent). If so, Focus (and other such properties), when present, must be overtly indicated

\subsection{Movement}

Superficially, languages do look different in ways other than the type of realization heads may have, that is, abstracting away from the affixal or the conflated nature of heads. Ordering is the most visible case as such. We need to provide other plausible sources for the observed differences. A partial answer compatible with what was said so far is inherent in (1). The relative ordering of a functional or a lexical head with respect to other material in a clause will be affected by whether it is overt, and in the former case whether the head is a syntactically bound morpheme. If it is, it might precede material that an independent head would follow. For example, the respective order of a head and one of its arguments could change as a result of the head appearing before its arguments, instead of after, because as a syntactically bound morpheme it must appear incorporated in another - possibly covert - head to the left of the argument (see, for example, the alternation an interesting one/someone interesting).

Ordering differences are not limited to alternation between a head and some other material. We also find such alternations between phrases. Combining ideas of Chomsky (1993) and Kayne (1994), we can reduce this type of variation to the first one, that is, to properties of heads.

Examining the properties of head-initial/head-final languages, Kayne (1994) notes that the expected mirror image distribution of properties in head-initial and head-final languages is not found. Instead, bias toward initial headedness is found. He proposes to account for this asymmetry by postulating that all languages are essentially head-initial and that the appearance of final headedness is given by post-head arguments of a head moving around this head (that is, to its left) overtly. This position is more restrictive than the more usual head initial/head final parameter, and I will adopt it.

Dependencies between two positions exhibiting movement properties are not always realized as overt movement, as the literature on LF Wh Movement illustrates. A reasonable account of this observation postulates that nonovert movement dependencies between two positions showing movement properties are cases of covert movement as suggested, for example, in the case of $W b$ Movement in Aoun, Hornstein and Sportiche (1981), or Huang (1982). 
We may assume then that different constructions in the same language or the same construction in different languages can differ in whether they involve covert movement instead of overt movement. Kayne's proposal concerning the head-initial/head-final alternations can be straightforwardly integrated with Chomsky's (1993) proposals by construing head final languages, namely, languages moving (some) arguments around to the left of their heads, as languages doing overt rather than covert movement.

There is an alternative approach to the question of covert movement that simply denies its existence while maintaining that movement is involved by postulating that "covert movement" constructions actually involve overt movement of a covert element, as the work of Aoun and Li (1993), Watanabe (1992), and Cheng (1992) points out in the case of $\mathrm{Wb}$ Movement. This approach looks quite plausible in such cases. If it could be extended quite generally, crosslinguistic variation based on overt versus covert movement could be entirely eliminated, obviously a desirable move if the overt movement/covert movement distinction was reduced to some independently necessary property. ${ }^{12}$ Although I believe there is some advantage to an approach eliminating covert movement, I will keep to the familiar overt/ covert assumption, making occasional remarks on the alternative.

The remaining question asks what exactly differentiates overt movement constructions from covert movement constructions. In the cases he looks at, Chomsky, pursuing his minimalist ideas, suggests that overt movement constructions involve overt movement because some phonological property of some head must be licensed. He calls this diacritic property of heads "strong" and the lack thereof "weak." If indeed this could be maintained (and it is surely conceptually desirable - movement must feed the phonology because some phonological property is involved - even though it is most unclear how to do it precisely), it would reduce the overt movement/covert movement distinction to the phonological property of some head, that is, it would fall within the range of parametric properties listed in (1).

\subsection{Summary}

In sum the general picture that emerges is in effect a generalized version of the Universal Base Hypothesis in which there are no crosslinguistic differences in the syntactic structures of the various levels of syntactic representations. A given ordered set of properties exhaustively instantiated as a string of (possibly covert, possibly affix-like, possibly conflated) heads in any language is associated with a unique syntactic structure. Observed variations arise either (a) because of morphophonological properties of the string of heads involved as in (1), conflation, or (b) because some movement dependency involved is instantiated overtly instead of covertly - quite possibly a subcase of (a). This set of constraints may appear too restrictive to handle the observed crossconstructional or crosslinguistic variations. The opposite is probably 
closer to the truth. It is easy to realize that an extremely large number of (but not every possible) cases of ordering and concatenation can be generated, given a universal (and possibly invariant) clausal structure augmented with the possibility of leftward movement of phrases and of heads.

Movement plays a prominent role in such a view. I will argue that this role extends to more cases than is customarily acknowledged but at the same time that possible types of movement are radically restricted. More specifically, I will propose that (almost) all syntactic dependencies should be analyzed the same way, say, as movement dependencies: $:^{13}$

(3)(a) There is only one type of non-local syntactic dependency.

(b) There is only one type of local syntactic dependency.

In the rest of this article I will explore ways to substantiate these proposals. Essentially, I will explore the possibility that the only non-local syntactic dependency is movement to specifier of some designated projection and that the only local dependency is incorporation.

\section{Types of syntactic dependencies}

\subsection{A preliminary inventory of syntactic dependencies}

The following list illustrates the variety of syntactic dependencies.

1 Movement (landing site, trace): the moved item may be a head or a phrase and must command its trace.

2 C-selection or subcategorization: a lexical category imposes categorial identity on some phrase that it commands.

3 S-selection: a word-level category imposes a particular property on some head that it commands. ${ }^{14}$

4 Anaphor/pronominal binding (antecedent, anaphor/pronoun): the antecedent must command the anaphor/pronoun.

5 Scope (scope "position," quantifier): the scope position of a quantifier must command the quantifier.

6 Clitics (clitic, argument position): the clitic must command the argument position it stands for.

7 Polarity items (polarity licenser, polarity item): the licenser must command the polarity item.

8 Quantifier split constructions (English only, even or French beaucoup): the quantifier modifies another constituent in its command domain. 
Recent work extends this inventory to include:

9 Case for DPs (Case position, theta position): the Case position commands the theta position. ${ }^{15}$

10 Agreement processes in general (Koopman (1992), Kayne (1989)) are construed as relations between a head and its phrasal specifier.

11 Number for DP (number determination, NP): the locus of number commands the NP position that has this number (see Ritter (1991), Valois (1991), Koopman (1993b)).

How many primitives are needed to describe these relations? If this sample is significant, it suggests that this set is quite narrowly constrained. Putting agreement processes aside, which I analyze as instances of specifier/head relationships, any of these dependencies D obeys two properties: ${ }^{16}$

(4)(a) D is a binary relation $D(x, y)$.

(b) One of $\{x, y\}$ must command the other.

where (4b) is stated in terms of some unique appropriate primitive of "command," which we will take to be i-command, roughly defined in (5): ${ }^{17}$

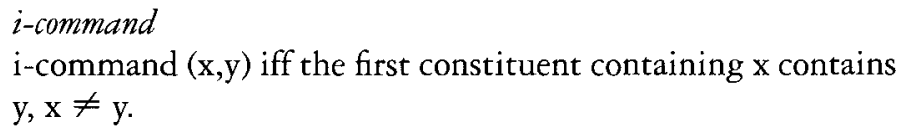

These two properties are neither syntactically nor semantically necessary. It is easy to manufacture a language, syntax and semantics, that would use different structural requirements for each of these dependencies. If this uniform characterization is correct, this uniformity needs to be explained. One way of explaining it, and the one that I will pursue, is that there is a central uniformity to the way in which the syntax of these dependencies ought to be construed. I want to pursue a line of explanation that at the most abstract level of analysis simply reduces all these relations to the same one. This approach is inspired by that adopted by Chomsky (1977). There he suggested that, if some constructions had a sufficient number of common properties, they should be analyzed as involving the same process ( $W h$ Movement in this instance). Carrying this further, I propose that if constructions have any property in common, they should, at the proper level of analysis, be analyzed as identical. Given that movement is essentially a binary relation and that movement is always to an (i-)commanding position, I will suggest that all these relations are cases of movement.

Naturally, these movement relations are not identical. The varying locality conditions constraining these relations illustrate one prominent difference. 
But this does not affect their being movement processes, as the difference between $A$-movement and $A^{\prime}$ movement demonstrates. Some differences, however, do seem to bear on the question to how to treat these dependencies. Consider again the examples of dependencies listed above, still leaving agreement aside. Each is of the type $\mathrm{D}(x, y)$, where $x$ (i-) commands $y$. This set can be subdivided into three subsets according to the categorial nature of $x$ and $y . X$ can be a phrase, as in phrasal movement, scope (Wh Movement), antecedent anaphor, or polarity licensing (none of them gives a damn). Or it can be a head, as with clitics (see Sportiche (1992; 1993a) and references therein for extensive justification), polarity licensing (be denies giving a damn), or scope (il ne regardera personne). Similarly, $y$ can be a phrase (XP movement relation, subcategorization) or a head (head movement).

Thus we find homogeneous relations (head/head) or (phrase/phrase) and heterogeneous relations (head/phrase). We consider each type in turn.

\subsection{On the domains of syntactic dependencies}

Postulating that all these syntactic dependencies are cases of movement derives both that they are binary relations and that they involve a command requirement. Some finer distinctions appear necessary, as we discuss now, since different dependencies take different sets as domains and exhibit systematically different properties.

\subsubsection{Homogeneous head/head relations}

Head/head relations are primarily exemplified by head movement constructions such as V to I (in French), I to C (in Germanic), P to V (in English reanalysis, Dutch, or Bantu), and V to $\mathrm{V}$ (in Romance restructuring or more generally "clause union" constructions). These relations are extremely local, a locality reducible to antecedent government imposed by the ECP ${ }^{18}$ (see Koopman (1984)) and usually described in terms of Travis's (1984) Head Movement Constraint (HMC), which requires that the trace of a head be $\mathrm{i}$-commanded by an antecedent without any barrier intervening, that is, that the trace of a head be governed by an antecedent:

Head Movement Constraint

The trace of a head must be governed by an antecedent of this head.

Head/head relations are also exemplified by s-selection (linked to theta assignment). Thus, a verb may require of the lexical head of one of its arguments that it be [+ animate] or [+ concrete]. S-selection as well is an extremely local relation (see Chomsky's (1965) strict locality). Apart from s-selection of its external argument by some predicate - to which we will return later - this 
locality condition requires that an s-selector i-command a head that it selects without any barrier intervening between them, that is, the s-selector must head govern its selectee:

\section{S-Selection}

S-Selection requires head government.

\subsubsection{Homogeneous phraselphrase relations}

$\mathrm{XP} / \mathrm{XP}$ relations comprise a variety of different relations allowing apparently less local dependencies. NP-raising as in John, seems to be likely to have been seen $t_{i}$, Wh Movement as in who do you think Mary saw $t_{i}$ or polarity item licensing as in Nobody demands that you do anything, illustrate the apparent unboundedness of these relations. Even though the movement dependencies are analyzed as a succession of small local steps, it is a kind of derivation that is not allowed for head movement. A head cannot move successively cyclically; at each step it incorporates into the next head up (which may be silent) and the combination may move. In another words, a particular head may only move once: ${ }^{19}$

$$
\text { (8) U } \quad\left[_ { W P } \left[[ _ { W } W + X ] \left[\left[_{X P}\left[{ }_{X} e\right]\right]\right.\right.\right.
$$

Once $\mathrm{X}$ has incorporated into $\mathrm{W}$, only $\mathrm{W}$ itself (excorporation) or $\mathrm{W}+\mathrm{X}$ can move to the higher head $U$.

\subsubsection{Heterogeneous $X / X P$ relations}

Heterogeneous relations split into two subsets. The first subset comprises the head complement relation such as is exemplified by c-selection. These relations, just like the head/head relations, are extremely local and the locality restriction appears to be the same, namely, head government: a head may c-select a phrase if it $\mathbf{i}$-commands it and no barrier intervenes.

The second subset comprises exactly the same relations as are found in $\mathrm{XP} / \mathrm{XP}$ relations - LF $W h$ Movement (dependency between a $[+\mathrm{Q}]$ particle and a $w$ h-phrase), polarity item licensing (e.g., deny ... anything), expression of scope (e.g., ne ... personne - compare with expression of scope in terms of movement, that is, in terms of an XP/XP relation) - and exhibit fundamentally the same properties (for example, the locality of polarity item licensing is independent of whether the licenser is a head or a phrase).

This raises several questions. First, is it accidental that binary relations are both heterogeneous in terms of their domain (a pair head/phrase) and heterogeneous as a set in terms of their properties? Second, why should some head/phrase relations exhibit a behavior similar to that of head/head relations, and other head/phrase relations exhibit a behavior similar to that of phrase/ 
phrase relations? Finally, why do phrase/phrase relations systematically appear to be expressible in terms of head/phrase relations (descriptively, these relations are the same: $w$-structures, polarity licensing, scope assignment)? This immediately suggests that some head/phrase relations are covert head/ head relations, and the other head/phrase relations are covert phrase/phrase relations. This is the line I am going to pursue.

\section{Unifying head/head relations}

\subsection{Generalized incorporation}

Let us begin with head/head relations. It is easy to reduce strictly local head/phrase relations to cases of head/head relations. Take c-selection, for example. It suffices to construe $c$-selection not as a relation between a head $\mathrm{X}$ and a phrasal category $P$, but rather as a relation between $X$ and the head of $P$. More generally, it suffices to construe the head complement relation not as a relation between a head $\mathrm{H}$ and a phrase $\mathrm{P}$, but between a head $\mathrm{H}$ and the head. Given the assumption that every category strictly conforms to the $\mathrm{X}$ ' schema, there are exactly as many heads as there are phrases.

But this still leaves one question unanswered. Why is strict locality expressed in terms of either the Head Movement Constraint or head government? If indeed we are dealing with some unitary phenomena involving both locality (no intervening barrier) and also a hierarchical requirement (i-command), there should be a unitary account underlying head government and the HMC.

Expanding on Koopman's (1993a) proposal, which considerably extends the scope of incorporation rules and discusses it in much more detail, we may derive a unitary account by eliminating head government altogether and replacing it by the Head Movement Constraint ${ }^{20}$ This raises no direct problem as the configurations of head government are identical to the configurations of antecedent government by a head, apart from the involvement of movement. In order to achieve this result I suggest that all cases of head/head dependencies are in fact cases of movement.

Consider first cases of s-selection or c-selection by some head $\mathrm{H}$. Let us adopt Stowell's (1981) idea of theta grid, augmenting it to include a notion of categorial grid in the following way:

A head $\mathrm{H}$ will c-select an XP by imposing the categorial feature $[+x]$ and s-select its head by imposing some property $[+p]$ iff the lexical representation of $\mathrm{H}$ contains a slot marked $[+\mathrm{x},+\mathrm{p}]$ that must literally be filled by movement of some $[+\mathrm{p}]$ head $\mathrm{X}$ into it by $\mathrm{LF}$.

For example, the verb witness takes as internal argument an NP (c-selection), whose denotation can be construed as an "event" (s-selection); call this property $[+$ event $]{ }^{21}$ These requirements will be instantiated as follows: 


$$
\left[\mathrm{V} \text { witness }\left[_{\left[+N \cdot-V_{-}+\text {event } t\right.}\right]\right] \quad\left[_{D P} D\left[_{N P} N\right]\right]
$$

Lexical encoding of this sort can be further elaborated. For example, the Theta Criterion can be reformulated as stating that (a) unincorporated (argument) heads at LF are deviant; this would replace "every argument must have a theta role," and (b) a head with unfilled slots at LF will be interpreted as an unsaturated predicate (and default rules might apply to fill this slot); this would replace "every theta role is assigned to one argument." Note that we do not need a uniqueness requirement - replacing "one" by "one and only one" because of the impossibility of moving two items into the same position or that of heads moving twice.

Elaborating still further the internal structure of the lexical representation of the verb, we may encode the hierarchy of arguments that predicates take (external, internal ${ }_{1}$, internal ${ }_{2} \ldots$ ) and enforce a particular hierarchy of syntactic realization of these arguments (we need to specify further principles by which these lexical slots can be filled). To give a concrete example, consider the transitive verb pour. Assume it imposes the complex of s- and c-selection $[+\mathrm{p}]$ on its external argument and the complex $[+q]$ on its internal argument. Then it will have the following lexical representation: ${ }^{22}$

$$
\begin{aligned}
& \operatorname{pour}\left({ }_{-}\right)=P(\underset{\mid}{(, Q} \underset{\mid}{(-)}) \\
& {[+p \quad[+q]}
\end{aligned}
$$

and enter in the following syntactic representation (modified from Larson's (1988) proposal): ${ }^{23}$

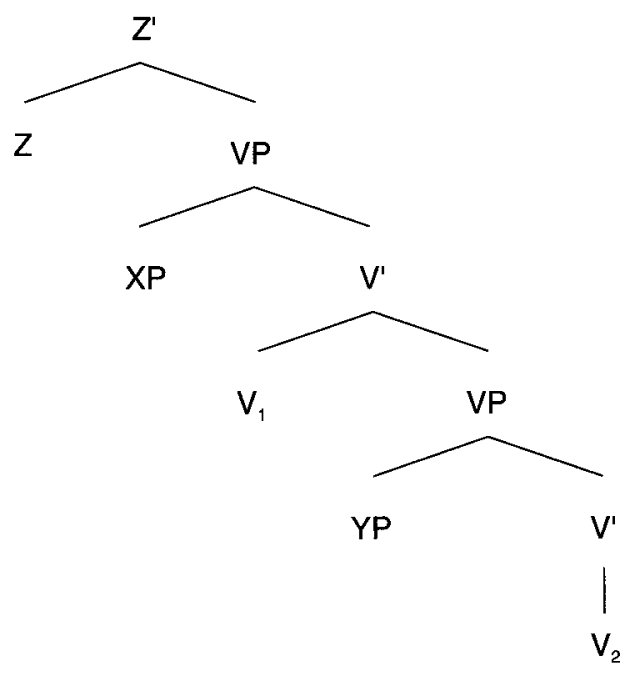


This representation will be well formed if $P$ contains a $[+q]$ head that can (and then must) incorporate into the $[+\mathrm{q}]$ slot of the $\mathrm{V}$ and XP contains a $[+\mathrm{p}]$ head that can (and then must) incorporate into the $[+\mathrm{p}]$ slot of the $\mathrm{V}$. Given that incorporation is movement, hence upward only, it means that the verb must move from $V_{2}$ to $V_{1}$ to allow the first incorporation and then from $V_{2}$ to some higher head $Z$ to allow for the second incorporation, namely, that of the external argument. ${ }^{24}$

One important result emerging from Baker's (1988) work on incorporation was that agglutinative languages could be reduced to underlying analytic languages by invoking analytic underlying structures and attriburing the observed compounding to a syntactic process of incorporation. The difference between agglutinative languages and analytic languages can be reevaluated, given the central role processes of incorporation are postulated to play in general, in particular, to license selection, for it makes all languages uniformly agglutinative at LF.

\subsection{Some cases and some problems}

\subsubsection{C-selection/incorporation/s-selection asymmetries}

If c-selection and s-selection are cases of incorporation, we expect all three to behave in the same way. In some areas they apparently do not. Although all arguments of a predicate are by definition s-selected by it, the external argument is neither c-selected by its predicate (see Chomsky (1965), Carter (1976)), nor can it incorporate into it (Baker (1988)). I have no explanation to offer for these facts (if indeed they are facts), nor do I know of any, however these various notions are construed.

In the case of c-selection it is easy to stipulate the answer in any number of ways. The usual way has been to require that c-selection is only of $\mathrm{i}$-commanded or sister material at D-structure. Such a description can also be stated on lexical representations in the present approach (the external argument slot is not categorially specified).

The case of incorporation is trickier, and I have only speculations to offer. Incorporation of the external argument is required, we claim, by LF. Baker (1988) and more recently Hale and Keyser (1991) contend that overt incorporation of an external argument is impossible (although some care is needed, viz. subject pronoun incorporation in VSO languages). Although this impossibility is typically attributed to a lack of antecedent government because the incorporee is higher than the verb in which it incorporates, it is in fact unclear how exactly this is supposed to work. This is because the relevant asymmetries are not present at LF, sometimes not even at S-Structure as, for example, in cases in which the verb raises higher than the subject. Possibly, if indeed the generalization is correct, external arguments are not lexical arguments of the predicate at all, that is, they would be neither 
c- nor s-selected. Their licensing then would have to be done differently (e.g., Spec/head).

I leave this as unresolved, simply making the following programmatic remarks. First, there is no evidence that external arguments do not incorporate at LF. I am led to postulate that they do, although I need to explain why they do not do it overtly. Notice that, given the decompositional analysis of non-monadic predicates (e.g., kill) implicit in Larson's (1988) proposal and reanalyzed here as involving a conflation of several monadic predicates (cause to die) - in fact several clauses - the notion that external arguments cannot incorporate appears highly dubious since the direct object of kill is indeed the external argument of die and may, as direct object of kill, in principle incorporate to kill. The problem then becomes why the highest argument of a $\mathrm{V}$ (whether conflated or not) cannot incorporate to V overtly. An obvious difference is the following: for incorporation of some argument into this $\mathrm{V}$ to take place, the verb must raise to a position higher than this argument (to guarantee i-command). Consider the structure in (11): the nominal head of YP may incorporate into $V$ if $V_{1}$ raises to $V_{2}$ and this is done without category change ( $V$ to $V$ raising). In fact, with conflated verbs it is arguable that it is the same $\mathrm{V}$. To incorporate the nominal head of XP, however, this raising must be by definition to a non- $\mathrm{V}$ category $\mathrm{F}$ (presumably a functional category). We may argue that the target slot within $\mathrm{V}$ is no longer available given the derived internal structure $\left[{ }_{F} \mathrm{~F}+\mathrm{V}\right] .{ }^{25}$ At LF, however, categorial distinction may be irrelevant and $\left[_{\mathrm{F}} \mathrm{F}+\mathrm{V}\right]$ is indistinguishable from $\left[_{\mathrm{V}} \mathrm{V}+\mathrm{V}\right]$, allowing the required incorporation.

\subsubsection{Postposed or preposed arguments}

If internal arguments must incorporate to their selector at LF, what happens when these arguments appear moved leftward or rightward? Some head part of this argument (the head noun for DPs) must incorporate, we claim, into its selector. How can this be reconciled with the overt movement that arguments may undergo to positions not governed by the relevant selector? The usual reconstruction option is plausible but not general enough. It would work for movement to an $\mathrm{A}^{\prime}$-position (left or right) but not for cases of A-movement, such as raising to subject, as A-movement does not display the typical binding effects associated with reconstruction. Chomsky (1993) offers a construal of movement rules as a copying process (plus PF deletion) - in effect an enriched version of Burzio's (1986) "layered trace" proposal - that provides a way to reconcile movement of a phrase with incorporation of a subpart of it into its selecting predicate. Traces are full copies of the moved phrases. Incorporation of the relevant subpart into its selector can take place exactly as if movement had not taken place. 


\subsubsection{Silent complementizers}

The previous proposal suggests an approach to a couple of puzzles concerning the distribution of silent complementizers in English. Complementizer "deletion" is allowed only in complement contexts:

(12)(a)*(That) Mary left bothered Louis.

(b) Mary said (that) Louis was too fat.

The central generalization concerning their distribution can be phrased as follows (Kayne (1984) or Stowell (1981)):

\section{(13) Silent Cs can occur only in head governed contexts.}

Stowell (1981) naturally attributed this restriction to Chomsky's (1981) ECP, requiring that silent categories be head governed by an appropriate head (a proper governor, here a head). Such an approach is especially desirable since a silent that seems to be allowed precisely in the $C$ projections that allow an intermediate trace of $W b$ Movement: that may be omitted only in complement position of bridge verbs. The problem with this approach, apparent throughout in Chomsky (1986), is that the ECP applies only to traces (i.e., actually involves antecedent government), not to silent categories in general, e.g., pro. But an omitted that appears to be the $C$ equivalent of pro rather than a trace.

This problem can be circumvented within the present approach. Consider a that-headed argument clause. Its complementizer that, expressing the tensed and declarative status of the embedded clauses, is selected by some higher predicate. It will have to incorporate into this predicate by LF. Suppose we look at a missing complementizer not as a silent allomorph of $t$ bat, but as the trace of a silent allomorph of that that has incorporated in the overt syntax into its selector. ${ }^{26}$ Silent that will occur precisely in contexts in which it can overtly incorporate into its selector in the syntax. It follows that this can happen only in contexts in which they are head governed by their selector or, to put it equivalently, in contexts in which this incorporation leads to a proper configuration of antecedent government of the trace of silent that by its incorporated antecedent. In terms of the list in (1) the difference between that and silent that is that the latter would have to be lexically designated as a bound morpheme requiring overt incorporation. This extends to cases of clauses that are complements of prepositions, such as before $\left[_{C} e\right] J o b n$ left, which select the silent option of the complementizer. ${ }^{27}$

As Koopman (personal communication) suggests, this approach can provide the beginning of a reason as to why only certain classes of verbs allow silent Cs. Manner-of-speaking verbs such as whisper do not allow silent Cs but verbs such as say do. Capitalizing on the idea of conflation (lexical 
decomposition), suppose that a verb such as whisper is in fact a conflated verb + manner adverbial combination, that is, it is syntactically projected as two projections - say, for concreteness here, an adverb heading an AdvP taking as complement a $\mathrm{V}$ heading a VP. ${ }^{28}$ To license its internal structure, the verb whisper = speak softly will have to appear in the Adv slot binding a silent $\mathrm{V}$ in the $\mathrm{V}$ slot: $\left[_{A d v p}\left[_{A d v}\right.\right.$ speak softly $\left.\left.\left[_{V P}\left[t_{i}\right]\right] 1\right]\right]$. This makes overt incorporation of the complementizer impossible, as the verb whisper is separated from $\mathrm{C}$ by an intervening head (the silent $\mathrm{V}$ ). ${ }^{29}$ Verbs such as say, which lack this internal structure, allow overt $\mathrm{C}$ incorporation. ${ }^{30}$

\section{Unifying phrase/phrase relations}

\subsection{Generalized Spec/bead licensing}

Let us turn now to XP/XP relations and non-strictly local relations of the form X/XP (e.g., polarity licensing). As discussed earlier, it is desirable to reduce them all to the same type of relation in order to explain why they are both binary relations and require the command requirement. There is no a priori bias in favor of unifying all these relations under the movement banner, except that which comes from the discussion of the previous sections; regarding head distribution, movement does seem to be a primitive. Why then introduce any other if we can avoid it? Many of the relations here might be argued to resemble each other because some sort of scope taking is involved (e.g., Wh Movement, negative quantifier licensing, polarity item licensing). However, a generalized scope approach does not seem to extend to cases of A-movement (subject or object raising) in any plausible way. It is precisely because movement is a formal relationship that we have the (dangerous) freedom to make it encompass everything. This freedom would not exist if all the dependencies were reduced to some general process with interpretive correlates (e.g., scope assignment). The existence of A-movement or head movement shows that we need the additional leeway.

Assuming then that they should be reduced to movement, an immediate difficulty is that it is not immediately obvious how to make head/phrase relations into phrase/phrase relations or vice versa The structural relations involved, although they all involve command, do not do it in quite the same way. Thus, in a head/phrase relation H/P, the phrasal projection of $\mathrm{H}$ contains P. In a phrase/phrase relation XP/P neither contains the other. In order to achieve this unification I would like to suggest that all of these relations actually involve three terms: two phrasal positions and a head. To instantiate this general idea in a way that also captures the i-command requirement, let us postulate the following approach. In each case the relevant property ( $w$ h, quantificational, polarity, specificity for clitics, anaphoricity, Case, number, being quantified over by only, etc.) must be licensed in the same way. The $\mathrm{XP}$ bearing the property $\mathrm{P}$ in question can only be licensed in an appropriate 
subject/predicate relationship with the proper predicate. To put it in contemporary terms, the element carrying the syntactic property $\mathrm{P}$ (i.e., a lexical property with syntactic reflexes) ${ }^{31}$ in question must, in order for $\mathrm{P}$ to be properly licensed, be in a specifier/head relationship with a head of the $\mathrm{P}$ type, that is, one whose only content is the property P. In other words, for each relevant property $\mathrm{P}$, a configuration of the following sort must obtain:

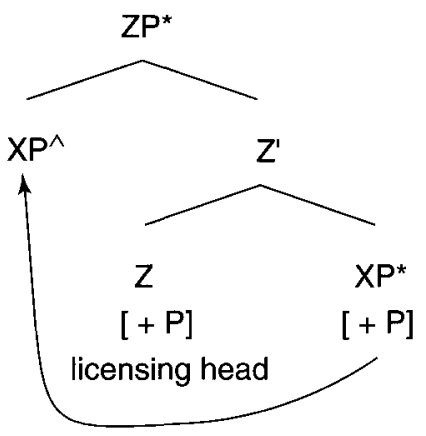

In order to ensure this state of affairs we postulate (15):

Generalized Licensing Criterion (GLC)

Syntactic properties must be licensed in Spec/head configurations

Movement is the means by which the licensing Spec/head relationship is realized in case the phrase to license is not generated in the appropriate position. This in general will be the case. The existence of movement is a reflection of another (unexplained) linguistic property, namely, that a word or a phrase does not express an atomic (syntactically relevant) property, but rather a concatenation of atomic properties, each of which has to be satisfied under the GLC. Note that under this view movement cannot be optional. It is a driven process. If it can apply - because some property needs licensing - it must (whether covertly or overtly).

A uniform analysis of these dependencies must still leave room to explain their difference of appearance (that is, what we see) and their difference of properties. Concerning the first one, it is easy to see that the variation is a function of which of the three heads involved in this ternary relation ${ }^{32}$ is phonologically realized (this is congruent with our earlier discussion of parametric variation):

(16) In the ternary relation $\left(\mathrm{XP}^{\wedge} \mathrm{H}^{0}, \mathrm{XP}^{*}\right)$ each of them may be overt or covert. 
The two types we have been discussing (phrase/phrase versus head/phrase) correspond to which of $\mathrm{XP}^{\wedge}$ in $[\mathrm{Spec}, \mathrm{HP}]$ and $\mathrm{H}^{0}$ is overt, respectively. But the typology is actually richer, as we may have cases where all three are overt: if both $\mathrm{XP}^{\wedge}$ and $\mathrm{XP} *$ are overt, it does not look like a movement relation (e.g., as in who saw what), but we would argue that it is. Furthermore, if $\mathrm{H}^{0}$ is also overt, ${ }^{33}$ we will have an overt ternary relation. With $\mathrm{XP}^{\wedge}$ in $[\mathrm{Spec}, \mathrm{HP}$ ] overt and XP* covert we have a usual movement relation. With $\mathrm{XP}^{\wedge}$ covert and XP* overt we will have a covert movement relation.

Other properties differentiating these dependencies will have to capitalize on particulars of each dependency as is standardly assumed:

(17)(a) Overt versus covert movement

(b) The properties of $\mathrm{XP}^{\wedge}$ and $\mathrm{XP} *\left(\mathrm{~A} / \mathrm{A}^{\prime}\right.$-position, Case/ caseless, etc.)

(c) Lexical properties of the moving item itself.

The contribution of the first two factors is clear. The third might play a crucial role in determining the appropriate locality domains. Thus, the binding domain for English anaphors (e.g., himself) is narrower than the binding domain of long-distance anaphors (e.g., Chinese iji or Japanese ibun). Neither of these domains is identical to the domain over which a relation between the French negative quantifier personne and its scope position marked by ne is allowed. Still, none of these is identical to the domain over which $W h$ Movement is allowed. Rather than attributing these differences to anaphoric binding relations not being movement relations, we have to attribute them to differences in the internal structures and properties of the moving items. For example, the internal structure and properties of lexical anaphors are different from those of $w h$-phrases; anaphors differ from each other - for example, "self" in Chinese versus "X-self" in English. The anaphorizing item may also differ - for example, "self" in Chinese or English versus "same" in French. Consequently the kind of "interveners" to which these movement relations will be sensitive will differ as well.

\subsection{Elimination of adjunction}

In this section I briefly outline, without arguments, some assumptions that I defend elsewhere but that I will use later on. I propose in Sportiche (1993b) that syntactic adjunction is not an available option. Neither is adjunction under movement (all movements are substitution in the Spec position of some projection along the lines of the GLC) and there are no (base-generated) adjuncts either, such as adverbs, adjectives, PPs, and extraposed clauses. The aspect of this proposal relevant to what follows is the latter, namely, the treatment of adverbs and adjectives. Informally speaking, I will assume that adverbs and adjectives are all dominated by a projection whose head takes the 
modifiee of the adverb or the adjective as an argument, that is, as complement (or sometimes as specifier). The general intuition is that adjectives and adverbs bear the same kind of relation to their modifiee that determiners bear to their noun phrases or predicates to their arguments.

A constituent such as the book is, since Abney (1987), analyzed as having a head/complement structure:

(18)(a) $\left[\left[_{D P}\left[L^{\prime},\left[_{D}\right.\right.\right.\right.$ the $\left[\left[_{N P}\right.\right.$ book $\left.\left.]\right]\right]$

(b) $\left[_{D P}\left[{ }_{D P}\right.\right.$ the $]\left[_{D},\left[_{D} e\right]\left[_{N P}\right.\right.$ book $\left.\left.]\right]\right]$

Although the structural skeleton of determiner phrases that this assumes is well motivated, the particular distribution of the lexical material within it indicated in (18a), as proposed by Abney (1987), is not obviously correct and might have to be modified to (18b). ${ }^{34}$ Adverbs and adjectives, I propose, should be treated in exactly the same way. This is illustrated in the following structures (where for each case it must be decided which of the two options is the correct one and what the identity of the complement is):

(19)(a) John will stupidly answer: $\ldots\left[\left[_{A d v P}\left[{ }_{A d v^{\prime}}\left[{ }_{A d v}\right.\right.\right.\right.$ stupidly $]\left[\left[_{v P}\right.\right.$ answer $\left.\left.]\right]\right]$

(b) John will answer stupidly: $\ldots\left[_{\mathrm{Advp}} \mathrm{L}_{\mathrm{vP}}\right.$ answer $] \mathrm{Adv}_{\mathrm{Adv}} \mathrm{L}_{\mathrm{Adv}}$ stupidly $] \mathrm{H}$

(c) A proud mother: $\ldots\left[{ }_{A P}\left[A^{\prime}\left[{ }_{A} \text { proud }\right]\left[\left[_{N P} \text { mother }\right]\right]\right]^{35}\right.$

(d) A mother proud of her son: $\ldots L_{A P}\left[_{N P}\right.$ mother $] L_{A^{\prime}} L_{A}$ proud $]\left[\left[_{D P}\right.\right.$ of her son $\left.\left.]\right]\right]$

(e) John always buys books: $\ldots\left[_{\text {Adv }}\left[\begin{array}{l}\text { Adv } \\ L_{A d v}\end{array}\right.\right.$

(f) John always buys books: $\ldots\left[_{A d v P}\left[{ }_{A d v P}\right.\right.$ always $]\left[_{A d v^{\prime}}\left[\left[_{A d v}\left[{ }_{A d v} e\right]\right]_{X P}\right.\right.$ buys books $\left.\left.]\right]\right]$

As a final remark on this topic, I also assume that the adjunct status of adjuncts is directly encoded by stipulating that these adjunct projections are totally transparent to government. In other words government (hence, head movement) may behave exactly as if the entire projection were absent.

\subsection{The program}

In the next section we will turn to what motivates this approach in one individual case. In the case of the typical A-movement dependencies this approach just recapitulates the currently adopted positions. Thus, NP-movement, as in the case of VP-internal subject raising, object raising, or passive, is motivated by Case licensing; that is, it is a tripartite relation between the 
moving phrase, the Case licensing head - the appropriate Agr projection - and the landing site - the specifier of that AgrP. It straightforwardly extends to theta assignment if we adopt a slightly modified version of Larson's (1988) VP shell proposal by requiring that all arguments of a verb, and more generally of a predicate $P$, be generated as specifiers and only specifiers of layered phrasal projections of this predicate $\mathrm{P}$, as illustrated in (11). In this case the ternary relation reduces to a binary relation as $\mathrm{XP}^{*}$ and $\mathrm{XP}^{\wedge}$ are identical.

We will therefore primarily concentrate on $A^{\prime}$ movement. I will illustrate this approach here with the well-known example - and we will conclude archetypal case - of $w h$-questions, discuss its syntax, modify some existing proposals concerning it (mostly Rizzi (1991)), and draw some consequences about characteristic properties of such dependencies.

\section{Questions}

The GLC is inspired by Rizzi's (1991) update of May's (1985) Wh Criterion; the former takes into account Chomsky's (1986) generalized $\mathrm{X}^{\prime}$ proposal extending the $\mathrm{X}^{\prime}$ schema to all projections: ${ }^{36}$

Wh Criterion or $Q$-criterion

At LF

A $[+$ wh $]$ head must be in a Spec/head relationship with a $[+$ wh $] X P$.

$A[+w h]$ XP must be in a Spec/head relationship with a $[+$ wh $]$ head.

The underlying motivation for this proposal is the syntactic attribution of scope to $w h$-quantifiers: the scope of a $w h$-question phrase is syntactically marked by the presence of a $[+Q]$ head. It is directly motivated by the syntax of $w b$-questions in English-like languages. In the present approach it is simply motivated by the need for the wh-property to be properly licensed by an appropriate head. Since there is no principled motivation for why the scope of $w h$-question words should be what it is observed to be, a primitive motivation in terms of scope is no better a priori than a syntactic licensing motivation, although a posteriori the two should obviously interact (moving $w h$-phrases has consequences for their scope properties). This proposal encodes in a simple manner a number of assumptions concerning the functioning of $w b$-question quantification and the nature of LF representations. It "derives" the existence of $\mathrm{Wb}$ Movement. It make all languages essentially identical at LF, regardless of whether $W b$ Movement is overt or not. Furthermore modulo minimal assumptions concerning the difference between selected clauses and unselected clauses, it derives the existence of $\mathrm{V}$ or I to $\mathrm{C}$ movement in $w \mathrm{~b}$ questions. Departing from Rizzi's proposal, I will suppose that the [+wh] feature is always a property of certain Cs indicating the question property. 
Remember, however, that the locus of a property and the locus of its realization in overt syntax are not necessarily the same (past may be generated on a V in the $\mathrm{V}$ slot head of VP even though it is a property of the head $\mathrm{T}$ of TP).

\subsection{English questions}

I will suppose without discussion a particular organization of the English auxiliary system. First, I will suppose that every verb (including modals) (see Sportiche (1993b), for justifications) is generated in a V slot. Second, I will suppose that main verbs may not overtly raise to $T$, unlike auxiliary verbs, which may (possibly because, as we interpret Pollock (1989) in terms of a clause structure containing $\mathrm{Agr}_{\mathrm{O}}$ below $\mathrm{T}$, they cannot even reach $\mathrm{Agr}_{\mathrm{O}}$ ) and must when tensed. Following Chomsky (1993) I assume that in English and more generally (given the existence of suppletion) Tense features are generated on $\mathrm{V}$ in the $\mathrm{V}$ slot of VP.

\subsubsection{Wh-questions}

Let us begin with embedded $w h$-questions:

(21) Mary asks [ + Q you should see who].

By LF both the $W h$ Criterion and recoverability must be satisfied: first, who must raise to $[\mathrm{Spec}, \mathrm{CP}]$; second $[+\mathrm{Q}]$ must be recoverable. In English the first requirement must in fact be met at S-Structure, that is, the $w b$-element moves overtly (when there is only one; if there are several wh-elements, only one must and may move). I know of no proposed explanation as to why this is so. ${ }^{37}$ English does not have an overt $[+Q]$ complementizer. What happens to the $[+Q] C$ property? Suppose that the English $[+Q]$ is a covert bound morpheme and, more precisely, a morphological affix or a syntactic clitic; that is, it requires morpholexical or syntactic incorporation. As such, it must incorporate into some other category. This incorporation must be into the verb ask itself, which in its lexical representation contains an open clausal slot specified [+ Q]; as I have earlier suggested following Koopman (1993a), this movement is the way selection is always satisfied. This is then similar to what happens with silent instances of the complementizer that. Note that the presence of this silent $[+Q] C$ is recoverable, since the $Q$ feature is realized on [Spec, CP]. Since the verb ask or the verb know allow both declarative and interrogative complements, the presence of $+Q$ is contingent. One of $+Q /-Q$ may be recoverable as the unmarked option. Since silent that can be covert without any visible reflex, it must be construed as the unmarked option. Hence $+Q$ must be visible. Complement clauses of certain verbs such as wonder require $\mathrm{a}+\mathrm{Q}$ setting. But they behave exactly like complement clauses 
of verbs such as know and ask. We must therefore take contingent (or optional) as meaning "paradigmatically contingent" (or optional), that is, contingent in general, even though it may be necessary for particular choices of lexical items.

The main clause case is more complicated. Here I will rely extensively on some of Chomsky's (1991) economy ideas. The first part of the account of (21) would apply unchanged; the $w$-word must raise in the syntax. The bound morpheme status of the $[+\mathrm{Q}] \mathrm{C}$ cannot be handled the same way. The option available in embedded clauses is excluded, since there is nothing to incorporate the $[+\mathrm{Q}] \mathrm{C}$ upward to (I am going to ignore intermediate heads such as $\mathrm{Agr}_{\mathrm{S}}$ and $\mathrm{Agr}_{\mathrm{O}}$, limiting the discussion to $\mathrm{C}, \mathrm{T}$, and $\mathrm{V}$ ):

\section{(22)(a) [ + Q Mary should teach what $\rightarrow$ What should Mary teach?}

(b) $[+Q$ Mary taught what $] \rightarrow$ What did Mary teach?

Consider first (22a). The tense morphology is generated on the verb should, which raises to $T$ overtly to license its $T$ features $($ say, $+T)$. How is the bound morpheme status of $+Q$ licensed? One way would be to generate $+Q$ on $T$. I would like to exclude this possibility by invoking a principle of "Paradigmatic Uniformity" (PA). If $+Q$ could in general be generated on $T$, causing overt $T$ to $C$ in main clauses, we would expect overt $T$ to $C$ in embedded clauses as well (at least with non-subject $w b$-phrases, given the lack of overt $T$ to $C$ in subject questions, viz. who left). Because of paradigmatic uniformity, I will assume - for the moment - that it never can. So, + Q is generated in $\mathrm{C}$ in main clauses, and its bound morpheme status requires that T raise to C overtly. This derivation violates Chomsky's (1993) Principle of Greed, which requires movement to be motivated solely by properties of the moving item, that is, for the purpose of licensing one of its own properties. Here, $T$ to $C$ is triggered by $C$, not by $T$. I suggest that we interpret Greed as an Economy Principle: it can be violated as a last resort. ${ }^{38}$

Consider next (22b). By paradigmatic uniformity, the tense features $+T$ must be generated on $\mathrm{V}$ (as simple declaratives show). If they are, $+\mathrm{Q}$ could not be licensed at all, since for whatever reason non-auxiliary verbs cannot overtly raise to $\mathrm{T}$, a fortiori not higher. No well-formed output is predicted possible. However, English has a dummy verb do, on which it can generate + $\mathrm{T}$. So $+\mathrm{T}$ is generated on $d o$. Do as an auxiliary verb may raise to $\mathrm{C}$, and raising to $C$ is required to license the bound morpheme $+Q$ in $C$.

A further complication arises in the case of subject $\mathrm{Wh}$ Movement:

(23)(a) [+ Q [Who taught calculus?]

(b) [Who $+\mathrm{Q}$ [should teach calculus?]

In either of the forms PA forces $+\mathrm{T}$ to be generated on $\mathrm{V}$. The bound morpheme nature of the $[+Q] C$ should force some head to raise to $C$, but 
this is the structure of neither example in (23). ${ }^{39}$ Why is this raising to $\mathrm{C}$ prevented? Koopman (1983) suggests a reason; she makes an analogy between the lack of raising to $C$ in the present case with the obligatory absence of that in Who do you think (*that) t left? Her idea is that the trace is illicit (due to the $\mathrm{ECP}$ ) if the $\mathrm{C}$ position is filled. Whichever implementation of this idea is the right one, this generalization applies to the present situation:

*Who should $_{\mathrm{j}}\left[\mathrm{t}_{\mathrm{i}} \mathrm{t}_{\mathrm{j}}\right.$ teach calculus?]

If $+Q$ was in $C$, forcing $T$ to $C$ in the syntax as in (23a), a violating configuration would be created (at S-Structure). This means we must relax paradigmatic uniformity. I suggest we weaken it by allowing violations of it as a last resort strategy. In the absence of any alternative toward a well-formed output, minimal departures from paradigmatic uniformity are allowed. In the present case PA can be relaxed with respect to either the $T$ features or the $Q$ features, yielding the two following minimal options: $+Q$ may be generated on $\mathrm{T}+\mathrm{V}$ in $\mathrm{V}$ instead of $\mathrm{C}$, or $+\mathrm{T}$ may be generated in the $\mathrm{T}$ slot instead of the $\mathrm{V}$ slot, both violating PA. The second option is ruled out, however, because it still leaves $+Q$ unbound (and raising to it is impossible as it would violate Koopman's generalization). The only option is to violate PA by generating $+Q$ on whichever verb bears $+\mathrm{T}$ :

(25)(a) [Who [t taught ${ }_{\mathrm{V}+\mathrm{T}+\mathrm{Q}}$ calculus?] $]$

(b) [Who $\left[t\right.$ should $_{\mathrm{V}+\mathrm{T}+\mathrm{Q}}$ teach calculus?]]

At $\mathrm{LF}, \mathrm{V}+\mathrm{T}+\mathrm{Q}$ will raise successively first to $\mathrm{T}$ and then to $\mathrm{C}$ to license the $+T$ and $+Q$ morphemes it bears.

Let us recapitulate the ingredients we need. We postulated that the English $+\mathrm{Q}$ morpheme is a bound morpheme, we introduced a Principle of Paradigmatic Uniformity, and we also mentioned Chomsky's Principle of Greed. Greed may be violated when PA is not. PA can be violated as a last resort if no well-formed output is derivable. This gives a ranking of strength among these principles:

Lexical properties ( $+Q$ is a bound morpheme) cannot be violated.

ECP (or whatever is responsible for that-trace effects) cannot be violated.

PA can be violated to avoid violations of the previous two.

Greed may be violated to avoid violations of the previous three.

\subsubsection{Yes/no questions}

Let us turn now to yes/no questions. Given Larson's (1985) arguments that the disjunction(s) introduced by a yes/no question marker in some clauses can 
affect any clause from within which operator movement could have taken place to the yes/no marker, we postulate, adopting his conclusions, that yes/no questions involve the movement of a (mostly covert) disjunctive or yes/no wh-operator to [Spec, CP]. Postulating such an operator explains why $w h$-questions and yes/no or disjunctive questions are incompatible. There is only one position: the yes/no operator and a wh-phrase cannot both overtly move to it. ${ }^{40}$ Thus, consider the sentences below:

(26)(a) Who did you see?

(b) What did Gloria write to Mary or Louis?

(c) Did Gloria write to Mary or Louis?

Even though (26a) exhibits both a preposed $w b$-word and subject/aux inversion, it cannot mean "who did you see or who did you not see." Similarly, (26c) is ambiguous (and disambiguated by intonation). It may call for a yes/no answer or for a term of the alternative answer (it may then be paraphrased as "Did Gloria write to Mary or did Gloria write to Louis?"). (26b), however, cannot be a request for both what was written and which of Mary and Louis it was written to (although, pragmatically, it may be answered this way).

Consider an indirect question. Assume that whether is the wh disjunction operator as suggested by its resemblance with wh-either (see Kayne (1991a) for recent arguments). Then the derivation of (27) below is not significantly different from the previous cases:

Mary asks [Q you should leave whether].

At LF, whether must have raised to the embedded [Spec, CP] and the [+ Q] $\mathrm{C}$ must have incorporated into the verb ask. Note that whether has the option of being silent in certain dialects. ${ }^{41}$ Incorporation of the $\mathrm{Q}$ morpheme into ask would leave the $[+\mathrm{Q}]$ value of the head $\mathrm{C}$ opaque. The $[+\mathrm{Q}]$ head must not incorporate into its selecting verb. The derivation invoked in (25) is not available either: if $+Q$ was generated on $V$ with $L F$ raising to $C$, the question nature of the embedded clause would be opaque as well. The last option, since the $\mathrm{Q}$ morpheme is a syntactically bound morpheme, is for raising to $\mathrm{C}$ to take place overtly in a way similar to main clause subject/aux inversion, yielding:

$$
\text { Mary asks }\left[\left[_{w b \text {-operator }} \mathrm{e} \mathrm{k}_{\mathrm{k}} \text { should } \mathrm{j}_{\mathrm{j}}\left[\text { you } t_{\mathrm{j}} \text { leave } t_{\mathrm{k}}\right]\right.\right. \text { ]. }
$$

In main clauses whether must be silent for unknown reasons (but cf. below). If it did not have to, we would presumably derive (29a). Otherwise, we must have raising to $\mathrm{C}$ exactly as in the previous case of non-subject $\mathrm{Wb}$ Movement, yielding (29b). 
(29)(a) [whether ${ }_{\mathrm{k}}$ should you $t_{\mathrm{j}}$ leave $t_{\mathrm{k}}$ ]

(b) $\left[\mathrm{E}_{w b \text {-operator }} \mathrm{e} \mathrm{e}_{\mathrm{k}}\right.$ should $\left[\right.$ you $t_{\mathrm{j}}$ leave $\left.\left.t_{\mathrm{k}}\right]\right]$

Finally consider a simple declarative such as They (should) sleep. Such a clause must contain a highest projection, headed by $[-\mathrm{Q}]$, indicating the declarative status of the clause. This we may assume is the default value and, since it triggers no word order changes at all, is analyzed as a silent independent morpheme. Its recoverable character follows from its being the default setting.

\subsection{French questions}

\subsubsection{Questions with word order changes}

French wh-questions and yes/no questions fundamentally function like English questions:

(30)(a) Quand Marie est partie/Quand (Marie) est-elle partie/ Quand est partie Marie?

When Mary has left/When (Mary) has-she left/When has left Mary?

(b) Lequel d'entre eux a mangé/Lequel d'entre eux a-t-il mangé? Which one of them has eaten/Which one of them has-he eaten?

(c) Il demande quand Marie est partie/*Quand (Marie) est-elle partie/quand est partie Marie.

He asks when Mary has left/*When (Mary) has-she left/ When has left Mary.

(d) (Marie) est-elle partie?/Je demande si Marie est partie.

Has Mary left?/I ask whether Mary left.

As (30a) illustrates, matrix $w b$-questions all have a preposed $w h$-phrase..$^{42}$ The rest of the sentence may stay unaffected, and may display Complex Inversion, Subject Clitic Inversion, or stylistic inversion. Stylistic inversion appears to be a French-specific (and poorly understood) construction, properties of which are discussed in Kayne and Pollock (1978) and Kayne (1972) and which I will basically ignore here. Complex Inversion and Subject Clitic Inversion are excluded in embedded questions. They have been traditionally analyzed as involving overt raising to C (cf. Kayne (1984), Rizzi and Roberts (1989)). I analyze them in Sportiche (1993a) as involving covert $\mathrm{T}$ to $\mathrm{C}$ only in main clauses. This is also true of yes/no questions. The yes/no operator in French is always silent. In embedded yes/no questions, the $[+\mathrm{Q}]$ property is spelled out on C as si. In main clauses this + Q C is silent. Sportiche (1993a) argues that French lacks overt raising to $C$ altogether. In effect, then, French main clause yes/no questions behave like English subject wh-questions in which 
overt raising to $\mathrm{C}$ is prohibited. As in the case of (25b), [+Q $]$ must be generated on $\mathrm{T}$ (which is generated on the verb itself), and $\mathrm{T}$ to $\mathrm{C}$ applies covertly.

Now recall the pattern of Standard English

1 In standard English [+Q], a bound morpheme, is always generated in $\mathrm{C}$, except when doing so yields violations of some principle.

2 In embedded $w h$-questions we have both a preposed $w h$-phrase and $[+Q] C$ incorporation into a higher head. In main clauses we have both a preposed $w h$-phrase and $\mathrm{T}$ to $\mathrm{C}$ movement to license the morphological requirement of the $[+\mathrm{Q}] \mathrm{C}$, except when the $w b$-phrase is the subject. In that case overt $\mathrm{T}$ to $\mathrm{C}$ is disallowed. Instead, we must resort to generating $[+\mathrm{Q}]$ on $\mathrm{T}$ and raise $\mathrm{T}$ to $\mathrm{C}$ covertly. Yes/no questions work exactly like $w h$-questions except for the fact that the main clause yes/no operator is silent.

French then appears identical to English except for the fact that French whether is always silent, and French lacks overt $\mathrm{T}$ to $\mathrm{C}$ altogether.

\subsubsection{Why main clauses whether, si are silent}

Why do we find main clause/embedded clause distinctions in the way yes/ no is marked (viz. the French alternation $\emptyset /$ si? A possible reason is the following: suppose, following Bhatt and Yoon (1991), that we distinguish the subordinating property of $\mathrm{C}$ from its clause-typing property. They argue that in certain languages these two properties are represented by different morphemes. The only way to make this idea compatible with the general assumptions we make is to split $\mathrm{CP}$ in two, replacing it by a subordinating projection SP taking a clause-typing projection TypeP as complement, and so universally. What they analyze as cases of conflation, for example, a unique English CP headed by $\mathrm{C}$, we must reanalyze as a case of two morphemes, Type and S, morpholexically incorporated to each other. ${ }^{43}$ Hence, the complementizer that is really morphologically complex (tensed declarative + subordination), is generated in TypeP, and must raise to SP to check its subordinating feature. Suppose that French $s i$ is such a conflated head (subordination $+Q$ ). What would we expect to see in main clauses? Clauses seem to be unique in that they are the only constituents that may lawfully appear unembedded (in non-elliptic contexts). It is not unreasonable then to expect that the subordination/non-subordination of clauses be marked in some way, for example, by the presence of SP. ${ }^{44} \mathrm{~A}$ correlate of this is that main clauses should lack SP. If French si indeed is $\mathrm{S}+\mathrm{Q}$, it cannot appear in main clauses. We could then state that the unincorporated form of the $+Q$ head of TypeP is a silent bound morpheme, like English 's. ${ }^{45}$

In what follows I will continue using $\mathrm{CP}$ and $\mathrm{C}$ as shorthand. 


\subsubsection{Questions with no word order change}

Some (non-echo) questions have the same word order as statements, such as intonation yes/no questions:

Louis a mangé un oeuf?

Did Louis eat an egg?

Compared with statements, the only change is intonational. For example, in (31a) intonation rises at the end of the sentence, unlike what happens in statements. Intonation plays a disambiguating or interpretive role in many constructions and in particular in other kinds of questions that I will not discuss. ${ }^{46}$

How should these kinds of questions be treated? I want to outline a proposal consistent with the kind of restrictive assumptions made in the first section and with the overall organization of the various components of grammar.

First of all, since intonation plays a role both in PF (obviously) and at LF (it provides the question property), it should be represented at S-Structure (spell-out), namely, syntactically.

How should it be represented? The null hypothesis, it would seem, is that it should be represented exactly as it always is, that is, as $[+Q]$ in $C$. This is also the only one consistent with the restrictive assumptions we made in the first section: if the question/statement distinction is sometimes represented on the head of a designated functional category, then it universally always is. This would mean that one of the realizations of the French $[+Q]$ head in main clauses has no segmental value but only a suprasegmental value (equivalent to a tonal melody in tone languages), which manifests itself on the intonational melody. Two properties need to be explained. First this question intonation is in complementary distribution with all the syntactic operations, such as Subject Clitic Inversion, diagnostic of a main clause question. Second, and quite surprisingly, the intonational effect is a rising intonation at the end of the sentence, whereas typically (care is needed here to avoid any parasitic contrastive focus), the $[+Q]$ property triggers a high effect on the element bearing the $[+Q]$ property or immediately preceding its syntactic position (thus Marie a t-ELLE mangé?/"Has Mary eaten?" and Avez-VOUS mangél"Have you eaten?"; similarly, QUI a vu ce film? "Who saw this movie?" and QUAND êtes-vous parti? "When did you leave?"

Consider then the syntactic structure of an intonation yes/no question such as (32):

$$
\left[_{C P}[+Q]\left[_{I P} \text { Louis a mangé un oeuf }\right]\right]
$$

Rules of phonological interpretation of syntactic information will have to readjust the effects of the presence of the question morpheme by interpreting 
its effects sentence-finally, that is, away from its syntactic position. This raises the question of how exactly the syntax/phonology interface works. One of the present practices, consisting of postulating rules of phonological interpretation that sometimes rearrange syntactic structures, seems a priori unacceptable. ${ }^{47}$ Its unacceptable character is illustrated by imagining what it would mean if it were applied at other interfaces, for example, the lexicon/syntax interface or the syntax/interpretation interface. Keeping again to minimal assumptions, the strongest approach concerning syntax-tophonology readjustment rules is that they do not exist at all. Phonological rules simply interpret the syntactic and lexical information present in their input congruent with the structure of the phonological vocabulary. If the phonological rules in general are faithful to syntactic information, but in some cases credibly appear to rearrange it, then taking phonological evidence seriously entails that the postulated syntactic structure is wrong. In the case at hand we would have to postulate either that an intonational Q-suprasegmental morpheme is generated sentence-finally or that it is generated in C but its phonological effects are shifted to the end of the sentence. Neither option is acceptable. I would like to suggest instead that the structure (32) is incorrect and that it should be replaced by (33):

$$
\left[_{\mathrm{CP}}\left[_{\mathrm{IP}} \text { Louis a mangé un oeuf }\right]_{\mathrm{i}}[+\mathrm{Q}]\left[t_{\mathrm{i}}\right]\right]
$$

in which the entire IP has preposed to [Spec, CP], that is, to a position preceding the $[+\mathrm{Q}]$ morpheme. The obligatoriness of this preposing can be made to follow from the lexical stipulation that the suprasegmental version of the $[+Q]$ morpheme is a leftward phonological clitic; that is, it needs material to its left. Since the question/statement distinction is a property of the entire IP following it, the silent question operator usually moving to [Spec, CP] (and sometimes lexicalized as whether in English) is an IP level modifier. Since the Q-morpheme needs phonological material to its left, the only way to produce a well-formed output is to pied-pipe the entire IP as in (33).

This structure explains some odd features of this intonation yes/no question. In simple clauses it derives the complementarity noted above between other phenomena involving $T$ to $C$ (such as Subject Clitic Inversion) and this intonational pattern, since the material that would potentially raise from $\mathrm{T}$ to $\mathrm{C}$ (recall that $\mathrm{T}$ to $\mathrm{C}$ occurs only at $\mathrm{LF}$ in French) is already higher than the $\mathrm{C}$. It also explains why these intonation yes/no questions, although they have the interpretive properties of questions, lack their syntactic properties. For example, polarity items are not licensed in intonation yes/ no questions. Thus, the polarity item qui ce soit "whoever" can occur as a bare object only in polarity environments, for example, those commanded by some negative item or by $[+Q]$. It cannot appear in an intonation question for lack of appropriate command: 
(34)(a)* Il a vu qui que ce soit (statement). He saw anybody.

(b) A-t-il vu qui que ce soit?/Il n'a pas vu qui que ce soit. Has he seen anybody/He has not seen anybody.

(c)* Il a vu qui que ce soit (question)?

Has he seen anybody?

Licensing by a commanding yes/no operator in [Spec, CP] at S-Structure is required and this configuration is not met in (34c), since the operator is embedded inside the preposed IP. ${ }^{48}$

\subsubsection{Multiple questions}

Consider multiple questions:

Who saw what?

Given the requirements imposed by the $W h$ Criterion, both wh-operators must be in a Spec/head relationship with the same $[+Q]$ head (since there is only one such head). Since the $w b$-property of both $w b$-operator properties must be licensed by a unique head, we must postulate the existence of a process reducing these two phrases to one. One way this rule may be functioning would be by reducing these two operators to one, namely, by creating a binary $w h$-quantifier out of two unary quantifiers so that there would be one relevant wh-property to Spec/head license. Call this "Absorption." Semantically, this might seem gratuitous because the $w h$-question operators are idempotent (i.e., have the property $\mathrm{x}^{2}=\mathrm{x}$ ), although it could explain why we get the pair-list reading (i.e., we cannot answer $w h$ and what independently as in who saw something and what was seen). The same phenomenon is observed in negative constructions (negative concord) with a clear case of non-idempotent operators (negation).

Although the treatment we have sketched above is the standard treatment for multiple questions, some of its properties are unclear. First of all, Chomsky (1993) has advanced some arguments for wanting to conclude that $w h$-movement should universally be overt. But many languages, including English and French, that tolerate multiple questions clearly exhibit both moved wh-phrases and wh-in-situ at the same time. How is this to be reconciled with the requirement that $W b$ Movement be obligatorily overt? One possibility could be to claim that the $+Q$ property of $C$ is strong in Chomsky's (1993) sense and thus requires a $w$ h-operator in $[\mathrm{Spec}, \mathrm{CP}]$ in overt syntax. Second, the precise mechanism by which absorption takes place is most unclear. It is a definitional property of syntactic positions that they can contain only one item at a time. Yet in the case of the standard account of multiple questions we want several $w h$-phrases to occupy exactly the same 
[Spec, CP] at LF at the same time. This suggests an alternative treatment along the following lines that takes advantage of the approach to $W b$ Movement to Japanese or Chinese $w b$-questions advocated by Aoun and $\mathrm{Li}$ (1993), Watanabe (1992) or Cheng (1992). Current analyses of DPs headed by English who, what or French qui assume that the wh-word is itself the question operator. A word like who seems to stand both for the question operator and for its range (here $[+$ human $]$ ). We may claim instead that the wh-operator in French or English is always covert and that the $w h$-words are wh-polarity items ([+human] nouns such as who, [-human] nouns such as what, determiners such as which, and so on) that must occur in the scope of these $w h$-operators. The structure of a $w h$-phrase would then be [wh-operator [whol\}. In a sentence with a unique $w h$-phrase, this $w b$-phrase must move to [Spec, CP]. When there are two, only one of them can contain a wb-operator (otherwise there is no possibility of satisfying the GLC) and it must move to [Spec, CP]. The other one must then be parasitic on the first one (i.e. exactly like a parasitic gap). The structure of a sentence such as (35) would then be as in (36):

$$
\left.\left[_{\mathrm{DP}} W h-o p_{j}[\text { who }]\right]_{\mathrm{i}}\left[t_{\mathrm{i}} \mathrm{saw}\left[\mathrm{DP}_{\mathrm{DP}}\left[{ }_{\mathrm{pg}} e\right] \text { [what }\right]\right]\right]
$$

Here movement of the $w$-operator must pied-pipe (in English but not with, say, French combien) the entire DP. Languages in which wh-words always are $w$-operators themselves (possibly conflated with the head noun or some other category) would then simply not allow multiple questions (Italian might be such a case; see Rizzi (1982)).

\subsection{Diagnostic properties}

This analysis of $w h$-constructions evidences a number of properties that we expect to see recur elsewhere and that we can use as diagnostic for the idea that something like the configuration mandated by the GLC is indeed met:

$1 W h$-question words (now including the yes/no operators) are related to a particular, designated and fixed position in the syntactic structure of a sentence.

2 It is a relation between a head $([+\mathrm{Q}] \mathrm{C})$ and a $([+\mathrm{wh}]) \mathrm{XP}$ that must be Spec/head at LF. Because this relationship is established by movement, the specifier of the $[+Q] C$, hence apparently the $[+Q] C$ itself, is in a possible movement relationship (i.e., subject to islandhood, etc.) with the wh-XP.

3 Doubly filled Comp effects may be found (which we can now analyze as a prohibition against filling both the specifier and the head position of any projection at the same time; call this the Doubly Filled Projection Prohibition). 
4 Absorption effects are observed.

5 The specifier of this $[+Q]$ head is an $A^{\prime}$-position (as exemplified by its licensing parasitic gaps).

\subsection{Furtber and future extensions}

I discuss elsewhere how this general approach extends to clitic constructions (Sportiche $(1992 ; 1993 a)$ ) and to negative constructions (Sportiche (1993c)). I hope to extend it in future work to a number of other syntactic constructions: anaphoric binding dependencies (binding of anaphors and hopefully pronominal binding), scopal and other properties of non-wh non-negative quantifiers (see Stowell and Beghelli (1994)), the syntax of Focus, etc. The general proposal we put forth allows very little analytical leeway, but I try to push it as far as I can. In each instance it forces us to postulate a designated projection to fulfill the role of $Z \mathrm{P}^{*}$ in (14). In the case of non-wh non-negative quantifiers, the way to take the analogy with $w b$-questions is straightforward and leads us to postulate designated Q-projections for each type of these quantifiers with concomitant movement. In the case of anaphor binding it leads us to postulate designated positions - for example, [Spec, ReflexiveP] - that anaphors have to move to to be properly licensed. The general idea is clear. It will lead to fairly abstract analyses of individual lexical items of the kind postulated by Klima (1964) in the case of negative polarity items. It will also lead to the view that the same property expressed in different sites of a single clause cannot be a property licensed in-situ. Take, for example, the case of Focus. In a simple French clause it may be marked prosodically in-situ but felicitously only on one constituent at a time. This uniqueness suggests immediately that the Focus property is associated with a particular unique position. We are then led to postulate both the existence of a Focus phrase with a Focus head, the morphemic content of which is responsible for the prosodic effect, and movement of the focused constituent to the specifier position of this phrase.

The abstractness price we pay for this kind of unification is reduced in some cases by the overt similarity found in some languages between constructions that are treated differently in English or French. Extremely significant in this respect is the work of $\mathrm{Li}$ (1992), showing that Chinese $w$-words ( $w$ b-one), polarity items (any-one), and existential quantifiers (some-one) are one and the same word, the interpretive import of which is contextually determined by the kind of elements in the scope of which they are found. It should come as no surprise that they should have closely related syntactic functioning, as we propose.

\section{Notes}

* Various parts of this work have been presented at the 1992 GLOW Colloquium in Lisbon, the University of Indiana at Bloomington, Cornell University and the 
European Science foundation in Strasbourg and in graduate seminars at UCLA in 1990, 1991, 1992 and 1993. Thanks to their audiences for their input. A grant from the UCLA Academic Senate partially supporting this research is gratefully acknowledged.

1 The discussion of conflation later on might be taken to suggest that even this kind of variation is even more superficial than it appears. For example, shallow might be analyzed in English as the conflated not deep.

2 As we will see, the conclusion of this section and all others in this article are consistent with a model of syntax comprising a unique level of representation conflating D-Structure and S-Structure as in Sportiche (1983). In fact, it is consistent with a model comprising a unique level of representation conflating DStructure, S-Structure. and LF, i.e., one lacking syntactic derivations altogether. I will continue talking in derivational terms to keep exposition on familiar terms.

3 This is a generalization to head movement of an approach that has been suggested for phrasal movement in Jaeggli (1980), who suggested replacing Case assignment by Case checking.

4 This distinction is reminiscent of Marantz's (1993) distinction between DStructure incorporation and S-Structure incorporation. Differences between the two incorporation processes are recently discussed in Sportiche (1993a) in connection with pre and postverbal subject clitics in French. For a more general approach to these questions see Koopman (1993a) and references therein.

5 This would depend mostly on whether strict compositionality is respected. In general with these cases, it is not.

6 Larson's proposal differs in that it would allow kill to project the agent as the specifier and the patient as the complement within the same unique VP.

7 In ongoing work (Sportiche (1993b)), building on Collins and Thráinson (1993), I argue that we can and should go one step closer to lexical decomposition analyses in postulating that there is full clausal structure per verb and even per VP: (a) each verb has its own full clausal structure and (b) each VP (of a VP shell) projection has its own full clausal structure; there is no VP shell literally. Thus, kill $=$ cause to die corresponds to two full clauses (see Collins and Thráinson's (1993) work arguing that Icelandic double object constructions are biclausal).

8 For example. the verb die is not present in the syntax at all as such and so cannot be modified by adverbs, as in cause-to-die. Note also that, correlatively, it is also possible to have a syntactic aspect to derivational morphology exactly as in the case of inflectional morphology, a conclusion with some merits (see Valois (1991)).

9 Lexical categories are $\mathrm{V}, \mathrm{N}, \mathrm{A}$, and perhaps $\mathrm{P}$, i.e., predicative categories. I use "lexical" also in the sense of idiosyncratic, as in lexical properties of some functional head, e.g., how future Tense is pronounced in French.

10 This means, for example, that every verb is always associated with a full clausal structure, as I discuss in Sportiche (1993b). Alternatively, there may be general principles predicting which, if any, of these functional projections is not present in given contexts.

11 It appears that which one is covert (statement, affirmation) is constant crosslinguistically, a generalization that has no explanation but demands one. 
12 For example, following Cheng's (1992) suggestion, we could analyze a Chinese $w h$-phrase as [OP $w b$-word], with overt movement of the silent operator OP, much as combien movement in French combien as-tu vu d'enfants. We could then treat English wh-words the same way but with OP movement of this null operator requiring pied-piping of the entire phrase in English; in the case of Chinese $W b$ Movement this pied-piping would be prohibited.

13 Note that, strictly speaking, it makes no sense, if we reduce all movement and binding relations to one type, to say that we have reduced them to movement (or binding). They now are all the same and, if the text is correct, of the type antecedent/trace relation. Saying that they reduce to movement is saying that the driving property is a formal one (properties are licensed in Spec/head relations) rather than an interpretive requirement (anaphors must be provided with reference, variables with a range, and so on).

$14 \mathrm{I}$ am for the moment ignoring selection for the external argument, to which I will return.

15 Although this is the usual account for NP Movement in passive or raising structures, it has been considerably extended recently by Chomsky (1991), Koopman (1992), Mahajan (1990) and Sportiche (1990).

16 It is quite possible that the following extends to all syntactic dependencies. For example, although there are superficial cases of, say, $n$-ary relations, essential cases are possibly non-existent. $N$-ary relations can always be factored as a conjunction of (n-1) binary relations, each involving a distinguished element among the $n$. Thus, although who saw what when could be seen as a relation between a scope position, who, what and when, it can be construed as three relations, each between a scope position and a $w h$-phrase. One possible irreducible case is the case of a plural pronoun bound by two independent quantifiers as in Every girl told some boy that they (i.e., be and she) should leave.

17 This is instead of the usual c-command or m-command (as discussed in Sportiche (1990)). Roughly for $x \neq y, c$-command ( $x, y)$ iff the first branching constituent (or its immediate projection) containing $\mathrm{x}$ contains $\mathrm{y}, \mathrm{m}$-command $(\mathrm{x}, \mathrm{y})$ iff the first phrasal constituent containing x contains y (see Aoun and Sportiche (1981) and references therein).

18 Throughout, I will assume a version of ECP stated in terms of antecedent government and barrierhood. Part of this could be translated in an economy approach in terms of shortest steps, as Chomsky (1993) has recently suggested.

19 See Koopman (1993a) for a discussion of these questions (long-distance head movement excorporation). See also Sportiche $(1990 ; 1992 ; 1993 a)$ for the particular case of pronominal clitics.

20 A similar suggestion was made by Kayne at the 1992 GLOW in Lisbon and a more restricted version of it by Chomsky at the Irvine Lectures, Winter 1993.

21 Some important questions are left unaddressed here. The complement is really a DP, but I would claim that this is a derived property. C-selection is of an argumental category (it gets a theta role) whose lexical content is nominal, i.e., NP (nouns are predicative categories). Ds make NPs into arguments. Thus, the conjunctive requirement nominal and argument will force the projection of a DP (see Stowell (1989; 1991) for discussion of these questions). On why the $\mathrm{N}$ of a DP may incorporate into its selecting V, see Koopman (1993b), who suggests that the NP raises to $[\mathrm{Spec}, \mathrm{DP}]$ first, whence incorporation takes place. 


\section{A REDUCTIONIST APPROACH TO SYNTACTIC VARIATION}

22 This internal structure completely follows from the internal structure of the verb pour. Because it may be very roughly analyzed as the conflated verbal category cause-to-flow (like a liquid), it is actually a sequence of two verbs, each with its own subject.

23 Actually, a biclausal structure if the proposals referred to in Note 6 are taken into account.

24 If an external argument must incorporate (but cf. discussion below), the necessary existence of the higher head $Z$ entails that every predicative category (i.e., the lexical categories $\mathrm{A}, \mathrm{V}, \mathrm{N}$, and perhaps $\mathrm{P}$ ) must be complements of some functional category.

25 Recall that I in fact take conflated verbs to literally comprise two (or more) verbs and that each must project a full clausal structure. This means that in kill =cause to die, a full clause, i.e., functional projections such as $\mathrm{T}, \mathrm{C}$, and so on, should intervene between the two verbs. However, because incorporation of die to cause is obviously selected and overt, these functional projections must count as non-intervening, i.e., as defective. If for some reason the $F$ of the text was defective, we would for the same reason expect external argument incorporation to be possible. I would argue that this is exactly what happens in agentive nominalizations, e.g., killer = one who kills, which I would take to be exactly a relative clause with a defective clausal structure and an incorporated agent (see Potter (1994) for relevant discussion of related Navajo and Cherokee facts).

26 Note that recoverability of this silent complementizer is obviously satisfied.

27 Below we will see another case of silent $C$ with similar properties. Unfortunately, the distribution of the silent infinitive complementizer that is not subject to restriction (13) (viz. I tried $f_{C}$ e) to win/ $t_{C}$ e) to win is easy) prevents generalizing the bound morpheme status of silent heads.

28 This approach to adverbial adjuncts is discussed and justified in Sportiche (1993b). See Section 4.3 below for a quick summary.

29 If covert incorporation is blocked as well, the that-clause complement of whisper will have to be treated as extraposed.

30 See Koopman (1993a) for discussion of other such cases. As for the other generalization explored by Kayne (ibid) and Stowell (ibid), i e., that verbs allow silent complementizers in tense complements if they are bridge verbs, it is tempting to relate it also to $\mathrm{C}$ incorporation: $\mathrm{CPs}$ would be opaque for extraction unless the $\mathrm{C}$ can incorporate to its selecting verb.

31 For example, being a $Q$ is a syntactic property: it is coded lexically but has syntactic, i.e., structural, relational consequences, namely, scope.

32 I use this term descriptively here. There are actually two binary relations: a Spec/head relation and a movement dependency.

33 We return later to apparent restrictions concerning co-occurrence of $\mathrm{H}$ and its specifier as, for example, in cases of $W b$ Movement and doubly filled Comps.

34 The first one is probably correct for French le livre, while the second one has some plausibility for the English case of the book.

35 Abney (1987) suggests this structure for certain adjective-noun combinations.

36 Throughout I will suppose that $[+$ wh $]$ or $[+Q]$ is an optional property of certain complementizers but nothing essential turns on this. As Bhatt and Yoon (1991) discuss, the subordinating function must be distinguished from the clause type 


\section{PARTITIONS AND ATOMS}

function. English or French conflates the two in C. It is thus likely that $+Q$ is a property of an independent declarative/interrogative head.

37 Chomsky (1993) suggests that Wh Movement is always overt.

38 The alternative, closer to Rizzi's (1991) proposal, would generate the $+Q$ feature on $\mathbf{T}$ - much as Tense may be generated on the verb in VP in English, as Chomsky (1993) suggests. Raising of $T+Q$ to $C$ would then not violate Greed. But this violates PA.

39 In the first case this conclusion is supported since the tensed main verbs follow VP adverbs. In the second it is, for example, supported by have contraction:

1 John should have taught.

2 John should've taught.

3 Should John have taught?

$4 *$ Should've John taught?

5 Should John've taught?

6 Who should have taught?

7 Who should've taught?

If the modal had raised to $C$ in (7) we would expect contradiction to be impossible as in (4) or (5).

40 We return below to the question of why multiple questions with one of them moved and the other in-situ are also excluded.

41 This is because in the paradigm of $w b$-question operators it is the only one that can be silent without an antecedent and is thus recoverable. Again, the question arises why it is this particular operator that can be silent.

42 With a complication concerning que/what. See Obenauer $(1976 ; 1977)$ for discussion.

43 They propose that in languages with two morphemes, SP is adjoined to TypeP, although they leave open the possibility we suggest. For English-type languages they assume because of the conflated head - and it is essential for their analysis of V2 - that there is one projection only: CP. If necessary, some of their results could be duplicated here by distinguishing languages incorporating Type to $\mathrm{S}$ (because of recoverability or morphological boundedness) from languages $\mathrm{V}$ (or more precisely $\mathrm{V}+\mathrm{T}$ ) to Type (for the same reasons).

44 This is why we would want SP to be higher than TypeP: TypePs would then be internally identical in main and embedded clauses, SP added on main clauses only. The alternative order is compatible with the test, even if conceptually less desirable. The data on the question is contradictory. The bimorphemic languages Bhatt and Yoon (1991) discuss, show the hierarchy S > Type. So does, for example, Spanish (viz. ¿Pregunto que a quién hablaste?/"I ask that who you spoke to $=\mathrm{I}$ ask who spoke to," now analyzed with que in $\mathrm{S}$ and a quién in [Spec, TypeP]). The Germanic languages seem to suggest otherwise. For example, Dutch embedded questions allow the co-occurrence of three morphemes. Thus, corresponding to the underlined CP material I wonder who Jobn saw, it is possible to have wie of dat/"who if that," i.e., a wh-phrase, a $[+\mathrm{Q}]$ complementizer, and a "that" complementizer co-occurring. The presence of the wb-word leaves no room but to analyze of as the clause-typing particle marking the question, which thus seems higher than the subordinating particle that.

45 The same analysis could be applied to English $\varnothing /$ whether alteration if, unlike what 
we have supposed, whether also is the conflation of $S+Q$, an assumption otherwise consistent with our analysis.

46 For example, in-situ normal $w$-questions versus echo $w h$-questions (Marie a vu qui/Marie a $v u Q U I)$ and $w$-questions from $w$-exclamatives ( $Q u e l$ tableau il a peint/Quel tableau il a peint).

47 This is not to say that all of syntactic structure is relevant. For example, some syntactic information might simply be irrelevant, but then, systematically so; e.g., syntax provides for infinite embedding possibility.

48 Left unexplained so far is why pied-piping must be of the largest IP viz. *[L(que)

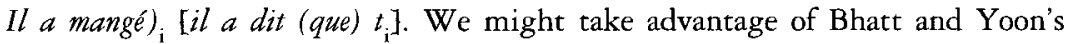
conflation idea: complementizers such as que conflate Type and S. Pied-piping a lower IP, in fact a lower TypeP, would carry the trace of que (raised to S) higher than its antecedent. This kind of situation is not allowed (it would be the equivalent of passivizing a DP whose $\mathrm{N}$ head has incorporated to $\mathrm{V}$ ).

\section{References}

Abney, S. (1987) “The noun phrase in its sentential aspect," Ph. D. dissertation, MIT.

Aoun, J. Hornstein, N. and Sportiche, D. (1981) "Aspects of wide scope quantification," The Journal of Linguistic Research 1: 69-95.

Aoun, J. and Li, Y.A. (1993) “Wh-elements in-situ: syntax or LF," Linguistic Inquiry 24: 199-238.

Aoun, J. and Sportiche, D. (1983) "On the formal theory of government," Linguistic Review 2: 211-36.

Baker, M. (1988) Incorporation. Chicago: University of Chicago Press.

Bhatt, R. and Yoon, J. 1991. "On the composition of COMP and parameters of V2," in D. Bates (ed.) Proceedings of the Tenth West Coast Conference on Formal Linguistics, Stanford: Center for the Study of Language and Information.

Borer, H. (1984) Parameter Syntax, Dordrecht: Foris.

Burzio, L. (1986) Italian Syntax, Dordrecht: Reidel.

Carter, R. (1976) On Linking: papers by Richard Carter, Cambridge, Mass.: MIT Center for Cognitive Science Lexicon Project.

Cheng, L. (1992) "On Wh-movement and wh-in-situ," Colloquium presented at UCLA.

Chomsky, N. (1965) Aspects of the Theory of Syntax, The Hague: Mouton.

- (1970) "Remarks on Nominalizations," Studies in Semantics in Generative Grammar, The Hague: Mouton.

- (1977) "On Wb-movement," in P. Culicover, T. Wasow, and A. Akmajian, (eds) Formal Syntax, New York: Academic Press.

(1981) Lectures on Government and Binding, Dordrecht: Foris.

(1986a) Barriers, Cambridge, Mass.: MIT Press.

(1991) "Some notes on economy of deriviation and representation," in R. Freidin (ed.) Principles and Parameters in Comparative Grammar, Cambridge, Mass.: MIT Press.

(1993) "A minimalist program for linguistic theory," in K. Hale and S. J. Keyser (eds) The View from Building 20: Essays in Linguistics in Honor of Sylvain Bromberger, Cambridge, Mass.: MIT Press. 


\section{PARTITIONS AND ATOMS}

Collins, C. and Thráinsson, H. (1993) "Object shift in double object constructions and the theory of case," unpublished manuscript, Harvard University.

Hale, K. and Keyser, J. (1991) "On Argument Structure and the Lexical Expression of syntactic relations," unpublished manuscript, MIT.

Huang, C.-T. J. (1982) "Logical relations in Chinese and the theory of grammar," Ph.D. dissertation, MIT.

Jaeggli, O. (1980) "Remarks on to contraction," Linguistic Inquiry 11: 239-45.

Kayne, R. (1972) "Subject inversion in French interrogatives," in J. Casagrande and B. Saciuk (eds) Generative Studies in Romance Languages Rowley, Mass.: Newbury House. (1984) Connectedness and Binary Branching, Dordrecht: Foris.

- (1989) "Facets of Romance past participle agreement," in P. Benincà (ed.) Dialect Variation and the Theory of Grammar, Dordrect: Foris.

- (1991a) "Romance clitics, Verb Movement and PRO," Linguistic Inquiry 22: 647-86.

_ (1991b) "Italian negative infinitival imperatives and clitic climbing," unpublished manuscript, CUNY.

_ (1993) "Toward a modular theory of auxiliary selection," Studia Linguistica 47: $3-31$.

(1994) "The antisymmetry of syntax," unpublished manuscript, CUNY.

Kayne, R. and Pollock, J.-Y. (1978) "Stylistic inversion, successive cyclicity and move NP in French," Linguistic Inquiry 9: 595-621.

Klima, E. S. (1964) "Negation in English," in J. A. Fodor and J. J. Katz (eds) The Structure of Language Readings in the Pbilosopby of Language, Englewood Cliffs, NJ: Prentice-Hall.

Koopman, H. (1983) "ECP effects in main clauses," Linguistic Inquiry 14: 346-51.

— (1984) The Syntax of Verbs: from Verb Movement Rules in the Kru Languages to Universal Grammar, Dordrecht: Foris.

_ (1992) "On the absence of case chains in Bambara," Natural Language and Linguistic Theory 10: 555-94.

- (1993a) "Licensing heads," in N. Hornstein and D. Lightfoot (eds) Verb Movement, Cambridge: Cambridge University Press.

- (1993b) "The internal and external distribution of pronominal DPs," unpublished manuscript, UCLA.

Larson, R. (1985) "On the syntax of disjunction scope," Natural Language and Linguistic Theory 3: 217-64.

- (1988) "On the double object construction," Linguistic Inquiry 19: 335-92.

Li, Y. A. (1992) "Indefinite Wh in Mandarin Chinese," Journal of East Asian Linguistics 1: 125-56.

Mahajan, A. (1990) "The A/A' distinction and movement theory," unpublished Ph.D. dissertation, MIT.

Marantz, A. (1993) "Implications of asymmetries in double object constructions," in S. A. Mchombo (ed.) Theoretical Aspects of Bantu Grammar, Stanford, Calif.: CSLI Publications, CSLI Lecture Notes No. 38, 113-150.

May, R. (1985) Logical Form, Cambridge, Mass.: MIT Press.

Obenauer, H.-G. (1976) Etudes de syntaxe interrogative du français, Tübingen: Niemeyer. 


\section{A REDUCTIONIST APPROACH TO SYNTACTIC VARIATION}

(1977) "Syntaxe et interprétation: que interrogatif," Le Français moderne 45: $305-41$

Pollock, J.-Y. (1989) "Verb movement, universal grammar, and the structure of IP," Linguistic Inquiry 20: 365-424.

Potter, B. (1994) "Navajo and Cherokee nominalization," unpublished manuscript, UCLA.

Ritter, E. (1991) "Two functional categories in noun phrases: evidence from modern Hebrew," unpublished manuscript, UQAM, Montreal.

- (1982) Issues in Italian Syntax, Dordrecht: Foris.

(1991) "Residual verb second and the WH-Criterion," Technical Report in Formal and Computational Linguistics 2. University of Geneva: Faculty of Letters.

Rizzi, L. and Roberts, I. (1989) "Complex inversion in French," Probus 1: 1-30.

Sportiche, D. (1983) "Structural invariance and symmetry in syntax," unpublished Ph.D. dissertation, MIT.

- (1990) "Movement, agreement and case," unpublished manuscript, UCLA, published as Chapter 3 in this volume.

(1992) "Clitic constructions," unpublished manuscript, UCLA.

(1993a) "Subject clitics in French and Romance, complex inversion and clitic doubling," unpublished manuscript, UCLA, published as Chapter 5 in this volume.

(1993b) "Adjuncts and adjunctions," unpublished manuscript, UCLA.

(1993c) "Remarks on the structural representation of negation," unpublished manuscript. UCLA.

Stowell, T. (1981) "The origins of phrase structure," Ph.D. dissertation, MIT.

- (1989) "Subjects, specifiers, and X' Theory," in M. Baltin and A. Kroch (eds) Alternative Conceptions of Phrase Structure, Chicago: University of Chicago Press.

- (1991) "Determiners in NP and DP," in K. Leffel and D. Bouchard (eds) Views on Pbrase Structure, Dordrecht: Kluwer.

Stowell, T. and Beghelli, F. (1994) "Direction of quantifier movement," GLOW 94.

Travis, L. (1984) "Parameters and effects of word order variations," unpublished Ph.D. dissertation, MIT.

Valois, D. (1991) “The internal syntax of DP," unpublished Ph.D. dissertation, UCLA.

Watanabe, A. (1992) "Wh-in-situ, subjacency, and chain formation," unpublished manuscript, MIT. 


\title{
APPENDIX: THETA-THEORY AND EXTRACTION
}

\author{
with Hilda Koopman
}

Recent work on long distance $w h$-extractions out of $w$-islands reveals two kinds of asymmetries with respect to extraction possibilities:

(a) within a language, depending on the categorial type and position of the extraction site (subject, object, NP, PP, V)

(b) across language with respect to a given position and/or category (subject in English versus subject in Italian).

In this article, we suggest that these asymmetries all result from the interaction of a single condition with independently motivated parameters of theta-role and Case assignment. This article first presents the basic condition governing extractions out of $w b$-islands, then proposes a generalization of this condition, and finally addresses more technical aspects of its integration within grammatical theory.

Recent studies indicate that it is universally impossible to $w$ b-extract from an adjunct position (cf. Huang (1982), Lasnik and Saito (1984)) or a Comp position (Rizzi (1978), Aoun (1984), Huang (1982)) inside a wh-island. The lack of typological variations in this respect suggests the following generalization:

(1) If $\alpha$ is a theta-bar-position, $\alpha$ cannot be a long $w$-extraction site.

In the first part of this article, we propose to extend this generalization into a necessary and sufficient condition:

(2) $\alpha$ is a possible $w$-extraction site (out of a $w b$-island) iff $\alpha$ is a theta-position.

This generalization captures the counterpart of (1), and seems to hold with overwhelming typological regularity, i.e. direct objects are extractable out of wh-islands. 
Prima facie counterexamples, i.e. cases of theta-positions which are not possible extraction sites, are commonplace: subjects in many languages, objects in some. We will argue that independently motivated assumptions on the structure of theta-marking and Case-marking show that these positions are always theta-bar-positions. Besides the recently argued proposals as to the adjacency condition on Case assignment (Stowell (1981)) and directionality of Case- and theta-marking (Koopman (1984), Travis (1984)), we introduce the theta-complex connectedness condition:

\section{(3) Theta-complex connectedness condition}

A theta-position must be adjacent to its theta-marker $M$ or to a theta-position theta-dependent on $M$.

\section{Some cases}

Dutch is a language for which it has been claimed that extraction out of $w h$-islands is uniformally impossible, thus contradicting (2). We will show that this is incorrect in that theta-marked PP extraction markedly contrasts with direct object extraction. The Dutch data, in conjunction with (3) suggest that direct objects are not in a theta-position. We argue that they move to a theta-bar-position because of Case. Since PPs do not require Case, the asymmetry with respect to extraction out of a $w h$-island of theta-marked PPs versus non-theta-marked PPs is the archetypal illustration of the effects of (2) in Dutch.

We extend the Dutch treatment of direct objects to subjects in the languages in which they are not extractable. Thus, we argue that the distinction between English, French or Dutch subjects on the one hand, and Japanese or Chinese subjects on the other, is the theta-bar versus theta status of the surface subject position. For example, we argue that the French sentence Jean voit Pierre has the structure in (4):

$$
\text { (4) } \left.\left[\left[_{N P} \text { Jean] INFL } \leftarrow_{X} \leftarrow_{N P} t\right] \text { VP }\right]\right]
$$

where NP is the surface subject position (in fact, [Spec, Infl] and NP* the underlying subject position meeting (3), the $\mathrm{X}$-constituent being a small clause with VP head (which appears embedded under causative verbs). This treatment will lead to a reinterpretation of the Italian data on subject extraction (Rizzi (1982)).

In the second part of our paper, we explore the obvious generalization of (3) stating that extraction out of domain $\mathrm{D}$ is possible iff $\mathrm{D}$ is a theta-position with respect to some predicate governing $\mathrm{D}$. We show that this generalization subsumes the Condition on Extraction domain (Huang (1982)) as well as various extensions of the Subjacency Condition (Chomsky (1984)).

We conclude by examining the similarities and differences between long 


\section{PARTITIONS AND ATOMS}

and short extractions. This poses the problem of the relationship between the condition we propose and the properties of empty categories and the Binding theory. In particular, we evaluate with which of the various recent alternative proposals about the Binding Theory (Aoun (1981), Chomsky (1984)) and the ECP (Aoun (1981) Chomsky (1981), Kayne (1984), Lasnik and Saito (1984)) it can be best integrated.

\section{Note}

1 This abstract was first published in its entirety as Koopman, H. and Sportiche, D. (1985) "Theta Theory and extraction," in GLOW Newsletter 14: 57-58.

\section{References}

Chomsky, N. (1981) Lectures on Government and Binding, Dordrecht: Foris.

(1984) "Barriers," manuscript, published as Chomsky, N. (1986) Barriers, Cambridge, Mass.: MIT Press.

Huang, C.-T. J. (1982) "Logical relations in Chinese and the theory of grammar," unpublished doctoral dissertation, MI'T.

Kayne, R. (1984) Connectedness and Boundary Branching, Dordrecht: Foris.

Koopman, H. (1984) The Syntax of Verbs, Dordrecht: Foris.

Rizzi, L. (1978) "A restructuring role within syntax," in S.J. Keyser (ed.) Recent Transformational Studies in European Languages, Cambridge, Mass.: MIT Press.

Stowell, T. (1981) "The origins of phrase structure," unpublished Ph.D. dissertation, MIT.

Travis, L. (1984) "Parameters and effects of world order variations," unpublished doctoral dissertation, MIT. 


\section{INDEX}

A-bar movement 109-10, 178, 275, 284

A-bar position 57, 82-3, 189; and clitics 286, 288, 289, 290, 291, 294, 295, 299; distinctive properties of $76,288-9$; head position as 190 ; and L-dependence 131; and object agreement 145-6; [Spec, ASPP] as 79-82; and subject position 77, 78-9; and Theta Theory 93-5

A-chains, Case-marked 164

A-movement 110, 173, 191, 214-15, 281, 395; dependencies 400-1

A-position 57, 82-3, 129; and Case determination in NP movement 190; distinctive properties of 75-6, 288-9; head position as 190; and L-dependence $131 ; \mathrm{NP}^{\wedge}$ as $76-9$; and object agreement 145-6, 167; [Spec, ASPP] as 79-82; and Theta Theory 93-5

Abney, S. 206, 216, 400

absorption 410,411

accusative Case 8, 331; and agreement $162-4$; assignment of, by verb 182 , 187; clitic 275, 276, 286-8, 289, 309 ; and inheritance 176 ; $l e$ as 371 ; and Scrambling 291-3, 298; and se constructions 201, 202-3; SSC effects of 191-205; structural vs inherent 193; surface position of objects in English 170-2, 173; surface position of objects in French 168-9; see also objects accusative voice 298

adjectival small clauses 346-7, 349-56

adjectives: and adjunction 399-400; agreement of 346; 'argument' 227, 229; possessive, as clitics 225

adjunct condition 118

Adjunct Projection Principle 20-1, 22, 24

adjunct small clauses $368-72$

adjunction 104-5; cannot trigger

agreement 81 ; elimination of 399-400; and incorporation into head 111-13; reductionist approach to 12 ; theory of 26,322 ; in VP small clauses 365

adverbs 5, 112; and adjunction 399-400; floating Qs as 20-3 affixes 189-90, 322-3, 381-2; agreement 102; applicative 185 ; Class L 183, 185; hopping 382; inflectional 102, 324; -ing 182, 183 , 185, 186; and lowering 325, 382-3; types of participial 185

agreement 7, 389; adjectival 346; and anti-movement constructions 173-4, 176-8; in Arabic 52-3, 174, 177; and Case 8-9, 158-215; and Case assignment 59-61, 67, 77, 78; and clitics 265, 296; and en 208-13, 220-1; and head and phrasal movement 187-90; and inflection 102; and inheritance 173-81; and internal structure of VP 167-87; in Kilega $79-81$; in middle 


\section{N D E X}

constructions 363 ; and restructuring 281-2; and Scrambling 298-9; and se 196-8, 199, 200; and surface position of NPs 168-87; see also object agreement; participle agreement

Agreement, Theory of 91, 146 agreement transmission 177-8

AGR 3 3, 8, 9, 71-3, 213-15, 381

AGR $_{s} 8-9,330,381$

AH see Auxiliary Hypothesis

all see tous

allomorphy 324-5, 382, 383, 396

alternation 386

anaphors $24-5,30-2,37,38,196$, 399; binding 388,412

antecedent government 390, 392, 394, 396

anti-movement constructions $173-5$, $176-8,180$

Aoun, J. 253

APs 346-7, 352; preposed 99; small clause structure 74-5, 98, 99, 101

Arabic: agreement 52-3, 174, 177; Case assignment 58-9, 60, 61, 78; clitics 252; Lebanese 268, 273; $\mathrm{NP}^{\wedge}$ as A-position 76, 77; surface position of external argument 172-3

argument adjectives 227, 229

arguments: generated as specifiers 401 ; and head/head relations $393-4$; postposed or preposed 395; and Projection Principle 21; see also external arguments; internal arguments

article: indefinite plural 207-8, 212; partitive 208, 212-13

ask $402-3,405$

aspect $48,63-4,357$

auxiliaries: aspectual 357 ; do 77-8, 403; English 33-4; in participial small clauses 356-60; and participle agreement 146-7, 149-51, 152, 157-8, 258; and Pesetsky's theory on $\mathrm{NP}^{\wedge} 77-8$; semantic properties 48; and VSO languages 51; see also avoir; être

Auxiliary Hypothesis 77, 78
AuxSVO languages 51, 103

avoir 146, 149-50, 151, 357-8; derivation 384; and sembler 365; see also auxiliaries

Baker, M. 394

Bambara 73,92 ; objects in $68,69,72$, $97,173,174,176$

Bantu languages 191; see also Kilega

Barrier Theory 6, 60

barrierhood 105-7, 112, 133; and extraction 121, 130; and object 194; revised definitions $195-7,200-1$, 215,218

base-generation analysis: of clitics 246-7, 251-4, 259-64, 270-1; of floating Qs 31-3, 36

believe 135

Belletti, A. 24, 25, 161, 179, 180, 186,373

Bhatt, R. 407

biclausal structures 366,367

binding, local 136

Binding Theory 74-5, 97-8, 113, 156, 422; and anaphors 399; and clitics $254,282-5$; and overt I to $\mathrm{C}$ in CI constructions 334-5; Principle A 90, 135, 155, 254-5, 285; Principle C $155,160,202,284,285,335$; and small clauses $346-7$

Borer, H. 379

Bouchard, D. 362

Brandi, L. 312, 313, 325

Burzio, L. 154, 395; Generalization 186-7, 192, 201

c-command 112, 113, 283

c-selection 388, 391, 392, 393-5

canonical position 44

Case: adnominal clause 119-20; and agreement 8-9, 158-215; for $\mathrm{DP}_{\mathrm{s}}$ 389 ; inherent $59,102-3,162,164$, 219; objective 59, 103-4; relative clause 119-20; see also accusative Case; datives; nominative Case; structural Case

Case assignment: agreement vs government 59-61, 62-3, 67, 77, 


\section{NDEX}

$78,103-4,162$; in Bambara 68; and Complex Inversion 325; and double subject problem 331-2; governed, and barrierhood 106-7; and infinitives 64-6; Infl and 58-64, 77, 103; and se constructions 201-5; and subjects 58-61; structural vs inherent $59,102-3,162,219$; by verbs 182-5, 186-7; and wb-extraction 420, 421

Case checking 175-6, 177-8, 181

Case Filter 102; violations 98, 99

Case inheritance 175-8

Case marking 219

Case Theory 36, 91, 98, 102-4, 272-3; and double subject problem 331; and modified idea of government 113 ; and raising 56-7, 58

Case transmission 178

Catalan 157-8, 168, 205, 274

causatives 137,185

CCL see Condition on Chain Link

CED see Condition on Extraction Domain chain formation $175,176,177,178$, 179

chaque/chacun 17, 19-20, 36

checking approach 382,384

Chinese 4, 26, 129, 399, 411, 412, 421

Chomsky, Noam 1, 2, 9, 12, 276; A/A-bar theory 75-6, 79, 83, 93-4; on affixes $322-3,382$; and Barrier Theory 6; on Case checking 175-6; Economy proposals 12,403 ; on extraction 126; on intervention 106-7; on movement 104-5, 387, 389,395 ; on multiple questions 410 ; on object agreement $71-2$; on parasitic gaps 285,287 ; on participle agreement 213, 214; Principle of Least Effort and Reading 197; proposal of morphological checking $270-1$; theory of adjunction 26 ; theta theory 49; Uniformity Condition 134; X-bar theory 91; see also Principle of Greed

CI see Complex Inversion

Cinque, G. 227, 268 clause boundedness effect 279, 281

clauses: embedded 51, 78; main, always IPs 78; 'necessary' vs 'contingent' 385; non-finite 57, 64-6; structure 47, 364-7; see also small clauses

clitic climbing $271,278,279-81$

clitic constructions 10-11, 309; left-dislocation 268, 297, 309, 409; parameters 267-8; participle agreement in 273-6; reflexive vs non-reflexive 266

Clitic Criterion 267-8, 272, 289, 294-6

clitic doubling 252-3, 264, 268, 271-3, 276, 285, 295, 296-7, 298, $299,309,316,336$

clitic movement 7-9, 91, 137-40, 264, 278, 279; in Dutch 122; and genitive en 140-3; as head movement 274-5, 279

clitic placement 139, 254, 256, 260, 264, 291; in French 128; and head movement 278-82; and locality 294; movement analysis of 258-9; and object agreement 144-5; stranding under 255

clitic projections 268-71, 295, 296, 298,309

clitic voices $264-71,293,296,297$, 298, 299

cliticization: and adjunct predicates 368-72; and complements 158-9; of direct object 143; and extraposed elements 352; morphological 323-4; and quantitative/partitive en 205-6; in reflexives $360-1$; syntactic 323 ; typology 323-5

clitics 244-307, 381-2, 388; and argument XP* (analysis) 264-82; and Binding Theory 282-5; complementarity 253-4; definition 310; dont 132-3; and Dutch Scrambling 291-4; en 128, 131, 140-3, 149, 161, 201-2, 205-13, $260,284,285,352$; as heads $137-8$, 139, 265, 278, 295, 296, 299, 312, 313 ; in infinitives 249 ; leftward, in French questions 409; leur 352; lui 


\section{N D E X}

255, 276; in non-imperative clauses 249 ; movement vs base-generation analysis 251-64; object 138-9, 291-3, 310-11, 315, 321-2; and parasitic gaps 275, 276, 285-8; and participle agreement 205; phonological 318-19; possessive adjectives as 225; postverbal 311 ; problems of analysis 244-7, 249-51; pronominal 69, 169, 244-6, 248, $282,310-11,351-3,354$; reflexive $11,193,361$; se $146-7,152-6,202$, 203-4; subject 248 ; syntactic 248 ; templates for 247, 310; types of 137; and Weak Crossover 289, 296, 297-8; see also predicate clitics; reflexives; subject clitics

COC see Condition on Chains coindexation: and clitics 196, 197, 198-9, 200, 202, 266

combien 350

comparatives $351-2,370$

complementarity 253-4, 263; distributional 314-16

complementizers 51, 326, 327-8, 407; quelqui rule 359 ; silent $196-7$, 396-7, 402

complements: and incorporation 111-13, 114-15; and participle agreement 158-60

complex forms 329-30, 382-3

Complex Inversion 138, 248, 271, 308, $314,316,325-35,336,406$ complex NP constraint 119-20 concatenation $323,324,325,333,382$, 383,398

Condition on Chain Link 7, 9, 107, 111; and se constructions 196, 197, 199; and super raising 136; and Wh-movement 117,121

Condition on Chains 154-5

Condition on Extraction Domain 90, 124, 421; and clitics 255-7, 276; and $W b$-movement 117-21

conflation 382-4, 395, 396-7, 407

conjunction: SCLs and 316-19

contingent 403

control structures 371
Coordinate Structure Constraint 317-18

Cordin, P. 312, 313, 325

constituency 342-3, 344

constituent coordination 344

count/mass distinction 212

Cortes, C. 157

CPs: adjectival small clauses as 354-6; le pronominalizes at $356,359-60$, $361,365,367,369,371$

CSC see Coordinate Structure Constraint

datives 299; and clitics 290-1, 309, 353-4; ethical 259-60; and participle agreement 158-60, 164; and Q-stranding 263-4; and Scrambling 293-4

de $127,132,219$

de-complements 158,160

definiteness effects $152,175,180$

degrees 349-51, 354-5

delaying 275-6

demonstratives 126

do (auxiliary) 77-8, 403

do-support 185, 186

domain 399, 421; see also Condition on Extraction Domain

domination 106

dont 132-3

Doubly Filled Projection Prohibition 411

DP hypothesis 206-7, 216, 220

DPs 337; and clitics 283-5, 290, 296, 299; in Complex Inversion 308 ; indefinite vs specific 336; and participle agreement 274; in participial small clauses 357-8, 359; problems of extraction and movement 221-6, 256-7; pronominal vs non-pronominal 330-1; and Q-stranding 4, 5, 261, 262, 264; silent vs overt 332 ; syntactic dependencies 389

Dutch: agreement transmission 177; Case assignment 61, 104; clitics 268, 296; extraction 121-3, 421; NPs as specifiers 92 ; objects $69-70,72,73$, 
IN DEX

97, 176, 193; overt incorporation 371-2; postpositions 123; restructuring 366; Scrambling 4 , 268, 288, 291-5, 298, 299

Dynamic Minimality 7

each 17-18, 19-20

ECM see Exceptional Case Marking

Economy/Minimalist Program 1, 12 ECP see Empty Category Principle E/M see Economy/Minimalist Program Empty Category Principle 369, 390, 396

en: 260, 284, 285; extracted 226; genitive $128,131,140-3,149,161$, 201-2, 220-1, 285-6; quantitative/partitive 205-13

English: accusatives 169, 170-2, 176, 193; affix hopping 178, 322; AGR 381; agreement transmission 177 ; anaphors 399; Case assignment 59, $61,62,78,104$; comparative constructions 351-2; double object constructions 191-2; eats/eatlate 382, 383; extraction 123-6; floating Qs 33-6; future of the present morpheme 381; gerunds and PRO 67; head movement and agreement 189; I to C 326, 327; Infl 52, 103; L-dependency 133; $\mathrm{NP}^{\wedge}$ as A-position 76, 78, 79; questions 331, 402-6; requires S-Structure Case checking 180 ; silent complementizers in 196-7; silent morphemes in present tense 342 ; [Spec, ASPP] in A-position 82; subject position 44-5; surface position of objects and subjects 169 , $170-2,173 ; w b$-island violations 121 equative structures 372 ergative constructions 28, 35 EST see Extended Standard Theory être 146-7, 149-51, 258, 356-8; with adjectives 349-54; in se constructions $147,152,362$; see also auxiliaries

Exceptional Case Marking 35, 57, 65, $103-4,135,219$ exclusion 106

excorporation 116

expletives: and agreement $177-8,180$; and predicate clitics 373 ; replacement 178,179

extended projection 93, 95, 363, 366

Extended Standard Theory 1, 44

external arguments: and incorporation 394-5; se treated as $193,195,196$, 198, 202; and small clause structure 100-2; and VPs $183,185,186-7$

extraction: and clitics 255-7; out of adjuncts $370,371,372$; out of NP 125-8; out of PP 121-5; out of subjects 129-33; and structure of NPs 220-6; subject, and tole of NomP 335-7; and theta theory 420-2; and wh-movement 117-21; see also Condition on Extraction Domain; wh-extraction extraposition constructions 372-4

Fillmore, C. 47

floating quantifiers 4-5, 16-43; adverbial analysis 20-3; anaphoric properties 24-5; in English 33-6; and Infl as raising category 53 ; leftward movement 24-7, 32-3, 37; linear distribution 18-23; modified 18; overlap with partitive 31,32 ; relation with $\mathrm{NP}^{\wedge} 23-7,82$; rightward movement 25-6, 27-31, 33,53 ; semantic analysis 37 ; and subjects in V 19-20; suitable NPs for 27-31; transformation vs base-generation analysis $31-3,36$

Focus 412

French: adjunct adverbial clauses 118; AGR 381; agreement transmission 177, 180; anti-movement constructions 174, 175; Case assignment 61, 331; CIP prohibition 315; clitic templates 247,310 ; clitics 11-12, 137, 138, 140-3, 146-7, 244-9, 252, 268, 270, 271, 276, 279, 291, 292-3, 295, 296, 381 ; clitics, predicate $342-78$; clitics, subject 308-12, 313-37; 


\section{N D E X}

comparative constructions 351,352 ; conditions allowing CI 333; conjunction of VPs disallowed 317 ; extraction 126-8, 129, 256-7, 421; floating Qs 4, 17, 18, 20, 23-33, 35-6; future of the present morpheme 381; I to C 327-8, 331, 333-5; idioms 55; impersonal constructions 186; indirect object reflexive 193; inheritance 176 ; interpretive adverbs 20 ; lack of definiteness effect 180; lack of inherent accusative 193; licensing 300; movement 189, 222, 262; negative quantifier $399 ; \mathrm{NP}^{\wedge}$ as A-position 76; object agreement 143-6; P-stranding 125, 255, 257; participle agreement 70-1, 72, 73, 81, 146-56, 158-67, 205, 257-9; passive constructions 28,35 ; pied-piping 13; possessives 224-5; NomP projection 332; questions 406-11, 412; raising 269-70, 309; replacement of postverbal SCLs 330; restructuring 366; silent DPs 332; silent subjects 319 ; subject condition 131-3; subject inversion in NPs 230 ; subject pronouns 310-11, 317-19; surface position of objects and subjects 168-9, 172, 173; $\mathrm{V}$-movement $51 ; w b$-island violations 121

Generalized Licensing Criterion 295, 296, 300, 398, 401, 411

Generative Linguist of the Old World $3-4$

genitives 140-3, 221-2, 227-9, 289; en $128,131,140-3,149,161$, 201-2, 220-1, 285-6

gerunds $67,182,185$

GLC see Generalized Licensing Criterion

GLOW see Generative Linguist of the Old World

government: antecedent 390, 392, 394, 396; and barrierhood 106-7; and Case 58-61, 62-4, 65-6, 67, 77, 78; head 391, 392, 396; and i-command 112-13

Government, Theory of: linked with Movement Theory 88-91, 107-10

Government Transparency Corollary 7

Greek 253, 297

Guéron, J. 161

Gur languages 22

Haik, I. 205, 206

Hale, K. 173, 383-4, 394

head/complement relations 391, 392

head government 391, 392, 396

head/head relations 390-1, 392-7

head movement $88,110-16,187-91$, 390; and anti-movement constructions 178; and barrierhood 105, 200; clitic movement as 274-5, 279; and clitic placement 278-82; and complex forms $329,382-3$; and excorporation 116; and genitive en; I to $\mathrm{C}$ as 327 ; impersonal constructions 180-1, 186; and incorporation 111-13, 114-15; and phrase/phrase relations 391; successive 115-16; and syntactic incorporation 323

Head Movement Constraint 90, 110-11, 115, 390, 392; and barrier theory 200; and clitics 269,271, $275,280,321,332$

head/phrase relations 392,397

heads: in A/A-bar positions 190; agreement on 190; and Case assignment $60-1$; clitics as $137-8$, 139, 265, 278, 295, 296, 299, 312, 313 ; covert vs overt 318 ; $e n$ as 206 ; functional, and syntactic variation $380-2,384-8$; grammatical vs interpretive 380-1; inflectional 379-80; inversion of adjacent 332; necessary vs contingent $385-6$; and postverbal SCLs 329-30; silent vs overt 381-2, 385-8; 'strong' vs 'weak' 330, 387

Hebrew 252, 261, 326

Hindi 298

Hoekstra, T. 161 


\section{N D E X}

Huang, C.-T. J. 88, 97, 99, 346 Hungarian 216

I to $\mathrm{C}$ : in postverbal nominative constructions 326-8, 333-5 i-command $60,112-13,143,187,389$ idiom chunks 54-5, 367-8

il 329,332

impersonal constructions 204, 208-10, 372-3

inclusion 106

incorporation 111-15, 331, 383, 392-5, 396-7, 402; in Dutch small clauses 371-2; external argument and 394-5; syntactic vs morphological/lexical 323, 325, 333, 383-4

infinitives 64-6

Infl: as Case assigner 58-64, 77, 103;

Case-marking, in Irish 173; infinitival 65; as a raising category 45-58; and subject clitics 312; tenseless 66, 67

inflection 379-80, 382; and agreement 102; and clitics 309

inherent constructions 198, 199, 203, 259-60

inheritance 173-81

internal argument: and se 198-9, $202-5$

interrogatives see questions intervention 105-6, 133

intonation 408, 409

intransitive constructions, impersonal 209

inversion 35-6; stylistic 406; see also Complex Inversion; Subject Clitic Inversion

Irish 51-2, 67, 180, 326; agreement 173, 174; Case assignment 58-9, 61

Italian 121, 173, 174, 175, 205; auxiliary selection 358 ; clitics 169 , 268, 274, 279, 280, 287, 297; clitics in northern dialects $312-14,315$, 316-17, 318, 319, 324, 336; extraction 128, 129; Fiorentino 324-5; inversion 35; lack of definiteness effect 180; participle agreement 157, 168, 274, 282; restructuring 366 ; se constructions 362 ; silent expletives and agreement transmission 177, 180; silent subjects 319-20; subject inversion 230; Trentino 312-14, 315, 316-17, 318,381

Jackendoff, R. 22

Jaeggli, O. 253, 313

Japanese 4, 26, 399, 411, 421

Kayne, R. 359, 384, 386-7; on clitics 137-8, 248, 252, 253, 254, 264, $272,273,274-5,278,279-80$; on participle agreement $8,143,160-1$, 213; on predicate clitics 348-9, 353-4, 357, 358, 359, 360, 363, 364,374 ; on subject clitics 310 , $317,322,331-2,337$

Keyser, J. 383-4, 394

Kilega 22, 79-82, 189

know 402-3

Koopman, H. 7, 8-9, 125, 172, 261, $292,298,367,373,392,396,404$

L-dependence 130, 131, 133

L-marking 101-2, 105

L-syntax level 384

landing site problem 325, 326-30

language groups: agglutinative/analytic 394; AuxSVO 51, 103; Bantu 191; Class 1/Class 2 45, 129; Gur 22; head initial/head final 386-7; Mande 68; Malayo-Polynesian 79; VSO 51-2, 103, 394; see also individual languages

language structure 50-1

Larson, R. 90, 91, 100, 181, 192, 405-6; on NPs 216-18; Theory of VP Shells and Adverbial Modifications 7, 343, 344, 366-7, 384,401

Lasnik, H. 136, 196

le (predicate clitic) 348-56, 359-60, 364, 365, 369, 371; idiomatic $367-8$

Leftward-tous rule 262 


\section{N D E X}

leur 352

lexical decomposition 384-5

lexical variation $379,380-1$

lexicon $54,383,385$

LF movement 276

licensing: Case 294, 296; generalized Spec/head 397-9; and I to C 327; PG 288; and postverbal SCLs 329-30; and predicate clitics 353; projection 296; specificity 294-5, 296; uniformity 299-300; see also Generalized Licensing Criterion licensing condition 5, 273

Like-Attracts-Like Constraint 26 local binding condition 136 locality 49, 390-1, 392; constraints 278 locatives $158,159,160,290,356$

Long Object Preposing 281

lowering $53-4,323,324,325,382-3$

lui 255,276

M-command 104

McCloskey, J. 173

Mahajan, A. 9, 298

Mande languages see Bambara mass see count/mass distinction May, R. 105-6

middle constructions 208,362 ; and se 198, 199, 203, 204, 361, 363

Milner, J.-C. 205, 206, 207

Minimalist Program see

Economy/Minimalist Program

Minimality effect 279

Mirror Principle 269, 270, 325

modifiers 20-1, 22

modularity 300

Mohammad, M. 53, 172-3, 177

Moore (Gur language) 22

morphemes, silent 342, 380, 382, 384; syntactically bound 386

morpholexical incorporation 383-4

morphological cliticization 323-4

morphological incorporation 323,325 , 333

morphology 308-9; conditioned epenthesis $324-5$

morphophonology 383

movement 388; dependencies 12 ; in
DP 221-6; object 8, 72; overt vs covert 291, 327, 386-7, 399; and phrase/phrase relations 397, 398-9; and small clauses 345-6; syntactic 107-36; and syntactic dependencies 389-92; verb 51-2, 325; see also A-bar movement; A-movement; anti-movement constructions; clitic movement; head movement; NP movement; $w$ b-movement

Movement, Theory of 6-9, 98, 137, 143,191 ; and A/A-bar positions 76; combined with Agreement Theory 146; and projections 104-5; unified with Government Theory 88-91, 107-10

movement analysis: and clitics 246 , 251-69, 271; and floating Qs 31-3, 36

negation: and Case assignment by Infl 64; and clitics $248,249,262-3$, 270, 284-5, 291, 292, 293, 295; and floating Qs 28-9, 32; as raising category 48

neutral constructions: and se 198, 199, 203

nominals 229; -ing affix 182, 183

nominative Case 331; and agreement 103,162 ; governed 77 ; as structural Case 59, 102-3; surface position 172-3

NomP projection: and subject clitics 335-7

nouns: and complements 140,141

NP movement 88, 133-6, 400-1; and A-position 76, 78, 94-5, 99; and AGR projection 213, 214-15; and agreement transmission 178; and $\mathrm{AGR}_{\mathrm{o}}$ 73; Case determined by highest A-position 190; and clitics 258-9, 278; and coindexed se 196-7; and floating Qs 28, 31, 35;

forbidden out of tensed clause (French) 262; passive 134-5; raising 135-6; most restricted type of movement 259; Sportiche's theory of 82,90 


\section{IN DEX}

NP-trace 27-8, 29, 31, 35, 36 NP* 16; covert vs overt 35-6; in non-movement situations 179 ; properties, and relation with VP 67-75; as [Spec, VP] 97; in there constructions 180

NP^ 75-83; as A-position 76-9; relation with $Q 23-7$

NPs 140; and agreement 162-3; Case-marking 219; and extraction 125-8, 129, 220-6; and floating Qs 18, 19, 27-31; Larsonian 216-18; postverbal, in impersonal constructions 204, 209; as specifiers 92; structure of 216-30; surface position of nominative and accusative 168-87; word order in 226-9; see also complex NP constraint null hypothesis $359,367,374,408$ null subjects 319-20

numbers: and extraction from NP 126; syntactic dependency 389

object agreement 70-1, 143-6;

A/A-bar status 145-6; and inheritance 176 ; lack of participle agreement 158-67; and reflexives 193-4; structural Case and 162-7; and surface position of accusative $168-72,173$

object constructions, double 172 , $191-2,193,366-7$

object movement 8,72

objects: in Bantu languages 191; clitics 138-9, 291-3, 310-11, 315, 321-2; and control structures 371 ; direct, and agreement 194-5, 196; in middle constructions 204-5; preposing 280-1; and raising verbs 192-3; and reflexives 193-5, 202; Scrambling 4; in spec VP 68-71; surface position 183; see also accusative Case; Agro

of 126

on (French) 317

P-stranding 118, 124-5, 131, 255-6; in Dutch 69, 121-3; in English 125
Paradigmatic Uniformity 403-4 parasitic gaps 275, 276, 285-8, 299 particle constructions 125 participial small clauses 356-63 participle agreement $7,8,70-1,72$, $73,81,144,145,146,205$; and AGR projection theories 213-15; and auxiliary selection 150-1; in Catalan 157-8; and clitics 257-9, 273-6, 278, 281-2; and head movement 189; in Hindi 298; impossible with postverbal accusatives 347-8; lack of, with objects 158-67; in passive constructions 147-8, 151, 154, $164-5$; in reflexive constructions $153-6,361$; in se constructions 152-3, 204, 362; skipping and delaying 275-6; subject 146-58; in unaccusatives 148-9, 358

participles: affixes 185; morphology 181-6, 200; see also participle agreement

passive constructions $203,208,363$; and floating Qs 28, 35; morphology 185 ; and NP movement 58, 134-5; and participle agreement 147-8, 151, 154, 164-5; and raising verbs 192

Pesetsky, D. 76-9

peut-être 320, 321, 328

phonology 409

phrase/phrase relations 391,392 , 397-401

pied-piping 13, 98-9, 277-8, 409, 411

PISH see Predicate Internal Subject Hypothesis

polarity items: licensing 388,391 , 409-10; negative 412

Pollock, J.-Y. 179, 269-70, 271

Portuguese 61, 173, 174

possessives 222, 224-6, 367

postmovement structures 31

postpositions, Dutch 123

PPs: extraction out of 121-5, 255-6; instrumental 69-70; extraposed $352-3$; theta-marked, and extraction 421

predicate clitics $11-12,342-78$; 
IN DEX

adjunct small clauses 368-72; avoir/être 356-60; clausal structure 364-7; in impersonal constructions 372-4; le 348-56, 359-60, 367-8; participial small clause 256-63; and raising verbs 372 ; reflexives $360-1$; structure of small clauses 343-8

Predicate Internal Subject Hypothesis 344-5; see also VP-Internal Subject Hypothesis

predicates, non-monadic 395

preposing: and small clauses 98-9, 344; VP 346; see also Long Object Preposing prepositions: Dutch 121-3; similarity to particles 125 ; see also P-stranding Principle of Greed 327, 328, 329-30, 403, 404

Principle of Least Action 197, 224

Principle of Least Effort and Reading 197

PRO: distribution of 66-7; and floating Qs 27-8, 29, 34-5 pro 66-7

pro-drop constructions 266-7

probably 20,21

proforms, subject 310-11

projection: AGR and participle agreement 213-15; extended 93, 95, 363,366 ; licensing 296; and movement 104-5; NomP 335-7; in participial small clauses 357-8; see also Adjunct Projection Principle; clitic projections

Projection Principle 57, 168, 185, 251, 379

pronominal binding 388

pronominals: as clitics 69 ; idiom chunks as $367-8$

pronouns: attraction to specifier position 172; clitics as $282,310-11$, $351-3,354$; featured and nonfeatured $66-7$

property 401-2

Q Criterion 267, 401

Q-float see floating quantifiers

Q-lowering 53-4
Q-movement constructions 172

Quantifier Raising 26, 262

quantifiers: determiner 17, 19-20, 32; in en constructions 209-10, 211; non- $w b$ non-negative 412 ; partitive 17-18, 19, 31, 32; postverbal 28, 35,36 ; separable 209, 210-12; split constructions 388 ; and there vs impersonal constructions 180-1; unselective 210; see also floating quantifiers; stranded quantifiers quantity operator $350-1$

que 326,328

quelqui rule 359

questions $316,320,326,401-12$; embedded interrogatives 326 ; English 402-6, 407; French 330, 406-11, 412; multiple 410-11; yes/no 330, 404-7, 408-9; see also wh-questions

raising 48, 322; and agreement 150-1; and Case theory 58; and idiomatic le 367-8; and Infl 45-58; and non-reflexive se 361-3; and NP movement 135-6, 259; to object $170-2$; in postverbal SCL constructions 332-3; and predicate clitics 372; and small clauses 99; to specifier 171-2; super 136; verbs 45-8, 49, 150-1, 192-3, 269-70, 271,332

Recoverability Principle 385-6

reflexives 11, 202, 358, 360-1; indirect object 193-5; and participle agreement 152-6; se 193, 195, 196, 197, 198-9, 201, 202-3, 266

relations: heterogeneous 391-2; homogeneous 390-1

restructuring 279-82, 366

Riemsdijk, H. van 121-2

Rizzi, Luigi 154, 169; on clitics 267, $280,287,290,295$; on quelqui rule 359 ; on subject clitics 312,313 , $317,319,325,326,331,334$

Roberts, I. 325, 326, 331, 334

Romanian 252, 253, 268, 296, 297, 335 


\section{N D E X}

root phenomena 326-30

Ruwet, N. 156

s-selection 388, 390-1, 392, 393-5

S-Structure chain 177, 180, 285

SCH see Strong Correlation Hypothesis

Schlonsky, U. 261

Schneider, Pat 253

SCI see Subject Clitic Inversion

scope 388, 397, 401

Scrambling 11, 297, 309; and agreement 298-9; dative 293-4, 295; in Dutch 268, 288, 291-5, 298, 299; and instrumental PPs 69, 70 ; and locality 294

se: and Case 201-5; clitic 146-7, 152-6, 202, 203-4; in middle, neutral and inherent constructions 197-200, 203; non-reflexive 361-3; reflexive $193,195,196,197-200$, 202-3, 266

seem 45-7, 364-5

semantic structure: correlation with syntax 5-6, 47-8, 190

sentential subject condition 119

sentential subject constraint 129

SI see syntactic incorporation

si 407

sign/meaning problem 1

silent categories: $A_{G} / A_{0} R_{s} 381$; complementizers 196-7, 396-7, 402; morphemes 342, 380, 382, 384, 407; whether (French) 407 sisterhood 21, 37, 49, 50, 67, 93 skipping 275-6

small clauses: adjectival 346-7, 349-56; adjunct 368-72; AP 74-5, 98, 99, 101 ; clitics in 137; as CPs 354-6, 370 ; and existence argument 50; external arguments and 100-2; and $l e$ clitic 365; in middle constructions 204; NP 101; with overt subjects 98; participial 356-63; and raising to specifier 171; and silent morphemes 342-3; structure of $95-102,343-8$; subject governed from outside 114 ; VP 96-100

sound/meaning problem 1
Spanish: clitics 252, 253, 268, 272, $273,279,296,297-8,299,335$, 336 ; and participle agreement 151 , 157

specificity 294-5, 296, 297, 335-6

Specified Subject Condition effects: of accusatives 191-205; and clitics 254-5

specified subject constraint 349

specifiers: restrictions on 224-6

SSC see Specified Subject Condition Stowell, T. 7, 50, 343, 344, 392, 396

stranded quantifiers $4,5,37$; in clitic constructions 276-8, 315-16; and dative constructions $263-4$; in L-tous cases 260-3; and small clauses 345 , 348,355

stranding: in le clauses 364; see also $\mathrm{P}$-stranding; stranded quantifiers

Strong Correlation Hypothesis 162; and accusative $162-4$

structural Case 59, 72, 102-3, 162, $164,219,331,380$; and agreement 162-7, 173-81; and anti-movement constructions $173-5,176-8$; assignment of $182,186-7$; and inheritance 175-8; and internal structure of VP 167-87

Subjacency Condition 90, 421; and wh-movement 117-21

'subject across categories' proposal 7

Subject Clitic Inversion 308, 320-5, $328,406,409$

subject clitics $11,308-37$; complementarity of distribution 314-16; and conjunction 316-19; double subject problems 330-3; lack of overt I to C 333-5; landing site problem 326-30; paradigmatic regularity and null subjects $319-20$; postverbal nominative 320-35; preverbal 313-20, 324; proximity problems 321-2; subject extraction and the role of NomP 335-7; syntax/morphology interaction 322-3; in Trentino Italian 312-14, 315, 316-17, 318, 381; typology of cliticization $323-5$ 


\section{N D E X}

subject condition 119; in French 131-3 subject extraction 335-7

subject pronoun clitics 310-11, 317-19

subjects: and Case assignment 58-64; clausal 129; distribution of PRO 66-7; double 330-3; extraction out of 129-33; and infinitives 64-6; Infl as a raising category $45-58$; move out of small clauses 345-8; and participle agreement $146-58$; position of 44-87; properties $55-6$; properties of NP* 67-75; silent 319-20; surface position of nominative 172-3; in Welsh 61, 62-4; see also VP-Internal Subject Hypothesis

substitution 104-5

Suñer, M. 336

suppletion 383

suppletive forms 323

surface position: of accusative objects

168-72; of nominative subjects 172-3; of objects in participial VPs 183

surface structure words 381

syntactic cliticization 323

syntactic concatenation 324

syntactic dependencies $12,388-92$

syntactic incorporation 323,383

syntactic structure: correlation with semantics 5-6, 47-8, 190

syntactic variation $12,379-419$; restrictive boundary conditions 379-88; theories 379-81

syntax/phonology interface 409

Tellier, C. 286

tense: affixes 185; Case assigning properties 185, 227; and clitic projections 270-1; complement structure of $364-5$

tenseless clauses; and clitic projections 270,271

there constructions 179-80, 367

theta assignment 148-9, 401

theta-complex connectedness condition 421
Theta Criterion 185, 227, 393

theta grid 392

theta-marking 101

theta role: and floating Qs 31, 35; in inherent clitic constructions 260; and reflexive se 155-6; and seem 45-6

theta theory $28,93-5$; and extraction 420-2; and NP movement 135; and raising categories 49,50

topicalization 34 tous $261-3,277,315-16,345-6$; as floating Q 17, 18, 23, 24, 25-6, $27-33,36$

traces 36, 395, 396; see also NP-trace; wh-traces

transitive constructions 209,358

Turkish 335

unaccusatives 183 ; absence of DP* projection 363; cannot assign accusative 182; impersonal 208; participle agreement in 148-9, 151, 152, 156, 164-5, 358; and predicate cliticization 372-4; reflexive constructions as 362

unergatives 358,373 uniformity 332, 342, 389-90, 403-4

Uniformity Condition 134

Universal Base Hypothesis 387

Universal Theta Assignment 148

$\mathrm{V}$ movement $51-2,325$

V2 effects 327: in German 331

Valois, D. 227, 229

Vata $51,125,318$

verbs: aspectual 48, 49; Case assignment by $182,186-7$; causative 137; conflated 382-4, 395, 396-7; ECM 135; intransitive 182, 186; lexical decomposition 383-4; manner-of-speaking 396-7; non-derived 183; raising 45-8, 49, 150-1, 192-3, 269-70, 271, 332; 'S-bar deletion' 135; transitive 165-7; unaccusative 148-9

Vergnaud, J.R. 159-60

VP-Internal Subject Hypothesis 2-6, 7, 57, 90, 181, 186, 344-5; and AGR 


\section{N DE X}

projection 213-14; arguments supporting 74-5, 81, 98, 346

VP-shell theory $7,343,344,366-7$, 384, 401

VPs: and agreement 163, 165, 166; object in spec VP 68-71; participial 181-6; and preposing 98, 99, 344, 346 ; relation of NP* to 67-75; structural Case, inheritance and agreement 173-81; structure of 95-102, 181-7; surface position of nominative and accusative NPs 168-73

VSO languages 51-2, 103, 394; see also Arabic; Irish; Welsh

Weak Crossover Effects 76, 289, 296, 297-8, 299

Welsh 51-2, 93, 173, 174; Case assignment 58-9, 61, 62-4; extraction out of subject 129,131 ; not subject to definiteness effect 180 ; subject in NP* 81

wh-Criterion 267, 273, 401, 410

$w b$-extraction $4,370,420-2$

$w h$-island condition 120-1

$w$-islands $88,282,420$

wh-movement $10,26,27,74,75,88$, 98-9, 117-21, 391; and A-bar position 76, 78; of APs 347; blocking 89, 90; in Complex Inversion 308; and degree comparison 350,351 ; and domain
399; in Dutch 122; in English questions 403-4; in French 128, $132,133,178$; the grammatical cases 117; in multiple questions 410-11; and participle agreement 81, 259, 276; and Q-stranding 263; subjacency and the CED 117-21; in VP small clauses 365

wh-phrases 267; in A-bar position 81; and agreement 82 ; in Case position 98 ; dative 290

$w h$-questions 12-13, 29, 268, 370, 401; diagnostic properties 411-12; English 402-4, 405, 407; French 406, 410-11; multiple 410-11

$w h$-traces $27,29,34$; as anaphors 88 ; in Case position 98

whetber 120, 405, 407, 409

will 48

X-bar theory 51-2, 68, 91-2, 104

$\mathrm{X}$-command 60, 104

$\mathrm{X} / \mathrm{XP}$ relations $391-2,397$

$\mathrm{XP} / \mathrm{XP}$ relations see $\mathrm{phrase} / \mathrm{ph}$ rase relations

XP*: and clitics 264-82, 299

XP^: and clitics 288-91, 294, 295, 299

Yiddish 79

Yoon, J. 407

Zribi-Hertz, A. 255-6 




